\title{
THE DEVELOPMENT OF A MUSIC THERAPY SCHOOL CONSULTATION PROTOCOL FOR STUDENTS WITH HIGH OR VERY HIGH SPECIAL EDUCATION NEEDS
}

\begin{abstract}
A Thesis Presented in Fulfilment of the Requirement for the Degree of Doctor of Philosophy in Music
\end{abstract}

New Zealand School of Music,

P.O. Box 2332, Wellington, New Zealand

\section{DAPHNE JOAN RICKSON}

2010 


\section{ABSTRACT}

Music therapy school consultation is positioned to become a significant practice for music therapists. Historically, music therapy work with children who have special education needs in New Zealand has focused on those who attend special schools or units and, according to the published literature, seems to have taken place in clinic settings or withdrawal rooms. The current emphasis on inclusive education demands that music therapists consider other ways of working. Further, a paucity of music therapists and the geographic isolation of many students who attend their local schools suggest that the large majority of students who would benefit are unable to access music therapy services. The aims of the current study therefore were for a music therapist to empower members of special education teams to use music experiences which had been especially planned to assist children to meet individual developmental or academic goals, and to describe how the process was perceived, understood, used, and valued by participants. A further aim was to develop and trial a protocol for music therapists undertaking consultation work.

Eight registered music therapists interviewed in stage one of the study, to aid the development of the initial protocol, had differing views and attitudes about consultation, and findings confirmed the need to clearly define the practice. The initial protocol was therefore fragile, based on limited understandings from sparse music therapy consultation literature and the author's previous experience of working with team members in isolated areas. In stage two, four consecutive case studies enabled the protocol to be trialled in the field and, using an action research approach, to be developed further. Accumulated learning outcomes led to the development of a music therapy school consultation protocol based on social learning theory which emphasises the interdependent relationships between the consultant's (music therapist), consultees' (identified team members), and clients' (students) behaviour, their internal personal factors, and environmental factors.

The establishment of collaborative relationships, and an ecological assessment which is based on the theory that human development is influenced by environmental systems (Bronfenbrenner, 1989), are critical components of the protocol. Thus the music therapist spends a full week at each student's school. Findings demonstrate that interacting with 
team members as they went about their daily lives led to deeper understanding of their needs and in turn enabled pragmatic, accessible, and meaningful music activities and strategies to be successfully implemented. A 'clinical' music therapy session remains an important part of the protocol, but findings suggest its primary significance is in highlighting students' strengths so that team members develop fresh understandings and increasingly positive views of students that enhance their mutual relationships. Team members became more motivated, energised, self reflective, and able to support as well as challenge their students' development. They were thus able to continue to use, develop and evaluate their use of music strategies, after the music therapist left the field.

Music therapists are currently unprepared for the triadic relationships and the emphasis on adult empowerment that is fundamental to consultation. The findings therefore have significant implications for music therapy practice and training. These implications, including areas for future research, are discussed herein. 


\section{ACKNOWLEDGEMENTS}

I wish to offer my sincere gratitude to:

My supervisors: Dr Alan Badley (Initial Primary Supervisor), Professor Donald Maurice (Subsequent Primary Supervisor), Professor Leslie Bunt, and Associate Professor Jill Bevan-Brown, who all willingly shared their expertise, inspired me with their passion for their particular subject areas and for research, and provided me with consistent support and encouragement ;

My colleagues and friends who have offered ongoing support especially Associate Professor Sarah Hoskyns who provided peer review, Dr Morva Croxson who has been my music therapy mentor, and Barbara Mabbett who provided editorial support;

My immediate and wider family especially Malcolm, Michael and Angela Rickson who loved me, kept me 'real' with their humour, and gave me the necessary time and space to do my work, and my nephews Tim and Colin and their families who taught me about bravery, commitment, and unconditional love;

All my friends who were willing to put up with my egocentricity or absence while I was completing this thesis;

The late Sir Roy McKenzie and the late Marion Rayward and their committees for the McKenzie Music Therapy Scholarship and Marion Rayward Memorial Trust Awards respectively, without which I would not have been able to complete this research;

The students, families, other educators and therapists I have worked with over the years who have taught me so much;

And most of all the participants in the current study - your courage and dedication have been inspiring. 


\section{TABLE OF CONTENTS}

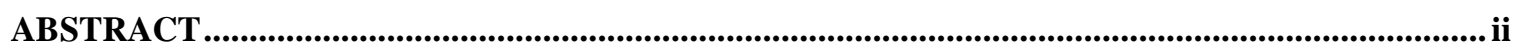

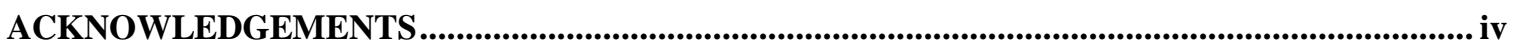

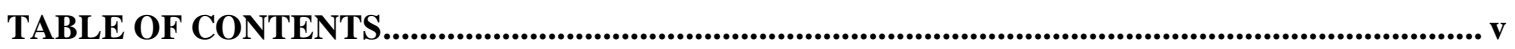

ALPHABETICAL LIST OF ACRONYMS ............................................................................................. viii

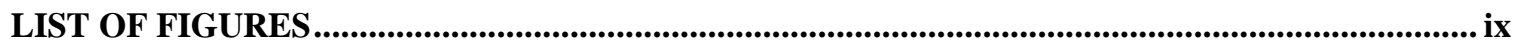

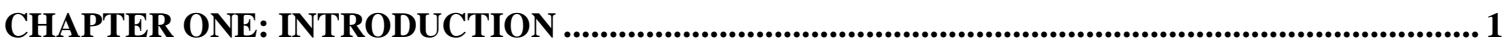

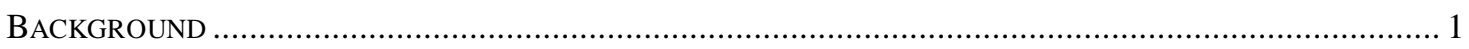

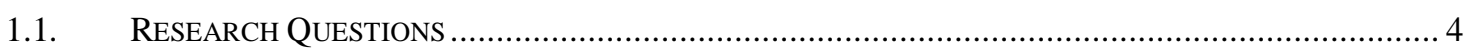

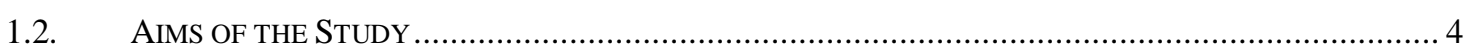

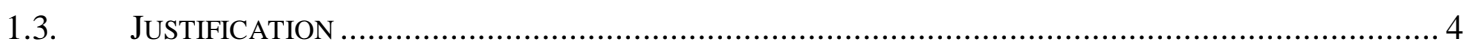

1.4. DEFINITIONS AND TERMINOLOGY USED IN THE THESIS ....................................................... 5

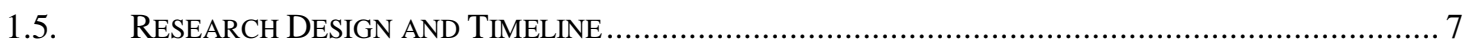

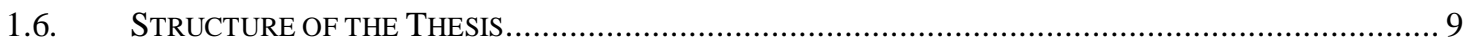

CHAPTER TWO: LITERATURE REVIEW ..................................................................................... 10

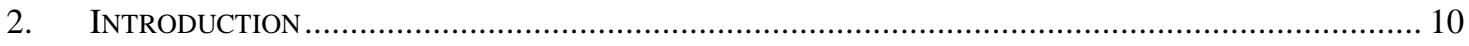

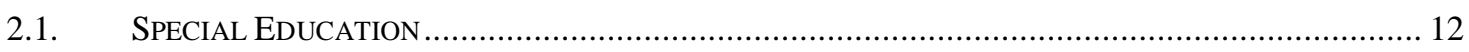

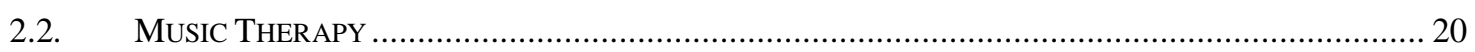

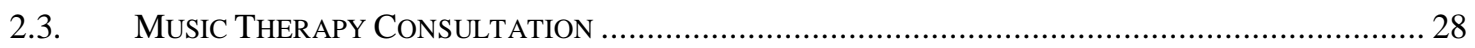

2.4. FurThER CONSIDERATIONS FOR MUSiC THERAPY SCHOOL CONSULTATION WORK .................. 40

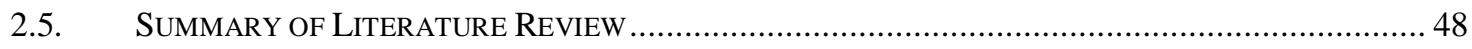

CHAPTER THREE: THEORETICAL PERSPECTIVES AND ETHICAL ISSUES ........................ 50

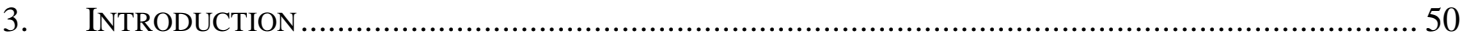

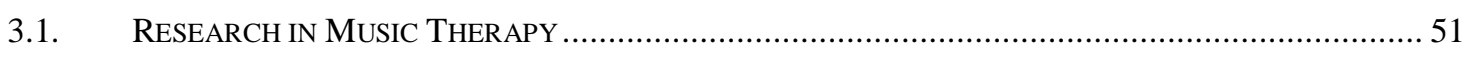

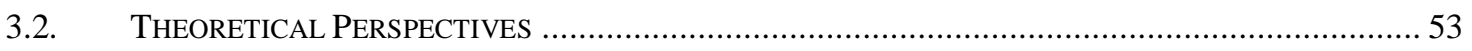

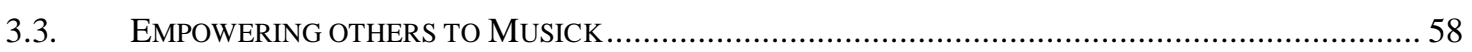

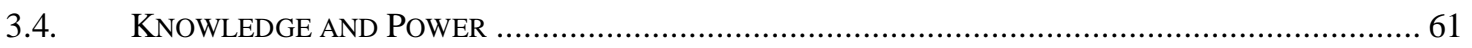

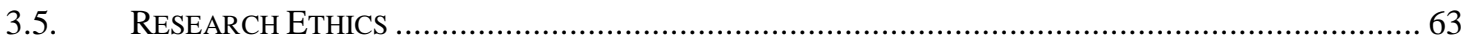

CHAPTER FOUR: METHODOLOGY AND METHODS ................................................................... 69

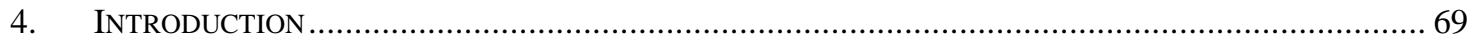

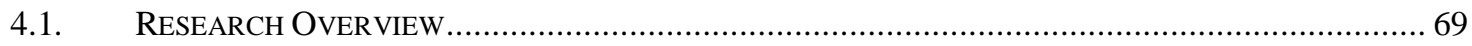

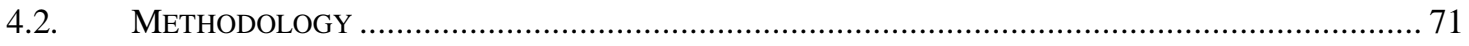




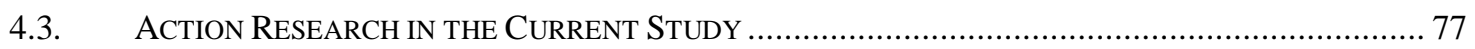

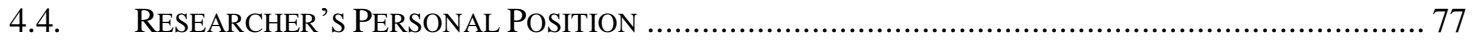

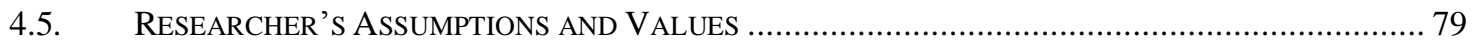

4.6. Choice of Methods and Data Collection TeChniques .................................................. 84

4.7. PROCEDURES FOR STAGE ONE - MuSIC THERAPIST INTERVIEWS …....................................... 88

4.8. PRocedURES FOR STAGE Two - CONSUlting WITH TEAMS .................................................. 90

4.9. Procedures for Stage Two - Developing a Consultation Protocol............................. 94

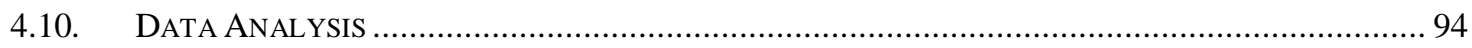

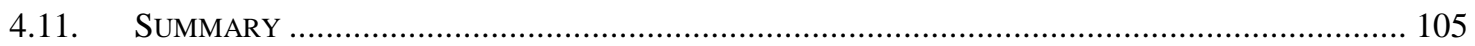

\section{CHAPTER FIVE: STAGE ONE. MUSIC THERAPISTS CONSIDER MUSIC THERAPY} ASSESSMENT AND CONSULTATION................................................................................................... 106

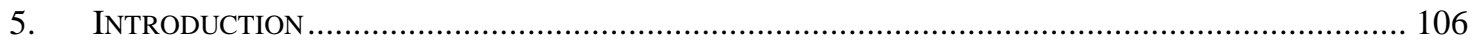

5.1. FINDINGS - THEMES FROM INTERVIEWS WITH MUSIC THERAPISTS ........................................ 107

5.2. EXPANSION ON THEMES FROM INTERVIEWS WITH MUSIC THERAPISTS ................................... 108

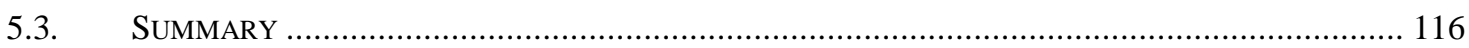

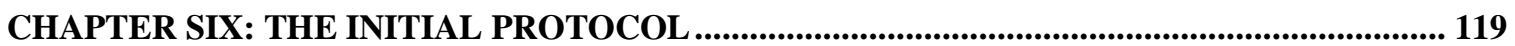

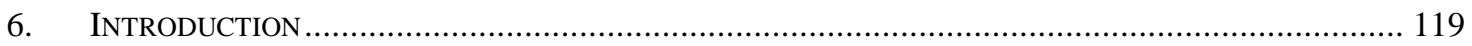

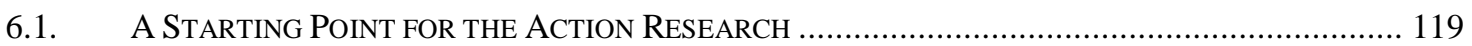

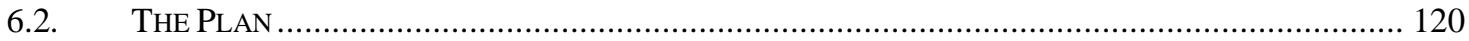

CHAPTER SEVEN: STAGE TWO. ACTION RESEARCH - CASE STUDIES .............................. 124

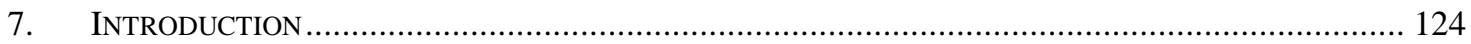

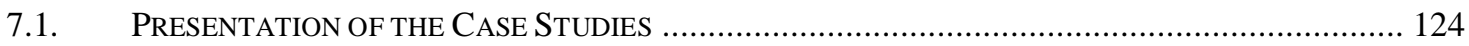

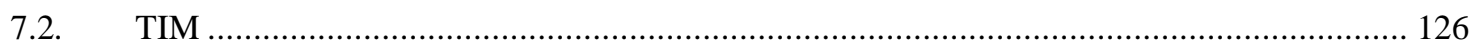

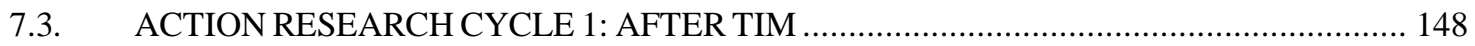

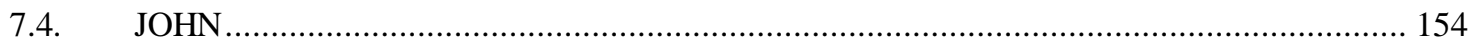

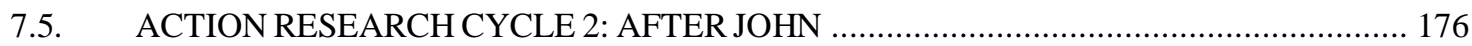

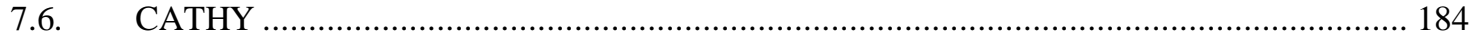

7.7. ACTION RESEARCH CYCLE THREE: AFTER CATHY ……..................................... 209

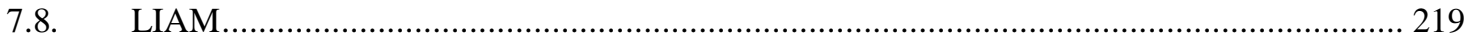

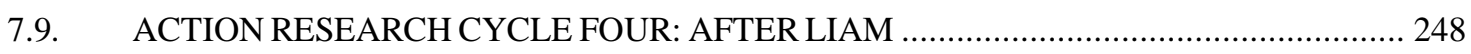

CHAPTER EIGHT: STAGE THREE. THE FINAL PROTOCOL .................................................. 252

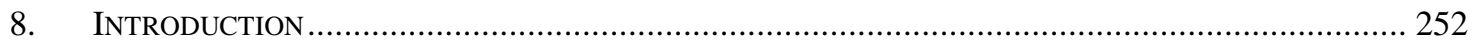

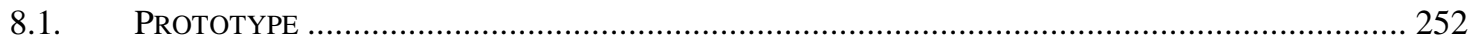

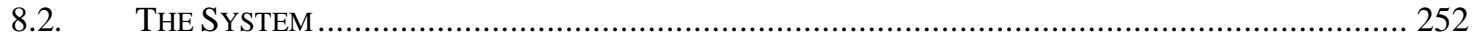

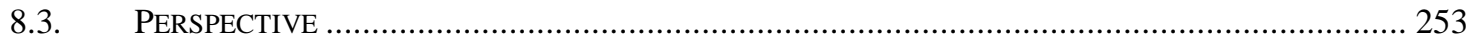


8.4. MODE

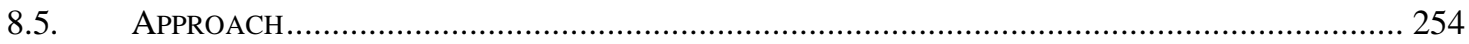

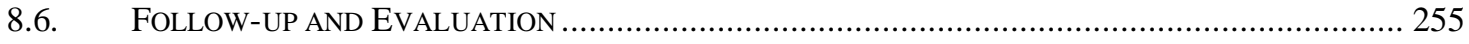

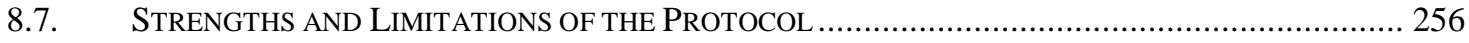

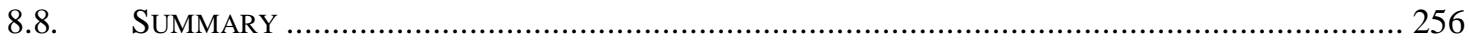

CHAPTER NINE: ANSWERS TO RESEARCH QUESTIONS ........................................................ 258

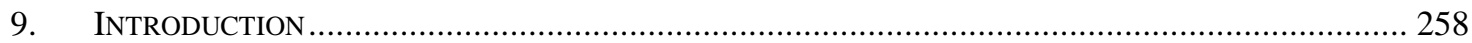

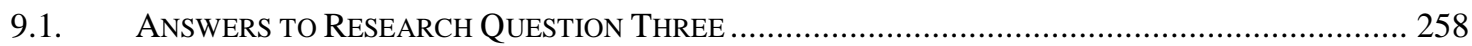

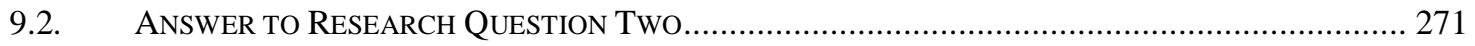

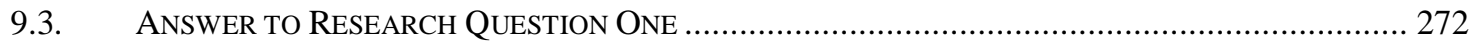

CHAPTER TEN: DISCUSSION AND CONCLUSION ..................................................................... 273

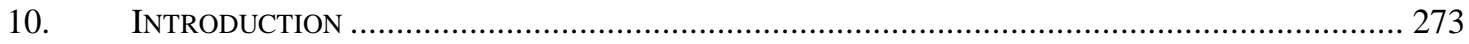

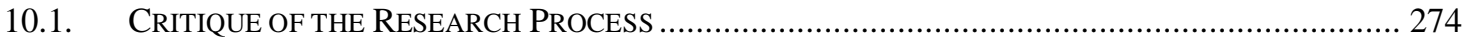

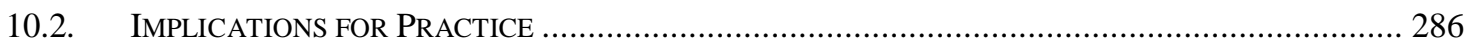

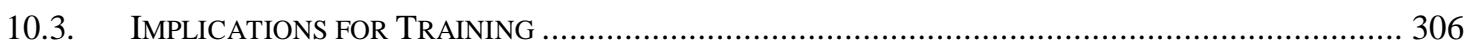

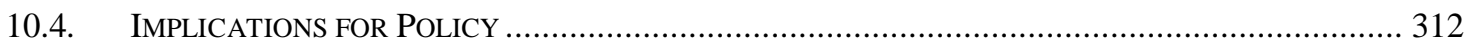

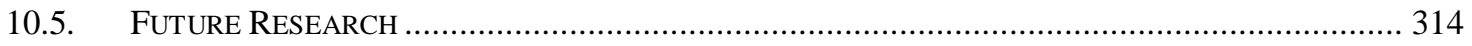

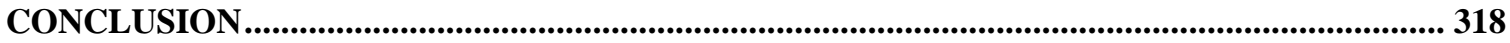

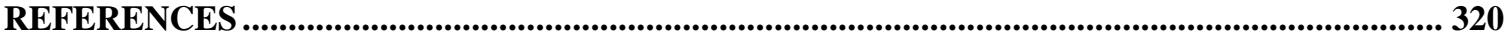

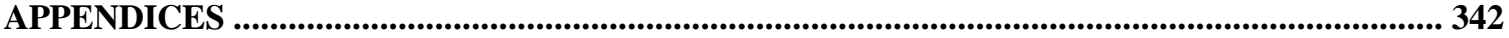




\section{ALPHABETICAL LIST OF ACRONYMS}

AR Action Research

ASD Autism Spectrum Disorder

CoMT Community Music Therapy

IAPs Improvisational Assessment Profiles

IDTM Identified Team Member (in info/consent forms, later referred to as 'consultee')

IEP Individual Education Programme

LO Learning Outcomes

MAP Music therapy Analyzing Partitura (Gilboa, 2007)

MLE Mediated Learning Experiences (Feuersteing \& Feuerstein, 1991)

MMusTher Master of Music Therapy

MoE Ministry of Education

MoE SE Ministry of Education Special Education

NZMTs New Zealand Music Therapists

NZSM New Zealand School of Music

NZSMT New Zealand Society for Music Therapy

ORRS Ongoing and Reviewable Resource Scheme

PAR Participatory Action Research

PECS Picture Exchange Communication System (Frost \& Bondy, 2002)

PMLD Profound and Multiple Learning Difficulties

PMP Perceptual Motor Programme

RMTher Registered Music Therapist

SCERTS Social Communication, Emotional Regulation, and Transactional Support

(Prizant, Wetherby, Rubin, Laurent, \& Rydell, 2006)

SE Special Education

SE2000 Special Education 2000

SEA Special Education Advisor

SEMTAP Special Education Music Therapy Assessment Process (Brunk \& Coleman, 2000)

SENCO Special Education Needs Coordinator

SES Special Education Service

SLD Severe Learning Difficulties

SLT Speech and Language Therapist

TEACCH Treatment and Education of Autistic and related Communicationhandicapped Children

TM Team Member 


\section{LIST OF FIGURES}

Figure 1: Research Design and Timeline ............................................................... 8

Figure 2: Elements of the Research Process (Adapted from Crotty, 1998, p. 4) .............51

Figure 3: Action Research Cycles ........................................................................... 70

Figure 4: Local Action Research Projects (with consultation stages in colour) ..............91

Figure 5: Procedure for Synthesis of Data from Each Case Study ............................... 104

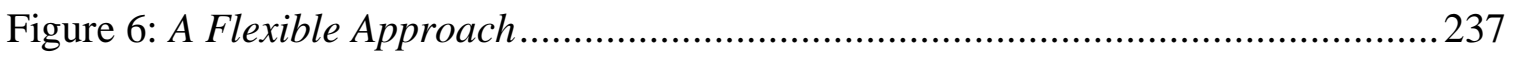

Figure 7: Multiple Factors in Social Learning Theory Consultation............................253

\section{LIST OF TABLES}

Table 1: An Initial Music Therapy Assessment and Consultation Protocol ................... 123

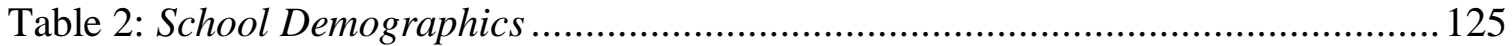

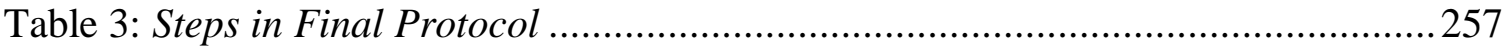




\section{LIST OF APPENDICES}

APPENDIX 1: Letter to New Zealand Society for Music Therapy ...................................342

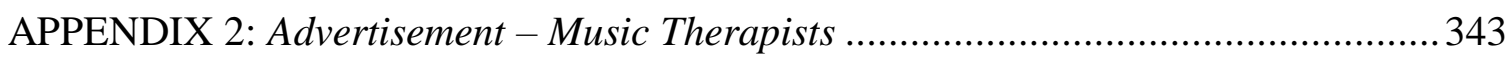

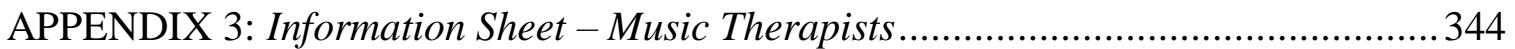

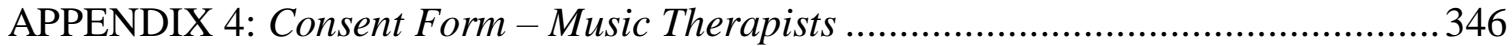

APPENDIX 5: Interview Guidelines - Music Therapists ............................................. 347

APPENDIX 6: Transcript Information Sheet - Renegotiating with Music Therapists ..348

APPENDIX 7: Transcript Consent Form - Music Therapists ........................................349

APPENDIX 8: Advertisement - Special Education Team Members ..............................350

APPENDIX 9: Expression of Interest: Special Education Team Members ......................351

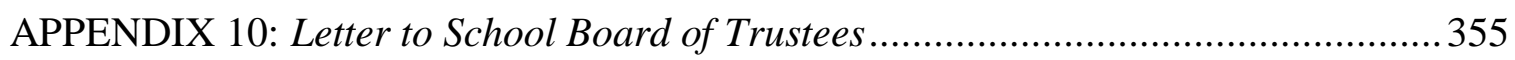

APPENDIX 11: Consent Form - School Board of Trustees ............................................ 357

APPENDIX 12: Information Sheet - Identified Team Member ...................................... 358

APPENDIX 13: Consent Form - Identified Team Member ...........................................360

APPENDIX 14: Information Sheet - General Team Member......................................... 361

APPENDIX 15: Consent Form - General Team Member .............................................363

APPENDIX 16: Information Sheet - Parents/Caregivers ..............................................364

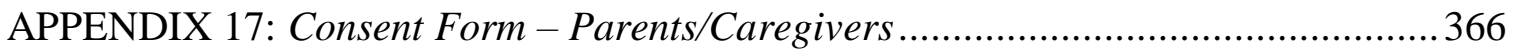

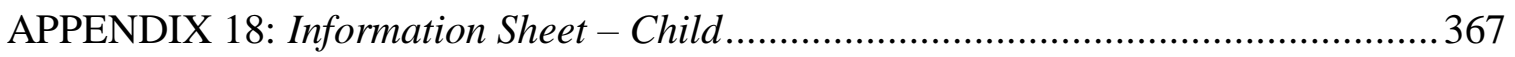

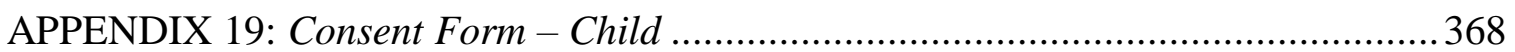

APPENDIX 20: Interview Guidelines - Identified Team Member ..................................369

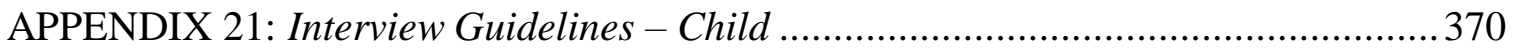

APPENDIX 22: Authority for the Release of Tape Transcripts .....................................371

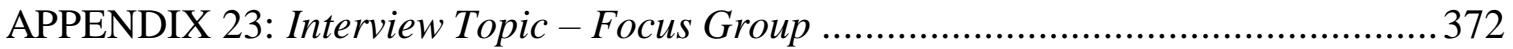

APPENDIX 24: Interview Transcript, Petra ………................................................. 373

APPENDIX 25: Preliminary Definitions of Categories ................................................. 391

APPENDIX 26: Category - What can the data tell me about helping others to use music...

APPENDIX 27: Interview with Petra, from code to category......................................... 404

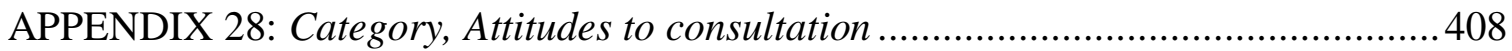

APPENDIX 29: Examples from Music Diary Entry Provided to Participants ............... 414

APPENDIX 30: Stage Two. Data sources and reasons for collection ..........................415

APPENDIX 31: Field Log. Tim's Case................................................................... 417

APPENDIX 32 : Thoughts from reflective journal after case study one ......................... 443

APPENDIX 33: Themes from second interview with Angela .........................................44

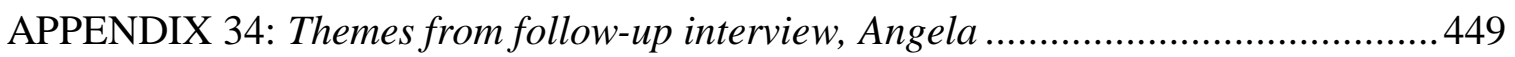

APPENDIX 35: 'Angela' [Tim's Case] example of category development ....................459 
APPENDIX 36: Example of Focus Group Interaction - Tim's Case ............................. 460

APPENDIX 37: Analysis of Tim's Music Therapy Assessment Session - Timeline ....... 467

APPENDIX 38: Indicators of Efficiency, John's Case .............................................. 488

APPENDIX 39: Reflections and Learning Outcomes from John's Case ....................... 490

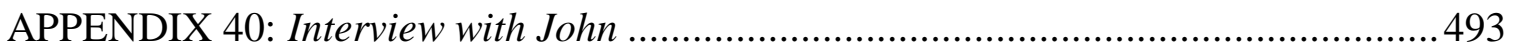

APPENDIX 41: First five pages of participant diary (Tim's case) ............................... 495

APPENDIX 42: Findings, Themes from All Four Case Studies...................................503 


\section{CHAPTER ONE: INTRODUCTION}

One of the best examples of working together is a musical ensemble... it is a united effort that creates the musical experience. Musicians of many instruments are not brought together to play the same note. Doing so would only make the music louder and not richer and more harmonious. (Collaborators) work in concert, not usually in perfect unison, to create an effective learning experience for students (Dettmer, Thurston, Knackendoffel, \& Dyck, 2005, p. 14).

\section{Background}

Music therapy work with students who have special education needs in New Zealand has historically focused primarily on students who have 'high' or 'very high' needs according to the Ministry of Education's Ongoing Reviewable Resource Scheme (ORRS). The primary reason for this focus is likely to have been that Registered Music Therapists (RMTher) are listed as specialists who can provide a service to ORRS funded students, thus creating opportunities for fund-holding schools to directly employ their own therapists. Music therapy has predominantly taken place in special schools or units, in 'clinic' settings or withdrawal rooms, with individuals or small groups of children.

However when this study began, more than two-thirds of students with high or very high special education needs were attending regular schools and being taught within a general classroom environment (Ministry of Education, 2004a). The withdrawal (or 'clinical') model of music therapy delivery needed to be challenged in the light of an inclusive education system which aims to support students within regular school and classroom environments. Further, in many cases where clinical music therapy programmes might be helpful for students, their geographical location prohibits regular therapy visits.

Further, there is still a paucity of registered music therapists in New Zealand - seventeen when this study began in 2004, and fifty in June 2009 - and many music therapists work with client populations other than children. This means that most of the children who would benefit from music therapy are unlikely to have access to regular services in the 
foreseeable future, especially if the clinical model of music therapy continues to prevail.

Moreover, following the introduction of the first tertiary music therapy training course in New Zealand in 2003 (the Master of Music Therapy (MMusTher) programme at Massey University, now based at the New Zealand School of Music), and the opening of New Zealand's first dedicated music therapy centre in Auckland in 2004, the positive profile of music therapy and the demand for music therapy service in this country increased. Music therapists are receiving rising numbers of requests for assistance from families who are unable to attend regular music therapy clinics, and education teams who want to know more about how music can be used to help students, especially those who have high and very high special education needs. Many of these people are already using music as a curriculum activity, to support students' developmental needs, and as a carrier of academic information. Music therapists are in a unique position to work with them to enable them to use music more effectively and extensively. Nevertheless, references to music therapy consultation are sparse.

On the other hand, workshops and 'one-off' music therapy consultations undertaken by the author in the past have resulted in unsolicited anecdotal evidence suggesting the work was valuable for motivating team members to successfully introduce music into students' programmes, and ultimately to help students to achieve developmental goals. However, this 'consultation' work was exploratory and no formal protocol had been developed; follow-up was limited to informal inquiry regarding progress and further support needs; and although various team members had been involved, it was not based on full collaboration with team members. The aims of this study were to find out, by capturing their experience of the process, whether music therapy consultation can empower participants to use and/or develop their intentional use of music and, in addition, to use those findings to improve practice.

It seemed important if the potential of music therapy consultation work was to be maximised, to examine the practice, find ways of improving it, and to communicate those findings in a way that would be useful not only to the author, but also to other music therapists. While findings from qualitative research cannot be easily generalised, they can be utilised more widely if music therapists can connect with at least some of the findings 
and use that resonance to examine their own music therapy practice. Further values incorporated into this research were the desire to uncover and build on a shared perspective, to incorporate the voices of those who participate in music therapy consultations, to gain an understanding of the culture in which each of those teams work, to highlight their social situations and values, and to base interventions on agreed understandings. Action Research provided the mechanism for this exploration (see Chapters Three and Four). The researcher, who is also a registered music therapist, consulted with team members to support their therapeutic use of music and, using knowledge gained from that experience, developed a music therapy school consultation protocol. 


\subsection{Research Questions}

1. Can music therapists, through a process of consultation, empower team members to use music therapy strategies in a sustained way in their work with students who have special education (SE) needs?

2. How do team members who are directly involved, perceive, understand, use, and value the consultation process?

3. What indicators of efficiency or learning outcomes can be identified through a process of action research to help others who engage in music therapy consultation with special education teams?

\subsection{Aims of the Study}

1. To help members of New Zealand special education teams to use music experiences which have been especially planned to assist children to meet developmental and/or academic goals.

2. To describe how the process was perceived, understood, valued, and used by participants.

3. To develop and trial a protocol for music therapists undertaking consultation work, and to produce findings that can be used to guide future practice.

\subsection{Justification}

The increasing emphasis on inclusive education, the geographic isolation of many students who have special education needs and the paucity of music therapists in New Zealand, strongly suggests that a consultative model of music therapy service needs to be developed. Further, the relatively new concept of 'Community Music Therapy' (CoMT) recognises the importance of acknowledging the social and cultural factors that influence music therapy work. This study will provide an important contribution to the CoMT literature base as research in this area is still in its infancy (Stige, 2005b). 


\subsection{Definitions and Terminology used in the Thesis}

\subsubsection{Action Research Cycles}

Following each consultation, I reflected on, evaluated, and planned the development of a music therapy consultation protocol. ${ }^{1}$

\subsubsection{Consultation}

My understanding of consultation shifted as I engaged with participants, and read about the consultation work of other professionals. I derived my initial working definition from the Oxford Dictionary, which defines 'consult' as "seek information or advice" (Soanes, Spooner, \& Hawker, 2001), whereas the second was borrowed from the human services and psychological consultation literature.

1. Consultation is the sharing of information and/or advice

2. "Consultation within the human service professions represents an indirect model of delivering educational and mental health services by which a professional with specialised expertise (i.e. consultant) and a staff member (i.e. consultee) work together to optimise the function of a client in the staff member's setting" (Erchul \& Sheridan, 2008, p. 3).

\subsubsection{Culture}

"Culture is the accumulation of customs and technologies enabling and regulating human coexistence" (Stige, 2002)

\subsection{4. 'Indicators of Efficiency' and 'Learning Outcomes'}

'Indicators of Efficiency' and 'Learning Outcomes' were drawn from each case study to answer research questions. 'Indicators of Efficiency' identify the positive aspects of the

\footnotetext{
${ }^{1}$ First person language has been used in various sections of this thesis to reflect the intimate involvement of the researcher in the research process.
} 
consultation, describing actions and outcomes that were valued by team members. 'Learning Outcomes' describe further issues uncovered during this study which are relevant and need to be considered in music therapy consultation.

\subsubsection{Music Therapy}

Music Therapy is the use of music and/or its musical elements (sound, rhythm, melody and harmony) by a qualified music therapist, with a client or group, in a process designed to facilitate and promote communication, relationships, learning, mobilization, expression, organization and other relevant therapeutic objectives in order to meet physical, emotional, mental, social and cognitive needs (World Federation of Music Therapy, 1996).

\subsubsection{Music Therapy Protocol}

The protocol describes the 'stages' or 'steps' involved in music therapy consultation. Although the word 'protocol' has been used by some writers to describe a set of 'rules', in the context of this study the development of a 'protocol' is not meant to imply that music therapists will adopt an inflexible approach. Rather, the intention is to produce a framework that provides guidance and enables a certain degree of replication to take place, yet is broad enough to encourage team members to collaborate and to adopt a unique approach that meets their needs.

\subsubsection{Music Therapy Strategies}

Strategies are "used by the therapist to engage the client in a specific aspect of the therapeutic process; or to accompany a specific methodological objective” (K.E. Bruscia, 1987 , p. 16)

\subsubsection{Music Therapy Techniques}

"An operation or interaction initiated by the therapist to elicit an immediate response from the client, or to shape his/her immediate experience" (K.E. Bruscia, 1987, p. 18). 


\subsubsection{Students who Have Special Education Needs}

The philosophy of inclusive education discourages the use of the term 'special' or 'special needs' which is associated with the devaluing and excluding of disabled students (Ainscow, 2000, in Kearney, 2008). Nevertheless, students who participated in this study are deemed to have 'special education needs' according to current government guidelines, and I have therefore chosen to use the term throughout this thesis.

\subsubsection{Team Member}

Team member refers to all those who provide direct or indirect service to particular students, including their parents and other caregivers, principals, classroom teachers, teachers' aides, special needs or resource teachers, therapists, psychologists, and so on. It is important to note that parents and caregivers are considered to be essential and central members of the team and there will be no reference to them 'alongside' the team in this thesis. Similarly, the student is considered to be a member of the team. However, the nature of consultation is such that the focus of the discussion is often on the adults (the consultees) so for ease of reading 'the team' or 'team members' will usually refer to the

adults. The information and consent forms refer to Multidisciplinary Team Member/s (MDT). The MDT has been described as one in which different agencies work alongside each other and do not interact (Twyford \& Watson, 2008b). Individual practitioners from a range of agencies were involved in this study and collaborated in various ways in each of the consultations. The term MDT has therefore been deleted from this thesis.

\subsection{Research Design and Timeline}

Figure 1 (p. 8) provides an overview of research procedures. Full details are provided in Chapters Three and Four. 


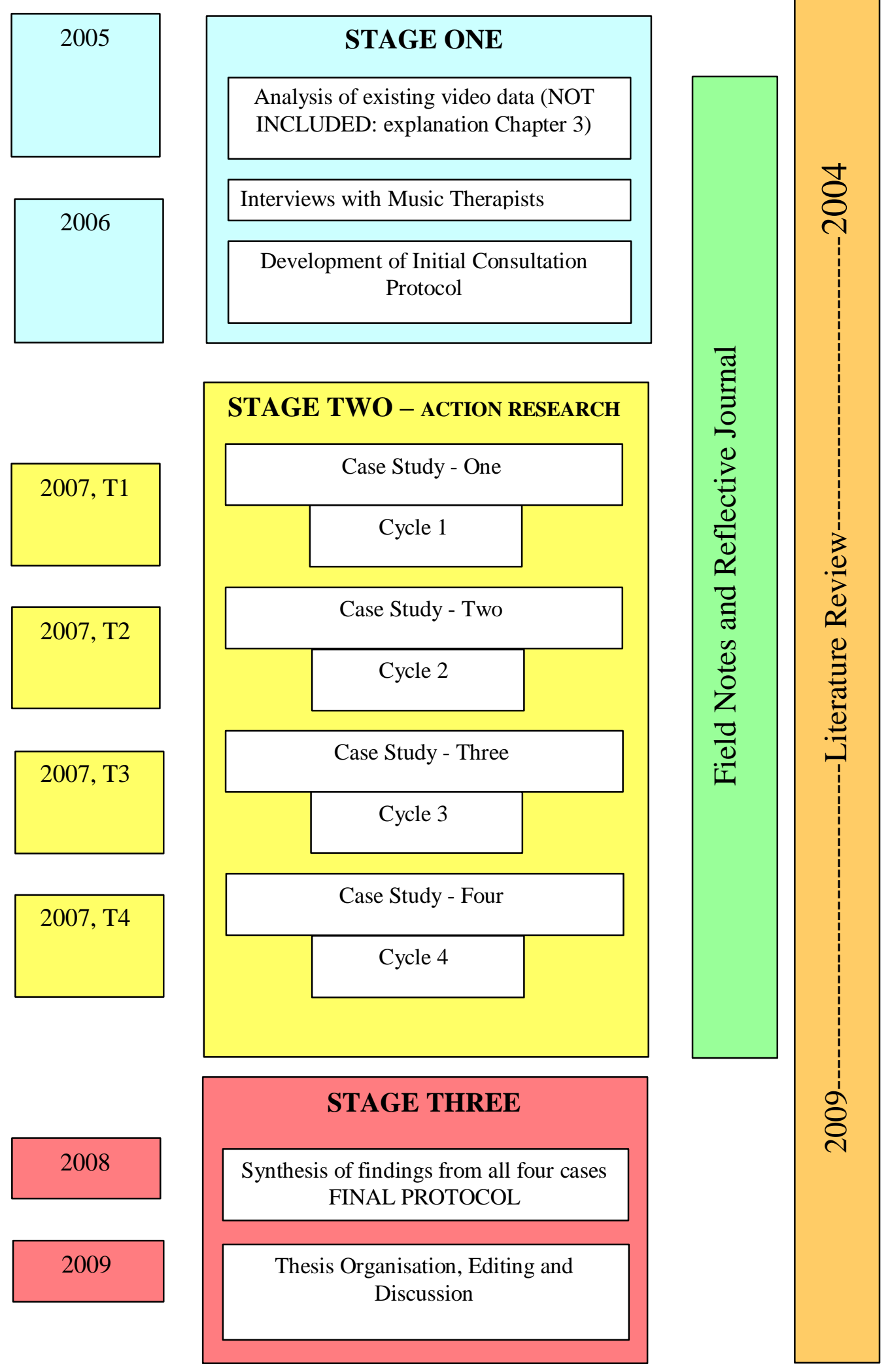

Figure 1: Research Design and Timeline 


\subsection{Structure of the Thesis}

A review of the literature is presented in Chapter Two. Chapter Three contains theoretical perspectives and ethical issues, while specific methodology, methods and procedures are outlined in Chapter Four. Findings from interviews with music therapists undertaken during Stage One of the study are presented in Chapter Five. These findings contributed to the development of the initial protocol. Chapter Five therefore concludes with a brief summary of interview findings, and further reflections are included in the initial protocol in Chapter Six. (A full discussion of interview and other findings is offered in Chapter Ten). Chapter Six outlines the initial music therapy consultation protocol.

Case studies (consultations) undertaken during Stage Two are presented in Chapter Seven. Each of the four cases is followed by an action research cycle. The action research cycles focus on the developing protocol which is presented in final form in Chapter Eight, p. 252. Answers to the research questions are presented in Chapter Nine in the form of themes. Findings are discussed in Chapter Ten. The discussion includes implications for practice, training, and policy, and recommendations for future research.

Several publications have already been generated from this work. Naturally it has been necessary to retain sections of published work in the thesis to provide context. Where this occurred, the text is appropriately referenced. 


\section{CHAPTER TWO: LITERATURE REVIEW}

\section{Introduction}

Literature was sourced from books, hand searches of music therapy journals, and electronic databases. Search platforms Web of Science, Web of Knowledge, and EBSCOhost were used and articles were sourced from Current Contents Connect, Journals@Ovid, MEDLINE, PsycINFO, Academic Search Elite, ERIC, JSTOR and ISI Proceedings. The initial literature search focused primarily on action research, special education policy and practice in New Zealand, and the music therapy research particularly as it related to consultation and assessment with children. However, while survey studies suggest that music therapists are engaged in consultation, many authors give the topic no more than a passing reference. It is rare to find descriptions of a music therapy consultation process although some seminal articles do exist. Thus several older references are included in this thesis to provide historical context.

In action research initial ideas are viewed as a starting point only (Elliott, 1991; McNiff, 1988). Music therapists have strong links with art, drama, and dance therapists, and allied health professionals such as speech and language, occupational and physio therapists as well as psychologists, social workers, and so on. Initially therefore I resisted too much engagement with literature from other fields believing that it could potentially reduce the possibility that I might develop a unique process for music therapy consultation in schools. Instead, I began the process of developing a consultation protocol for music therapists with an open mind, valuing the opportunity I had to draw on practice-based knowledge. Like Warner (2005) I believed that "reading as the process developed, and afterwards in reflection, was a more useful practice" (p. 36).

Therefore, in the interests of clearly demonstrating the action research learning process, I have introduced some of the literature in the action research cycles rather than in Chapter Two. By taking a more chronological approach I have been able to demonstrate the way my reading and reflection aided my learning, and to allow the thesis to flow more easily. For example, several new texts were published during the study period, including 
Dougherty (2005) who, after noticing work from Pellitteri (2000) and Register (2002), mentioned music therapists in his list of mental health professionals for whom consultation is or will be a 'cornerstone activity'. This connection, and my work in the field in 2007, led me to explore 'psychological school consultation', which predominantly describes the work of school psychologists, in more depth. Central themes from this body of literature linked significantly with music therapy consultation and have been referenced in the cycles of learning.

This chapter is divided into four main sections: Special Education, Music Therapy, Music Therapy Consultation, and Further Considerations for Music Therapy School Consultation.

The literature review begins with a brief summary of disability issues, particularly as they relate to education, and an historical overview of New Zealand special education service delivery. A discussion regarding the work of therapists from other disciplines highlights the need to carefully examine the process of moving from direct to indirect models of service delivery.

Section two begins with a brief history of the development of music therapy and highlights music therapists' increasing interest in Community Music Therapy, i.e. working in and with communities. This leads to a review of music therapy in special education which highlights the more traditional clinical model of practice, and music therapy assessment of children.

Section three considers music therapy consultation and begins by differentiating the terms collaboration and consultation. Then the practice of school 'consultation' in New Zealand and overseas is examined. The literature on music therapy school consultation with regular classroom educators is sparse, so examples from music education and early childhood settings have also been included in this section.

The final section begins with 'cultural considerations' to acknowledge the centrality of context and culture in consultation work. Finally, attention is turned to the potential for team members to use music and to be available for a music therapy consultation. 


\subsection{Special Education}

\subsubsection{Disability Issues and Inclusive Education}

Over the years, the education of students who have different learning needs has naturally been affected by various understandings of disability. These understandings have grown from and are reinforced by historical discourse. For example, the medical model views disability as a health problem or physical deficit, a problem residing in the person, which needs to be fixed (Ho, 2004; McArthur, 2009; Neilson, 2005; O'Brien \& Ryba, 2005). Within the medical model, professionals and other 'experts' have talked about children who have disabilities with great authority suggesting that 'highly specialised treatment' would be necessary to alleviate their problems.

In contrast, the social model of disability which emerged during the 1960s acknowledges that people with disabilities can be self-reliant, independent, and should have consumer rights (McArthur, 2009; Neilson, 2005). This includes the right of children who are disabled to receive the same education as those who are not disabled. These rights were confirmed in law in the United States in 1975 with the adoption of the Education for All Handicapped Children's Act, and, following strong parent advocacy to follow their lead (O'Brien \& Ryba, 2005), in New Zealand by the 1989 Education Act.

The United Nations Convention on the Rights of the Child (UNCRC) (UNESCO, 1989) stated that all children have the right to non-discriminatory education. The New Zealand Human Rights Act (New Zealand Government, 1993) reinforced the 1989 Education Act making it illegal for schools to refuse student enrolment or to treat students who are disabled less positively. The New Zealand Disability Strategy also looks to an inclusive society which eliminates the barriers to living that disabled people experience and advocates for equal opportunities for all children to learn and develop in their local and regular schools (Ministry of Health, 2001). Various declarations from the United Nations have outlined the rights of children to participate fully in education settings of their choice leading to the current UNESCO Policy Guidelines on Inclusion in Education (2009). 
Nevertheless, in education circles there remains a persistent assumption that students' special learning needs are related to their pathology (McArthur, 2009; O'Brien \& Ryba, 2005). Hall (in O'Brien \& Ryba, 2005) argues that this discourse has legitimised the special education system and led it to be what he describes as a self-perpetuating professional industry. Moreover, when regular teachers lack knowledge, skill and resources to work with students who have disabilities they react by moving the child into 'expert' hands (O'Brien \& Ryba, 2005). Economic, financial, social, and psychological barriers to inclusion exist, and the 'rights' approach insists that these barriers be removed (ibid).

Promoting inclusion (therefore) means stimulating discussion, encouraging positive attitudes, improving educational and social frameworks to cope with new demands in education structures and governance. It involves improving inputs, processes and environments to foster learning both at the level of the learner in his/her learning environment and at the system level to support the entire learning experience (UNESCO, 2009, p. 7).

\subsubsection{Special Education in New Zealand}

In 1996 the New Zealand Government introduced Special Education 2000 (SE2000) which aimed to provide the resources necessary to meet the specific physical, intellectual, sensory, social and emotional needs of students to enable them to maximise education opportunities, with the aim of achieving "a world class inclusive education system over the next decade" (Ministry of Education, 1996, p. 2). There is a range of resourcing components to the SE2000 policy.

One of these is the Ongoing and Reviewable Resourcing Scheme (ORRS) which aims to provide specialist programmes, equipment and materials, adapted learning environments, and therapy and extra teaching for individual students. Under this scheme students are verified as having 'high' or 'very high' levels of need because they are likely to require ongoing support and intervention, significant adaptation of curriculum content, assistance with face to face communication, and/or supervision with activities of daily living and support with mobility throughout their school years (ibid). Students who 
qualify for ORRS funds are required to have an Individual Education Programme (IEP) which lists the strategies necessary to help them to reach their potential (Ministry of Education, 2004b).

Initially, where schools or clusters of schools had a significant number of students with high or very high needs they could apply for bulk funding to resource their students, whereas the responsibility to resource students at other schools would remain with the Special Education Service (SES), an independent Crown agency. Government driven research to evaluate the impact of special education reforms (Wylie, 2000) highlighted parent and school staff frustration regarding resource allocation and an associated view that inclusion placed increased burden on schools. The findings from Wylie's research contributed to the disestablishment of SES and responsibility for special education was returned to the Ministry of Education.

Registered Music Therapists in New Zealand are recognised as specialists who can provide a service to ORRS funded children (Ministry of Education, 2004b). To date, most music therapy work has taken place within fund-holding special schools and units using ORRS funding. In contrast, the current research focuses on ORRS funded students who have high or very high needs who do not attend special education facilities. Students who attend regular schools are usually resourced by Ministry of Education Special Education (MoE SE) visiting staff. A few music therapists have been employed by MoE SE and have begun to find ways of working within this system but for this to continue to grow it will be crucial to have music therapy research which draws on the philosophy of inclusive education and is conducted in regular education settings.

Also as part of SE2000 reforms, in 1999, 'Resource Teacher: Learning and Behaviour' (RTLB) positions were established "to assist schools and teachers to achieve the best learning outcomes for their students who have moderate special education needs" (Ministry of Education, 2006a, p. 5). The role of RTLB was considered to be a challenging one, requiring a high degree of professional expertise, knowledge and experience (Berliner, 1988, in D. Brown et al., 2000). Brown et al. noted that preparing RTLB to work within an ecological paradigm and to promote effective learning environments would involve a focus on inclusive teaching philosophy, an 
educational/ecological approach to assessment and intervention, and a collaborative consultative model of problem solving. However, the ministry did not clearly outline collaborative problem solving as the service delivery model to be used until 2007 (Ministry of Education, 2007).

More recently, the RTLB collaborative consultation model has been found to be feasible for the New Zealand context and effective in bringing about improved academic and social outcomes for students (Thomson, 2008). Thomson found that RTLB were committed and enthusiastic about collaborative consultation and that teachers reported high levels of satisfaction and the development of new skills and strategies which they were able to incorporate into their teaching repertoires. Thomson also suggested that relationships formed between the RTLB and the teachers are critical to the success of the model (ibid).

The Ministry of Education is currently conducting a review of special education services (Ministry of Education, 2009b). Additional funding has been promised for ORRS funded students and the ministry aims to ensure best use is made of this extra funding. However, the review will primarily focus on ensuring that services provide value for money and that best outcomes are achieved with existing resources. The principles that currently guide special education in New Zealand acknowledge that all students have the right to the same education and all have individual learning and developmental needs (Ministry of Education, 2009c). The stated aims in the review guidelines are to ensure that children reach their potential through the fair and equitable provision of resources, the provision of high quality professional services, the development of a confident and capable workforce who support parents and teachers, and the coordination of agencies who can work effectively and collaboratively to achieve best outcomes. The importance of meeting the multi-cultural needs and interests of students and their families, the rights for family and whanau to have choices and be actively involved in their child's education, and for disabled students to attend their local school, are also reinforced.

In line with the social model of disability, SE2000 calls for educators to focus on students' abilities, and on ways in which social and cultural systems as well as the physical environment can be improved to accommodate difference, and to facilitate 
participation and learning (Armstrong \& Moore, 2004; McMenamin et al., 2004; Ministry of Education, 2006b, 2009c). Inclusion has been shown to provide positive gains for students, teachers and society in general (McArthur, 2009; McMenamin et al., 2004). Increasing numbers of students who have high or very high special education needs are choosing to be educated in regular schools in New Zealand. About 7000 students receive ORRS assistance at any one time (Ministry of Education, 2009a) and in $200470 \%$ of the 6884 eligible students were educated in 'mainstream' settings (Ministry of Education, 2004a).

Students with special education needs attend mainstream classrooms and early childhood centres, kohanga reo, kura kaupapa Māori, attached classrooms or units, residential special schools, hospital schools, home schooling, and the Correspondence School. However, they continue to be excluded from and within school in a number of ways (Kearney, 2008). Inclusive practice goes far beyond writing policies and locating students with special education needs in regular classrooms (Mentis, Quinn \& Ryba, 2005) and the main focus needs to be on values rather than on specific 'inclusive' practices (McArthur, 2009). It involves increasing the capacity of schools so teachers can respond to student diversity (McArthur, 2009) and a move away from individually allocated resources to the provision of school-based resources (O'Brien \& Ryba, 2005). Teachers will need professional development to aid their understanding of ecological practice and specialist staff will be required to work alongside educators within schools to support individual and system level developments (ibid). School diversity will lead to schools working towards inclusion in different ways (McArthur, 2009), but in all cases collaborative problem solving will be important (McArthur, 2009; O'Brien \& Ryba, 2005).

Withdrawing students for therapy encourages the view that "(children) are problems to be fixed by experts" (McArthur, 2009, p.17). Further, O'Brien and Ryba (2005) point out that the very act of labelling students as having 'special needs', in order to provide them with the extra support they need to break down those barriers, has remnants of medical discourse. These authors note that SE2000 “...exemplifies a tension between the individual or biological model and that of an ecological or social model... ...the use of classification for ORRS-funded students reinforces the pathology of difference" (p. 35). 
In the meantime, the ministry's position is that when specialist intervention is required the least intrusive option should be chosen. It should take account of the physical environment, students' interactions with significant others, and lead to increasing independence (Ministry of Education, 2009a). Thus students with special education needs are continuing to receive clinical music therapy in various, but predominantly special education, settings. In regular schools however, it can be difficult to provide clinical music therapy to individual students because therapeutic space and appropriate equipment is rarely available. Further, the cost of providing direct therapy to children in regular schools is likely be higher than that in special units or schools because of additional time and travel related expenses. Whether and/or how students who attend regular schools will have access to clinical music therapy in future is an extremely important issue for music therapists but is beyond the scope of this thesis.

On the other hand, music therapy consultation is in line with inclusive philosophy as it aims to increase teachers' capacity to support students with diverse learning needs. Professional development that is perceived to be practical and specifically related to classroom practice can boost staff's confidence and enjoyment of working with students who have special education needs (Kearney \& Poskitt, 2001, in O'Brien \& Ryba, 2005).

\subsubsection{Therapy in Schools}

The concept of inclusive education has affected the development and delivery of a range of therapy services worldwide. Collaboration and consultation are increasing as practice moves beyond the withdrawal-for-therapy model to an ecological approach where therapy is integrated into naturally occurring school tasks and routines (Carlsson, Hocking, \& Wright-St Clair, 2007; Dettmer et al., 2005; Dougherty, 2005; Gascoigne, 2006; Hanft \& Place, 1996; Hasselbusch \& Penman, 2008; Law et al., 2002; McCartney, Ellis, \& Boyle, 2009).

However, few studies are available to guide the more philosophical aspects of therapists' school-based practice (Carlsson et al., 2007). Therapists have described difficulties defining their role within the changing context of the general school environment, especially if they have previously worked in health settings (Bowler, 2005; Carlsson et 
al., 2007; Gascoigne, 2006; Hanft \& Place, 1996; Hasselbusch \& Penman, 2008; Law et al., 2002; Penman \& Caswell, 2007). Further, workload pressures impact on their ability to allocate the necessary time to their students (Hasselbusch \& Penman, 2008; Law et al., 2002), they feel unprepared for teasing out whether to attend to student, teacher, teacher's aide, principal, or parent voice first (Carlsson et al., 2007), and lack specific skills and knowledge such as complex problem-solving skills and the flexibility to juggle many factors and to make on the spot judgements and responses (Hasselbusch \& Penman, 2008). They acknowledge defending existing practice, and wondering if new ways of working were really 'therapy' (Penman \& Caswell, 2007). Nevertheless, practicing therapists have been noted to adapt quickly and to embrace inclusive philosophy and practice (Carlsson et al., 2007; Hasselbusch \& Penman, 2008; Law et al., 2002; Penman \& Caswell, 2007). Writing from the USA, Hanft and Place (1996) noted that consultation and direct service models provide distinctly different but equally important ways for occupational therapists and physiotherapists to contribute their expertise to enhance children's performance. Therapy professions now need to pay attention to the importance of preparing graduates for practice in non-medical settings (Carlson, 2007).

McWilliam (1995) presents a continuum of six 'consultation models' ranging from 'individual pull-out' to 'collaboration' that are used in early childhood settings by occupational therapists, physiotherapists, and speech-language pathologists, and special education teachers in the USA. Therapy can be provided in the context of routines, in or out of the classroom, and/or can provide foundation skills that help the child to participate in routines in different contexts. McWilliam notes that consultants take various roles depending on where the therapy is provided, the involvement of other children, routines and activities, adult-child initiations and intervention styles, goals, and how the therapist/consultants' role is viewed or negotiated. She argues that critical differentiations in the way location can be used depend on whether other staff members are present and/or actively involved. Similarly, when other children are present they can either be active or passive, interacting with the child, or working alongside.

From the UK, the Royal College of Speech and Language Therapists (Gascoigne, 2006) suggest that the delivery of services to students in schools has two moveable dimensions: with management responsibilities lying on a continuum of therapist-led interventions to 
interventions led by others, including schools, and with the focus of the intervention at one end targeting student impairments and at the other improving child participation (Gascoigne, 2006). Speech and language therapists are encouraged to adopt a model of service delivery where professionals carry out tasks that require professional skills and delegate others tasks to assistants and support workers (Gascoigne, 2006). It is assumed that if intervention and support are effective, the child will follow a typical pathway from speech and language therapist led to school-led services, with the spotlight initially on 'barriers', then on 'participation'.

UK SLTs are committed to basing their services within schools and to planning jointly with education professionals to provide integrated services in schools (McCartney, 2009). However, learning opportunities can be limited by restricted opportunities for coprofessional working and the complex and challenging language environment of the mainstream classroom (McCartney et al., 2009). Further, consultancy approaches for speech and language therapists in the UK typically involve giving advice and guidance to school staff and families who carry out any language learning activities with the child (ibid). SLTs are concerned about universal applications of indirect service, particularly since paucity of resources restrict opportunity for genuine professional collaboration (Law et al., 2002).

Studies have shown that language intervention, offered via the withdrawal model, can be equally effective when offered by an SLT or an SLT assistant, whether offered individually or in small groups (Boyle, McCartney, Forbes, \& O'Hare, 2007). On the other hand children who received language intervention delivered by SLTs or their assistants made more progress in expressive language than similar children who received intervention delivered by education staff (Boyle et al., 2007; in McCartney et al., 2009). That education staff were unable to give the same amount of time to delivering the intervention as specialists was a contributing factor (McCartney et al., 2009). On the other hand allied health and education professionals can improve their skills and attitudes to working with students who have speech and language difficulties following specific training in the implementation of strategies (O'Toole \& Kirkpatrick, 2007). 


\subsection{Music Therapy}

Although music has been associated with wellbeing and healing since the beginning of documented history, it was not until the $20^{\text {th }}$ century that it gained recognition as a professional discipline. The profession has developed according to the psychological, social, and medical thinking of the time, growing out of, and interacting with, culture and society (Stige, 2002). It has been used in recreation programmes for mentally ill patients, medical experiments, remedial care of children who have disabilities, and in education. It draws from numerous fields including psychology of music, psychology, physiology, biochemistry, sociology, and education.

For over two decades however, music therapy has predominantly been described in psychotherapeutic terms whether the model be primarily behavioural, humanistic or psychoanalytical. For example music has been used as a stimulus and contingent reward for promoting imitation (Underhill \& Harris, 1974), speech (Harding \& Ballard, 1982), and for reducing inappropriate behaviour on school buses (McCarty, McElfresh, Rice, \& Wilson, 1978). Music therapists also describe work which focuses on possibilities for music to enable children and adolescents to grow and reach their potential by being together with others in music (e.g. Aigen, 1997; Robbins \& Robbins, 1991), while others focus on the way students' inner conflicts can surface in behaviour or emotions and can be 'contained' by and managed in musical and/or group interaction (e.g. Sutton, 2002).

According to Ansdell (2002) the pioneering models of music therapy shared several features including a move from 'receptive' music listening to 'participatory' shared music making, the use of improvisation to enable spontaneous music making, consideration for the musical interpersonal relationship, and an increasing focus on individual work with 'patients' or 'clients'. Thus the 'Consensus Model' of music therapy (Ansdell, 2002), with its psychotherapeutic understandings and boundaries, has been taught in various training centres and expounded in the literature for several decades. Music therapists have used music to develop therapeutic relationships with clients predominantly to change their intrapsychic state, which in turn improves their relationships in other contexts. 
However, they are increasingly likely to work in different ways according to their philosophy, training, the needs of the clients they are working with, and the community or facility in which the therapy takes place. Regardless of the setting and the client group involved, music therapy has always been difficult to define and to describe (Ansdell, 1996; K.E. Bruscia, 1989; Rogers, 1995). Now "we can no longer simply state that music therapy is 'such and such' a practice, described with the help of 'such and such' theories, without addressing a crucial third bit: context" (Pavlicevic, 2004, p.45).

\subsubsection{Community Music Therapy}

Music therapists are broadening their work to take account of clients' political, cultural, and social contexts (Pavlicevic \& Ansdell, 2004a). The concept of Community Music Therapy (CoMT) (Ansdell, 2002; Pavlicevic \& Ansdell, 2004a; Stige, 2004b) has emerged as the focus of inquiry has shifted from trying to define music therapy as a profession to questioning what one might do in a particular contextual setting. While many music therapists had begun to question their own and other's 'unconventional' practices because they did not fit into current theoretical models (Aasgaard, 1999) it is now acknowledged that music therapists work broadly across the individual-communal continuum in order to address the needs of their clients. CoMT is an approach to working musically with people in context which acknowledges the influence of social and cultural factors on health, relationships and music (Ansdell, 2002). Clients are helped to access a variety of musical situations, and to move between 'therapy' and music making in wider social contexts. CoMT takes account of how physical surroundings, client preferences and cultural contexts shape the work (ibid).

Several authors have indicated that defining Community Music Therapy is not only difficult, but that attempting to do so can be unhelpful (Pavlicevic, 2004; Pavlicevic \& Ansdell, 2004b; Proctor, 2004; Stewart, 2004; Stige, 2004a). Rather, it can be understood as a set of responses to challenges given by contemporary developments in society and culture, such as individualisation, specialisation, and professionalisation (Stige, 2004b). The role of clients and music therapists in relation to each other and to society at large are being negotiated in new ways and within new contexts (Stige, 2004a, 2006). 
Community Music Therapists pay considerable attention to how material, social and cultural situations influence their practice and the ways in which they can help individuals and the 'circumstantial community' (hospitals, schools, residential facilities) in which they work (Ansdell, 2002). Clients should not be viewed as isolated individuals, but as cultural individuals in their communities and complementary goals should be set, at varying levels, for individuals, groups, organisations and institutions (Stige, 2002). Musical relationships are formed with clients, families, and staff. Music therapy can take place in corridors, halls and common rooms, treatment rooms or out of doors (Aasgaard, 1999). Other musicians can also be recruited to work with the therapist (Pavlicevic, 2005) and transdisciplinary and interdisciplinary work with other educators and health professionals (e.g. speech, occupational and physiotherapists) is highly valued (Tait, 2005). Tait's action research with indigenous communities in Australia for example, marries the techniques and processes of music therapy, music education and performance with the priorities of indigenous teachers, health professionals, families and children.

There is no need for rivalry between individually directed music therapy and one mainly directed toward the environment (Aasgaard, 1999). Decades ago, the respected music therapy team of Nordoff and Robbins described moving easily between intense clinical work, and school wide and public performances of music works (Nordoff \& Robbins, 1977). In NZ, a longstanding definition for music therapy has been 'the planned use of music to meet identified need' (New Zealand Society for Music Therapy, 1989), and there are many intrinsic assumptions associated with this simple definition. 'Identified need' for example, can easily include clients' needs in relation to the communities in which they live. Māori, the indigenous people of New Zealand, have a holistic concept for health which looks to physical, emotional, spiritual and whanau (wider family) wellbeing (Ministry of Health, 2009). Building on this, it has been logical for music therapists in New Zealand to work with children in wider contexts than clinic settings.

The process of music therapy consultation in special education settings involves building relationships with the special education community - entering 'the field' with an open mind to learn about participants' strengths and needs, collaborating to determine what their needs are in relation to their use of music, and providing support in a way that 
acknowledges and values team members' skill and effort and encourages them to use music effectively in a sustained way. The work is music centred, will take place in the contexts and environments in which students 'live', and will be responsive to school and classroom culture and the needs of team members, and can thus be viewed as CoMT. The 'performance' referred to in CoMT literature in this context might be seen as a metaphor for the functioning of team members, particularly their ability to implement music strategies to help students.

\subsubsection{Music Therapy in Special Education (Traditional Practice)}

Bruscia (1989) suggested that therapists working with children have a different perspective on music therapy than those working with adults and, drawing on the psychotherapeutic thinking of the time, argued that in education settings music therapy is practised quite differently than it would be within rehabilitative, psychotherapeutic or medical settings.

During fifteen years of practice with children and adolescents, the author drew on Bruscia's (1989) Developmental Music Therapy model which encompasses broad clinical goals and a process that "focuses on assisting (students) to accomplish a wide variety of developmental tasks that may have been delayed or thwarted at various periods in the life span" (p.73). In this model the therapist is concerned with the child's physical, intellectual, social and emotional development, as well as their personality. Family and medical history is gathered as part of the assessment. Following data gathering, assessment, and planning, sessions would usually be undertaken with the music therapist and the child meeting regularly, often weekly, in the same therapeutic space, for approximately 30 to 45 minutes.

Programmes can run for several months or sometimes years, depending on the needs of the child and the contribution that music therapy can make to his or her development. Central to traditional music therapy work is an acknowledgement that music therapy is a process involving multiple relationships between the therapist, client, and their music. A therapeutic relationship between the therapist and student is considered to be a crucial ingredient in music therapy in school settings (Rickson, 2006b) and this emphasis has 
helped differentiate music therapy from the therapeutic use of music.

However, the literature appears to suggest that in school settings the primary differentiation between music education and music therapy is that music therapists focus on the functional use of music to support non-musical goals, and there are significant overlaps in terms of content (Langhan, 2009). Music therapy is portrayed as the use of music activities to enhance, or as an alternative to, traditional teaching methods. Bruscia (1989) claimed that this practice falls within the boundaries of music therapy because it addresses educational problems that require therapeutic intervention or needs that have important implications for the student's overall development. Like Nordoff and Robbins (1983), Bruscia noted that the concept of music therapy in special education invites the therapist or teacher to "use music to help handicapped students gain non-musical knowledge and skills essential to his or her education” (K.E. Bruscia, 1989, p. 72).

There are many examples of research demonstrating the effectiveness of music therapy in school settings including, since 2000, studies by Bertolami \& Martino (2002), Boisvert, Labbe, and Kane-Benveniste (2000), Booth (2004), Brotons (2001), Brownell (2002), Coleman (2002), DeBedout and Worden (2006), Jackson (2003), Johnson (2002), Kennedy and Scott (2005), Rickson (2002; 2004; 2006a), Rickson and Watkins (2003), and Wilson and Smith (2000). There is also general agreement that music has an important role to play in the education and personal development of children with severe or profound disabilities (Boxill, 1985; Bunt, 1994; Chester, Holmberg, Lawrence, \& Thurmond, 1999; Daveson \& Edwards, 1998; Jellison, 2000; Ockelford, Welch, \& Zimmermann, 2002; S. Wilson, 1991). In her content analysis of music research with children and youth who are disabled (1975-1999), Jellison (2000) found many diverse studies across populations and numerous examples which demonstrated the different functions of music to bring about changes in academic, motor, social, and verbal behaviour. A few of the studies also looked towards music outcomes, ranging from simple imitation and perception tasks to more complex activities such as music reading or playing an instrument. 


\subsubsection{Music Therapy Assessment}

A music therapy assessment which enables the team to have a comprehensive picture of students' responses to music will be an important feature of music therapy school consultation.

An effective music therapy assessment will have "clear objectives; be conducted by a qualified music therapist; use unique music therapy tools; utilise effective methods of data collection; produce reliable data; draw valid conclusions; and maintain ethical standards" (K.E. Bruscia, 1988, p. 5). However, each music therapist's personal beliefs about the role of music in music therapy, their treatment philosophy, the population they work with and the institution in which they work, greatly influence the decision of what and how to assess (Isenberg-Grzeda, 1988). For example, clinicians have developed evaluation or assessment tools which focus on specific diagnoses e.g. children who have severe emotional disturbance (Layman, Hussey, \& Laing, 2002) or autism (Wigram, 2000) while others are much broader (e.g. Baxter et al., 2007; Chase, 2004). For example, the Individualized Music Therapy Assessment Profile (IMTAP) (Baxter et al., 2007) is a comprehensive and systematically developed paediatric assessment scale that aims to ensure clinicians are providing the most appropriate therapy programmes and that client progress is accurately evaluated (Baker, 2008).

Music therapists also use assessment tools which are not specifically designed for music therapy. Walworth (2007) for example argues that the music therapy setting provides unique opportunities for observing children's interaction as part of the Social Communication, Emotional Regulation, and Transactional Support (SCERTS) (Prizant, Wetherby, Rubin, Laurent, \& Rydell, 2006) assessment. The importance of including ecological assessment which include a variety of holistic approaches such as family interviews, observations of learning styles and parent/child or educator/student interaction has also been acknowledged (Chase, 2004).

Developmental data obtained from music therapy sessions can provide a valuable comparison or addition to that obtained by other professionals (Bunt, 1994; Gantt, 2000; Loewy, 2000; Molyneux, 2002; Oldfield, 2000; Wigram, 2000). Music therapists can 
anticipate different responses from a child in a music therapy environment by understanding that despite significant disability or neurological injury a patient's response to music can be preserved (Gantt, 2000; Nordoff, Robbins, \& Marcus, 2007). The music therapy session can therefore be viewed as an opportunity to view children's strengths and to evaluate their responses in terms of their potential to respond to other forms of therapy or intervention (Molyneux, 2002; Oldfield, 2000; Wigram, 2000; Wigram, Pederson, \& Bond, 2002).

Music therapy assessment tools designed specifically for use in special education contexts are emerging. The Special Education Music Therapy Assessment Process (SEMTAP) (Brunk \& Coleman, 2000) for example is designed to provide a direct comparison of a student's performance on IEP objectives with and without music, specifically to determine whether music therapy is an appropriate intervention for a student. Langhan (2009) developed a tool with Australian music therapists which incorporates specific curriculum outcomes and music therapy outcomes. The tool uses education terminology to help educators gain a perspective on how music therapy relates to education and an understanding of the complexity of music therapy processes.

However, highly structured assessment tools can have a negative impact on the assessment process. Goodman (2007) describes the SEMTAP as clear-cut and "almost common sense" in terms of adaptability, but notes that relying on stated IEP goals and objectives as a basis for decision-making may be short-sighted. She argues while the SEMTAP is relatively systematic and provides procedural ease in terms of the data collection, observation, targeting objectives and devising music therapy tasks, it limits process-oriented music therapy assessment sessions. Proceeding step-by-step to administer specific activities precludes following the child when they become interested in something else.

Many music therapists prefer to use a spontaneous, creative and open-minded approach to assessing individuals and each assessment session is therefore quite different. For example by examining the way children respond to specific elements within improvised music, as well as the interpersonal relating that occurs within musical dialogue, children's capacity for and progress towards non-musical relationships can be evaluated (Bunt, 
1994). Clinical improvisation provides information about how children communicate with instruments (including voice) as well as their movement, body language and facial expression, and whether they are initiating or following, stopping on cue, appear to be noticing changes in the therapist's music, allowing accompaniment, leaving space for the therapist to contribute, or engaging in turn taking dialogue and so on. Further, music therapy improvisation enables the therapist to gauge the child's emotional condition.

Some music therapy assessment profiles are generated primarily from musical data, which is interpreted and considered in terms of communicative intention (K.E. Bruscia, 1987; Gilboa, 2007; Nordoff \& Robbins, 1977; Wigram, 2000). Musical material can be interpreted qualitatively, or the length or frequency of events recorded quantitatively (Wigram, 2000; Wosch \& Wigram, 2007). However, it can be difficult expressing musical material in 'words' and interpreting the meaning of an aesthetic experience (Aigen, 1999; Aldridge \& Aldridge, 2002; Ansdell, 1996, 1999; S. Brown, 1999; Gantt, 2000; Pavlicevic, 1999; Stige, 1998; Streeter, 1999). Music therapists have found limitations in commonly used assessment tools that focus on musical material.

For example, assessment scales developed by Nordoff and Robbins (1977) to measure the 'Child-Therapist Relationship in Musical Activity' and 'Musical Communicativeness' are potentially unreliable when used by therapists untrained in that particular approach, because of the specific definitions used (Bunt, 1994). Brucia's (1987) Improvisational Assessment Profiles (IAP) have also provided possibilities for music therapists to analyse musical material in a similar way, and thus to share findings, yet Wigram (2000) described the profiles as a "complex, detailed, and extensive method of analysis" (p17) which can be off-putting to the practitioner. Nevertheless, Wigram successfully utilised just two of Bruscia's six profiles, those of 'autonomy' and 'variability', in his diagnostic and assessment work with children who have significant social and communication disorders. Despite the difficulties, there has been increasing recognition of the potential for musical material to be used for assessment and evaluation purposes (Wosch \& Wigram, 2007).

Description also appears to be an accepted and valued method of music therapy assessment (Loewy, 2000; Molyneux, 2002; Oldfield, 1993, 2000; Rickson, 2001). 
Loewy (2000) argues that "descriptive documentation substantiates, challenges and redirects the way in which music therapy inferences are conceptualised and translated into words. As clinical description is written, studied, and edited, we increase our ability to understand the process and ground it in meaningful theory" (p.48). Despite being considerably subjective, Loewy's 'hermeneutic' approach to assessment was validated by a peer checking process in 1994 and again in 2000 (cited in Loewy, 2000).

Music therapists have differed in their opinions regarding the need for standardisation of assessment tools, and some clinicians have begun to advocate for a standardised procedure rather than a standardised assessment tool (Ala-Ruona, 2005; Brunk \& Coleman, 2000). This is logical given the need to accommodate creativity in assessment sessions, Loewy's (2000) report that 94\% of music therapists surveyed in 1994 were not using the music therapy assessment tools that were available to them, and Wigram et al's (2002) claim that "clinicians have developed their own very effective and appropriate ways of analysing and recording change in music therapy" (p. 257). Further, the music therapists in Chase's (2004) survey who used predominantly self-created assessment forms had many positive comments to make about using an individualised approach.

\subsection{Music Therapy Consultation}

\subsubsection{Collaboration and Teamwork}

Consultation and collaboration are both important activities, but the terms are not interchangeable (D. Brown, Pryzwansky, \& Schulte, 2001; Dettmer et al., 2005; Dougherty, 2005; Register, 2002; Wesley \& Buysse, 2006). Collaboration is the process of working jointly with others in an intellectual endeavour to bring about change, and it implies shared responsibility (Register, 2002). It involves the interactive exchange of resources, interdependence, systematic planning, shared definition of goals or visions leading to the identification of targeted outcomes, and joint problem solving (Dougherty, 2005; Register, 2002). Music therapists engage in various levels of collaboration with

team members from communication, interaction, facilitation/observation, to fully integrated work which involves simultaneous and combined treatments with other 
professionals (Twyford, 2007). McCartney (2009) notes that writers have tended to classify models of co-professional working according to who works with the student, who retains responsibility, the direct or indirect nature of the work, whether relationships are egalitarian or supportive, and who sets the targets. Consultation involves professionals co-working in nominally egalitarian relationships (ibid).

Consultation tends to be collaborative as consultant and consultee share expertise to solve a problem. Collaborative school consultation can help students to develop to their fullest when general, special education, and support personnel, related services, families of students, and the students themselves work together and share their diversity of knowledge and expertise to define needs, plan, implement, assess, and follow-up (Dettmer et al., 2005). However, the consultee carries out the plan and the consultant remains on call to assist if necessary. SLTs in the UK note that the term 'consultant' is used to define both activity and degree of expertise, and that in reality collaborative opportunities are limited (Law et al., 2002). Rather, undertaking direct assessments and modelling techniques have become important aspects of consultation work (ibid).

Music therapists surveyed in the USA report significantly higher levels of 'collaboration' versus 'consultation' (Register, 2002). Collaboration and teamwork has continued to come to the fore in recent years (see Twyford, 2007; Twyford \& Watson, 2008a) and the 2005 British Society for Music Therapy Conference was dedicated to groups, partnerships, and teams in music therapy. In contrast, although music therapists are increasingly writing about consultative models of practice with children in early intervention and music education settings and there are some examples in regular education settings (see below), overall references to consultation in school settings are sparse (Register, 2002). Consultation might be mentioned in passing (e.g. in Goodman, 2007; Johnson, 2002) but the process is rarely described.

\subsubsection{The New Zealand Situation}

The New Zealand music therapy literature gives the impression that music therapists here are primarily offering programmes directly to clients in schools and/or early intervention programmes (Archer, 1995; Rickson, 1997, 1998, 2000, 2001, 2002; Rickson \& Watkins, 
2003; Stevenson, 2003; Warren, 1997). Papers focusing on music therapy with children and adolescents who have disabilities predominantly describe the direct delivery of music therapy programmes in 'clinic' settings.

There are a few exceptions. Croxson (1999), for example, writes of opportunities to provide advice to school staff, firstly at a special school in a city and secondly at a mainstreamed rural school, as a follow-up to her work with individuals and families in clinical settings. Rickson (1997) described various uses of music in school settings, but differentiated music used to help learning in the regular classroom (music in education), adaptations and special techniques used to assist children who have special needs to reach their potential in classroom music programmes or private tuition (special music education), and classroom teachers' use of music with children who have special education needs to enhance overall development (music in special education), from music therapy. Twyford (2009) describes a model of music therapy practice which involves individual sessions with students, leading to group work with peers. This is followed by a 'hand-over period' during which school staff are encouraged to continue with music activities begun in the therapy setting. However, none of these examples describe how adults are empowered to facilitate music planned to meet specific students' needs.

Nevertheless, music therapists in New Zealand have been working in partnership with schools and families to promote the use of music with children who have special needs since 1974. During that year, a visit from world renowned composer Paul Nordoff and special needs teacher Clive Robbins prompted a small band of music therapists and supporters to form the New Zealand Society for Music Therapy (NZSMT), now known as Music Therapy New Zealand. Since that time, music therapists worked with members of this society and its branches to provide regular workshops, conference presentations, and individual consultation sessions to promote the use of music for children who have special education needs. Further, while there are few references to consultation in the New Zealand music therapy literature, Warren (2005) also informally describes supporting and supervising music teachers, teachers' aides, classroom teachers in the use of music and to develop IEP goals with students, and considers that work to be consultation. 


\subsubsection{International 'Models' of Music Therapy Consultation in Schools}

Changes in education approaches have led the re-evaluation of music therapy programme delivery in the USA. The American Music Therapy Standards of Practice (American Music Therapy Association, 2009) state that music therapists may provide consultancy services to other professionals in music therapy and related disciplines and to others directly involved with the client, provide resource information regarding music therapy techniques and materials, or may design music therapy programs for clientele in various settings. Collaborative, interdependent work with other team members has been considered essential in the school setting with teachers and teachers' aides as 'central players' for some time (Snell, 1996).

On the other hand music therapists have approached the inclusive model cautiously. In 1998 in the USA, even though music therapists overwhelmingly knew about and expressed willingness to provide services in inclusive educational settings, they predominantly worked in segregated settings. Only $13 \%$ provided consultative services (D. S. Smith \& Hairston, 1999) even though it was clear that the use of music therapy in integrated settings could facilitate the interaction between children with and without disabilities (Gunsberg, 1988; Hughes, Robbins, McKenzie, \& Robb, 1990; Humpal, 1991; Jones \& Cardinal, 1998). Literature from the USA therefore describes various ways music therapy can be delivered and learning can be transferred to education practitioners, but studies have not produced information that can be generalised across a wide range of settings (Register, 2004). Consultation methods listed by several authors include books and publications, lectures, workshops/seminars/in-services, observations with written/oral feedback, and one-to-one meetings, as well as onsite services mostly delivered in small group or individual settings and often on a weekly basis (Register, 2002; Reuer, 1996; B. L. Wilson, 2002).

Reuer states: "a consultant is simply anyone who gives advice or performs other services of a professional or semi-professional nature in return for compensation... This means that regardless of an area of interest or expertise, one can become a consultant" (Reuer, 1996, p. 17). Register (2002) also writes that consultation allows for the dissemination of information to an individual or group in order to educate and advise on a given topic or 
methodology. On the other hand she also understands consultation as a collaborative process involving a trained consultant who assists 'consultees' to make decisions and carry out plans to support clients or patients. Register states that "the goal of consultation is to improve the functioning of the client while enhancing the functioning of the consultee" (p. 309). Music therapists view themselves as collaborators and are therefore in a good position to consider engaging in collaborative consultation with school staff (ibid). However, consultation processes in music therapy are not well articulated.

Snell (1996) for example designed experiences to "support the team's approach" but does not articulate precisely how this was achieved. Rather he describes facilitating music therapy groups which allowed for 'music therapy consultations' with teachers. Snell observed that teachers valued the discovery of students" "hidden skills", and their frequent demonstration of education, physical, and emotional "firsts" during music therapy assisted experiences. His case examples suggest that modelling and the provision of materials enabled teachers to continue the activities. However, he stressed that successful implementation was reliant on the music therapist's skill in making 'real time adjustments' to the music and being able to convey these to teachers. How teachers were empowered to do this work and how it was scheduled is unclear but a case example mentions observing a child during problematic transitions which suggests that time may have been available for ecological assessment and work in context.

Johnson (2002) names four different categories of 'consultative models' i.e. consulting to facilitate (a) inclusion, (b) music education, (c) a musical performance, and (d) consulting with non-music education staff, while Coleman (2002) writes of 'consult-student' and 'consult-programme based' models. In the consult-student model the music therapist undertakes a music therapy assessment, develops goals and objectives, and provides regular weekly sessions for a set period, while the teacher observes and is trained to carry out a specifically designed programme for the student/s. The music therapist provides regular monitoring (fortnightly or monthly) as the teacher implements the programme. Coleman claims this model can be effective if the teacher is able to carry out the programme as designed and is responsive to ongoing supervision and consultation (Coleman, 2002). 
Coleman's consult-programme model, first published in 1996, was expanded by Chester et al. (1999). The model is similar to that used by Snell, described above, where the music therapist designs and 'demonstrates' how a group music therapy programme might be facilitated with the aim of motivating and up-skilling teachers to implement the programme. It is important to note that models such as these which involve music therapists facilitating weekly sessions with students are essentially 'direct delivery' models which cannot address the needs of isolated students. Nevertheless, Chester's model does involve ecological assessment and, while the music therapist functions as a member of the interdisciplinary team, the student's IEP does not contain a music therapy specification. Instead, the intervention is specifically provided as resource for the teacher or classroom (ibid).

While very little has been written about the process of music therapy consultation in general classrooms, the tasks for the music therapist may be similar to those described in music education settings (see below). For example, Threlfall (2005), an Australian music therapist, provides a list comparable to that provided by Jellison (1979) which notes the importance of observation in the classroom; talking to teachers and other therapists in order to find a shared purpose; inviting their input into intervention planning to ensure programmes are acceptable to them; and allowing final decisions regarding programming to be made by the teacher. In Threlfall's model, when the school community begin to implement the agreed programme the therapist supports by evaluating and designing any necessary changes. Working in a collaborative way can enable more time for music therapists to make classroom visits and more time for therapists and teachers to meet both formally and informally as well as encouraging teachers to be explicit about what the music therapy was able to contribute to classroom communities (ibid).

On the other hand, Jellison (2000) notes that collaboration is particularly difficult for those music therapists who travel among several schools, serving children with the most severe disabilities in separate classrooms, because their schedules leave little time for substantive involvement in the child's inclusive education programme. The success of collaborative models lies in the frequency with which music therapists can meet with other professionals. 


\subsubsection{Working with Music Educators}

A large proportion of the references to music therapy consultation relate to music therapists working with music educators, to support the inclusion of children who have different learning needs in mainstream music classes (Darrow, 1996, 2002; Jellison, 1979; Johnson, 2002; Steele 1977). Music has been identified as an ideal subject to integrate children with special education needs and their peers, because of its flexibility and potential to aid development and learning (Brotons, 2001).

The move towards inclusion places many higher functioning students with special education needs in the music room of the music educator (Goodman, 2007; Snell, 1996) and increasing importance is being placed on the consultancy aspect of the therapists' work in schools (Darrow, 2002; Heine, 2002). Music therapists are trained to work with students with diverse needs and can function as excellent resources for the general music teacher, either as consultants or as facilitators of simulation or information training (Brotons, 2001; Coldwell, 2001, in Brotons 2001) helping the music educator select appropriate musical materials, musical activities, instructional approaches, and ways to maintain positive affect among students in the classroom (Duerksen \& Darrow, 1991).

The selection and prioritisation of functional educational objectives has always been an important topic in special education and has implications for programmes in music education and music therapy (Jellison, 2006; Walworth, 2007; Wolery, Sigalove Brashers, \& Neitzel, 2002). Jellison (2000) writes that therapists should aim to develop skills that can be generalised and will ultimately lead to students' greater independence and improved quality of life outside of the school day, support the acquisition of a new behaviour, meet learning objectives, and/or facilitate positive attitude changes in peers, parent/guardians, or teachers. Jellison also argues for the selection of behaviours that are valued by peers and adults in the student's social and educational settings.

Choosing the most beneficial functional music and non-music tasks will most often occur during collaborative efforts among parents/guardians, music educators and music therapists For example, music educators, music therapists, and others can work together to help encourage at-risk students to attend school and to reap the benefits of the school 
experience (Duerksen \& Darrow, 1991). Music therapists were urged to work with parents and colleagues and to seek opportunities for behaviours to be observed in the natural environment of home, school, and community before developing a music curriculum for students who have special education needs, decades ago (Jellison, 1979). Jellison provided a comprehensive list of steps necessary in what she described as the development of functional, appropriate instructional programme strategies. These included reading records; finding out about home school and community music resources; learning about students' chronological age-appropriate preferences and activities; seeking to interact with significant others in the students' lives; and conducting a comprehensive assessment of the students' current music skills and preferences.

Around the same time, Steele (1977) described consulting with music teachers for thirty minutes per week to help them discover ways to design programmes of effective individualised instruction which would engage students who have special education needs in music programmes. Steele's approach was similar to action research, involving observation, planning, implementation and evaluation, with students actively contributing to the development and implementation of the plan. The music therapist also facilitated individual and group sessions for students who needed extra support, with students moving easily between therapy and education groups. Steele noted the consultations were not $100 \%$ successful, with inconsistencies, frustrations, and failures being experienced. On the other hand the teachers found the consultancy useful for problem solving and many techniques were used in an ongoing way.

Much later, high levels of music therapy services were still delivered in segregated environments (Jones \& Cardinal, 1998) and there were still few studies describing the work of music therapists in music education settings even though the limited evidence was positive (Darrow \& Grohe, 1996). Children were known to respond differently in music education (regular), and special music education/music therapy (separate) settings (Kostka, 1993; Jellison and Gainer, 1995), suggesting that collaboration between music therapists and educators would be valuable. Music therapists were encouraged to advocate for a role in inclusive environments alongside other support services that had already made or were making this transition. During this same period, interviews with music teachers regarding students' ability to access the music curriculum reinforced the 
need for music therapists to serve as facilitators and inclusion specialists in music education programmes, be responsible for liaising and providing information between home/school parties, provide in-service for music teachers and para-professionals, give direct classroom assistance, and coordinate practicum experiences for music education majors (Darrow, 1999). Nearly all the music educators interviewed in Darrow's study identified a need for collaboration or consultation with special educators, music therapists, or others knowledgeable about students with disabilities.

Music educators often think that they lack the training, understanding, and effective teaching strategies to meet the educational needs of children with disabilities (Brotons, 2001; Darrow 1999; Steele, 1997). They may lack information, be unaware of students' needs, and/or be unable - because of time and/or skill limitations - to adjust music to support students (Darrow \& Loomis, 1999; Snell, 1996; Steele, 1977). Further, they might have difficulty maintaining momentum and enthusiasm for teaching under somewhat less than optimum conditions (Darrow \& Loomis, 1999; Facchini, 2001; Heine, 2002; Steele, 1977). Nevertheless, educators in Snell's (1996) study recognised that the things they were able to do for students who had special education needs also supported music learning for others in the classroom. She argues that it is up to music therapists to define and frame music therapy's unique role in helping children access education in the least restrictive environment.

Short-term in-service programmes are one of the most frequently used and highly rated ways to integrate students who have special education needs into music classes (Heine, 2002). Heine (2000) notes that the provision of information can positively affect the attitudes of peers and professional music educators towards individuals with disabilities, and is especially helpful when a practicum/experiential component is included as part of teacher courses. However, interaction and collaboration seems more effective than textbook information, which requires teachers to determine what is applicable to them (Heine, 2002).

Music therapists who have provided in-service training in school and early intervention settings (Brotons, 2001; de I'Etoile, 2001; Facchini, 2001; Heine, 2002) found that teachers valued new resources and skills, and acknowledged the need to adapt materials 
and adopt new teaching strategies to deliver music programmes to students who have special education needs. However, Heine (2002) asserts that it is not helpful to assume what teachers need; strengths-based approaches that acknowledge and allow teachers' skills and experience guide in-service content and format should be employed, and support should be practical and relevant. Evaluations that determine whether in-service programmes are meaningful and meet the needs of the participants are needed (Heine, 2002).

Teacher attitude is an important factor in the successful integration and learning success of students with disabilities in music classes (Darrow, 1999; Heine, 2002; Neilson, 2005). The success of students who have disabilities in the music classroom is often determined by their social skills, teachers' expectations, and peer acceptance (Jellison, 2000). When supporting child-care personnel with their use of music, de I'Etoile (2001) noted considerable improvements in caregivers attitudes and overall knowledge about music activities for children. Benefits were noted for both caregivers and children as the caregivers developed the ability to successfully implement activities and to increase child engagement.

Finally, there has always been confusion and different opinions regarding the role of the music therapist as opposed to that of the music teacher in special schools (K. E. Bruscia, 1998; Duerksen, 1991; Langhan, 2009; Markou, 2004; Woodward, 2000). Robertson (2000) proposed a continuum between music therapy and music education, and suggested that music teachers could be given sufficient training to deliver music therapy in education, a concept he describes as 'educational music therapy'. He posited that students who have a mild to moderate level of need can be guided towards learning about music as a result of therapeutic musical interaction, with the aim of 'contributing to' rather than 'coping with' their environment. Pethybridge and Robertson (2010) did indeed find that once children were able to contribute to the music making environment and to take ownership of their musical interaction within small groups in the school context, they were more able to address both musical and non-musical educational objectives. Separate pathways for music therapists working exclusively in education are still not available (Pethybridge \& Robertson, 2010). There is however a need for music therapists to offer training opportunities for music and general teachers, and for music therapists to 
learn more about key principles and practices of music education (ibid). Further, acting as a consultant to other professions can help music therapists to clearly define the principles of the therapeutic use of music (Register, 2002).

\subsubsection{Working in early childhood settings}

Encouraging parents and/or caregivers to observe and/or participate in early intervention programmes with a view to supporting or continuing the work is increasingly considered to be 'usual practice'. Only studies in which music therapy work is approached primarily as a consultation process have been included.

Kern (2004) for example drew on McWilliam (1995) to describe various potentials for music making in an early childhood setting. Among others, these included 'group activities' that can take place in the classroom or playground in small or large groups, 'musical interaction' with the child during individual routines in classroom or playground wherever they might be, and 'consultation' in or out of the classroom. In the group therapy model, the music therapist shares professional knowledge, involves a teacher in planning and implementing the work, and leads or co-leads the music making. When the music therapist is providing individual therapy within the child's ongoing routines, they will share professional knowledge with the teacher, train the teacher to use music therapy strategies, and exchange information with a teacher before and after routines (Kern, 2004).

Consultation on the other hand, according to McWilliam's model, can occur in or out of the classroom, and involves the music therapist working primarily with the teacher (Kern, 2004). Parties collaborate to agree the approach teaching staff should use in order to meet the child's identified goals. Peers are present if the work is occurring in a school setting. The role of the music therapist can range from 'expert' to 'guide' who provides information and training on the use of therapeutic techniques and to 'collaborator' who develops a relationship of equality, and works with the teacher on defining the problem, identifying the goals of intervention, planning the intervention and engaging in follow-up (Kern, 2004). 
When composing individually tailored songs to improve the performance of children with Autism Spectrum Disorder (ASD) in childcare settings, Kern (2004) took into account their individual education goals, along with strategies commonly used with children who have autism. Collaborative consultation and staff development enabled teachers to implement music therapy interventions successfully and meaningfully in ongoing centre activities and routines. In a further study, Kern and Aldridge (2006) used embedded music therapy interventions to support outdoor play of young children with autism in an inclusive community-based child care programme. They noted that the intervention produced desirable peer interaction, and the collaborative consultation approach enabled teachers to implement interventions successfully in ongoing playground routines. Kern, Wolery, and Aldridge (2007) used songs to promote independence in morning greeting songs with young children who have autism, and taught the children's teachers to sing the songs during the routine. The results indicate that the songs assisted the children in entering the centre, greeting the teacher and/or peers and engaging in play.

Stige (2002) described his role as consultant in an early childhood setting, supporting the teacher to facilitate music with a student who had special education needs. Although geographical barriers prompted the work to a certain extent, integrating activities carefully into kindergarten life was considered important. Drawing on Feuerstein's concept of a mediated learning experience (MLE) (Feuerstein \& Feuerstein, 1991) which recognises social interaction as an important part of the learning process, Stige empowered the teacher to focus the work in meaningful play and eventually to independently expand it. The student was able to participate competently in the planned and developed activities and was thus rewarded for his efforts. Stige used video evaluation as part of the process and found that viewing the tapes enabled the preschool teacher 1) to reflect on his part in the process and to become more sensitive to the student's musical participation and 2) to share detailed information with the student's mother and thus to stimulate positive communication between kindergarten and home. Stige noted that the teacher's development in self-reflection and attitude towards the student was significant because it suggested positive cycles of interaction could be established.

Pasiali (2004) was also able to empower others to use music to promote learning, in 
natural settings. She introduced prescriptive songs with children who have autism using the guidelines for writing the text of 'social stories', a strategy for modifying behaviours of children with ASD. However, one of the mothers in Pasiali's (2004) study indicated that a lack of musical training prevented her from using a prescribed song or other music activities with her child and compensated by reading the lyrics of the song to help her child. This reinforces Kern's suggestion that caregiver training and ongoing consultation is critical. However, the level of ongoing support that can be offered to children in family or inclusive education settings in an ongoing way is subject to time and resource limitations.

Register (2004) compared three groups of early childhood educators who received a variety of types of instruction and guidance on the implementation of music over the course of the school day. Findings suggested that providing a model for implementation and support may increase the amount of music used by the classroom teacher and that having a mentor present to help shape teacher behaviours may have far more beneficial effects than just a workshop. Register also noted that a mutual relationship developed between the early childhood professionals and the music education students who were involved in the work, with each group gaining knowledge and insight through the process of working together. Further evidence suggests that children benefit from support in context which capitalises on naturally occurring learning opportunities and can provide consistent treatment towards the generalisation of skills where they are needed (B. L. Wilson, 2002). High levels of musical play occur in early childhood settings providing plenty of opportunities for music therapists to undertake collaborative consultations in the natural environment, with both teachers and parents or other caregivers.

\subsection{Further Considerations for Music Therapy School Consultation Work}

\subsubsection{Cultural Considerations}

Stige (2002) writes that culture is inevitable in human development; it is a product of human biological adaptation (phylogeny) and individual experience of genetic, environmental and cultural factors (ontogeny). Individuals are situated in evolving social 
contexts which influence them, and on which they have influence (ibid). We all participate in the creation of culture; it is cumulative and changing, newly created and transmitted by, and over, generations. Nevertheless, communal interactions between persons and their environs lead to the development of cultural norms; values, beliefs and systems of symbols to which they attach particular meaning (Crotty, 1998; Yehuda, 2002) and which underpin their 'knowledge' and subsequent understandings of events (Annan, 2005; Stige, 2002). Community music therapists are interested in how individuals and communities constitute each other reciprocally, and how individual and communal identities are negotiated in social and cultural contexts (Stige, 2006).

Collaboration and negotiation will therefore be central to the consultation process because what counts as 'improvement' can only be decided in context and in relation to team values and norms (Stige, 2002). Further, consultation relationships will be influenced by individual team members' unique understandings developed from their experiences of being members of many cultural groups. Consultants need to adjust their services to address the varying needs of consultees (Tarver, Behring, and Ingraham, 1980, in Ingraham \& Meyers, 2000). When consultants impose their own values on the process, consultees' professional growth can be restricted (Crothers, Hughes, \& Morine, 2008; Dougherty, 2005). Cultural competency involves making hypotheses, rather than premature judgments about culturally different people, knowing when to generalise and went to individualise (Dougherty, 2005).

Collaborative consultation involves empowering team members to contribute equally; but there is a critical difference between taking part in an empty, ritualistic, or compliant way, and having real power and commitment to affect the outcome of an involved process (Leonard \& Leonard, 2001; Stige, 2006). Cultural sensitivity, which is essential to the work, grows from knowledge of one's own cultural biases and communication style and how that can contribute to the development of power relationships (Conoley \& Conoley, 1992; Crothers et al., 2008; Dougherty, 2005; Stige, 2002). Prior assumptions can be invisible or preconscious, deeply rooted, resistant to change, and have a profound effect on our interactions with others (Leonard \& Leonard, 2001; R. M. Smith \& Nevin, 2005). 
Being aware of cultural differences in communication styles is essential to successful consultations (Hanft \& Place, 1996). Various ethnic and disability groups have different values regarding levels of eye contact, interpersonal space, use of non-verbal communication (e.g. nodding and smiling), how much self-disclosure is appropriate or acceptable, the loudness and tempo of verbal interaction, interruptions or pauses during the conversation, directness during conversations and so on (Brown et al, 2001). Using active listening techniques with all cultural groups in order to provide feedback regarding what has been communicated and to demonstrate understanding is essential (ibid). Acknowledging and exploring differences to gain understanding of the ways individuals can most usefully and productively share information will lead to more meaningful relationships, and reduce the risk that the consultant will feel and/or be viewed as an “outsider".

An important role for consultants can be to help build schools' capacity to meet the needs of an increasingly diverse population (Crothers et al., 2008). Consultants also need to be aware and respectful of culture-specific needs of school staff and students (Hanft \& Place, 1996). Keen observation skills are essential to gain knowledge about classroom and school etiquette which might be explicitly expressed or implicitly understood. The concepts involved are too numerous to list but include asking to be introduced to all students so the context for the visit can be explained in reassuring and age-appropriate language; knowing not to talk about students when they or others might hear, unless it is appropriate to include them in the conversation; being unobtrusive and knowing how to respond and redirect students who are distracted by the music therapist's presence; asking whether it is acceptable to take notes, and so on. Finding the most appropriate way to communicate with team members, determining whether interpreters are required, whether someone might have hearing or other difficulties, how people prefer to be addressed (i.e. whether first names are appropriate) and so on contribute to cultural safety (Hanft \& Place, 1996).

Crothers et al. (2008) note that a common problem affecting culturally diverse students is the difference between the values and expectations of home and school. School partnerships with families are therefore essential. Differences in traditional roles and understandings, and individual perceptions and agendas of team members can affect 
outcomes (Pellitteri, 2000; Register, 2002).

The perceptions and values of educators have more influence on student success than any inherent characteristics or differences individuals have (R. M. Smith \& Nevin, 2005). When a person experiences impairment, while it is a real and important part of daily life, it imposes considerably less handicap than what society imposes due to assumptions of deficit and incompetence (ibid). Examining relationships between the individual, the educators, and the culture that defines the tasks and settings within which the individual is required to perform is therefore crucial (R. M. Smith \& Nevin, 2005; Stige, 2002). Pellitteri (2000) found that teachers and therapists can both benefit from collaboration and discussion of approaches but notes that therapists interpret and respond to students' behaviours differently. Behaviour which is viewed as disruptive in class, for example, can be understood by the therapist as an expression of a child's psychological and emotional needs. He posits that music therapy consultants can assist teachers to adopt a therapeutic philosophy and create a therapeutic milieu in the classroom, by explaining and modelling alternative frameworks for understanding psychological dynamics, but cautions that teaching staff need to be receptive to learning different ways of working with students (ibid).

However, while traditions and culture are usually to be celebrated, they can also be inhibiting (Stige, 2002). Register (2002) noted that the culture of an organisation and roles of participants prior to the consultation may affect the collaborative efforts of the team and overall outcomes. Individual perceptions and bureaucratic agendas of team members can hinder the success of collaborative effort (Register, 2002). Collaboration requires people to work in new ways, and challenges them to truly understand the other's world view. This is not always easy particularly where an existing culture of how to work with families or classroom teachers prevails (Kelly, 2008).

The Treaty of Waitangi, the founding document of Aotearoa New Zealand, with its emphasis on partnership, inclusion, and 'standing alongside', provides a foundation for inclusive education (O'Brien \& Ryba, 2005) and logically for consultation activities. Kaupapa Māori pedagogy can help too, as it is a way of understanding situations and people in relation to the social, historical, cultural, physical and spiritual environment in 
which they develop (Annan, 2005). When consultation and collaboration are viewed as human relationships, the respect, dignity, and welfare of the parties involved become paramount (Dougherty, 2005). Consultants need strong interpersonal skills which allow them to listen, adapt to the cultural background of the consultee, and to be able to determine sometimes whether the consultee is demonstrating resistance or displaying behaviour appropriate to his or her cultural background (Crothers et al., 2008).

Cultural differences can be viewed as fresh perspectives, rather than impediments (Dougherty, 2005). Threlfall (2005) argues that a working knowledge of the underlying values and beliefs at work in each classroom will help ensure that music therapy programmes are implemented, and that students receive the full benefit of therapy interventions. Music therapists working with children who have special education needs share with other team members at least some elements of 'special education culture'. Finding commonalities (Ingraham \& Meyers, 2000) and drawing on similarities is a helpful starting point (Yehuda, 2002). Teachers who hold similar beliefs and values about education are more likely to collaborate (Leonard \& Leonard, 2001).

\subsubsection{Team Members' Use of Music}

Specialists in a variety of professions, including parents and other educators, are using music to support students in special education programmes, and increasing numbers are requesting assistance and professional development opportunities from music therapists, to enhance their work. O'Regan (2007), for example, who is a classroom teacher, psychologist and music adviser in New Zealand, encourages teachers to use music to improve achievement. The music work she has done with children who have special education needs has been heavily influenced by a number of eminent music therapists, and O'Regan declares that it has been the most enjoyable and often the most exciting of all her experiences as an educationist.

Lefevre (2004) applies music therapy principles and techniques in her social work practice alongside other creative, non-verbal modes of expression and communication. She argues that many music experiences can be incorporated into social work programmes by non-musical practitioners. Positive interactions allow a relationship of 
trust to develop, providing the child with an experience of safety and enabling the worker to gain both a better understanding of the child's responses, needs and views, and to carry out appropriate direct interventions (ibid). Lefevre suggests the worker's own comfort level with playing and singing and lack of self-consciousness is crucial in modelling for the child the aim of expression and communication rather than aesthetic achievement. This suggests a music therapist's involvement would be helpful although Lefevre noted that the music therapy literature did not appear to have informed and enriched direct social work practice in the way that play and other creative arts therapies had.

Music programmes with parent and child attending result in greater communication between parent and child and strengthening of the relationship (Archer, 2004; Berger \& Cooper, 2003). Yet children's musical play is often not valued (Matthews, 2000, in Berger \& Cooper, 2003), parents often disregard sound exploration as unrelated to music (Fox, 1989, in Berger \& Cooper, 2003), and children's musical play can be extinguished when adults correct children's actions (Berger \& Cooper, 2003). In contrast, Berger and Cooper found that adult willingness to participate in child directed activity, encouragement and valuing of all musical behaviours, providing adequate time and offering appropriate tools, enhanced play. They concluded that educators should avoid imposing traditional musical ideas on children's musical play through continuous corrections.

Although it seems clear that some staff or carers can be supported to successfully implement planned music experiences, others may lack confidence when initiating music activities. Nevertheless, regardless of whether music is being taught for its own sake or being used as a tool to meet non-musical goals, it is essential that the facilitator has a degree of musical skill and confidence. Some have argued that generalist primary classroom teachers in New Zealand and Australia lack competence and confidence to teach music beyond the simplest level because of their own poor arts experience at school and inadequate teacher training (Jeanneret, 2006; Webb, 2005), and that there has been a lack of well designed and taught music education programmes in primary schools in this country (Orams, 2003).

Nevertheless, musical confidence can have a more profound impact than formal training 
(Giordano, 2002; Jeanneret, 2006). Giordona's view is that "one of the greatest fears that teachers face is their perception that one must be an art specialist, that one must be 'talented' in order to have an arts presence in the classroom" (Giordano, 2002, para. 3). Teachers do not need to be proficient in the arts to employ them in the classroom, but do need to accept that all people have an innate capacity to play creatively, and to understand the importance of the work for their students and the role it plays in enriching the curriculum (ibid). Music consultants could therefore make a real difference to the effectiveness of the generalist in relation to music education (Jeanneret, 2006).

\subsubsection{Team Members' Availability}

Regardless of their skill or enthusiasm levels, supporting team members to use music in their work in schools might not be easy. Team members may be under considerable time and energy constraints and might not be able to add another major task to their workloads (Cardno, 2003; Hartas, 2004; Sanders, 2003). Educators resist collaboration because they feel threatened and/or are avoiding conflict; are competitive; have time constraints, or fragmented vision (Leonard \& Leonard, 2001). However, as noted earlier, when they have common goals or similar beliefs and values they are more likely to collaborate.

Pasiali (2004) had difficulty recruiting parents to be involved in her study which investigated the use of songs with autistic children. She suggested that some may have felt unable to meet her criteria, i.e. to identify a specific high frequency behaviour occurring daily that might be suitable to address through prescriptive song. However, she also argued that a twenty-eight day commitment to recording data may have discouraged parents from becoming involved. Nevertheless, none of the three sets of parents who eventually opted in indicated the data collection was tedious or difficult. In addition, although this study did not involve them in the implementation of music therapy treatment, two of the three parents continued to use music experiences when it concluded. Those parents were likely to be highly motivated and dedicated caregivers, who were possibly already musically confident.

Although the aim is to work with teachers, participants in the current project are likely to be teachers' aides who are overworked yet eager for support and therefore to participate 
in what will be a time consuming undertaking. Tutty and Hocking (2004) captured the experiences of teachers' aides working in regular classrooms with children identified as having high needs, in New Zealand. They described

- Work that was physically demanding;

- Being unprepared for the responsibilities, and relationships that developed with the students;

- Feeling as if they had no choice or control;

- Feelings of loneliness and isolation;

- Feeling guilty for taking time for simple and necessary routines such as going to the toilet;

- Feeling at risk of criticism, even when they believed their actions were safe;

- Being in a mothering role, sensing the students' needs and feelings and embodying their feelings as their own;

- Feeling guilty as a result of the child's behaviour e.g. when they are difficult to console;

- A profound commitment to the student;

- Taking home work preparation, worries and concerns;

- Difficulty relinquishing responsibility.

Adding to their stress by asking them to implement and closely monitor music strategies could be counterproductive. On the other hand, the musical interaction might be helpful for students and teachers' aides. For example, one of Tutty and Hocking's participants described not knowing what to do at times, and the use of silence as a retreat... "I might put (the child) on the swing and push her a bit. And not talk much" (p.5). In contrast, another participant described singing with her student regularly as a way of comforting them both when times were tough.

Tutty and Hocking (2004) also emphasise the importance of professionals taking time to support each other in ongoing ways. They describe teachers' aides "catching people on the run" in an attempt to discuss concerns, a situation which left them feeling undervalued and less important than other professionals. They write that "making a difference may depend on teachers' aides perceiving there is mutual respect and shared understanding of their reality because this is the starting point of working 
collaboratively" (p.7). On the other hand, they caution that "working directly with teachers' aides as the primary source of information about the student and recipient of education and behavioural strategies may perpetuate a situation of teachers being unable to take responsibility for students" (p.7). While they argue that the current model of funding makes finding a solution difficult, they advocate strongly for professional seminars that require teachers' aides and teachers to attend together. They logically conclude that having a teachers' aide full time with a student may be counter-productive and suggest inclusive strategies to reduce their dependence on one adult. Classroom music programmes adapted as inclusive practices, and smaller music group work programmes, might help to address this issue.

\subsection{Summary of Literature Review}

The social model of disability and the inclusive model of education focus on students' abilities and recognise that students who have special education needs can learn alongside their peers. Educators are now employing ecological approaches which consider the impact of the physical environment and social and cultural systems on student participation and learning. Specialists are encouraged to collaborate with teachers who support students who have diverse needs, and to focus on removing barriers to learning and development rather than on 'fixing' students. Therapists throughout the world from a range of disciplines have described difficulties or inefficiencies relating to the provision of indirect service to students in inclusive education settings. However, the benefits of providing service to teachers and others who support students who have special education needs have also been widely reported.

To date however, music therapists have predominantly employed a clinical model of music therapy in school settings, which involves withdrawing students from classrooms for treatment and highlights their pathological differences, thus reinforcing the medical model of disability. Nevertheless, the need for music therapists to consider contextual and cultural issues in music therapy has been articulated, and it is now acknowledged that music therapists work in and with communities, not just in clinic settings. Research has demonstrated that clinical music therapy can meet a wide range of children's 
developmental needs and support learning. Students' strengths are highlighted in music therapy settings and music therapy assessment can therefore highlight ways in which music might be used to support students in other contexts.

Music therapists have begun to write about the need for consultative models of music therapy. However, although music therapists collaborate with other professionals, provide resources, facilitate workshops and provide other in-service training, there are few examples of the process of consultation in school settings. The few available reports regarding music therapy school consultation are positive, but most relate to direct work which is observed by consultees or indirect work with music teachers or early childhood teachers rather than general educators in school settings. Further, it seems that consultation work is more likely to take place with educators who support students described as 'high functioning'. On the other hand, those who work in early childhood settings describe opportunities for working in natural environments with teachers, parents or other caregivers and provide examples of processes which are linked with theoretical perspectives such as 'mediated learning experiences', include practical strategies such as the use of video evaluation, and demonstrate that team members can implement music therapy interventions successfully.

Consultation from an ecological and cultural perspective suggests that consultants need strong interpersonal skills to build collaborative relationships which will in turn enable them to empower others to use music in their work. An awareness of the impact of one's own culture and respect for the cultural values of others will be fundamental to the consultation process. Meanwhile educators are interested in using music with their students and are likely to benefit from the support a music therapist can provide.

While it is clear that more research is needed, the reports regarding music therapy consultation are positive and there is an opportunity for expansion of music therapy services in this field (Kern, 2004; Register, 2002). However, music therapists need education regarding the potential benefits of collaboration and consultation (ibid). It is time to investigate music therapy school consultation, to describe the process, and determine how the practice might be used and valued by team members who support students who have special education needs. 


\section{CHAPTER THREE: THEORETICAL PERSPECTIVES AND ETHICAL ISSUES}

\section{Introduction}

How do we solve a problem? We have a way of viewing the world (ontology) which gives us a particular frame for developing knowledge (epistemology), on which we build a theoretical perspective. This in turn guides our research process, plan of action, choice of strategies (methodology) and the techniques or procedures used to gather and analyse data (methods) (Crotty, 1998).

This study set out primarily to gain some understanding of the experience of music therapy consultation from the participants' perspective, and to describe process, and is therefore qualitative in nature. 'Seeking to understand' rather than to 'know' derives from relativist ontology, i.e. the philosophy that 'reality is socially constructed'. The epistemological stance is constructionism (see p. 53). The study draws on ecological and critical theories and uses the specific ethnographic methodology of action research. Figure 2 (p. 51) demonstrates how all the concepts in this paragraph come together.

In this chapter a brief history of music therapy research is followed by explanations of constructionism, ecological theory, and critical theory. This leads to a discussion about power issues and other ethical issues that would be encountered in the research. The specific methodologies of ethnography and action research, as well as methods and procedures, are outlined in Chapter Four (p. 69). 
Ontology: Relativist

Epistemology: Constructionism

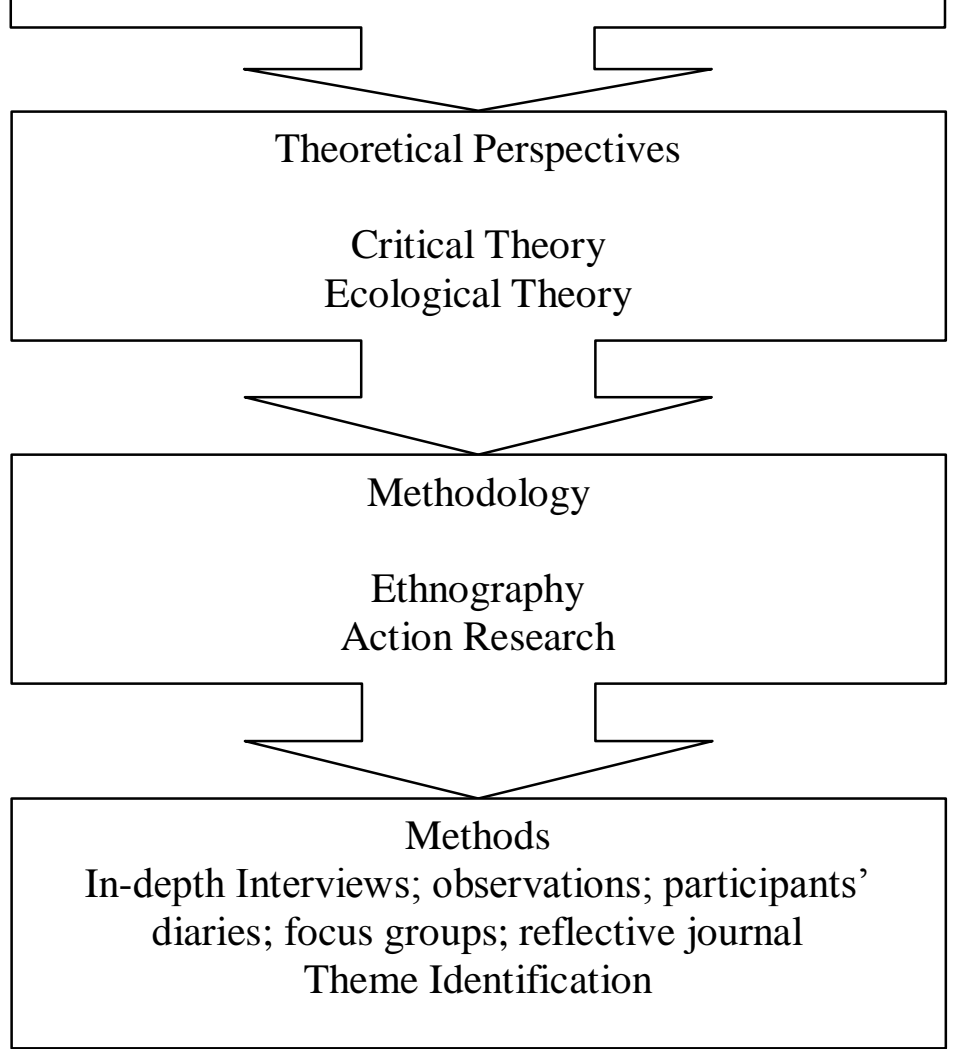

Figure 2: Elements of the Research Process (Adapted from Crotty, 1998, p. 4)

\subsection{Research in Music Therapy}

Research has always been considered to be essential for the profession of music therapy as it provides theoretical frameworks for practice and the evaluation of clinical interventions (Rickson \& McFerran, 2007). Rickson and McFerran note that early research into music therapy in special education drew heavily on behavioural principles and was predominantly positivist. In the positivist view of the world, objects have meaning independent of consciousness. Scientists are able to uncover 'objective truths' and the meaning already inherent in objects, by distancing their experiments from the influences of everyday life. Meaningful reality is considered to be value-neutral, unaffected by history and cross-cultural influences (Crotty, 1998). Positivist research often focuses on specific and minimised variables and involves large numbers of participants. Thus music therapists strived to demonstrate music therapy's effectiveness by objectively measuring observable change in clients that had been caused by musical 
interventions. The efficacy of music therapy for children and adolescents with psychopathology has been demonstrated (Gold, Voracek, \& Wigram, 2004). However, although positivist research can show that there is an effect as a result of music therapy it does not describe subjective effects (Smeijsters, 1997).

Twentieth-century philosophers challenged the positivist view by highlighting that science was limited by the 'way in which humans know what we know'. Social scientists in particular became dissatisfied with over-reliance on an objective stance and the need for researcher 'detachment'. Further, positivist research was noted to result in elitist academic research which had little relevance for the participants, or the contexts in which it was undertaken (Lincoln, 2001). Similarly, while acknowledging that the study of effects remains important, music therapists have become increasingly interested in naturalistic research which enables them to capture their clients' subjective experiences and to describe personal processes. Aldridge (1993) argues that music therapy is a transpersonal process, and therefore what happens cannot be separated from the therapist. Music therapists are challenged to demonstrate the ways in which meaningful change occurs for patients and their families (ibid).

Stige (2002) suggests music therapy 'clinical research' focuses on both process and outcome and aims to improve practice through the development of theories on practice, and to support practice through documentation of its effect and value. Music therapy researchers are asking more complex questions and increasingly rely on in-depth qualitative analysis and description that can capture the richness of clients' experiences and/or interpret the musical dialogue, which is the primary communication mode between the therapist and clients in music therapy sessions. Qualitative music therapy research has been presented as a distinct form of scholarly inquiry published in the English language literature for more than twenty years, and a clinically relevant body of knowledge has now been generated (Aigen, 2008).

However, the study of clinical practice also needs to include study of social and cultural practice - "the researcher needs to know (as much as possible of) what the actors know... all agents are involved in acts of meaning-making and interpretation” (Stige, 2002, p. 255). Yet Stige found, at that time, ethnographic perspectives were rarely integrated in 
music therapy clinical research. He argued that music therapists' self-understanding and sensitivity would be served with the establishment and cultivation of a tradition of music therapy research which is informed by ethnography. Moreover, he notes that while many music therapists might believe that endeavours towards social change are beyond the responsibility and competence of a music therapist or clinical researcher, others argue that a focus on health and well-being of people cannot neglect the issue of social change. Possibilities for music therapy to contribute to social and cultural change need to be examined in real-life settings (ibid).

Neither has music therapy developed a strong critical tradition - feminist and postmodern perspectives are rarely articulated in music therapy research (Stige, 2005a). However, Stige argues that "contemporary social and intellectual developments will probably become more influential in music therapy in the future and this will certainly increase the relevance of participatory action research" (p. 413). More recent discussion and debate regarding the concept of Community Music Therapy (see page 21) has led to an increase in literature which emphasises possibilities for community participation and opportunities for empowerment through music therapy (Ansdell, 2005; Proctor, 2004; Rolvsjord, 2006; Ruud, 1998; Stige, 2004a, 2005a, 2006) and examples of action research have emerged (Baker, 2007; Hunt, 2005; Smeijsters, 1997; Tait, 2005; Warner, 2005; Williams, 2006). Warner (2005) for example involved residents (all of whom had severe learning difficulties and challenging behaviour), and staff of a community home, as coresearchers in an action research project to evaluate group music therapy.

\subsection{Theoretical Perspectives}

\subsubsection{Constructionism}

The failure of conventional research methods to address meaningful programme change led to the emergence of constructivist inquiry (Lincoln, 2001). In contrast to the objectivist view, constructionist understanding is that meaning is not discovered but constructed through human interaction with the world. People perceive objects differently depending on the context of their viewing and can develop diverse understandings of the 
same phenomenon. "All meaningful reality (therefore)...is contingent upon human practices, being constructed in and out of interaction between human beings and their world, and developed and transmitted within an essentially social context" (Crotty, 1998, p. 42).

Only when people consciously engage with their world can they create meaning. Constructionists employ the concept of 'intentionality' which occurs when the mind becomes aware of something and consciously reaches out to interact with and shape that object. Therefore, although there can be useful or liberating interpretations, there can be no one true or valid interpretation (Crotty, 1998). Thus in constructivism objectivity (the world) and subjectivity (experience) are brought together and interact. Constructionists need to employ a large range of tools and methods; to 'dialogue' with, and interrogate the materials, i.e. to self reflect; and to interpret and reinterpret, eventually to offer multiple realities.

While there are a range of subtle differences in the ways practitioners employ both constructivist inquiry and action research, "there are several instances where action research and constructivism might be considered indistinguishable, either in theory or practice" (Lincoln, 2001, p. 126). Both focus on action, acknowledge constructed realities, and are committed to ensure the research is as useful to the participants as the researchers themselves (ibid). More information on action research is presented in Chapter Four.

\subsubsection{Ecological Theory}

While constructionist theory recognises the mutual effects that subject and object have on each other, and emphasises the researcher's role in constructing knowledge, sociocultural or ecological theory stresses the role that environmental factors play in human development, and thus the impact that 'context' has on social research outcomes.

Vygotsky (1896 - 1934) and Bandura (1977) both emphasise the influence of social and environmental factors on human development and learning. Bandura's (1977) main focus was on the importance observational learning which takes place when tasks are modelled, 
and Vygotsky's on learning though social interaction (Vygotsky, 1978). Vygotsky's argument that accumulated knowledge of children's communities is highly influential in their development and that learning is a reciprocal process situated in social and cultural contexts highlights the relevance of collaborative learning. Kurt Lewin (1952) also argued that behaviour is a function of the person and their environment.

Building on the work of Vygotsky and Lewin, Bronfenbrenner (1979) noticed significant differences between the challenges faced by families in varying socio-economic groups, and posited that these variations have a considerable impact on human development. Thus he developed an Ecological Systems Theory positing that human development is variously and highly influenced by five environmental systems, each containing roles, norms and rules. Significantly, Bronfenbrenner does not consider the individual a passive recipient of experiences in these settings rather that individuals help to construct the settings.

Firstly he describes a 'micro-system' which involves biological factors as well as immediate environments such as family, school and childcare facilities, peers, and local community. Secondly he describes a 'meso-system' which involves the interaction of micro-systems. For example, the quality of parent-child relationships at home is believed to impact on the child's teacher-student relationships at school. Thirdly, Bronfenbrenner describes an 'exo-system' where influences such as parents' work commitments can impact on a child's development. Fourthly, a 'macro-system' which involves wider cultures and subcultures such as ethnic, economic, political and disability cultures is included (ibid). Finally, and more recently, Bronfenbrenner added the idea of the 'chrono-system', which describes the patterns of environmental events over time, and the historical impact of significant life events such as divorce (Bronfenbrenner, 1989).

This research recognises that 'school' is a complex social system (involving home, school and wider contexts) and that students' learning and development is impacted by the physical environments they encounter and by the interactions they have with people in those environments (families, teachers, support staff, peers, specialist staff and others). Thus interventions have an impact on, and are impacted by, the entire social system. A music therapy assessment which takes account of the child's ability to manage and 
respond to music in naturally occurring environments, rather than in a clinical setting or withdrawal room, will be central to consultation work.

\subsubsection{Critical Theory}

The positivist argument that the social sciences should emulate the natural sciences was also rejected by critical theorists, who posit that social conditions are connected to the production and application of knowledge (Stige, 2002). Critical pedagogy for example therefore takes account of the social context of education (Freire, 1972). Critical theorists do not aim to establish and demonstrate theories about reality; rather they subject all theory to critical review to determine how well the theory withstands the criticism.

While the origins of critical theory can be traced to Kant (1724-1804) and Marx (18181883), the strongest influence arguably came from philosophers during the 1920s who were associated with the early Institute for Social Research at the University of Frankfurt am Main (often referred to as the 'Frankfurt School'). The revolution in Western Europe, which failed to improve the conditions of working-class people, and the rise of the Nazi regime, led these philosophers to note that groups who have social and economic power can strongly influence what counts as knowledge and reason (Crotty, 1998; Morrow \& Torres, 2002; Stige, 2005a).

Critical theorists recognise that people in dominant roles set the criteria for what is considered to be 'normal' and those with less power tend to accept the worldview of those in power, an ideology referred to as 'repressive trains of thought' (Stige, 2002). Habermas (born 1929) noted for example that being liberated from hunger and misery does not necessarily mean liberation from servitude and degradation. Repressed people can become dehuminised, lacking hope and even fearful of the autonomy and responsibility that come with freedom. In critical theory cultural norms, social processes, and power structures are highly implicated in real world problems.

A primary aim in the current study is to empower participants to use music with students who have special education needs, with initial action facilitated by the music therapist. It is anticipated that many of the participants in this study will not be attempting to use 
music with their students because they do not view themselves as 'musicians' (see p. 58). Some might recognise themselves as musicians because of their formal training or considerable experience with music but limit their interactive music making with students who have special education needs because they believe that requires the skills of an expert. It is anticipated that by recognising the concept of 'musician' as a socially constructed view, participants will be able to more readily engage in music making with their students. Further aims are for participants and the researcher to develop new perspectives of day to day school life; and to recognise and take advantage of the potential to effect change in agreed areas. New 'realities' will thus be created through collaborative work and become new forms of knowledge.

Critical theorist Freire (1921-1997) argued that language is the foundation of knowledge. He acknowledged that when communication is lacking, more powerful parties begin to dominate, dehumanize, and alienate and marginalise. Freire and Habermas both agree that "dialogue presupposes certain possibilities of communication that open the way for processes of emancipatory individual development" (Morrow \& Torres, 2002, p. 34). Noting that words are symbols of reflection and action, they argued that relationships of mutual trust can be built through empathic dialogue, and that analysis of dialogue in context can therefore reveal the origins of what has come to be known as 'truth'.

In the critical research context participants enter into dialogue with a shared desire for mutual understanding and recognition based on consensus. The aim is for the voices of all participants to be heard, valued, and incorporated into the findings. The very act of speaking makes the claim that the speaker has a right to speak, is sincere in wanting to be understood, and is speaking the truth (Park, 2001). A synthesis of comprehensibility, factual truth, sincerity, and rightness (practical reason) leads to valid knowledge (Morrow $\&$ Torres, 2002).

It is anticipated that highlighting participants' voices in this text will give credence to the development of new knowledge. However, critical theory acknowledges that the voices of less powerful people can echo the voices of people with more power, which in this case could be the 'expert' music therapist. Nevertheless, liberation is demonstrated not only in what people say, but in their adopting alternative forms of action. It is anticipated 
that participants in this study will be mobilised into interacting musically with their students, and will be able to demonstrate this by recording their actions in a daily diary during the follow up period. However, the process will be iterative; while the musical interaction might enable the development of new knowledge, the new knowledge will enable participants to determine the action they will take.

On the other hand, action "may represent blind action, rather than action which is informed by self-conscious awareness and analysis of one's own reality" (Gaventa \& Cornwall, 2001, p. 76). Once again, an aim of the current study is to use participants' voices to demonstrate their process of critical reflection i.e. ways in which they might act, develop new knowledge, analyse the consequences of that action and develop further knowledge. As a potentially more powerful actor in this situation, the most significant aspect of the researcher's learning is likely to come from witnessing and sharing in other participants' processes, particularly their ability to critically reflect on and thus make changes to their own situations.

However, power can also be viewed as positive; something that is grown from within and not limited by others (Gaventa \& Cornwall, 2001). Foucault (1977) suggested power is embedded in all social relationships and in all discourses, institutions and practices, and is not only related to knowledge production and sharing but to 'position'. Thus, rather than remaining with the idea that power/knowledge produces inequalities, he was able articulate a more productive view of power as "the right... to rediscover what one is and all that one can be" (cited in Gaventa \& Cornwall, 2001, p72). Foucault's theory highlighted possibilities for empowerment through action, and possibilities for action by some to enable further action by others.

\subsection{Empowering others to Musick}

Human perception of music is strongly influenced by cultural history (Stige, 2002). This research begins with an acknowledgement that the Western view of music as a cultural artefact produced by the elite of the community has led to a dominant discourse that limits opportunities for participation in active music making to a privileged few. It 
assumes that those who have not had formal music training and/or experience in music making with others in ongoing ways throughout their lives are likely to view music as an 'outcome' which results from skills they do not have. Traditions that cultivate musical perfection can promote exclusivity with music making being reserved for the select few that have particular talent and discipline (Stige, 2006).

Yet music is found in all cultures and in many non-Western countries singing and dancing is considered to be as natural as speaking. Further, while colonial New Zealanders have been influenced by historical and culturally determined elitist attitudes developed in their countries of origin, Māori arguably have a more 'natural' relationship with music viewing it as part of everyday life. Moreover, researchers have now found that the music we meet in our daily lives is a cultural development of proto-musical components first developed in mother-infant interaction (Dissanayake, 2001, in Stern, 1985; Stige, 2006; Trevarthen, 2000, 2002, 2005). Mothers and babies communicate through sound and movement with synchronicity and mutuality of body and voice, rhythm and melody. These authors have argued that humans have a capacity and basic motivation to make music from birth and one can therefore assume that that music should be accessible in various ways to all who choose to participate. The highly influential ethnomusicologist Christopher Small (1998), a New Zealander, argued that music is not a 'thing' but an activity, and that its meaning is produced through shared action in context. Thus he coined the phrase 'musicking'. In the act of musicking music and context reciprocally shape each other.

The act of musicking establishes, in the place where it is happening, a set of relationships, and it is in those relationships that the meaning of the act lies. They are to be found not only between the organised sounds which are conventionally thought of as being the stuff of musical meaning, but also between the people who are taking part, in whatever capacity, in the performance; and they make, or stand as a metaphor for, ideal relationships as the participants in the performance might imagine them to be (Small, 1998, p. 13).

Nevertheless, in the Western world music is considered to be an 'art', and is thus linked to power and privilege with the term musicality being used to indicate that a person has particular musical talents (Stige, 2002). Further, varying musical genres that are 
embedded in Western culture are often valued differently and the meaning of music is presumed to reside in the object of the music, or the performance. This emphasis has led individuals to deny or suppress their inherent ability because they have not had the opportunity or motivation to develop musical confidence (Austin, Renwick, \& McPherson, 2006) or have had it destroyed as a result of unhelpful interactions with others (Berger \& Cooper, 2003). Facilitating team members to introduce music to enhance students' development and learning is likely to involve supporting them to reframe their understanding of music from something that people trained and/or experienced create, to a communicative act that is natural and accessible for all.

It has been argued that music strongly influences our values and beliefs and is a powerful channel through which people develop their personal and social identities (MacDonald, Hargreaves, \& Miell, 2002). Music as a means of communication can mediate the construction and negotiation of developing identities in the same way that language can. Trevarthen (2002) posits that music functions to enhance the quality of individual experience and human relationships, and that its structures reflect patterns of human relations. The utilisation of the music therapy consultation protocol will therefore rely as much on team members developing their musical identity, regaining or developing musical confidence and/or refining musical skills so they can use music freely with students who have special education needs, as on their learning new skills from an 'expert'. A highly collaborative consultation approach can be considered to be effective when participants are able to sustain their use of music with students after the music therapist leaves the field.

On the other hand, 'music therapy' as defined in this thesis requires a highly trained music therapist to engage a child in the process of music therapy. Participants in this study will be made aware that music therapists could engage students in different and/or more in-depth therapy work; and participants' musical interactions with students will not be defined as music therapy. Nevertheless, they will be strongly encouraged to use music with their students, and to think about how their musicking might assist students' development and learning. While highlighting this differentiation will be important, it might also to contribute to the music therapist being perceived as an 'expert' in a more powerful role. 


\subsection{Knowledge and Power}

Knowledge and power are closely linked (Gaventa \& Cornwall, 2001; Park, 2001) and 'power relationships' can occur in a variety of ways. For example, a power relationship is evident when people are able to make others do something they wouldn't normally do, when they minimise or prohibit others' involvement, and when they keep information from others. Knowledge is a resource which is used in decision making. Powerful people can make use of knowledge, regardless of who produced it and the impact it might have on others, to make changes in the lives of less powerful people; can control the production of knowledge to create an agenda that excludes others; and can control the production of knowledge so that it gives an inaccurate or incomplete picture thus influencing others' consciousness of the agenda in the first place (Gaventa \& Cornwall, 2001; Park, 2001).

University academics are often perceived as being the top end of the hierarchy of knowledge and power and are therefore presumed to 'know better' and to be able to make decisions regarding the actions others need to take. Team members might anticipate an expert is coming to 'teach' them. Further, while there will be a range of professionals in each team, many of the team members are likely to be teachers' aides who often experience a position of subordination and weakness. It will be important to build positive relationships with them to help them acknowledge their skill and experience, to participate in the co-construction of knowledge, and to develop a new awareness of and ability to act for themselves. The aim of the study, to 'empower' participants to use music with their students, will be a constant reminder for the music therapist/researcher to hold the position of learner. Sympathetic and enabling professionals, who are aware of the effect of their positions, can work alongside others and, by listening and learning, shed their dominant position (Gaventa \& Cornwall, 2001).

Various forms of knowledge are used in the construction of truth. For example, participatory research can create 'interpretive' knowledge by describing, explaining and bringing understanding to a phenomenon. The interpretive process calls for the complete involvement of participants who bring open attitudes in order to 'come to know' the 
phenomenon on its own terms (Park, 2001). Park writes that "interpretive knowledge is synthetic and integrative, rather than analytic and reductive” (p. 83). New understanding through interaction produces changes in the parties involved. Interpretive knowledge can therefore contribute to the development of empathic relationships and relational knowledge.

In the current study it is expected that engaging in music-making will lead participants to develop new understandings, and the confidence to work independently. Teams at each school will engage in their own projects, collaborating with the researcher to plan and implement music experiences for students who have special education needs. The researcher will encourage shared ownership of the research to promote maximum participation. These projects have much in common with Participatory Action Research (PAR) (see Chapter Four) because of the emphasis on shared problem solving through the direct and active involvement of all parties (Park, 2001; Stige, 2005a) and will build on relational knowledge.

Relational knowledge gives us power through a sense of solidarity and is important in strengthening communities (Park, 2001). Habermas' theory that being rational involves drawing on objective knowledge as well as acting according to moral order and personal experience, contributed to the concept of 'relational knowledge'. However, Habermas focused on 'sincerity' as the factor that enables bonding to occur and consensus to be reached, rather than the sharing of feelings and experiences (ibid). Nevertheless, affectivity enables us to understand the world and to 'know' each other as friends and lovers (ibid). Relational knowledge "resides in the act of relating and shows itself in words, expressions, actions and other forms of doing relationship" (Park, 2001, p. 86) and grows from reciprocal interaction such as that which occurs during music therapy improvisational exchanges. It involves respect, caring, sincerity, authenticity and trust, which are concepts familiar to therapists.

Reflective knowledge, which enables us to have the confidence to change our world, will also be highly relevant in this study. Participatory research such as this seeks to "strengthen community ties and heighten transformative potential through critical consciousness which can be thought of as a form of knowledge as well" (Park, 2001). It 
aims to create new kinds of emancipatory knowledge - participants can use their 'insider' knowledge to generate new solutions to their problems without the support of expert professionals. Intensive commitment to produce change requires participants to engage in conscious reflection, to critically analyse and evaluate their personal situations, the morality issues they face and the values they hold, and to determine the action that is required. Nevertheless, reflective knowledge can also be the product of group activity, and as such reflects the capacity that participants have to "act with determination, confidence and resourcefulness that is made possible by, and expressed in, the interaction and interdependence embedded in human communities" (Park, 2001). It is emancipatory and practical in that people are considered to be free and autonomous to act on their own behalf, albeit in the context of interdependent relationships (ibid).

Action is a fundamental of reflective knowledge - the experience of action produces the knowledge. In the current research it is anticipated that high levels of involvement in the research means the participants will not only become more self-determining, but will be able to make sense of the data in relation to their own observations, insights and values (Bray, Lee, Smith, \& Yorks, 2000) and thus to continue to learn after the 'researcher' leaves the system (Bray et al., 2000).

This study therefore draws on critical theory which acknowledges that power and knowledge are closely linked. I aim to minimise dominance by remaining alert to the existence of power relationships, adopting the stance of learner, and involving participants fully in their team projects. The complete involvement of participants and a cohesive team approach will enable interpretive and relational knowledge to emerge and critical reflection will give us confidence to take action.

\subsection{Research Ethics}

Social science research involving human subjects brings with it significant ethical responsibilities. This research has the approval of the Massey University Human Ethics Committee (HEC: WGTN Application - 05/59), and during the planning and execution of the work I referred to Massey University's Code of Ethical Conduct for Research 
Involving Human Subjects (Massey University, 2004), and the Code of Ethics for Music Therapists in New Zealand (New Zealand Society for Music Therapy, 2006). This would enhance my ability to view the research through the eyes of the other participants and to ensure the research was ethically safe.

Research, the practice of music therapy, and the practice of consultation all carry ethical responsibilities. Consultants need to be culturally competent, to act with integrity (e.g. maintain confidentiality) adhere to an ethical code, engage in consultation only within their professional limits, maintain personal and professional growth, have the intent to help, effectively cope with the stress of consultation, avoid manipulation of others, use effective writing skills, and use power for legitimate purposes only (that is, using one's skills to influence others appropriately) (Dougherty, 2005).

Different research contexts demand different considerations (Tolich \& Davidson, 1999). Five core ethical principles for research are presented below with discussion regarding their relevance to this study. Further discussion regarding ethical considerations in consultation is offered in the action research cycles and in the discussion (p. 273)

\section{First Do No Harm}

At first glance research questions often seem innocuous but it is highly likely that any research involving humans will pose risks in one way or another (Tolich \& Davidson, 1999). By aiming to become close to people in order to learn about their experiences and to empower them to make changes in their lives, the current research had the potential to uncover information or to have participants see things about themselves that might be uncomfortable. A tension existed between the need to describe the 'problems' that people were experiencing while remaining sensitive to how that exposure might affect them. The participatory nature of the research helped in this regard - participants were able to identify problems themselves and in doing so were comfortable to articulate them in the research context. In articulating problems they were more able to plan and act to reduce or eliminate their difficulties and thus to rebalance the risks and benefits. 


\section{Voluntary Participation}

Participants in Stage One, were eight registered music therapists. Participants in Stage Two, from each of the four school teams, and who all signed consent forms, were:

1. Tim's parents, classroom teacher, three teacher's aides, and the .2 ORRS teacher.

2. John's parents, his special needs teacher, two teacher's aides, classroom teacher, special education needs coordinator, and speech and language therapist.

3. Cathy's parents, classroom teacher, neurodevelopmental therapist, visual resource teacher, special education advisor, and teacher's aide.

4. Liam's parents, principal, classroom teacher, .2 ORRS teacher, special education advisor, speech and language therapist, and two teacher's aides.

Before participants agree to take part in research, they need to know what is involved, and their participation should be voluntary.

In stage two, a call for expressions of interest helped to identify potential participants who were then provided with information sheets. Help was sought from the MoE Special Education Team Leader: Research, Professional Practice, to support the ethical process of recruiting special education team members who provided indirect support to students (Rickson, 2009b). However, the iterative nature of action research allowed stage two participants to consent only to the principles, procedures, and aims of the study. Participants were given as much information as possible (see information and consent forms in appendices) and close negotiation and collaboration ensured that they were fully informed throughout the research process and as co-researchers they had the opportunity to change the direction of the research as needed.

However, while participation was voluntary, team members who desired to be involved in a music therapy consultation would be unlikely to have the opportunity to do so without being involved in this research. Their choices might therefore be limited. It would be inappropriate to use this knowledge to coerce people to be involved. On the other hand, rather than being coercive, the research could be viewed as motivating when potential participants recognise the likely benefits of being involved in their own way. 
Participants were free to withdraw consent for their data to be used in the development of the consultation protocol until the time they had completed interviews and reviewed their transcripts. They were also engaged in informal discussion regarding the way the data from their projects would be interpreted but could not be fully informed about this aspect of the work. For example it would be possible to discuss with the first team how findings from their project might be interpreted in the music therapist's first cycle of learning. However, the nature of action research is such that the ongoing cycles of learning led to accumulative understandings; i.e. findings would not emerge specifically from an individual team. In the development of the consultation protocol therefore, the music therapist researcher would be the 'expert', and participants' limited knowledge of music therapy theory and practice meant they would be less able to directly contribute to this aspect of the work. While there was little that could be done about this dilemma, participants were given the opportunity to consider the issues and in giving informed consent to participate agreed that, for them, the benefits of being involved in the music therapy project outweighed the risks.

\section{Avoid Deceit}

Deliberately deceiving informants is unethical and constitutes serious misconduct (Tolich \& Davidson, 1999). Participants in this study were not deceived in any way either through the provision of misleading information, or the withholding of relevant information.

\section{Anonymity and Confidentiality}

Participants in the study were not anonymous. That is, the close liaisons between researcher and participants meant that the researcher always knew who said what. On the other hand, throughout this research every effort was made to maintain confidentiality, and participants' identities were not made public. Pseudonyms have been used throughout this thesis and all associated publications and presentations.

Nevertheless, the small size of the music therapy and special education communities meant that it would be likely that at least some of the participants would be identifiable 
both within and outside the school setting. Participants were informed that confidentiality could not be guaranteed, and issues were negotiated as they arose. For instance, in stage one of the study demographic information was not requested from music therapists because of their small number and the high risk that they might be identified. Nevertheless, participants offered information regarding the type of facilities they had worked in and some referred to specific and easily identifiable client groups they had worked with. During initial analysis procedures it became clear that it would be helpful to include some of this data to contextualise other information they shared. Participants were informed of the increased risk of identification, and were given the opportunity to refuse or to re-consent to the use of their interview material (see Appendix 6).

In stage two, work took place in very small communities, and involved Māori. Both groups expect people to share information about their background and connections and there was naturally some tension around the need to maintain confidentiality. Ethical principles are not rules that must always be rigidly adhered to, but they provide checks for the researcher (Tolich \& Davidson, 1999). It was necessary to negotiate particular issues with team members and to take a pragmatic approach. For example, at small country schools team members agreed it was more appropriate for me to be introduced to all staff and students so I could explain my presence sensitively, rather than allowing speculation and gossip to develop. Further, there was a risk that locals would wonder and talk about the 'stranger' staying at the only motel in town and visiting the primary school. It would be natural for young children to share that 'the lady was with Tim' and for Tim, the only child with special education needs in the school, to be easily identified. In another example, a music therapy colleague who had recently moved to a remote district was asked to do further work with one of the students involved in the study. The team deemed it important to share music therapy information, but in doing so the student was exposed as a participant in the research project. Action needed to be pragmatic but also carefully considered and negotiated with those who were affected by it.

\section{Qualitative Rigour}

An ethical study produces useful findings as a result of rigorous systematic enquiry. As a qualitative action research project, the study involved collection of evidential data, a 
search for understanding within context, and the production of a descriptive and interpretive research report. The extensive data provided opportunity for 'thick description' (see Ethnography, p. 71) situated in the life context of the participants, thus enabling their actions to be understood in light of their experiences. Further, data was gathered from multiple sources and triangulated (see p. 102).

\subsection{Summary}

Genuine concern was maintained for the welfare of participants throughout the whole of the research process. Harm was avoided at all times and the coercion, exploitation, abuse, misplaced negative (malicious or malevolent) judgement or misuse of power or privilege was avoided. During the research process I consciously addressed ethical issues in both clinical and research supervision contexts. This section has provided examples of some of the issues encountered and the way they were managed. 


\section{CHAPTER FOUR: METHODOLOGY AND METHODS}

\section{Introduction}

This chapter describes the methodology (research process, plan of action, choice of strategies) and methods (techniques or procedures used to gather and analyse data) used in this study. It begins with an overview of procedures followed by introductions to ethnography, action research, and participatory action research. Then the researcher's personal position and initial assumptions are presented. Finally the choice of methods is explained before specific procedural steps are outlined.

\subsection{Research Overview}

As indicated in Figure 1, p. 8, the research had three stages.

1. Stage one interviews with music therapists acknowledge the importance of drawing on existing knowledge and assisted the development of an initial protocol for use in stage two.

2. a) In stage two four consecutive consultations with special education teams enabled the protocol to be developed further. Each team planned their music projects and engaged in their own action research, (see Figure 4, p. 91), and were supported to develop confidence and skills as necessary, with the aim that they would be empowered to continue to develop their work through cycles of learning after the researcher left the field.

b) The researcher analysed and reflected on the outcomes of each consultation to determine what changes, if any, could be made to improve the developing protocol (see Figure 5, p. 104). In line with the Action Research process, findings from each consultation enabled a cycle of learning to take which informed the next consultation.

3. Finally in stage three, the potential and limitations of the music therapy protocol were drawn from all data.

A diagram depicting the cycles of Action Research is presented in Figure 3, p. 70. The diagram should be read from bottom to top. 

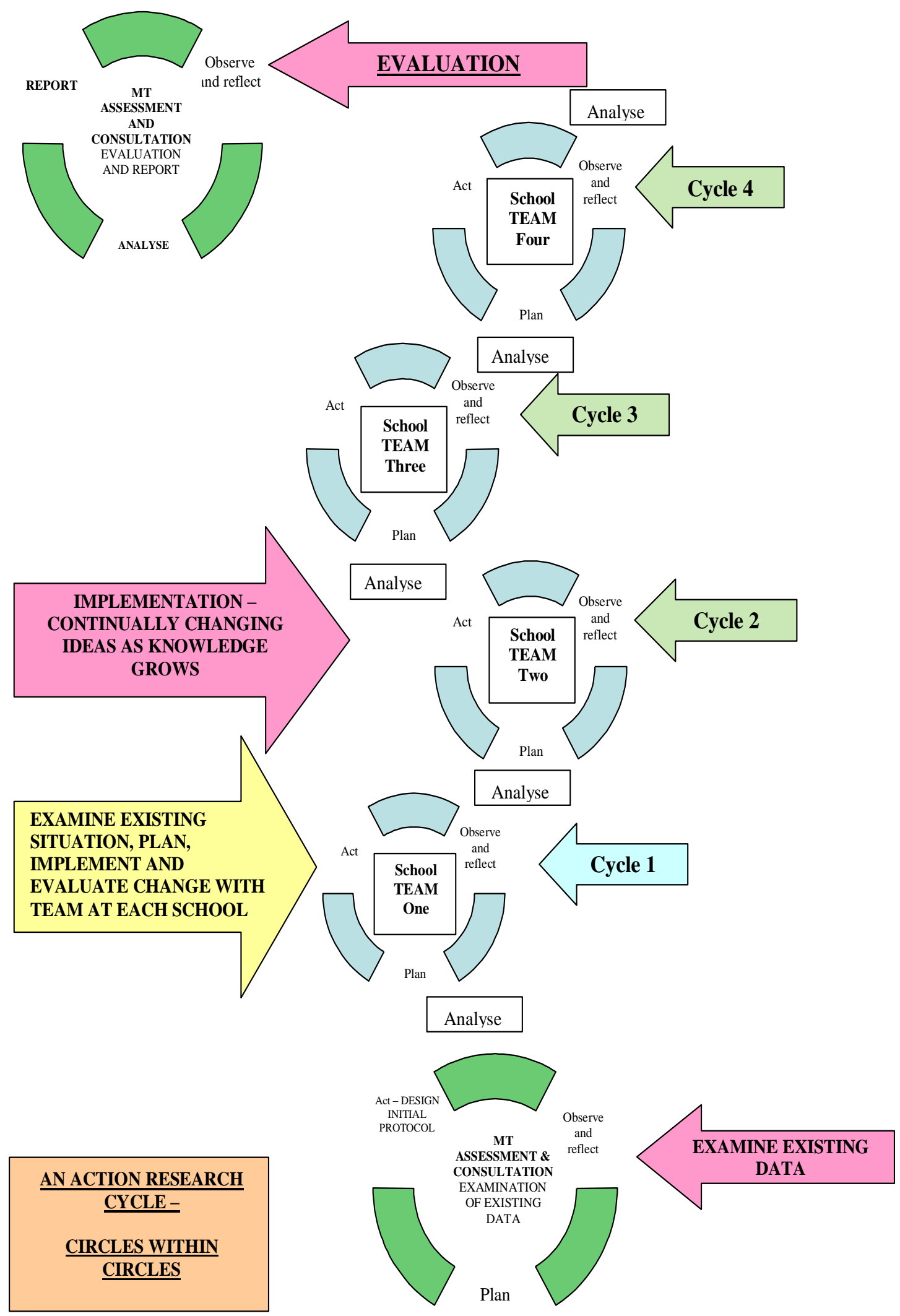

Figure 3: Action Research Cycles 


\subsection{Methodology}

\subsubsection{Ethnography}

Critical theorists stress the need for research to focus on emancipation and empowerment of individuals; and for researchers to acknowledge and accept social responsibility by undertaking research that is normative, practical, and self-reflexive (Stige, 2002). Critical researchers therefore often use an ethnographical approach which involves prolonged engagement in the field in order to develop close relationships with participants, with a view to understanding their world. Ethnography developed from the interest of colonial powers to describe the natives in their colonies. Joseph Banks for example offered the first detailed ethnographic account of Māori (cited in Beaglehole, 1963). Now various forms of ethnographical research are undertaken in a range of 'everyday' settings including schools and hospitals. Bland's (2003) critical ethnography of residential aged care in New Zealand is one such example.

A deep and rich understanding can be obtained through immersion in the culture of another. Ethnographers are able to view the world through the eyes of the participants rather than his or her own cultural lens; are able to remain focused on the issues that are important to participants; and apply findings in a way that benefits those participants and the community in which they work. On the other hand, ethnographers recognise that regardless of how open they try to be, the assumptions they bring to their work will impact on their research. Cultural understandings are deeply embedded. Therefore, in order to understand 'others' it is necessary to assume a position of humility and willingness to learn, to closely examine assumptions before beginning the research, and to continue to examine how and to what extent the researcher's assumptions are influencing the work. Ethnographers therefore need to outline their own cultural perspective, their beliefs and values, and to maintain an ongoing internal dialogue regarding the influence they have on the research process in order to develop 'authenticity' (K.E. Bruscia, 1998). In this thesis these are offered in paragraphs beginning on p. 77. 
Participant values and beliefs influence the role the researcher takes. Negotiated roles lead to various levels of participation and observation at different times in the process. In the current study participants' attitudes towards music, their perception of themselves as musicians, and their understandings and expectations about how they might be supported in their work would have an impact on the roles that the therapist/researcher played out. Ethnographical accounts therefore do not merely describe what happened, but include information about the various contexts in which situations occur, thus producing what is termed 'thick description'. Behaviour which is described in context becomes meaningful to an outsider and potentially leads to understanding. However, ethnographers acknowledge that in choosing the data that will be included, and in constructing the research account, they have considerable influence on the text that is produced. They therefore maintain a strong focus on self-reflection and self-criticism, and acknowledge the influence their values and beliefs have on the text (see Reflective Journal, p. 85). Further, the text does not claim to reveal the 'truth', but a representation of the truth as it appears in particular contexts.

\subsubsection{Action Research}

Action Research (AR) is one of the ethnographical approaches which can enable music therapy researchers to obtain a detailed and clear description of a music therapy process and to engage in rigorous investigation through systematic classification (Bunt, 1994). AR draws on critical theory and therefore emphasises the importance of critiquing and changing society rather than simply trying to understand and explain it.

Various writers have contributed to the development of AR. Dewey for example (1927, in Johansson \& Lindhult, 2008) argued that knowledge is created through interaction and testing the consequences of that action. Nevertheless, there is general acceptance that action research emerged primarily from the work of Lewin (1946) who described possibilities for research into the conditions and effects of social action using cycles of planning, action, and fact-finding about the result of the action. Findings from collaborative efforts within a mutually acceptable ethical frameworks can contribute to the goals of science (McKernan, 1991; Rapoport, 1970). 
AR provides a basis for learning and knowing which is born from everyday practice and thus narrows the gap between theory and practice (Abraham, 1994; Elliott, 1991; McTaggart, 1997; Piggot-Irvine, 2003a, 2003b). It "produces... knowledge which is contingent on the particular situation, and which develops the capacity of members of an organisation to solve their own problems" (Susman \& Evered, 1978, p. 601). It is a cyclic approach which begins when something happens which causes wondering (Newman, 1998), or there is a dissonance or discrepancy between what is happening and what we might expect will happen in practice (McNiff, 2005). The existing situation is examined before change is implemented, and the intervention is constantly evaluated through a process of critical reflection and dialogue with participants, leading to further or alternative action.

Each cycle involves investigation and analysis, planning and action, and evaluation and reflection (Cardno, 2003). The dynamic nature of action research means that the initial concern or idea should be viewed as a starting point only (Elliott, 1991; McNiff, 1988). Initial interventions are based on current understandings of the social and cultural context of the work and are modified during the research process. The immediacy of applying research findings to active intervention is unique to this methodology (Cardno, 2003).

Action research has been increasingly applied in international education and management research over recent decades. Thus it has grown to mean many things to different people and its meaning and function need to be reinterpreted as it is used in particular contexts (Cardno, 2003). For example over the years, practitioners have variously emphasised the role of critical theory in action research. Kemmis (2001) differentiated three kinds of action research in education settings, two of which are relevant to this study. The first is a 'practical' form in which "practitioners aim not only to improve their practices in functional terms, but also to see how their goals, and the categories in which they evaluate their work, are shaped by their ways of seeing and understanding themselves in context" (p. 92). Thus the current study would help me understand and change my music therapy consultation practice.

Also relevant is a 'critical' form of action research which aims to improve outcomes and self understanding as well as assisting practitioners to critique their social or educational 
work and work settings. This form of AR recognises that there may be collective misunderstandings about what people do, and aims to help practitioners to understand the way people and settings are shaped by history, culture, and discourse (ibid). Thus it was anticipated that team members in the current study would understand and overcome some of the social and historical barriers that impacted on their use of music with students who have special education needs.

Although AR is a broad term for a variety of approaches (Armstrong \& Moore, 2004; Reason \& Bradbury, 2001), several elements are common to all action inquiry models. Specifically AR should: 1) construct new knowledge on which new forms of action can be based; 2) ensure the research 'population' is central to the research process; 3) involve the systematic collection of data based on the experience of participants; and 4) focus on generating improvements in "professional practice, organisational outcomes, or social democracy" (Brooks \& Watkins, 1994, p. 12). Winter (1996, in Armstrong \& Moore, 2004) also highlights investigating and debating work in context, and collaboration, where everyone's view is accepted as meaningful, as characteristics of AR. In addition he suggests that distinguishing features of the approach include the ability of researchers to reflect critically and be aware of their own biases; to risk disturbance by submitting their own processes to critique; to articulate multiple possibilities rather than an authoritative interpretation; and to view theory and practice as mutually dependent and complementary stages in the research process.

\subsubsection{Participatory Action Research}

The Participatory Action Research (PAR) process has been described as "steps toward social change through a series of collaborative action-reflection cycles" (Stige, 2005a, p. 409) which, like other forms of action research, involve assessment, planning, action, evaluation and reflection. PAR aims to liberate participants by valuing individual knowledge and experience and empowering people to take action to make change in their own lives. It has been adopted by practitioners who hold the goal of fostering learning how to learn among participants as well as solving problems and generating valid knowledge (Bray et al., 2000). One of the aims is for participants to begin to define themselves differently and to realise that their circumstances are not necessarily 
inevitable. In the current study, the aim is for participants to begin to define themselves as musicians who are able to use music in a planned way to facilitate students' learning and development.

Participatory action research involves participants sharing ownership of the research and their voices are heard throughout the process (Stige, 2002). Stige (2005a) referred to the polyphonic nature of multiple perspectives, the likelihood that conflict and resistance might occur, and the need for the participatory action researcher to have excellent interpersonal skills and ethical standards. He argued that there can be problems with regard to participation in jointly owned research suggesting that participants might use the research process to promote an agenda in ways that lead others to lose sight of their own ideas and values. He warned that researchers must be committed to the values of critical research and actively monitor the work with the aim of minimising dominance. In the current research, the music therapy researcher's position would be one of equality with other team members both in clinical and research terms. Participants would work together with the common goal of improving a particular situation for a student.

Collaborative inquiries vary in the extent to which control over the research is shared among participants, including inquiry design, methodology, data analysis and interpretations, and dissemination (Bray et al., 2000). Bray et al. claim that although researchers aim to help the group become self-determining, the differences in their formal training inevitably influence the direction of the investigation. Shared values and visions do not necessarily mean that participants will have the same values and visions, but rather implies they are explicitly communicated to group members as they develop. While original values and ideas are held, each assessment of the situation provides opportunity to incorporate new facts and to guide future action.

To a great extent the PAR model seems to involve a transfer of skills and abilities. While each participant's experience is equally valid and useful, there can be a need for specialists to support teams because of a particular skill that they bring. The instigator might be differently resourced, and hold different knowledge. For example, music therapy consultants will bring expertise in the use of music with diverse groups of children, and experience of working in a range of special education facilities. However, team members 
know their system, the environment and the people who work in that context, i.e. they have experience and knowledge of that particular school's culture. Further, they bring individual knowledge and expertise from various lived experiences which can be invaluable towards practical intervention as well as the building of shared knowledge. Moreover, while collaboration might lead to a degree of collective knowledge, it is accepted that people will continue to experience, and therefore to 'know', different things. That is, multiple realities exist.

McTaggart (1997) differentiates 'involvement' and authentic participation, arguing that the latter means participants share in the way research is conceptualised, practised, and disseminated. He notes that in the cogeneration of knowledge participants may reinterpret and reconstitute the research methodology itself. Further,

the same group does not have to be involved in every aspect of the project... the action researchers are the people nominated as the core research group who are willing to see the project through to the final reporting stage (Cardno, 2003, p. 20).

Ritchie (1996) also refers to diversity among PAR participants and suggests that the instigator may come from outside an organisation and may be from a different subculture "if that person is better resourced and more highly educated than the other participants" (p.207).

Stige (2005a) notes that some participants may experience serious barriers to participation in the research process because they have limited cognitive or communication abilities. Further, while AR has a basic tenet of doing research with, as opposed to on or about people, and the researcher values the views, perspectives, opinions, prejudices and beliefs of the informants (Cardno, 2003; Heron, 1996)... "this does not mean being naïve or credulous - informants may lie or be deluded or misinformed themselves" (Delamont, 1992, p. 7). Rather the aim is to find out how they understand their world and the focus is on what actually goes on rather than what ought to go on.

The aim of PAR is to focus on an area of interest to the group (in this case music making with students who have special education needs) and for the research process to be a 
learning experience for all participants. In each of the case studies team members would be able to decide with the music therapist how they might use music to meet student goals, and monitor the effectiveness of their interventions. In this respect they would be involved in the design, data collection, and analysis of their own projects. Each team would be responsible for deciding how they used the information obtained from their individual 'music projects'.

\subsection{Action Research in the Current Study}

This study used two linked Action Research frameworks.

Firstly, I would use action research as I continued to develop my own practice in music therapy school consultation as I gained knowledge and skill from cycles of practice with four special education teams (see Figure 3, p. 70). Four cycles of learning would be constructed in turn after engagement with each of the teams.

Action research was also used with team members, to empower them to use music in a planned way with their students. Team members at each school determined what music experiences they would introduce, how they would do it, and the way in which it would be evaluated (see Figure 4, p. 91). These highly collaborative projects therefore had much in common with Participatory Action Research.

\subsection{Researcher's Personal Position}

I trained as a music therapist with the New Zealand Society for Music Therapy. As a candidate for their 'Professional Accreditation of Music Therapists' programme I developed music skills and studied essential topics such as the psychology of music, human development, and Māori culture and music, at my local university and polytechnic. Music therapy topics were taught in block courses by invited international

music therapists acclaimed for their expertise in specific areas. Music therapists were proud of the eclectic nature of this programme, and the variety of knowledge and expertise that could be shared within the local music therapy community (Rickson, 
2009a). I was therefore not strongly schooled in any particular theoretical model of music therapy.

I am also the parent of a congenitally profoundly deaf child, and began training with an interest in working with children who have disabilities and an already established reputation as a musician in special education settings. My experience parenting a child with special education needs, and thus being a consumer of special education services, would enable me to bring valuable insight to this research, as well as assumptions which needed to be regularly reviewed. From my extensive work as a clinician I knew the importance of reflecting on the impact my personal experience has on my professional life and I would continue to value that reflection as an essential part of this research experience (Rickson, 2009b).

Clive and Carol Robbins were significant contributors to my training and I have predominantly drawn on a humanistic framework and the Nordoff Robbins Creative Music Therapy approach in my clinical work. More recently when working with adolescents who had social and emotional difficulties, I looked to psychodynamic theory. Nevertheless, the work, students' needs in context, always led the way. Further, pioneers do not have the luxury of working, or perhaps the burden of needing to work, within established 'ways'. We do not, and neither do our government agencies, institutions, families/whanau and clients, have strongly held preconceived ideas about what music therapy 'is'. We build relationships and set out to determine what we can offer each other. The way we define music therapy changes with each new encounter (Rickson, 2009a). Croxson (2002) confirms that New Zealand music therapy has had a conscious desire to be eclectic, there has been no favoured school of thought, and music therapists have drawn on the approach most appropriate at any given time.

During my fifteen years of clinical music therapy practice I have predominantly worked with students who have 'high' or 'very high' needs according to the MoE SE guidelines, and most of my work took place in segregated education settings. However, although I am personally drawn to work with children who have significant needs and with whom 'clinical' improvisational music therapy is often a primary mode of communication, my music therapy work within education settings was varied. It included group music 
making, workshops, musical visits, concerts and shows, and music learning - all the musicking possibilities described in the Community Music Therapy literature (Wood, Verney, \& Atkinson, 2004). While I have predominantly worked with individuals in withdrawal rooms (e.g. Rickson, 1998) and small groups in designated music therapy rooms in schools (e.g. Rickson, 2002; Rickson \& Watkins, 2003) I have also been able to involve students in wider school activities or take them to events out of the school setting according to their individual needs (Rickson, 2004). I have also responded to invitations to do 'one-off' music therapy assessments with students who cannot access music therapy, predominantly in more remote areas of the country. It was this experience that motivated the current study, and provided considerable data for the initial protocol (see Chapter Six, p. 119).

During the past five years, I have been employed by the New Zealand School of Music to teach and research in the field of music therapy. My contact with children who have special education needs has been predominantly via the music therapy students I was supervising or examining as part of their practicum experiences. I consistently desired to maintain my own music therapy practice and felt I wasn't able to do enough of that. By choosing to engage with an Action Research project I would be able to link my passions for music therapy practice, teaching, and research. My starting point for this research was therefore pragmatic. I wanted to be doing music therapy, to improve my practice, and to be able to share my findings with colleagues and students.

\subsection{Researcher's Assumptions and Values}

I therefore began this research with experience and knowledge of working with students with special education needs and their teams in special school settings, as well as a parent's perspective of the 'system'. Believing that music therapy activities ranged from boundaried clinical work in the psychotherapeutic tradition to work with groups in community settings, and that 'consultation', or helping others to use music in their work, was part of that continuum, I also called myself a 'consultant'. Further assumptions regarding consultation work have been captured in the Initial Protocol, outlined on p. 119. 
I began this research believing that music therapists have flexible skills which enable them to do a wide range of tasks effectively, and I could therefore use my existing skills and knowledge to undertake consultancy work. Engaging in regular clinical and research supervision would enable me to practice safely. However, I was viewing consultation through a 'clinical' lens; and I had an 'outsider's view' of life in regular schools.

In $\mathrm{AR}$, focus of the research can change. In this case, a change of focus would mean some of the data collected in stage one could not be presented in this thesis. Therefore, although the following paragraphs signal some of the findings, they are necessary at this stage to explain the data exclusion. The original working title of the thesis was "...Developing an assessment and consultation protocol" and my emphasis would be on describing and potentially improving the music therapy 'clinical' assessment that was dominant in my previous consultation work. My sessions were student-centred and predominantly free-flowing - I rarely used a music therapy assessment 'tool'. I believed that a thorough clinical assessment (albeit single-session) would provide the foundation for the work, and that it would be necessary to make my assessment process explicit in order to be able to draw extensively from it with participants during the consultations. Therefore, during stage one of the study I would undertake a thorough analysis of videotape from four previous single-session assessments for 'consultation'.

However, it became clear that team members would not want or need 'lists' to guide their interactions with their students. Further, my focus would need to be on the adults (the 'consultees') rather than the student (the 'client') and I would need to observe and respond to what they were already doing. Analysing music therapy assessment sessions in depth would also reinforce the things a music therapist could do that team members could not. Rather than being empowering, highlighting the complexity of my interactions in clinical assessments would have the potential to intimidate team members. I may have emphasised the 'expert' role, perhaps not for egocentric reasons but to demonstrate 'music therapy expertise', and thus protect the profession. The findings from video analyses of previous assessment sessions undertaken in stage one, with informed consent, have therefore not been presented in this thesis but will be offered for publication in future. Readers who wish to have access to this information should contact the author for 
details about how it can be obtained.

To do this research I would have to make a conscious effort to remain 'alongside' my participants, allowing them to prioritise their concerns and determine the purpose of their individual projects. I imagined inevitable tensions between my role as researcher and therapist as I thought about allowing the teams to take their projects in unforeseen directions. When setting up a small group for an action research project, it is inevitable that a lead researcher or co-ordinator will emerge (Armstrong \& Moore, 2004). My task would be to avoid automatically becoming the 'lead' researcher while accepting the possibility that it might be necessary; and to encourage others to lead while ensuring I still carried out my work ethically, and with integrity.

For example, before beginning the work I had occasion to talk with a teacher of low vision and blind children, and found the interaction difficult. Although she was asking about music therapy, she seemed to have strongly formed opinions and ideas that were hard to negotiate. Reflecting on this I realised that I was primarily concerned with my ability to gain her trust and sensitively have her see my point of view, rather than to find out how I might draw from her understandings. Thinking ahead to my research tasks, I realised I it might not be easy for me to accommodate team members' ideas, and leave aside the 'expert' role in order to be an effective collaborator.

Further, as I revisited literature relating to discourse theory and power relationships I became aware that my own discourse had tended to marginalise students who had special education needs. My exposure to 'medical model' discourse, had led to the perception that music therapists were 'experts', who provided a 'treatment intervention'. This is perhaps not surprising because music therapy had been consistently portrayed this way in the literature until the recent emergence of 'Community Music Therapy'. Most music therapists still work with 'patients' or 'clients' - terminology I now find problematic. While I have always valued students' individual strengths, I would become aware of my tendency to report on the difficulties they experience and the ways in which music can address those 'needs'. Further, while I had good reason for limiting inclusion criteria to ORRS funded students - music therapists predominantly work with these groups - I would become increasingly aware that using these categories as inclusion criteria for the 
study highlighted participants' 'difference'.

My daughter was fully mainstreamed. I believed she would be able to manage this because she was 'different' from students I worked with in special school settings. For example, she did not have significant behaviour issues, could manage her own activities of daily living, and progressed well with the technology and personal support available to her. Other children with special needs seemed 'worse off', to need more intensive support and therefore to require 'special' schooling. My judgement about whether students might be mainstreamed was based on the 'problems' they had. Now, although I do not take the extreme position that special schools be abolished, I would advocate for improved attitudes and increased support systems, with the removal of barriers so that families have a real choice about where their child might be educated.

Only after I consistently and explicitly examined my position in the light of new experiences would I be able to recognise some of the assumptions and attitudes I brought to my work. My cognitive understanding of different perspectives and ability to offer carefully framed professional responses would give way to genuine re-conceptualisation. In particular I believe it is easier for me to see beyond the students' disabilities to the less complicated people beneath. I now genuinely struggle with 'labelling' and the contradiction between 'differentiating' students for inclusion in a music therapy programme, and the principles of inclusive education.

During the research process I would become increasingly aware that my support for special schools had been partially related to a perceived need to protect the 'home' for the music therapy clinic. Music therapists are more readily employed in special school settings. The environment, with physical and human resources on hand, makes many aspects of the work easier. The model of work is more familiar, and 'comfortable'. However, while clinical music therapy will continue to be valuable for some students, I would become more aware of the need to carefully examine the decision to work in this way to ensure it is not primarily about what is convenient for the music therapist.

My appreciation of the diversity between and within teams would grow as I embraced the opportunity to work closely and develop deeper relationships with team members. I 
would develop 'softer' attitudes towards some of the approaches that might have seemed unacceptable to me in the past, as I realised what they had to battle with on a daily basis. Genuine collaboration with team members would enable me to relinquish some of the individual responsibility I was harbouring in the beginning, to be comfortable with and appreciative of relatively 'small changes at the grass roots level', and to acknowledge the encouraging 'ripple effects' that grow from positive experiences.

I value the inclusion of students' voices and had hoped to interview them. Armstrong and Moore (2004) wrote

In the context of participatory action research, based on emancipatory principles, all those who are implicated in change would participate in identifying and planning that change and monitoring and evaluating it, planning the next stage, and so on. However, in the context of education this presupposes a very high level of democracy in settings in which students' voices are clearly audible and valued (p.5).

On the other hand, I realised that I had doubts about being able to capture the "clearly audible voices" of the students in this study given their 'high' or "very high' level of disability, and I would struggle with even introducing the idea to teams who worked with students with extreme communication difficulties. On the other hand, throughout the study it would become increasingly evident that students communicate their views adequately in other ways, and I have aimed to give them a 'voice' throughout this thesis by presenting a sensitive portrait of each student's presentation and their responses to intervention.

I would need to be alert to the roles I found myself in as I engaged in this work. As well as being a researcher, music therapy consultant, and music therapy clinician, I would find myself variously being a 'stranger', 'visitor', 'musician', 'new friend', 'another parent', 'team member', 'defender of the music therapy profession', 'child advocate', 'parent advocate', and 'liaison person' (between school and ministry of education personnel). I would even become a 'sister-in-law' (when team members discovered I had family living in their district) and would have to manage the demands of building relationships with people who live in very small communities, and with Māori, who consider it culturally inappropriate and/or rude not to share information about backgrounds and connections; 
with negotiating and maintaining confidentiality. Further, I would have to balance the responsibility of ensuring that team members gained maximum benefit from being involved in the consultation process, with my research interest to generate findings that resonate with, and could therefore be useful, to wider communities.

\subsection{Choice of Methods and Data Collection Techniques}

Ethnographers use three main data gathering techniques - participant observation, interviews, and interpretation of artefacts (Stige, 2002). Ethnographical accounts include concrete observations, appropriate quotes, and examples of dialogue from the field. In the current study the research fields would be school environments and, to a lesser extent, students' homes. In music therapy, the predominant artefact is the music that is produced. In this study music examples are included, although the primary emphasis is on describing interpersonal interaction rather than musical product.

\subsubsection{Participant Observation}

An ethnographical approach would enable me to immerse myself in the everyday experiences and activities of the consultees and to experience and learn from events as they experienced them. I would be able to observe and talk with them in order to understand their reality in context. Ethnographers do things with and for people while they are in the field, and describe these experiences and observations, as well as perceptions and interpretations of particular situations, in 'field logs' (see below). Ethnographic accounts have limited generalisability because of the small numbers of participants.

\subsubsection{Researcher's Field Logs}

Naturalistic observations of the students at school would involve watching as well as interacting with team members and participant children in the classroom, playground and other school environments, to gain an understanding of the context in which they worked and to support as appropriate. The field logs would include factual information about 
events, dates, and people; and provide a time-line to chart progress and to illustrate general points, including successful or unsuccessful action and reflection on this (McNiff, Lomax, \& Whitehead, 2003). They would hold anecdotal evidence and passing conversations; and comments about personal experiences, thoughts, and feelings.

\subsubsection{Researcher's Reflective Journal}

During the period of this research I would engage in a process of constant critical reflection on how I was shaping the work, and keep a reflective journal. I would type my thoughts directly into the electronic journal or make handwritten notes to include in it later. Maintaining a continual process of writing (description, analysis, interpretation, negotiation and rewriting) can enable negotiated situated meaning to be established, and communicated to the scholarly community (Stige, 2002).

\subsubsection{In-depth Interviews}

In stage one of this study, in-depth semi-structured interviews would be undertaken to gather information from music therapists regarding their consultation work, or other work with students who have special education needs, which would contribute to the development of the initial protocol. In stage two in-depth interviews would be undertaken with team members who were identified as primary 'consultees' by the teams themselves.

In-depth semi-structured interviews are favoured by qualitative researchers who want to explore more general themes and "aim to explore the complexity and in-process nature of meanings and interpretations that cannot be examined using positivist methodologies (Rice \& Ezzy, 1999, p. 53). In-depth interviews allow social processes and negotiated interactions to be studied and new understandings and theories to be developed (ibid). Broad questions linked to the overall design are used to create a frame for the interview, and prompts are used to keep the interview on topic (Rubin \& Rubin, 1995). Subjectivity and bias cannot be avoided. Qualitative researchers acknowledge that meanings are continually constructed and re-constructed in interaction, that their presence and contribution influences the research, and they therefore become co-participants in the discourse (ibid). The advantage of using semi-structured interviews in the current study 
would be that interviewees would be able to 'tell their stories' and rich data would be obtained.

In-depth interviews take considerable time and energy, and sometimes incur considerable cost. Further, because of the time involved, participants' understandings and experiences can be developed from interview to interview (Rice \& Ezzy, 1999), and when many participants are involved the focus of the interviews may vary. It can therefore be difficult to compare their responses. Finally, in-depth interviewing can be difficult to do well requiring persistence and sensitivity to the complexities of interpersonal interaction (Rice \& Ezzy, 1999). Nevertheless, carefully facilitated one on one interviews give people safe opportunities to discuss sensitive issues and participants often find the experience rewarding (ibid). Moreover, the time and energy that is invested in in-depth interviewing can also result in rewarding and enriching experiences for the interviewer.

\subsubsection{Participant Diaries}

In this study, in addition to follow up interviews, participants would be asked to keep a diary of the musical interaction they did or didn't have with their students in order to determine whether they were empowered to use music in an ongoing way. They would be asked to describe how they felt about the work with particular reference to any barriers they encountered. This method would minimise the possibility that they would not remember important details during interviews, and what they would actually able to do could be compared to what they had intended to do, or said they had done.

\subsubsection{Member Checking}

In order to reduce the impact the researcher would have on the work, the interpretations would also be checked with participants, a process called member checking. In this study, interview transcripts would be checked for accuracy, and interpretations (music therapist interview findings and case studies) would be also be reviewed by and negotiated with participants. Thus they would be able to confirm that they were comfortable with the way they were represented and the researcher's influence on the research would be kept to a minimum. 


\subsubsection{Video Data}

In the current study video data of music therapy sessions would serve several purposes. Firstly it would be a clinical tool, assumed to be a valuable aspect of consultation practice which would enable participants to learn about the strategies and techniques that a music therapist uses, to help them to determine what they might be able to do, and to refer to in an ongoing way when they need reminding about certain aspects of the work. Video data would therefore also become part of the students' school records. Secondly it would serve as a focal point for the focus group; and artefact for participants to observe and discuss (see below). Thirdly it would serve as an artefact for the research, subjected to rigorous analysis in order to provide the reader with a rich and meaningful account of the students' responses to music.

\subsubsection{Focus Groups}

Focus groups aim to elicit the perceptions, interpretations and beliefs of a group of people, ultimately to gain understanding of a particular issue from their perspective (Rice \& Ezzy, 1999). In the current projects, teams would be shown video data of the music therapist working with the student they were supporting and asked to discuss the clip. This would enable the music therapist researcher to gain an understanding of what they valued about the session, and what aspects they felt might be useful to develop in their own work. Typically focus groups comprise a small group of people who share similar experiences or concerns. In this case they would be a team of educators, support staff and/or therapists who had a particular interest in using music with their student.

Focus groups rely heavily on the interaction between participants and are therefore different to group interviews. In this study, the group process would enable participants to explore and clarify particular points of view, which is an important feature of focus groups (Rice \& Ezzy, 1999). While one limitation of focus groups is that topics are generated by the researcher and therefore might not always reflect participants' interests, in this case the topic would reflect the shared interests of the team. However, the focus groups in this case would not be typical because the researcher was also the music 
therapist, and participants would seemingly engage more actively and ask more questions from her than they might an 'external' facilitator. On the other hand, an external facilitator's presence and activity is also likely to influence participants' interactions in various ways (Rice \& Ezzy, 1999).

In addition to the advantage of producing in-depth information through interaction, focus groups can uncover unexpected information or information that might be too sensitive to discuss one-on-one. In contrast, some information might be too sensitive for sharing in the group setting. Nevertheless, focus groups provide opportunities for participants to compare and contrast information, can enable shy people to contribute more, and reduce possibilities that participants might misunderstand questions (Rice \& Ezzy, 1999). They are particularly valuable for gathering information from marginalised groups, assisting the empowerment process and enabling participants to be active in the research process (ibid).

On the other hand, focus groups cannot capture the intricate beliefs and practices of individuals, or the actual behaviours of individuals or groups of people (Rice \& Ezzy, 1999). Participants might not do what they say they do, and/or might agree with others for the sake of conformity or to please the facilitator. Finally, focus groups generate large amounts of data which are complicated and time consuming to analyse.

\subsection{Procedures for Stage One - Music Therapist Interviews}

\subsubsection{Recruitment}

The total pool of twenty Registered Music Therapists (RMTher) were contacted via their professional newsletter, and those who had worked in SE settings or had been engaged in music therapy consultation work were invited to contact the researcher to express interest in participating. The eight who responded were provided with information and consent forms, all gave informed consent to be interviewed and all were included in the study.

(Advertisement, Expression of Interest Form, Information Sheet, and Consent Form are presented in Appendices 1-7). 
Meaningful response rates cannot be generated from this data because the work contexts of non-respondents are not known. However, all eligible music therapists were invited to comment on findings from the interviews, published in the New Zealand Journal of Music Therapy (Rickson, 2008). Forty were registered at the time of publication and no feedback on the findings was received.

\subsubsection{In-Depth Semi-structured Interviews}

In-depth interviews have been described on p. 85. They were undertaken with music therapists at a time and place which was convenient for them. In some instances I travelled to their homes, while others chose to be interviewed around the time of an event such as a music therapy conference. Thus the interviews were spread over a period of approximately six months during 2006. In all cases the interviews were conducted in a comfortable, relaxed, but private environment. Broad interview questions provided a focus for the interviews, but interviewees were encouraged to talk about other relevant topics as they arose. The interviews lasted for approximately one hour. Audio recordings were taken and transcribed by the researcher. The interviews aimed to determine what music therapists do when assessing students for consultation purposes, what can be learnt about a child from just one assessment session, and how that information might be used to help others use music in their work.

\subsubsection{Reflective Journal}

While preparing for and during stage one of the study I reflected on my previous consultation experiences. When interviews were completed, codes and analytic memos were created from the journal and relevant data was combined (point five below) with interview data. Thus when findings were returned to participants (point six) they had full access to emerging findings. 


\subsection{Procedures for Stage Two - Consulting with Teams}

Each team developed their own project to improve their use of music with students. In each of these projects the researcher was also the music therapy consultant (See Figure 4, p. 91).

\subsubsection{Recruitment}

An advertisement was placed in the New Zealand Education Gazette inviting teams who were working with students identified as having 'high' or 'very high' needs according to the ORRS verifiers, and who could identify at least one team member (the identified team member/s', or IDTM) who would be willing to implement and monitor the planned use of music with their student, to express interest in participating. ${ }^{2}$ Potential participants were asked specific questions on the expression of interest form (see Appendix 9) to enable the researcher to choose teams that would capture the diversity that would be encountered in music therapy consultation practice. Responses were received from seventeen teams, and four teams were chosen according to the order in which they were received, the variety of professionals in each team, the geographical location of teams, the special education setting in which the learning was taking place, student diagnosis, and student gender. Relevant demographics preface each case study in Chapter 7. School demographics are presented on p. 125. The desired level of diversity was evident in the first seven expressions of interest. Four of those teams were invited to participate. Team members were sent information and consent forms.

Advertisement, Expression of Interest, Information Sheets, and Consent Forms are presented in Appendices 8-19.

\footnotetext{
${ }^{2}$ The IDTM from here on are referred to as 'consultees'.
} 


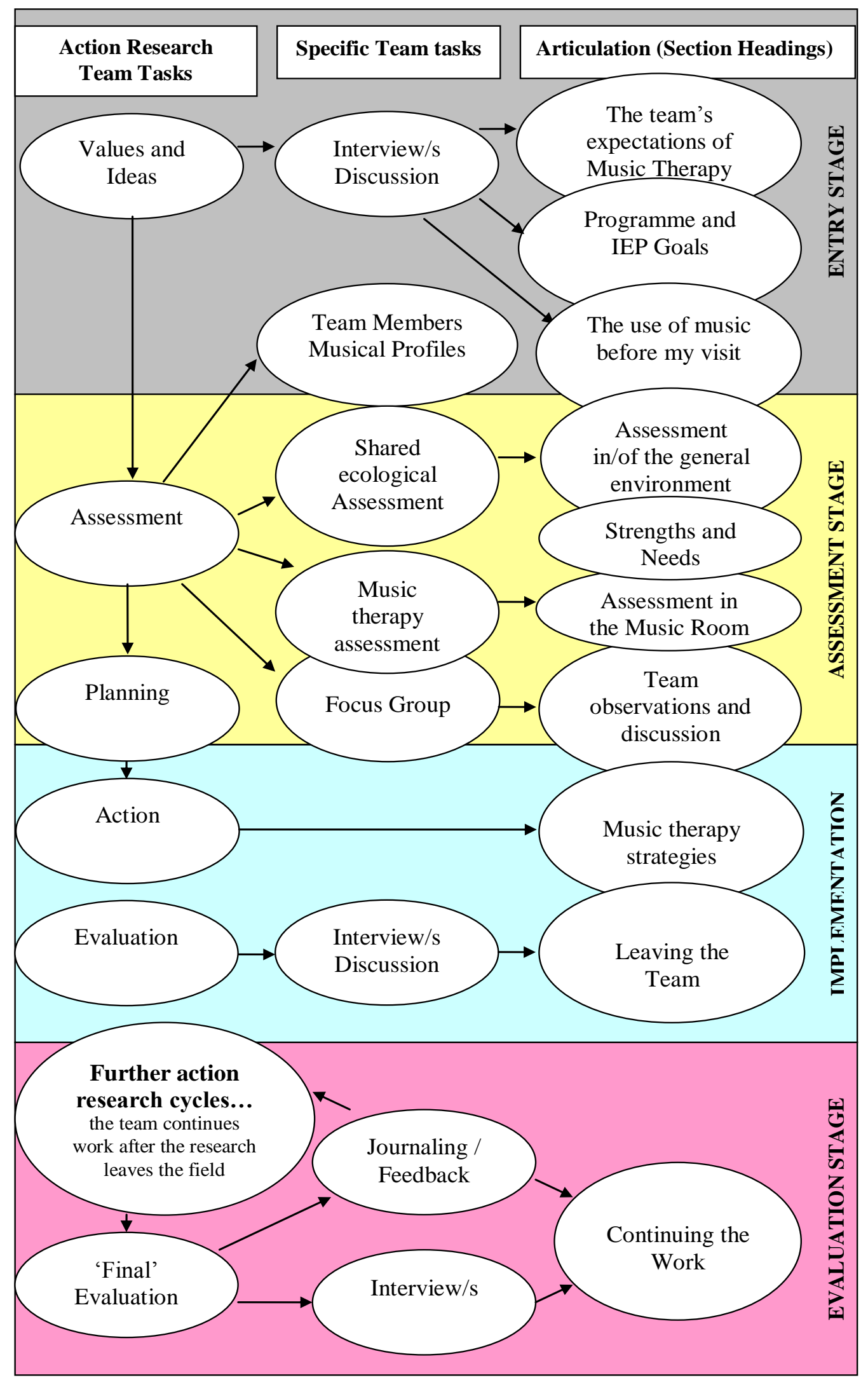

Figure 4: Local Action Research Projects (with consultation stages in colour) 


\subsubsection{Data Collection}

Each case study describes the music therapy consultation from the perspective of team members, using data from:

- participant observations in school settings (Researcher's Field Log);

- clinical data gathered from school records and a music therapy assessment session, undertaken by the music therapist, which was videoed;

- in-depth interviews with consultees at the beginning and end of each consultation (see below);

- focus group data from each team meeting during the video viewing;

- diary entries from consultees over a twelve-week period following consultation;

- follow-up in-depth interviews with consultees after their diary entries ceased;

- researcher's reflective journal

\subsubsection{Video Data}

During the week of the consultation, approximately mid-point, the student was engaged in an individual music session with the music therapist, which was videoed. Sessions lasted between eighteen and forty-five minutes.

\subsubsection{In-depth Semi-structured Interviews}

Three in-depth interviews (see p. 85) were undertaken with each of the consultees. The first two interviews were held during the week of their consultation in a quiet space in the school. The first, lasting approximately one hour, was to gain an understanding of participants' needs and what their expectations of the consultation might be, and to draw out existing values and ideas that could be openly discussed and negotiated before the shared work began. The second took place at the end of the week to gain their impressions of, and reactions to, the work. Second interviews were mostly shorter (approximately thirty minutes) because considerable informal discussion had taken place

during the week. Nevertheless, the process of being interviewed a second time also helped consultees to evaluate how their projects were progressing. Consultees were 
interviewed a third time in the follow-up period regarding their ongoing music work, for approximately an hour, by telephone. The follow-up interviews, especially alongside participant diaries (see below), would help determine whether planned music experiences were introduced in a sustainable way by team members.

It was intended wherever possible, and with the consent of child, legal guardian and with general agreement from the team, to interview participant students to determine how they experienced the procedure. Informed consent was obtained to interview one child.

\subsubsection{Focus Groups}

All team members who had given informed consent to participate in the research were invited to view videotape of the student's music session with the music therapist/researcher, to discuss what they saw, and to consider how music might be used to meet IEP objectives. This discussion took the form of a focus group and was audiorecorded. The focus groups lasted approximately one hour. The purpose was to learn about the team's process as they 'unwrapped' and tried to made sense of the music therapy assessment session. Their observations and the discussions that ensued helped determine the issues that were of most relevance to them. Finding out how a music therapy consultant might best support this interaction, i.e. by offering specific expertise, making links and/or drawing from the expertise of team members, was of particular relevance to the research.

\subsubsection{Participant Diaries}

Consultees and other participants who chose to make diary entries were provided with a 'Music Diary'. They were asked to set aside some time each day to enter information about what they did with music, how they felt about the interaction, and what they thought they might do next. They were also explicitly encouraged to write about times when it was difficult to do music and/or musical experiences were not going well. Sample pages in the diary gave examples of the sort of thing they might record (see Appendix 24). 


\subsection{Procedures for Stage Two - Developing a Consultation Protocol}

The data (i.e. interviews, clinical assessments, field observations, focus groups, participants' diaries) were collected at various stages in the consultation process from each team and, along with less formal interactions, enabled participants to articulate values and ideas; and to negotiate, plan, and evaluate their work (see Figure 4, page 91). Data were not formally analysed until after the researcher left the field.

Themes uncovered from each case study were articulated in rich descriptive form and were re-examined to uncover more precise indicators of effectiveness and learning outcomes. After each consultation I reviewed the indicators of effectiveness and learning outcomes, my reflective journal data and relevant literature, and made further notes in my reflective journal. This data formed the basis for the action research cycles, helping to determine how the consultation protocol might be improved before the following case study began.

When all four cases were completed the indicators of effectiveness and learning outcomes were compared and contrasted to answer the research questions in stage three.

\subsection{Data Analysis}

The data were subject to thematic analysis (Rice \& Ezzy, 1999) involving both inductive and deductive identification of codes from the data and, at least in stage two of this study, an ongoing dialogue between pre-existing theory as new insights were generated. Thematic analysis is similar to grounded theory (Glaser \& Strauss, 1967) but does not include theoretical sampling (ibid). The primary task in thematic analysis is the process of coding, sorting and organising data, in an attempt to relate various data to each other or to the data as a whole (Dey, 1993). In this study QSR-N6 software (QSR International Pty Ltd, 2002), for supporting the analysis of qualitative research, was used to store and organise data; and exploration and interpretation was done manually. 


\subsubsection{Stage One - Data Analysis: Interviews with Music Therapists}

In Stage One the primary quest was to uncover existing knowledge from the experience of the participants. The findings would provide initial 'data' for the development of the protocol, and theory would be formed both inductively and deductively as interviews were compared and contrasted over time, and relationships between categories were identified. The first step in the analysis of any qualitative data is to identify 'units of analysis', also known as 'concepts', which may be "meanings, practices, encounters, narrative structures, organisations, or lifestyles" (Rice \& Ezzy, 1999, p. 193). The initial units of analyses for stage one of this study, drawn from the research questions, were "tasks associated with assessment for consultation" "outcomes from single session assessments" and "helping others use music in their work".

\section{Transcription}

I began by transcribing the interviews, from the audio recordings, verbatim; including line numbers (see APPENDIX 24, 373). I chose to include fillers, pauses, laughs, and so on, even though this method of transcription is more applicable to discourse analysis rather than thematic analysis, to assist me to recall the tone of the message when the audio was not to hand. They were later removed for ease of presentation. I read each transcript through several times, and annotated initial ideas (margin notes) on the transcript. Manuscripts were returned to participants inviting their checking, editing and additional comments.

\section{Initial Reading of the Interviews}

The interviews were conducted over a six-month period and the more detailed analysis of each began as soon as it was approved by the participant involved. Individual transcripts were coded, i.e. portions of data were labelled according to their likely relevance to the research topic, and further analytic memos were made. 


\section{Analytic Memos}

Analytic memos were created to identify emerging themes (Ely, Vinz, Downing, \& Anzul, 1997). However, in addition, I wrote freely and creatively in memo form as I recorded a range of observations and ideas about the data. Dey (1993) argues that memos are essentially private, "like diary impressions of the data" (p. 89). Thus my memos were usually suggestive, even provocative, and often built on reflections that were recorded in my journal. But I also used them to remind myself of other pieces of data or literature, to raise questions, and to challenge my assumptions. Thus writing analytic memos was a constant task in the analytic process.

\section{Open Coding: Creating Categories}

Each transcript was interrogated independently, in the first instance, to begin the process of grouping concepts to form categories (meanings, feelings, actions). The nature of the research questions led to the creation of several 'middle-order' categories. Dey (1993, p. 104) argues that these are logical to "draw some broad preliminary distinctions within the data, which may be based on fairly common sense categories around which the data can be organised quite effectively". For example, 'Work Context', 'Music Therapists' Previous Experience', and 'The Purpose of Assessments' (see APPENDIX 25, p. 391) were all natural categories to develop.

Further, during the transcription of later interviews I had already developed several fairly persistent categories and was conscious of the need not to 'force' data into these categories, but rather to let new ideas emerge from the data. Once all the interviews were complete, I was careful to explore the data in varying ways, such as searching categories by keyword, as an aid to shifting focus. During the process of open coding, "normal ways of conceptualising events and interactions are put aside as the analyst attempts to conceptualise the data in a way that exposes social processes" (Rice \& Ezzy, 1999, p. 196). As I read and reread I asked myself questions such as "what can the data tell me about helping others to use music in their work?" (see APPENDIX 26, p. 393). Variously 'moving in and out' of the data, reading and rereading, examining sentences closely before looking at the paragraph as a whole, led to the naming and renaming of categories, 
and emergent subcategories (see APPENDIX 27, p. 404). Comparisons were made between various categories, and between various categories and subcategories. Paragraphs, sentences, and observations were taken apart and renamed. Metaphors were helpful in the conceptualisation. When discussing music therapy consultation Petra, for example, referred to "losing the whole essence" of music therapy (see again Petra $152-$ 170, APPENDIX 24, p. 373). This phrase seemed to provide a powerful representation of how she felt.

\section{Axial Coding}

Axial coding involves specifying the developed codes more rigorously, and representing the data new ways by making connections between a category and it's subcategories, and scrutinising codes to make sure each is fully elaborated and delineated (Rice \& Ezzy, 1999). For example, Petra's fear of "losing the whole essence of music therapy" was described as:

The thought of losing the "whole essence of music therapy" was frightening. Employers would expect too much, and she would lose her professional identity. Team members' activities would not be therapeutic, and could perhaps even be counter productive.

During the axial coding stage the analysis continued to involve inductive and deductive exploration as I tested my theory against the insights that had been generated.

\section{Developing Theory}

Grounded theorists use a process of selective coding which involves combining categories around a central core category (Strauss \& Corbin, 1990). A comparison of codes and categories enables a central code to be identified, providing a theoretical point of integration for the study. For example while Petra was frightened of "losing the whole essence of music therapy", other music therapists were, for example, "uncomfortable about some aspects of consultation, yet valued being able to support others in their use of music' (Cara) or felt that 'consultation is integral to the work' (Lisa). The data might have been worked more thoroughly to develop a core category, but I preferred to maintain broad categories to allow for the existence of "complexity and multiplicity of 
interpretations" which Rice and Ezzy (1999) argues is preferred by many researchers. I used these broad categories as headings, and was able to express a variety of themes, and exceptions, in the theoretical description (see APPENDIX 28, p. 408).

\section{Peer Debriefing}

Lincoln and Guba (1985) describe peer debriefing as "a process of exposing oneself to a disinterested peer in a manner paralleling an analytic session and for the purpose of exploring aspects of the inquiry that might otherwise remain only implicit within the inquirer's mind" (p. 308). A colleague from the New Zealand School of Music was able to review the analysis process, and to discuss and challenge emerging findings.

\section{Member Checking}

The findings were mailed to participants who were asked to clarify, expand or confirm interpretation of the data, and their comments were incorporated into the findings.

\subsubsection{Stage Two: Data Analysis (Introduction)}

In stage two, data generated from action research with team members in their schools, and data generated for the larger action research project to develop a music therapy consultation protocol, was managed separately. A total of thirty-five stage-two documents were entered into QSR-N6. The way in which the data was gathered and synthesized is summarised in Figure 5, page 104. I begin by describing the management of the data which came directly from the school setting. A table of research questions and data sources is provided in the appendices (see APPENDIX 30, p. 415).

\subsubsection{Action Research (Case Studies)}

\section{Data sources}

The action research projects undertaken by four school teams have been presented in Chapter Seven as case studies. Thus they represent a condensed version of the most 
relevant data from each case, and can be described as 'coded case studies' (K. E. Bruscia, 2005). Each of these projects generated the following data:

1. Researcher's field log

2. Researcher's reflective journal

3. Initial interview with team member/s

4. Video of music therapy assessment

5. Focus group data

6. Second interview with team member/s

7. Participants diary of music activity

8. Follow-up interview with team member/s

Field Log

The researcher's field log was transcribed (see APPENDIX 31, p. 417). The steps in the analysis were the same as for interviews, described above. Once again, some categories (such as descriptions of the 'school environment' and 'observations of student's responses') were self evident, while others could only emerge from the data.

\section{Reflective Journal}

The journal was constantly referred to, and entered into, and data from the source contributed significantly to other data sources such as the field log and analytic memos, and the cycles of learning in the wider action research project. Moreover, after each case study themes were drawn to consider alongside case study data and the literature search as cycles of learning were developed (see APPENDIX 32, p. 443).

\section{Interviews (Initial, Second, and Follow-up)}

The thematic analysis approach described for Stage One was also used for Stage Two interviews (see APPENDIX 33, p. 444; and APPENDIX 34, p. 449). Each of the interviews in Stage Two was undertaken for a specific purpose. For example, initial interviews which were done at the beginning of the consultation week were undertaken primarily to gain an understanding of the interviewee's concerns including the student's 
strengths and needs, their current use of music, and their expectation of music therapy consultation. Thus, initial categories were easily labelled 'student strengths', 'student needs', 'music past and/or present', and 'expectations of MT'. Nevertheless other categories, such as the 'wishes' of parents, emerged from the data. Second interviews were undertaken at the end of the week in the field to find out how the interviewees had perceived the music therapy consultation. Thus the categories that emerged from the data were unexpected, rich, and diverse. Follow-up interviews aimed to find out what strategies the teams had found valuable, and whether music making might be sustained over time, so the categories 'music strategies' and 'managing music in future' were anticipated, while others were less so (see APPENDIX 35, p. 459).

\section{Video Data}

The music therapy assessment session was videoed to provide a significant focal point for each of the teams. Data from the session were analysed and presented in the case study to add richness to the description of the student, and the consultation context. The data were chosen to 'represent' the child, rather than to highlight a specific problem or skill. Thus the first ten minutes of the session was chosen, but in two cases other more representative samples were also analysed (see process below). The approach taken can be described as 'ethnographic descriptive approach to video microanalysis' (Holck, 2007). Holck argues that the ethnographic approach is "very useful in recognising small indicators of communication and social interaction in music therapy with clients with severe communicative limitations". How the data is transcribed is left to the researcher, but might include narrative, conventional notation, or graphical notation. While Holck describes a horizontal and vertical analysis which involves detailed description across time on the horizontal plane and, for example, facial expression, direction of gaze, playing hand, instruments used, posture and facial expression, on the vertical plane. This study used an adapted version of Holck's description; including narrative description and conventional notation (see 467

The procedure in each case involved:

- viewing the session as a whole to note significant interactions and overall impressions; 
- viewing the first ten minutes of the session using Windows Movie Maker, stopping the recording every five seconds to note who was acting (student or therapist), what they were doing, and 'how' things happened - in bullet point form;

- rewriting the bullet point findings (from step two) in descriptive form;

- viewing the session as a whole to determine whether enough of the significant interactions and overall impressions (see step one) are conveyed in the description; and

- where it was determined that the description did not convey enough of the significant interactions and/or overall impressions, other sections of video were chosen according to their significance for further analysis (repeating steps two and three)

- excerpts of the description were chosen for inclusion in the case study

\section{Focus Group Data}

Audio recordings of the focus groups were transcribed verbatim. Initially the intention was to allow the categories to emerge from the data. While exploring the data from the first case, when it seemed that the group were predominantly asking direct questions of the music therapist, it seemed as if it would be helpful to explore the data from an interaction perspective. It could then help to determine how participants in this situation, i.e. viewing video of their student at music therapy, were able to actively contribute and to what extent they might become self-determining. Thus the data from the focus groups was analysed deductively and initially forced into categories of 'therapist reinforcing ideas', therapist introducing ideas', 'participants' ideas', 'participants' questions', 'therapist answering questions' and 'participants developing ideas' (see APPENDIX 36, p. 460). While this may seem rather restricting, the subcategories that eventually emerged contributed to the richness of the study.

\section{Participants Diaries}

The participants' diaries were also fully transcribed (see beginning of diary from Tim's 
team, APPENDIX 41, p. 495) and subjected to thematic analysis.

\section{Triangulation}

Data were gathered from multiple sources, across time. As indicated above, data were compared and contrasted at several stages in the research process to verify themes (Piggot-Irvine, 2004).

\section{Peer Debriefing}

A colleague in the music therapy department at the New Zealand School of Music provided 'peer debriefing' - that is, she would be consulted to provide support, scrutiny of data, feedback on interpretations and guidance in the uncovering of insights during the research process. Our discussions would contribute to my reflection and give confirmation to the findings which would be considered to be meaningful to the music therapy profession.

\section{Presentation of the Case Studies}

Data were synthesised into 'chronological' categories (see again APPENDIX 35, p.459). As explained earlier, specific data were collected to determine what participants' perceptions were at a particular point in the consultation. It was therefore logical to assume that a higher proportion of the data from initial interviews would be coded to 'expectations'. Each of the final categories was elaborated in the individual case studies. That is, the category label became the heading for the paragraphs within the overall description.

\section{Synthesis of Case Study Material}

The figure in APPENDIX 42, p. 503, demonstrates how the data was analysed for one case study. When all four studies were complete, the 'themes from all data after a week in the field' from each case were compared and contrasted to generate final findings. The initial units for the final analyses were 'indications of empowerment', 'the use of music 
therapy strategies', 'team members perception and understanding', 'indicators of efficiency' and learning outcomes', whereas the emerging categories were 'existing resources to draw on'; 'time availability and management'; 'team meetings'; 'empowering others'; 'music therapy strategies'; 'belief in music'; 'new perspectives'; 'student responses'; and 'commitment to continuing the work'; 'a model of music therapy consultation'; 'time availability and management'; 'building relationships and empowering others'; 'differing approaches and the dilemma of inclusion'; 'cultural issues'; 'collaboration'; 'providing adequate support for teams'; 'student need for ongoing therapy'; and 'programme planning' (see Chapter Nine, Findings). 


\section{CASE ONE, Example of Data Gathering}

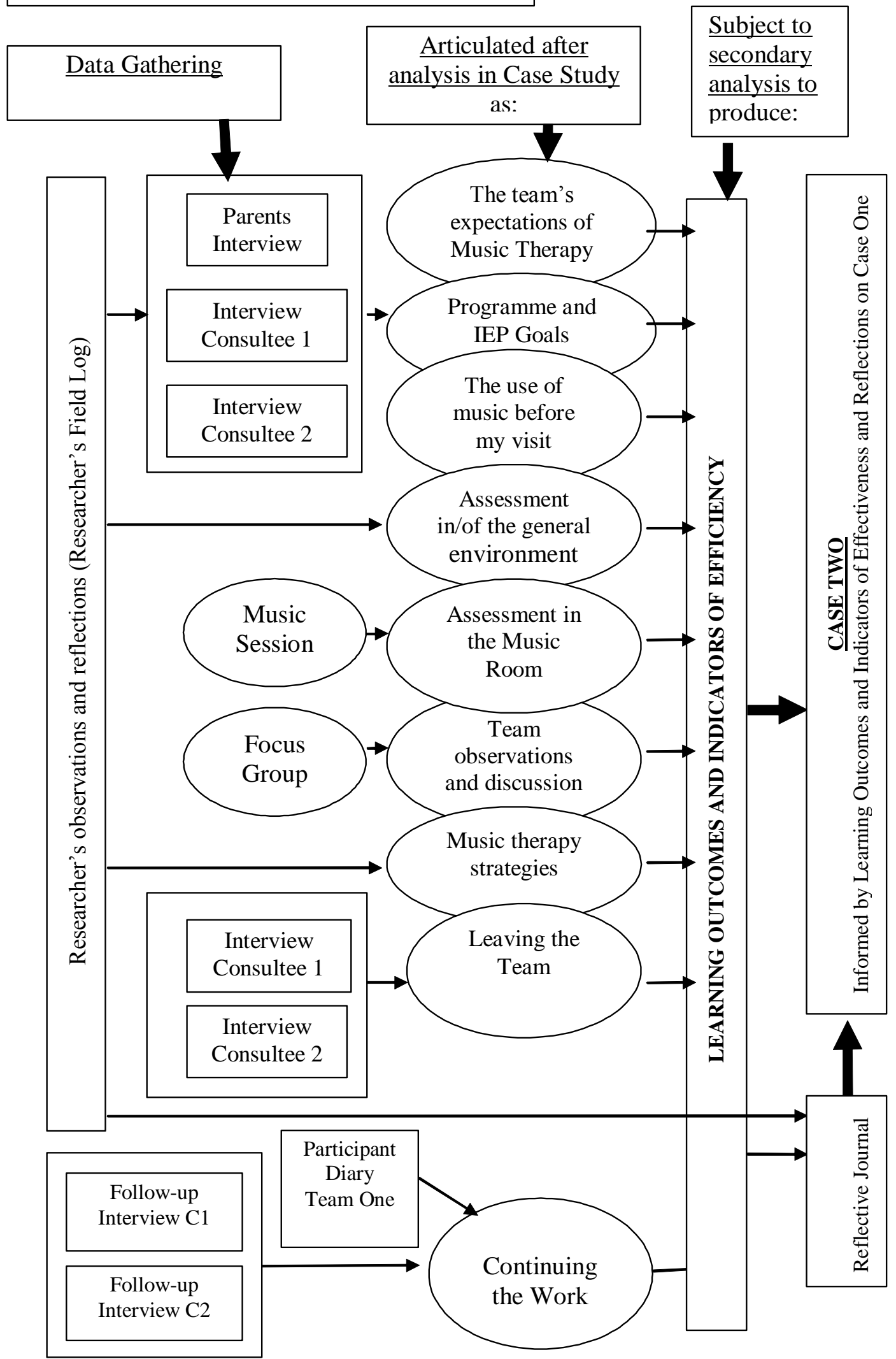

Figure 5: Procedure for Synthesis of Data from Each Case Study 


\subsection{Summary}

Data collected for this study came from the experience of the participants and have been "systematically collected" (Brooks \& Watkins, 1994). The research agenda for each school project was negotiated with the relevant team of participants. Informal discussion and debate were encouraged. Interview transcripts were checked for accuracy by interviewees and the integration of their perspectives, and that of other participants, has been promoted through consistent collaboration and member checking, i.e. participants were asked to compare what they intended to convey of their experiences, with the way their communications had been interpreted. Much of the interpreted data has also been substantiated through triangulation.

All research findings are based at some level on the researcher's interpretations of a phenomenon (Abrams, 2005). I have conscientiously inquired into my own relationship to the research, taking ownership and responsibility for my perspective, assumptions, motives, values, and interests. The research especially values my relationships with participants and my role as a participant, and the first person writing style adopted in this and other sections of this thesis acknowledges this. My thoughts, feelings, reactions and concerns were recorded in a reflective journal and have been communicated explicitly throughout the research report. Writing helped me to clarify my thoughts in relation to the thoughts of others and, in the process, to be challenged to develop those thoughts further. I engaged in several contrasting roles during the course of this research. The implications and management of multiple roles have been acknowledged throughout this thesis. 


\section{CHAPTER FIVE: STAGE ONE. MUSIC THERAPISTS CONSIDER MUSIC THERAPY ASSESSMENT AND CONSULTATION}

\section{Introduction}

Interviews were undertaken with music therapists to find out what music therapists do when assessing students for consultation purposes, what they can know about a child in just one assessment session, and how that information might help others use music in their work. Eight music therapists who had assessed children who have special education needs, or had been engaged in music therapy consultation in New Zealand, participated. The interview questions were:

- What do you do when assessing students for consultation purposes?

- What can you learn about a child from just one assessment session and how can that information be used to help others use music in their work?

Surprisingly, none of the interviewees had specifically assessed students for consultation purposes. Rather they had diverse experience working in a range of settings with children, and/or consulting with other populations. Similarly they rarely undertook single-session assessments. The precise research questions have therefore not been answered. However, following initial interviews, it became clear that interviewees had a particular interest in the issue of whether or how they might help others use music in their work. While they talked about their experience of music therapy consultation across populations and age groups, and assessment work with children undertaken for various purposes not just consultation, interviews predominantly focused on whether and/or how music therapy school consultation might be undertaken. As noted in paragraphs 4.6.4 when many participants are involved in in-depth interviews the focus of the interviews may vary. 


\subsection{Findings - Themes from Interviews with Music Therapists}

Interviewees had differing views about what a consultancy role might entail and the value of that work as they understood it. Similarly, they gave a diverse range of responses regarding assessment procedures although several key tasks were identified. The interview findings therefore affirmed the need for a broad but explicit protocol which included referral and data gathering procedures, a clinical assessment opportunity, provision of resources, and evaluation and follow-up procedures. Only one interviewee referred to the value of an ecological assessment. The following themes emerged.

- Interviewees had various understandings of and diverse attitudes to 'consultation'

- Consultants were viewed as 'expert advisors'

- Consultation was not described as a 'process'

- Interviewees had varying opinions about what activities can be called 'music therapy', but believed that music therapy could only be practised by people who have training in the discipline

- Interviewees believed that team members generally do not have the confidence or musical skills to actively engage students in music making

- Interviewees experienced inconsistent and often unclear processes for referral to music therapy

- The purpose of music therapy assessments need to be clearly defined

- Music therapy sessions identify students' strengths and provide unique opportunities to assess emotional well-being

- Sharing information and working with team members are considered to be important aspects of MT work

- Interviewees experienced limited opportunities for follow-up work, and overall music therapy service evaluation

- Interviewees identified the potential for tension to develop between therapists and educators due to philosophical differences

- Interviewees acknowledged limitations on quality of practice due to paucity of time and money

- The importance of ecological assessment had generally not been overtly considered by interviewees 


\subsection{Expansion on Themes from Interviews with Music Therapists}

\subsubsection{What is Consultation?}

Interviewees had contrasting views about what consultation might involve. For example, some saw it as an educative role, a way to inform people about music therapy such as first visits with families who want to find out about music therapy; sharing information about ongoing music therapy with other professionals, responding to questions from other professionals about the use of music or music equipment, and informing staff and/or carers at meetings, workshops and/or conferences what they might do in music with their students. Others emphasised receiving information, describing interaction with teams to find out what goals and aims they were working on and taking those into music therapy. To some, consultation meant helping others 'take over' aspects of music therapy work when programmes closed, particularly as a way to ease pressure on caseloads. The interviewees also discussed facilitating classroom music or large group work while team members watched and/or supported; choosing, making or gathering resources for team members; composing, adapting and/or directly teaching songs or musical activities for specific purpose. One also viewed consultation as a way of providing a service to geographically distant students and another of supporting teachers to provide a more holistic service to children who have special education needs.

Regardless of their understanding of the role, it was evident that interviewees predominantly viewed consultants as 'experts' who give professional advice rather than a collaborator who works alongside other professionals to meet a common goal. Two music therapists who were also educators believed there would be overlaps between teaching and school consultancy work, and/or they would draw on their educative skills to engage in consultation.

It was clear that interviewees worked both in and out of the regular classrooms with other therapists, classroom teachers, teachers' aides, parents and music teachers and their work was at times highly integrated with that of these professionals. Music therapy was often 
recorded on the IEP as a method or a 'resource' to support other programmes. However, although interviewees described a wide variety of work outside the music therapy 'clinic' including collaborative work with other professionals, some talked freely while others were cautious or even anxious about acknowledging this aspect of their work as 'music therapy'. It seemed they had differing and sometimes strongly held views about what constitutes 'legitimate' work for a music therapist.

\subsubsection{Attitudes to Consultation}

Kath was an educator and music therapist who worked alongside a music teacher with students who were not coping well with the regular music programme. ${ }^{3}$ She enjoyed bringing her music therapy skills to different roles, and valued working with students with a wide range of abilities.

I've got a drumming group at a primary school. Anybody can come. About four of those sixteen that turned up this year are referred children on my caseload... ...for lack of attention, lack of motivation, unable to express themselves in the classroom and so on... (Kath)

Kath was aware of increasing opportunities for collaboration with other professionals yet also described regularly encountering "stifling" role defensiveness from those who liked to 'wear their own hats'. Nevertheless, she argued that it is the right time for music therapists to extend their involvement with other professionals believing an increase in professional collaboration would enable a wider range of people to access services. Her practice example demonstrates how music therapists can work effectively in inclusive settings but provides little evidence of how teachers might be empowered to continue with some of the work.

In contrast, Lisa saw consultation as an integral part of her music therapy practice with adults. She believed it could be a very effective way to help staff work with clients who have not been to music therapy, to support music therapy sessions, or continue with therapeutic music activities once the clinical work has closed.

I'm doing more and more consultation work and I think that is valuable because

\footnotetext{
${ }^{3}$ All names are pseudonyms.
} 
we have a waiting list and we can (only) see people for a limited amount of time. It's a way to extend things that have worked for them into other environments, into their home and day programmes...... (And) can also be a really good way to enhance the relationships between the staff. (Lisa)

Prior to training as a music therapist, Ava had been a teacher. She described herself as a music therapy consultant but continued to notice an overlap with music education. She recounted supporting a four-year-old child with autism who had been "very alert and alive" in the music therapy setting, with his transition to school. When the teacher claimed she was unable to sing, Ava encouraged her to sing the roll.

I went to observe at the end of that class and there she was singing her little heart out - it was wonderful ... then a little while later to see how they were getting on and again to workshop with some ideas - the third time just give them confidence really. (Ava)

Yvonne suggested she valued ongoing clinical work more highly than consultancy aspects, and was sometimes frustrated by the increasing emphasis on short term work due to employer regulations and funding restrictions, and the limited services that could be offered to geographically distant students. Several interviewees appeared to resist consultancy work, and if they did work outside the clinic they implied they were pressured into it, viewed it as something other than 'music therapy' (e.g. education), or took it on as a way to get to the 'real' work. 'Petra' for example was highly resistant to the idea that others might be empowered to introduce planned music experiences into their work. Although she worked outside the clinic with wider school activities such as song-writing, making CDs, and musical productions, she could not, initially at least, imagine other people using music with students who have special education needs without the support of a trained music therapist. Petra described having to close clinical programmes because the "money's run out" but still could not contemplate the idea of engaging in collaborative consultation. During the interview she was able to concede that it might be possible for others to use music but empowering them to do so would draw on her skills as an educator.

I haven't done that and I hesitate to do that - I don't believe in that particularly...I just don't think that music therapy works without a music 
therapist... (it's) about the relationship between the therapist and the client... ...definitely those (facilitation) skills could be transferred to someone with a little bit of musical skill... with a bit of guidance ...but I think there's a real crossover with music education, early childhood education, and music therapy ... I would be very hesitant to call it music therapy. By transferring all those ideas would I be using my therapeutic skills or would I be using my education skills...? (Petra)

In recognising that she would be using new and/or different skills as a consultant Petra was disinclined to "call it music therapy". But this reluctance also seemed clearly associated with a fear of losing her identity as a music therapist, and losing control over what her music therapy practice might entail. She believed that future employers might demand too much of music therapists and that consultancy options would reduce opportunities for music therapists to facilitate 'clinical' music therapy and in turn students would not realise "ultimate" outcomes. It seemed that the perceived enormity of a potential consultancy role produced an overwhelming sense of anxiety in her, making it difficult for her to consider realistic possibilities.

It's a little bit frightening - the few music therapists that we have would then be sitting in an office writing these programmes... (I could) be shared amongst the 300 people that have (special education) funding......It personally scares me. I just think if that becomes the expectation, we are actually losing the whole essence of music therapy. (Petra)

Other music therapists were not as strongly outspoken about their concerns, yet certainly conveyed a cautious approach to the concept of consultation.

I think it is tricky because I do feel a little bit uncomfortable saying to people "Oh well, you haven't got a music therapist there but you can run a group like this"... But I also recognise the value of being able to say to teaching staff or therapists "Use music". Yes. "Use music - and here are some of the things you can do. You can't recreate a music therapy situation but this is going to be really helpful"... I think that is part of our role as music therapists - I don't think we should be exclusive and 'protective' - although I feel that sometimes, that I want to protect it and hold onto it. (Cara) 
Others agreed that team members could be encouraged to use music, but had little confidence in their ability to do it well referring to team members' likely paucity of music skills, particularly their inability to improvise. While they talked about providing activities, recordings and resources, only Ava suggested that it might be possible to empower team members to develop and use their own natural musicality to enable spontaneous musical interaction to occur between them and their students.

Music therapists are trained to work with individuals and small groups, and might resist working in classrooms. 'Myra' argued 'I don't call it music therapy when I'm seeing ten children at once". In one instance, instead of facilitating class music as requested, she negotiated to assess one child each week and write up ideas for the teacher to use.

Robin was keen to describe the advantages of clinical music therapy for students in special school settings. She advocated strongly for a safe non-judgemental environment where students could express themselves constructively and was reluctant to consider work in other contexts. Nevertheless, she described encountering and joining students' spontaneous play in a classroom setting.

(We had) one of those kind of magical moments where they were spontaneously patterning this little ostinato in unison - it wasn't something I instigated... eventually I joined them and there was just a sense of joy in the spontaneity of that interaction. (Robin)

\subsubsection{Referral for Assessment}

Interviewees did not experience a consistent process of receiving a referral, undertaking an assessment and making decisions about ongoing therapy or consultation. They rarely had input into an IEP meeting until a student was already on their caseload. That is, the assessment was not requested as part of the IEP process to help the team to decide what the best course of action might be. Rather, referrals for music therapy came from principals, teachers, therapists and family or caregivers often as a result of the IEP meeting where informed professionals had already observed the student's positive responses to music. Some music therapists who worked within a strongly integrated team took students straight into a programme of therapy with ongoing assessment as part of 
treatment.

Some interviewees had been asked to work with groups of children because funding or time pressures did not allow for individual assessment. Although they did not have the opportunity to formally assess or 'group' students according to perceived need, they were able to 'screen' children for music therapy programmes. Moreover, interviewees believed teachers were able to observe and pick up activities and strategies from the sessions.

\subsubsection{The Purpose of the Assessment}

Most of the 'consultation' work described by interviewees was seen as a part of ongoing work, or as a transitional task which involved the music therapist passing on ideas before music therapy programmes closed. Students were not referred for consultation, and they were therefore unable to discuss assessment which might be undertaken specifically with consultation in mind. One exception was a music therapist who worked for a specialist team which enabled students to have 'one-off' access to a variety of assessment services including music therapy. In that case, reports with ideas for ongoing music were sent to the students' local support teams but facilitation and ongoing support for the introduction of those resources was not part of the service.

Interviewees agreed the primary aim of music therapy assessment is to find out how a student responds to music - the way in which they manage in the music setting can help others to more accurately determine their overall developmental level. However, they also suggested it is particularly useful to discover how students are operating emotionally. It helps to identify their strengths, and new ways of addressing their needs. It can create a new and positive picture of a student, motivating educators and carers to engage optimistically with them. When the music therapist is available for ongoing work, assessment helps to determine whether an ongoing music therapy programme is needed; whether group or individual work is warranted; to identify changes over time; and to determine and/or prioritise the music therapist's caseload.

Some of the music therapists wrote up programmes for teachers to use, but usually for students who were already known to them through involvement in regular therapy 
sessions. In those cases it was not considered necessary to undertake full assessments for consultation because the student's responses in the music setting had already been fully described in clinical reports. None had been able to do full ecological assessments with students to determine ways in which music might be used, or used more effectively, throughout the day at school and/or at home, although one expressed disappointment that this was not possible.

\subsubsection{Sharing Information and Working with the Team}

Interviewees generally reinforced the importance of having good knowledge of the students' backgrounds before working with them. They gathered details about students' history and why they were being referred from various sources including medical, therapy, education records, and from families or managers of residential facilities. Discussion with team members was highly valued. Further, although it was rarely possible, interviewees anticipated that having the opportunity to see students in other contexts would be very beneficial. On the other hand, some also acknowledged that it could be advantageous to do the music therapy assessment in a clinical setting without too much background information, because prior knowledge has the potential to prejudice the process or the interpretation of responses.

Receiving information in an ongoing way can be equally important. Teachers' aides were mentioned as being particularly helpful at music because they were with the students for long periods each school day, and knew them very well. Some interviewees undertook transdisciplinary assessment work, working together and at the same time with other professionals and the student. Yet they conveyed a lack of confidence that the majority of team members would have the level of self-belief, motivation, or skills to use music effectively themselves.

Information from music therapy assessment sessions was shared with team members. Family and/or staff were sometimes invited to observe or participate, although music therapists working within a psychotherapeutic clinical model of music therapy considered observers might disturb or delay the development of their therapeutic relationship with the student. Similarly while video could be considered as an alternative, the intrusion of 
the camera is likely to be equally distracting at times.

Music therapy reporting procedures are variable in special education settings. Some interviewees produced full reports while others integrated brief progress notes with other school records; some reports contained precise data while others described developmental findings in a more general way. Reports describing student responses in the music therapy clinic were shared with assessment teams, with various authorities involved with the student, with Boards of Trustees, and with parents. Some music therapists produced written plans describing music therapy activities for team members, while others met to discuss what colleagues were doing, or might be able to do, with music.

Interviewees did not mention collaboration; building and maintaining positive relationships with team members; and/or including them in discussions about how a consultation might proceed and how ongoing communication, decision-making, and disagreements might be managed.

\subsubsection{Follow-up of Students and Programme Evaluation}

After programmes closed music therapists might receive anecdotal comments from families and colleagues about specific students. Only two of those interviewed were occasionally able to actively approach teams to do follow-up work with individuals or groups and to provide more support when it was needed. Those who work within teams might also receive anecdotal feedback from colleagues about the value of music therapy within their particular service, and agencies or facilities have certain processes which enable staff within them to receive information about music therapy and to make their own judgments about its value.

However, while all music therapists kept clinical records of student progress none of the music therapy programmes discussed were formally evaluated. Further, interviewees did not identify any formal methods for evaluating music programmes facilitated by colleagues. In general, while assessment of individual client or group work was integral to music therapy practice, overall service evaluation appeared to be neglected. 


\subsubsection{Philosophical Differences}

Music therapists in clinical practice with students who have special education needs found educators often had different understandings and expectations of music therapy, and what the role of the music therapist might be. Interviewees suggested they had to balance clarifying their role and convincing others to allow them to concentrate on meeting the emotional needs of the student for example, with focusing on the more easily defined educative goals and outcomes their colleagues expected. Although there was clearly an element of negotiation taking place, they did not suggest that role clarification or any other problem solving was undertaken collaboratively with team members.

Interviewees accepted that the practice of inclusive education had impacted on the practice of music therapy. Moreover, they generally agreed that the music therapy profession needs to respond at least to a certain extent to universal changes in models of practice including an increasing emphasis on short term therapy work.

\subsubsection{Time and Money}

Music therapists agreed that their programmes should take place at school, in school time, and noted that schools wanted more students to have access to music therapy programmes. However, despite increasing acknowledgement of the value of music therapy, limited funds led to music therapists being pressurised to move students on and to fit other individuals into that space. They were often asked to undertake group work regardless of student need. Programmes closed because schools ran out of funds and usually, as mentioned above, there was no budget (or time) for follow-up or evaluation procedures. There were differences in the amount of time that music therapists were able to allocate to assessments across settings and this variation had an impact on the way in which the assessments were carried out.

\subsection{Summary}

Interviewees had come from a variety of trainings and work settings and therefore offered 
diverse responses to interview questions. They agreed that 'music therapy' can only be undertaken by music therapists who have extensive training and high levels of musicianship, and that this differentiated them from others who might use music in their work. They gave the impression that using this training and skill was what defined 'music therapy' rather than the specific practice they engaged in, although the therapeutic relationship was also referred to as a defining criterion.

Consulting with other team members was considered to be part of a music therapist's clinical role and it seemed that interviewees believed that consultancy work paralleled or emerged naturally from direct clinical work. While they expressed diverse understandings of consultation, there was a general view that a consultant would be an 'expert' who imparts information or teaches others new skills. The notion of consultation as a 'process' involving authentic collaboration did not come to the fore during the interviews. Further, while most interviewees already worked outside the clinical setting and were engaged in supporting or working alongside team members using music in various ways, some expressed reluctance to accept this as part of their 'music therapy' work. Moreover, several of the interviewees believed that team members would not generally have the confidence or musical skills to engage students in active music making.

Interviewees have experienced tensions in their work in schools due to the philosophical differences that are associated with the concepts of therapy and education. Furthermore, music therapists have always struggled to define their practice and it seems that some interviewees feared that empowering other professionals to use music with students who have special education needs might weaken this definition further, threaten their identity, and limit music therapy employment opportunities. The majority appreciated the need to engage in multiple roles but none wanted to lose possibilities to undertake in-depth clinical work, which Petra described as 'the whole essence of music therapy'.

None of the interviewees had assessed students for consultation purposes and few had done single-session assessments. The purpose of an assessment determines how it is undertaken, so it was not possible for interviewees to discuss how a single music therapy session might contribute to assessment for school consultation. However, they were able to identify tasks that they believed were important as part of any music therapy 
assessment. Specifically these involve receiving a referral, gathering data from students' health and education records, discussion with team members, and music therapy clinical interaction with the student. Interviewees noted that the clinical music therapy session highlighted students' strengths and provided unique opportunities to assess their emotional well-being. The value of undertaking ecological assessment was mentioned by one interviewee.

The process of music therapy consultation is likely to involve considerable variations to current practice and, as Petra demonstrated, the enormous potential for community work can feel overwhelming. Although most of the interviewees described interacting musically with clients outside of their 'music therapy' practice, they were not used to having observers in their music therapy sessions. Most did not prepare programmes, activities and resources for others to use. Only one interviewee had experienced preparing full developmental reports based on a single clinical session. None were used to evaluating team members' musical abilities, supporting their use of music, evaluating their progress and providing follow-up. Time and money limitations prohibited follow-up work and programme evaluation.

The findings from interviews also highlight the need for formal evaluative procedures at the programme level for all forms of music therapy practice in New Zealand. Outcome evaluations would be likely to validate the service and could be used to identify unique aspects of music therapy and give confidence to the profession as a whole. It seems therefore that there is a need to attempt to 'define' and describe the practice of music therapy school consultation which would enable consultation practice to be explicitly outlined and transparently evaluated. These findings give impetus to the development of a music therapy consultation protocol. 


\section{CHAPTER SIX: THE INITIAL PROTOCOL}

\section{Introduction}

Critical reflection on the interview themes and my previous consultation experiences, led to the construction of an initial consultation protocol. ${ }^{4}$ However, as noted earlier, interviewees engaged in consultation tasks as part of their ongoing work but only one described herself as a consultant. Therefore, while interview findings contributed, the initial protocol was mainly generated from critical reflection on my own consultation experiences. I chose not to employ a specific theoretical construct for the initial consultation protocol preferring to draw on practice-based knowledge. The practice described below was influenced by the writing of music therapists and colleagues in allied fields who described a 'continuum' of therapy practice for therapists in schools, which includes direct one to one or group work in the music room, small groups in the classroom, work with the teacher in the classroom, work with the student and possibly peers in the playground, whole school activities and so on.

The following paragraphs describe the initial understandings I took into my action research journey. Thus my 'existing situation' is outlined before change is implemented. The specific steps I would follow during the stage two implementation phase are outlined in Table 1 (see p.123).

\subsection{A Starting Point for the Action Research}

In the past I have provided assessments for students in mainstream settings, particularly in isolated areas, and have been able to give advice to team members about how they might use music with these students. Travel and accommodation was arranged to allow a full day with the student and their team. The process involved reviewing the student's IEP prior to the visit, talking with the team, facilitating a music therapy session with the

\footnotetext{
${ }^{4}$ Videotapes of single-session assessment sessions from previous consultations were also analysed to support the development of the initial protocol but findings have not been included (as explained on p. 80).
} 
student, providing resources such as an original song for a specific purpose, interacting briefly with team members and students as they attempted a musical exchange, preparing an extensive reporting package including ideas for ongoing musical interaction with the student. Whenever possible I also made connections with local people, such as a music teacher, who could support the work in an ongoing way.

Although the people I worked with valued my contribution, I was aware that improvements could be made. The main issues that I wondered about included 'time to build relationships', 'how to ensure the best information is gained from an assessment', and 'how the process might be formally evaluated'.

\subsection{The Plan}

\subsubsection{Building Relationships}

Maintaining healthy professional relationships is important in all music therapy practice. It will be essential for me to develop and maintain positive relationships with team members to ensure their input is valued, ideas are taken on board and planned music experiences are utilised in a sustainable way. One day of interaction has not seemed enough in the past. I anticipate the one week 'block of time' allowed for each consultation in the context of this research will enable me to move from negotiating plans of action to engaging with teams in authentic in collaboration.

When the emphasis shifts from negotiation to collaboration there is more opportunity for learning about each others' priorities and planning programmes together. Thus opportunities will arise for developing mutually beneficial ways of working which embrace the philosophies of both music therapy and education. Beginning with what the teacher and/or teacher's aide is already doing, and developing existing programmes taking into consideration what skills they have, the IEP goals for the student, and the available resources, seems a logical starting place. Even so, high workloads experienced by team members might prohibit their involvement at desired levels. 


\subsubsection{Assessment for Consultation}

The purpose of an assessment influences the type of information generated and the relative importance of that information for client, clinician and other team members. While there are no guidelines as to what 'assessment for consultation' might involve, some 'standard' assessment tasks including receiving a referral, data gathering, choosing an assessment method, facilitating an assessment session to observe the client's ability and interest in music experiences, evaluation or interpretation of the session, and reporting outcomes, should be included in a music therapy school consultation protocol.

I will need to gain consent to access school records and to plan time to review these early in the process. I will meet with students' families and/or caregivers, at home if possible. Extensive observation and interaction with the student and consultees in a range of settings will be essential to the success of the music therapy consultation. I will need to find out what team members can do, discover ways to expand their musical experience, and encourage their use of music to meet students' needs.

The clinical music therapy assessment will be central to the consultation process. While a range of assessment tools are available for music therapy assessment with children, many practitioners use therapist-constructed tools and/or descriptive methods. The individual sessions will be student-centred, predominantly improvised and free-flowing. However, the use of precomposed familiar music is also likely to be introduced to find out what the students enjoy and what they can remember from other settings. Further, familiar music can help a child to relax, "rather like having a musical hand to hold" (Flower, 1993, p. 42).

Students' strengths are often highlighted in the clinical setting. Evaluation of clinical interaction can also highlight the students' specific responses in relation to the music therapy strategies and techniques that are employed. Ongoing musical interactions can be planned based on these responses. When assessing for consultation, I will therefore need to welcome observers and/or ensure that videotape is available to enable other professionals to gain insight, knowledge and skill from the work. Team members will be invited to watch the video and to discuss ways in which music might be incorporated into 
the student's programme. Descriptive methods will be used to evaluate and report on the clinical assessment

\subsubsection{Developing Resources and Sharing Information}

I will also need to support team members to develop musical activities to use at home and/or school, and/or recommend ways in which students might increase participation in their regular music programmes at school. I will need to produce a report that includes a full developmental description based on the clinical assessment, observations and musical interactions with the student in a variety of contexts, and the resources, activities and strategies developed in collaboration with the team.

\subsubsection{Evaluating and Providing Follow-up}

I will need to monitor team members' progress and offer to provide further advice and resources after leaving the school. The amount of follow-up needs to be realistic in financial and personal terms, and should be pre-determined as part of a contractual agreement for assessment and consultation. At present it is unclear how much would be reasonable and achievable but I have chosen a time-frame of one term which, in the context of this research, enables me to complete my work with one team before engaging with the next.

Team members will be invited to telephone or email me in the follow-up period with questions, and to send video of their interactions for feedback on their work. They will be asked to keep a diary of their musical interactions with students to share with me at the end of the follow-up period. I will also request to interview them at that time and the findings, along with the diary entries, will provide an indication of whether they have been empowered to continue and/or to develop their music work. While these tasks are also part of the research protocol, they will be practice-based tasks in the context of music therapy school consultation. 
Table 1: An Initial Music Therapy Assessment and Consultation Protocol

\begin{tabular}{|c|c|c|c|c|}
\hline $\begin{array}{l}\text { Data Gathering for a Special } \\
\text { Ed MT Assessment }\end{array}$ & $\begin{array}{l}\text { Additional data Gathering } \\
\text { for Assessment and } \\
\text { Consultation Purposes }\end{array}$ & $\begin{array}{l}\text { The Assessment Session - } \\
\text { Music Making and } \\
\text { Observing }\end{array}$ & $\begin{array}{l}\text { Interpreting in the light of } \\
\text { ITM Skills/Opportunities }\end{array}$ & Planning \\
\hline $\begin{array}{l}\text { Request family history and } \\
\text { child development } \\
\text { information, including } \\
\text { diagnosis and recent medical } \\
\text { and therapy reports }\end{array}$ & $\begin{array}{l}\text { Find out about the skills of the } \\
\text { IDTM/consultee who will take } \\
\text { responsibility for the } \\
\text { therapeutic music programme. }\end{array}$ & $\begin{array}{l}\text { Engage student in listening, } \\
\text { playing, singing and moving } \\
\text { with precomposed or } \\
\text { improvised music; and engage } \\
\text { in discussion if appropriate. } \\
\text { Observe musical and non- } \\
\text { musical behaviours. } \\
\text { Determine which types of } \\
\text { music therapy experiences are } \\
\text { engaging for the child. } \\
\text { Identify strengths and needs. }\end{array}$ & $\begin{array}{l}\text { Think about how the child's } \\
\text { motivation for specific music } \\
\text { experiences might be used to } \\
\text { address developmental goals }\end{array}$ & $\begin{array}{l}\text { With the IDTM/consultee } \\
\text { consider what specific } \\
\text { experiences activities might be } \\
\text { employed when the therapist } \\
\text { has gone and how the } \\
\text { intervention will be monitored } \\
\text { in relation to student goals. }\end{array}$ \\
\hline $\begin{array}{l}\text { Request education information, } \\
\text { including latest IEP, } \\
\text { communication systems, } \\
\text { positioning requirements, any } \\
\text { other special equipment, etc.; } \\
\text { and experience of and } \\
\text { relationship to music. }\end{array}$ & $\begin{array}{l}\text { Find out about the } \\
\text { environment - what space is } \\
\text { available, what instruments are } \\
\text { available. }\end{array}$ & $\begin{array}{l}\text { Use all resources available to } \\
\text { you \& bring in additional } \\
\text { equipment if required. The } \\
\text { session should mimic what } \\
\text { would occur in a 'clinic' } \\
\text { setting as much as possible in } \\
\text { order that the MT might } \\
\text { 'come to know' the child. }\end{array}$ & $\begin{array}{l}\text { Think about how the student's } \\
\text { interest/motivation can be } \\
\text { fostered in the light of the } \\
\text { musical skills of the } \\
\text { IDTM/consultee }\end{array}$ & $\begin{array}{l}\text { With the IDTM/consultee think } \\
\text { about what staff development } \\
\text { and/or resources might be } \\
\text { necessary to maximise their } \\
\text { potential use of planned music } \\
\text { activities. }\end{array}$ \\
\hline $\begin{array}{l}\text { Find out about the child's } \\
\text { timetable, including other } \\
\text { programmes they might be } \\
\text { involved in and where those } \\
\text { take place. }\end{array}$ & $\begin{array}{l}\text { Extend observation and } \\
\text { discussion, to determine how } \\
\text { music can be employed in } \\
\text { other settings. Consider how } \\
\text { the implementation of } \\
\text { therapeutic music might } \\
\text { impact on other students. }\end{array}$ & & $\begin{array}{l}\text { With the whole team, think } \\
\text { about where music making } \\
\text { might take place (classroom } \\
\text { group work, playground and } \\
\text { other environmental } \\
\text { 'incidental' and/or } \\
\text { withdrawal). }\end{array}$ & $\begin{array}{l}\text { With the whole team, } \\
\text { reconsider and negotiate } \\
\text { timetables as necessary and/or } \\
\text { appropriate. }\end{array}$ \\
\hline $\begin{array}{l}\text { Talk to staff and observe the } \\
\text { child in other settings to find } \\
\text { out how they respond in a } \\
\text { 'non-musical' setting }\end{array}$ & & $\begin{array}{l}\text { Involve all team members in } \\
\text { observing the music therapy } \\
\text { assessment session so they } \\
\text { have opportunity to note } \\
\text { child's responses to music. } \\
\text { Identify strengths and needs. }\end{array}$ & $\begin{array}{l}\text { With the whole team think } \\
\text { about how music might be } \\
\text { used to enhance the wellbeing } \\
\text { or education of other students. }\end{array}$ & $\begin{array}{l}\text { With the whole team, consider } \\
\text { how the implementation of the } \\
\text { programme might be } \\
\text { monitored and evaluated } \\
\text { overall (including the impact } \\
\text { of the changes on staff and } \\
\text { other students) }\end{array}$ \\
\hline
\end{tabular}




\section{CHAPTER SEVEN: STAGE TWO. ACTION RESEARCH - CASE STUDIES}

\section{Introduction}

This section describes four consecutive case studies undertaken in 2007. Each involved a week in the field with the team, and one school term (approximately ten weeks) of follow-up. The case studies were undertaken:

1. To introduce the music therapy consultation protocol with the aim of empowering team members to use music therapeutically with their students. Each team collaborated with the music therapist to develop, introduce, and evaluate their own project.

2. To find out how the consultation was received and valued by each team and as that information came to hand to modify the protocol accordingly.

\subsection{Presentation of the Case Studies}

Participants in each case were mindful of their role as action research participants. The way in which these steps in the action research cycle link with the presentation of findings is illustrated in Figure 4, p. 91.

Each case is presented in the same chronological format (see Figure 4, p. 91), beginning with a background to the case, an introduction to the team, and a brief account of ethical issues specific to the study. This is followed by an articulation of negotiated values and ideas, described as 1) the team's expectations of music therapy, 2) programme and IEP goals and 3) and the use of music before my visit. Material relating to assessment is presented as 1) ecological assessment 2) identified strengths and needs 3) assessment in the music room, and 4) team observations and discussion of the assessment material (a fuller example of focus group interaction is provided in APPENDIX 36, p. 460). ${ }^{5}$ After that 'Planned Music Experiences' are described and their initial evaluation is presented

\footnotetext{
5 'Assessment of the general environment' does not include detail of team members' musical interactions with students. This information seemed to sit more comfortably with 'agreed planned music experiences' and 'introducing the strategies'. Further, although some references to musical responses are included in 'identified strengths and needs', more extensive elucidation of students' musical strengths surfaces from 'assessment in the music room'.
} 
in sections entitled 'Introducing the Strategies'. Finally, team members' continued use, modification, and valuing of planned music experiences are captured in the section entitled 'Continuing the Work'.

As each case study was completed, it was analysed further 1) to find ways to improve the protocol (action learning cycles are presented after each case) and 2) to extract themes to answer the research questions. Space limitations prevent indicators of efficiency and learning outcomes from all four cases being presented separately. Instead, they are synthesised in the findings section and examples from an individual case are provided in APPENDIX 38, p. 488, and APPENDIX 39, p. 490.

To protect team members' identities, demographic information is presented away from the case studies, in Table 2 below, and all names are pseudonyms. In each of the case studies drawings of students demonstrate real interaction but disguise facial features.

Table 2: School Demographics

\begin{tabular}{|l|l|}
\hline Schools & Town/Village \\
\hline Decile 6 & Coastal tourist town \\
Catholic Integrated Primary & 3,500 \\
130 Pupil & Closest city by car 3 1/2 hours \\
$75 \%$ Māori & \\
\hline Decile 5 & Outside city \\
Full Primary & 6,000 \\
750 Pupil & Close to city and special education \\
$40 \%$ Māori & resources \\
\hline Decile & Rural coastal town \\
Contributing Primary School & 5,000 \\
250 Pupil & Closest city $21 / 2$ hrs away by car \\
$95 \%$ Māori & Rural town \\
\hline Decile 5 & 2,200 \\
Full Primary & Closest city 1 hr away by car \\
320 Pupil & \\
$4 \%$ Māori & \\
\hline
\end{tabular}




\subsection{TIM}

\subsubsection{Background}

Tim was a seven-year-old boy with a diagnosis of global developmental delay, and many symptoms of Autism Spectrum Disorder. He was nonverbal, had very high needs according to the ORRS guidelines, and attended school with thirty hours of teacher's aide support. Tim's cultural identity was recorded as 'New Zealander'. The team hoped the music therapy consultation would help develop their mutual understanding of Tim.

\subsubsection{Introduction to the Team}

The referral was made by Tim's Special Education Adviser (SEA), a MoE SE staff member. The participating team consisted of Tim and both his parents (Lee and Patrick), the classroom teacher (Mary), three teacher's aides (Sally, Siobhan, and Valerie) and the .2 ORRS Teacher (Angela). Others who worked with Tim "once or twice a term" included physio, occupational, and speech language therapists, as well as the SEA. Angela, Sally and Siobhan all agreed to be consultees, to introduce planned music experiences with Tim and report on progress, with Angela as team leader.

\subsubsection{Ethical Issues with Regard to Participation}

Although the initial referral was made by the SEA, no other MoE SE staff members were listed as potential participants in the project. However, sharing information and experiences with colleagues seemed critical to gain maximum understanding of the potential for music to assist students. Since teamwork was seen as integral to consultation practice they would typically be actively encouraged to participate, particularly in viewing the video of the clinical assessment session and the ensuing discussion. However, in a research context it felt less appropriate to actively solicit colleagues support in case they felt pressured or 'coerced' to participate in the research project. On the other hand, it was important to ensure that the assessment process was not compromised because of the research. People needed to be involved in whatever way they might under 'usual practice' conditions.

The MoE research division agreed to forward letters to their Service Managers to 1) 
request that $\mathrm{MoE}$ staff be given permission to collaborate in the consultation process as part of 'usual practice' and 2) suggest that from a research perspective it would be helpful to have a range of cross-disciplinary views about the work and 3) seek permission for staff to give informed consent to be involved as participants in the study should they choose to do so. In Tim's case none did, and feedback from service managers suggested they perceived the workload would be too high to allow staff to be involved.

\subsubsection{The Team's Expectations of Music Therapy}

Angela anticipated the consultation process would involve the music therapist "walking alongside" the team to highlight any occasions when music could be used to help Tim manage and enjoy opportunities for learning. They knew he loved music and believed they could use it to communicate with him, but didn't think they had the skills to use music to best advantage.

\subsubsection{Tim's Programme and Individual Education Plan Goals}

Primary goals for Tim related to behaviour, communication, sensory difficulties, and socialisation. The strategies and adaptations listed in his IEP included a 'sensory programme', 'swimming', 'biking', a 'physio programme', and 'music therapy'. There was no other reference to the way in which music might have helped Tim achieve his long term goals or teaching targets. His IEP identified twenty teaching targets which included reducing aggressive behaviours, staying in the classroom and/or at a workstation or desk with decreasing teacher's aide support, making appropriate transitions to new activities, sitting and/or playing alongside peers, developing use of Picture Exchange Communication System (PECS) (Frost \& Bondy, 2002), and making choices. $^{6}$

The team was using the principles of Floortime (Greenspan and Wieder, 2006) to engage Tim at his developmental level, and to build on his strengths. Floortime and

\footnotetext{
6 PECS is a communication programme for children who have limited functional speech. Using knowledge from the fields of applied behaviour analysis and speech-language pathology, it promotes communication within the social context, beginning with teaching the child how to make a spontaneous request by producing a picture which symbolises the desired object/activity.
} 
music therapy share the premise that carefully timed exchanges of non-verbal signals form the basis for learning and emotional development. The two approaches are therefore highly compatible. Further, music was one of Tim's primary reinforcers, could calm him, focus and hold his attention.

Floortime is incredibly important to us because we are focusing on trying to establish some sort of recognition or participation... Because a lot of the times we're working with Tim he doesn't show any desire or any recognition of the need to initiate or to use verbal or any physical gestures... ... Floortime is play and play is interaction and music is in there totally - it just all meshes in together. (Angela)

It seemed likely that an improvement in communication skills would lead to less challenging behaviour and an increase in positive socialisation. However, communication requires motivation to interact and the ability to attend, both of which were difficult for Tim.

I just think anything that's going to help Tim to develop as a person and any skills that can help us to reach him and to give him some more independence, choice, and a better quality of life would be fabulous... any way that we can reach him. (Sally)

As music was one of his primary reinforcers, the music therapy referral seemed highly appropriate.

\subsubsection{Use of Music Before my Visit}

Music played a big part in Tim's home and school life and team members had all witnessed his positive response to it in various ways. Lee took him to infant and toddler music classes and, because of his limited communication and mobility as a preschooler, frequently 'entertained' him with finger plays, singing and action songs. She felt he had certain rhythms that he liked, knew that music could calm, focus and hold his attention, and that he particularly enjoyed the music of baroque composers, especially Vivaldi. Although Patrick agreed that music was important he was less confident with his voice, and rarely sang to Tim.

Angela was musically confident. She played the violin for six years as a child, and studied piano briefly in her adult years. At the time of the consultation she was not 
playing musical instruments for her own pleasure but her husband and son were both active musicians.

We've got a house where we have music going while we're doing the housework and everybody's singing - it's a pretty loud sort of house. And I come from a family of eleven kids so we have complete confidence in our voices! (Laughs) ...I don't think it matters how good a singer you are or what your knowledge is what I think is important is that you grab moments with Tim where he is in there with you, because there are plenty of moments when he is not... (Angela)

Sally said her musical experience was limited to "singing in the bath" but she appeared comfortable using music spontaneously with Tim. When he was upset she said she might "sing a little song" and massage his head.

But (when) he's happy... I can just sit down in front of him and we sing and I do lots of reciprocating of his verbal sounds. (Sally)

Siobhan could play accordion and piano and felt she could 'hold a note' with her voice, but seemed shy about singing with Tim. She was new to working with him at the time of the consultation and had not begun to use music in spontaneous ways perhaps because her relationship with him was still developing.

Music was used at school to calm Tim down towards the end of the day when he frequently became upset. Music CDs and DVDs were available to him in the library. Prior to my visit staff would also sometimes take him into the music room to play various musical instruments and/or sing with him.

Sometimes he will lick or hit the instrument against his skin - other times he'll go for the tambourines and he'll just rattle them. And he LOVES songs! (Siobhan)

\subsubsection{Assessment Activities}

\section{Ecological Assessment}

The school environment was compact and tidy, and the general atmosphere was warm and relaxed. A room with a piano, guitar and limited percussion instruments could be accessed for music making. Team members interacted with Tim in all areas of the 
school as he attempted to draw letters in play-dough, played with bubbles, blew whistles and engaged in his perceptual motor programme (PMP). The PMP programme involved a range of gross motor activities using various pieces of equipment including spinning on a 'turning chair' to provide vestibular stimulation. Tim loved his 'turning chair' and it was frequently used as a reward. I observed Tim spontaneously stamping his feet, running away, hiding, climbing steps and stairs, vocalising and singing. I saw his fascination with wheels, and the comfort he gained from carrying plastic containers with him.

As a team we gradually began to introduce familiar and improvised songs and chants to support this work. We rehearsed timing the music to Tim's actions, pausing for his response, and the use of lyrics, melody and rhythm to support his movement. We also considered how we might use an original song to help him to 'stop' if he was running towards the school gate.

We watched Tim trying to manage 'mat time' and physical education with the other students, and at morning tea and lunch times. We often observed him sitting away from his peers, apparently lacking interest in them. Tim also struggled to transition from one activity to another variously sitting on the ground and refusing to budge, wandering away, or was removed from class activities because he was not managing to be alongside the other students. Team members would frequently physically move him to where he needed to go. We therefore introduced 'standing up' and 'walking' songs (see agreed music experiences, p. 136) to increase his independence with transitions.

It seemed that recorded music was predominantly used in the classroom although I also observed Mary encouraging student leaders to call the roll using a 'Roll Rap'. This focused Tim, especially when the rhythm was strong. Team members also engaged Tim in an individual music session away from the classroom (see agreed music experiences, p. 136).

\section{Identified Strengths and Needs}

Tim's team quickly identified his strengths and frequently communicated how much he was loved at school and at home. His parents had a very positive view of him and high expectations for his future, wanting to draw out the best in him because they believed he 
had more potential than he was able to demonstrate. Lee and Patrick were determined to help Tim to develop a relatively independent lifestyle and the skills to contribute to society in a positive way. Underlying this was their desire for him to have a sense of belonging and positive self esteem. Nevertheless, they were realistic and aware that Tim would need to take a multitude of small steps towards the long term goals they had for him.

\section{Communication Profile}

Few experiences were motivating for Tim, and although he focused well on things that interested him (e.g. the empty containers he carried with him) his interpersonal skills were extremely limited. He had a very short attention span, and was described as 'living in his own world'. Although he appeared to understand more than he could express, developing his communication skills was a high priority for the team. Tim was able to make choices, but had a quirky sense of humour and it seemed he often chose not to engage or respond.

Tim used a Big Mac, with hand-over-hand support, to communicate news to his classmates, and had begun to use the PECS, inconsistently, at a basic level. He vocalised spontaneously using varied intonation, a range of vowel, consonant and blended sounds, and could say simple words, particularly negatives such as "na!" and "no!" He had progressed steadily, albeit very slowly, through developmental stages of sound making and was increasing the number and varying the type of sounds he used.

\section{Physical and Sensory Profile}

Tim was mobile, and was developing physical strength. At the time of the consultation he had only been walking for two years but was able to 'stamp' his feet, propel himself on a swing, and get onto his trampoline independently. He was variously over or understimulated with visual, auditory, and tactile information, and persistently sought vestibular stimulation. He enjoyed physical contact on his terms, but often resisted touch or startled suddenly when touched without warning. Although he was often nonresponsive, or responded slowly, to interesting auditory stimuli, he was also readily distracted by extraneous environmental sounds. Tim had an independent spirit and high levels of determination which led staff to believe they had no option but to physically 
move him when he didn't want to go somewhere.

\section{Emotional and Social Profile}

Tim was very independent in the sense that he knew what he wanted, and was described as a strong individual. His lack of expressive language led to increasing frustration and appeared to contribute to outbursts of physical aggression that were causing team members concern. Tim experienced emotional dysregulation, had no awareness of danger and frequently ran away from adults who were trying to keep him safe. ${ }^{7}$ His behaviour was generally regarded as very challenging, particularly because he was unpredictable.

Tim's difficulty with social reciprocity meant he was usually unable to acknowledge or respond to other students. Nevertheless, he was 'popular' at school and children were approaching and interacting with him in the playground, keen to develop friendships.

\section{Activities of Daily Living}

Despite his stubbornness, Tim understood and was beginning to comply with routines such as sitting on the mat. He had also begun to help with the steps involved in simple tasks, such as making a cup of Milo.

\footnotetext{
${ }^{7}$ Emotional dysregulation refers to an emotional response that is poorly modulated and falls outside the conventionally accepted range of emotive response. The response is not necessarily negative, and can also be seen within the positive range of emotions. Severe mood dysregulation is known to occur in conjunction with many childhood disorders, including ADHD and ASD.
} 


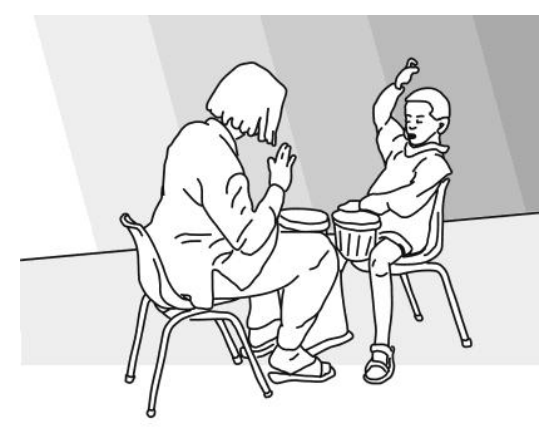

During my 18 minute individual assessment session with Tim we sat face to face, on small chairs, playing percussion instruments and/or singing. This level of physical containment seemed essential to maintain his engagement.

Throughout the session I was interested in Tim's ability to attend and interact. I was looking for signs of focused listening, indications of interest in any particular activity, song, or instruments, the initiation of sounds, and/or the production of sounds in response to musical stimuli.

A close analysis of a few minutes of the session demonstrates the predominant quality of the interaction we were able to have...

Tim smiled as he sat easily on the chair, legs dangling, and gave me direct eye contact. He began to twirl the cluster bells by hanging them over his thumbs and moving his arms in a rolling motion. I sang a simple Hello song in the same tempo as his movement. At the end of the second phrase Tim stopped twirling, gave me direct eye contact again, and laughed. He continued to laugh through the repeat of the first phrase, only playing his bells sporadically. But during the repeat of the second phrase he resumed turning them in time to the music, stopping and laughing only at the end.

As we continued with the bells I began to sing an adapted personalised version of jingle bells. Tim was engaged: many times he 'stilled' or turned towards me, or laughed when I imitated him. He vocalised more during the session than he had prior to music, and at one point offered an approximation of the word "more". Occasionally he sang clearly pitched tones such as a falling third from F-Db, and was noted to vocalise around the tonic note I was producing in my song. He allowed me to hold the bells with him and to coactively shake them - still laughing, even giggling at times, and smiling. Eye contact increased during the exchange.

When I offered him a pocket shaker from the table he did not get up to fetch it, but took it from me when I brought it to him. As I waited I heard him sing "uh uh uh uh" ... 


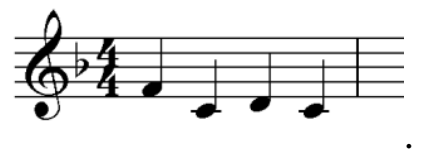

Then, with the pocket shaker in his hand,

Tim was tapping his feet together in time with the steady beat, but stopped immediately when I finished imitating his phrase. He became still, listening, and looking directly at me. He waited until I came to the end of my improvised song before standing and turning as if to leave. As I held him at the hips and guided him to sit down again he was smiling. He began to 'flap' in time with my singing, still smiling, and it seemed as if he was dancing. His 'dance' slowed almost to a stop at the end of the second phrase but gradually built again with the music and was energetic for a while - he seemed unable to stop. He was giggling and might have been 'teasing', or simply enjoying himself. He put his hands down as if he was 'finished' but added a little flamboyant 'coda' to his dance, and a vocal call - an anacrusis on $F$ and a clear tone on $G$. We had a brief vocal exchange before I repeated the song. As the original tempo of the Shaker song returned Tim turned toward me and resumed his dance.

We continued with the pocket shakers for several minutes and Tim engaged in several turn taking exchanges before I introduced the bongo drum. He continued to offer musical responses. Tim was definitely not a young man 'in his own world' during music sessions, as the following example demonstrates.

\section{Timothy - Bongo Drums}
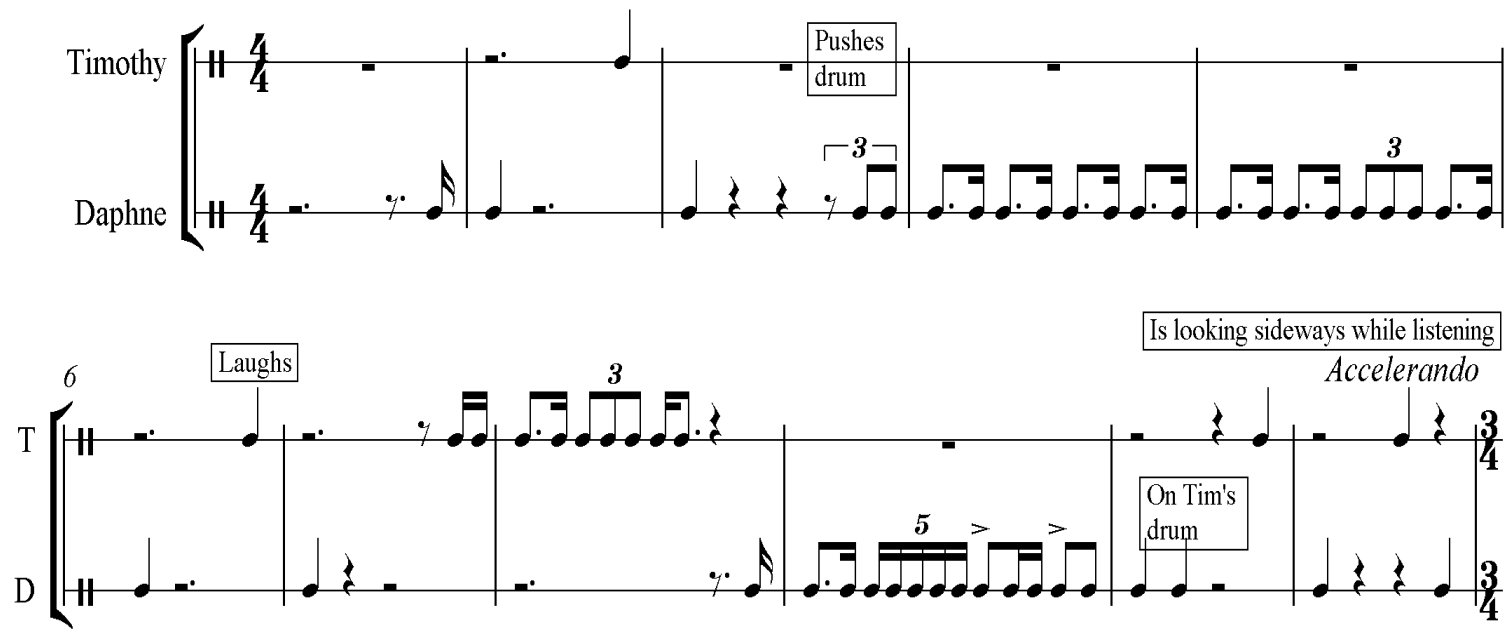


\section{Summary of the Individual Assessment}

Tim demonstrated interest in other people in the music session and offered good quality eye contact which increased in duration throughout the session. He noticed when music matched his movement/playing, and was aware of simple phrasing. He was able to keep a steady beat; imitate and extend a relatively complex rhythmic pattern; move in time to music, and produce short melodic vocal phrases with some clearly pitched tones. He had begun to initiate and improvise and build on musical phrases. He cooperated during coactive instrument playing and was able to turn-take several musical exchanges. Tim enjoyed musical games, and demonstrated his sense of humour.

\section{Team Observations and Discussion of the Assessment Session}

After viewing video of the music session, team members conversed about his potential to communicate. They made links with suggestions and ideas introduced during the assessment period and accepted direct critique of their interaction with Tim. They clarified what some of his observed behaviours might mean in terms of his overall development. However, they acknowledged that it was not always possible to know what was going on and considered ways they might enjoy interacting with Tim at his current developmental level.

Team members were able to reaffirm their current practice. They recognised and reinforced the value of the music they were already doing with Tim; noted that they each brought different strengths to the team and gave each other encouragement and confidence. They identified issues to refer to other professionals (such as the occupational therapist).

The team renegotiated long term goals, and identified some underpinning skills Tim would need to meet them. They learnt the reasons for making particular choices with regard to musical interaction, made connections between theories of ASD and their practice, and learnt some basic principles of music therapy. They clarified why various music activities might be used and how they might be carried out, considered how Tim might be challenged to progress yet still experience success, and began to make plans for how they might include music in Tim's timetable. 
The team agreed they would continue to use music spontaneously but would include short structured sessions as well, while acknowledging that the work was not always easy, that Tim would respond differently at various times, and that the music activities might not always be successful.

\subsubsection{Agreed Planned Music Experiences}

Observations and interactions with the team over the period of the week enabled me to discuss and model a variety of activities and strategies they could use with Tim during the course of each school day. For example my field log notes:

Tim sat on the ground and would not weight bear when lifted. I sang

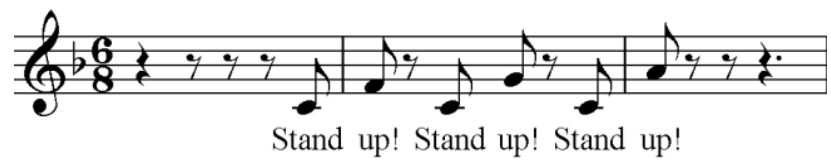

while giving him a prompt by touching his shoulder. Once he was on his way, we also sang a walking song. While not an 'instant fix' both of these songs seemed helpful. Tim was showing more willingness or ease of movement as we repeated the songs... Further, as I sang the walking song phrase "Walking to the Classroom", I heard him imitate the scale:

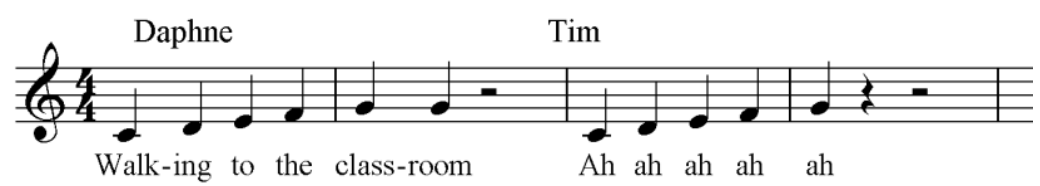

And later...

Taking a cue from Angela, I sang "Stand up" as above, stamping with the beat. The second time I sang I tapped Tim's back, and the third time I give him under arm support. He still did not weight bear but laughed and vocalised

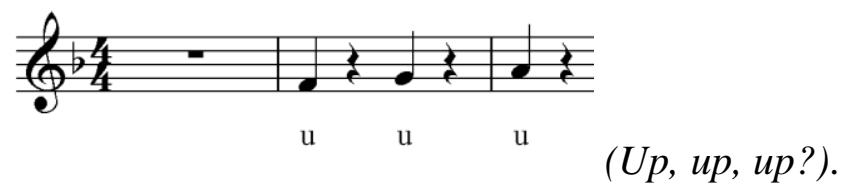

I laughed with him and repeated the sequence, and this time he stood willingly. He walked to the toilet as we sang the walking song, which offered him a strong clear beat to follow.

It was soon clear that team members would value anything to assist Tim to transition 
from one activity to another, without needing physical support. And although they were mostly musically confident, they had not often considered using live music during difficult moments. However, they planned an individual session of music making with him which gave me the opportunity to demonstrate the use of musical instruments as an interactive communicative medium with Tim.

In the library Valerie introduced a box containing various simple percussion instruments. She held out a drum and Tim beat the rhythmic pattern:

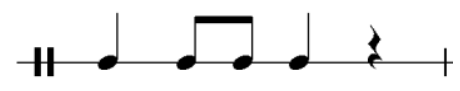

Unfortunately Valerie didn't seem to notice his response and I got the feeling she was playing to him instead of with him. I asked her to listen to him and respond to what she heard and she quickly realised that a musical communication was possible.

Over the week I noticed that some of the staff demonstrated heightened awareness of Tim's responses, listening and interpreting them as important communications. They were already using and enjoying the success of some of the music activities and strategies.

When Tim vocalised

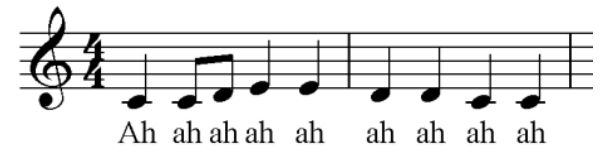

Sally and I simultaneously recognised it as a possible rendition of "Down at the Station" (see below). She reported that it was a favourite DVD... (Was he requesting it by singing?)

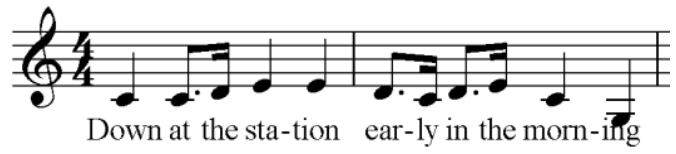

(Later) Sally sang 'Winnie the Pooh' (as Tim ate his honey sandwiches) in a warm and calming voice. She moved her body to the gentle rhythm, employed an appropriate level of accent, paused occasionally and kept him calm and interested.

Tim's positive response to the 'Roll Rap' prompted his classroom teacher to use it regularly. A greetings song was also introduced to increase his socialisation and 
communication with peers in the classroom. Team members were encouraged to memorise an original song recorded on $\mathrm{CD}$, and facilitate the activity live with the class. When we introduced the song Tim expressed enjoyment and interest in staying with the group. Each day I joined the class to help them maximise the opportunities the song presented, for example highlighting how musical elements could gain his attention, and the structure could help him anticipate when he was to respond. Team members and peers also learnt to give him more time to respond.

Music was also introduced to support Tim's perceptual motor programme (PMP) with good results. The team believed he was demonstrating very good levels of engagement during the (PMP with music) session and the time he was able to stay in the gym (45 minutes) was excellent. They were keen to continue with music in this context. The songs were notated and recorded on $\mathrm{CD}$, with a copy for each team member.

\subsubsection{Introducing the Music Therapy Strategies}

\section{Learning the Importance of Timing}

After observing me with Tim, Angela saw that recognising and specifically acknowledging Tim's efforts to communicate were important keys to improving his communication skills.

Imitating Tim's efforts is something that we've done occasionally but we've never used it in the way we've seen it this week. So that is REALLY important for us ...giving him some recognition of his communication attempts. (Angela)

She was impressed with the opportunities music provided for Tim to anticipate or predict a response and the idea of leaving a gap for his participation. Team members gained a heightened awareness of the need to balance 'intruding on Tim's space' with giving him time to communicate what he wanted, and had learnt to wait for Tim, to allow him the time to process what is going on.

In fact what I really think I've learnt most is to stop... and to be patient... rather than to do ANYthing else"... ... "Seeing somebody else WAIT for him and allow him the time to process what is going on has given US enough skill to show us how we might go forward. (Angela)

They began to appreciate the opportunity music afforded for reciprocal communication. Sally described her observations of my interaction with Tim in a very musical way. 
...that backwards and forwards rhythm for Tim is really important... you just seemed to have this real knack of tuning in to him, getting on his wavelength I think...(I've seen) the importance of giving him that chance to sing back to you and reciprocate the tune... (Sally)

\section{Student Response - Reducing Physical Prompts}

To reduce their physical handling of Tim, the team began to use songs to motivate and prompt him to negotiate his environment with more independence.

That is really important to us... We've tried a number of different strategies over the three years and the LEAST physical prompting is a priority for us. So that's really positive... (Angela)

One reason that Tim was able to respond to me was a communication style which conveyed my trust in him. I believed that he would understand the musical prompts, and expected that he would respond. Angela recognised that the balance of singing and speaking was crucial for Tim to distinguish the type of request that was being made.

"It seemed really important when you changed from the singing to the speaking... when you were dealing with different instructions or a different type of request that you were making. And I think once again that's something we can do ..."

\section{Playful Musical Communication}

Team members became more playful in their individual sessions with Tim, and began to anticipate his musical responses.

The bongo drums could be a REAL asset to us! I particularly like the way that it can differentiate territory and then you can intrude on someone's territory and they can intrude on yours - and then it can become a shared territory. I really like that idea for Tim... and some of the other special needs kids I work with. (Angela)

Angela was also keen to try using the drums as an echo chamber because she noticed that Tim liked the feel of the vibrations on the skin when I sang into them or tapped them.

I've never done anything like that with him before and he responded really 
nicely in that situation - it's that turn taking once again indicating that he's communicating something. (Angela)

\section{Revitalising the programme}

Angela and the team seemed relieved to have new ideas to try. They were aware that not all would be helpful, or might only work for a while, but it was important for them to have fresh input and the confidence to try new things which might help to rejuvenate their own creativity and generate new ideas.

Well for us it's great...We've got strategies we can use... it doesn't matter if $50 \%$ of them don't work. One new strategy with Tim is really important because we're here on our own going "what DO we do next?" And I believe that we will learn more about Tim from using new strategies. (Angela)

It also gave them confidence to continue with the music they were already using, and add new ideas that seemed particularly appealing.

It's reinforced that I'm doing a really good job with Tim and given me lots of other little strategies that I can intersperse with him during the day... the 'Standing Up' song is a really great one - a really, really good one! ... and the emphasis on keeping with his rhythm... (Sally)

\section{Modelling Strategies}

The team found it helpful to see me working directly with Tim. Angela believed that observing strategies being modelled was far more effective than a professional development course or learning from books. Because the team was isolated, usually only one team member could go to any particular course and by the time they relayed what they had learnt the information had become 'diluted'. She valued the opportunity for everyone to be involved in the work and to take different things from it that they could share.

When Tim wasn't going to come in I needed to see exactly how you were going to deal with that ... if I've seen it then I can think, "Oh, ok well I could do it this way or that way, and this is what else I could do and I could adapt this here and there" - but it makes a big difference when you see somebody who is really skilled sitting down and doing it with you here. And I think that has been 
essential! Because ALL of us saw what you did. And all of us took different parts from that. (Angela)

\section{Music Becomes Integral to the Programme}

Tim's team saw music as an integral part of his day. They agreed they could use music to motivate him to attempt tasks, and to master steps in activities of daily living. A greetings song might encourage interactions with peers, and they intended to use this activity at 'mat time' each morning, as well singing it with peers in the playground as opportunities arose. Music would be used to support Tim's movement. Song offered a resource to help him to stand and walk to another activity, and to work through his perceptual motor programme in the gym. Rhythm could support his initiation of movement, and melody could support directionality. Team members were very willing to sing but not everyone found dynamic timing or spontaneously singing an improvised melody easy. Several seemed more able to put new lyrics to a familiar tune and thus to concentrate on matching their timing to Tim's movements more effectively. They were encouraged to continue with simple songs that 'fit' an activity e.g. "This is the way we wash our hands", to use lyric substitution, or to use chant so they could concentrate on appropriate timing.

The way the music therapy assessment session was facilitated was considered to be a good 'fit' with the sensory play the team enjoyed with Tim as part of the 'Floortime' programme. They were also keen to allocate short periods of time daily for planned music experiences in an environment where he was less distracted, and musical instruments were available.

When Tim was engaged in solitary play, he frequently tapped or flicked in a stereotypic way. The team were encouraged to consider how they might interrupt musically and use this to engage him in reciprocal interaction. His mum quickly recognised this useful strategy.

He thinks that's quite funny - 'I can do the same as you or something different from you...' He likes the tapping the drum thing - he thinks that that's funny when you copy it and he'll do it again. (Lee) 


\subsubsection{Continuing the Work}

\section{Music Activities and Strategies Employed by the Team}

Spontaneous and playful music making is now seen to be important in facilitating Tim's pre-verbal communication at the level of attending, imitating and reciprocal turn taking and songs are being used to motivate him to begin or continue an activity.

Strategies like a little song make a big difference to Tim - we can get him to do things now because we can just jolly him along with a song. (Angela)

Although they use pre-composed and improvised songs with Tim, Sally and Siobhan continue to use his personalised $\mathrm{CD}$. While the use of live music might be preferred in many circumstances, the familiarity and predictability of the recording enabled Tim to show the team that he was able to remember, anticipate, and eventually to choose tracks and activities from the CD.

\section{A New Perspective}

The team has found the new ideas they picked up from the music therapy assessment and consultation process refreshing. They communicated clearly that the outcome they desired from the consultation i.e. to identify where there might be missed opportunities, was achieved.

The process has made us stop and look at his education from another viewpoint - from another lens...Because we know that there were gaps that we were not getting to. It's helped US identify some. YOU were able to identify some when you were here but we are also able to identify some now that you are not here because we have a platform to work from. (Angela)

To work on the same goals for a very long period, they needed a variety of creative activities and constant rejuvenation to stay enthusiastic. An 'injection' of simple planned music experiences stimulated them and enabled them to take a new approach to their work with Tim.

I think the process of looking at the goals for the IEP (over past years) has been quite difficult because we have had the same goals... (now we're) a step further and considering the types of strategies we can use, particularly sensory and 
music therapy strategies, it changes the WAY that we teach. (Angela)

The team are developing the music strategies themselves, being creative and checking things out with Tim. They are spontaneous and playful and he is responding similarly to them regularly enough for the team to be very encouraged.

When we're doing music if we're actually copying his physical gestures he becomes incredibly excited...And he will actually stop what he's doing and laugh and laugh ... He is stopping and engaging with us ... Now that's huge for us because we haven't DONE it in the past...it is a strategy we haven't used. (Angela)

Tim's motivation and ability to attend and listen to others seems to have increased. There has also been an improvement in his general behaviour. Angela intimated that opening up the communication between Tim and those who work with him has had a very big impact... and the changes are on both sides.

...the music therapy strategies have opened up the potential for communication and that's HUGE for us...because in some ways we shut it down.

Success does breed success. Tim was not easy to work with and over time some team members seemed to have lost some confidence both in their ability to manage, and in Tim's ability to cope with what was being asked of him. Being involved in the music therapy consultation gave them an opportunity to step back and look at the whole situation from a different perspective. It strengthened their conviction that he would continue to make progress.

Sometimes we questioned ourselves because we hear that 'he's at this end of the scale'... but suddenly the music therapy strategies have justified to us our thoughts. We actually believe in the potential that he is showing... ...It's giving him a new awareness of himself and it's giving US a new awareness of OUR selves and OUR role. It's made us realise how limiting we can be without even meaning to be ... ...We are seeing this huge spike in interactive intent. So I do think the (music therapy) visit has made a difference - the whole process of us looking at ourselves as well. (Angela)

And Patrick, Tim's dad, is singing to him! 


\subsubsection{Student Progress and Potential}

The first two weeks after the music therapy consultation visit were difficult for Tim, but when he became used to new routines, activities, and strategies, things settled down dramatically, communication opened up, and his level of compliance was much higher. Angela thought this might be because those who worked with him were "listening to him more." She excitedly shared that there had been a 'spurt' in his general development over the period of the term and the team were delighted to be able to change some of his goals for the first time in three or four years. They strongly believed that music opened up the potential for communication with Tim and they could build on this by utilising the support that others could offer. Angela reported Tim had begun to talk to the SLT and a hi-tech communication device was being investigated for him.

As Tim became increasingly familiar with the songs on his personal $\mathrm{CD}$, he demonstrated to the team that he could remember the sequence of activities and actions.

He's getting his hands ready for the song that's coming on. So you know that is awesome for us because we now know that he understands the routine... he predicts that the song is coming on and he gets his hands ready for that music ... that's fabulous because it shows he's thinking about it. (Angela)

Tim was imitating and repeating rhythmic patterns in interactive play. The team became aware he was attending even when he was not looking at them or the activity, because he had begun to copy them. He seemed increasingly aware of himself and others, and had begun to seek the company of other children.

There were some boys being 'naughty' in the class... Tim was looking from the side as he does. He stopped what he was doing and watched the boys and laughed ... three times he looked, and three times he laughed... He knew they were going to get in trouble. He knew that what they were doing was wrong... (And) it came through in the IEP really clearly from everybody ...that Tim is actually seeking other children. And that's a massive step forward for us...to see any sign that he is actually seeking play with somebody else. (Angela)

The team were able to re-evaluate Tim's IEP goals based on his improvement in motivation. Angela was particularly excited about his willingness to sit at his desk when 
offered musical support.

I KNOW his motivation has increased ... I have videoed him sitting at his desk since he came to school and in the last four weeks he has doubled the time he can stay at his desk. So I actually have clear data - it has become a realistic goal and we're aiming for one minute more each week and we're getting it! Sally's using song to settle him at his desk and then she's moving away. So when I'm talking 4 1/2 minutes of sitting - I'm talking about no teacher's aide support. It's fantastic... absolutely amazing. (Angela)

Some things that Tim had been doing at home were now being generalised to school and gave Angela "real hope" for where they were going with their programmes.

He's just been fabulous! He's just been gorgeous doing all these little incidental things and just letting us know that he is part of our world. (Angela)

\subsubsection{Relationships Within the Team}

Angela argued there was nothing difficult about the music therapy consultation process or the ongoing work, especially as they could refer back to the video of the assessment session as they needed to. However, the process of looking closely at Tim's programme highlighted some "difficulties in our organisational structure".

Team members recognised problems in identifying and clarifying who was responsible for Tim. Angela felt this had been an underlying issue in the school for some time, but it had only been highlighted when they began to concentrate on his new programme which incorporated activities for inclusion. They wanted him to spend as much time with the other children as possible.

It has brought up a bigger issue. When a child is mainstreamed inclusion means they are part of the class - that they will be included... ...Simple things like "ok lets sing the 'Hello' song to the whole class" - that's not a difficult strategy to put in place - BUT there is resistance from some people at times who see that as interfering with the daily timetable... We've had to work through these issues.

Angela intimated that the team was eventually strengthened in their united approach to determine roles and responsibilities, especially since the principal of the school was supportive of every child in the school being fully included. 


\subsubsection{Managing Music in the Future}

Tim's team see promise in the planned music experiences and increasing potential in Tim, which suggests ongoing success.

Suddenly for us a big light's gone on for us and it's fabulous. ... I do believe that the strategies we've used (recently)... have pushed that door open a little bit wider for us... ...The music therapy report was fabulous. For me the amount of information you gave was almost a difficulty because there was SO much that we needed to do and yet I couldn't see all of the ways I could fit it in. So I've had to step back and say 'Ok, no. Let's go back to one goal and work from one goal'...but the reality is that music's just become immersed in his day anywayit's used in behaviour management; it's used in communication; it's used in sensory (programmes)... ...The teacher's aides are actually taking it a step further by saying 'hold on... I know that this worked really well so why don't we bring in this song?' and 'why are we using this?' So they're starting to reflect. (Angela)

\subsubsection{Valuing the Process}

Angela reiterated the importance of being able to participate directly in the music therapy assessment and consultation process; having someone model how to deal with Tim in difficult situations; and having new ideas to try especially when they had been working on the same goals for some time. She re-emphasised the team's seemingly desperate need for ongoing and regular support

I believe that the family and school have not received as much support (as they deserve)... we have really done it on our own...And I have found it really difficult at times to watch Lee and Patrick searching for support and it is not always forthcoming ... We contribute to this in that people will ask "what do you need" and we'll say "nothing" because we've had nothing so we don't see any point in somebody extra coming around if it's not going to make any difference.

The most striking thing for us is that the 18 minutes we saw you working with Tim (in the music session) is probably the most 'hands-on' we've seen ANY professional work with him...so for us we consider you have 'made the grade' as 
far as being accepted as part of the team because you've stayed there when he HAS refused to do something and you've shown us other strategies. (Angela)

Angela believed the team would consult a music therapist again to work with Tim.

I can already see that his levels of compliance are much higher - I think (because) we are listening to him more... but I think that as he goes forward we probably will want some more support. (Angela)

The planned music experiences were viewed as a positive way of working and were therefore likely to be sustained over time.

We're isolated ... and it's the first time we've seen his communication really open up so this is working...we are now looking at this (work with the music therapist) as the 'START' of a journey with Tim - It's not going to be just a 'project' it's going to be part of how we look at things in the future. (Angela) 


\subsection{ACTION RESEARCH CYCLE 1: AFTER TIM}

Three concerns, to have time to build relationships, gain optimal information from the assessment, and formally evaluate the consultation process were outlined in the plan (see 6.2) for this cycle of learning. 'Time' enabled the concerns to be addressed. It enabled collaborative relationships to develop, and an ecological assessment to take place. Being able to work with Tim and his team throughout the school day, observing, making suggestions, and modelling interventions in a timely and relevant way was fundamental to the positive outcomes. A term of follow-up during which participants recorded their musical interactions and a final interview gave clear indications of their potential to use music in an ongoing way.

\subsubsection{Relationships and Identities}

The collaborative nature and clearly stated tasks associated with action research, namely the need to define issues and roles and develop a mutually agreed action plan, contributed to the success of the consultation. One of my roles as instigator of the action research project was to ensure that participants had appropriate opportunities to contribute to and share responsibility for the project. I was therefore concerned to keep my input balanced; not to assume the expert role, 'take over', or offer too much advice. Maintaining this position is also important for consultants.

I began the consultation 'passively' observing Tim interacting with others, but immediately realised this was unhelpful. Team members turned to me with a look of desperation when he sat down and would not budge. A relationship of trust could not develop until they gained some understanding of how I might manage difficult situations. Demonstrating our various 'expertise' early in the relationship meant that we developed a reciprocal understanding that we had something to offer each other. Team members will see music therapy consultants as a resource that they are eager to draw from. I recognised that in future consultations I would be able to trust my instinct about how much input is needed, because team members would ultimately draw from me the help that they required. 
That I would be working primarily with adults surprised me. I perceived the referral for music therapy consultation as a request to work with the student and for team members to be part of that process. My musical interaction with the student would enable me to identify their strengths and needs. Then the team would decide with my help what they would continue to work on. Perhaps I thought they would predominantly 'watch and learn'. However, I learnt that music therapy consultation will primarily involve focusing on the needs of the adults who support the students.

Tim's team seemed to learn most from my interaction and communication both with them and Tim. I was not used to using such high levels of verbal interaction and wondered whether my work was music-centred enough to be 'music therapy', even within a community music therapy framework. However, I noticed the musical phrases team members used to describe my interactions (e.g. 'being in tune' with Tim) and realised that trying to separate the tasks of interpersonal relating and empowering musical interaction is a folly when one recognises that music therapists are likely to 'perform' their improvisational skills in relationship contexts. My particular way of being in the world is the 'performance' of my identity as a music therapist (Aldridge, 2005; Ansdell, 2005; Stige, 2004a).

I recognised that as a musician I have experience communicating with listeners and listening to myself, through music. As a therapist, I am a keen observer and listener; have a particular interest in non-verbal communication, and have developed the ability to demonstrate unconditional positive regard by 'being with' people in my work. My music therapy work and the extensive interactions I have had with deaf people have enhanced my ability to notice and use non-verbal communication. I 'perform' as a music therapist whether I am in the music room or playground. This 'music therapy way of being' is likely to be common among, but unique to, music therapists and an important distinguishing feature for music therapy consultants.

With support, team members were able to recognise Tim's musical identity too, his 'performance' as he improvised his musical communication with us (Aldridge, 2005; Ansdell, 2005; Stige, 2004a). He was taking part in a performance that was meaningful to 
him, and the musical offerings of the adults around him encouraged him to improve his performance (Vygotsky, 1978).

\subsubsection{Ecological Assessment}

I had anticipated that the clinical assessment would be central to consultation work and watching and discussing videotape of the music therapy session would enable participants to identify specific music strategies that they might choose to work on. The videotape was crucial in helping the team members to identify Tim's strengths, gain new perspectives and increased respect for him, but work in context had considerably more influence on their planning. Further, lists of activities and strategies I had prepared for the protocol seemed inappropriate and were not introduced.

Taking an ecological approach, team members were able to observe and interact with Tim, sharing ideas and interpretations as meaningful events occurred. A natural process occurred where interactions with Tim in the playground, classroom and other school environs became increasingly musical as team members observed and discussed his needs in the context of their current interventions. Therapeutic music activities were embedded into his daily routines. An ecological approach enabled us to focus on social interaction and to create a more effective learning environment for Tim. Team members began to identify environmental precipitators for his behaviour. They listened to him more, and he contributed more in return. Ecological assessment is a reciprocal process situated in social and cultural contexts and I became aware that it is a continuous rather than discrete activity in the consultation process.

\subsubsection{Empowering Others}

While team members were clearly empowered in their use of music I wondered if workshops might have added value to the consultation. Would attending a music therapy workshop have helped Tim's teacher to gain confidence to use more music in the classroom? Tim's mother was a classroom teacher herself, was keen for and had good ideas about how Tim might be included in classroom activities. How can parents be empowered to contribute more, especially when they have such knowledge and skill? 
Could music therapy workshops facilitate closer working relationships between parents and staff?

\subsubsection{Evaluation}

Before I left Tim's team, they did not specify which of his goals they would be addressing through music; rather his special education teacher felt music had "just become immersed in his day". I therefore did not anticipate specific measures of student outcome as a way to evaluate the effectiveness of the consultation. The team believed however that the proposed strategies would lead to meaningful and relevant outcomes for Tim and his team members and thus had 'functional value' (Jellison, 2006). Moreover, team members had high levels of acceptance and belief in the significance of the music therapy consultation which suggests the protocol has 'social validity' (Bonner \& Barnett, 2003). Further, their diary entries and interview comments provided evidence that they would be highly likely to continue the work.

\subsubsection{Setting Boundaries in Consultation Work}

Tim's team reported being frustrated at times because their ongoing individual music work with him was being interrupted. If 'withdrawal' is considered necessary, would it be the music therapy consultant's role to ensure that team members' timetables allow for the necessary uninterrupted time? O'Brien and Ryba (2005) suggest specialist staff will be required to work alongside educators within schools to support individual and system level developments. Music therapy consultants will need to consider whether it is appropriate and/or possible to engage in systemic issues such as this in the time available.

Tim's team had particular needs associated with living/working in 'remote' areas. They attributed their perceived lack of support to their 'isolation' but also confessed to 'not asking' for help because they did not expect a response, or because they did not believe the level of support they would be offered would make a difference. This signals the need for music therapy consultants to be clear about the limits of their service, so teams do not have too high expectations of the process and/or become disappointed and discouraged from seeking help. It also suggests there may be a problem with special education 
service delivery on a broad scale, and once more highlights systemic issues that need consideration but might be beyond the influence of the practising music therapy consultant.

I was asked to provide advice on resources and equipment for Tim's programmes (e.g. music/sound in a purpose-built sensory environment; positioning students in class). Being able to offer some advice about what resources might be helpful, and how they might be accessed, increased my professional credibility. But I was careful to explain the limits of my expertise, and to maintain adequate professional boundaries. It seems music therapy consultants will be asked to consider issues that are slightly outside their field, especially in schools that are underserved due to their geographical location. How would I have managed this when I was a new graduate?

\subsubsection{Pragmatic Issues}

Tim's case also highlighted practical issues that need addressing in the protocol:

- Some students will benefit from a photograph of the music therapist prior to their arrival so team members can help them anticipate the visit;

- It will be important for music therapists to consider the timing of specific events such as the viewing of the video (e.g. to accommodate parents' needs regarding childcare issues; and to ensure there is enough time left in the week to plan, prepare resources, and facilitate the implementation of the music experiences;

- The amount of information that is generated during the consultation has the potential to be overwhelming;

- It is essential to check not only whether a student has been involved in music therapy programmes, but whether he has 'seen' a music therapist before... e.g. for a single-session assessment;

- It is important to explicitly state that parents are integral team members as some may be used to experiencing their role as peripheral;

- Tim's team seemed to be comfortable using the existing home/school notebook, instead of a consultation diary, because it is part of their 'usual' practice. Team member diaries could be requested as part of music therapy consultation practice 
as a way of evaluating the practice;

- The research context enabled detailed analysis of the individual music therapy session. Previously unseen communicative potential was revealed, but this level of analysis might not be realistic in clinical or consultancy practice. It will be important to consider how data is gathered and reviewed in relation to the time that will be available to a music therapist consultant, rather than a researcher.

\subsubsection{Summary}

Spending a week with consultees allowed collaborative relationships to be established and ecological assessment to take place. Ecological assessment is a continuous rather than discrete activity in the consultation process. Consultee diary entries over the period of a term and a follow-up interview provided evaluation data for the protocol.

Collaborative relationships develop when parties are interested in, listen to and respond to each others' needs. I feel easier about allowing my intuition to guide my decisions about when it is appropriate to observe or support. During the next consultation I will consider whether a staff workshop would add value to the consultation. However, my primary concern for the next cycle of learning is to consider boundaries in consultation work.

1. Will it be possible or desirable to influence inclusive practices, i.e. to work at a macro-system level? Is it more desirable to promote inclusion by encouraging inclusive activities or to help team members who choose to withdraw students to work in that setting more effectively?

2. Is it necessary or possible to identify specific tasks that are 'off limits' to music therapy consultants because they are beyond music therapy scopes of practice? Is it more desirable to offer what support one can because it is not available from other practitioners? 


\subsection{JOHN}

\subsubsection{Background}

John was a ten-year-old boy diagnosed with Autism Spectrum Disorder (ASD), who had very high needs according to the ORRS guidelines. He experienced global developmental delay; epilepsy which was reportedly well controlled with medication; and processing difficulties affecting all sensory domains. John identified as 'New Zealander'.

\subsubsection{Introduction to the Team}

The referral was made by the Special Needs (SN) Teacher, via the SEA. He was the primary coordinator of the school team, but was absent due to illness during the consultation period. However, teacher's aides (Dianne and Barbara) jointly facilitated a music group for students with special education needs, and had agreed to be the consultees. A third teacher's aide (Rae); the Special Education Needs Coordinator (SENCO) (Penny), who was also the deputy principal; a speech and language therapist from MoE SE (Edith); and John's parents (Ivan and Mandy), also participated.

\subsubsection{Ethical Issues with Regard to Participation}

The SEA had shared information about the study with the MoE SE team, and Edith asked to participate. Initially I was not aware that she was not John's primary SLT, but SE staff collaborated closely and agreed to Edith 'representing' them on the research team.

I was not aware until I met with John's classroom teacher that she had not received information about the study. Nevertheless, she readily agreed to my presence in her classroom and was eager to contribute information. Her contribution to the research has been negotiated using a process of informed decision making.

Informed consent was obtained to interview John but data has not been included because very little information was gained (see Appendix 28, p. 493). 


\subsubsection{The Team's Expectations of Music Therapy}

Dianne and Barbara wanted new ideas, to 'refresh' their music sessions with John and his peers. They were frustrated that students were not moving forward academically and were looking for ways to enhance their development. They felt they could improve the quality of the students' time at school by building on what students enjoyed and could be successful with. They were aware that music helped them to connect and communicate with the students, but felt limited because they lacked qualifications in music.

\subsubsection{Programme and IEP Goals}

John's team was helping him to develop social conversational skills, specifically to remain on a single topic during a short conversation, to listen and take turns in small group discussion, and to articulate his thoughts and feelings. Increased compliance, cooperation, and more effective inclusion were primary goals.

The team had begun to use social stories to help John understand what was required of students such as remaining in the classroom during class time, and eating lunch in a nominated area. Social Stories have been accepted as an effective way of delivering information to children who have ASD (Brownell, 2002). The technique involves developing a short story constructed for a specific individual, purpose and objective. The stories adhere to a particular format and guidelines which describe a person, skill, event, concept, or social situation, using four basic sentence types - descriptive, perspective, affirmative and directive (Gray, 1994). Musically Adapted Social Stories have been used by music therapists (Brownell, 2002); more information is provided on page 168.

\subsubsection{The Use of Music Before my Visit}

Dianne was initially nervous about participating in the research project because she had 'no musical background' and thought her singing 'might be very hard on the ears'. Nevertheless, she reported that she loved music and it had been a big part of her life. 
My mother was a good singer - fantastic singer. Dad was shocking - he was absolute tone deaf... I love music personally - I would love to be able to sing... REALLY sing, or to be able to play a musical instrument... I took the violin up as a seven-year-old and nearly killed the neighbours so that was probably my only venture into real music. But I just love music. (Dianne)

Barbara had not had formal training either but was enthusiastic and willing to take risks.

I love music as well. I'm the one who sings really loud in the car much to everyone's embarrassment but I love music...there's always music playing (Barbara)

John's mum was an amateur musician and music listening had featured strongly in his life. He also occasionally played spontaneously on a keyboard that was available at home.

The school had a well-equipped music room and a full-time specialist music teacher. John's class went to music lessons with him once a fortnight and were working on notation tasks, listening to and identifying various elements of music (fast/slow etc.), recorder playing, and composition using the computer programmes 'Garage Band' and 'Classics for Kids'. Unfortunately John didn't manage this mainstream music class well, and the music teacher was unable to see how John might contribute. John was therefore usually offered alternative activities outside of the classroom.

\subsubsection{Assessment Activities}

\section{Ecological Assessment}

John's day involved a range of activities, several of which were repeated many times so he could enjoy the familiarity of tasks with which he was successful. Although there was a high emphasis on activities of daily living such as fetching and returning his lunch box and reading material from his bag, and tidying the staff room, John also had an academic programme that was adhered to as rigorously as he would allow.

Sometimes he sat at his desk but was often sitting or lying on the floor or couch in the 
classroom. He was most engaged with maths tasks and could reportedly do forty minutes of maths work when he was not tired. His favourite activity was inserting different coloured shapes into a peg-board, matching the patterns to various templates illustrated in a book. He sometimes listened to stories in a very relaxed manner, tracking the words (with occasional prompts), and answering questions about the text. John was also invited to interview one of his peers each day. This activity promoted social interaction, as well as speech and language development as he was prompted to ask peers simple questions while his teacher's aide repeated and scribed their answers.

John also spent a lot of time out of the classroom. He was regularly removed because he wasn't coping, but outdoor play was also offered as a reward. John enjoyed using climbing equipment, spending time in the shade of the bushes, and ball play. We noticed that he seemed to find rhythmic chant helpful to support his timing as he attempted to catch a soccer-sized ball.

I helped Dianne and Barbara facilitate music sessions with John and a small group of his peers. In this setting, many opportunities for developing students' skills were not being seized. We shared ideas about possibilities for improving students' speech and language, or developing their specific movements, by thinking about the tempi of the songs, using visual resources, and/or carefully timing the phrases to enable students to contribute purposefully and meaningfully.

John sang as he walked to the staffroom, seemingly eager to begin his job of wiping the tables. He counted the tables and verbalised enthusiastic phrases such as 'let's wipe!'. However, on other occasions he became suddenly extremely angry and upset - screaming and crying loudly. Sometimes he seemed to have an idea that he could not get across. At other times when his articulated ideas could not be carried through, he tried desperately to argue his case until he again became exceedingly distressed. Staff members felt unable to deal with this behaviour and several different approaches were employed at various times. Most often John was removed to a more solitary environment until he was able to calm down but he was physically difficult to extract and his behaviour was disruptive and upsetting for staff and students alike. 
Throughout the week we used spontaneous songs, or humming, to help John to relax, distract him, reinforce what he was doing, or direct him to new activities. We also discussed possibilities for John to listen to music occasionally via headphones to reduce the impact of environmental noise.

\section{Identified Strengths and Needs}

\section{Sensory Profile}

John has variable responses to auditory and tactile stimulation, and poor awareness of body temperature. His vision and hearing were understood to be within normal limits although he had a history of recurring ear infections (to age four years), and tonsillitis. Sometimes he appeared not to hear; at other times he demonstrated heightened sensitivity to sound. Unless particularly interested in an activity he was easily distracted by noise and occasionally played with his ears, folding them down. In the past, he had made frequent noises, e.g. repetitive singing and humming, talking himself through tasks, and singing himself to sleep and it is possible he used these behaviours to mask sound. It is also possible that he was hypersensitive to particular frequencies that he encountered perhaps even some sounds that other people around him were not aware of. In contrast, his teacher reported that he previously used a loud voice and needed help to reduce the volume. Moreover, he loved the sounds of the lawn mower and vacuum cleaner and responded positively to music.

\section{Communication Profile}

John's significant impairments in listening, auditory comprehension and expressive language skills impacted on his verbal communication. His speech had followed a natural course of development, but he had severe articulation delay and used only short phrases to communicate his wants and needs. His receptive language was supported when

questions and comments were kept short, simple, and repetitive, and when pictures were used. He could follow a visual timetable with support. 


\section{Physical Profile}

John was mobile and liked physical play, but had poor coordination and balance skills. His fine motor skills were delayed and inconsistent. John was unable to write or cut and paste independently, but could manage a mouse for computer work.

\section{Activities of Daily Living and Cognitive Profile}

John's independent dressing, toileting, feeding, and play skills had been slow to develop, and as a toddler he had manipulated toys in unusual ways. He still required monitoring with activities of daily living predominantly because he was easily distracted, and was often uncooperative when assisted. However, he liked helping with practical tasks such as wiping tables.

John had grown to enjoy ball games, but favoured using the computer, playing Playstation games and watching television, especially enjoying programmes which involved music. He often sang along, jumped or bounced to the rhythm. John had always loved reading books which employed rhythm and would often get fixated on them. He was able to read and understand basic text and liked to copy parts of his reading story onto the computer. He could also write some of his own ideas with one-to-one assistance and enjoyed solving basic addition and subtraction sums. John reportedly took a long time to learn new skills, needing frequent repetition to retain information.

\section{Emotional Profile}

John had a good sense of humour. However, he had difficulty regulating responses and expressing his emotions and demonstrated periods of extreme tiredness at school, which affected his ability to cope with the demands of classroom and playground activity. He resorted to temper tantrums in various situations, shouting, screaming, crying and dropping or throwing himself on the floor, especially if he was denied an activity of his choice or was frustrated with solving a problem and unable to request help. John frequently sought reassurance and approval from others. He did not always adhere to rules and routines yet had difficulty adjusting to change and strived for consistency. John 
required constant close adult supervision because he often wandered or 'darted' off; had poor road safety awareness; and tended to get lost easily in unfamiliar environments.

\section{Social Profile}

It seemed that John wanted to be with his peers but did not always approach them in appropriate ways because he was so intently focused on his own needs. He tended to perseverate on topics which did not interest others and had difficulty following the conversations of his peers. He liked to be the centre of attention and appeared to have difficulty with empathy. John also demonstrated oppositional behaviour, apparently needing to 'test' people's reaction to his demands. On the other hand, he occasionally initiated 'casual' interactions which seemed very natural e.g. spontaneously greeting a friend by name with a smile and a wave. He was sometimes able to talk happily with the children in his class and could take turns with adults and peers in structured activities, albeit with supportive prompts to wait for his turn and/or to allow turns to be completed. He did not always complete simple tasks or allow activities or communications to come to an end before moving away.

John had a high number of staff working with him and had difficulty adapting to each person's different interaction styles (see p.161). The difficulty John experienced understanding and managing school routines placed particular demands on staff to ensure that he was included safely in class and school activities. John enjoyed being with the other children, just watching or interacting with them albeit predominantly on his own terms, and participating in classroom activities alongside them. The team was making a considered effort to help him to stay in the classroom but his difficulty managing routines and rules and his tendency to be disruptive meant that his inclusion was an issue for them.

\section{Inclusion Issues}

Although his classroom teacher described John as a 'neat kid' she seemed at a loss as to how to include him or move him on academically or developmentally. She described him as "sitting aside" and his team as "a class within a class". She believed a lot of things "work better when he is out of the classroom" and appeared to delegate considerable 
responsibility for his education to her aides. Nevertheless, she expressed regret at not having the opportunity to have more input into his programme.

John's part time 'Thursday teacher' was similarly unable to manage stating that "he is not suited to be in a class with his chronologically same-aged peers". His dad suggested that school for John seemed to be about trying to find a way to 'fit in' - he always seemed to be “'behind the 8-ball' - a square peg trying to fit into a round hole”. The school team may have thought they were supporting his inclusion but in practice were finding the task extremely difficult.

We've definitely been told to keep (the students with special education needs) in class this year... not in terms of the music session obviously... It's probably one of the few times (they're out) really... So we want it to be really fun. (Barbara)

\section{Relationships within the Team}

For various reasons, it seemed that relationships within John's team were strained. There were a high number of people who had direct contact with him for short periods throughout the day and maintaining a consistent approach to managing him was difficult.

On a regular basis during just one day he could have five different 'teachers'. The reason cited for such a high number of staff was to 'share the load' since John was difficult to manage and people needed a break from him. Although they appeared collectively to agree that they found the work difficult, comments from some individuals suggested they felt personally inadequate and/or experienced a loss of confidence because they were not able to manage. Further, specific techniques employed by particular staff members were not used consistently by others, and in some cases staff felt as if their work was being undermined.

The SN teacher retained responsibility for John and his team, and had been overseeing the teacher's aides' work. However, various factors including his absence at the time of the consultation resulted in communication difficulties within the team. And while the SN was responsible for John's programme, the classroom teacher had to find ways to include him in classroom activities. Although she had minimal direct involvement with him she 
was also the team member who was able to have the most contact with his parents.

Ivan observed that team communication had not been optimal and that good ideas were articulated in the IEP process but not always followed through. While he was appreciative of the support that staff were providing John, he felt that they were 'winging it' at times. He sensed that the implementation of strategies tended to be reactive, i.e. set up to deal with problems - with staff viewing John as a problem - rather than establishing strategies to help him cope and therefore to learn.

\section{Assessment in the Music Room}

The following paragraphs provide a glimpse into the individual music session, which lasted for 42 minutes. Throughout the session I was interested in John's ability to:

1. use music as an expressive medium;

2. tolerate various timbre, pitch, and levels of sound;

3. attend to and remain with a task that he had chosen;

4. sustain his play until the end of a phrase, short section of music, or song (i.e. until a clearly defined cadence point);

5. engage with a task of my choosing;

6. acknowledge me as a music partner;

7. engage in shared or reciprocal music making.

John did not demonstrate any adverse responses to sounds during the session. In contrast, he seemed surprisingly oblivious to very high volume music and noise in the hall next door when a large group of students began doing 'Jump Jam' exercise to music, even though the extreme levels of sound were distracting at times for me.

Throughout the session John was directive and controlling. He indicated several times that he preferred to play on his own and I should be silent, but was prepared to invite me to play on his terms. Playing musical games with 'rules' e.g. taking turns to be the 'band leader', helped John to share his music with me. He enjoyed being a 'showman' calling "look at me!", and presenting his work for the peer who was filming, and Dianne who was also present, especially when he was doing well. 
John showed some interest in a drum and guitar, swapped places with me at the piano, and tried other instruments with encouragement but showed little commitment to them. John 'flitted' from one thing to another, not finishing songs, changing his mind about what song he wanted to play or what instrument he would use, sometimes before he had even begun. My task was to try to focus his attention; to join him and help him sustain his play thus enabling him to have the experience of sharing and communicating in music.

He darted to the guitar, gave it a strum, and moved back to his xylophone. I reminded him to say "1,2,3 - go" (our play rules) and he contributed "1, steady, go!’. As I began to sing and play guitar he joined me steadily beating on his xylophone and on the last phrase lifted his arms up to slow fleetingly before coming down with a demonstrative accent on the final beat.

He listened as I praised "Nice stopping" but the beater in his left hand dropped to the floor from his loose grasp. He bent to pick it up as I asked "What's next?" J: "You play the guitar and I (inaudible) you... you play the guitar and I sing a song ok?”

D: "What song are we going to sing?"

J: "You play drum" (pointing to the hand drum)

D: "I'll play guitar. What song?"

J: "And I'll play the xylophone" (Already tapping lightly with beaters, both hands)

D: "Yes" ... John has already begun his song.

However, once John discovered the xylophone he was 'hooked' on it. He played musically using expressive techniques such as slowing at the end of a song, creating at one point a momentary sense of stillness. John also explored melody and rhythm in interesting ways and his music often sounded cheeky or playful. He looked for cues and direction and regularly invited my response although it was more difficult for him to stay on task with a musical partner. Nevertheless, despite his impulsivity and distractibility John was able to adjust the way he played in response to changes in my music, at times. Further, he showed good attending skills at times.

...John began to play single notes in an ascending scale on his xylophone while 
reading and singing the letter names from the instrument. I paused and waited. When he got to the top of the instrument he declared "Now we've finished".

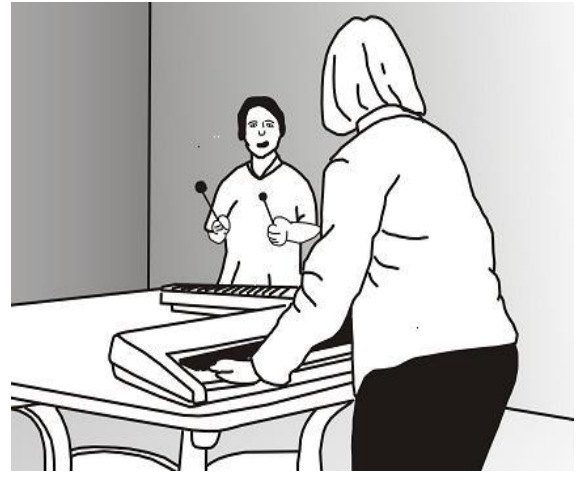

We engaged in an improvisation involving frequent use of glissando and a pattern of 2-3 staccato notes but John also introduced new ideas including a slower more melodic sequence of three notes and the sung phrase "now it's time to stop". He giggled and smiled and gave excellent anticipatory eye contact throughout.

...He reintroduced his song, singing and playing it steadily through, stopping with a flourish at the cadence. I waited for him, and he offered a glissando to signal the beginning of another improvisational exchange.

This time we challenged each other a little more. After an exchange of glissandos John boldly offered two notes played simultaneously on his xylophone, a melodic interval for me to respond to. However, he did not build on my imitative response, instead returning to making glissando movements with both hands in contrary motion (as I 'rebelled' with clusters of notes grouped in threes). His next challenge to me was a melodic pattern of quavers:

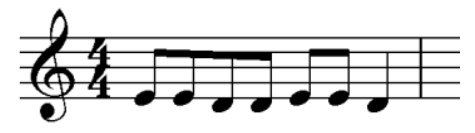

I picked up his rhythm but did not incorporate any features of his melody. He seemed to 'give up on me' and returned to his song.

... When we stopped he laughed out loud and declared "It was fun eh! (D: "It was fun!') Let's go again!” We played the song again together, and as I played the final note he laid his beaters down. 


\section{Summary of Individual Assessment}

Throughout his time in the music room John's need to maintain control persisted but his obvious interest and potential for success in music activity, and his playful response to being challenged musically, suggested that he could be motivated to increase his attention to task and to develop reciprocity in the music setting. He had begun to acknowledge possibilities for sharing music and was enjoying that experience. It seemed that he would benefit from an ongoing music therapy programme if/when it became available, because it provided him with a motivating forum for rehearsing his communication skills.

On the other hand, John was also able to demonstrate potential for developing more formal music skills in the way he used the xylophone. Although he played in a predominantly percussive and/or atonal way, I noted his exploration of single notes and simple scales and I considered that he might like to learn to play tunes by ear or with music (see Agreed Planned Music Experiences, p. 166).

Although it was not a primary focus of the assessment, I was also aware of the way John manipulated instruments, noting for example that he grasped the beater loosely and dropped it, indicating a need for further work on fine motor skills. In future sessions one might monitor how he manages the shape and weight of various beaters.

\section{Team Observations and Discussion of the Assessment Session}

The team was only able to view ten minutes of video due to technical difficulties. Dianne had been present at the session however, and others were eager to discuss what they had observed of the session and what they already knew of John. They agreed that music seemed 'easier' for John, something he might be successful at - a possible strength. Importantly, his parents were delighted to see how much he enjoyed himself.

You can see the real joy on his face... he just loves it...It would be nice to see more of that 'natural' emotion...You don't see that in his face much, do you? (Mandy)

No in his life I think there's not that much real fun. Everything is much more effort than it is for other kids and it was nice to see him have a good laugh there- 
he's really enjoying it. (Ivan)

They observed that he was interested in me as a communicative partner and that his eye contact with me was exceptional. They were able to talk about the patterns of behaviour they observed (typical and atypical), and to note that despite his obvious engagement and enjoyment, John's presentation in the music setting often mirrored how he was in other settings. They also discussed some of the reasons for some of these behaviours and perhaps came to a better understanding of him.

The video examples enabled discussion of sensitive issues. They offered illustrations of alternative ways of interacting with John, and gave me opportunities to critique the various communication styles I had seen staff employ with him during the days prior. The team noted John's response to structured and free play and observed how playful learning can be. They acknowledged how overwhelmed they had been by John's needs at times. And while they agreed that it would take time to achieve meaningful outcomes, they acknowledged his potential to learn. They gave reasons for discarding ideas that had been introduced in the past and were able to accept constructive criticism from each other.

They discussed the possible introduction of musically adapted social stories and the content that would be most helpful. This led to further understanding of John's needs and to the clarification of some behaviour management issues.

You've just got to see his reaction today to know that it's worth giving it a shot ...Until we can get John's behaviour moderated...he's not actually going to learn an awful lot. So let's see if we can make this a goal - (to) get to him through the music (and) through the social stories so he's in a space to assimilate new information. (Penny)

\subsubsection{Agreed Planned Music Experiences}

Seeing and hearing John's response to the xylophone made me wonder whether he might learn to play tunes by ear or with music. His ability to match shapes and to undertake simple maths tasks, and his interest in reading the letter names on the instrument, 
indicated that he might be able to read simple notation. I prepared some resources for him and when we trialled this activity with him he was very engaged, tracking the notation as I pointed and played, and also tracking from page to xylophone as he played. It was clear that he did not always read the notes, instead preferring to sing a few notes and play by ear. Nevertheless, his concentration, request for repetition, smiles and calls for approval, indicated that learning to read music and play the xylophone would be a motivating and successful activity for John. I discussed possibilities with the team and a music programme which could take place in music time with peers was agreed for trial.

However, we agreed the primary focus of their project would be the use of 'musically adapted social stories'. The exact method for constructing Social Stories has been described in detail (Gray, 1994; Swaggart et al., 1995) and proper use of the technique involves careful data gathering, construction, implementation, review and eventual 'fading'. Social stories are usually presented in verbal and pictorial form. The detail offered suggests that formal training would be necessary to ensure it is carried out appropriately. Nevertheless, Gray (1994) agrees that common sense underwrites every Social Story, and the basic premise has been used by others for creating a similar method which John's team called 'Learning Stories' (SEA, 11/5/07). ${ }^{8}$

Singing meaningful lyrics has also been identified as an effective way of helping people with autism to learn and retain information (Davis, Gfeller, \& Thaut, 1992). Brownell's (2002) findings from four case studies suggest that Musically Adapted Social Stories, i.e. singing the story, could be more effective than merely reading it to the child. John's team agreed that his positive response to music suggested that 'learning songs' with lyrics composed with Social Story guidelines in mind would be an effective teaching tool. Edith, SLT, was particularly interested in the concept believing that learning songs would be valuable because of the ease with which people are able to learn lyrics to a song, compared to their ability to learn a speech or a piece of prose, and the ready recall of lyrics on hearing the melody of a song.

\footnotetext{
8 'Learning Stories' is also a term used to describe an assessment technique for early childhood education. In that context learning stories are narratives that capture the child's experience in the context of the learning environment and enable educators to identify positive elements or constraints to learning.
} 
Six songs, relating to areas of need identified by the team, were composed and recorded for John. Two social stories already in use, to help John with lunchtime routine and to stay in the classroom, were set to music. John's parents asked for assistance with two others, specifically to help him to take his gloves off, and to stop when called. However, the whole team agreed the "issue of the red tee-shirt" was also of primary and immediate importance (see below) and requested a song to support John with removing and storing his tee-shirt when requested to do so.

When I presented the recordings and lyrics of the learning songs to John, he sat alongside me, pointed to the lyrics, turned the page, sang occasional words, and smiled at the lyric "stop" when the song abruptly ended. He would evidently enjoy the songs.

\section{'My Bright Red Tee Shirt' - A Learning Song}

John's fifteen hours per week teacher's aide time was insufficient to cover morning tea and lunch times. But John was a 'runner'. He liked to explore the very large playground and had been over the fence and on to the busy road. When he was wearing his dark green school uniform it was difficult to distinguish him from the other students and to spot him when he went hiding in the bushes. So the team agreed, for safety reasons, that John and several other students who had special education needs would wear bright red tee shirts when they were outside. John loved his red tee-shirt and was often extremely distressed because he could not wear it inside.

The team had already drawn up an action plan which agreed amongst other things that:

1. John would wear his tee-shirt only for fitness, PE, playtime and lunchtimes.

2. He would have a special box to store his tee-shirt in when he is inside

3. His Special Needs (SN) Teacher would write a Social Story to go with the shirt removal

4. Consistency of approach, structure and routine were important. 


\section{A Learning Song}

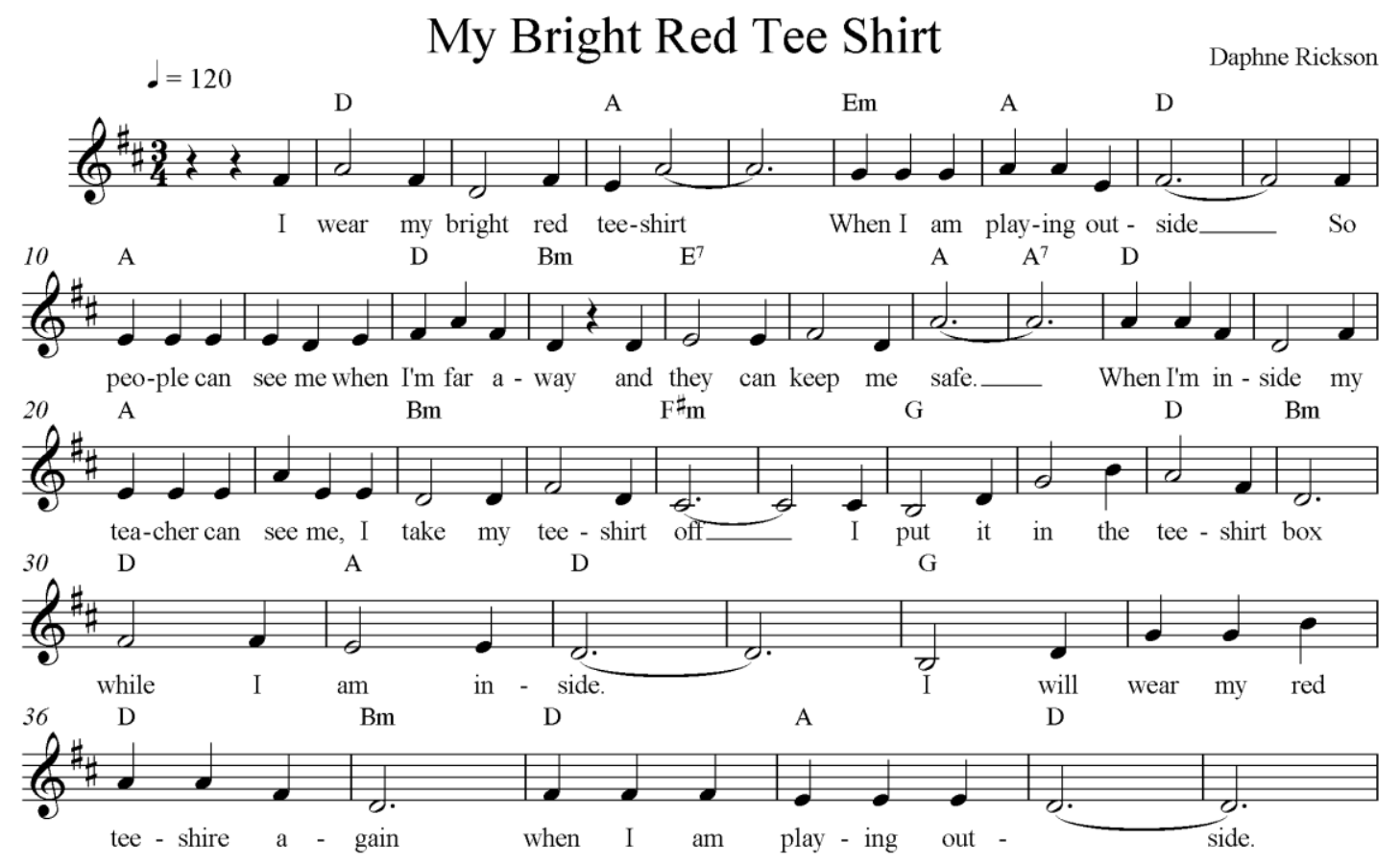

\subsubsection{Introducing the Strategies}

\section{Practical, Effective Strategies}

Dianne was pleased that the planned music experiences were practical. She believed they helped John immediately and that they would be "invaluable" for him because he had such a passion for music, but helpful for other children too. She confessed to feeling quite inadequate, until she realised that the strategies built on what she was already doing particularly with the music group that she had always facilitated.

(Now I know how to use each of the activities) - it was never explained to us in that way when we took it on... (Dianne)

\section{Affirmation and Confidence Building}

Barbara and Dianne both experienced a boost in confidence after they received positive feedback and could acknowledge they were already doing well.

I think I have learnt a lot more than... (I thought I would). Initially I thought this 
was a bit daunting and 'does this lady know that I'm not a music specialist' and you know, I felt quite inadequate. But now that you've taught us... it's just so practical, and I can see that it works! (Dianne)

I think it's made us aware of how much we do use music and the rhythmic talk and all that stuff already that we can perhaps emphasise even more. (Barbara)

\section{Enthusiasm for and Belief in the Work}

Further, the affirmation they received and John's positive responses increased their enthusiasm for continuing with and developing the work.

(I'll use...) definitely the marching and DEFINITELY all of the songs that you recorded... (Dianne)

And I can't wait for the Tee-Shirt one! ... (Barbara)

I think the 'stop and coming back' thing - that's huge. (Dianne)

There's a stop/go game as well which we must (try to find)... It got talked about at that teachers' aide thing we went to? We might be able to speak to (the SEA) about exactly how that is done. (Barbara)

Definitely the xylophone and the bongos that he seems to have picked up on... (Dianne)

The SLT observed Dianne and Barbara's enthusiasm too.

What I really LOVED about the process throughout the week was seeing the teacher's aides really begin to believe in the music therapy. I think this came about due to you involving them so heavily in the assessment process and consequent therapy sessions - getting them to have a go. This way they saw first hand (what they could do) - (they) experienced a new way of communicating with John. (Edith)

\section{Using Local Resources}

Although I did not have the opportunity to view John in the mainstream music setting I was able to discuss possibilities for his partial participation in music with the specialist 
teacher who was prepared to trial some ideas with him. Once the teacher's aides were alerted to the possibility of including John in mainstream music classes they realised that the music teacher was a resource in the school that they could use more. They began to look forward to going with John to his next music class.

\subsubsection{Continuing the work}

\section{Learning Songs}

Dianne's and Barbara's diary entries indicate that the learning songs were well utilised. John reportedly enjoyed listening to the tape and learnt the lyrics easily. Most importantly the lyrics were meaningful for him and the "Stop!", and "Red T-Shirt" songs in particular were deemed to be very successful in helping him to manage desired behaviours.

He responds really well when you say John "stop!" he loves that song - and so do we all...As soon as he hears it he knows what's coming. So that's been a really good one. (Dianne)

The Tee Shirt songs and things - they're going really well now. There's been a big improvement there... (Barbara)

It's good that he is listening - I'll keep reinforcing the songs. (Dianne)

\section{Staff Members are Improvising 'Little Ditties'}

Dianne and Barbara gradually adopted a musical way of being with John, and felt increasingly comfortable improvising songs as well as spontaneously "singing little ditties” in context such as 'One, Two, Buckle my Shoe', 'It's Raining, It's Pouring', and 'The Marching Band'. They gave examples of using improvised song to help him transition to new activities, to sit down, to eat lunch and return his lunchbox to his bag, and to look at people when they are talking. They sang about choosing books, and about books they had already read.

When we got back to class he didn't want to sit at his seat but I sang 'sit on your seat' and it went well - he sat down and completed his work. It was awesome! 
(Barbara)

I've got him putting his own shoes on now whereas he would never ever do that before. He would just thrust them at you and say "you put them on" in a whiney voice. I made up a song about 'putting on my shoes' and he puts his shoes on now. (Dianne)

\section{Music is the Way You Can Converse with John}

(Music is) sort of like - that's the way you can converse with John... Many times when you ask him to do something you can just tell that he hasn't heard a word that you've said. Yet when it becomes musical he engages. Now I'm no psychologist so I don't know how much he really understands but given that so many of his actions actually follow through on the songs I'm assuming that he understands... It's a pleasant way for him to hold a conversation. (Dianne)

\section{John is Spontaneously Singing}

Arguably the most exciting outcome was John improvising his own spontaneous songs. When staff sang a marching song, John added a 'jumping' verse. He made his own songs to match his activity including "going to the computer", and "kicking a ball". He sang a story about having a bath; and even introduced his own learning song.

John was singing a 'Don't forget to watch where you're going' song. I don't know who made it up. I'm using a variation... Ifeel more and more confident with using music as a social tool with him. He can't get enough! (Barbara)

\section{Improvising with instruments}

The teacher's aides continued to use bongo drums and xylophone with John, in playful improvisation as well as in more structured ways, setting aside a regular time to do these activities. They offered him the opportunity to 'practise' with the adapted notation I had left them, but when he was not able to concentrate on the more formal music making they allowed him to explore freely or played semi-structured games such as 'move when the drum plays'. 


\section{Turning his Mood}

Team members' flexibility has ensured that John continues to enjoy the instruments and the opportunity to play them can now be used as a motivator to help 'turn his mood around'. They were particularly impressed with John's initiative and creativity in making up songs, but also the way in which music "changed his attitude".

(When I sing) a big smile breaks out on his face... He'll join in and do a complete turn around in his behaviour. (Dianne)

If you say you're going to do music it's a good thing for him. You need to find the thing that is going to be able to move him on... And usually I can stop and listen, and if I start singing the song hopefully he'll follow along. (Barbara)

...He has been a difficult wee boy to handle this year. And since you've left I've found him - I've found him easier in many ways. ... Yeah it is wonderful. It's been better than I expected...I've got more out of it than I probably thought I would. I've learnt more. (Dianne)

\section{Inclusion}

The team had very little opportunity to develop John's inclusion in school music class because of his, and the music teacher's, absence from school. John's class is scheduled to attend music on a fortnightly basis only, so if one session is missed, a whole month passes before the next appointment. He did attend one music class early in the term but was not able to be included with other students and the experience was not particularly positive because he had to leave his peers when he wasn't keeping up.

We took his recorder into the hall as children were following a tune. He 'lost everything' and went to the library where he spent most the afternoon. (I) sang a song about going to music but no response. He was very upset and crying. (Barbara)

Nevertheless, Dianne and Barbara believed that the sharing of music activities had 
facilitated positive peer relationships which extended into the playground.

We're doing music with $S$ and $H$ as well and like on the playground, he's actually playing with $S$ now. I think it definitely helps turn taking... I guess because they are having a bit of time with just two of them together rather than the big group of kids...(Barbara)

Further, some of the experiences that had been introduced specifically for John were found to have a positive impact on other students' behaviour.

One of the students absolutely loved (the songs you recorded for John)! The CD just completely calmed him down! You know how he would do that awful screaming and he would be banging his hand against his mouth... the minute I put your CD on he would just completely relax and I've never seen him do it with any other music. He would just lay there calm as and the screaming would stop and the hand motions would stop. And that happened lots and lots of times. (Dianne)

\subsubsection{Managing music in future}

It is clear that the success of the activities, and a heightened awareness of what made them successful, will motivate staff to continue with many of the ideas while they are relevant to John's needs. He has been allocated his own glockenspiel and bongo drums for school, and his dad has purchased a xylophone for home. It will be important for Dianne and Barbara to continue to consult with specialist music staff to ensure that the equipment is used to best advantage, and that programmes are at an appropriate level for enhancing John's development and learning.

Barbara was also prepared to suggest they might include music strategies on John's IEP in future but would consult with a music therapist to ensure the programme was appropriate, and that they were carrying it out effectively in the long term.

I've found it really helpful! ... We'll keep the things that we're doing-definitely. (Barbara)

We were using music to a small degree, and we weren't very aware of that until you came and when we thought about it we realised that we were already doing a 
small amount but it was subconscious - and after you left we ... it's something you can introduce consciously...And it's also been something we can use that HAS taught him. (Dianne)

John's dad found the consultation process extremely valuable for the team, describing it as "a breath of fresh air". 


\subsection{ACTION RESEARCH CYCLE 2: AFTER JOHN}

Two concerns were identified for cycle two (see 7.3.7): to consider whether it would be desirable to promote inclusion by encouraging inclusive activities or to help team members who choose to withdraw students to work in that setting more effectively, and whether specific tasks are 'off limits' to music therapy consultants because they are beyond music therapy scopes of practice.

\subsubsection{Ecological Theory and Inclusion}

John was trying to manage in an environment that was not always conducive to his learning. His behaviour was having a significant impact on team members' ability to cope, and they in turn were responding in ways that sometimes exacerbated his difficult behaviour. Drawing on social model of disability and inclusive philosophy I knew it was important to try to work with the full team (teachers as well as teacher's aides) to help them to recognise that John's 'problems' were not necessarily with him, but with the way the environment was set up or people interacted with him. On the other hand, his teachers did not view him as a member of the class, and there were no clear cut answers to some of the dilemmas we encountered as a team.

For example the red tee shirt highlighted John's 'difference' and writing a song to encourage its use has the potential to reinforce exclusion. On the one hand, John's increased independence in the playground provided opportunities for him to eat lunch and interact freely with peers without close adult supervision. It was not surprising however that John's parents were resistant to the red tee-shirt strategy and eventually agreed that it could be worn outside only and removed during class times. But John loved his red teeshirt and was often extremely distressed because he could not wear it inside!

Staff members were desperate to resolve this issue but divided about how it might be done. They knew that consistency of approach by and across team members was very important for John, but the violent resistance they sometimes encountered when trying to

remove the tee-shirt was also counter-productive to his socialisation and learning. They 
wanted to respect his parents' wishes, but in doing so were not able to respect John's own wishes. Further, the lack of team agreement regarding management of the issue led to staff conflict and left some members feeling that their work was being undermined. While some tried to 'follow the rules', others tried to 'keep the peace'.

In both Tim's and John's cases team members had difficulty agreeing on how their inclusion in school activities might be facilitated, and Tim's case demonstrated that when teams are not cohesive, the consultant's intervention has the potential to highlight or even strengthen team members' differences of opinion. Nevertheless, while John's red tee shirt might have signalled him as being 'different', the song we introduced ensured he was aware of what was expected of him and he managed the task more easily. In doing so he reduced his emotional outbursts which in turn could make him more acceptable to his peers and aid his social inclusion.

John's teacher's described him 'sitting aside' and 'his' team as 'a class within a class' suggesting he was operating within his own micro-system. One of the principles of ecological thought is that changes in one system may lead to changes in another (Stige, 2002). Thus improving the functioning of John's micro-system is likely to lead to his integration into the classroom system.

Consultation can aim to increase concordance between the behaviour and attitudes of people and the settings in which they work in a purposeful attempt to increase the possibility of system change, the competence of individuals, and the congruence of individuals with the settings (Smith \& Nevin, 2002, p. 17).

Consultants are likely to need to work at administrative or political levels when particular aspects of the work require ongoing involvement with school administrators, and to convince policy makers that music therapy school consultation is worthy of promotion and support. However, time constraints on the 'practice' of music therapy school consultation allow for no more than careful negotiation with school administrators and MoE SE before entering the schools. 


\subsubsection{Setting Boundaries in Consultation Work}

In John's case there were occasions when I felt like a mediator as team members talked to me about their co-workers. At other times I was a confidant as they shared information regarding their own perceived difficulties. I recognised their need to be heard; overtly acknowledged the difficult situations that they were in; listened and offered carefully considered responses; highlighted positive observations and tried to keep them focused on their work with John. Once again I wondered how I would have managed this with less experience. I had drawn on my experience as a parent of a child with special education needs to develop genuine empathy with John's parents, and believed that it helped me to respond appropriately. The personality of the consultant, the approach they take, and the relationships they build can have more impact than content knowledge. Dougherty (2005, p. 54) argued that "who you are as a person can affect the outcome of consultation or collaboration as much as what you do when you engage in the service".

Nevertheless, I needed to have a stronger framework for future consultations. I had been relying on my training; reflective awareness; my links with the cultures of special education and schools; disability and ecological theories; and action research frameworks to guide my consultation work. I considered each of my actions carefully, and negotiated with team members consistently. Yet in addition to concerns already voiced, my tradition of 'boundaried' music therapy left me feeling uncomfortable with some of the activities I was undertaking, such as having a meal with John and his family in their home, even though I knew that was an important thing to do in this context. Although other music therapists had cleared pathway for community practice, not knowing where my particular trail would lead concerned me.

Moreover, working with adults to facilitate their work with students was beyond what I had been trained for. If I was primarily to work and build relationships with adults, what was my relationship with the student? Who was the 'music therapy client'? The music therapy consultation literature predominantly emphasised 'modelling' music therapy practice (direct work with students) and 'teaching' adults to facilitate music activities rather than issues of empowerment; and was therefore not helpful in this situation. 
On the other hand, the 'human services' and 'psychological' school consultation literature was relevant.

Collaborative school consultation involves personnel in general education and special education, related services and support personnel, families of students, and the students themselves working together and sharing their diversity of knowledge and expertise to define needs, plan, implement, assess, and follow-up on ways of helping students develop to their fullest (Dettmer et al., 2005, p. 9).

The consultant does not usually work directly with clients; the aim is ultimately for both consultee and client to benefit from the consultation; and by increasing the consultee's skills it is anticipated that benefits will be experienced by a wider number of clients (D. Brown et al., 2001; Conoley \& Conoley, 1992; Dettmer et al., 2005; Dougherty, 2005).

\subsubsection{Human Services or Psychological School Consultation}

The role of the consultant varies with the consultation model employed and the needs of the consultee/s (Dettmer et al., 2005; Dougherty, 2005). The amount of direct contact a consultant has with the client is to a certain extent negotiable (ibid). However, the primary purpose of consultation is to help others work more effectively, to change their behaviour or their client's behaviour in some way (D. Brown et al., 2001; Conoley \& Conoley, 1992). Was I achieving that as efficiently as I might? I wondered for example about the balance of direct work with students, and the work with adults. I wondered about aligning my work with a specific 'model' of consultation, testing the 'fit' to find and utilise a more precise frame.

Various models of consultation stem from 'mental health' (Caplan, 1970) and 'behavioural' theories (e.g. Bergan, 1977; Bergan \& Kratochwill, 1990), and there are several variations on each of these models. These include system-centered approaches which concentrate on organisational change within schools to improve programmes for students; teacher-centered approaches that focus on the difficulties a teacher might have in managing a student; and child-centered approaches which focus on a student's specific academic or behavioural difficulties (D. Brown et al., 2001; D. Brown \& Schulte, 1987; Conoley \& Conoley, 1992; Dettmer et al., 2005; Dougherty, 2005). 
The Social Learning Model of Consultation (D. Brown \& Schulte, 1987) is based on Bandura's (1977) theory that learning can occur through observing others and can be stored and drawn on in the future; that thinking can change behaviour, and behaviour influences future learning and the environment, i.e. internal and external influences are reciprocal determinants of human behaviour (Bandura, 1977). In this model the functioning of the consultant (music therapist), consultee, and client (student) is believed to be dependent on their behaviour, internal personal factors, and environmental factors the locus of change is with the interdependent relationship between these variables (D. Brown \& Schulte, 1987). The goal is to alter the relationships between the variables to ultimately increase consultees' motivation to problem-solve and to establish self-efficacy and independence (D. Brown et al., 2001; D. Brown \& Schulte, 1987).

Consultants help consultees to set goals and create situations that will develop their confidence, and lead to the anticipation of positive outcomes. They offer physical demonstrations, verbal descriptions, or use other media to help consultees develop new skills and/or perceptions; explicitly model self-evaluation statements, and facilitate situations that enable consultees to experience the consequences of their interactions (D. Brown \& Schulte, 1987). The consultant also helps the consultee to estimate the importance of situations, to develop expectations regarding what they can achieve, and to evaluate their progress based on those expectations. When the assessment is complete the consultee prioritises goals with minimal input from the consultant, and both collaborate to choose appropriate interventions (D. Brown et al., 2001). When the intervention has been decided the consultant models the chosen intervention, the consultee practices and the consultant provides feedback until both are confident that the consultee can implement the strategy effectively with the client (ibid).

Brown and Schulte (1987) posit that within the social learning model "the educational process in consultation can be enhanced by what has traditionally been viewed as a therapeutic relationship - that is, one characterized by warm, open, non-defensive communication" (p. 284). Given my strengthening commitment to taking an ecological perspective, and my skills in developing therapeutic relationships, this seemed a good model from which to draw. However, it was new for me to be considering therapeutic relationships with the adults who work with students who have special education needs, 
rather than the students themselves. On the other hand, true to the model, I had been collaborating with team members to assess what they believed motivated students' behaviour, and had considered environmental factors (D. Brown et al., 2001). I observed them working with the student, and had interacted with them formally and informally to assess their own beliefs regarding their self confidence, motivation, and ability to implement suggested music strategies. We had jointly identified issues and goals, although the ultimate decision lay with team members, and I helped them to determine how well they understood and/or would be able to manage the introduction of therapeutic music strategies. In the social learning model, whenever possible, the client is involved in describing his or her own behaviour, identifying antecedents, cues and reinforcers, providing an opportunity for the consultee to suggest alternative responses to the situation (D. Brown et al., 2001). While neither Tim nor John had the skills to contribute verbally, we were able to observe their behaviour to determine the antecedents, cues, and reinforcers that influenced their responses.

In this model, the consultant's 'power base' comes from his or her 'interpersonal attraction' and ability to communicate competence when establishing an effective consultation relationship "by demonstrating a knowledge of the consultee's environment and of the clients with whom the consultee works and by modelling practices that can readily be adapted to both the client and the environment" (D. Brown \& Schulte, 1987, p. 284). These ideas resonated with me. I had identified in the first action cycle that interpersonal skills seemed to impact highly on my ability to influence team members; and their statements confirmed that the music strategies were realistic and accessible.

The assessment process should result in statements of problems and goals in relation to clients (in this case through the IEP), as well as consultees. Interventions are designed to increase self-efficacy of consultees and/or clients through the mastery of a particular behaviour, in this case the introduction of planned music and, significantly, consultants do not take primary responsibility for any stage within the process, rather they take a responsive approach.

Consultation is successful when consultees seek out the consultant for information and support, feel more confident and skilful in their work, and use problemsolving approaches with new problems...Client change may come a while after 
consultee change however, so evaluation strategies may focus on consultee attitudes and behaviour more than on clients (Conoley \& Conoley, 1992, p. 9).

Evaluation involves determining whether there has been a change in consultees' and clients confidence', self-belief, ability to appraise a problem situation, and their expectations of outcomes (D. Brown et al., 2001; D. Brown \& Schulte, 1987). Statements of self-efficacy provide good evidence about whether team members have been 'empowered' to use music in their work in a sustained way. For example, John's teacher's aides began the process believing that their singing might not be attractive and were judgmental about what constituted 'good' or 'real' music; yet they became enthusiastic about their singing after observing John's positive responses. Their statements regarding their increased use of spontaneous musical games and songs, and social learning story songs, indicated they were more relaxed and enjoying their work, yet providing consistency and developing higher expectations for John. Their feedback provided evidence of increased confidence and motivation for them, and John.

\subsubsection{Pragmatic Issues}

John's case also highlighted pragmatic issues that needed to be considered in relation to the protocol. Prior to future consultations I would ensure that the classroom teacher was expecting me, that information regarding my visit had been received by all interested parties, and determine whether there is a music specialist in the school.

\subsubsection{Summary}

The social learning model of consultation, outlined within the psychological school consultation literature, provides a promising frame for the work I am doing. Applying the model was clarifying. Firstly, the primary relationship is with the identified team members' (the consultees) who agreed to be team leaders and to introduce or support the introduction of music with students. Secondly, the assessment will identify both student and adult goals. Thirdly, the success of the consultation is evaluated in terms of whether change occurs in the adults and it is acknowledge that student change takes longer. Statements of self-efficacy provide good evidence about whether team members have 
been 'empowered' to use music in their work in a sustained way.

My concern for cycle three will be to consider whether the social learning model of consultation continues to support my music therapy school consultations. 


\subsection{CATHY}

\subsubsection{Background}

Cathy was a sixteen-year-old Māori student with very high needs who had a rare genetic disorder which resulted in significant intellectual and physical disabilities, and limited vision ${ }^{9}$. She was educated in a classroom with just one other student who was hearing impaired.

\subsubsection{Introduction to the Team}

The referral was made by the SENCO, Rona, who is Cathy's classroom teacher. Other participants were Debra and Joe (Cathy's parents), Dana (neurodevelopmental therapist subcontracted from a District Health Board Child Development Services to MoE SE, Gina (visual resource teacher), Aaron (special education advisor), and Rose (teacher's aide). Rona agreed to be the consultee.

\subsubsection{Ethical Issues with Regard to Participation}

Before the research began, I asked for guidance from kaumatua to ensure that the process would be culturally appropriate, and was given full support to introduce the project to the school.

\subsubsection{The Team's Expectations of Music Therapy}

Cathy demonstrated positive responses to music and her team believed that music could be a pathway to increase or enhance her participation and learning. Cathy had previously been assessed by two music therapists, twelve years and eighteen months ago respectively. Reports from these encounters were on file.

\footnotetext{
${ }^{9}$ The specific diagnosis has been withheld as disclosure is likely to lead to the student being more easily identified.
} 
The assessment undertaken prior to Cathy beginning school indicated that she was stimulated by music, and became increasingly responsive as the session progressed. She was able to hit and sound the piano keys, and to use both hands at an electronic keyboard (although the left seemed to be just resting). She reached out to sound the autoharp and lyre, scratched and hit a drum, held beaters in both hands and was successful beating with the right. Cathy played the chimes with her foot, and was amused by this activity. During the session she vocalised in a conversational turn-taking way. The music therapist encouraged ongoing use of music for relaxation, sensory stimulation, and to promote speech.

During the more recent assessment, Cathy was reportedly alert and attentive, smiling frequently throughout the hour long session. However, the music therapist describes coactive movement and the use of "bell gloves" to motivate movement of the hands, in contrast to the independent reaching, hitting, and beater use reported earlier. A feature of both sessions was Cathy's response to changes in auditory stimulation, such as a change in tone colour on the keyboard, or in people's voices. The second music therapist also encouraged the ongoing use of music for relaxation and sensory stimulation, but focused more on opportunities for non-verbal expression, and added a recommendation for the use of songs for learning routines.

Rona therefore understood that a music therapist would aim to engage a student in activities that were connected with music, or the elements of music, while looking for reactions and responses, and would harness or 'shape', and encourage, responses.

\subsubsection{Programme and IEP Goals}

Goals for Cathy included the development of meaningful choice making and communication skills, the extension of early literacy skills and experiences, gross motor skills and ongoing mobility training, sensory stimulation, and social interaction. Specific communication objectives included the use of yes/no cards and a single switch, and the development of concepts such as up/down, fast/slow, stop/go. Objectives for the gross motor domain included increased trunk control, particularly in sitting. All other goals were matched with objectives that required staff to introduce specific activities, rather 
than anticipating Cathy's active response. For example, in the sensory stimulation domain the objectives were for staff to establish a tactile exploration programme, and to participate in a music therapy research programme. Many of the same goals and objectives had been recorded in previous IEPs, indicating that progress was difficult and extremely slow.

Consistency, predictability, anticipation, and practice were key concepts in Cathy's programme. Routine activities have been recommended as the basis for instruction for students with multiple disabilities who are blind (M. Smith, 2002), because they can be developed to encourage maximum student participation. Music can provide consistency and predictability, which enables the development of expectations and provides opportunities for making a particular response, and repetition. The structure of music could provide framework for Cathy's programme of routines.

Each step of Cathy's eating, airbed, visual awareness and tracking, and communication programmes, was carefully documented. Her timetable was specific and detailed, although it was difficult to adhere to it strictly in the context of 'regular' school life. Further, Rona felt that Cathy's learning opportunities had been somewhat neglected because there had been a high turnover of professionals who offered good ideas, but had little time for follow-up or feedback.

\subsubsection{The use of music before my visit}

Rona was an experienced musician who had an associate level diploma in piano, and had taught music many years earlier. Nevertheless, she was anxious about the consultation.

(Before I met you) I thought 'Oh gosh - have I got to do all these things... you're going to look at me and I'm going to be totally inadequate' (Rona)

Over the years, Rona had planned and attempted programmes that she was unable to sustain, and had decided against developing ideas that she might not be able to manage long term. She had put music 'aside', and placed considerable store in my ability to come up with the 'right' programme for her and Cathy.

I was planning a lot of things and never actually doing them so I think at the 
moment (I've decided) that if I'm actually not going to do it then I won't waste my own time... and I must admit, because I knew you were coming to do this music... I thought, well I'm not even going to worry about music because when Daphne comes she'll sort out all my problems! (Rona)

Despite her musical experience and training, and the input she had from other music therapists, Rona did not have a programme of music for her students. However, many of the activities she was engaged in with Cathy and/or other students did involve music. For example, she sang occasionally, they attended musical assemblies, and were involved in the fitness with music programme, 'Jump Jam'. Cathy was also involved in daily 'Jolly Phonics', a language learning programme that had a musical component.

\subsubsection{Assessment Activities}

The consultation involved Cathy in two individual assessment sessions (one to assess her ability and motivation to contribute to music activity using a single switch), and group work. We also observed and interacted with Cathy as she engaged in school programmes.

\section{Ecological Assessment}

During assembly, as her peers sang waiata, Cathy had her hands clasped. She stretched them out in front of her, lifting her head for a moment during each phrase of the song. She smiled and searched for other children, and seemed to be listening hard. She put her hands together several times when the teacher encouraged paki paki (clapping). During verbal exchanges her head was down, but she lifted it again when the children began to chant. As she 'marched' out in her wheelchair alongside her peers, she held her head high.

I observed Cathy attempting to participate in 'Jump Jam' from her wheelchair. The music and movements of the other children were fast and the sequences initially seemed complex. Rona was unsure of how to involve Cathy in this experience, yet knew it provided a good opportunity for her to be included with her peers. We discussed ways of simplifying actions to enable Cathy to be more involved and explored co-active 
movement using motions that were congruent with the music, and with what the other students were doing. We agreed that simple, repetitive movements could become meaningful for Cathy, and outlined a routine that involved arm and wheelchair movements. However, when we prepared to trial our ideas the leader surprised us with new songs and movements. Still, Rona decided to liaise regularly with the leader, obtain the music to be used, and to choreograph routines using the ideas we had discussed, so Cathy could begin to recognise the music, anticipate the co-active movements, enjoy the activity (especially being with the other students) and increase her independent movement.

In the classroom, mat time cognitive routines helped students become more aware of their environment. Activities included observing who was present, how many people were there, remembering their names, considering the weather, and finding out what day of the week it was. Each day, Cathy was also assisted to sit on a classroom chair, supported, positioned in a hoist to give her the experience of upright posture, and engaged in physiotherapy stretches to help minimise her contractures and maintain her range of movement. Team members were encouraged to sing to help Cathy to hold her head up, or when they were physically supporting her movement e.g. transferring her from her wheelchair. Singing, for example "up, up, up, up, up" using a rising pitch as she rose in the hoist, would help reinforce spatial and body awareness.

Cathy was also laid daily on an airbed. Rona put varying pressure on and around the airbed to provide her with a range of movement opportunities, and vestibular stimulation, in order to stimulate her sensory system. The aim of the programme was to raise Cathy's alertness, and in turn to increase her motivation to learn. While she was on the air mattress, there was a marked increase in her energy and activity levels, which could be sustained for a short period following the experience. The airbed and mat routines were repetitive, enabled Cathy to anticipate sequences, and provided opportunities for choice making and other communication, e.g. asking for 'more'. The activities lent themselves well to a musical framework.

Cathy was able to make purposeful choices using two single switches. Noting her successful use of single switches at meal times led me to consider the possibility of using 
switches to enable her to activate a musical note, or notes. Rona's musical ability suggested she had the potential to engage Cathy in music activities which required her to anticipate and make a specific musical response. However, we had little success with this activity. We agreed that Cathy may have been confused because the switches were more usually associated with food. Nevertheless, Rona would discuss Cathy's potential to use a switch in musical activity, with the Neurodevelopmental Therapist.

At Rona's request, but in her absence, I facilitated a music group which included students from another classroom. Activities included a greeting song; playing and passing instruments (particularly those that Cathy enjoyed and could actively use, such as bells, drums, tambourine, and shakers); a movement activity during which I assisted Cathy gently but firmly to lift her arms (giving her similar experience to her peers while also engaging her in a therapeutic stretch); and a simple dance with a partner. This involved another student taking Cathy by the hand to greet her, turning her wheelchair on the spot, and pushing her in a larger circle, following the children as they skipped. It seemed that supervised, structured, wheelchair dancing would be a useful and enjoyable activity for Cathy to engage in with peers.

During the group session Cathy smiled, lifted her head frequently, held instruments with her right hand, and also mouthed them. She independently held and shook cluster bells, held the tambourine, allowed coactive beating, and lifted her arms. I observed that one of her teacher's aides tended to put instruments in her hand instead of encouraging her to reach for them, offered coactive support rather than waiting for an independent attempt, and prompted unnecessarily. Team members were encouraged to give Cathy more time to respond.

Rona was finding her work extremely difficult due to her isolation and lack of support. High levels of special education staff turnover, and changes in the ways resources were funded, felt uncomfortable and unprofitable for her. She noted that some staff tried to compensate by offering wide-ranging support, but this was not always without difficulties.

Even when we get regular visits, new people come. So you're getting constantly maybe an assessment, and they come up with some things and either you hear 
nothing or it's someone else and you feel like you're going backwards. (We had someone who) was good in the sense that there was some continuity there and he was quite prepared to cross boundaries - but that can create other problems for other professionals. (Rona)

It was clear that Rona valued the input of individuals when they were able to come to the school. Nevertheless, the challenges of working with a profoundly disabled student who made little or extremely slow progress seemed to leave her doubting her own skills and abilities, and constantly searching for answers from other professionals who were rarely able to respond. It was unsurprising that she felt disempowered and sometimes directed frustration at specific events or people associated with those events. Further, Cathy's limited ability to respond to Rona's efforts to stimulate her left them both relatively impotent.

Cathy's mum chose to observe Cathy participating in a group music session, and joined the focus group to discuss her individual session, but work obligations prohibited her being more closely involved. It seemed that she had a confident, trusting relationship with staff at the school and would be comfortable with the ideas they chose to initiate.

\section{Identified Strengths and Needs}

\section{Medical Profile}

Cathy had spastic quadriplegia, which was more severe on the left side. She used a wheelchair, and was dependent on others for all activities of daily living. She was nonverbal, had limited vision, and required consistent one-to-one support. Cathy's general health was very good and she was rarely absent from school.

\section{Physical Profile}

Cathy needed her wheelchair for mobility, and required full trunk support for sitting. Her head was often down, probably partially due to the limited visual reward she obtained for her physical effort. She was able to use her upper limbs for some tasks, usually with 
assistance, to grasp and release small objects in her right hand, and to maintain her grasp for long periods if the texture of the material was tolerable for her. Cathy enjoyed using her right hand to hold, shake and hit things, and to communicate in other ways, e.g. a shake for "more". She was able to activate a switch with her right hand and would cross the midline to do so. The team was keen for Cathy to use her left hand more than she was doing, and to increase the frequency with which she was willing to pick up and explore objects or toys.

\section{Intellectual and Communication Profile}

Cathy had difficulty demonstrating understanding, although it was clear she could comprehend some simple statements and verbal prompts. She made definite choices using "Yes/No" indicators and single switches, and could indicate "more". She also used vocalisations and facial expression to communicate, including a non-response for "finished" or "no". She was able to understand a sequence of activities if given a consistent communication pattern. Rona felt Cathy's quality of attending changed, and she became more alert, when listening to certain pieces of music.

\section{Sensory Profile}

Cathy's hearing could not be adequately tested but her mother believed it was unaffected. She had a cortical visual impairment, and nystagmus. She had better vision in her left eye but turned her head both left and right to locate sound. In the past Cathy poked her eyes, especially when she was under-stimulated. She continued to mouth objects but this reduced when staff members were actively engaging her in specific tasks.

Cathy demonstrated tactile defensiveness and found some textures difficult to hold. However, her limited opportunities for exploration, and her lack of visual cues to help her anticipate the feel of various materials, may have contributed to her discomfort. It was important to give a verbal cue before touching her so she might anticipate what was going to happen. 


\section{Orientation and Mobility Profile}

Cathy's Orientation and Mobility Programme included routines such as the airbed, an activities course, and a visual awareness programme. Identification of clear starting and finishing points, and the use of consistent carefully-planned language and symbols to reinforce her movements and routes around the school, enhanced her understanding of herself and her environment. Movement experiences such as 'up' and 'down', 'forward' and 'backwards', 'bumpy' and 'smooth', needed to be verbally labelled. Drawing Cathy's attention to sounds that occurred regularly on route to familiar places helped to establish a pattern she could anticipate, and gave her some measure of control. Playing games involving 'fast' and 'slow' in the wheelchair, and including timing cues such as " 1,2 , 3... go!" were also recommended.

\section{Activities of Daily Living}

Cathy was dependent on others for dressing, eating, washing, and toileting. She could eat very small pieces of soft food placed in her mouth or spoon fed to her. She was encouraged to help by holding the spoon.

\section{Emotional and Social Profile}

Cathy appeared happy at school, particularly when stimulated with one to one interaction. She smiled frequently during group and individual music activities, and giggled occasionally. However, she sometimes seemed profoundly moved, for example crying when listening to music.

Cathy enjoyed the company of other students, and was noted to be more alert in group situations such as assembly, fitness, or group music. She had a classmate who was a particular friend, attentive, supportive and gentle with her. Cathy was not highly responsive to her attentions, but was clearly comfortable in her presence and occasionally looked for her. Cathy had also been noted to search for people who had been interacting with her, after they had gone. Team members were encouraged to give her a clear indication when conversation was intended to involve her. Rona wanted Cathy included 
with her peers in meaningful ways. She described how easy it was to 'forget' her because she was so quiet, and other students demanded all the attention. She also valued time alone with Cathy so she could focus fully on her when facilitating her programme.

Because Cathy was 16 years old, the team were arranging for her transition to the local high school in approximately two terms' time. This was a particularly difficult decision for her mother, who was very happy with Cathy's current placement. However, it was agreed that interaction with students her own age, and the stimulation of the high school environment, would be helpful for Cathy's ongoing development.

\section{The individual music therapy assessment session}

The following paragraphs provide a glimpse into Cathy's individual session, which lasted for 47 minutes. A range of experiences was planned to evaluate Cathy's potential to engage in various developmental tasks. Her physical limitations prompted a more detailed plan than might be prepared for more active students (see p. 162). That is, I

anticipated that I would have to take more of a lead introducing the experiences, because Cathy would be less able to communicate her choice of activity. As expected, the description of the session has a strong focus on my intervention and intentions as I attempted to motivate her to respond to a range of musical stimuli. Throughout the session I was interested in Cathy's:

1. ability to attend, to offer overt and subtle signs of engagement;

2. responses to sound, any visible reactions indicating ability to detect, recognise, and locate a sound source;

3. interest in sound and music. What would motivate her to interact, and what would help her attempts to adjust her posture and/or physically engage;

4. ability to make choices, take control, and be aware of cause and effect. I therefore offered instruments for Cathy to play, and/or to prompt me to play or to stop playing;

5. response and reaction to touch, coactive movement, and wheelchair dancing;

6. ability to manipulate equipment, including grasp, and use of the specially adapted ' $\mathrm{T}$ ' beater. 
Cathy enjoyed sound making and seemed aware that she could share in the music making. Although she mouthed her hand or instruments often, she ceased when stimulated in other ways. She appeared especially interested in drums, maracas and other instruments she could shake, such as the hand bells.

Cathy began to stir. I lifted the bells above her head and she immediately responded, raising her head high to track them, and holding it there for eight seconds. When I finished humming the verse I gently placed one of the bells down. I offered Cathy the other bell, placing it carefully in her right hand. She shook it immediately and I surreptitiously reach for the second bell. I resumed my song and joined her as she played. Sometimes, as she attempted to shake, her bell didn't ring, but she persisted and was successful. Soon Cathy paused, attempted to mouth the bell, and put it on her face seemingly interested in the cold metallic feel of the instrument. I paused, waiting for her, and she resumed shaking the bell.

Cathy rang her bell enthusiastically, and although her head was down I could see her smiling as I began singing. While still listening and attempting to play, she placed the bell against her mouth - once more restricting opportunities for it to sound. I hummed the last phrase. As I paused on the penultimate note, waiting for her to contribute, she dropped the bell. I sensed that she might have done this deliberately but it was hard to tell. I hummed the last note and rang my bell. Cathy immediately lifted her head but just as quickly allowed it to drop again.

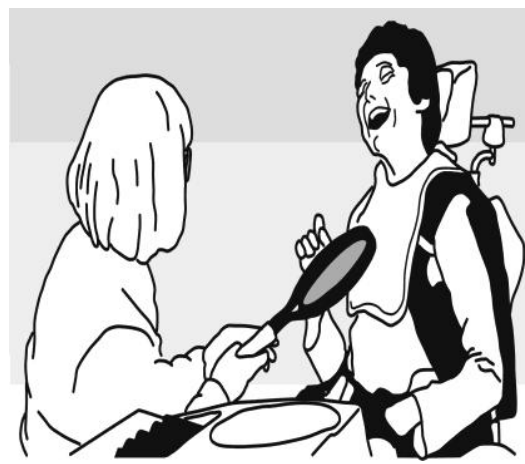

It was very often difficult to interpret Cathy's responses, especially to determine what stimuli she was responding to. Was she smiling at my face, my voice, or the bells? Could she even see my face? Sometimes though, her emotional reaction was strong and it was clear that she was enjoying the opportunities for interpersonal communication that music afforded her.

Although her head was down, there was only a brief pause before she moved her hand to the drum and began to tap. I resumed the song, increasing the pace to match her rapid tapping. Her head came up immediately but she stopped playing 
and shook her head from left to right and back, smiling. I wondered if this was a habitual 'blindism'. I stopped singing and waited for her. When her head stilled I sang the next phrase, strongly. Cathy resumed shaking her head and this time I wondered if it was her way of joining the 'dance'. Nevertheless, again by way of inviting her to play, I paused at the end of the line "here comes the drum for...." Her open right hand seemed poised to strike. I tapped the drum against it lightly to remind her of the game.

Cathy resumed her rapid tapping for two brief phrases before suddenly stopping. Her head was down while playing but she sat tall as she waited for my response. I stopped singing at the break in her drumming, and we waited together for a few seconds. Cathy seemed to be wondering what was happening before suddenly realising that she could take control. She tapped rapidly again for just one phrase, stopped and laughed.

After a brief pause she began playing again, seemingly now aware that she could lead the music, and 'control' my singing. Her playing became strong and 'bold' but again, at the end of the second phrase, she seemed to stop to 'test' my response. She paused too at the end of the final phrase, when I changed the melody to include a 'turn around' suggesting a repeat. Cathy began to giggle! Her chuckle became louder and she lifted her head, laughing. Suddenly she resumed her rapid playing and her head dropped, although her smile could still be detected. The tempo steadied and stopped."

Later,

I returned with an adapted ' $T$ ' beater, held it out and offered it with verbal encouragement, to Cathy. She looked, took it immediately and began to shake her hand as if wanting to drum. She played with enthusiasm and vigour. Her beating was rapid and she was generating exciting music which I matched with my singing. The expression on her face demonstrated enjoyment and commitment. Her exuberant music continued for fifteen seconds before suddenly stopping, and I wondered if a sudden jump in the melody of my sung accompaniment surprised her. 
Cathy seemed interested in my singing, vocalised occasionally during the session, and at one point was able to engage in two exchanges of vocal turn taking with me. I also worked with her in movement activities, introducing a simple wheelchair dance in structured folkdance style, as well as freer coactive movement with upper body.

\section{Team observations and discussion of the assessment session}

Much of the discussion addressed what we might expect Cathy to be able to do, and how the team might interpret her responses. I explained the techniques I employed during the session.

Seeing the session on video was very worthwhile because really sitting back and analysing what is going on is something we don't often do. (Dana)

The team noticed how much Cathy enjoyed being involved in music making and how relaxed she was during the session. They talked about the importance of encouraging her to use both arms, observed her spontaneous use of left hand, and acknowledged the importance of hand-over-hand support and its gradual reduction.

This is a crucial step in the development of reciprocity for her - it isn't a matter of expecting simple imitation to occur. (Ian)

Team members noted Cathy's discomfort using her hands on a drum, and that she might prefer to use a beater. They observed her particular interest in bells, and thought she might enjoy the session even more when other instruments became more familiar to her. They agreed they might be able to expect more from her, noted their need to be more persistent in waiting for a response, and to sing with conviction.

Music is a very promising medium to get more communication ... we haven't tried it very much in the past at all... its fascinating! (Aaron)

\subsubsection{Agreed Planned Music Experiences}

Rona saw potential in using music with Cathy to enhance the routine activities she had to manage on a daily basis. Although she noticed positive responses from Cathy during the 
individual improvised music therapy session, and intended to use more musical communication in her spontaneous interactions with her, Rona preferred to focus on the use of especially composed songs to reinforce cognitive concepts. I therefore composed and recorded a programme of songs for mat time and the airbed routine.

Cathy would be encouraged to request "more", indicate "finished", smile and/or laugh, and vocalise during the routines. The consistency and repetition of the songs and/or instrumental music would help Cathy to understand what was going to happen to her and to demonstrate that she could anticipate events. Team members were encouraged to pause and exaggerate the end of a phrase before changing movement and/or direction with her, to exaggerate the end of a verse when singing "stop" and to pause for Cathy to indicate "more" before continuing. They would warn her "one more" before singing the last verse in any activity, use the lyric "finished" at the end of a song before changing the activity, and sing directional lyrics when rocking or moving Cathy ("side to side"; "forward and back"). Music was recorded on a CD to help Rona learn the songs, but the importance of singing songs live and timing the music with Cathy's movements was emphasised. She would also use simple improvised techniques to support movement such as singing ascending and descending scales when lifting and placing Cathy, and would play selected recorded music while giving Cathy's hands a massage.

The morning 'mat' routine provided opportunities to present cognitive information in a consistent and repetitive but enjoyable way, and for Cathy to respond with maximum independence. Songs were composed and/or adapted to use in conjunction with each of the cognitive concepts being taught. Two examples are provided below.

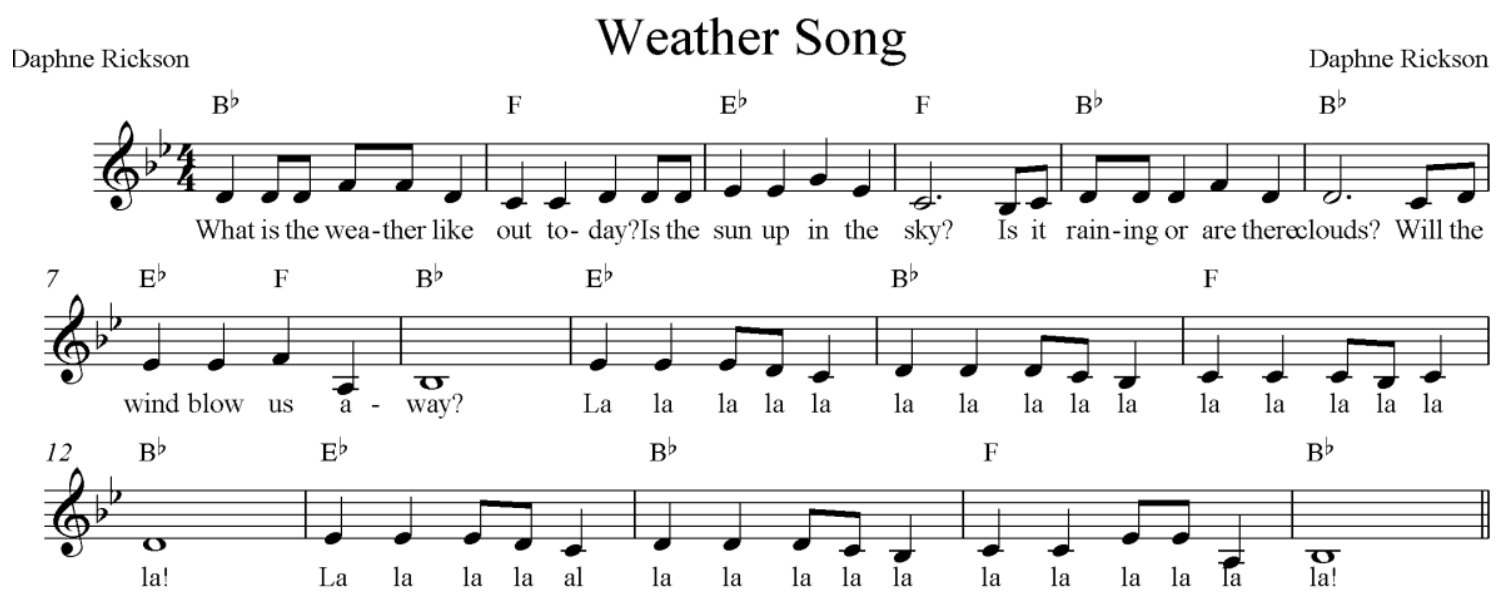


At the appropriate time in the song below, students would be encouraged to count people in the group or on the photo chart, slowly, from left to right. Staff members would emphasise one-to-one correspondence by pointing and counting simultaneously, to help with pre-reading skills. Cathy would be encouraged to indicate "Yes/No" in response to a question, and to vocalise. Staff members would give a single verbal prompt e.g. "Cathy, your turn" occasionally, as necessary. Rona recognised the need to allow a good amount of time for the whole mat time experience, so she could give Cathy and others time to respond within that.

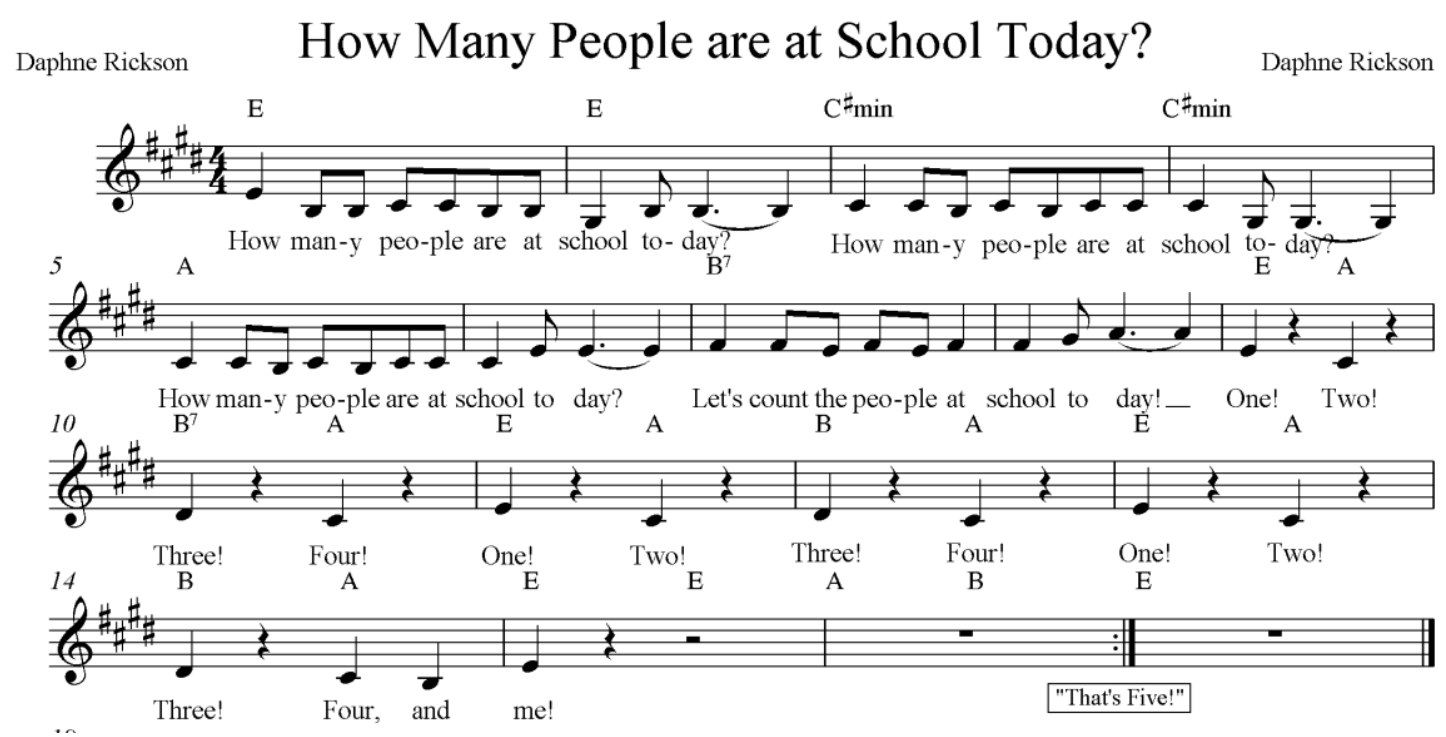

Rona suggested the routine fitted her way of working and she was likely to use the activities because they were integrated into what she was doing regularly. She strongly indicated that she needed to have the music experiences and the expectations for herself, and Cathy, clearly laid out.

(Because) when you don't get any support or feedback, and if you don't really know what you're doing in the first place, it can fall off... you think you will remember things but you don't? So yes... break it up as small as you like - it's good for me! (Rona)

Rona had no inhibitions about singing after the music therapy consultation, realising the introduction of planned music experiences was well within her capabilities.

Sometimes actually starting is the hardest part of it. ...Having someone doing it was good, and watching you work with the group for example was so simple I 
thought "Oh I can do that!" whereas if someone does something that is hi-tech or whatever you can have a bit of a sinking feeling thinking 'how am I going to be able to do that?' It seemed accessible. (Rona)

Rona is a robust advocate of the need for professionals to make programmes realistic, meaningful and purposeful, to have enough time to build relationships with staff at the grass roots, and to provide follow-up to the programmes they implement so the momentum continues.

(Another professional working with Cathy) just gave us HEAPS of things to do and I actually said "this isn't working” - it wasn't that the activities were 'wrong'... it was too much. You've got to do things that suit people... (Later) he worked quite a while with the teacher's aide ... and it allowed a bit of a relationship to grow because he spent that time with her. So she started listening... and I think he incorporated some of her ideas so it gave her a bit of a boost... she was feeling valued. (Rona)

Rona stressed the importance of follow up to keep her motivated, but also believed that she would be more committed to continuing the work because it was being done in a research context.

I guess what I thought would be good about this (music therapy procedure), is that it's part of a learning thing YOU'RE doing that that will make me (continue with it)... When you've got a little bit of structure and you've got to do a followup, it gives you a kind of boundary or a marker that instead of just falling away...gives you the motivation to do it because that's what you've agreed to do. (Rona)

\subsubsection{Introducing the Strategies}

\section{Attitude to Using Music}

Despite her increasing musical confidence, Rona still expressed some reservation about her ability to sustain the music making long term. She gave little indication that she was excited about the work or that it would be potentially enjoyable. Nevertheless, she did express commitment, believing that having to complete a diary of her activities would 
increase her motivation to keep up with and to develop the work, including "making up songs". Rose and Rona were the only two team members on site, so would continue the work in isolation. Rona argued her work could be enhanced, and she would maintain more enthusiasm, if she could keep in contact with other members of the team who had been involved in the assessment process.

The effort required to engage Cathy and her peers in consistent and repetitive activities was taking its toll on Rona, and it was important that introducing music activities did not add to her burden. However, she acknowledged that the consultation opened up her mind to new possibilities for working with Cathy, and believed she would be able use simple planned music experiences in an ongoing way. She felt more able to work with music activities because she had musical confidence, the simple ideas had evolved in context, and they seemed relevant.

Because for me... it's not that music is 'easy' but I feel I know something about it - I know I can sing...I feel I don't need any (extra skills)... it's kind of 'every day' (music) - if you can talk you can do it. (Rona)

\section{Modelling Strategies}

Rona valued the collaborative nature of the consultation highly, and believed that observing me and our trying activities together gave the written music therapy report meaning.

When someone comes and works with Cathy, say for an hour, I might not even be there then they mail me a report three weeks later ...I just understand it better because when you say "do such and such" we've done it together so I know what you mean. ... If you just gave me a CD and said "do it" I probably wouldn't do it. (Rona)

\section{Increasing Team Member's Confidence}

Rona's intrinsic motivation was clearly low. I was concerned about how hard it seemed for her to continue with activities introduced by other professionals when she did not 
have regular contact with them, and that she would not be able to sustain the work.

If you don't feel confident or competent at some things you might really value it but just not know what to do (and) when you encounter a problem...if you don't know how to remedy it you just throw it to the back of the cupboard I guess. (Rona)

\section{Benefits for Other Students}

On the other hand, Rona believed the planned music experiences would be helpful not only for Cathy, but for other students she was working with too. And she had begun to think about how she might use music spontaneously to engage some of the other students. It's just started me thinking that maybe something like this could be good for $K$. too and actually the other day I just started singing to him "come on let's jump down", or something like that, and he did do it. I just think sometimes that maybe singing makes it 'fun'. (Rona)

\section{Therapist Relationship with the Team}

My relationship with Rona was very good, and she felt this was essential to the success of the visit. Further, she was pleased I was able to engage the other students she worked with, and felt my presence made a positive difference in her classroom.

I think it was good that we had common ground. It could be quite difficult if we clashed - you know people can be really different and have different approaches... ...so I think that is quite important that you have that rapport with a person so they can really hear what you are saying. ...(The students) were very accepting of having you there. And $H$, remember I said he used to be really shyit was like pulling hen's teeth to get information out of him - and he was just chatting (with you)...(briefly overcome with emotion) that was so amazing! (Rona)

\section{Time Spent with the Team}

Despite Rona's clear appreciation, I needed to negotiate the amount of time I spent in the 
classroom to ensure she did not become overwhelmed by my presence. She reinforced how important it is for everyone to be flexible when engaging in collaborative work, and noted that it was helpful when I took time out to make resources and write reports. Although she agreed that it was important for me to be available for the full week, she found the process tiring and was ready for me to go.

Yesterday it was actually nice to just have a bit of space...its like when you have visitors in your house it's nice to have a break from them even when you love having them there. ...I guess it is the tension between the work we have to do together and the other things I have to do. (Rona)

Rona also recognised that for Cathy to be able to show me what she was capable of, it was important I should have time to build a positive relationship with her.

\section{Revitalising the Programme}

Rona believes that the impact of support visits has a lot to do with the personality of the professionals who offer their services.

Having someone who's livelier ... it sort of gets you going. You know how you can sort of feel a bit (give big sigh) 'ho hum', and then someone comes and you can just think "Oh yeah, yeah!" And I do think that its part of the isolation here... (Rona)

\section{Relationships with 'Distant' Team Members}

After seeing the advertisement asking for expressions of interest in the music therapy research project, Rona was proactive in investigating the potential for her student/s to be involved. In contrast, when she receives visits from other professionals she often feels unable to make the most of their skills because of the way the services are offered.

I do feel a bit of tension with specialist people coming because often they come when it suits them ... that might be totally unsuitable for me but we are so desperate to get the Speech and Language Therapist or something, we say 'yes'. (Rona) 
Rona had begun to feel powerless and to accept her situation as inevitable, even though she believed she deserved more help. She was somewhat sceptical about whether it would even be worth asking for music therapy services for Cathy or other students in the future.

Quite often, with the way the referral systems go, it's almost like you don't askyou just get what comes if you know what I mean... ...some of the services that would be reasonable to think would be available just aren't available for various reasons. (Rona)

Further, unless information is routinely forwarded to staff members who work with students who have special education needs, they might not know what services are available.

I sort of think, who do you ask? This might sound really stupid but if you don't know it is there, who do you ask? (Rona)

\subsubsection{Continuing the Work}

Rona's diary indicated that she engaged cautiously with the music activities at first. Tasks such as passing copies of the songs to Cathy's mum were carefully reported. Programmes were introduced one song at a time, and Cathy was initially only supported to participate in a few movements at 'Jump Jam'. On several different days the music could not happen because Rona was at meetings, or did not have any teacher's aide support. She reported feeling 'muddled' with some of the proposed activities, and requested that several of the songs on the $\mathrm{CD}$ be re-recorded for various reasons.

Interruptions continued. Cathy or Rona were sick or there was no Jump Jam due to bad weather and so on. However, Rona gradually began to write of 'adding new words', 'making up different moves', and 'having a go' at different music activities, and reported introducing her own gentle recorded music for movement with Cathy. She began to describe feeling 'great', 'very relaxed', and having 'great fun', and to add smiley faces to her diary. She even wrote about continuing the mat routine when they had a visitor, pleased that "he sang along". The songs "helped to carry the activities along" and she loved the "special time" she had together with Cathy in music. She reported gaining confidence as the songs became easier to remember.

(Doing the routine was) very enjoyable. The music seems to make it easier to do 
and to remember the correct sequence (;) ). (Journal Entry)

Rona reported positive and increasingly detailed responses from Cathy, and it was clear that the success they both experienced added quality to their relationship. On at least one occasion, Rona confessed to playing music in the background "probably more for myself'. It appeared that she was increasingly aware of the power of music to affect the classroom environment, and understood that being relaxed was important for her work. Rona was encouraging the teacher's aides to use planned music experiences, and noticed they were becoming more confident in their musical interactions with Cathy.

\section{Music Strategies}

Rona felt the music for the airbed routine was the most successful music experience, citing the consistency that was provided by the framework of music as being really helpful for both her and Cathy. Further, having different music for gentle and more vigorous movements helped to make the contrast more distinctive for Cathy. Rona was introducing new music for additional tasks such as standing. Interestingly Rona also reported that sometimes when Cathy was in her standing frame she had to withdraw the music because Cathy had a strong physical response to it.

Rona began to give herself permission to use music for its own sake, and to enjoy being with Cathy as they listened to recorded music together. She began to recognise that music enhanced the time they spent together and was extremely valuable for developing their relationship.

I kind of don't care any more what other people think... I feel I'm more confident. And things like touching Cathy's hand and stroking her face and listening to the music... it's just what I'm doing and I don't care what anyone else says. I don't know if that's the right thing to say but I feel it is the right thing to do. And I could justify it to someone if I really had to. (Rona)

Singing to Cathy and closely observing her responses encouraged Rona to recognise the subtle communication Cathy was offering, and to increasingly value her efforts. 


\section{Student Progress and Potential}

Rona's diary entries indicated that Cathy was increasingly alert when music was used as a stimulus; and was continuing to develop purposeful movement. For example, Cathy was 'stilling' and throwing her arms out; banging her hand on the equipment or floor, or scratching, to indicate 'more'; arching and stiffening her back in anticipation; rocking side to side in her chair, 'dancing'; vocalising more, sometimes 'singing out' or 'calling out'; listening; enjoying her work routines to music (laughing and smiling often); and generally seemed more relaxed.

Rona believed that using music with movement routines helped her to be more consistent, which in turn helped Cathy to anticipate what was going to happen next.

It helps me because the tracks are timed; I know if I listen to these two songs that that's about ten minutes instead of looking at my watch all the time. But Cathy definitely reacts to it - she laughs and she actually sways to the music... (Rona)

The Jump Jam musical fitness programme was offered somewhat erratically during the study period, and Rona did not believe that Cathy was any more aware of the other children than she had been the previous term. She wondered whether that was because focusing on the music and the physical activity might be all that Cathy could cope with at that stage. However, Rona was certain Cathy was enjoying being out amongst the action.

(When they used new music for the fitness routine) I decided to move forwards and backwards with the beat, but went a lot further than usual with a longish 'stop' in between. Cathy seemed very alert. One teacher said she had never seen Cathy so alert (in three years!). Her posture was good, and she had her head upright. (Rona)

It seemed that the music therapy consultation had encouraged Rona to look closely at, and to more readily trust, Cathy's subtle responses. Rona noticed that her slower, more deliberate communication provoked Cathy to offer a more speedy response. This was particularly evident when she was being fed - Cathy seemed to be more aware that Rona was waiting for her to choose and gave quicker indication, via her switch, that she was ready for more. 
I think ...that it's actually helped me with her switches... I give her morning tea and I think I have more confidence to use them properly and to encourage the teacher's aides to do it properly. (Rona)

It seems clear from Rona's report that Cathy is responding well to the musical support, and that the quality of interaction is greatly improved for both of them. However, it would be extremely difficult to determine whether or how much the music activities support Cathy's development over time, because of her severe physical difficulties.

\section{Increased Confidence}

Rona found using music for routine activities most useful for 'adding interest'. She reported initially feeling a 'bit foolish' singing while jumping up and down, but believed the confidence I exuded gave her the assurance she could do it too. She continued to develop self-belief as she engaged with the work after I left the field, and saw this as an exciting outcome of the consultation process. Significantly, she was affirmed in her personal development by her school principal who suggested during her appraisal that the music therapist's influence on her confidence was very clear. Further, Rona suggested that a snowball effect was occurring - her conviction was influencing the teacher's aides.

I think it's just a confidence thing. And I think the more I do it, the more confident they (teacher's aides) become... ... they've got a positive attitude to it. (Rona)

\section{Consultee Relationship with Student}

Using music to support routines gave Rona and Cathy a focus and timeframe for their work, and enabled Cathy to learn about, and to anticipate, the steps in her tasks. However, over time the music became the foundation for new ways to connect, relate, and to communicate.

Sometimes I just do simple things with music in the background... even just holding her hand or stroking her hand while the music's playing - little things like this... touch, that human contact, that's probably her most important communication mode if you like... ... it's a very universal thing...it's almost on an instinctive level... (Rona's voice becomes tearful) ...I'm crying now... it's just one of those things. 
Like you look at someone like Cathy and you could say, you know, that her life is 'useless' but when we're listening to music together (breaks down again) it's actually really beautiful. ... And maybe if you don't have those kinds of experiences you don't actually understand it. (Rona)

\section{Time}

Initially, Rona suggested there was not always time to introduce the programmes she had planned. However, as the term progressed and she began to use music more spontaneously, it was evident that she was increasingly able to seize the moment to have precious communicative musical interactions with Cathy. Moreover, apart from the 'Jump Jam' programme, many of the therapeutic music strategies were embedded in daily routines, and little extra time was needed to use them effectively.

\section{Benefits for Other Students}

Rona felt that in many ways the programme we set up was just as helpful for another student who was deaf.

Because of the kind of things we're doing like counting and whatever... it's definitely been better for (the student) in terms of giving her structure and giving her cues about what's going to happen next... And I do wonder sometimes too whether singing is easier for her than just listening to someone talking. (Rona)

\section{Managing Music in Future}

As Cathy's programme changed, Rona used the ideas introduced during the consultation to alter or add new planned music experiences. While she was aware of Cathy's need for consistency, Rona's developing confidence enabled her to take initiatives too. She planned to bring recorded music from home to play to Cathy to see how she might respond to it. Further, she was committed to including Cathy in the music and movement programme, even though it involved considerable extra work for her in finding out what music was going to be used each week, and developing an adapted movement routine to suit Cathy's abilities. 
I don't know what's going to happen next term because sometimes they change the routine, but I think for me a big part of it is not be worried about it. It's a very big thing for me to just accept that life's not perfect and there may be days when you do hardly anything - you know, but as long as you know you've done your best... that's it really. (Rona)

Rona shared how easy it can be to forget how a song goes when it hasn't been introduced or listened to regularly. She valued the recordings of the songs, and video of group work, as a resource that she could return to for specific activities or ideas. It was evident that Rona now considered music management to be an important aspect of her work, and that it would continue despite the difficulties she encountered.

Cathy had an IEP last week and with her moving to the high school ...one of the things we talked about was that we wanted to continue the music. (Rona)

\section{Postscript}

Rona felt impelled to send a note on Cathy's progress approximately four weeks later. She advised that when Cathy is standing in her hoist they listen to music.

I stand near her and reach out my hands to hers. Sometimes she waves her arm and pats against my hand, like a high five, in her own rhythmic pattern. Occasionally she grasps and holds my hand. I call this our 'dancing'... interesting that mostly when I take her hands, she pulls back - when we 'dance' she is the instigator not me! (Rona) 


\subsection{ACTION RESEARCH CYCLE THREE: AFTER CATHY}

My concern for cycle three (7.5.5) was to consider whether the social learning model of consultation continued to support my music therapy school consultations.

\subsubsection{Testing the 'Fit' of the Social Learning Model of Consultation}

The social learning model of consultation still provided an appropriate frame for the work, in that the focus was on helping Rona to set goals and create situations that would develop her confidence, and lead to the anticipation of positive outcomes. I offered physical demonstrations, verbal descriptions, written and audio resources and modelled self-evaluation statements to help her develop new self-perceptions. However, Rona also requested that I undertake high levels of direct work, even while she was away doing other things.

Teachers will continue to expect children to be withdrawn from the classroom for specialist support (Hanft \& Place, 1996; Wesley, Buysse, \& Skinner, 2001). Nevertheless, I thought it would be unusual for a consultant to facilitate a group while the teacher did other work, and was aware it would not be helpful if my active participation resulted in consultee dependency and no new coping skills. Further, Conoley and Conoley (1992) note that while consultants actively participate to model skills, demonstrate a range of options, increase opportunities for success, or to show concern and investment, it is necessary to know how to limit active involvement, and when to fade out implementation plans. Consultees generally view the consultant taking the lead and providing direction as positive, but the degree of directiveness needs to be tested and reactions monitored (Dougherty, 2005).

Polsgrove and McNeil (1989, cited in Wesley et al., 2001) defined the consultants' role as a continuum in which consultants provide direct, hands-on service to the child at one end, and collaborate with consultees to effect change at the other. However, these authors note "with little empirical information or training in consultancy to guide them, consultants

often must walk a tight rope in relating to consultees" (p. 113). In order to develop and 
maintain a therapeutic relationship with Rona, I balanced, and carefully timed, the tasks of enabling her to attend to her own needs; having her observe and co-facilitate; or inviting her to independently introduce the therapeutic music strategies.

Like team members in previous case studies, Rona chose strategies that were embedded in existing programmes. I tried to make the programme realistic, meaningful, and purposeful, and fit with her way of working. "Working collaboratively with the teacher in therapy or instruction and weaving the therapy or instruction into everyday routines might be the most critical aspect of service delivery" (McWilliam, 1995, p. 33). Further, if there were too many expectations, I feared she might do nothing. Consultees are free to do whatever they wish with the consultant's suggestions and recommendations (D. Brown et al., 2001; Conoley \& Conoley, 1992; Dettmer et al., 2005; Dougherty, 2005). I was concerned to provide all reasonable support to maximise the potential for the music strategies to be sustained. That Rona continued to seek support when she was feeling anxious about her ability to cope was positive, although Williams (2000, in Dougherty, 2005) noted that consultees often ask for assistance as a 'last resort'. Further, the effectiveness of consultation is limited if, among other things, participants do not believe it will be helpful (Conoley \& Conoley, 1992).

On the other hand, if the consultant and consultee have similar perceptions and feelings about the student, and about the issues involved, and if the intervention fits consultees' theoretical beliefs, and is considered to be worth the time and effort involved, consultees are likely to engage with the process (Conoley \& Conoley, 1992). Moreover, Cartledge and Johnson (1996) argued that teachers' personal and professional attitudes and fears regarding students with disabilities can be addressed by directly teaching or modelling necessary competencies. So although the starting point for the music therapy consultation was not optimal I could still anticipate positive outcomes.

\subsubsection{Managing Resistance}

In the beginning, Rona appeared exhausted and gave the impression she was 'stuck', disempowered by, and frustrated with, previous 'specialist interventions'. She lacked confidence, was not using her musical skills, and worried that I would 'judge' her. She 
was not a risk taker. Rather than taking initiatives, she tended to rely on other professionals to produce programmes for her. In contrast to previous participants, she preferred clear directions and the consultation activities to be outlined in writing, and requested further resources from me during the follow-up period. Her intrinsic motivation was low, and her initial commitment was to the research project rather than the consultation.

Despite Rona's tendency to devolve responsibility for Cathy's progress to other 'experts', by communicating the goals of consultation clearly I was able to facilitate a collaborative relationship which enabled her to take responsibility for choosing the music strategies she would implement. However, I was naturally concerned with her initial statement 'when Daphne comes she'll sort out my problems'. "Team members may expect consulting therapists to work miracles... educators and parents often hope that someone else holds the key to positive change..." (Hanft \& Place, 1996, p. 112) and "we can find ourselves cast as a rescuer, or someone with all the answers - the expert, the concert performer, and therapist to all" (Sutton, 2002, p. 199). Idealising special educators or therapists who work with children who have special education needs has served to disempower regular educators, and reduced their motivation and the need to develop new skills (SaponShevin, 1996).

Resistance or conflict occur for various reasons and are typical in consultation relationships (D. Brown et al., 2001; Conoley \& Conoley, 1992; Dettmer et al., 2005; Dougherty, 2005; Hanft \& Place, 1996). Nevertheless, they can be minimised by:

- obtaining support from school leaders;

- having clear goals for the consultation and ensuring the consultee knows what they need to do for it to be successful;

- explaining the relevance and potential benefits of interventions without guaranteeing success;

- demonstrating how potential gains can exceed costs;

- gaining the commitment of team members; maintaining high levels of collaboration and choice;

- being alert to the possibility that resistance and conflict might occur, and when it 
does finding the reasons for it;

- using examples or 'stories' to illustrate points;

- fairly distributing the workload;

- ensuring consultees have the skills they need to implement strategies;

- challenging unhelpful patterns of thought

(D. Brown et al., 2001; Dettmer et al., 2005; Dougherty, 2005; Hanft \& Place, 1996).

\subsubsection{The Consultation Relationship}

Rona referred to the importance of having 'common ground', 'rapport', and 'someone who's livelier to get you going', thus emphasising the primacy of the therapeutic relationship, and the personality of the therapist. I was constantly aware that I needed to consider my emotional presentation carefully ('should $\mathrm{I}$ in this moment be modelling lively and enthusiastic practice, or inviting calm reflection on issues of concern?'). I also noticed that I was sharing personal and professional stories with her - something I had rarely done in practice in other contexts. However, Conoley and Conoley (1992) suggest that consultants should be self-disclosing, that they are there to enhance the experiences of the consultees and therefore can share personal information that will inform or support them. "Consultants can build referent power... influence based on the perception that the consultee and consultant are enough alike that the consultants suggestions are likely to be valid" (p. 35). Yet inappropriate sharing, generalisation, and stereotyping can result from over-enthusiastic communication (Dettmer et al., 2005) and finding a balance in selfdisclosure is difficult (Conoley \& Conoley, 1992). Once again I wondered about how inexperienced music therapists might manage this task.

Rona also shared personal information with me, and I felt concerned to support her appropriately so she could get on with the work, without crossing boundaries. In school consultation humanistic concepts are mirrored in the therapeutic relationship, but it is not therapy, counselling, or supervision (D. Brown et al., 2001; Dettmer et al., 2005; Dougherty, 2005). Consultation should not focus on the psychological problems of the consultees, although it might be necessary to address issues when they restrict consultees ability to deal with certain problems (D. Brown et al., 2001). Rather, consultation should 
focus on the issue of how to facilitate the consultant to take care of student learning and development in the school setting (Conoley \& Conoley, 1992; Dettmer et al., 2005; Dougherty, 2005). It does not involve emotional probing, especially about past emotional history; rather, consultants help consultees to cope whilst remaining alert to the possibility that they will be vulnerable and in need of psychotherapy (Conoley \& Conoley, 1992).

Taking time to build a therapeutic relationship with Rona meant she was eventually able to engage with the music therapy consultation process. Teachers have indicated that one of the most important factors impacting their perceptions of the consultative process is the amount of time the consultant devoted to them (Athanasiou, Geil, Hazel, \& Copeland, 2002). These authors found that teachers valued emotional support, and having time to express their concerns and share ideas. Although in some cases intervention effectiveness was not obvious, teachers still perceived the consultative process as positive. Caplan's (1970) 'mental health consultation' theories came to the fore when I was reflecting on Cathy's case. Caplan recognised that caregivers experience difficulties which stem not only from lack of skills and knowledge, but also from poor self esteem and loss of professional objectivity. He believed that consultees often needed emotional support as much as they needed answers, and that the consultant's role is often to help them reduce or reframe constricting thoughts about a particular client or issue (a concept he labelled 'theme interference'). Rona's statement "you could say... that her (sic. Cathy's) life is 'useless' but ... it's actually really beautiful" (see p. 207) suggests she may have experienced constricting thoughts, which were beginning shift as a result of using music with Cathy.

\subsubsection{Evaluating the Process}

Building on what I learnt from previous consultations, I focused on helping Rona to recognise the positive aspects of her work and to trust her ability to engage in therapeutic music activities with Cathy. The case study demonstrates emerging statements of selfefficacy such as "I can do that", "I know I can sing”, and "I feel I don't need any extra skills". As a result, Rona's confidence increased to the point where the school principal remarked on the changes he had observed in her. More importantly, she was able to 
accept that "life's not perfect...but as long as you've done your best... that's it really". Moreover, arguably the most important outcome was not related to the simple daily routine strategies that were implemented, but to Rona being 'given permission' to build a musical relationship with Cathy, to sit and gently touch her while listening to music - an experience she perceived as beautiful. Teachers perceived to be the most effective are those who believe that teaching can change children, and have confidence in their own ability to teach difficult students (Landrum and Kauffman,1992, cited in Cartledge \& Johnson, 1996); and those who are comfortable using many different teaching techniques, and readily enjoy and value all of their students (Cartledge \& Johnson, 1996).

Consultation is about changing adult perception, attitude, and behaviour as demonstrated above. When Rona began to use music with Cathy, she noted that the work was sometimes 'probably more for myself' and made things 'more interesting for both of us'. New skills, insights and experiences can be generalised, and can benefit other students in consultees' care (Conoley \& Conoley, 1992; Dettmer et al., 2005). Rona's experiences would likely have a 'ripple effect' with other students benefiting from her newfound confidence.

\subsubsection{Other Possibilities}

Consultants need to vary their interaction styles according to the circumstances and situation they are managing (Dettmer et al., 2005). Successful consultants understand the internal motivational states of consultees (mental health consultation); are concerned with ways in which environmental conditions affect behaviours and accomplishments (behavioural consultation); interact well, and can facilitate positive interaction between others (process consultation), (Conoley \& Conoley, 1992). Although the social learning model still seemed an appropriate frame to use, it appeared that different cases would prompt me to look to the wider literature, and that I would be able to draw helpful concepts from various models. Consultation processes are affected by context and there is therefore no 'best way' to consult (Dettmer et al., 2005; Dougherty, 2005).

Dettmer et al. (2005) write that the various theoretical, problem-based and blended consultation practices that exist, have created a tangle of philosophy and terminology that 
can be problematic for educators. They recognise that school consultants often initiate integrated models. They therefore recommend informal discussions at the planning stage to determine which type of service is best and why, who will be involved, what is expected to occur, where, and for how long, and how plans will be implemented and evaluated. Once these parameters have been set, planners can consider ways in which 'systems', 'perspectives', 'approaches', 'prototypes', 'modes', and 'models' can be structured to create the most appropriate method for the context (ibid).

As noted earlier, the 'system' can include the school, family, other specialist services such as GSE, and community agencies. Consultations can be undertaken from a 'purchase perspective' (buying particular knowledge or resources), 'doctor-patient perspective' (diagnosis or advice-giving), or 'process perspective' (where the consultant helps the consultee perceive, understand and act on the 'problem'). The 'approach' can include formal processes such as workshops, IEP meetings, discussions in the staff or classroom, or 'on the run' in corridors, and so on. 'Prototypes', or patterns of practice, such as the 'mental health', and 'behavioural' consultations described earlier, emerge. Behavioural consultation based on social learning theory is probably most familiar to educators and therefore most easily introduced into schools (Dettmer et al., 2005). 'Modes' for the delivery of school consultation include direct service delivery to clients (students), or indirect consultation to consultees (team members). A 'model' of consultation provides an example that others can study to adapt or replicate.

To explain the concept of a model, Dettmer et al. use the basic triadic model, which involves the consultant working with the consultee to improve the wellbeing of the client. However, these authors caution that it is important to include role description when examining models because, for example, it would be possible at times for the music therapist to become the consultee if they sought information or expertise from the teacher. Collaborative consultation models are triadic, but the communication between consultant and consultee is not hierarchical, a sense of parity exists, and both parties work directly with the student. 


\subsubsection{Ethical Issues in Consultation}

When the consultation elements outlined above (Dettmer et al., 2005) are put together in flexible ways, multiple variations regarding roles and relationships in consultation practice emerge. Individual teams therefore need to consider the ethical issues that might arise in each consultation situation, and how they might be managed. For example, whether a consultant is considered to be 'internal' or 'external' to the school is important in terms of confidentiality. If a school is paying for the consultation, can the principal expect to receive information about the consultee's performance? Music therapists already encounter similar issues when providing professional supervision to colleagues and know that boundaries need to be clearly outlined prior to the commencement of the work. When the consultant is internal, issues such as status and role differences, the existence of hierarchical relationships, the possibility that consultees might be expected to participate, and to accept advice, can be openly acknowledged (Caplan, Caplan, \& Erchul, 1994). However, collaborators cannot automatically assume consultant/consultee confidentiality given the pragmatic need to share information within the school setting.

Consultants working with teams who support students who have special education needs are working with sensitive information relating to students and adults. If team members are clearly identified at the beginning of the consultation, they can agree to keep information confidential within that team. On the other hand, it is clear that in some cases it would be more productive to keep information regarding adult coping skills confidential between consultant and consultee. In Cathy's case it was important to explain to Rona that the focus of support, and thus the reported outcomes, had moved from student to adult.

Consultation is complex, and there is a lack of professional research on ethics in consultation (Harrison, 2004). While the law represents the minimum standard of behaviour that society will accept, codes of ethics provide boundaries for what is deemed to be acceptable professional behaviour, and represent 'ideal' circumstances. Moreover,

all codes of ethics need to be viewed within cultural frameworks, and consultants may need to abide by several ethical codes. The Code of Ethics for Music Therapists in New Zealand (New Zealand Society for Music Therapy, 2006) expresses music therapists' 
core values and beliefs. It outlines ethical principles to guide the responsible practice of music therapy in this country, and aims to give due protection to the rights and interests of both clients and therapists. The term 'client' is broadened to include research subjects, and/or the legal guardian, parent, or representatives of the client. Music therapists acknowledge responsibilities to colleagues, employing bodies and the wider community but, quite naturally given the innovative nature of the service, have not yet considered the complex ethical issues associated with collaborative consultation. With this in mind, as I undertake this work I am reflecting on both my professional and research codes of ethics to determine whether my practice is ethically sound, and considering whether my actions would seem appropriate to 'ordinary reasonable people', that is those who are reasonable, prudent, careful, informed, and maintain standards in their work (Harrison, 2004).

Cultural variables can adversely affect communication in consultation (Dougherty, 2005). Cathy's mother attended part of the group music session at school, and the team meeting where video of Cathy's individual music session was viewed and discussed, but her input was comparatively limited. Parental involvement had been encouraged via the information and consent process, direct invitation from the teacher, and later the music therapist consultant, at school. I offered a home visit and/or meeting at school, at any time during the week of the consultation, and encouraged Cathy's parents to make an approach. Nevertheless, I wondered if this Māori family might have found it easier to be involved if they had routinely received a visit from the music therapist consultant, to their home, at the beginning of the consultation week to korero (talk) about the work. To a certain extent Rona and my research supervisor (who is Māori) allayed my concern that I needed to pay more attention to ethnic differences in this case. After all, her mum and dad had work commitments too. Consultants need to take into account individual differences while resisting overemphasis on cultural variables, and acknowledging that culture goes beyond characteristics such as race and language (Dougherty, 2005).

The concept of applying cultural sensitivity simultaneously at student, consultee, and systems levels is complex. It involves coming to understand and being sensitive to the unique experiences of all parties, negotiating and using multiple perspectives productively when problem-solving (Wesley \& Buysse, 2006). It seemed important to 
include waiata (Māori songs) in Cathy’s programme, and I suggested several. Rona preferred to use English songs, and it seemed as if this was simply 'easier' for her. If there were more time, this would be something we could work on together. But in the short term, it seemed more important for Rona to feel comfortable with the choice of materials, to ensure her musical interactions with Cathy were sustained.

\subsubsection{Summary}

Exploring the social learning model of consultation in the context of music therapy school consultation enabled some helpful links to be made, suggesting the model does provide a useful frame for the work. However, consultation is complex. The management of resistance and ethical dilemmas have been highlighted in this cycle of learning. A broad understanding of a range of models has given me a better appreciation for the model I have chosen to use. Nevertheless, consultants need to vary their practice according to the consultation circumstances. Applying the model to another cycle of learning will reinforce or weaken my premise that the social learning model of consultation is an appropriate frame for my work. 


\subsection{LIAM}

\subsubsection{Background}

Liam was six years old, had been diagnosed with severe Autism, and Attention Deficit Hyperactivity Disorder, and had very high needs. He was nonverbal. Liam was identified as a New Zealander.

\subsubsection{Introduction to the Team}

The referral was made by a MoE SE psychologist on behalf of a visiting SLT. By the time of the consultation there had been several staff changes with MoE, and some who had expressed interest in participating in the study had moved on. Nevertheless, the principal (Denis), classroom teacher (Joan), .2 ORRS teacher (Molly), two teacher's aides (Kerry and Jill) and Liam's parents, (Carol and Jason) were keen to go ahead with the project, with support from the MoE SEA (Jane) and his current SLT (Lisa). Molly and Kerry requested to be consultees.

\subsubsection{Ethical Issues with Regard to Participation}

It was necessary to invite the 'new' participants to be involved, or to take new roles, in the research project after it had been set up. My explanation that others had agreed to be involved, but were now unavailable, had the potential to be coercive. I had to consider the potential for participants either to be genuinely enthusiastic about being involved, or to feel it was their duty to fill in. I also had an ethical responsibility to parents and staff already recruited, to continue with the work if possible.

\subsubsection{The Team's Expectations of Music Therapy}

Molly and Kerry initially claimed to have no preconceptions of what the music therapy consultation might involve. Nevertheless, Kerry had learnt of another child with ASD who 'could sing every word in a song but couldn't talk', and was 'hoping Liam might 
pick up something like that'. Molly took this idea a little further, noting that Liam's vocalisations had become more sophisticated, and wondering whether music therapy might contribute to the ultimate aim that he would speak.

I think the family were told a while ago by a speech therapist, who is not here any more, that he would never speak, which could have been quite distressing for them because he was only five at the time - and he will speak ... somewhere down the track. (Molly)

I was initially concerned about the team's seemingly high emphasis on Liam's ability to acquire speech, preferring to focus on musical interaction to develop his pre-verbal communication skills, which also underpin speech. However, although the team's long term goals for Liam seemed ambitious and optimistic, Kerry and Molly believed that music would be intrinsically motivating for Liam, and hoped he would 'really take to music', and might 'open up a wee bit'. Their immediate wishes for him were therefore simple, and expectations realistic.

It would just be nice for him to be comfortable and happy and motivated probably with our help. ...We've been quite frustrated up until now with how little we've been able to do for him because of the mood swings and the behaviour ... (but) you can just see the potential - he will benefit from any input, let alone musical input. (Molly)

\subsubsection{Programme and IEP Goals}

Priority aims for Liam were to improve his interpersonal skills, particularly attending behaviour and turn-taking with adults, and to help him to understand and manage school routines. Liam experienced emotional dysregulation. His behaviour was therefore unpredictable, and staff found it difficult to introduce and maintain structure in his day. This in turn made it difficult for them to use specific objects and/or pictures linked with different activities, to help him understand routines.

Further, there were few known motivators for Liam. He required very high levels of external reinforcement, including small pieces of food items as well as verbal praise and reinforcement, to help him to attend to educators. Despite their wish that Liam would 
develop speech staff accepted his immediate need was to be more motivated to attempt interaction, and to develop positive strategies for self expression. They were aware that his seemingly aggressive behaviours were an attempt to communicate.

He (has been) particularly unhappy and quite distressed...It was his lack of being able to communicate which probably was so frustrating for him. We would get down on our hands and knees - I put my arms around him one day and said 'it's alright, it's alright' and that's when I got bitten. He didn't bite me because he was cross with me; it was because he was frustrated. He would be pushing us away, but pulling us at the same time as if he was saying 'can't you understand what it is I want you to do?' (Molly)

Team members were using the Social Communication, Emotional Regulation, and Transactional Support (SCERTS) (Prizant et al., 2006), as their curriculum model for Liam.

\section{SCERTS Model of Assessment}

The SCERTS has a developmental, social-pragmatic focus, and has been promoted for children who have Autism Spectrum Disorder (ASD) (Prizant et al., 2006). It recognises relationships between communication, social-emotional development, and emotional regulation, and the need to understand the intention and function of a child's behaviour. It draws on person-centred philosophies, and from recent understandings of the learning styles of people who have ASD, as well as family-centred philosophies which advocate for supporting the child's family and education community.

The main goal of the SCERTS model is to facilitate children's successful participation in developmentally appropriate activities across settings with family members, adult partners, and peers. Transactional supports, such as environmental accommodations (e.g. reducing auditory stimuli), implemented across settings by all who are involved with the child, are considered crucial. In the SCERTS model, functional and developmentally appropriate goals, objectives, and strategies, address family priorities. They build on children's capacity to initiate communication with a pre-symbolic or symbolic communication system, and their abilities to develop skills of attention, and to control 
arousal and emotional responses.

Music has the potential to engage and motivate a child to initiate musical communication, and to affect emotions. The music therapy session provides an exceptional setting for observing a child's ability to communicate and to self-regulate, especially when they are nonverbal, and the music therapist is a particularly valuable asset to the SCERTS team (Walworth, 2007).

\subsubsection{The Use of Music before my Visit}

Kerry had learnt the piano to grade one many years ago, and felt comfortable singing. She had worked with Liam at kindergarten where she experienced a lot of singing, and had been able learn a good repertoire of children's songs. Kerry and Molly both sang spontaneously with Liam and were able to make up or adapt songs to suit the activity they were engaged in. They had noticed that Liam attended more readily, and responded more positively, when they sang to him.

(I've always done music, but) subconsciously probably. I hadn't actually realised that I was doing it as much as I was until we had books, or we've been to courses, where they've talked about how important it is and I thought "oh, we're doing it anyway”. It's something I don't find difficult to do. (Molly)

We generally (sing) when we go to the toilet or wander round the school... When you want him to wash and dry his hands, just sort of put it into a tune. Sometimes you sing nursery rhymes when you're changing him......If kids are struggling to do something, you just naturally start singing it and... it eases, it calms them in some ways. (Kerry)

On the other hand, although they were comfortable working with Liam musically in private, Molly and Kerry both seemed to have a level of self consciousness that may have been placing limitations on what they did at other times. Kerry expressed relief that the invitation to be involved in the music therapy project stressed that the participants did not have to be trained musicians and, despite her apparent natural musicality and confidence, and genuine interest in being involved in the project, she 'hoped she wouldn't have to 
sing in front of the class!' Molly too laughed, slightly embarrassed, when she said:

I talk a lot and sing a lot... (slightly embarrassed laugh)...it's not particularly wonderful but it's the way he looks - he likes it. (Molly)

I enjoy music - (but I'm) not overly confident - that's why this will be good. There will be someone giving me directions - not me thinking 'what do I do now?' You'll be giving us directions about what to do with Liam and I'm quite happy to do that. (Molly)

Kerry and Molly used music spontaneously and naturally, but rarely anticipated musical responses from Liam.

We don't really do any music sessions with him or anything like that... ... I think probably when I am working with him I am singing to him - I don't actually expect a particular response except for the fact that what I am saying to him might be easier for him to listen to. It's a bit more interesting with the music. But what I call a real musical experience we haven't done. (Molly)

\subsubsection{Assessment Activities}

\section{Ecological Assessment}

Without exception, team members used a warm, gentle, and positive approach with Liam. School staff were supportive of each other and shared tasks willingly. Joan was familiar with autism, and felt very comfortable having Liam in her classroom. She regretted having to allocate teacher's aides to work with him, as she would have liked to spend more time with him herself. At the time of the consultation Liam was able to attend school during mornings only, because funding constraints meant there was not enough staff to cover the constant one-to-one support he required. Further, Liam and his team had been allocated two smaller rooms alongside his classroom, referred to as the 'work room' and the 'calm room', and he spent considerable time in these rooms away from his peers.

The 'work room', sometimes also referred to as the 'quiet room', was set up for withdrawal teaching. Staff believed Liam needed a quiet space away from other children, 
to maximise his potential to learn. A range of simple percussion instruments was left on a semi-permanent basis in the work room. Molly used them to help Liam 'learn about cause and effect', and Kerry and Molly both used recorded music in quiet settings hoping to reduce his auditory sensitivity. In contrast, the calm room was bland and contained no pictures or equipment. Plans were in place to provide a bed and a duvet, because wrapping Liam up could be a way to help his sensory regulation. Conversations with staff indicated that the room had been vital as 'a place to take him when he lost it'.

Liam was reportedly frequently emotionally dysregulated, and was anxious, frustrated, and prone to excessive emotional outbursts, especially when he was experiencing sensory overload, and/or having difficulty managing a task. However, in general, during the week of my visit he appeared to be a happy boy who enjoyed being with the people who supported him. When I met him, he was sitting easily on a teacher's aide's knee, turning frequently to reach for and hug her. He looked at me, appearing curious, vocalising, and his intonation sounded like 'talking'. He laughed several times as I smiled at him, and he rocked and flapped briefly. Liam startled as someone slapped a book down, made a lot of sound when the room was noisy, and put his fingers in his ears. He also held, flicked, and mouthed, a coloured 'gosh' ball, and intermittently mouthed his left hand index finger and thumb, his whole hand, or bit his knuckle. He placed his hands over his eyes, and staff thought he might be 'hiding'. However, he also squinted at times and was possibly visually over stimulated.

Staff advised in the two weeks since term began, including the one when I was present, Liam had been remarkably settled at school. He was offering increased eye contact, and had begun to touch staff gently on the face. He took them by the hand to lead them somewhere or to ask for help, and accepted a hand when it was offered to him. However, although he was having a 'good week' he occasionally grabbed clothes, pulled hair, and bit people, seemingly without malice.

Workmen were repairing the road outside Liam's classroom. The noise they created with concrete drills and other equipment was potentially extremely disturbing. However, while he demonstrated minor distress at times, Liam was able to manage by using ear muffs to reduce the noise. Staff also introduced cordless headphones for the first time. We 
anticipated that if he were able to tolerate them, music could be used to mask unpleasant noise, as well as to calm Liam, thus increasing opportunities for learning.

Staff introduced sensory play (e.g. bubbles; action songs; water play at the sink), or helped him work at his desk, while other students were engaged in group activity. They attempted to engage him in handwriting with a variety of sensory tools e.g. fat crayons on corrugated card or whiteboard markers on laminated page; reading from picture books; doing puzzles; language exercises using photographs or objects; counting with an abacus; listening to music; and massage. Liam demonstrated that he understood simple instructions by, for example, immediately removing an object from his mouth when told 'not for eating'. He responded positively when told 'No. Sit down', and resumed his activity when told 'Not finished'. Much of his time was spent in toilet routines, and he was also encouraged to undertake purposeful activity away from the classroom, for example to go to the library, or to fetch musical instruments from the hall.

Throughout each day, team members interacted musically with Liam. They spun him on a swivel chair, chanting. They sang adapted or improvised songs to help him manage his toilet routine, while he was being massaged, during reading activities, and to help him with language and/or body awareness, e.g. singing about his nose. He was frequently jiggled, patted, and supported hand-over-hand to clap, in rhythmic ways. I helped staff to pause to indicate a response was expected, and Liam began to take his turn. While playing 'Row Row Row the Boat' with Liam, Kerry paused at the end of each verse to ask if he would like more. Liam requested "more" by using 1) a vocal approximation of "Row Row Boat" 2) the action associated with the activity i.e. rocking, and 3) patting her hand to prompt her to begin again.

Joan engaged students in rhythmic chants at various times while Liam was present. She encouraged them to vary dynamics but was careful to avoid very loud or shouting levels; sensitive to Liam's potential to respond negatively. He was engaged by these rhythmic games, and it seemed likely that group music would provide opportunities for inclusion and social interaction.

The other day we did 'Ring-a-Ring-a-Rosie' Liam and me, just by ourselves, then one of the other children joined in and we did it again and again and again ... he 
Chant was also frequently used to keep him on task and/or to help him move, e.g. when walking to the classroom. Team members were already imitating Liam's sounds, particular his vocal offerings. I noticed that he was singing; his vocalisations were musical - melodic and rhythmic motifs were emerging. Liam was particularly vocal in the toilet, or when he was wearing ear muffs, probably responding to the timbre of an environment that 'echoes'.

Liam responded relatively well to visual cues, and seemed particularly aware of the importance of following staff and other children during transitions. Further, he seemed to be listening to, and perhaps understanding, adult conversation at times. When staff members were discussing the men working on the road outside, Liam was referencing each of the speakers, and the men outside, in turn.

Liam was frequently an observer in his classroom. He enjoyed watching the other students play, but also spent long periods of time moving around the room, looking at pictures on the wall. During the short periods he was with the group (e.g. ten minutes of mat time when he arrived at school) he needed to be seated on a chair, while the other students were on the mat. Although he ate his morning tea outside as did other students, he needed to be separate from them to prevent his over-stimulation. After he had eaten he was given the freedom to wander and interact with his peers, but was required to wear a bright yellow road safety vest so staff could locate him easily.

Liam appeared aware of me being a new person in his environment, observing me with an expression of curiosity at times. He had always taken to new people really well, and was beginning to understand the facial expressions of staff when they were frustrated or upset. Sometimes his expression appeared mischievous, as if he knew a lot about what is going on, and it seemed he was beginning to look for a reaction from those who worked with him. 


\section{Home / school relationships}

Lindsay and Joan had worked hard to ensure that Carol and Jason received all relevant information from school, and that communication between parties was open and honest. However, despite everyone's best efforts, effective communication had not been easy. For example, school staff were concerned they did not receive important information from home regarding changes in Liam's medication and diet. Parents were concerned that the paucity of teacher's aide support restricted Liam's access to school. The risk of him being stood down was distressing for both parties. Liam's parents regularly visited the school to drop him off or pick him up, but it was hard for other team members to talk at those times. The recent process of completing an ORSS application, which necessarily highlighted Liam's difficulties rather than strengths, may have led parents to believe that their child was not valued enough, and to feelings of hopelessness.

\section{Identified Strengths and Needs}

\section{Medical Profile}

Carol reported that Liam's early development was normal, and that he had begun to use single words at age 15 months. Around that time he was given his infant vaccinations, and also contracted Rotavirus, a common paediatric 'stomach bug'. Following these events, his speech became unclear and he eventually stopped talking. His toileting skills also deteriorated, and are still a primary concern for Carol. At age three Liam began to attend kindergarten, and his developmental needs were drawn to the attention of special education services.

Although he picked up colds easily, Carol stated that Liam was generally very well. For several months prior to the assessment his family had trialled the use of Ritalin with him, but during the research period he was no longer taking medication.

\section{Physical Profile}

Liam's gross motor skills were normal, and he was physically confident. He had good 
balance, muscle tone and strength and did not tire easily. However, he was frequently restless and needed to walk, and could become over excited when engaged in movement activities. Liam had immature fine motor skills, and hand dominance had not been established.

\section{Sensory Profile}

Liam had a problematic sensory profile. He was hyposensitive to tactile and vestibular stimulation, and consequently sought movement activities. He enjoyed rough and tumble play. He had a weighted vest, which he wore for approximately two 20 minute spells each day at school, to provide him with deep pressure to calm his nervous system. Liam also experienced a high degree of auditory sensitivity (as described above).

\section{Cognition and Communication Profile}

Liam's recent ORRS application stated that he rarely initiated communication or engaged in reciprocal interaction, and did not cease inappropriate behaviour as a result of a change in a partner's expression. He had begun to use adults as a tool to get basic needs met, but did not initiate other actions such as fetching food when he was hungry. Nevertheless, Molly believed he had a strong awareness of who he could trust, and who trusted him.

Liam had not yet mastered an alternative communication system, and was therefore unable to communicate his basic wants and needs in a functional manner. He had begun to use the Picture Exchange Communication System (PECS) at an emergent level, with staff presenting the pictures to let him know what would happen next. Liam did not find meaning in any table-top tasks, staying for approximately ten seconds only, and was still developing an understanding of object permanence, cause and effect, and same and different. He occasionally looked at photographs, but did not appear to understand that the picture told a story. Liam was able to turn a page with assistance, had begun to use a palmer grip to hold a fat crayon, and to make marks on paper independently. He required hand-over-hand assistance to participate in matching or categorising activities. 


\section{Activities of Daily Living}

Liam was able to finger feed himself, but required supervision while eating, and at other times, because he could not differentiate non-food items. He was fully dependent for toileting, and when he was distressed two staff members were required to help him with toileting routines. He was able to assist an adult to dress or undress him when he was well regulated.

\section{Social Skills Profile}

Liam was able to sit on a chair during mat time for up to 10 minutes, with adult support, in ideal conditions. Although he appeared interested in his classmates, he rarely interacted with peers, and when he did he could be aggressive - biting, grabbing, pinching, and hair pulling. It was difficult for him to play with other children. Nevertheless, some students from his class had begun to make a carefully considered effort to play with him. Adult support was required to facilitate and monitor this interaction.

\section{Emotional Profile}

At the time of the consultation Liam was settled, but had historically been prone to severe often unexplained emotional outbursts. He occasionally self-harmed, and often hurt others. He needed constant supervision to ensure his and his peers' safety.

\section{Assessment in the Music Room}

Liam's music therapy session lasted 25 minutes. I was interested in his motivation and ability to engage in music making.

Liam was keen to enter the music room when he heard me singing and playing the keyboard, and throughout the session was particularly attracted to the keyboard. As I accompanied him, he appeared aware of the shared nature of the music, and was able to engage in moments of reciprocal music making. However, he continued to demonstrate 
various forms of resistance, finding it difficult to tolerate physical touch, and adult direction, for example.

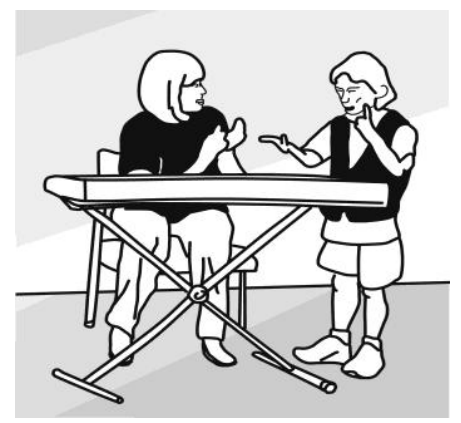

The following description demonstrates the way in which Liam acknowledged that his musical contribution was important. He has resumed playing keyboard after a pause, while looking up at me and smiling "almost as if he is saying 'there you are then, I'm playing,'”.

I continued improvising a greeting song to accompany Liam's playing, hoping to develop a shared keyboard improvisation. His solid and rapid playing slowed, and became gentler. When he stopped playing he kept his hand on the keys and turned slowly to look at me, seeming curious, before resuming his earlier style of playing. I accompanied by clapping to his beat, and singing nonsense sounds such as "dee dee dee deedle dee dee dee". Liam looked at me while he continued to play, but stopped suddenly and began to twiddle his fingers. My singing immediately became slower and softer. Liam rubbed his hand horizontally on the keys seemingly enjoying the roughness. Again he looked at me, and resumed playing briefly at the bottom end as if to 'prompt' me to increase speed and dynamics.

Suddenly though, he flapped, turned away from me and the keyboard, and began to flick rapidly on the arm of a chair. I did not match his tempo this time, but slowed to an unfinished cadence as if to ask "have you finished?" Liam came back to the keyboard, resumed his playing, and his beating was more sustained. He looked relaxed and gave me eye contact, seemingly aware of our shared experience. Our playing together continued, with Liam stopping only briefly to 'check out' what I might do in response. For a brief period, he played steadily and purposefully, referencing me, aware of our shared music making.

Although he was able to make brief musical connections, Liam found it difficult to remain on task, and wandered the room frequently. He demonstrated a relatively high degree of resistiveness, preferring solitary activity to sharing. On the other hand, his 
communication sometimes seemed to indicate that he 'couldn't be bothered' with me if I didn't understand his wishes. The session involved constant negotiation between us, a stream of invitations, acceptances, quitting, and refusals.

I picked up his rapid beat when it was established, and introduced a rhythmic pattern against it. Liam looked at me and his beat immediately slowed and was lost. I continued to tap lightly using the previously established tempo and rhythm, stopped and held the drum out to him. He took this as a cue to recommence at the piano but stopped again as I tried to join him with my drum. I held it out to him, and he resumed his piano playing. I now had a real sense of his resistance to parallel or reciprocal play!

On the other hand, we made real attempts to share music. Liam was spontaneously and intuitively caught up momentarily in the music, which resulted in fleeting periods of togetherness and musical turn taking.

We played together, with Liam holding a relatively steady beat at the piano while I introduced simple rhythm on the drum. Fleetingly, as he stopped and I continued, he resumed and I stopped, he stopped and I resumed, there is turn taking. When we both finished, Liam made a tiny vocalisation "a ha!", intoning musically, and I imitated...

Liam was distracted at times by his need for tactile sensory stimulation, and seemed to enjoy the instruments for their feel as much as their sound. The structure of music helped him to actively engage in music making.

I began to improvise in a more structured way singing "It's Liam's Turn to Play the Drum", while gently and steadily beating. At the end of the first phrase I paused, waiting for Liam to stop flicking. As he reached out to rub the drum I began the second phrase. After I paused a second time, Liam began to beat the drum strongly. This time he stopped at the end of the phrase, waited for my brief pause, and played the fourth phrase in different 'style', flicking at the edge of the instrument.

When he was interested in an instrument, Liam was willing to request and/or accept help from me. 
I encouraged him to hold the string as I tried to share his triangle, but Liam was more interested in placing the beater "through the hole!" I engaged playfully with him as he dangled the instrument by the string, and took the beater to strike the triangle. Responding to his interest, I persisted in helping him to manage the instrument either on his own or with me, while framing the activity with an improvised song, "The triangle". Liam relaxed, and together we sounded the triangle at the beginning of each bar.
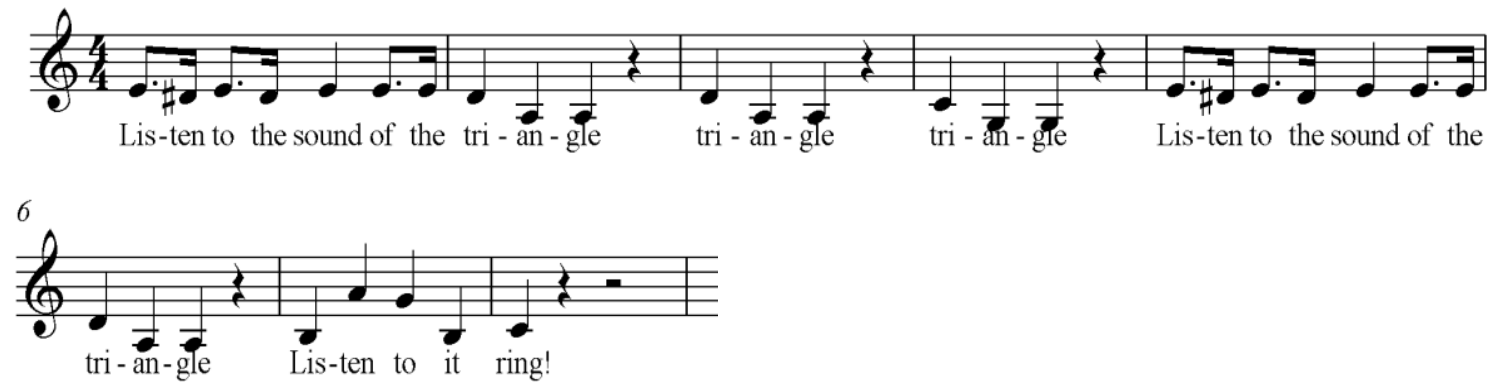

Other noteworthy interactions occurred in the latter part of the session. Liam approached me as I waited kneeling on the ground, quite a distance from him, and raised both his hands for a high five. He remained with me, patting my hands in a body percussion game for several seconds. In a similar exchange, he took a clave and tapped it against the one I was holding. He also 'discovered' the cabassa.

At a point where it seemed he had 'had enough' of the music session, Liam suddenly took the cabassa from the floor, ran his hands over it briefly, and took it with him as he sat on his chair. It seemed that he liked the feel of the beads but was unaware of its potential for sound. However, he allowed hand-over-hand support, before attempting to play independently. He asked me for help twice, reaching toward me to indicate his request, before eventually mastering the task. During this three minute interaction, I was improvising a song, timing it carefully so each sound of the cabassa fell on the beat.

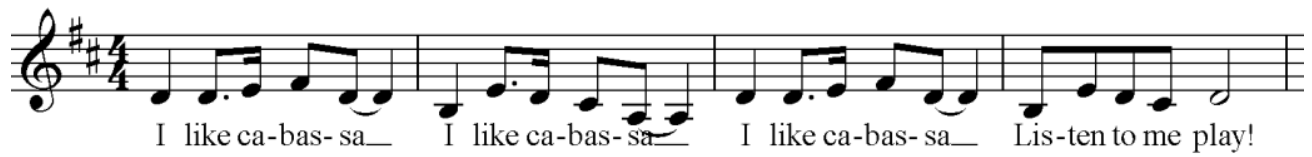

Although he wandered the room frequently, approached Kerry (who was operating the camera) twice, and made several attempts to leave, Liam also went back to the piano a 
number of times, and played briefly each time. On one of these occasions the quality of his music changed considerably.

As he began by 'banging' at the top end of the piano I joined him, and he played gently, pushing the keys rather than striking them, and attempting to isolate his fingers instead of playing clusters. As he began to walk away I played a glissando. He hesitated, smiled, and then left.

\section{Summary of Individual Assessment}

It was necessary to decide 'in the moment' what needed to be done to ensure that experiences were positive and rewarding for Liam, and yet elicited the most useful information about his presentation in a music setting. This involved building a therapeutic relationship by 1) creating musical connections, 2) allowing him to 'just be' for brief periods, 3) following his lead and using his interests and/or behaviour to engage him and develop his activity further and 4) challenging him to attempt new activities or new levels of participation.

Team members were having difficulty challenging Liam to attempt tasks because of his historical volatility. The music therapy assessment session was helpful in demonstrating that in a music session Liam could feel safe and supported enough to reach optimal performance as long as he was not under constant pressure to perform.

\section{Team Observations and Discussion of the Assessment Session}

While watching the video, team members observed Liam's excitement and enjoyment in the activities. They noted the spontaneous eye contact he offered, and his ability to cooperate. The speech therapist was particularly excited by the communicative aspects of music therapy interactions.

I just think it's really interesting the way that communication is really built into everything within the music therapy session. Like the nice face to face interaction, all that imitation ... he was taking turns, and just the nice repetitions, the really simple language, lots of simple sounds - there was lots there ...It's just interesting to see how easily or how well he engaged in it all ...he was showing so much 
Team members noted that Liam had a particular interest in piano and drums. They agreed that he had demonstrated an understanding of object permanence, and talked about developing cognitive skills they had seen in other settings. They noticed the importance of providing physical boundaries to help contain Liam, and observed me being consistent, using musical techniques to prompt Liam, allowing time for him to respond and make choices, and giving space for him to walk away. They recognised that their own interactions with him were cautious, and acknowledged the need to build on his abilities by expecting a response. They noticed that the music kept Liam calm and on task, and agreed songs might help him to remain emotionally regulated, or to learn routines. They talked about his favourite recorded music, and possibilities for it to be used to mask environmental sounds. They considered using spontaneous music, as well as planned sessions, in future, and requested recordings of some of the songs that were used during the assessment session so they might continue using them.

(We could be) making up little ditties to fit those songs appropriate to what the activity is... ... (We'll use) a bit of everything really. The spontaneity is quite nice because you can do it any time... you don't have to be thinking 'in five minutes I have to do this' but a structured music session would be very good for him, even if it was just 15 minutes. (Molly)

Understandably, team members were cautious about 1) the type and volume of noise Liam was exposed to, and 2) the level of demand they were willing to place on him. I suggested their singing was uniformly quiet and often 'unconvincing'. They recognised they were using music predominantly to keep Liam distracted, calm, entertained, or to gain and maintain his attention. Music was also used as a 'teaching' tool, a carrier of language and academic concepts. However, the team had not considered that it might be an activity he could participate in.

\subsubsection{Agreed Planned Music Experiences}

Team members wanted music activities to promote interaction, particularly those they could do one-to-one with Liam. They needed reassurance that Liam would be able to 
manage increased auditory stimulation, before they would feel comfortable including him in group music situations. One-to-one musical interaction would give opportunities for working on his primary goal of developing and rehearsing interpersonal communication, and allow time for mutual trust to develop.

We therefore explored ways of sharing music with Liam. Staff would develop higher expectations that he would be willing and able to actively engage, and offer specific responses, in a musical interaction. Molly and Kerry wanted to introduce ten-minute structured music sessions throughout the day, using simple repetitive material presented in consistent ways. They would also listen to his sounds and imitate them, and watch his movements and match them with a musical response. They would leave space for his response, and expect him to respond in his own way, thereby setting up possibilities for reciprocal interaction based on Liam's 'musical offerings'. We discussed how they might use hand-over-hand support occasionally but sparingly, to prompt him to begin a musical activity (e.g. co-active clapping). They might put instruments 'in his way', so he strikes them accidentally at first, anticipating that he will repeat the action if he likes the sound.

They would use the structure of simple songs to help Liam understand when an activity begins and ends, introducing 'stop' at the end of the song when they wanted him to have the option of requesting 'more', and introducing 'finished' when the activity needed to close. They were encouraged to try to pre-empt his need to finish by stopping the activity while he was still engaged, so he had successful experiences. They might also vary the tempo when taking a turn e.g. singing faster to help Liam to wait successfully for 'his turn' to come round. They would use their name and Liam's name in song, to help him know whose turn it currently was, or would be next.

Team members would use pre-composed, adapted, and improvised songs, as well as chants and body percussion, to engage Liam, and as a 'carrier' of information he needs to learn. They were encouraged to use their voices demonstratively, sometimes exaggerating intonation e.g. "big sigh", and to sing clearly and with confidence. However, they also needed to monitor the volume of their voices and to avoid sudden loud sounds which might be over stimulating for Liam. They would continue to 'play musically' with him e.g. to play pat-a-cake on his shoes. They might introduce structured body percussion 
games with very simple repeating patterns, e.g. pat, pat, clap..., to help him to remember and anticipate a sequenced action. They would sing to accompany his physical activities, using pitch to reinforce directionality e.g. "down, down, down, down, down" in a clear descending scale, and rhythm to match his movements. They would continue singing or using recorded music during gentle activities, such as massage. They would also take opportunities to play the keyboard or piano with Liam, and help him to use black notes if he was comfortable to do so thus enabling less dissonant music making. In addition, musically adapted social stories might be developed collaboratively with Lisa (SLT), if and when they were required during the follow up period.

After noting how contained Liam was during the music session, team members were interested in how they might help him to feel safe and yet still challenge him to try new things. We described a flexible approach that could be used during music sessions to contain him, yet encourage his continuing development (see Figure 6, p. 237). The image helped us understand the importance of 'holding him', having expectations, but also allowing for the possibility he would not always manage to meet expectations, and to give him some leeway if he becomes over-stimulated or dysregulated. This approach was presumed to fit well with the SCERTS philosophy.

The diagram suggests a boundaried but flexible approach would be used for each music activity, but this kind of flexible structure would also be applied to the music session as a whole. For example, the expectation might be that Liam would come to music after reading (this could be part of his visual schedule). Staff might anticipate that he would be able to participate in five or six short but specific activities. However, as he enters the room they might see that he might only manage Hello and Goodbye songs, and allow him to choose his own music activities, perhaps as few as one (from instruments, or other visuals), between these two songs. On the other hand, they might feel he is able to cope with all the things they have planned, and could therefore give him a full visual schedule outlining their expectations for him. 
A FLEXIBLE APPROACH TO 'CONTAINING' - The

therapist/teacher/teacher's aide holds one end of the 'elastic' experience and the student is able to stretch and relax while still engaged with the activity.

The line represents the 'activity' or 'therapeutic experience'. There are many steps to 'completing' the activity - while the student is engaged in some way he is working towards successful 'completion'. Put another way, the therapist's expectations of what the student can achieve will vary according to how he is managing at any given time.

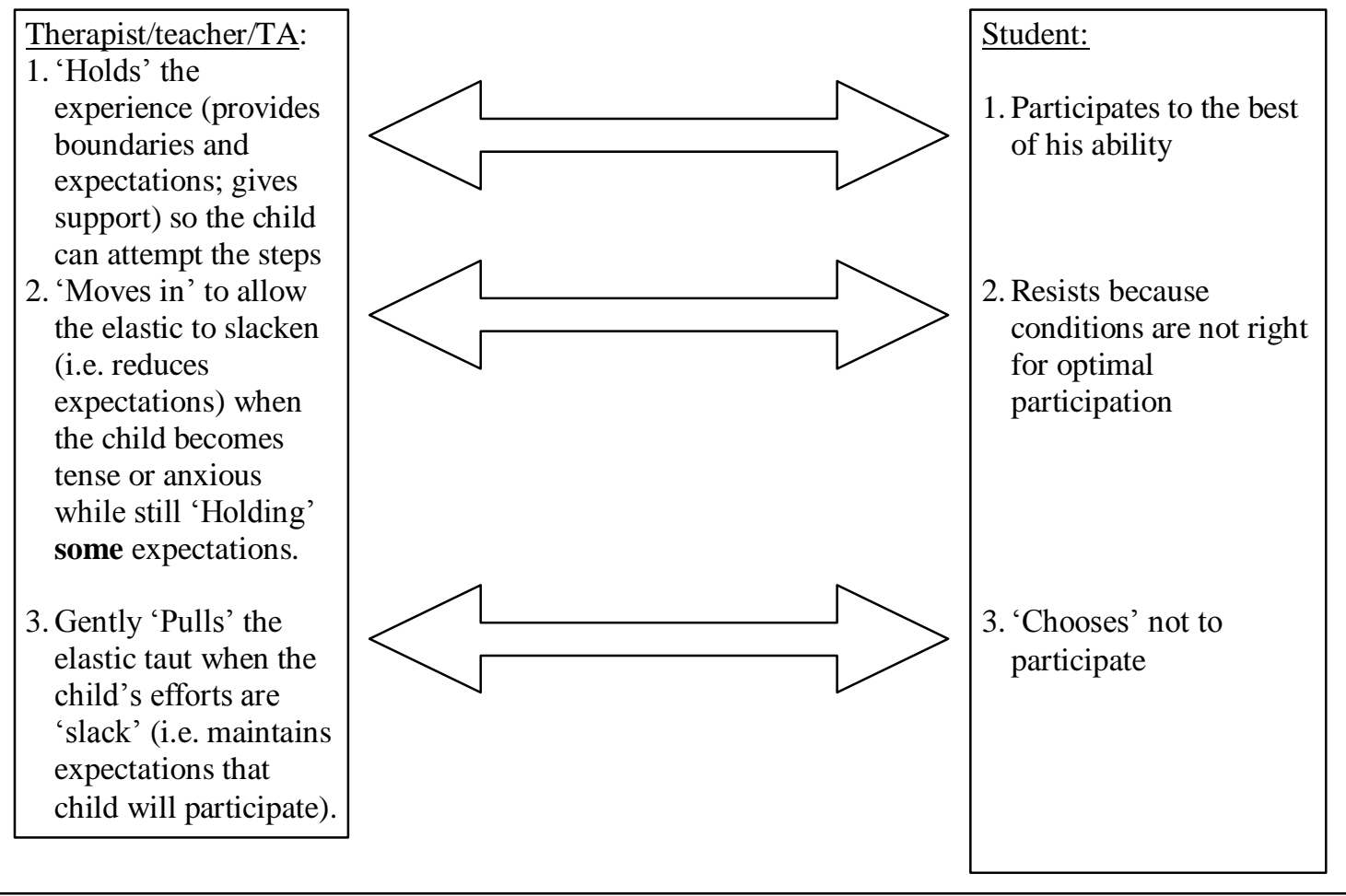

Figure 6: A Flexible Approach

While some flexibility is important for students who have variable responses to sensory stimulation and difficulties with emotional regulation, predictability and consistency are also important to help children who have ASD to understand, anticipate, and tolerate environment demands. Walsh Stewart (2002) also describes giving opportunities for choice and creativity within clearly structured programmes for children who have ASD, similar to the model I outlined for Liam's team. She combined the techniques of pychodynamic music therapy and the structure of the Division TEACCH programme, to create "a structured and a flexible environment for the children" (p.169). In addition to improvised music making and the opportunities that afforded for the development of an 
affective relationship, attention was paid to consistency of time, place, duration and content of sessions.

A programme of nine simple songs, to support instrument and movement activities, lasting a total of ten minutes, was recorded on $\mathrm{CD}$ for staff to refer to. The songs were examples only, and team members were encouraged to use other similar material adapted from familiar songs for example - as long as they were consistent in what they introduced. It was intended that the songs be learnt from the CD and sung live - the recording was not for Liam. Staff were aware of the importance of singing unaccompanied so they could be flexible in the way they presented and used the songs, and respond to Liam's communications.

\subsubsection{Introducing the Strategies}

\section{Student Response}

Observing Liam's positive responses to music motivated Molly and Kerry to continue the work. They were optimistic that they could build on what they had learnt from the music therapy consultation, despite their concerns that he would not always be as emotionally regulated as he had been during the week of my visit.

I think once we're comfortable with the music and doing that regularly and getting the responses - it does make a difference that he's feeling better in himself which makes us feel better about ourselves too, which I think he knows... ... It's definitely going to help him and it's going to help us to help him. (Molly)

\section{Attitude to Using Music, and Managing in Future - Increased confidence}

Molly expressed a genuine willingness to persevere with attempts to engage Liam in active music making. The music would give him pleasure, while improving his interpersonal pre-verbal communication skills.

I know I am confident to do those sessions at least once a day - or more depending on who's coming and going and what's happening... And I will get more confident singing the wee songs and not looking at the paper and thinking 
“what am I doing next?” That will all just quietly fit into place. (Molly)

Kerry too was confident moving forward in music with Liam. She realised a musical way of being comes 'naturally' to her. But it was hard sometimes for her to describe what was happening in her interactions with him and, although she was keen to learn new and specific strategies to use with Liam, I was concerned that overtly 'analysing' their music making might impact on her natural way of being with him.

\section{New Strategies - Allowing Time and Space}

Kerry had been surprised at Liam's auditory tolerance during music sessions, and was going to carefully increase the volume of her sound making interactions with him, and monitor his responses. She was also pleased to have learnt how some of the percussion instruments such as the Cabassa might be played, and used with Liam. Molly thought learning to engage Liam sensitively in music making gave her, and others, strategies that would extend beyond the music room and music activities.

(I learnt) not to anticipate that he's going to do something and do it for him. I think that we tend probably not to use as many teaching moments as are available because we're directing him off to do something else. And I think we need to learn to stop - if he runs off into the toilet and he starts to turn the tap on ... to let him play with the water - let him do those things and sing a little song to him...He was responding to that... ... (We need to) take more of a lead from what he is choosing to do - not what we are choosing for him to do... ... We assumed up until now that he needs us to do as much for him as possible - but he doesn't. I think that will reflect in other areas of the school - not just the music. (Molly)

\section{Modelling and Direct Instruction in the use of Music Strategies}

Molly enjoyed co-working with me, and suggested that receiving direct instruction, as well as having the work modelled for her, was particularly helpful. She was delighted with Liam's responses to the short music sessions she had begun facilitating.

I think I wasn't sure to be honest what to expect; what would come out of it - that quarter hour that we spent with Liam in our wee room just now is the icing on the cake really! It's taken a week for all that to sink in and to read some more 
information and to watch Liam, and then that session in there today - that was lovely. (Molly)

\section{Time spent with the Team}

Although working closely with team members for a full week had the potential to be overwhelming and invasive, Liam's team did not view the experience this way. They valued time to process information, rehearse strategies, and clarify specific issues.

(Your presence was) absolutely very unobtrusive - no fears of being watched for 'what is she doing' or 'she shouldn't be doing that' or whatever. No, no feelings like that whatsoever. It's been very comfortable having you around, and I can't believe that it's been a whole week... ...It's been beneficial because it's been ongoing, we've thought about it, we've contributed, we've watched you, you've watched him, you've watched us - it's been an ongoing thing all week. And probably it's been quite tiring for everybody - you included - but much better that than 'I'll see you in a month's time and we'll go through something else', because I don't think we would feel as confident about participating in this programme with him if it had just been a 'one-off' every now and then. (Molly)

It's been good to have you here 'cause sometimes people come and they're here for a day and then you think of a question after they've gone ... whereas this time, we've been able to see you the next day and ask you different things. ...I've needed you there to help me, to see if I'm doing it right. (Kerry)

\section{Relationships with Geographically Distant Team Members}

Liam's team was frustrated at the limited amount of support MoE SE were able to provide. They were grateful to the individual therapists and advisors who had worked with them, but felt the system did not provide enough contact with these professionals. This, and the high levels of staff turnover in their GSE team, meant that building relationships with specialist staff was difficult.

We haven't had anything like this before for him - nothing of this nature before... It's just been quite a year of frustration because somebody is going to come 
because we need help with this, that, or the other - and then the meeting is cancelled because they're not able to come. Then we have to wait until the next meeting and go through (goodness knows what!) - Probably the last major meeting we had was when Liam's behaviour was just so uncontrollable that the (safe) room was set up. We needed support! (Molly)

\subsubsection{Continuing the work}

\section{Using Valuable Time Productively}

While at school Liam was predominantly working one-to-one with staff, away from other students. Molly and Kerry began to use music in a planned way with him every day, helping them use that time productively. Molly predominantly based her interventions on an adapted version of the ten-minute structured programme demonstrated during the consultation, while Kerry more often engaged Liam in spontaneous musical play but in a more considered way; observing his responses, reflecting on the work, and adjusting her contribution accordingly.

Liam's physical health had been variable during the follow-up period, and this impacted on his attendance and ability to progress at school. Although he was still able to make headway, Molly believed that the looming long school holiday period over December and January would set him back, and the regression would involve her 'starting again' with his programme, to a certain extent, the following year.

\section{Valuing the Process}

Molly found the music therapy consultation process particularly helpful for programme planning. Music experiences could be introduced regularly with Liam, and helped her to understand and value the responses she could anticipate from him.

Having another professional work alongside her heightened Kerry's awareness of what she was already doing well, helped her to build on that, and to take more notice of Liam's responses to her input. She felt the feedback I gave to the comments she made in her 
participant's journal during the follow-up period, was helpful and encouraging.

I look for more opportunities to interact with him and build on them (now). Especially in the music - I build on anything he does. Like on the moon-hopper when he started tapping on it rather than bouncing on it, we just worked with that... ...And the other day the classroom was empty ...so I just brought him back to his classroom, and turned the cassette on and starting dancing. And he grabbed my hand and started going round and round in circles. So it's about taking opportunities to do things with music. (Kerry)

\section{Student Progress and Potential}

Liam demonstrated increasing interest in the music sessions, and singing continued to engage him. He began to observe and imitate actions in music and movement activities, and to explore musical instruments purposefully. Molly stated that by the end of term he did not drop instruments distractedly as often as he used to.

Molly reported that Liam enjoyed listening to a $\mathrm{CD}$ of music composed for young children with development difficulties (Rickson, 2003) and, in the beginning at least, allowing him to do so was helpful. He initially remained somewhat cautious about committing himself to a task, and Molly continued to allow him to be a passive observer and listener for much of the time. However, her tactic appeared reassuring because Liam was increasingly motivated to approach her to request interpersonal interaction.

He loves the singing! ...for a half hour he was listening to the music, walking around and looking at the pictures but coming back and sitting down. I didn't have to say come and sit down. I just let him walk around and come back when he was ready. And that was quite lovely. So we've continued using the CD...He just absolutely loves that. He's more receptive to that, at this point, than just going in and saying 'music time - put instruments out and play the cabassa' or whatever. ...when we got to the boat ride song...he got on my knee facing me and rocked from side to side. And when it finished he got off - he was quite up close to me but not anxious or anything. (Then) we did 'Stomp, stomp, stomp'he stood like a statue and looked at my feet and when it came to the next one, 'Jump, Jump, Turn Around' I stood up and did that one and he laughed and fell 
on the floor...I don't know if he thought it was like 'Ring-o-Rosie'! (Molly)

Molly had begun to use the CD as 'background' music when she was doing cognitive activities with Liam, believing it helped him to engage and remain on task. She reported his ability to be 'in the moment' appeared to have improved, and was sure the music had contributed to this.

I think he's concentrating a wee bit more. We can sit for quarter of an hour doing a drawing... ... playing the music in the background... He will complete a drawing whereas before he would do a little squiggle, then move away. He's sitting for longer and when I say 'not finished yet' he will stay. He doesn't push past me to get away. (Molly)

Kerry found that Liam initially required a lot of hand-over-hand support, and observed " $a$ couple of days I've gone into the quiet room to do music and it - just nothing - he won't even sit down". She often found herself making choices for him because he was not willing or able to initiate music activity. As his anxiety decreased, he was more able to remain at an adult directed activity, to engage and to concentrate, and this in turn allowed the team to consider working on specific cognitive goals. He was also more able to use learning materials without the need to frequently mouth or play in the wet materials.

Molly observed that Liam had demonstrated no violent, and very few agitated, responses while working with her during the follow-up period. In contrast, he had begun offering and seeking affection. Molly was aware that his requests for physical interaction, including kisses, had to be carefully managed in the school setting. However, the blossoming relationship also meant that she could increase her expectations of what he might be able to achieve at school.

I think before if he resisted we would be inclined to let him go, because we were scared of the fallout... because he would get himself in such a state. I think now we are just pushing him a bit further, and we're gaining strides in that respect, because he's hardly spending any time in the calm room.

Liam was not demonstrating the same level of anxiety as he previously expressed in noisy situations, and his auditory sensitivity appeared to be having less impact on his 
ability to function. His vocalisations and the range of sounds he was producing increased, and he did not need ear muffs to mask environmental sound as he had done in the past.

I think it's just that he doesn't appear to need them quite as much. He's choosing not to have them on because he can cope with everything. ...He might have his fingers in his ears if there's suddenly a lot of noise... (But) he'll sit for longer... ... he'll sit for the whole of assembly now.

His more settled presentation enabled team members to introduce his new programme confidently, and he was more able to benefit from it. Liam also began to use the toilet. It is possible that his reduced anxiety, and increasingly positive relationships with staff, contributed to this achievement.

\section{Music Activities, Strategies and Resources}

Molly and Kerry used the agreed music activities flexibly according to Liam's anticipated needs, as well as their own strengths and teaching styles. Kerry appeared to be less insistent that Liam engage with or complete planned musical tasks, and seemed more inclined to abandon them in favour of spontaneous musical interactions. She did not find specific activities to be particularly helpful or motivating for him, rather she acknowledged his positive response to music by engaging him readily and easily in spontaneous musical play. Molly, on the other hand, found the consistency of musical structure helpful.

I am more confident going into the quiet room where we do our work, knowing that I've got something that's set out... because I know we're going to be doing something productive, and something that I know he will enjoy. (Molly)

Kerry took the CD of Liam's songs home and listened to them regularly so she knew them well, and could bring them to mind quickly in music sessions with Liam. She did not use the recorded music during sessions. In contrast, Molly used both recorded and live music in sessions, and continued to find recorded music helpful for calming Liam. They both sang spontaneously in a variety of settings.

When I see him somewhere and I'm not working with him, I sing (the hello song) and I sing the same thing, and I sing it right through - or if he's going somewhere I'll sing goodbye - and it doesn't matter where we are I always do it! (Molly) 
The team considered feedback in the follow-up period to be very helpful for their ongoing work, because it enabled them to modify how they were executing the programme, or simply reinforced the positive aspects of their work. Their independent decisions, based on their knowledge of and relationship with Liam, were also very important to the success of the music intervention. For example, while the CD was provided so that action songs would be selected and used live with him, Molly used selected tracks as background music for academic tasks. Although I would not have recommended the $\mathrm{CD}$ be used for this purpose, it seems to have been successful for her and Liam.

\section{Managing Music in the Future}

Both Kerry and Molly seemed committed to their music programmes, and certain to continue the work in future. Lisa, SLT, was enthusiastic about the likelihood that Liam's communication skills would progress through the use of music.

It has been fascinating and very valuable for me to be involved in this project... it was very interesting to see how music and accessing musical instruments crosses over so many different areas of Liam's development, and encouraging to see some of the different ways he is communicating with others. (Lisa)

Molly found it difficult, however, to identify any skills that she might have developed from the music therapy consultation, which she could use with other students.

I'm just concentrating on Liam and I haven't thought that far ahead. I would be concentrating on using music as often as I possibly can because of the calming influence it has on him...It's quite a relaxing time for him and he can sense that I'm comfortable, I can sense that he's comfortable, and it works really nicely. So I think it is something we will always use (Molly)

Kerry stated that "every day is different" and she often had to be flexible about what activities she might employ with Liam. But while she admitted that there have been "days we haven't been able to do anything", it seemed clear that Kerry's relaxed musical spontaneity would bode well for his future, and for other students she might work with. 
We often go and do music in the quiet room after morning tea. Or I try other things. Each day I try to do something. It might be to walk down to the hall to play the piano or (to play) with the moon hopper - he uses that like a drum. Or we go to the library and get out the drums just for a change. We try to get a bit of music - even if we do 'patty pat pat, and clap'. (Kerry)

\section{Inclusion}

Liam's peers were respectful of him, and their calm understanding was positive for all involved with him. However, it seemed that there would be many opportunities for Liam and his peers to be supported or educated about how to respond to each other, particularly with regard to beginning sustainable interaction, and music activities would likely provide a good base for this.

We sat opposite each other in the classroom and did 'Pat Pat Pat and Clap' lots of times - sometimes on my knee and sometimes on his. He kept showing me he wanted more. A couple of girls came and spoke to us while we were doing it. (Participant Journal)

Joan used greeting song each morning to promote social interaction in the classroom, but there had been few other opportunities for Liam to experience music making with peers, because he was engaged in so much one-to-one tuition. Further, although he had enough physical and emotional resources to remain at school over the lunch period, he was still not attending school for full days, and was not able to participate in group activities such as school picnics and other end of year events, unless additional support was provided by parents. Nevertheless, his reducing anxiety, positive relationships with staff, and emerging ability to manage adult direction, suggested that he would be increasingly able to participate with his peers.

\section{Consultees Relationship with Student}

Molly gave Liam opportunities to listen to music passively, and to engage spontaneously in action songs. However, she also began to directly request that he remain at an activity for longer periods, until a sense of completion was achieved. Liam appeared to be testing 
out the 'new' boundaries, to discover how flexible Molly would be in her approach. She noted that her quiet, calm and increasingly confident way of dealing with him, particularly when he was beginning to get anxious or distressed, almost always drew a positive response from Liam. His reduced anxiety enabled Molly to have higher expectations of what he might be able to achieve and the confidence to "push him a bit further" without fearing a negative emotional response. Molly's relationship with Liam seemed to have taken a positive turn since the music therapy consultation.

When I go and get him and say 'come with Molly' he takes my bangles and we go out the door, and I just take a step back and he walks into the work room. He was running away (before) ...These last three of four days I say 'off to the work room', and I don't hold him and I don't guide him. He just walks around there! (Molly) 


\subsection{ACTION RESEARCH CYCLE FOUR: AFTER LIAM}

Did this cycle of learning reinforce or weaken my premise that the social learning model of consultation is an appropriate frame for my work (7.7.7)?

\subsubsection{Making Links with Social Learning Theory}

It was easy to see the interplay between the way in which team members approached Liam (their behaviour), their confidence and skill levels (interpersonal factors), and his responses (the environment in which consultees worked) and thus to link the work with social learning theories. Prior to the consultation both consultees were cautious in their approach to Liam, even though they were confident in their ability to create an interesting musical environment. Liam was often calmed or distracted by their music making, but his aggressive responses in non-musical situations reinforced their caution, and discouraged them from attempting to engage him in interactive music making. They were open to having their work critiqued, and valued being given advice about how they might modify their music making, especially in individual sessions with him.

\subsubsection{Evaluating the Process}

The first goal of consultation is to assist consultees to cope more effectively with the difficulties they are confronting in the moment; and can be defined as successful when consultees perceive the process as meaningful and presenting problems to be reduced or removed (Zins \& Ponti, 1996). In this case, consultees judged the work as successful because they were more able to follow Liam's lead, which in turn motivated him to approach them, and provided them with opportunities to engage him in interactive musical play. The second goal of consultation is to enhance the ability of consultants and consultees to achieve preventive objectives, by providing 'content' (knowledge regarding the presenting problem) and 'process' information that pertains to problem solving (Zins $\&$ Ponti, 1996). In this case, the consultation focused on helping consultees understand the impact Liam was having on their behaviour, and providing them with the resources and additional skills they needed to enhance their practice. Their new understandings of 
various aspects of interpersonal relating are preventive measures that would aid their work with Liam, and other students.

From the beginning, Kerry and Molly were able to improvise simple songs. They also used other strategies such as imitating Liam's vocal sounds to let him know he had been heard. The next step towards maximising their use of music involved carefully managing such things as planned pauses, changes in dynamics or tempo, sudden stops and so on, to motivate his musical interaction. Using these therapeutic techniques effectively required them to make sophisticated observations and interpretations of Liam's responses, and to manipulate the musical elements with immaculate timing, in the moment. During the follow-up interviews Kerry and Molly did not mention these specific techniques. It is possible that Kerry in particular had incorporated the strategies naturally into her interactions with Liam, but it is also possible that neither consultee were able to master these skills in such a short consultation time frame. Videotape of consultees working with students during the follow-up period would have enabled further evaluation and/or support to be provided. Sending video in the follow-up period for learning purposes was encouraged, but was not insisted upon fearing it would create too much work for participants. None did.

I was aware that Liam was having a 'good week' when I was with him, and that I had only a limited view of the team's difficulties because I had not experienced his historical aggression. I valued the team's knowledge and the experience they had of Liam, and appreciated their need to find their own way to work with him - especially since he had been unwell following the consultation. I recognised that consultees will not always be able to implement suggested strategies in the precise way that is agreed at the time of the consultation.

\subsubsection{Improving the Model for the Future}

Team members might have benefited from a longer period of training, some of which might have been able to be offered through a series of workshops. However, although it seems realistic to assume workshops could offer basic training to staff and parents in the general use of music with students, those who work with students who have high or very 
high special education needs are more likely to need specialist training within integrated services, support with delivering programmes, and direct specialist intervention for students. The consultation protocol proposed herewith might still be the best option for training, but teams are likely to benefit from regular, perhaps annual, consultations.

On the other hand, Liam's principal had considered cancelling the music therapy consultation because the team believed Liam's behaviour might have been too difficult for me to manage. Music therapists will need to find ways of making people aware that music therapy programmes can be designed to meet the full range of need, and can perhaps be most powerful when things are 'difficult'.

Advising on, and supporting, Liam's team's decision to facilitate individual music sessions away from other classmates felt contrary to inclusive philosophy. Given a longer time in the field, or return visits, it might have been possible to support music activities specifically to promote inclusion. However, having explored my role with regard to systemic issues in previous cycles, I was more accepting of what I could do in the limited time available. An ecological approach requires specialists to be seen and used as a resource for team members, enabling team members to draw from specialists what they need (Bourke, 2006). That Liam's team were able to take programme ideas and resources, and adapt and use them in ways that suited them, was positive. It was interesting too that both consultees benefited in different ways from the consultation the importance of valuing existing skills and encouraging team members to build on what they do well, was reinforced.

School staff openly acknowledged that communication between them and Liam's parents was strained, and the team was severely frustrated with the lack of access to, and frequent changes in, special education support staff. They believed they only received support when they were at the desperation stage. Therefore, this case too suggests that consultants will need particular experience and skill to deal with interpersonal issues that are likely to be encountered in the work. Communication problems among team members can be due to both professional differences and personality differences (Dettmer et al., 2005). It was important for me to understand and value team members' comments in context, and keep them involved in open dialogue with me. "The greatest 
team success will come from making sincere efforts to respect members' differences, value contributions, and communicate in ways that respect and accommodate a variety of verbal and non-verbal styles" (Dettmer et al., 2005, p. 64).

\subsubsection{Pragmatic Issues}

Technical difficulties experienced with video playback equipment during the team meeting suggest consultants should be cautious about relying on technology.

Team members' suggestions that Liam was likely to lose some of his gains over long holiday periods implied that undertaking a music therapy consultation in the fourth term might be less effective than at other times in the year, because teams do not have time to consolidate the work before the long break.

\subsubsection{Summary}

This cycle of learning reinforced the social learning model of consultation as a frame for music therapy school consultation. It has been possible to develop a protocol to guide practice (see Chaper 9). Findings suggest that the protocol should be used flexibly specific tasks may need to be added or changed according to teams' circumstances. 


\section{CHAPTER EIGHT: STAGE THREE. THE FINAL PROTOCOL}

\section{Introduction}

The follow paragraphs describe a 'model' for music therapy consultation that others can study to adapt or replicate. Prior to each consultation, the referrer and consultant discuss whether this is an appropriate way to deliver the service, and if so, broadly what will occur, and who will be involved. The headings used are consultation 'parameters' suggested by Dettmer et al. (2005).

\subsection{Prototype}

Music therapy school consultation is about helping adults to use music more effectively with the student/s in their care. The aim is to assist team members to cope more effectively with the difficulties they are confronting in the moment, as well as enhancing their ability to manage in the future. The protocol draws on triadic and collaborative consultation models, and is predominantly based on social learning theory (Bandura, 1977; D. Brown, Pryzwansky, \& Schulte, 2006).

Social learning theory suggests the functioning of the consultant (music therapist), consultee, and client (student) is dependent on their behaviour, internal personal factors (also know as intrapersonal factors e.g. motivation, determination, resilience, and so on), and environmental factors (see Figure 7, p. 253). Altering the interdependent relationship between any of these variables can lead to complex and interrelated changes. Given the complexities of these multiple variables it would be extremely difficult to represent the interaction in graphic form.

\subsection{The System}

The music therapy consultant will be external to the school system, but works within 
school (and possibly home) contexts. Involvement with family, and other specialists, also predominantly takes place at school.

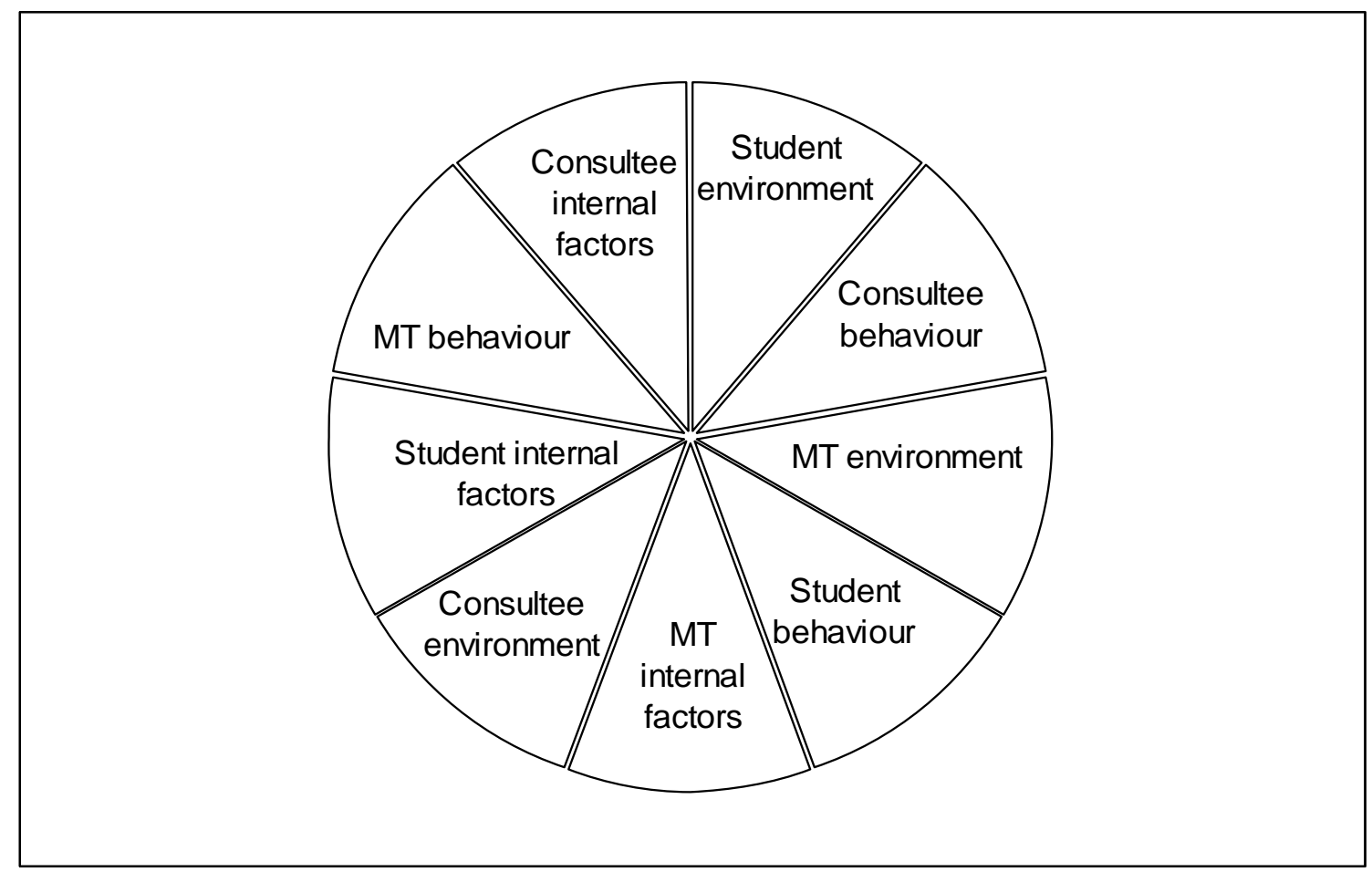

Figure 7: Multiple Factors in Social Learning Theory Consultation

\subsection{Perspective}

The music therapist takes a process perspective, helping team members to understand how they might use music to support student development and learning and to implement agreed strategies. Participation is assumed to be voluntary; consultees are free to accept or reject advice and/or the implementation of the strategies.

\subsection{Mode}

School consultation is predominantly an indirect service, with direct service being limited to assessment and modelling ideas, although in particular circumstances it might be prudent to undertake more direct work. 


\subsection{Approach}

Consultant and consultee work together for a week at the school; gathering data, interacting in formal and informal situations, and observing each other work. They clarify issues, negotiate and formalise roles, and undertake a collaborative assessment. They then jointly develop action plans, and agree on and implement data collection plans. Student goals are adjusted and/or reinforced, and consultation plans revised as necessary, during the process. The consultant models strategies, the consultee implements them. The consultee evaluates student outcomes, and the consultant evaluates the consultation process.

Nonlinear stages include establishing therapeutic relationships with consultees, collaborative assessment and goal setting, implementation, and follow-up and evaluation. Relationships are based on empathy, respect, and trust. Boundaries, including confidentiality issues, need to be clearly established. Consultants and consultees will have different personalities and needs, so adopting an appropriate communication style is crucial. Consultants and consultees can engage in a certain amount of carefully considered self-disclosure, or story telling, to demonstrate points. Consultation is a unique activity: it is not therapy, counselling or supervision.

All parties need to be committed to the consultation process. The music therapist should work alongside colleagues as an equal, relinquishing the potentially disempowering 'expert' role as much as possible. Although demonstrating a certain amount of expertise enables trust to develop, the primary focus of the consultation is on creating an environment where parties can contribute different perspectives, and have equal influence on outcomes. It is unhelpful when consultants are perceived as 'miracle workers'. Resistance and conflict is typical in consultation relationships. Consultants need to make sincere efforts to respect cultural, personality, and communication differences, and will need expertise in managing interpersonal conflict with, and amongst team, members.

An ecological assessment is central to this approach. However, a clinical music therapy assessment session is also crucial as it helps team members identify the student's 
strengths, and gain new perspectives of them. The music therapist and consultee, with other team members, watch and discuss video of the clinical assessment in a formal meeting situation. The music therapist might also formally interview the consultee as part of the data gathering process, to learn about their musical confidence and skill. Following the team meeting goals for adults, as well as for students, are jointly agreed.

Music strategies need to be accessible, meaningful, time efficient, and cost effective. It is highly desirable, therefore, for consultants to build on what team members are already doing with music. The music therapist supports the implementation of therapeutic music strategies, and team members take from the consultation what they are able to manage. The consultant will work to develop their confidence by modelling strategies and/or techniques, providing resources, and/or directly teaching music therapy skills. It is unrealistic to anticipate that sophisticated music therapy skills can be mastered in a short period of time, but basic strategies and techniques are nonetheless valuable for team members. It is important for music therapy consultants to carefully judge the most appropriate levels of directiveness to use with consultees. Pre-consultation workshops may improve consultation outcomes, and should be considered if the school's geographical location does not make the idea too prohibitive. Annual consultations which would enable them to continue to build on the skills they have acquired might be considered.

\subsection{Follow-up and Evaluation}

The follow-up period spans approximately twelve weeks after the therapist leaves the field. The consultee completes a music diary during this time, describing their attitude toward and ability to manage musical intervention, and might also video their work in order to gain feedback from the consultant. Consultees are interviewed at the end of the follow-up period. Diary data and interview findings are used for evaluation purposes. Because positive student outcomes are assumed to follow improvement in consultee effectiveness, evaluation focuses on whether consultees have changed their thinking and behaviour, rather than on student outcomes. 
While some direct work with students is necessary, the primary emphasis is on working with the consultees, and other core team members. The protocol promotes acceptance of cultural norms, and advocates music therapists' work within each school system to improve students' situations by enhancing the positive aspects of the environment. Some issues, such as changing negative attitudes to inclusion, are likely to be too difficult for consultants to manage in the time available. Music can be used to maximise students' potential to participate in school activities in or out of the classroom, thus increasing the likelihood of them being more fully included in future.

\subsection{Strengths and Limitations of the Protocol}

The music therapy consultation protocol described herein appears to have 'social validity', and be of 'functional value', for students and team members. However, because it provides a broad framework only, music therapy school consultants need to be familiar with a variety of 'human services' and 'psychological consultation' models, and to draw from them what is relevant for their specific contexts. Further, they should be fully aware of ethical issues associated with consultation, and maintain an ethical practice at all times. Consultants should know the limits of their competence, and have the knowledge, skills, attitudes, and values necessary to practice consultation. Clear communication is essential; values should be made explicit and the misuse of power avoided by engaging with participants in honest discussion and respectful negotiation.

\subsection{Summary}

The steps in the protocol are presented in Table 3, p. 257. The chronological approach suggests the emphasis for the first two days will be on gathering data and ecological assessment, the third day on music therapy assessment, and the last two days on introducing music therapy strategies. In reality the 'stages' are not so clearly delineated, rather these activities span the week. 
Send the child a picture of yourself with a letter about the visit

Negotiate a camera operator, to help with Wednesday session

Ensure all interested parties are aware of impending visit (e.g. parents and other professionals; negotiate involvement of GSE staff if necessary).
MUSIC THERAPY CONSULTATION PROTOCOL FOR STUDENTS WHO HAVE SPECIAL EDUCATION NEEDS

\begin{tabular}{|c|}
\hline MONDAY \\
\hline DATA GATHERING AND ECOL \\
\hline $\begin{array}{c}\text { Meet with team to discuss } \\
\text { timetable \& mutual } \\
\text { expectations for the week. } \\
\text { Negotiate time for team to } \\
\text { watch video - class cover } \\
\text { and/or babysitting issues are } \\
\text { important! }\end{array}$ \\
\hline
\end{tabular}

Observe student during day one - gradually increasing participation as appropriate. Note the student's communication systems, positioning requirements, any other special equipment, etc.; and their experience of and relationship to music. Observe the relationship between student/consultee and other, and consider impact of consultant's presence.
TUESDAY

TUESDAY

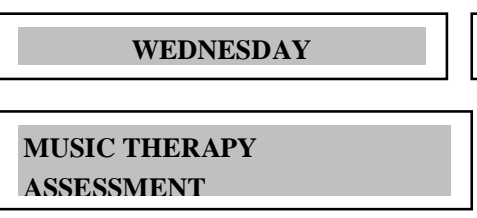

Undertake a child-centered music therapy assessment session - video the session.

Continue with ecological

assessment, observing and interacting with team members during daily routines. Notice what space and what instruments are available.

Find out about the skills of the 'Identified Team

Member/s' who will take responsibility for the therapeutic music

programme. Consider how the implementation of therapeutic music might impact on other students.
Involve all team members in

observing the music therapy

assessment session so they

have opportunity to note

child's responses to music. TOGETHER identify

strengths and needs and think about how the child's

motivation for specific music experiences, and team members music skills, might

be used to address developmental goals

Prepare necessary resources

e.g. compose song, source instruments and so on.

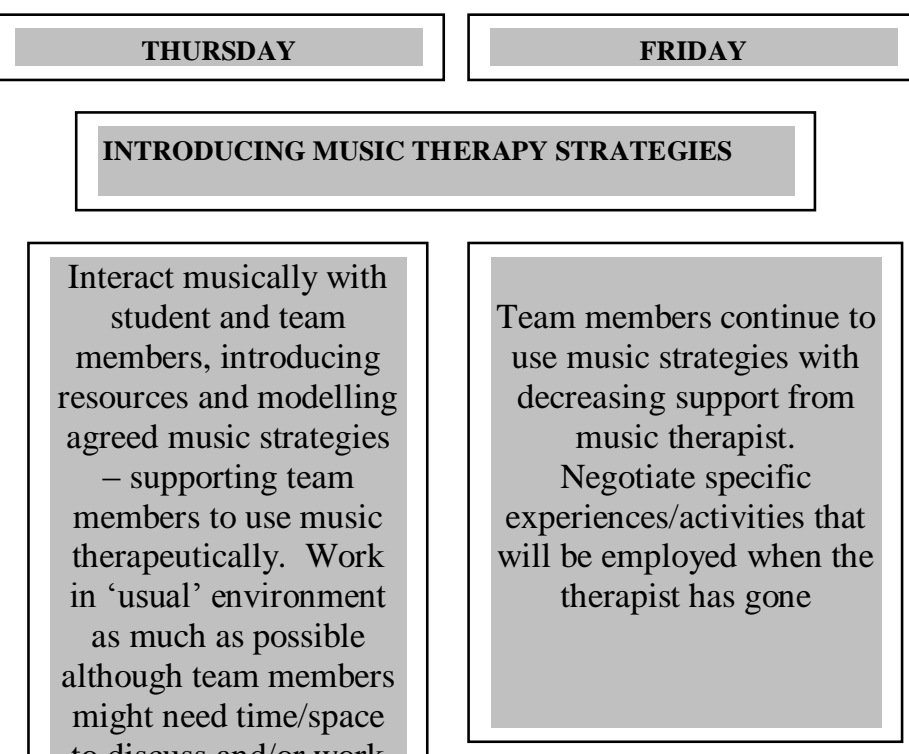

to discuss and/or work

on strategies without the student present.

Meeting to discuss ongoing music goals and their evaluation, consultation outcomes, follow-up and evaluation procedures

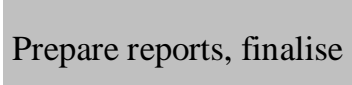
resources

Follow-up for one school term, participant keeps diary and is interviewed for evaluation nurnoses 


\section{CHAPTER NINE: ANSWERS TO RESEARCH QUESTIONS}

\section{Introduction}

After each action research cycle, 'indicators of efficiency' and 'learning outcomes' were identified and considered in relation to the development of the protocol. When all four case studies were completed, data were combined to answer the research questions.

The initial synthesis was undertaken to determine what 'indicators of efficiency' or other 'learning outcomes' could be identified, and thus provided answers to question three. These findings also hold the answers to questions two, and one. I have therefore reversed the order, and will demonstrate how the findings have gradually been refined as I answer question three, followed by question two, and finally question one. The newly developed protocol which emerged from the action research cycles, which is also a 'finding', is presented in Chapter Eight, and findings are discussed in Chapter Ten.

\subsection{Answers to Research Question Three}

What indicators of efficiency or learning outcomes can be identified to help others who engage in music therapy consultation with SE teams?

The following categories were derived from all four cases. The themes which were derived from these categories are presented in APPENDIX 42, p. 503.

\section{Indicators of Efficiency}

Categories that emerged were 'existing resources to draw on'; 'time availability and management'; 'team meetings'; 'empowering others'; 'music therapy strategies'; 'belief in music'; 'new perspectives'; 'student responses'; and 'commitment to continuing the work'. 


\section{Learning Outcomes}

Categories that emerged were 'a model of music therapy consultation'; 'time availability and management'; 'building relationships and empowering others'; 'differing approaches and the dilemma of inclusion'; 'cultural issues'; 'collaboration'; 'providing adequate support for teams'; 'student need for ongoing therapy'; and 'programme planning'..

\subsubsection{Indicators of Efficiency}

\section{Existing Resources to Draw On}

Teams had a range of physical and personal resources to draw on. For example, all schools had a good supply of musical instruments. One had a fully equipped and dedicated music room where the student could be included in music activities with peers, and from which instruments could be borrowed for individual work. In this, and at least one other case, money would be made available to purchase further equipment if it was required. In all cases space could be found for individual work when it was required.

Most of the team members were already making music spontaneously, e.g. singing with their students, albeit in private situations. All were willing to take new ideas on board but while some were confident and enthusiastic, others needed more encouragement to take risks. Team members' level of confidence did not appear to be associated with previous musical training and/or experience. Most had realistic expectations of the music therapy consultation process and what they might be able to achieve, by using music in programmes, over time.

One of the classroom teachers regularly used music in her classroom in a spontaneous and confident way, and was able to introduce strategies to encourage students who had special education needs to be included with their peers. A second was supporting her participant student to be involved in music activities in the wider school, and observed that the music therapy consultation increased her confidence to use music with students in a class group.

The best resource available to teams was arguably the students themselves. 
They were all reported as being highly responsive to music, with individual descriptions ranging from "music has always been a big part of his life" (Tim), "she enjoys sound making, and can be stimulated to actively participate in music activities" (Cathy), "he is attracted to, emotionally affected by, and aware of the shared aspect of music making" (John), and "he expresses sensitivity and real joy in music making" (Liam). Further, in addition to musical responsiveness, each of the students possessed strength of character, including determination, persistence and a quest for autonomy, which was difficult to manage at times but which were necessary attributes in order to achieve success with their developmental and academic goals. They were interested in their peers, or developed an interest in them during the period of the consultation. Further, peers mostly had a positive view of the participant student, and the potential for friendships to develop was evident in three of the cases. Cathy's teacher believed that the music therapist consultant's skill in building relationships with Cathy's peers was an important factor in improving the milieu of the classroom and therefore to the success of the consultation.

\section{Time Availability and Management}

Teams valued highly the length of time the music therapist consultant was able to spend in the field, and findings indicate that spending five days with each was crucial to the positive outcomes. It was possible to see, firsthand, students' strengths and needs in context, and to begin to interact with them and their teams as they went about their daily routines. From the first case it was evident that the time would be essential to enable relationships to be formed and to discuss, model, and problem-solve strategies with team members, as well as giving direct advice. Team members felt the consultation was just long enough for them to absorb information, read some more, observe the student, and try ideas. Members of Tim and John's teams particularly, believed the time was especially valuable and necessary to work collaboratively through moments or periods of extreme stress when the students, and staff, were having difficulty managing.

The week at each school was carefully planned and structured to ensure best use was made of the time available. While data gathering and adaptations to programmes continued, the individual session and team meetings needed to be undertaken early enough in the week so decisions could be made about the strategies that would be employed, while still ensuring there would be enough time to support staff in their 
facilitation of these. Moreover, in Liam and John's cases, collaborative consultation involved designing programmes with other visiting professionals and a great deal of flexibility was needed to accommodate their schedules.

\section{Team Meetings}

Team meetings, or focus groups, were well utilised by participants in this study. Having video material as a central focus enabled teams to warm up quickly and easily. Most team members were perceptive, made good observations from the video and used these to develop in-depth conversations about a variety of issues that affected their students, from general developmental progress to specific strategies that might be considered, and from political issues to their own team dynamics. In Liam's case, the team was able to address sensitive issues, which had resulted from poor team communication and which had previously been mostly avoided, openly but tactfully. Generally observations were diverse, but each team noted their student's positive interaction with the music therapist and viewed their engagement with music as one of their strengths.

\section{Empowering others}

Team members recognised and embraced new ways of working through collaboration, but also from observing the music therapist's approach to and interactions with the student. The music therapist had the role of infusing musical confidence in a way that facilitated the ongoing use of the music strategies that were thought to be helpful for the student. Most of the participants in this study were already using music in their work, but had not thought about, or had little confidence in, the way their use of music might positively impact on their student's development. Several believed that their singing might not be attractive, yet they were aware that their student 'tolerated' it well. An important aspect of the consultation process was helping them to observe the students responses more closely, and to consider and adapt their music accordingly so they might feel empowered to use music confidently in an ongoing way. As a result of the music therapy consultation, team members appeared to experience a heightened awareness of their own strengths and needs, as well as that of their students. It was clear that all teams exhibited growing confidence and an increasing sense of optimism as the consultation progressed. 
The teams and the individuals within them took different but equally successful approaches to the work based on their style of work with their student. While their initial levels of confidence and previous experience of using music may have had some impact on the way in which they initially approached the work, the most successful strategies were those which fitted their personalities and the way they interacted with their student generally. Team members felt that the work they were already doing was affirmed through positive feedback, and they gained confidence during the week of the consultation as they observed and participated in the use of music therapy strategies. The music therapy consultation was perceived as being unobtrusive and nonthreatening.

Team members variously learnt to use songs to motivate and support action, and to teach routines, to use music as a communicative medium in spontaneous play, to improvise during planned sessions with instruments, to maintain playful interaction, to develop musical turn-taking with the student, to use predictable and familiar live (and occasionally recorded) music to enable students to anticipate and prepare a response. Significantly, several team members, and one of the students, began to develop their own music strategies following the consultation. In Cathy's case, listening to and making music together had a particularly positive effect on the teacher student relationship. Her teacher's increased confidence, optimism, and enthusiasm following the consultation was profound.

\section{Music Therapy Strategies}

Music therapy strategies were built into existing programmes including the SCERTS, Floortime, Social Stories, and 'Routines' associated with Orientation and Mobility. Team members recognised that music supported many areas of development. They appreciated that suggestions were holistic and encouraged physical, emotional, social and cognitive responses from students. They also appreciated the ability to choose from a range of music experiences to promote stimulation or relaxation, active participation, or shared listening. Participants confirmed that strategies helped to address current teaching targets, and the reasons behind the introduction of the strategies, and the ways they might be established were clear. The strategies built on what team members were already doing, and were valued for their 'practicality' and 'accessibility'. Further, staff declared that the fresh ideas added interest, motivation, and vitality for themselves and 
their students, to activities or programmes that already contained intrinsic goals. Most importantly, in all cases students were observed to be able to manage at least some of their tasks more easily when the music strategies were applied. Teams planned to include music strategies on their student's IEPs as a tool for learning, in an ongoing way.

\section{Belief in Music}

Although most were unsure what to expect at the beginning of the consultation process, team members believed that the music therapy strategies contributed appreciably to students' progress towards, or achievement of, developmental or IEP goals particularly in the areas of communication, or emotional regulation. John's team for example, recognised that he engaged more readily and understood more when offered musical communication, and, when they observed his positive response, were convinced that the music therapy strategies would be helpful. Specialists from outside the core school teams, e.g. Speech Language Therapists and the Neurodevelopmental Therapist, also expressed confidence that music strategies would support communication programmes. Several team members, across teams, also suggested that they thought the strategies would be helpful for other students. On the other hand the strong belief held by the majority of team members that music would be a powerful tool to help students achieve their goals, may have biased their perception of outcomes.

\section{New Perspectives}

Each of the teams who participated in this study was, in their own way, going through a particularly difficult period with their students. Although positive outcomes are described, initial observations suggested that student presentations were complex, the teams' work was not easy, and that each was struggling to find a way to move their student forward and to maintain an optimistic view of their task.

Teams consistently reported new and positive perspectives of their students from the music therapy consultation, and this affected their work in very fundamental ways. For example, Tim's team noticed they had implemented a whole new approach to the student which "changed the way we teach". Across teams, members noted that the consultation helped them to recognise the importance of observing their students more 
closely and identifying and acknowledging their communication attempts, of taking their lead from him or her, and allowing time for a response.

In their individual music therapy sessions, students revealed many aspects of their personality as well as their pathology, and in many ways their responses resembled those they offered in other settings. Nevertheless, it was evident that when the students were engaged musically they were able to demonstrate previously unforeseen skills or to perform skills at a higher level than formerly observed. In recognising their strengths, team members seemed to become more respectful of their students, to have a more positive view of them, and higher expectations of what they might be able to manage. They became variously more relaxed, with increased optimism, energy, and enthusiasm for their work with the students. Specialists from outside the core teams were also impressed by the new and positive perspective they gained from viewing students engaged in music activities, and the way in which musical interaction in a therapeutic context could reinforce their own programme goals.

Team members gently but firmly increased their demands and expectations of what their student could manage on a daily basis. Liam's team, in particular, was cautious of him because of their experience of his very aggressive behaviour; yet following the consultation they readily increased their expectations of what he could manage. In contrast, Rona seemed to have developed a level of impotence following long periods of work with Cathy during which she noticed little progress being made, yet as she incorporated music into her tasks she became increasingly enthusiastic in her work. Team members were comfortable with a consultation process that helped them look at themselves as well as their students. Tim's team in particular was impressed with the way their approach changed following the consultation. Team members anticipated their students would continue to make progress and described having "new ways of communicating" (Tim) and "real hope for the future" (John).

In helping team members choose the music therapy strategies they would use, they were reminded that despite their positive response to music, students with very high levels of need were likely to take small steps forward and progress could be expected to be slow. Regardless, teams consistently reported that seeing what their students were capable of, or noticing their joy in music making, reinforced what they were already doing, motivated them to continue with the work, and to include music whenever it seemed 
appropriate to do so.

\section{Student Responses}

Across cases, students were reportedly more able to engage in adult directed activity, and to spend more time on task with musical support. Three of the students developed various cognitive skills essential to communication, including attending, making requests, imitating, and sequencing during the follow-up period of the consultation. John, who was verbal, began to make up his own songs and to sing himself through tasks. Tim began seeking the company of other children, and Liam developed peer relationships through music which were generalised to the playground environment. All four students were reportedly less anxious or more relaxed, and three were more able to regulate emotions, comply with requests and manage routines during the follow-up period of the consultation.

In addition, Cathy was increasingly alert when music was used as a stimulus and had begun to demonstrate a new quality of listening. She was vocalising more, and her purposeful movements increased. Liam's tolerance for auditory stimulation appeared to improve and his team believed that the gentle increase in exposure to a wide variety of sounds and music during and following the music therapy consultation contributed to this. John learnt to read music from simple score while playing the xylophone.

\section{Commitment to Continuing the Work}

All teams indicated that they would continue to use music with students in an ongoing way. Tim's team for example, was adamant that the music therapy consultation work would not be viewed as a 'project', but rather that it signalled the start of a new journey with him. John's team described enjoying sharing musical instruments with John and argued that this helped them to develop positive relationships with him. They were therefore excited about the ongoing work. His dad described the music therapy consultation as "a breath of fresh air", and this, along with their commitment to buying him a musical instrument for home use, suggested that the family would be sustaining the music work at least in the short to medium term. Rona, Cathy's teacher, was choosing music more purposefully and was committed to continuing to use music with all her students in a planned and spontaneous ways, and was encouraging other team 
members to do the same. The collaborative nature of the music therapy consultation meant that team members could draw on each other for ongoing support. Teams intended to include music therapy strategies in their IEP documentation in future, and three teams suggested they would draw on a music therapy consultation for their student in future when it was indicated and if it were available.

\subsubsection{Learning Outcomes}

\section{A model of music therapy consultation}

The social learning model of consultation provides a promising frame for music therapy consultation. In this model the primary relationship is with the adult team members, and the success of the consultation is determined by considering changes in the consultee. The consultation protocol, which is also a major 'finding' from this study, is presented in Chapter Eight.

\section{Time Availability and Management}

Teachers need plenty of advance warning in order to plan for a consultation visit of this type, so they can fit it in with other commitments. It was important to communicate and negotiate details well in advance of the proposed consultation. Further, although the collaborative aspects and the time involved for the consultations were highly valued by all teams, Rona was somewhat relieved when the music therapist took time away from the classroom to make resources, write reports etc. She also enjoyed the opportunity to do other things while her students were involved in music sessions with the therapist.

\section{Building Relationships and Empowering Others}

Building positive relationships with team members enabled effective collaboration to occur. Team members' previous experience with professionals (see paragraphs on providing adequate support for teams) meant this was not always an easy task. There was friction within them, and between them and outside agencies. Effective and ethical communication, for example being supportive while maintaining a level of neutrality when teams expressed disappointment regarding the service other professionals are able to provide, was essential. In John's case it was necessary to take somewhat of a 
mediator role to avoid team members 'splitting', and to gain their support and commitment to the music therapy strategies.

Family and other team members wanted to disclose personal information to a caring person, and seemed to believe the music therapist consultant could meet that need. Music therapy consultants might be vulnerable targets for 'needy' staff and/or parents because they have good helping skills, desire to build close positive relationships in a short period of time, and are viewed as safe confidants because they are 'transient' i.e. they will be leaving the team within a relatively short period of time. It was important that the music therapist consultant knew how to manage this type of situation, and was able to maintain appropriate professional boundaries.

Team members had a variety of working styles too, and these needed to be respected and accommodated. For some, it was necessary to break their tasks (as well as the student's tasks) down into small steps, to record these in the reporting package, to make extensive resources to support the work, and to adapt or add to these in the follow-up period. Other team members were more comfortable working in flexible ways, taking on board broad ideas that they could try with the student and vary or adapt using their own initiatives. Therefore the music therapist consultant could not bring her 'own way of doing things' to the team but needed to be willing to help in a way that was most comfortable for each team member.

\section{Differing approaches and the dilemma of inclusion}

In this work the music therapist encountered team members who had differing philosophical beliefs, or who practiced in ways that differed from the approach the music therapist consultant might take in the same situation. The role of the consultant was to stand alongside other team members to consider what approach might be best for the student in relation to the school's culture, i.e. 'the way we do things round here', but it was also necessary to challenge team members to accept new ideas when they seemed important. Conversely, there were times when it seemed more appropriate to help teams to do their work in the way they had chosen to approach it, when potential positive outcomes for the student could still be identified. Although the work consistently complied with the Code of Ethics for Music Therapists in New Zealand, and personal and ethical integrity was maintained, some issues caused a dilemma. 
The way in which teams approached the issue of inclusion is one such example. The majority of the music therapy consultation work was done out of the classroom, and many of the strategies that were introduced would be used in an ongoing way in a oneto-one or withdrawal situation. It felt uncomfortable at times to be separating the students from their peers and regular classroom teacher, and yet many of the priority goals, such as toileting, needed to be addressed in private settings.

On the other hand there were times when it seemed that it would be possible to introduce team members to classroom or small group work, yet it was clear that the music therapy consultation would be more useful if it helped team members to relate to and work effectively with their students individually, at least in the first instance. Given a longer time in the field, or return visits, it might be possible to implement more music activities that support inclusion.

\section{Cultural Issues}

The one case in this study which involved a Maori student highlighted the possibility that the music therapist consultant might need to consider more face to face rather than written communication with team members, including parents. An increase in personal contact with whanau may have enabled the music therapist consultant to learn more about their wishes with regard to programme content and delivery. For example, the student's teacher in this case, like the music therapist consultant, was a Pakeha and although some attempt was made to introduce waiata and children's songs in te reo, the teacher chose to focus more on English material. More time and commitment would be needed to achieve mutual understanding and genuine collaboration in cross cultural work.

\section{Collaboration}

The findings of this study suggest that a collaborative approach to music therapy consultation is essential. Spending a week in the field enabled collaborative relationships to be established. Moreover, that the work was taking place in context was critical. The music therapist consultant worked alongside colleagues as equals, rather than as a 'specialist' or 'expert'. Nevertheless, being equal does not mean holding the 
same knowledge and it is possible that the credibility of the work depended to a certain extent on how helpful the music therapist consultant appeared to be in matters relating to 'sound' and 'music'. Team members asked direct questions about a wide range of education and developmental programmes, e.g. auditory stimulation in multi-sensory rooms; and the use of resonator boards. While it was not necessary for the music therapist consultant to know all the answers, it seemed that knowledge and experience were important. Similarly, the music therapist consultant needed to draw on the knowledge and experience of colleagues in order integrate musically adapted social stories; use of alternative communication systems and so on, in music programmes.

Teachers' aides had a dominant role in working alongside the music therapist, and facilitating the appropriate inclusion of others, such as teachers and parents, was delicate. Teacher's aides, and others, were at times inclined to 'over help' a student, and needed encouragement and support to notice and allow time and/or space for the student to respond.

\section{Providing Adequate Support for Teams}

Three of the teams expressed strong feelings about the lack of support they perceived they received from other professionals. Visits were rare and unpredictable. Cathy's teacher felt unable to implement some of their suggestions without ongoing support. Further, the professionals who did arrive to help were often 'new' to teams, and of course to the student, and the lack of continuity was disturbing for team members.

Although team members consistently attributed the lack of support to their geographical isolation, and were quick to explain that individual professionals were not to blame, the evidence suggests that team members across schools were experiencing extreme feelings of frustration and abandonment. They variously confessed they didn't ask for help because they didn't expect a response or because they didn't think the level of support they would be offered would make any difference to their ongoing situation, or, as in the case of Liam's team, became increasingly vocal until they received help when they were at the "desperation" stage. In contrast, Liam's school principal confessed that he had been very sceptical about what the consultation might achieve when Liam was having extreme difficulty managing, and considered cancelling the appointment until Liam's behaviour was more manageable. 


\section{Student Need for Ongoing Therapy}

Although music therapy strategies were employed effectively by team members, the music therapist was able to use relatively complex improvisatory techniques which were precisely delivered, and learnt from years of training and experience, to engage students in musical communication in a clinic setting. The clinical music therapy session gave each of the students a new communicative experience and it was unlikely that team members would be able to emulate this work. Ongoing therapy would have been recommended for the students if it were available.

\section{Programme Planning}

Some team members began with little understanding of the possibilities for music to support a student's developmental or academic programme, and student referral information did not always reflect what was eventually identified as teams' most urgent need/s. The assessment process uncovered unexpected opportunities for music to support student growth and learning. The ways in which music could be used therapeutically in students' programmes were many and varied. Programmes were planned collaboratively to ensure team members maintained a sense of ownership of the work and were thus deemed to be realistic, meaningful, and purposeful. Including peers in music therapy programmes was a useful strategy to promote social interaction. Considering and responding to the needs of other children in the referred child's community was an important aspect of the work.

Team members were generally unable or unwilling to negotiate highly specific measurable goals for students they engaged with in this study. Rather music was used to reinforce the IEP goals that were already in place and in many cases their articulation of how students might progress towards these goals was generally very loose. The evaluation processes they used were mostly qualitative, and anecdotal evidence featured highly in their reporting. 


\subsection{Answer to Research Question Two}

How do team members who are directly involved perceive, understand, use, and value the consultation process?

Although team members who were directly involved in the music therapy consultations perceived the process to be different to what they had anticipated, it was overwhelming considered to be helpful. Team members were empowered to use music regardless of their previous musical experience or training.

Team members valued knowing the reasons behind the introduction of the strategies, and having clear explanations of the ways they might be established. They confirmed that strategies helped to address current teaching targets and were practical and accessible. Further, they variously declared that the fresh ideas added interest, motivation, and vitality for themselves and their students, to activities or programmes that already contained intrinsic goals. Music was perceived to fit easily into existing programmes and activities. The teams and the individuals within them took different but equally successful approaches to the work. For example, music therapy strategies were built into existing programmes including SCERTS, Floortime, Social Stories, and 'Routines' associated with Orientation and Mobility. However, much of the work also took place during activities of daily living and frequently away from the classroom and peers.

Team members recognised that music had the potential to support many areas of student development, and appreciated the ability to choose from a range of music experiences to promote stimulation or relaxation, active participation, or shared listening. They variously learnt to use songs to motivate and support action, and to teach routines, to use music as a communicative medium in spontaneous play, to improvise during planned sessions with instruments, to maintain playful interaction, to develop musical turntaking with the student, to use predictable and familiar live, and occasionally recorded music, music to enable students to anticipate and prepare a response. As they observed their students' responses to music they developed new and positive perspectives of them. They were thus able to gently but firmly increase the demands they made on 
students, and to revise their expectations of student achievement. Peers were included at times, and team members believe that the music strategies would also be helpful for other students. Across teams, members noted that the consultation helped them to recognise the importance of observing their students more closely and identifying and acknowledging their communication attempts, of taking their lead from him or her, and allowing time for a response.

Team members also felt that the work they were already doing was affirmed through positive feedback, and they gained confidence during the week of the consultation as they built on this through the use of music therapy strategies. They variously reported being more relaxed or energetic, and experiencing enthusiasm in their work, and developing hope and optimism for the future. Within teams, members indicated they would draw on each other for ongoing support, and indicated that they would continue to use music with students. They planned to include music strategies on their student's IEPs as a tool for learning, in an ongoing way. The music therapy consultation was perceived as being unobtrusive and non-threatening.

\subsection{Answer to Research Question One}

Can music therapists, through a process of consultation, empower multidisciplinary team members to use music therapy strategies in a sustained way in their work with children who have special education needs?

The findings from four case studies indicate that team members were able to introduce planned music experiences, and to use music therapy strategies and techniques within those, to support student learning and development. Further, as they witnessed their students' positive responses, they became increasingly confident and committed to continuing and developing the work, which strongly suggested it would be sustained over time. 


\section{CHAPTER TEN: DISCUSSION AND CONCLUSION}

\section{Introduction}

Findings from four case studies suggest team members working with students who have special education needs can be empowered to use music strategies in their work, in a sustained way. Team members gained new perspectives of their students, and were energised and enthused by the consultation process. They gained confidence, motivation, and optimism as they recognised the value of what they were already doing with music, and as they observed their students' positive responses to the introduction of the collaboratively planned music strategies. They valued strategies that were realistic, meaningful, purposeful and accessible. They also valued the block of time approach as it more readily enabled relationships to be formed, collaborative ecological assessment to take place, proposed strategies to be modelled and explained, and consultees to attempt tasks, ask questions, and revise their approach, and so on, in my presence. Team members believed that the music strategies contributed to students' progress towards their goals.

Nevertheless, the initial frame for the consultations was fragile, based on limited understandings from sparse music therapy consultation literature predominantly from the early childhood field, interviews with New Zealand music therapists, and my previous experience of working with team members in isolated areas. As well as examining each consultation to learn how it was being perceived and valued by participants, I systematically engaged with the literature and my reflective journal to determine how subsequent consultations might be improved.

The discussion begins with a critique of the research process. Implications for practice, training, and policy are considered, and recommendations for future research are made. 


\subsection{Critique of the Research Process}

\subsubsection{Critique of the Action Research Process}

The task of examining whether music therapists can empower team members to use music strategies in their work through a 'process of consultation' while the 'process' itself is morphing, is clearly complex. Potentially, the action research process could have led to a series of significant changes to the protocol to the extent that several 'protocols' existed; thus prohibiting or at best severely complicating any attempt to describe how 'the protocol' was perceived and valued by participants. Further, the diversity of teams and contexts encountered could have led to a finding that each case was unique, thus requiring an individualised approach which would be unable to significantly inform the next.

In contrast, although there were considerable shifts in the emphasis placed on various aspects of the consultation process, and it was clear that a solid empirical base was needed for the work, the evidence suggested the consultation tasks outlined in the initial protocol were, with minor adjustments, consistently helpful and therefore appropriate for team members with a wide range of needs and in various contexts. As a result it has been possible, for ease of reading, to make a rather false distinction between the case study evaluations, and the development of the protocol.

The study enabled a protocol to be developed which is based on sound theoretical and empirical foundations laid by other professions, and on findings uncovered during a rigorous examination of practice. The case studies provide evidence of the way team members perceived, understood, used, and valued the consultation process; and cycles of learning describe my reflective learning and changes in perspective over time. After the first, I recognised the significance of the ecological assessment, and potential for the clinical assessment to help team members identify students' strengths, and gain new perspectives of them. I also recognised that my work was fundamentally about building relationships with adults, and felt disempowered when my primary skill of communicating musically with students seemed less to the fore. I worried about the extent of my role as a consultant, and whether specific systemic issues were within my realm of responsibility. 
I reflected on ethical issues, and recognised that the iterative and negotiated action research process would also contain the practice of consultation. Action research and collaborative consultation each involve 'observation'; 'assessment'; 'planning'; 'action/implementation'; 'reflection/ evaluation'; and 'interpretation/planning'; in order to improve practice. Action researchers are committed participants who are also stakeholders with a vested interest in the use of the research for problem solving. A rigorous and reflective approach to 'research activity' therefore helped to ensure ‘collaborative consultation activities' were carried out appropriately.

After the second case, I recognised the need for a consultation framework, uncovered a variety of 'human services' and 'psychological consultation' models, and began to draw on Social Learning Theory Consultation. When beginning the study, I had limited understanding of what consultation involved, and although I had genuine intention to develop collaborative relationships, the participant information and consent forms (presented in the appendices) seem to indicate that participants would primarily be observing and discussing a music therapy assessment. Certainly I believed that the clinical assessment would be central to consultation. The suggestion that all core team members who had chosen to be involved would be able to introduce music in planned and intentional ways following the consultation, albeit with support from the consultee, signals a "watch and learn" approach. As the significance of the relationship with the consultee was realised, the role that other core team members would play needed to be renegotiated.

Unfortunately it was necessary for me to engage with team members who were not 'consultees' on a more superficial level. Nevertheless, all team members had genuine interest in collaborating to learn how music might be used to enhance students' ongoing development, and were motivated to work closely to negotiate their levels of involvement, exchange information and/or resources, and engage in interpretive discussions. For example, in Liam's case it seemed particularly helpful to have the principal involved in the core team. His understanding of the process ensured ongoing support for the teachers' aide consultees and the continuing provision of resources where they were needed. However, the therapeutic relationship was with the teachers and teachers' aides who were consultees. 
After the third case I was reminded that consultees would have different personalities and needs, that consultation styles will vary with context, and that music therapy consultation might involve drawing on various theoretical frameworks in order to understand the practice and execute it safely. I encountered resistance, recognised the considerable emotional support that team members might need, and the importance of understanding and maintaining boundaries between therapy, counselling and supervision. I observed how consultees can become disempowered by visiting 'experts'. I noted that empowerment is a consistent theme in consultation work, and that statements of self-efficacy are therefore extremely relevant as indicators of consultee change.

The triadic nature of the work was reinforced. The fourth case study highlighted the interdependent relationships between the way in which team members approach students, their confidence and skill levels, and student responses. The complexities of music therapy consultation which aims to assist consultees to cope more effectively with the difficulties they are confronting in the moment, and to enhance their ability to manage in the future, were emphasised. This reinforced my emerging argument that this work is for experienced music therapists.

My new perspectives regarding the importance of ecological assessment, and the shift in focus from student to adult participants, has left me somewhat uncomfortable about the way the case studies have been laid out in this thesis. Initially it seemed appropriate to position students at the centre of the work. I have retained this traditional format for consistency but in future consultation case studies, adults will be positioned as primary participants.

\subsubsection{Power and Empowerment}

"The political implications of inquiry can vary according to whose interests the inquiry serves" (Bray et al., 2000, p31). The current project had several motives. As a music therapist I was interested to improve my practice with students who have special education needs; as a music therapist trainer in the advancement of other music therapists' practices; and as a $\mathrm{PhD}$ student in submitting a scholarly thesis at the highest academic standard. 
The management of ethical issues, including power relationships, involved all team members reflecting on and keeping student wellbeing at the forefront of our minds throughout the research process. I felt consistently comfortable within the relatively familiar culture of 'special education', where diverse workers in a variety of contexts demonstrate a genuine commitment to student wellbeing and progress, despite having different approaches and understandings about how that might be achieved. When it seemed that there might have been a tip in the power balance, we tried to put ourselves in other's shoes. Viewing the situation through the eyes of the students for example, enabled adults to position themselves differently in relation to each other. Further, that the 'success' of the project was to be measured in terms of whether participants were empowered, motivated me to consider and work very hard to ensure that my approach enabled that to occur. My role as consultant involved using 'referent power', persuading and influencing participants through the relationship bond (Crothers, Hughes, \& Morine, 2008).

Nevertheless, power relationships are particularly complicated in consultation because it is relationship focused and it was not possible to maintain equal relationships at all times. Although I felt accepted as a team member I was variously an 'insider' and 'outsider'. While in the field I was a team member, but my relationship with participants was temporary and brief, and I essentially remained an 'outside' researcher and consultant. Further, although we all brought particular expertise to the process, some team members seemed to revere music therapy expertise too highly at times.

Authentic involvement in PAR involves participants sharing in the way the research is conceptualised and carried out. In the current studies, although participants took primary roles in planning and implementing action within the frame of the consultation protocol, the protocol drove the research procedures to a large extent, and consultees' influence on its development could not be apparent until after the cycle. So it seemed that while the teams' projects had 'much in common with PAR' they could not be described specifically as such.

Further, while they were clearly empowered as music makers, participants continue to work in systems that disempower them in other ways on a daily basis: systems bound by structures and rules so strongly entrenched in school contexts and cultures that they 
could not be moved in the time available to us. Student exclusion, for example, is disempowering for staff and students, and reinforces the need for 'expert' input to alleviate difficulties. Nevertheless, participants were able to develop ongoing cycles of reflection, planning and action related to their music making with students. This seemed comfortable for most of them since they were familiar with reflecting on student responses, adapting plans and trying again, as part of the IEP process. But being part of an action research project enabled them to reflect more on their influence on student learning and behaviour.

This study identified several instances where IEP goals were articulated as 'programme' goals, i.e. what educators will do, rather than measurable goals for the individual student, and teams were generally unable or unwilling to negotiate highly specific measurable goals for students they engaged with in this study. Rather music was used to reinforce developmental and academic goals that were already in place, and in many cases their articulation of how students might progress towards these goals was loose. The evaluation processes they used were mostly qualitative, and based on anecdotal evidence. It seems that it is 'usual' for team members who work directly with students to share intimate first-hand knowledge of the student, and for events to be documented and progress determined largely from this source. In this respect the projects they engaged with for this research closely resembled usual practice.

Social learning theory emphasises the reciprocal influence of consultant, student, and environment. The action research process aimed for participants to improve their practice by understanding how context and history influenced the way they viewed their work. Their involvement therefore enabled participants to demonstrate significant shifts in thinking. For example, several team members suggested they had been working towards IEP goals for long periods because of their students' behaviour. Later they realised that when they took responsibility for listening to and/or observing the behavioural communication efforts of their students, faster progress was made. While each of the students experienced actual impairment that impacted significantly on their daily lives, principles of critical pedagogy and disability studies suggest they will experience less 'handicap' now that team members are able to see their competencies and focus on their strengths. 
At first, the majority of participants did not consider themselves to be musicians. Most were already using music in their work, but had not thought about, or had little confidence in, the way their use of music might positively impact on their students' development. Interestingly, although several believed that their singing might not be attractive, they were aware that their student 'tolerated' it well. An important aspect of the consultation process was helping them to observe the students responses more closely, and to consider and adapt their music accordingly so they might feel empowered to use music confidently in an ongoing way.

As a result of the music therapy consultation, team members appeared to experience a heightened awareness of their own strengths and needs, as well as that of their students. It was clear that all teams exhibited growing confidence and an increasing sense of optimism as the consultations progressed. While they developed awareness of the importance of considering why, what, how, and when music strategies might be introduced, they also learnt students were non-judgmental and responded consistently with relative enthusiasm to their efforts. Across all four cases, it was evident that team members were empowered to continue using music in their work with the participant students, and in many cases it seemed that they might generalise that confidence and enthusiasm to using music in their work with other students.

Most team members were already making music spontaneously, such as singing with their students. All were willing to take new ideas on board. However, while some were confident and enthusiastic others needed more encouragement to take risks. This study is too small to determine any association between consultees' confidence levels for engaging in music making with students, and their formal training in music or experience of playing musical instruments. However, the finding that a consultee who had been a music teacher initially seemed to use less music throughout the day than others, suggests it is possible that those who have more formal training might have different expectations and more difficulty relaxing and being musically playful with students. Clearly, consultees who did not initially consider they were musicians in the formal sense could engage well with the work.

\subsubsection{Significance of the Study}

This study, involving the development and evaluation of a protocol for the innovative 
practice of music therapy school consultation, is likely to make a major contribution to music therapy, special education, and consultation fields. The protocol will enable music therapists to take a systematic approach, which is needed to enable validation of music therapy work (Ala-Ruona, 2005; Brunk \& Coleman, 2000; Sabbatella, 1999), while encouraging them and the teams they work with to devise programmes that suit their unique communities. It draws on and sits comfortably alongside the practice of psychological consultation, since music therapy is most akin with psychological and counseling services (Pellitteri, 2000), and parallels music therapy consultation in early childhood (Kern, 2004). The protocol is based on social learning theory, which emphasises internal and external influences as reciprocal determinants of human behaviour and the impact of environment and culture on learning. Such ecological approaches are increasingly being discussed in relation to both music therapy and special education fields.

No studies were found that examined a music therapy consultation process with teams who work with students in regular school settings. Further, interviews with New Zealand music therapists support Register's (2002) finding that music therapists have not had a clear conceptualisation of collaboration or consultation. Register concluded that research should seek to define these concepts as they apply to music therapy. In addition she suggested research was needed to describe the impact of music therapy consultation on the consultee. This study begins to address these issues.

Researchers in other fields have noted that few studies examine the consultation process to identify what works best under which conditions (Dougherty, 2005; Wesley \& Buysse, 2006). Furthermore, a lack of rigour characterises most consultation studies (Hylander, 2004, in Wesley \& Buysse, 2006) and it is important for consultants to become researchers by conducting ongoing evaluation of the consultation process (Dougherty, 2005; Wesley \& Buysse, 2006). Ingraham and Meyers (2000) observed the contextual variations and influences of cultural aspects of consultation, and suggested qualitative methods may be helpful in clarifying the relationships among important variables. This study is an important first attempt to describe the variables encountered in music therapy consultation.

In addition to knowing whether consultation goals are achieved, Wesley \& Buysse (2006) note that qualitative evaluation enables the examination of ethical issues such 
as 1) the renegotiation of action 2) the maintenance of the therapeutic relationship and 3) timing and closure, and can enable the improvement of consultation practice as it occurs, rather than at the end of the process. Monitoring consultees' reactions to suggestions, clearly defining their concerns regarding the process and their perceived difficulty when implementing strategies and so on, provides useful evaluation data to guide subsequent interactions (Wesley \& Buysse, 2006). Using an action research approach enabled the above issues to be incorporated into the current study, and qualitative evaluation enabled consultee responses to be richly captured.

\subsubsection{Limitations of the Study}

The protocol has come to fruition through the reflective action of one music therapist and as such is a theoretical framework to be tested in the field. Although some of the findings from music therapist interviews resonate with the case study findings, the paucity of consultation practice and the originality of the protocol mean it is not possible to draw on other music therapists' experiences to situate the current work. ${ }^{10}$ Nevertheless, links can be made with the experiences of other professionals and these have been drawn into the discussion throughout this chapter. It will be crucial for music therapists who undertake school consultations to engage in ongoing debate regarding the utility of this, and perhaps other, protocols, and to contribute to an evidence base for the practice of Music Therapy School Consultation.

A preliminary motivation for the development of the protocol was to serve the needs of geographically isolated students. While it is likely to be a cost effective way of ensuring that these students receive a music therapy service, it will also be of value to students regardless of where they are schooled. The protocol is likely to be predominantly suited to primary school settings, since this is where it originated. A different systemic focus is anticipated in work involving preschool children, or students in secondary schools. For example, early childhood work is more likely to involve more intensive family work, since it is widely accepted that primary caregivers should be included in work with young children (Greenspan, 1992). Further, the protocol assumes that key people will work with students for sustained

\footnotetext{
${ }^{10}$ Research is already underway which investigates consultations, based on the current protocol, undertaken by another NZ music therapist.
} 
periods each week (e.g. primary teachers; and/or teachers' aides) whereas in secondary schools, students work with a variety of teachers for shorter periods of time. Consultations in secondary schools might focus on workshops for teachers, or with those who have a particular interest in using music with students (e.g. itinerant music teachers).

The protocol was developed with teams who support students who have high or very high special education needs in mind. Different approaches might be appropriate for teams who work with students who have less severe needs. On the other hand, the collaborative consultation approach employed by RTLB in their work with students who have moderate needs has also been found to be highly successful in the New Zealand context (Thomson, 2008). This suggests music therapists are also likely be able to work effectively with this population.

The music therapist was also able to engage students in musical communication in an individual session, in ways that were not able to be emulated by untrained professionals. The musical communication involved incorporating the use of relatively complex improvisatory techniques which were precisely delivered, and learnt from years of training and experience. The sessions gave each of the students a new communicative experience, which had the potential to be developed further in future therapy sessions. While this does not reduce the value of the consultation, students would benefit from both clinic and consultation input.

\subsubsection{Whose views are they, and how clearly are they represented?}

Findings from each of the case studies are not necessarily generalisable. Rather they are contextually bound to the researcher/music therapist, and to the schools and teams who participated. Nevertheless, while information is generated from action research primarily for the purpose of creating change within the social context in which it is generated, the data can also be examined for relevance to other contexts.

Significant amounts of data were gathered, enabling the generation of rich findings that highlighted the diversity of participants within and between schools, yet allowed for some common themes to emerge. The findings articulate understandings and carefully considered and critiqued opinions, which are likely be helpful to other music 
therapists and special education team members who engage with this type of work. However, despite my best efforts to engage in rigorous data analysis, it is possible that findings may have been positively skewed. Alternatively, in an effort to maintain high levels of research integrity, I may have under reported positive findings.

Although a total of thirty-five team members participated in the case studies, the primary focus was on eight team members or 'consultees', who provided the majority of feedback through formal interviews and/or research diaries. The consultees became team leaders who involved others in the work, and were therefore able to provide indepth information about their own experiences, as well as general comments about how others had developed their use of music with students. Where there was more than one consultee in a team, information regarding their personal progress was shared openly during face to face exchanges, and in one case they engaged in a joint interview. It is possible that in the presence of others they might have been less able to disclose difficulties, and/or individual views might have been overshadowed.

There may be differences between teams who expressed interest in being involved in the study, and those who did not. Consultees most likely represent a section of the special education community who are dedicated to providing the best possible outcomes for individual students, are committed to their own professional development, work in supportive schools, and already have an interest in using music with students in some way. Teams had physical resources they could draw on including space, instruments, and in some cases they had money to buy specific instruments that the students had expressed interest in during the consultation assessment.

Students were known to respond to music. Each of them had characteristics such as determination, persistence, and a quest for autonomy, which were difficult to manage at times, but which were necessary attributes in order to achieve success with their developmental and academic goals. They were interested in their peers, or developed an interest in them during the period of the consultation. Further, peers mostly had a positive view of these students, and the potential for friendships to develop was evident in three of the cases.

There will be other teams who might have vastly different experiences if they were to 
be involved in a music therapy consultation. There will be school administrators (Boards of Trustees and Principals) who will resist staff involvement in music therapy school consultation work. Teams in this study may have requested support because they were not able to receive specialist help from other avenues.

A major risk with self report is that participants might not be doing what they say they are doing. However, diary entries as well as interviews enabled team members to provide clear evidence that they continued with the planned music programmes, as well as implementing other music ideas that had been discussed. They all described their experiences on a regular basis, included qualitative evidence from informal team meetings and discussions, and were able to report progress with confidence. Quantitative and qualitative evidence relating to student progress was also gathered by teams. However, what was reported was much more than whether stated objectives had been met - the development of positive relationships, students and staff appearing to have improved self esteem, confidence, motivation, and so on, were described. Methods of evaluation that capture only measures of precise goals miss the rich impact that music therapy can have on participants' lives.

There is, however, also a risk that the regular affirmation team members were given to help increase and/or maintain their confidence levels, might have led to over zealous reporting of positive outcomes, and/or that team members may have reported positive outcomes to please me. Participants were therefore strongly encouraged, and supported with examples, to report difficulties. The diaries consequently describe a range of extraneous circumstances that impact on their ability to carry out programmes. For example, students were often absent from school due to illness and/or family events. At school they were reportedly affected by the weather, their physical health, seizures and general tiredness, and unidentified irritants, and by other children's individual or group actions, or whole classes interrupting their one-to-one activity in designated withdrawal spaces. Changes and/or surprises can be highly distressing for students who have special education needs, especially those who have autism, and can have a significant impact on students' potential to progress. One teacher's aide was left feeling as if she and her student were 'always getting in the way', and several expressed a strong desire for their students to 'fit in' better at school. 
Participants took responsibility for determining how they would use music in their settings. Enabling the reciprocal shaping of projects and contexts promoted autonomy, empowered participants, and facilitated sustainable change. However, people can choose not to be empowered (Clegg, 2004), and outcomes are dependent on the various cultural factors that influence their potential, including the difficulties they encounter in their daily lives. O'Brien and Ryba (2005) suggest that when schools lack capacity and capability they become separated from the 'system'. Each of the four case studies has some aspect that resonates with this suggestion, and it is possible that the music therapy consultation was a final attempt at reintegration for at least one of the schools.

When a child is capable of forming his or her own views, they have the right to express those views freely in all matters affecting them, and for those views to be fairly taken into consideration (UNCRC, 1989). Further, "disability may be understood best by listening to 'disabled people' tell about their lives and the real-life experience of living with a disability" (R. M. Smith \& Nevin, 2005, p. 270). Nevertheless, the difficulties associated with recording children's views have been well documented (Lewis \& Porter, 2004; Porter, Ouvry, Morgan, \& Downs, 2001; Whitehurst, 2007). In this study an attempt was made to interview one of the students. The interview technique built on the student's usual way of communicating and was informal, conversational and non-intrusive (see Appendix 28, p. 493). However, minimal data was obtained, and his apparent lack of comprehension meant it could not be analysed and interpreted with enough confidence to ensure that his views were represented authentically.

On the other hand, the behavioural communication of the students meant their collaboration was still possible. As Smith and Nevin explain, "the expertise of the student lies in what he or she is trying to express, and the expertise in the professional lies in the ability to be a detective and interpreter of the student's 'language"' (2005, p. 278). The students' positive responses to music therapy consultation activities suggest the process was highly valued by them. 


\subsection{Implications for Practice}

\subsubsection{Professional Identity and Protecting the Profession}

The case studies demonstrate that team members are able to use music in an intentional, planned, and sustained way. However, it is crucial to convey that consultees are not practising music therapy. In the current studies, some of the student IEP's had 'music therapy' listed, perhaps in anticipation of the consultation, but this seems inappropriate especially since the consultation goals were increasingly adultfocused. Pellitteri (2000) drew on the three-way relationship (therapist, client, music) oft quoted in music therapy, to argue that although music therapy interventions can be adapted and used by teachers and related service clinicians, the situations in which they do so are not music therapy per se.

'Music therapy is the use of music... by a qualified music therapist, with a client or group...' (World Federation of Music Therapy, 1996). But can 'indirect' service be music therapy? Therapists from various disciplines who work in schools have questioned whether consultation is really therapy. Themes from the CoMT literature, and music therapist interview findings in this study, suggest the debate is likely to continue. However, there is no doubt that consultation will be an important role for a music therapist, even though it involves using additional and/or different skills.

Nevertheless, music therapists in school settings already need to work actively to maintain their identity as a therapist (Sutton, 2002; Twyford, 2007) and, like their colleagues from other disciplines, are likely to have difficulties transitioning from a medical model to an inclusive model of practice. Further, music therapists might find the move away from psychotherapeutic model/s of music therapy difficult because they are losing the clear frameworks and boundaries which differentiate the role of music therapist from, for example, music educator or special education teacher.

While they are prepared to align their work with the expectations of fund holders to a certain extent in order to grow a 'realistic' practice, music therapists interviewed during this study, and others (Robertson, 2000; D. S. Smith \& Hairston, 1999; B. L. Wilson, 2002), convey concern that consultation will diminish their role and identity, and impact on the service they can provide. Some therefore, at least initially, are 
likely to reject or proceed cautiously with the protocol. Twyford (2007) suggests it might be valuable to undertake further research "to fully understand protectionist attitudes amongst music therapists as this influences how we are perceived by the workforce in which we are employed" (p. 29). While this is undoubtedly a good suggestion, sometimes reasons for concern are obvious. For example, people who do not have appropriate qualifications refer to their music making as 'music therapy' (e.g. Feltham, 2004) and in New Zealand, where the title of music therapist is not protected by government legislation, this poses a risk to the integrity of the profession.

On the other hand, sharing the work more widely by practising outside the clinic setting clearly has benefits for the profession of music therapy. Collaborative approaches have the potential to open new pathways in the development of music therapy, as therapists disseminate information and educate other professionals (Register, 2002; Threlfall, 2005; Twyford, 2007). But for this to be achieved, music therapists must be confident in what they do, and be committed to theoretical orientations while remaining flexible in their application of music therapy (Twyford, 2007). Further investigation regarding the effects of collaboration may facilitate the growth of music therapy as a more widely recognised method of treatment (Register, 2002). That is certainly one of the aims of this thesis and the protocol developed herein.

\subsubsection{Cultural Issues}

Music therapy consultants will be working in schools where students, staff and families do not necessarily have mutual values and/or work collaboratively towards a shared vision, but may have various difficulties related to power struggles within their community, and/or with outside agencies. Values feature highly in the lives and interactions of education stakeholders (Leonard \& Leonard, 2001). While the world of special education has its own broad cultural features, music therapy consultants will encounter a new sub-culture with every new referral. Groups of people in each school or classroom will have their own ideas about 'the way we do things around here' (Ingraham \& Meyers, 2000; Threlfall, 2005).

Furthermore, music therapist interviewees and others (e.g. Pellitteri, 2000; Sutton, 2002; Woodward, 2000), expressed concern that the different philosophies that 
underpin education and therapy have the potential to impede their work in schools, because the theoretical training can be at odds with the way the children's educational progress is measured. Sutton notes that music therapists are able to think developmentally and in terms of types of behaviour, but usually focus on the psychoemotional aspects of a child's life, which is not the focus of the school curriculum.

Families are possibly more likely to support the introduction of psychosocial goals for their children. In the current study, parents who viewed video of their children in music therapy identified their joy and having fun, as crucial aspects of the music therapy session. However, despite the government's commitment to including parents, they might have little meaningful involvement in the planning, implementation, or direction of their children's programmes. It will be important for music therapists to develop and maintain a genuine commitment to empowering parents to be part of the music therapy school consultation process.

Nevertheless, findings from case studies suggest that music therapists might have a particular way of relating to and approaching students which is highly valued by team members, and which to a certain extent can be emulated by them. Children cannot learn if their basic needs are not met (Sanders, 2003). Music therapy consultation led to students having increased opportunities for non-verbal expression through musical play which, among many other things, improved adult/student relationships, provided foundation skills for other forms of communication, and supported the emotional needs of the child.

The deficit model of disability is reinforced by the ORRS system, and to a certain extent by the IEP process. The categorisation which is necessary in order to receive the appropriate resources to meet students' individual needs is at odds with an inclusion policy which aims to focus on the positive aspects of diversity among students. This study confirms that teachers still expect students to be withdrawn, so individual programmes can be implemented, or to be tested so that progress can be demonstrated, and resources justified. Further, some team members viewed their student with special education needs as a 'problem'. Such views are often shared by team members, and can become a cultural norm within the school. When this happens, team members can ignore their own impact on the students' behaviour, deny their responsibility to facilitate change, and readily exclude students from school 
activities. The music therapy consultations appeared to help team members, over the consultation period, to become less focused on their students' problems and struggles, and more focussed on how environmental or interpersonal exchanges and relationships impacted on the students' ability to manage, and to identify opportunities for growth and change.

The delegation of responsibility for students who have special education needs from teachers to teachers' aides, seems widespread in New Zealand. There is clearly a need to address the potential for teachers to be more directly involved with students who have special education needs, in mainstream classrooms. However, given the skills many of them possess, there also seems to be a case for increasing the status - and thus conditions - of teachers' aides. Regardless, teachers' aides experiences generally appear to be frequently distressing (Tutty \& Hocking, 2004) and the finding that their wellbeing can be improved through music therapy consultation seems very important. Therefore, while it will be necessary to strive to increase the involvement of teachers in music therapy consultations, empowering teachers' aides to use music in their work is clearly valid. On the other hand, while trust and respect are possible in relationships regardless of status, teachers' aides are unlikely to be able to support teachers in their use of music, which might be possible if roles were reversed, because the teacher is in a position of higher authority.

Music therapists must pay careful attention to whether the student should be withdrawn for therapy or direct intervention provided by 'specialists', within an inclusive education system. However, the case studies highlight the value of intimate one-to-one work in a quiet space, because it allows team members and students to notice and respond to subtle but extremely important communications from each other. Further, many students with special education needs respond positively to the containment of the therapy room. Clinical music therapy is therefore likely to continue in schools when teams agree it the best option for student/s, and where there is space and equipment available to practise in this way. While music therapists must continue to work in the least restrictive environment, it is evident that withdrawal work, perhaps for 'enrichment through music' rather than 'therapy', will continue to be valid.

Music therapists working as consultants in New Zealand schools will also encounter 
people who differ from them in terms of age, gender, ethnicity, disability, and so on. The music therapist consultant needs to consider communication issues carefully. For example they should reflect on the likelihood that more face to face communication with whanau would be helpful when the student is Māori. Personal contact might be necessary to empower whanau to express their wishes with regard to programme content and delivery. It is also important to ascertain what cultural elements need to be incorporated into programmes. Time and commitment are needed if team members are to achieve mutual understanding and genuine collaboration in cross cultural work.

Consultation involves engaging team members in conversations that highlight and clarify each person's values, and being able to work with those values respectfully. Nevertheless, while it is clear that an individual should not impose their values on another, applying the concept of cultural sensitivity simultaneously at student, consultee and systems levels is complex, as it involves coming to understand and being sensitive to the unique experiences of all parties, and negotiating and using multiple perspectives productively when problem-solving.

\subsubsection{Professional Comfort}

Music therapists interviewed for this study were somewhat polarised in their views regarding the value of music therapy consultation. Moreover, general commitment to the idea seemed somewhat limited. Yet arguably the most significant aspect of effective music therapy school consultation will be the music therapist's commitment to the process. This in turn implies it will be necessary for music therapists to have a thorough understanding of disability and education philosophy, and consultation procedures (see training needs, p. 307).

Chester (1996) argues that the programme-based consultative music therapy model allows a maximum number of students to benefit from music therapy intervention, thus increasing cost effectiveness. However, consultation should not be viewed as an option which enables therapists to see more children. The potential exists for music therapy consultants to receive high numbers of referrals, especially while music therapist numbers are low. Initially, one of the primary arguments for developing a consultative model of music therapy was the 'paucity of music therapists'. That is, it was suggested it would allow students who might benefit, but are unable to access 
music therapy, to receive some form of service.

The findings clearly contradict this argument, since there are likely to be few music therapists who are able to commit the time necessary to be an effective music therapy consultant, as portrayed in this thesis. Other ways of working exist, but compromising time also compromises opportunities to build and maintain relationships. Different types of approaches have different 'transaction costs' and staffing implications (Law et al., 2002). Law (2000, p. 154) argues that "severe capacity limits have motivated the move towards consultancy services as a 'pragmatic solution to the problem of coverage" for SLTs in the UK, resulting in caseloads three times the manageable number (Law, 2000, in McCartney, 2009).

Findings suggest music therapy school consultants need to be familiar with consultation theory and a range 'human services' and 'psychological consultation' models, to choose a frame that matches their personality, skill levels, and values, and that will be appropriate for the contexts they find themselves in. Effective consultation involves examining the contribution and interaction of many variables (D. Brown et al., 2001; Dettmer et al., 2005). Consultants need to be aware that team members' expectations of possible and/or desirable consultation activities might differ from music therapists' understandings, and be able to describe the consultation process carefully, including examples from practice if possible. A contract should be established with the consultee prior to beginning the work.

Team collaboration, flexible funding, technology, training, access to experts, and knowing the outcomes of consultations, can improve the comfort levels of consultants (Wesley \& Buysse, 2004). Music therapists will need to ensure that they have enough time to build trusting relationships which enable them to work alongside consultees. Tim's teacher fervently reinforced the need for music therapists to actively help, particularly when things are tough, maintaining that it was not only essential from a practical point of view, but that it cemented the relationship and increased acceptance of the consultant as a team member. Professionals can be uncomfortable with colleagues who emphasise the 'expert' role, and are perceived as 'unwilling to get their hands dirty' (Wesley \& Buysse, 2004). On the other hand, 'expert' or specialised knowledge and/or skill are necessary to establish credibility in the eyes of consultees. Early childhood consultants report feeling pressurised to provide current accurate 
information, and even measured their effectiveness in terms of the technical correctness of the information they provided (ibid). However, expectations that music therapists will have in-depth knowledge about the full range of disabilities, and answers to all the challenges likely to be encountered are not reasonable. Rather, developing collaborative working relationships and communicating effectively with team members is a more realistic goal to ensure an effective consultation process.

The diversity of activities and approaches embraced by consultees in the current studies, reinforce Wesley \& Buysse's (2006) suggestion that consultees ultimately determine what they can realistically undertake given their resources, philosophies and beliefs, and that follow-through is likely to be enhanced when intervention strategies fit with the context. Consistent and overt valuing of their input in assessment and planning stages, at whatever level they were able to manage, enabled team members to readily identify their priorities, and to describe existing programmes and activities where planned music experiences might be situated.

Consultants expect that consultees will experience professional growth through contribution, and will be responsible for the implementation, and evaluation, of the strategies. However, care needs to be taken to ensure consultees do not perceive too many demands and expectations, tasks as difficult or complex, and/or their potential involvement as overwhelming. The experience of Rona, and her need for precise and detailed resources, indicates that an individual and flexible approach to planning is essential. However, despite her tendency to report minor barriers when implementing activities, Rona's diary entries became increasingly positive as she noticed Cathy beginning to develop a musical relationship with her, and her teacher's aide.

The unique nature of assessment for consultation has been highlighted in this study. Music therapists need to spend more time observing in classrooms, and meeting with teachers, to develop shared purpose, engage in collaborative planning, and jointly design and implement programmes (Threlfall, 2005). While interacting musically with students as they went about their daily activities, I was able to learn a great deal about their individual learning and development from others, and they about musical concepts from me. We learnt together as we musicked in natural and playful contexts. Studies illustrate that play activities exert strong influences on children's development (Berger \& Cooper, 2003: Vygotsky, 1978), and children experience and demonstrate 
musical growth during free play music sessions (Berger \& Cooper, 2003). During play, children participate in intrinsically motivated pleasurable actions where the process, not the product, is the goal (ibid). Clinical music therapy can of course be similarly child focused and playful, but consultees are not therapists. However, it is likely that some team members would be less natural and playful in a planned one-toone music session with a student.

Although the clinical music therapy assessment did not take primacy in the consultations as initially anticipated, the session remained a crucial aspect of the process. In the clinical setting, the music therapist can improvise music to encourage, support, and challenge students to enable them to demonstrate their potential for interpersonal relating. In the current study, recognition of this potential had great benefits for the participants. The success of music therapy consultations depends on team members recognising the benefits of using music, and believing that their musical interaction will make a positive difference in their students' life.

However, the untrained onlooker is often unaware of the music therapists' intentional use of various musical elements. The coordination and/or synchronicity that can be obtained between therapist and student might seem effortless (Snell, 1996). Videotape of the clinical session can be used to demonstrate specific strategies that would be helpful for team members to adopt, or improve on, in their music making. Still, it seemed that drawing out too much of the detailed interaction between music therapist and student would be intimidating, and unnecessary. Similarly, a substantial review of video material from single-session assessments, undertaken in stage one of this study, produced findings too detailed to be helpful to team members.

Importantly, conditions are optimal for building musical relationships in the clinical setting, even though the therapist might be a relative stranger to the student, whereas in more natural settings, interaction between the therapist and/or other team member/s and student is complicated by increased distractions, and/or more complex tasks. Thus the findings from clinical assessments demonstrate student skills that can potentially be developed in, but might not be immediately generalised to, other settings. Care needed to be taken therefore to ensure that team members did not feel inadequate when they are unable to elicit similar responses from their students. 
It is not possible in a study of this kind to differentiate the impact of the therapist's therapy skills, music skills, or other attributes that are brought to the work. However, some of the quotes from Tim's team suggest that they recognised my 'musical way of being' that is arguably likely to be a music therapist's particular way of being. Referring to her involvement in group music making, Pavlicevic noted that her music therapy skills of 'listening, tracking, witnessing, and becoming 'part of'... are activated almost automatically" (p.19). Perhaps music therapists have a unique presence, developed from extensive training and experience in observing and listening, and conveying non-verbal (usually musical) information in sensitively timed and emotionally charged ways. That some of these qualities (particularly those related to observing and listening to students, and carefully timing responses) were noticed and to a certain extent emulated by the teams, seems a very important finding.

Consultation involves the daunting tasks of discovering the strengths and needs of consultees as well as students, and the impact of the environment on their functioning. This reinforces the importance of collaborative efforts which use the diversity of knowledge, skills and experience within teams. However, self understanding is a prerequisite for coming to understand others, and consultants therefore also need to reflect regularly on their own observations, initial reactions, and behaviour, and to remain aware of the impact of their cultural values on interpretations. It is important to recognise 'uneasy' feelings, and rather than turning away from them, to reflect, come to an understanding, and make a plan of action. Reflexive journalling can be a useful clinical as well as research tool (Barry \& O'Callaghan, 2008; Rickson, 2009b), and is highly recommended as a typical collaborative consultation task, along with regular professional supervision. Given the complexities involved in the work, new music therapy consultants should also receive mentoring from peers experienced in consultation work.

\subsubsection{Time and Timing}

The five-day block of consultation time was highly valued and fully utilised by team members, purportedly more useful than attending workshops, and is a crucial element in the proposed music therapy school consultation protocol. However, it is likely that the amount of time needed to do consultations well will be challenged because of funding limitations, and that it will be necessary to continue to demonstrate value for 
money. Nevertheless, consultation success is likely to correlate with the amount of contact time between consultant and consultee (Jellison, 2000; McCartney et al., 2009).

The time is necessary to observe dynamic relationships at play in the classroom and other school settings, and to gain some understanding of the context for the work. Further, the block-of-time model is most helpful for developing therapeutic relationships with consultees. Closer interactions are likely to thrive where there is continuity of staffing, joint responsibility, and time to plan and discuss (McCartney, 2009). DiMeo et al. (in McCartney, 2009) suggest that building a collaborative relationship is like building a personal friendship, requiring time to develop and sustain. Positive working relationships can obviously be developed long term between professionals in stable appointments and positions who meet regularly. However, revisiting is likely to involve consultant and consultee having to warm-up and become re-acquainted with each other and the work context, to a certain extent, each time. One local music therapist recently suggested that it can take up to two years of regular visits before relationships with team members develop to the extent they are able to take on board her suggestions. The case studies demonstrate that the block-of-time model can enable trusting relationships to be developed within just one week, to a point where team members felt they could disclose personal information and/or strong political messages, which in turn aided my understanding of their situations. The relationships might be compared to 'holiday friendships', where intense interactions allow important connections to be made and sustained.

Further, while unexpected things do occur, it will be important to ensure that careful planning enables the time available to be used most efficiently. Preliminary arrangements need to be carefully negotiated with administrators, so that all core team members are aware of and can prepare for the visit, and can set aside time to engage with the work. Catching people 'on the run' can devalue the interaction (Tutty \& Hocking, 2004). Consultants will want to meet with specialist team members who are not necessarily based on site. These meetings need to be considered and planned in advance. The level of satisfaction that team members experience at the beginning of the process is likely to set the scene for the success of the consultation.

A further meeting with key people at the school on arrival, to clarify and negotiate 
more specific arrangements, will contribute to the smooth running of the consultation. Care should also be taken in managing time spent in consultant and consultee face-toface interactions, with or without the student. The intensity of the work can be reduced when each takes time out to complete independent tasks. Each of the case studies had unique pragmatic issues that needed to be attended to. For example, sensitive consideration needed to be given to issues such as the teachers' need for classroom cover, or parents' needs for childcare for students' siblings. The team meetings to view video of the music therapy assessment session are central to the consultations, but considerable negotiation and coordination is necessary to ensure their success.

Follow-up is also essential for providing support for consultees, and for evaluation purposes (see Evidence-based Practice, p. 297). The amount of contact initiated by consultees in the follow-up period was manageable for a music therapy consultant. A longer period might have revealed more information about the sustainability of the work and/or the support needs of team members.

\subsubsection{Systemic Issues}

Initial tasks for team members involved in music therapy school consultation include agreeing about where the work will take place (classroom, playground, withdrawal room and so on); which adults and children will be involved (principal, teacher, teachers' aides, therapists, referred student and/or peers and siblings); and clarifying, acknowledging and valuing different professional roles and areas of expertise.

Music therapy consultants will be undertaking assessment and providing direct intervention, only for the purposes of modelling strategies for consultees. Using the World Health Organisation taxonomy (2001) which suggests possibilities for a balance of focus between impairment, activity, and participation, the work is predominantly at the activity level, helping consultees to use music to support students to manage school tasks. Working at the level of the impairment, therapists engage in assessment, diagnosis and direct intervention; at the activity level they address the impact of the impairment on the individual's activities; while at the participation level they ensure that the individual's opportunities for participating fully are maximised (Gascoigne, 2008). The protocol suggests music therapists 
should always consider their options and influential potential in terms of ensuring that students' opportunities to participate fully are maximised. For example where schools are struggling to include students, the music therapist would be able to support by openly sharing his or her values, and offering practical ideas about music strategies that support inclusion. But it would be unrealistic to assume that team members' strongly held values could be influenced in such a short period of time, and inappropriate for the consultant to impose values upon them. The current findings suggest school and consultee cultural norms will position the work, and the most therapeutic solution is not always the most inclusive one (Carlsson, 2007).

\subsubsection{Evidence-based Practice}

Evaluation will be an ongoing duty for music therapy consultants in order to situate music therapy school consultation as an evidence-based practice. Evidence-based processes, drawn from the best available research, as well as experiential knowledge and understanding of families and professionals, should be used to make decisions about consultation services. Evaluation of music therapy school consultation aims to uncover whether team members are able to use music to support student learning and development, in an ongoing way. Positive student outcomes are presumed to follow, although to date other professionals have no strong evidence to show ongoing benefits for students (McCartney, 2009). Consultees are the ones who must judge effectiveness, because the implementation of strategies occurs after the consultant has left the field. However, it is also necessary to determine whether the strategies are being implemented in an appropriate way. Team members might resist ongoing implementation depending on the resources available to them, particularly if they are feeling isolated and/or unsupported. Rona, for example, disclosed a history of nonimplementation of programmes, for precisely these reasons.

Written evaluations and interviews, such as those conducted in the current study, provide opportunities for consultees to outline perceived benefits or difficulties that occurred during the consultation week, including their level of satisfaction with the process and content, and the consultant's interpersonal manner. Diary entries can provide evidence of how and when they engaged in ongoing implementation of the agreed intervention, and their experience of doing so. Statements of self-efficacy and commitment to ongoing music making are important indicators of consultee change, 
and in the current studies provided evidence of consultee learning and personal growth. Although the primary focus of consultation evaluation is on adult change, team members were also able to report positive outcomes for students that they associated with the introduction of therapeutic music activities.

None of the team members in this study resisted the idea of making a daily record of their musiking, and agreed that it was a manageable task. This suggests diaries will be a viable evaluation tool, particularly valuable because variables that are known to commonly impact on the effectiveness of programmes, such as student illness, staff absences, change in classroom programmes, family circumstances, and so on, can also be noted, and taken into account. One team chose to write in and photocopy pages from the 'daily diary' which is a typical resource in New Zealand, used for communication between home, school, and other professionals. Given that this is part of 'usual practice', the inclusion of information about the use of music, copied to the music therapist on a weekly or fortnightly basis, seemed a logical and user friendly way to exchange information.

Participants may have been highly motivated, and/or willing to make diary entries because they had committed to a research project. Register (2004) found child-care personnel who were taught to use music in the classroom were unable to implement and track new clinical practices, because scheduling constraints and mounting facility expectations took precedent. They did not feel that they had time during the day to document changes or assess their progress, forgot, or were unsure about what they should write.

In the current study, semi-structured interviews and focus groups provided opportunities for team members to learn about each other, develop a collaborative relationship, and evaluate progress made during the week of the onsite consultation. A follow-up interview also enabled the consultant to find out how team members managed after the consultant left the field. Wesley and Buysse (2004) suggest that 'interviewing' can also be part of the consultation process. The protocol therefore recommends an interview, timed to occur ten to twelve weeks after the consultant leaves the field, be undertaken for consultation evaluation purposes.

Although consultation data from journals/diaries and/or interviews would not have to 
be analysed as rigorously as is necessary for research purposes, it would be important for consultants to draw general themes, and to report findings appropriately to fund holders. Self-employed consultants will need to audit their records to help improve their practice, and publish them to help determine the effectiveness of consultation work. Published case studies also validate the work. In this study, videotape analyses of the individual music therapy sessions were undertaken at a detailed level. The analyses yielded significant data that was useful for research purposes, but is probably not sustainable as an ongoing practice as it is too time consuming.

While evaluating effectiveness it is an important part of a music therapy consultation protocol, it might be considered the most challenging because of the complexity of layers involved. It will not always be possible (or necessary) for music therapy consultants to effect change at a systems level, but it will be necessary to keep bigger issues in mind and to discuss how collaborative efforts might address these. In several of the case studies under discussion for example, inclusion issues became 'bigger' than initially anticipated. It would be possible for music therapists who are not inclusionists to implement the protocol. However, special education policy is leading music therapists to create contexts in which they can collaborate with colleagues to address the specific issue of using music to facilitate inclusion.

\subsubsection{Reporting Outcomes}

Music therapy school consultants should prepare written reports which describe the musical responses of the student, the agreed activities and resources, and the strategies that will be used to elicit positive student responses. These reports can then be made available to consultees and the students' parents or guardians, who would then be invited to present these to the six-monthly IEP meeting in order to determine how ongoing music or music therapy support will be provided.

On the other hand, information regarding consultee progress is confidential and should not be included in reports to other team members. It is the consultant's ethical responsibility to oversee the professional well-being of the consultee/s (Dougherty, 2005). Consultees might choose to share information and/or other team members might observe changes that they attribute to the consultation process, as Rona's principal did. In New Zealand, music therapy school consultants are likely to be 
employed by MoE SE and/or directly by schools, opening up the possibility that those who pay for the service will request information regarding consultee progress. Music therapists are aware of similar conflicts of interest that occur when professional supervision is paid for by employers. In order to maximise consultee engagement and openness during the consultation process, specific information regarding consultee progress should remain confidential and reporting should be limited to sharing timetables of shared activities and simple statements that confirm the consultee has engaged with the consultation process.

In extreme cases where teams seem dysfunctional or interventions appear to be carried out inappropriately, consultants will need to consider whether they can respect team members' choice to operate in a particular way, and still maintain their integrity. They might be in a position to bring a new and respected perspective, and have the power to influence practice. On the other hand, the consultant's input could be considered interfering and a nuisance, thus negating the potential for positive change, or the consultant's intervention could even highlight or strengthen their differences of opinion. Great care must therefore be taken in negotiating action. However, consultants also have a right, and responsibility, to break confidentiality when the safety of others is at risk. Ethical stances should be negotiated and clearly laid out in the consultation contract.

\subsubsection{The Development of Codes of Ethics and Professional Standards to Include Consultation}

Publication of this protocol will assist music therapists to engage formally and confidently with consultancy work. However, consultants need to know the limits of their competence, and music therapists will therefore need to consider how they might determine whether someone is competent to engage in music therapy school consultation. Findings from this study will be shared with Music Therapy New Zealand's Education, Training, and Professional Practice forum, and could contribute to the development of formal standards of practice. This process will involve music therapists determining what they value about consultation and being committed to those values. In the meantime music therapist 'consultants' should proceed with caution. The complexity of school consultation with its emphasis on triadic collaboration is mirrored by complex ethical issues relating to consultant, relationship, 
and process (Parsons, 1996, in Harrison, 2004; Wesley \& Buysse, 2006).

Ethical codes are often unspecific to allow for individual interpretation (Harrison, 2004). Ethical practice in music therapy school consultation can only be obtained through committed and considered reflection on issues such as ecological assessment, cultural contexts, and inclusion. For example, the case studies highlighted the probability that during the course of their practice music therapy consultants in New Zealand will interact with people who know them from other contexts, and that they might have had other personal or professional relationships with. Some consultees may have had previous professional or personal relationships with the music therapists' colleagues. The consultee might also begin to regard them as a friend. These scenarios highlight the importance of the music therapist consultant consistently applying reflective ethical thinking to their practice.

One of the consultees in the current study noted that in an attempt to reduce the effects of isolation, professionals had 'crossed boundaries' by addressing issues that were not strictly in their field of expertise. If not managed well, this could lead to professional rivalry and jealousy. Nevertheless, it is evident that music therapy school consultants will also need to comment on a variety of issues which may be the concern of professional colleagues, where they feel competent to do so. This strengthens the argument for close collaboration with allied professionals.

Informed consent prior to entering a consultation relationship ensures that consultees understand, and are willing to engage in an agreed process. However, as in action research, safe practice involves rigorous negotiation of ethical issues as events unfold. Ongoing reflective engagement between consultant and consultee can allow the process to evolve and take new directions. The potential for the misuse of power within the relationship is minimised, and the consultant is made more aware of ways in which they can appropriately help the consultees to adopt new perspectives and influence their participation, when issues are overtly discussed and negotiated.

\subsubsection{Managing Resistance and Conflict}

Findings suggest consultants need to make sincere efforts to respect personality and communication differences, and will need expertise in managing interpersonal 
conflict amongst team members, between team members and outside agencies, and possibly between consultant and consultee. They also need to remain aware of the roles that are being played out, and to avoid moving beyond empowering consultees to improve their work situations, to therapy or counselling relationships. Family and other team members wanted to disclose personal information to a caring person, and seemed to believe the music therapist consultant could meet that need. Music therapy consultants might be vulnerable targets for 'needy' staff and/or parents because they have good helping skills, desire to build close positive relationships in a short period of time, and are viewed as safe confidants because they are 'transient' i.e. they will be leaving the team within a relatively short period of time. Knowing how to manage this type of situation will be important, and might be more difficult for young or inexperienced music therapists.

Team members had a variety of working styles too, and these needed to be respected and accommodated. For some, it was necessary to break their tasks (as well as the student's tasks) down into small steps, to record these in the reporting package, to make extensive resources to support the work, and to adapt or add to these in the follow-up period. Other team members were more comfortable working in flexible ways, taking on board broad ideas that they could try with the student, and vary or adapt using their own initiatives.

If a problem is identified, the risks and benefits of each course of action need to be considered in the light of participant values, and existing practice and procedures. Once a course of action is decided it is important to clarify who will take responsibility for the outcome, and how it will be evaluated. All parties in a consultation need to take responsibility for the consequences of their decisions or actions. Ethical practice involves renegotiating when things don't seem to be working out, and correcting mistakes.

Several team members in the current studies felt isolated and abandoned by other visiting professionals, because visits were rare and unpredictable, and staff turnover was high. They were therefore frustrated, or disempowered and accepting of their lot. After feeling well supported by the consultant, team members may resist carrying on the work alone, stop doing it, and/or request excessive support during the follow-up period. Whilst empowerment primarily involves developing consultee's internal 
resources, they can also be supported in their work when connections are made with local resource people, such as the specialist music teacher exampled in John's case.

\subsubsection{Who will do the Work?}

There are considerable potential benefits in consulting for the music therapist, including the ability to determine how many hours and when he or she will work, the number and type of clients, and the extent and type of practice-building activities (Reuer, 1996). On the other hand, the need to spend a full week in the field will limit the number of music therapists who are able to undertake school consultations using the protocol herein. While some music therapists might be able to accept an occasional referral, those who have a clinical practice, work with clients on a regular basis, and/or have family or other commitments, will rarely have the opportunity to spend a full week away from home.

Moreover, the findings of this study suggest that inexperienced music therapists should be very cautious about engaging in consultation work. Reuer (1996) notes that creating and building an independent practice takes confidence, and suggests that particular personality traits and characteristics are necessary to succeed in independent practice. The protocol is therefore likely to be useful for experienced self-employed music therapists who choose to become known as consultants, in order to receive a steady stream of referrals.

However, given the highly positive findings of this study, agencies such as MoE SE should also be encouraged to consider employing music therapists who are able to work in this way (see 'Promoting Music Therapy Consultation', p. 304) perhaps balancing work close to home with time away doing week-long consultations.

\subsubsection{Consultation in New Zealand}

Interviews with New Zealand music therapists identified a paucity of consultation knowledge and practice. However, over recent years, there has been more discussion about music therapy consultation in New Zealand with increasing numbers now considering themselves to be music therapy consultants to children who have special education needs. The music therapy community is small, and growing awareness of 
the current study and the impact of the interviews themselves may have precipitated this discourse. The mode of delivery they describe usually involves regular visits to schools and appears to have less emphasis on empowerment and little or no reference to ecological assessment. The model espoused in this study does not claim to be more effective than other practices, but few other music therapists are writing about their work. Alternative frameworks need to be more formally described and published to allow for more debate.

\subsubsection{Promoting Music Therapy School Consultation}

Music therapy school consultants will need to ensure that potential service users understand the range of services that music therapists can provide, know about the clients they service, and can access case vignettes that exemplify the work, in order to understand the interventions that may be offered. Until they understand the impact music can have on student functioning, they are unlikely to request a music therapy service. For example, a school principal in this study confessed to being very sceptical about what could be achieved from a music therapy consultation if the student's behaviour was extremely difficult, and considered cancelling the appointment until his behaviour was more manageable. Moreover, special education teachers sometimes erroneously conclude that the student 'doesn't like music' (O'Regan, 2007) when they may have been over-stimulated in particular music settings. Further, some team members in the current study had little understanding of the possibilities for music to support a student's developmental or academic programme, and referral information did not always reflect what was eventually identified as teams' most urgent need/s. The assessment process uncovered unexpected opportunities for music to support student growth and learning. Nevertheless, there is general understanding that the majority of children respond positively to music and music therapy school consultation is likely to be a highly valued service. Educating other professionals about music therapy consultation processes and outcomes is likely to result in increased referrals, and more support from colleagues in other professional disciplines.

The current study highlighted discrepancies between what team members expected from visiting specialists, and what they were able to provide. Teams expressed strong feelings about the lack of support they perceived they received from other 
professionals, rare and unpredictable visits, and high staff turnover resulting in a disturbing lack of continuity. Although they attributed the lack of support to their geographical isolation, and were quick to explain that individual professionals were not to blame, people across teams were clearly experiencing extreme feelings of frustration and abandonment. Some were disempowered to the extent that they confessed they didn't ask for help because they didn't expect a response, or because they didn't think the level of support they would be offered would make any difference to their ongoing situation. Others became increasingly vocal until they received help when they were at the 'desperation stage'.

This signals the need for music therapy consultants to be clear about the limits of the service, so teams do not have too high expectations of the process, and/or become disappointed and discouraged from seeking help. On receipt of each referral it will be important for the consultant to explain the process and the relationships involved, and to identify the services they are qualified to offer. Where students have the potential to receive direct ongoing music therapy and/or indirect consultation, decisions regarding the services they receive should be based on thorough assessment of his/her school environment as well as their responses in a session with a music therapist.

\subsubsection{Getting the Team Together - Involving GSE Staff}

The assessment and consultation work undertaken prior to this study grew from referrals which had come from MoE SE as 'special projects', and staff eagerly contributed to the process. However, in Tim's case MoE managers supposed the workload would be too high to allow staff to be involved. This is possibly because of the research component, but the situation highlighted the need to investigate the role that MoE staff might play not only in the research process, but also in supporting a music therapy consultant, especially when referrals come from outside their own service. This is also relevant for students who have their funds administered by fund holding schools that in turn arrange for or provide specialist services.

Schools are likely to hold copies of MoE documents, but music therapists will want to meet professionals from other disciplines face to face in many instances, given their strong preference for collaboration. This is unlikely to occur unless SE Service Managers are approached in the first instance. It would be helpful if music therapists 
were able to attend the SE 'intake meetings' where the referrals are shared with the team. Intake meetings enable teams to pick up on the special relevance of a referral and determine who should be involved. Music therapists who receive referrals directly from families and/or schools should ask them for permission to contact the appropriate SE Service Manager to establish who should be involved in the process.

As noted above, teachers' aides take a prominent role in the education of students who have special education needs, and are likely to be frequently nominated to work with music therapy school consultants. While supporting teachers' aides' development and helping them to feel valued, thus improving their work with students, is clearly important, teachers have primary responsibility for students. When music therapists work directly with aides rather than teachers, inappropriate delegation of responsibility can be reinforced. It will be important for music therapists to continue to work towards involving classroom teachers and other team members as consultees.

Close collaboration with particular team members poses the risk that others 'on the fringe' may feel marginalised. Students have large numbers of support people, and not everyone can be closely involved in the music therapy consultation process. However, the findings suggest that the involvement of the 'core' team alongside the consultee/s was crucial to the success of the work. School teams are likely to decide internally who will be involved, and what role individuals will take.

\subsection{Implications for Training}

\subsubsection{A New Knowledge and Skill Base}

To date, there has been considerable confusion within the music therapy profession regarding what consultation involves. Some professionals (Hanft \& Place, 1996; McWilliam, 1995; Twyford, 2009) describe a continuum in which withdrawal and hands-on therapy is still considered to be consultation if the therapist addresses goals the teacher has identified and reports back after therapy, or ensures that therapy recommendations are incorporated in meaningful ways in a student's daily school activities.

Collaboration was identified in the current study as a critical factor in maximising the 
possibility that the consultations would be perceived as valuable, relevant, and useful by those on the receiving end of, or affected by the outcomes. Music therapists have strong interpersonal skills which can solidly underpin their efforts to build collaborative relationships with education team members. However, music therapists traditionally do not work within mainstream classrooms or natural environments, with large groups of typically developing children, or collaborate with adult educators. Collaboration is increasing (Register, 2002; Twyford, 2007; Twyford \& Watson, 2008a), especially among music therapists who are more experienced and confident in their practice (Register, 2002; Twyford, 2007). The literature also makes a clear distinction between collaboration and consultation.

The words 'consult', 'consultation' and even 'consultant' are frequently used in colloquial and/or rhetorical rather than professional terms. The use of small ' $c$ ' and big ' $\mathrm{C}$ ' might help to clarify this issue. Music therapists engage with out-of-class work which involves 'consulting' with team members by passing and receiving information in both formal and informal settings and/or via written notes or reports, and so on. Music therapists will continue to consult or collaborate with others as part of their clinical or community practice; or they might work as a 'Consultant'. However, 'Consultation' with capital ' $\mathrm{C}$ ' is a process, much more than advice-giving or conversing with another (Parsons \& Kahn, 2005). Rather, it is a unique paradigm of service conceptualisation and delivery, requiring changes in professional attitudes and orientation and additional skills (ibid). Music Therapy School Consultation is therefore likely to become a specialised practice, undertaken by experienced music therapists, trained in consultation.

\subsubsection{Training Needs of Music Therapists}

Inclusive philosophy and practice demand a change in professional roles, and the knowledge base and practices that have provided guidance to music therapists in the past are now inadequate. Music therapists who deliver direct service to students in clinic settings might not be able to easily transfer that expertise to working in the natural environment, in collaboration with others. School-based consultants are not just 'experts' who make recommendations or apply good process skills (Dougherty, 2005). The primary activity in music therapy consultation is to empower adults to work with students. This is a complex endeavour which involves applying social 
influence to build team members confidence, problem-solving how to embed planned music experiences in daily routines, and providing professional development to ensure team members have the skills to use music strategies effectively. Further, consultation work demands that music therapists be empathic diplomats, understanding and managing when, for example, teachers 'don't have time for a student with special education needs'. Not least, music therapy consultants will need to be 'specialists' and 'experts' who acknowledge the potential for power relationships to develop, yet can collaborate effectively to minimise the impact.

Other professionals have experienced uneasiness as their role changes to one of consultancy (D. Brown et al., 2000; Hanft \& Place, 1996; Wesley, 2002). Like music therapists, they are generally trained to work in their own clinics or classrooms, are challenged by the unique skills required for consultation work, and recognise the need for the introduction of professional development and standards in consultation practice. Early childhood consultants, for example, have been reportedly frustrated and uncomfortable when consultees have differing knowledge, skills, attitudes, and role expectations (for example, differing expectations about where the 'treatment' will take place, whether their teaching would be 'monitored', and whether consultants could provide 'immediate solutions' to problems). Providing consultation in contrasting environments was also described as a challenge. Their discomfort originated from the characteristics of specific programmes, and a feeling that programmatic factors, such as inadequate staff-child ratios, inappropriate curriculum, poorly trained staff, and high staff turnover, were out of their control (Wesley \& Buysse, 2004; Wesley et al., 2001). Similarly Brown et al. (2000) noted that NZ RTLB found the move to a consultancy role difficult, and a small but significant minority resisted the change.

Wesley and Buysse (2006) note that consultants need to operate simultaneously on two planes. Music therapy consultants will need to manage 'content', which involves considering students' developmental and learning needs with their musical responses, in context; and a 'process' which involves influencing, supporting and empowering consultees. The case studies reinforce arguments that it can be challenging for consultants to know whether to focus on child, family, teacher, or programme, and how collaborative efforts might be maximised to achieve lasting change (Wesley \& Buysse, 2006; Wesley et al., 2001). These challenges increase when consultees do not 
know what to expect from the process (Wesley \& Buysse, 2006).

Mental health professionals, including music therapists, are well positioned to develop collaboration as a preferred mode of delivery which involves sharing leadership, allowing for multiple experts and facilitating combined ownership of the problem and the solution (Dougherty, 2005). However, while music therapists are well schooled in interpersonal relationships, there are many tasks they will not be prepared for. Additional training will be required in consultation stages and strategies, cultural, ethical and professional issues, and adult skill acquisition. Music therapy school consultants will need a thorough understanding of education and special education philosophies, and have up-to-date knowledge of special education policies and procedures. Music therapists working alongside teachers also require a firm understanding of both teaching and therapy practise (Woodward, 2000). They will need to be well informed regarding teaching methods, curricula, and strategies to facilitate inclusion. Moreover, they will need to be committed advocates for the inclusion of students, through their consultation practice. They will need insight into teacher attitudes, and to know how to deal with, and resolve, interpersonal conflict. The problem-solving process involves both parties drawing on personal and professional theories to reflect on the issue/s as they perceive them, but the consultant also needs to understand the consultees perspective and consider how it might be expanded (Wesley \& Buysse, 2006).

Music therapists will also need training and experience in improvisational assessment procedures for consultation. Consultations rely on the therapist being able to make detailed observations; quickly bring meaning to those observations; simultaneously consider what music strategies might be helpful for meeting the child's needs and which of these would staff be able to employ; feeding back ideas to parents and staff using the appropriate professional approach; and managing personal, political, and sometimes powerfully emotional issues raised by team members. These skills are developed over time through clinical work and extensive contact and interaction with other professionals, including families.

Music therapy training programmes need to address the theoretical and empirical foundations of consultation, and provide opportunities for students to develop practical skills necessary for collaborative work. Kampwirth (1999, in Register, 
2002), describes consultation as a collaborative process in which a trained consultant assists one or more consultees in efforts to make decisions, and carry out plans, that will be in the best interest of their clients or patients. Music therapists interviewed during the current study felt strongly that appropriate skill and training was what defined them as music therapists. Applying the same analogy to school consultation, it is imperative that music therapists are specifically trained to work as consultants. Given the specialised nature of the work, and the suggestion that experienced music therapists are more likely to undertake the work, it might be most appropriate to offer additional training to music therapists who have had the opportunity, through several years experience of working with children, to assimilate their music therapy clinical training. Writing about SLTs in the UK, Law (2002), noted that less experienced therapists are more likely to undertake direct work, and argued for specialist recognition of practitioners who are very experienced in working in educational settings. Focusing on 'expertise' however fails to acknowledge the complexities and concerns regarding power relationships that also need to be addressed (Law et al., 2002).

Further, specific training in consultation does not guarantee that music therapists will have similar skill levels and experience, and their personalities will continue to vary. Consultant and consultee characteristics influence the selection of a consultation approach (Crothers et al., 2008), and music therapists are therefore likely to approach or carry out various consultation tasks differently, and this in turn is likely to influence outcomes. Further, consultee perception of the consultant (influenced by various factors such as age, gender, personality, ethnicity, and so on) is a significant indicator of the potential effectiveness of the consultation process (ibid).

\subsubsection{Training Other Professionals (Building Communities of Practice)}

Literature relating to community involvement in schools highlights the need to prepare professionals for working partnerships. Resistance can occur if schools fear public scrutiny; or if staff members have burnout, have accepted the norm of isolation, and/or have negative perception of students' communities (Sanders, 2003). Establishing partnerships with other professionals or communities outside of the immediate school can be challenging for staff. Not all teachers will value collaborative teamwork, particularly if they perceive it is being imposed on them. 
Therefore, it will be important for music therapist consultants to contribute to inservice teacher training, and to work with support staff, to help them to engage most efficiently in the music therapy school consultation process and effectively implement interventions. Moreover, combined workshops for music therapists and teachers (of music and regular classes) to explore possibilities for music therapy consultation could be highly beneficial, since it would model the beginnings of the collaborative process, and enable parties to learn from each other.

Wesley and Buysse (2004) note that the lack of comfort experienced by early childhood consultants and consultees raises questions about the effectiveness of consultation, and argue that there is an urgent need for professional development which brings together educators and consultants. In addition, findings from interviews with music therapists suggested that in general those interviewed had a tendency to hold on to the medical model of disability, and to take the 'expert' position of providing advice to school staff. This is probably because time is not available for ecological assessment and the development of therapeutic relationships with consultees. The practice of consultation is likely to be greatly enhanced when participants already have a broad understanding of what each can offer the other. Preconsultation workshops that bring teachers, teachers' aides and school therapists together to discuss what they need and what they can offer each other will provide a solid foundation for their collaboration and would improve consultation outcomes.

Music therapists also have a role in 1) training regular educators to work with students who have special education needs and 2) in training regular educators, as well as others training for careers in special education, to use music in their work. Robertson (2000) argues that a significant clinical dimension should underpin and inform music teacher training to prepare trainees for working with students who have different learning needs. Teachers and music therapists are likely to benefit from shared professional development opportunities, which aim to help them adapt and implement the music curriculum while accommodating the wide range of individual student need within the classroom. Moreover, this study has demonstrated that music can be a powerful tool to support students to access a variety of curriculum activities, not just music. Specific training and/or workshops can help educators and other specialises to use music in their work. This would also be a way of educating people about the 
difference between music therapy, and the planned use of music strategies by untrained professionals.

\subsection{Implications for Policy}

The issue of inclusion has had a significant impact on therapists in many fields, particularly those who have traditionally worked under a 'health' umbrella, and much can be learnt from their experiences. All music therapists need to pay attention to disability issues and inclusive philosophy, and reconsider the way music therapy is delivered. Community Music Therapy provides a welcome space for these considerations.

Specialist staff should work alongside educators within schools to support individual development (O'Brien \& Ryba, 2005). This study demonstrates that music therapists can provide considerable support for consultees and their students. Music therapy school consultation must therefore be promoted with policy makers to ensure it becomes a realistic option for team members who work with students who have high or very high education needs, and perhaps others.

However, in music therapy and other fields worldwide there is a lack of clarity regarding the practice of school consultation. Like others, New Zealand music therapists will need to consider issues at the student, practice, process, strategy, and governance levels. At the practice level, it is likely that some music therapists will welcome and draw on the findings to improve practice, others will find a number of the challenges articulated in this thesis uncomfortable. While the study attempts to open up new roles for music therapists who work with children who have special education needs, the work might be challenged by the music therapy community because 'traditional' 'clinical' ways of working are not being highlighted, and the

primary focus is on the needs of adults, albeit for the benefit of their students.

In addition, the findings imply the need for new ways of doing things in schools, which will also take time and money. Staff might need to be involved in professional development opportunities, as well as facilitating ongoing extra activities with students; resources might need to be made and/or purchased; and precious physical space might need to be found for the work. Further, closely examining school settings 
to identify the existence of resources and barriers to the successful implementation of a music therapy consultation process, might highlight areas for individual or school development that could cause team members discomfort. Nevertheless, it is possible to challenge assumptions about the supposed limits of inclusive education through collaborative and participatory practice (Armstrong \& Moore, 2004). The process involves sensitive team work which engenders trust, and which enables members to communicate any concerns to the group who will then be encouraged to collectively own and address them.

It is clear that one of the outcomes of the current research would be to examine possibilities for developing music therapy within MoE SE, an idea which has funding implications for this body too. However, if every ORRS-funded child who would benefit from music therapy input were considered eligible for service, regardless of their geographical location, it is clear that costs associated with the consultative model of music therapy (one-off travel, accommodation and living expenses; travel time in addition to the forty hours of contact, report writing, and production of resources; and follow-up time), would be less than travelling each week to every school to provide a period of ongoing clinical work.

However, examination of the potential costs involved in music therapy school consultations was deliberately avoided in this study. Comparing the costs of contrasting models would seem to be unhelpful, especially when they serve different purposes, and/or there might be significant differences in their perceived levels of helpfulness. Tim's team, for example, reinforced Register's (2004) argument that consultation is more effective than having one person attend a professional development course and being asked to relay the information to others. Further, although cost analysis identified indirect therapy as the least costly of speech and language intervention modes (Dickson et al., 2009), time limitations have been found to affect teachers' ability to provide direct input, and the amount of tailored activity that is carried out by consultees is directly related to outcomes (McCartney, 2009).

Finally, consultation should not be used as a way of maximising the numbers of students who can be seen, regardless of their needs. When working for government or private agencies, the music therapist consultant will need to consider and negotiate a model of work which ensures that teams receive an adequate service which is of 
benefit to each of the students on their caseload, and which enhances the reputation of the music therapy profession. Students who are isolated do not currently receive adequate therapy support, and music therapy consultation was decidedly instrumental in improving the quality of living and learning for students in this study. The MoE aims to provide high quality education to all students, including those who have high or very high learning needs, in the parent or caregiver's choice of setting. Students are entitled to the support they need to maximise their developmental and learning opportunities while at school, and a music therapy consultation protocol which offers support to educators to help them do this, should be keenly utilised.

Findings from this study reinforce the need for music therapy to be an integral service within MoE SE who, at the time of writing, have only employed two music therapists, in part time positions. Registered Music Therapists are recognised as specialists who can provide a valuable service to ORRS funded students. Music therapy school consultations should therefore be available to students via ORRS fund holders. That is, they should be mainly provided by music therapists working for, or subcontracted to, MoE SE.

\subsection{Future Research}

Consultation and collaboration have become important roles for many professionals, and practice has become increasingly research based (Zins, 2002, in Dougherty, 2005). However, there is no widespread agreement on the definition of consultation, and the continued ambiguous use of this term, makes quality research on the subject difficult (Dougherty, 2005). Further research will be useful to gather evidence about which consultation models are most effective, in what contexts, and for whom; what constitutes good practice; and how results might be measured in terms of student, consultee, and/or programme outcomes (Wesley \& Buysse, 2006).

From a music therapy perspective, in the short term more information is needed about current practice and/or consumer needs to determine the potential need for music therapy consultants. It would be useful to collect demographic data from music therapists working in schools which details numbers students seen regularly, occasionally, or who are on waiting lists; how long they wait, are in therapy, or are followed up; whether a designated person continues with music, and so on. A survey 
to all schools, to determine their potential interest in music therapy consultation, would provide much needed information for policy makers and fund-holders.

The protocol developed in this study focuses on students who have high or very high special education needs in primary school settings. It is possible that the way music therapy school consultants approach the work will be affected by students' levels of ability, and whether they are included at primary, intermediate, or secondary schools. For example, more able students are more likely to be included in mainstream music classes, and the consultant might provide high level curriculum support through the provision of resources, and focus less on consultee/student interaction. It will be important therefore to explore 1) whether and how the current protocol is adopted or adapted by music therapists, 2) the utility of any significantly different protocols and 3) the relationship of the protocol to the specific needs of consultees and/or students (e.g. students with moderate needs).

Consultation evaluation can be undertaken at the student, consultee, or programme/systems level. Demonstrating how a student benefits from an intervention that focuses on improving the knowledge and skills of another team member, is a major challenge to both practitioners and researchers (Wesley \& Buysse, 2004). Nevertheless, researchers should continue to aim to document the way in which music assists students' to manage their learning and behaviour in their daily routines in the classroom, playground or other environs.

Well documented IEPs could provide helpful data for research purposes, and single case studies using IEP data might provide an indication of the impact a consultation has had on student progress. However, with multiple professionals involved in working with the students it would be very difficult to attribute change specifically to the music therapy consultation. On the other hand, qualitative studies can uncover the impact of music therapy input on consultee and student lives, and action research has proven to be a useful tool in this regard. Issues of quality of life and inclusion may require designs that allow reports of long-term effects as well as transfer and generalisation of outcomes (Jellison, 1979). It will be important to continue to strive to obtain staff and student experience of being involved in music therapy consultations. 
Further case studies based on the utilisation of the protocol outlined in this research, by other music therapists working with students who have high or very high needs in geographically isolated areas, are needed since the consultants' personalities and skill levels are likely to have an impact on outcomes. In time, investigations which focus on consultant communication styles, for example the level of directiveness used with consultees, and how that might affect consultation outcomes, would be valuable additions to the literature.

Case studies will be improved with the inclusion of more intensive and longer periods of follow-up, which involve observation rather than consultee self-report, to learn more about consultees' abilities to maintain and develop their therapeutic use of music, and to investigate whether a music therapy consultation makes a difference to consultee/student outcome in the long term. It would also be helpful to focus on the specific needs of parents and/or whanau involved in home based consultations. Music therapy consultants might also introduce the protocol into their work with other populations (e.g. Lisa with adults, p. 109).

Consultants are concerned with learning about communication between parties, how roles are being played out and professional boundaries are managed, whether adequate resources are available, the level of team members' responsiveness, and so on (Wesley \& Buysse, 2006). As consultation is more widely practiced, researchers should draw on the collective expertise of teachers and music therapy consultants to find out what they believe contributes to consultation effectiveness, how that knowledge can be used to support the training needs of music therapists, and subsequently to improve practice. An investigation into how collaboration and consultation techniques are acquired and implemented by music therapists could answer questions about how these skills can be taught. Over time meta-analysis of rigorous and well presented case studies could provide answers to many of the above questions.

It will also be important to determine if and how the protocol outlined in this thesis might apply to multicultural and cross-cultural experiences and what theoretical and/or practical changes are needed to provide effective consultation services with diverse populations. Consultations based on the protocol have already been undertaken at a bilingual school in Thailand where the students were familiar with 
Western music styles. The sharing of music helped bridge the cultural gap, and the process was supported by committed staff who were enthusiastic and believed in music as a therapeutic medium (Rickson, 2009c). However, although inclusive philosophy has been mandated by the Thai government, the medical model of disability still appears to prevail in Thailand. Developing a culture of collaboration and empowering staff and parents to contribute equally was difficult, as staff and parents consistently deferred to the music therapist's expertise. While designated staffs are continuing to facilitate music strategies, they might have difficulty adapting or introducing new ideas to sustain the work beyond the short term.

When music therapy school consultation is well established, music therapists might engage in cross-disciplinary research assisting educators such as school psychologists in implementing reform efforts. 


\section{CONCLUSION}

Music therapy school consultation can be an effective way for music therapists to provide a service to students who have special education needs. Findings demonstrate that building collaborative relationships with team members while taking account of the contexts in which they work, empowered them to introduce music into their programmes in planned and intentional ways. Their use of music enhanced their relationships with students, and in turn engendered improved opportunities for student learning and development.

Nevertheless, the findings are based on the work of one music therapist and further exploration is needed. For example, the different personalities of music therapists might lead them to draw on other theories to support their consultation work; and the protocol may need to be adapted when working with students with different abilities, those in intermediate and/or secondary school settings, from different cultures, and so on. Further, there are still questions to be answered regarding practice possibilities. More research is needed to scope the current demand for music therapy school consultation and the costs involved; and policy makers and consumers will need to be made aware of the potential service. However, before the potential for music therapy school consultation is increased, it will be important to gauge music therapist interest in the work and to attend to their training needs. The findings suggest music therapists are unprepared for consultation work, and that it will be most suitable for experienced music therapists following advanced training.

It is appropriate to introduce a protocol based on social learning theory at a time when ecological approaches are increasingly being discussed in relation to both music therapy and special education fields. Nevertheless, while the concept of community music therapy provides a space for the work described in this thesis, the idea that other professionals can be empowered to use music therapy strategies is still likely to be challenged by some music therapists. Music therapist resistance to consultation work is likely to be due at least in part to fears that consultation will reduce opportunities for clinical work. However, music therapy input should be consistently based on student need determined after a full assessment. As the profession of music therapy continues to grow it must strive to offer a full range of music therapy support options including direct intervention as well as consultation services. That it might be difficult to define consultation as 'music therapy' is acknowledged in the thesis, yet 
given the positive findings the possibilities for music therapists to work as consultants is vigorously defended.

As Twyford (2007) noted, in addition to new insights researchers gain new enthusiasm for practice when they achieve a synthesis of practice and research. Despite a keen interest to support team members to use music in their work, this study began cautiously with the knowledge that it was likely the concept of consultation would be difficult for music therapists to accept. Throughout the process caution gave way to a passion for music therapy consultation and action research processes, and a conviction that both will be crucial for the successful growth of the music therapy profession in the future. 


\section{REFERENCES}

Aasgaard, T. (1999). Music therapy as milieu in the hospice and paediatric oncology ward. In D. Aldridge (Ed.), Music Therapy in Palliative Care. London: New Voices.

Abraham, S. (1994). Exploratory action research for manager development. Upper Mt Gravatt, Australia: Action Learning, Action Research \& Process Management Society (ALARPM) Inc. and Gibaran Management Consultants Pty Ltd.

Abrams, B. (2005). Evaluating qualitiative music therapy research. In B. L. Wheeler (Ed.), Music therapy research (pp. 246-258).

Aigen, K. (1997). Here we are in Music. One Year With an Adolescent Creative Music Therapy Group (Vol. 2). Saint Louis, MO: MMB Music, Inc.

Aigen, K. (1999). The true nature of music-centred music therapy theory. British Journal of Music Therapy, 13(2), 77-82.

Aigen, K. (2008). An analysis of qualitative music therapy research Reports 1987-2006: Doctoral studies. Arts in Psychotherapy, 35(5), 307-319.

Ala-Ruona, E. (2005). Non-structured initial assessment of psychiatric clients in music therapy. Music Therapy Today, , 23-47. Retrieved from http://musictherapyworld.net.

Aldridge, D. (1993). Music therapy research: Research methods suitable for music therapy. Arts in Psychotherapy, 20(2), 117-131.

Aldridge, D. (2005). Looking for the why, how and when. In D. Aldridge (Ed.), Music therapy and neurological rehabilitation. London: Jessica Kingsley Publications.

Aldridge, D., \& Aldridge, G. (2002). Therapeutic narrative analysis: A methodological proposal for the interpretation of music therapy traces. from http://musictherapyworld.net.

American Music Therapy Association. (2009). AMTA Standards of Clinical Practice. Retrieved 21st July 2009, from http://www.musictherapy.org/standards.html

Annan, J. (2005). Ecological practice. Kairaranga, 6(2), 10-18.

Ansdell, G. (1996). Talking about music therapy. A dilemma and a qualitative experiment. British Journal of Music Therapy, 10(1), 4-16.

Ansdell, G. (1999). Challenging premises. British Journal of Music Therapy, 13(2), 72-76.

Ansdell, G. (2002). Community music therapy and the winds of change. Voices: A World Forum for Music Therapy, Retrieved from http://wwwvoices.no/mainissues/Voices.

Ansdell, G. (2005). Being who you aren't; doing what you can't: Community Music Therapy 
and the paradoxes of performance. Voices: A World Forum for Music Therapy, Retrieved from http://www.voices.no/mainissues/mi40005000192.html.

Archer, C. (1995). Music in early intervention: A survey. Annual Journal of the New Zealand Society for Music Therapy, 46-54.

Archer, C. (2004). Music therapy and early intervention: The parent-child relationship is centre stage. New Zealand Journal of Music Therapy, 36-49.

Armstrong, F., \& Moore, M. (2004). Action research: Developing inclusive practice and transforming cultures. In F. Armstrong \& M. Moore (Eds.), Action research for inclusive education: Changing places, changing practices, changing minds (pp. 116). London: RoutledgeFalmer.

Athanasiou, M. S., Geil, M., Hazel, C. E., \& Copeland, E. P. (2002). A look inside schoolbased consultation: A qualitative study of the beliefs and practices of school psychologists and teachers. School Psychology Quarterly, 17, 258-298.

Austin, J., Renwick, J., \& McPherson, G. E. (2006). Developing motivation. In G. E. McPherson (Ed.), The child as musician (pp. 213-238). Oxford Oxford University Press.

Baker, F. (2007). Enhancing the clinical reasoning skills of music therapy students through problem based learning - An action research project. Nordic Journal of Music Therapy, 16(1), 27-41.

Baker, F. (2008). The Individualized Music Therapy Assessment Profile. Nordic Journal of Music Therapy, 17(2), 174.

Bandura, A. (1977). Social learning theory. Englewood Cliffs, NJ: Prentice Hall.

Barry, P., \& O'Callaghan, C. (2008). Reflexive journal writing: A tool for music therapy student clinical practice development. Nordic Journal of Music Therapy, 17(1), 5567.

Baxter, H. T., Berghofer, J. A., MacEwan, L., Nelson, J., Peters, K., \& Roberts, P. (2007). The Individualised Music Therapy Assessment Profile. London and Philadelophia: Jessica Kingsley Publishers.

Beaglehole, J. C. (Ed.). (1963). The endeavour journal of Joseph Banks: 1768-1771 (2nd ed.). Sydney: The Trustees of the Public Library of New South Wales.

Bergan, J. R. (1977). Behavioral consultation. Columbus, OH: Merrill.

Bergan, J. R., \& Kratochwill, T. R. (1990). Behavioral consultation and therapy. New York: Plenum.

Berger, A., \& Cooper, S. (2003). Musical play: A case study of preschool children and parents. Journal of Research in Music Education, 51(2), 151-166. 
Bertolami, M. D., \& Martino, L. A. (2002). Music therapy in a private school setting for children with multiple handicaps who are visually impaired or blind. Voices: A World Forum for Music Therapy, 2(1), Retrieved from http://wwwvoices.no/mainissues/Voices2(1)Bertolami.

Bland, M. (2003). All the comforts of home? A critical ethnography of residential aged care in New Zealand. University of Newcastle.

Boisvert, S., Labbe, L., \& Kane-Benveniste, M. (2000). Music therapy in the Quebec school system: Association Quebecoise de Musicotherapie.

Bonner, M., \& Barnett, D. W. (2003). Intervention-based school psychology services: Training for child-level accountability; preparing for program-level accountability. Journal of School Psychology, 42, 23-43.

Booth, R. (2004). Current practice and understanding of music therapy in Victorian special schools. Australian Journal of Music Therapy, 15, 64-75.

Bowler, J. (2005). Ecological and developmental issues in the practice of educational psychology. Kairaranga, 6(2), 49-54.

Boxill, E. H. (1985). Music therapy for the developmentally disabled. Austin, Texas: Pro-Ed, Inc.

Boyle, J., McCartney, E., Forbes, J., \& O'Hare, A. (2007). A randomised controlled trial and economic evaluation of direct versus indirect and individual versus groups modes of speech and language therapy for children with primary language impairment. Health Technology Assessment, 11(25), 1-158.

Bray, J. N., Lee, J., Smith, L. L., \& Yorks, L. (2000). Collaborative inquiry in practice: Action, reflection, and making meaning. California: Thousand Oaks.

Bronfenbrenner, U. (1979). The ecology of human development. Experiments by nature and design. Cambridge, Massachusetts: Harvard University Press.

Bronfenbrenner, U. (1989). Ecological systems theory. Annals of Child Development, 6, 187249.

Brooks, A., \& Watkins, K. E. (1994). A new era for action technologies: A look at the issues. In A. Brooks \& K. E. Watkins (Eds.), The emerging power of action inquiry technologies. New directions for adult and continuing education (Vol. 43, pp. 5-16). San Francisco: Jossey-Bass.

Brotons, M. (2001). Music therapy in the school system in Spain: A pilot project. Bulletin of the Council for Research in Music Education, 148, 19-30.

Brown, D., Pryzwansky, W. B., \& Schulte, A. C. (2001). Psychological consultation and collaboration: An introduction to theory and practice (5th ed.). Boston: Pearson 
Education, Inc.

Brown, D., Pryzwansky, W. B., \& Schulte, A. C. (2006). Psychological consultation and collaboration: An introduction to theory and practice (6th ed.). Boston: Pearson Education, Inc.

Brown, D., \& Schulte, A. (1987). A social learning model of consultation. Professional Psychology: Research and Practice, 18(283-287).

Brown, D., Thomson, C., Anderson, A., Moore, D. W., Walker, J., Glynn, T., et al. (2000). Resource Teachers Learning and Behaviour: an ecological approach to special education. Australasian Journal of Special Education, 24(1), 5-20.

Brown, S. (1999). Some thoughts on music, therapy, and music therapy. British Journal of Music Therapy, 13(2), 63-71.

Brownell, M. D. (2002). Musically adapted social stories to modify behaviors in students with autism: Four case studies. Journal of Music Therapy, 39(2), 117-144.

Brunk, B. K., \& Coleman, K. A. (2000). Development of a special education music therapy assessment process. Music Therapy Perspectives, 18(1), 59-68.

Bruscia, K. E. (1987). Improvisational models of music therapy. Il: Charles C. Thomas.

Bruscia, K. E. (1988). Standards for clinical assessment in the arts therapies. The Arts in Psychotherapy, 15, 5-10.

Bruscia, K. E. (1989). Defining music therapy. Phoenixville: Barcelona Publishers.

Bruscia, K. E. (1998). Defining Music Therapy (2nd ed.). Gilsum, NH: Barcelona Publishers.

Bruscia, K. E. (1998). Standards of integrity for qualitative music therapy research. Journal of Music Therapy, 35(3), 176-200.

Bruscia, K. E. (2005). Data Analysis in Qualitative Research. In B. L. Wheeler (Ed.), Music Therapy Research (2nd ed., pp. 179-196). Gilsum, NH: Barcelona.

Bunt, L. (1994). Music therapy, an art beyond words. London: Routledge.

Caplan, G. (1970). The theory and practice of mental health consultation. New York: Basic Books.

Caplan, G., Caplan, R. B., \& Erchul, W. P. (1994). Caplanian mental health consultation: Historical background an current status. Consulting Psychology Journal: Practice and Research, 46(2-12).

Cardno, C. (2003). Action Research: A developmental approach. Wellington: New Zealand Council for Educational Research. 
Carlson, G. A. (2007). Who are the children with severe mood dysregulation, a.k.a. "rages"? American Journal of Psychiatry, 164(8), 1140-1142.

Carlsson, C. S., Hocking, C., \& Wright-St Clair, V. (2007). The 'why' of who we are. Kairaranga, $8(2)$.

Cartledge, G., \& Johnson, C. T. (1996). Inclusive classrooms for students with emotional and behavioral disorders: Critical variables. Theory Into Practice, 35(1), 51-57.

Chase, K. M. (2004). Music therapy assessment for children with developmental disabilities: A survey study. Journal of Music Therapy, 41(1), 28-54.

Chester, K. K., Holmberg, T. K., Lawrence, M. P., \& Thurmond, L. L. (1999). A programbased consultative music therapy model for public schools. Music Therapy Perspectives, 17(2), 82-91.

Clegg, J. (2004). Practice in focus: A hermeneutic approach to research ethics. British Journal of Learning Disabilities, 32(4), 186-190.

Coleman, K. A. (2002). Music therapy for learners with severe disabilities in a public school setting. In B. L. Wilson (Ed.), Models of music therapy interventions in school settings: from institution to inclusion (pp. 197-199). Silver Springs, MD: National Association for Music Therapy Inc.

Conoley, J. C., \& Conoley, C. W. (1992). School consultation: Practice and training (2nd ed.). Needham Heights, Massachusetts: Allyn \& Bacon.

Crothers, L. M., Hughes, T. L., \& Morine, K. A. (2008). Theory and cases in school-based consultation: A resource for school psychologists, school counselors, special educators, and other mental health professionals. New York, NY: Routledge.

Crotty, M. (1998). The foundations of social research: Meaning and perspective in the research process. St Leonards: Allan \& Unwin.

Croxson, M. (1999). Music therapy and families. Annual Journal of the New Zealand Society for Music Therapy, 40-49.

Croxson, M. (2002). New Zealand and music therapy - A synopsis of a new scene. In C. Kenny \& B. Stige (Eds.), Contemporary voices in music therapy (pp. 183-186). Oslo, Norway: Unipub.

Darrow, A. A. (1999). Music educators' perceptions regarding the inclusion of students with disabilities in music classrooms. Journal of Music Therapy, 36(4), 254-273.

Darrow, A. A. (2002). Research on mainstreaming: Implications for music therapists. In B. L. Wilson (Ed.), Models of Service Delivery and their Relation to the IEP (pp. 4167). Silver Spring, MD: The American Music Therapy Association Inc.

Darrow, A. A., \& Grohe, H. S. (1996). Music therapy for learners who are deaf or hard-of- 
hearing. In B. L. Wilson (Ed.), Models of music therapy interventions in school settings (pp. 291-311). Silver Spring, MD 20910: American Music Therapy Assn, Inc.

Darrow, A. A., \& Loomis, D. M. (1999). Music and Deaf culture: images from the media and their interpretation by deaf and hearing students. Journal of Music Therapy, 36, 88-109.

Daveson, B., \& Edwards, J. (1998). A role for music therapy in special education. International Journal of Disability, Development and Education, 45(4), 449-455.

Davis, W. B., Gfeller, K. E., \& Thaut, M. H. (1992). An Introduction to Music Therapy: Theory and Practice. Dubuque, IA: W.C.Brown.

de I'Etoile, S. K. (2001). An in-service training program in music for child-care personnel working with infants and toddlers. Journal of Research in Music Education, 49(1), 620 .

DeBedout, J. K., \& Worden, M. C. (2006). Motivators for children with severe intellectual disabilities in the self-contained classroom: A movement analysis. Journal of Music Therapy, 43(2), 123-135.

Delamont, S. (1992). Fieldwork in educational settings. Methods, pitfalls and perspectives. London: The Falmer Press.

Dettmer, P., Thurston, L. P., Knackendoffel, A., \& Dyck, N. J. (2005). Collaboration, consultation, and teamwork. Columbus, Ohio: Pearson.

Dey, I. (1993). Qualitative data analysis. A user-friendly guide for social scientists. London: Routledge.

Dickson, K., Marshall, M., Boyle, J., McCartney, E., O'Hare, A., \& Forbes, J. (2009). Cost analysis of direct versus indirect and individual versus group modes of manual-based speech-and-language therapy for primary school-age children with primary language impairment. International Journal of Language \& Communication Disorders, 44(3), 369-381.

Dougherty, A. M. (2005). Psychological consultation and collaboration in school and community settings. Belmont, CA: Thomson Brooks/Cole.

Duerksen, G. L. (1991). Music class for the at-risk. Music Educators Journal, 78(3), 46-50.

Duerksen, G. L., \& Darrow, A. A. (1991). Music class for the at-risk. Music Educators Journal, 78(3), 46-50.

Elliott, J. (1991). Action research for educational change. Milton Keynes: Open University Press.

Ely, M., Vinz, R., Downing, M., \& Anzul, M. (1997). On writing qualitative research: 
Living by words. Bristol, PA: The Falmer Press.

Erchul, W. P., \& Sheridan, S. M. (2008). Overview. The state of scientific research in school consultation. In W. P. Erchul \& S. M. Sheridan (Eds.), Handbook of research in school consultation (pp. 3-12). New York, NY: Lawrence Erlbaum Associates.

Facchini, D. (2001). Music therapy in the educational system. Paper presented at the Music Therapy in Europe - Vth European Music Therapy Congress, Naples.

Feltham, S. (2004). Where special is normal. Education Gazette, 83(18), np.

Feuerstein, R., \& Feuerstein, S. (1991). Mediated learning experience: A theoretical review. In R. Feuerstein, P. Klein \& A. Tannenbaum (Eds.), Mediated learning experience (MLE). Theoretical, psychological and learning implications. London: Freund Publishing House Ltd.

Flower, C. (1993). Control and creativity. In M. Heal \& T. Wigram (Eds.), Music therapy in health and education (pp. 40-45). London: Jessica Kingsley.

Foucault, M. (1977). Discipline and punishment. London: Allen Lane.

Freire, P. (1972). Pedogogy of the oppressed. New York: Herder and Herder.

Frost, L., \& Bondy, A. (2002). The picture exchange communication training manual (2nd Edition ed.). Delaware: Pyramid Educational Consultants.

Gantt, L. (2000). Assessments in the creative arts therapies: Learning from each other. Music Therapy Perspectives, 18(1), 41-46.

Gascoigne, M. (2006). Supporting children with speech, language and communication needs within integrated children's services. London: Royal College of Speech and Language Therapists

Gascoigne, M. (2008). Change for children with language and communication needs: creating sustainable integrated services. Child Language Teaching and Therapy, 24, 133-154.

Gaventa, J., \& Cornwall, A. (2001). Power and knowledge. In P. Reason \& H. Bradbury (Eds.), Handbook of action research. Participative inquiry and practice (pp. 70-80). London: Sage Publications.

Gilboa, A. (2007). Testing the MAP: A graphic method for describing and analyzing music therapy sessions. Arts in Psychotherapy, 34(4), 309-320.

Giordano, E. (2002). The dynamics of arts integration. Music Therapy Today, Retrieved from http://musictherapyworld.net.

Glaser, B., \& Strauss, A. (1967). The Discovery of Grounded Theory. Chicago: Aldine. 
Gold, C., Voracek, M., \& Wigram, T. (2004). Effects of music therapy for children and adolescents with psychopathology: a meta-analysis. Journal of Child Psychology and Psychiatry and Allied Disciplines, 45, 1054-1063.

Goodman, K. D. (2007). Music therapy groupwork with special needs children. Springfield, Illinois: Charles C. Thomas Publisher, Ltd.

Gray, C. (1994). The new social story book - illustrated edition. Arlington, TX: Future Horizons.

Gunsberg, A. (1988). Improvised musical play: A strategy for fostering social play between developmentally delayed and nondelayed preschool children. Journal of Music Therapy, 25(4), 178-191.

Hanft, B. E., \& Place, P. A. (1996). The consulting therapist: A guide for OTs and PTs in schools. San Antonia, Texas: Therapy Skill Builders.

Harding, C., \& Ballard, K. D. (1982). The effectiveness of music as a stimulus and as a contingent reward in promoting the spontaneous speech of three physically handicapped preschoolers. Journal of Music Therapy, 19(2), 86-101.

Harrison, T. C. (2004). Consultation for contemporary helping professionals. Boston: Pearson Education, Inc.

Hartas, D. (2004). Teacher and speech-language therapist collaboration: being equal and achieving a common goal? Child Language Teaching and Therapy, 20, 33-54.

Hasselbusch, A., \& Penman, M. (2008). Working together. An occupational therapy perspective on collaborative consultation. Kairaranga, 9(1), 24-31.

Heine, C. C. (2002). Inservice training: A major key to successful integration of special needs children into music education classes. In Models of service delivery and their relation to the IEP (pp. 78-110). Silver Spring, MD: The American Music Therapy Association.

Heron, J. (1996). Co-operative inquiry: Research into the human condition. London: Sage.

Ho, A. (2004). To be labelled, or not to be labelled: That is the question. British Journal of Learning Disabilities, 32(2), 86-92.

Holck, U. (2007). An ethnographic descriptive approach to video microanalysis. In T. Wosch $\&$ T. Wigram (Eds.), Microanalysis in music therapy: methods, techniques and applications for clinicians, researchers, educators and students London ; Philadelphia: Jessica Kingsley.

Hughes, J. E., Robbins, B. J., McKenzie, B. A., \& Robb, S. L. (1990). Integrating exceptional and nonexceptional young children through music play: A pilot program. Music Therapy Perspectives, 8, 52-56. 
Humpal, M. E. (1991). The effects of an integrated early childhood music program on social interaction among children with handicaps and their typical peers. Journal of Music Therapy, 28(3), 161-177.

Hunt, M. (2005). Action research and music therapy: Group music therapy with young refugees in a school community. Voices: A World Forum for Music Therapy, 5(2), Retrieved April 9, 2006, from http://wwwvoices.no/mainissues/mi40005000184.

Ingraham, C. L., \& Meyers, J. (2000). Introduction to multicultural and cross-cultural consultation in schools: Cultural diversity issues in school consultation. School Psychology Review, 29(3), 315-319.

Isenberg-Grzeda, C. (1988). Music therapy assessment: A reflection of professional identity. Journal of Music Therapy, 25, 156-169.

Jackson, N. A. (2003). A survey of music therapy methods and their role in the threatment of early elementary school children with ADHD. Journal of Music Therapy, 40(4), 302323.

Jeanneret, N. (2006). The national review of music in schools and the endless debate about music in primary schools. Australian Journal of Music Education, 2006, 93-97.

Jellison, J. A. (1979). The music therapist in the educational setting: Developing and implementing curriculum for the handicapped. Journal of Music Therapy, 16(3), 126137.

Jellison, J. A. (2000). A content analysis of music research with disabled children and youth (1975-1999): Applications in special education. In Effectiveness of music therapy procedures. Documentation of research and clinical practice (3rd ed., pp. 199-264). Silver Spring, MD 20910: American Music Therapy Assn.

Jellison, J. A. (2006). Including everyone. In G. E. McPherson (Ed.), The child as musician (pp. 257-272). Oxford: Oxford University Press.

Johansson, A. W., \& Lindhult, E. (2008). Emancipation or workability? Action Research, $6(1), 95-115$.

Johnson, F. (2002). Models of service delivery and their relation to the IEP. In B. L. Wilson (Ed.), Models of music therapy interventions in school settings (2nd ed., pp. 83-107). Silver Spring, MD: The American Music Therapy Association, Inc.

Jones, L. L., \& Cardinal, D. N. (1998). A descriptive analysis of music therapists' perceptions of delivering services in inclusive settings: A challenge to the field. Journal of Music Therapy, 35(1), 34-48.

Kearney, A. (2008). Barriers to school inclusion: An investigation into the exclusion of disabled students from and within New Zealand schools. Unpublished Doctor of Philosophy in Education, Massey University, Palmerston North, NZ. 
Kelly, A. (2008). What works for one: Practice considerations for supporting a child or young person with autism spectrum disorder, drawn from participatory action research. Kairaranga, 9(Special Edition), 54-60.

Kemmis, S. (2001). Exploring the relevance of critical theory for action research: Emancipatory action research in the footstepts of Jurgen Habermas. In P. Reason \& H. Bradbury (Eds.), Handbook of action research. Participative inquiry and practice (pp. 91-102). London: Sage Publications.

Kennedy, R., \& Scott, A. (2005). A pilot study: The effects of music therapy interventions on middle school students' ESL skills. Journal of Music Therapy, 42(4), 244-261.

Kern, P. (2004). Making friends in music: Including children with autism in an interactive play settings. Music Therapy Today, 5(4), Retrieved from http://musictherapyworld.net.

Kern, P., \& Aldridge, D. (2006). Using embedded music therapy interventions to support outdoor play of young children with autism in an inclusive community-based child care program. Journal of Music Therapy, 43(4), U1-294.

Kern, P., Wolery, M., \& Aldridge, D. (2007). Use of songs to promote independence in morning greeting routines for young children with autism. Journal of Autism \& Developmental Disorders, 37(7), 1264-1271.

Langhan, D. (2009). A music therapy assessment tool for special education: Incorporating education outcomes. Australian Journal of Music Therapy, 20, 78-98.

Law, J., Lindsay, G., Peacey, N., Gascoigne, M., Soloff, N., Radford, J., et al. (2002). Consultation as a model for providing speech and language therapy in schools: A panacea or one step too far? Child Language Teaching and Therapy, 19, 145-163.

Layman, D. L., Hussey, D. L., \& Laing, S. J. (2002). Music therapy assessment for severely emotionally disturbed children: a pilot study. Journal of Music Therapy, 39(3), 164187.

Lefevre, M. (2004). Playing with sound: The therapeutic use of music in direct work with children. Child and Family Social Work, 9, 333-345.

Leonard, P., \& Leonard, L. (2001). Assessing aspects of professional collaboration in schools: Beliefs versus practices. Alberta Journal of Educational Research, 47(1), 423.

Lewin, K. (1946). Action research and minority problems. Journal of Social Issues, 2(4), 3446.

Lewin, K. (1952). Group decision and social change. In G. E. Swanson, T. M. Newcomb \& E. L. Hartley (Eds.), Readings in social psychology (pp. 459-473). New York: Henry Holt. 
Lewis, A., \& Porter, J. (2004). Interviewing children and young people with learning disabilities. British Journal of Learning Disabilities, 32(4), 191-197.

Lincoln, Y. S. (2001). Engaging sympathies: Relationships between action research and social constructivism. In P. Reason \& H. Bradbury (Eds.), Handbook of action research. Participative inquiry and practice (pp. 124-132). London: Sage Publications.

Lincoln, Y. S., \& Guba, E. (1985). Naturalist Inquiry. Beverly Hills, CA: Sage.

Loewy, J. (2000). Music psychotherapy assessment. Music Therapy Perspectives, 18(1), 4758.

MacDonald, R. A. R., Hargreaves, D. J., \& Miell, D. (2002). Musical Identities. Oxford: Oxford University Press.

Markou, K. (2004). The relationship between music therapy and music education in special school settings: The practitioners' views. Paper presented at the 6th European Music Therapy Congress, Finland.

Massey University. (2004). Massey University code of ethical conduct for research involving human participants. Palmerston North, NZ: Author.

McArthur, J. (2009). Learning better together. Working towards inclusive education in New Zealand schools. Wellington, NZ: IHC.

McCartney, E. (2009). Joining up working: Terms, types and tensions. In J. Forbes \& C. Watson (Eds.), Service integration in schools. Rotterdam, The Netherlands: Sense Publishers.

McCartney, E., Ellis, S., \& Boyle, J. (2009). The mainstream primary classroom as a language-learning environment for children with severe and persistent language impairment - implications of recent language intervention research. Journal of Research in Special Education Needs, 9, 80-90.

McCarty, B. C., McElfresh, C. T., Rice, S. V., \& Wilson, S. J. (1978). The effect of contingent background music on inappropriate bus behaviour. Journal of Music Therapy, 15(3), 150-156.

McKernan, J. (1991). Curriculum action research. A handbook of methods and resources for the reflective practitioner. London: Kogan Page.

McMenamin, T., Millar, R., Morton, M., Mutch, C., Nattall, J., \& Tyler-Merrick, G. (2004). Curriculum policy and special education support. Christchurch: School of Professional Development, Christchurch College of Education (for Ministry of Education, NZ).

McNiff, J. (1988). Action research: Principles and practice. Hampshire: McMillan Education Ltd. 
McNiff, J. (2005, 1st November ). Action Research. Paper presented at the HERDZA workshop, Wellington.

McNiff, J., Lomax, P., \& Whitehead, J. (2003). You and your action research project (2 ed.). London: RoutledgeFalmer.

McTaggart, R. (1997). Participatory action research: International contexts and consequences. Albany: State University of New York Press.

McWilliam, R. A. (1995). Integration of therapy and consultative special education: A continuum in early intervention. Infants and Young Children, 7(4), 29-38.

Ministry of Education. (1996). Special Education 2000. Wellington: Ministry of Education.

Ministry of Education. (2004a). Count of ORRS students by institution type. Retrieved Edumis Database 25/5/2004

Ministry of Education. (2004b). ORRS guidelines. Wellington, New Zealand: Ministry of Education.

Ministry of Education. (2006a). Resource Teachers: Learning and Behaviour 2005 - Annual Report. Wellington, NZ: Ministry of Education.

Ministry of Education. (2006b). Specialist service standards. Wellington, New Zealand.

Ministry of Education. (2007). Resource Teachers: Learning and Behaviour (RTLB): Policy and toolkit. Wellington, NZ: Ministry of Education.

Ministry of Education. (2009a). The ongoing and reviewable resource scheme. Retrieved. from

http://www.minedu.govt.nz/educationSectors/SpecialEducation/ServicesAndFunding /ORRS.

Ministry of Education. (2009b). Review of special education. Retrieved 2nd November 2009. from

http://www.minedu.govt.nz/theMinistry/Consultation/ReviewOfSpecialEducation.asp $\underline{x}$.

Ministry of Education. (2009c). Special education guidelines. Retrieved 2nd July 2009. from http://www.minedu.govt.nz/NZEducation/EducationPolicies/SpecialEducation.

Ministry of Health. (2001). The New Zealand disability strategy: Making a world of difference - wakanui oranga. Wellington.

Ministry of Health. (2009). Te whare tapa wha. Retrieved. from www.maorihealth.govt.nz/moh.

Molyneux, C. (2002). Short term music therapy within a child and adolescent mental health 
service - a description of a developing service. Unpublished MA Dissertation, Anglia Polytechnic University, Cambridge.

Morrow, R. A., \& Torres, C. A. (2002). Reading Freire and Habermass: Critical pedagogy and transformative social change. New York: Teachers College Press.

Neilson, W. (2005). Disability: Attitudes, history and discourses. In D. Fraser, R. Moltzen \& K. Ryba (Eds.), Learners with special needs in Aotearoa New Zealand. South Victoria: Thomson Dunmore Press.

New Zealand Government. (1993). Human Rights Act. Retrieved 28th November 2009. from http://www.hrc.co.nz/home/hrc/humanrightsenvironment/humanrightsinnewzealand/ humanrightsact.php.

New Zealand Society for Music Therapy. (1989). Music therapy. Wellington: NZSMT.

New Zealand Society for Music Therapy. (2006). Code of ethics for the practice of music therapy in New Zealand. Wellington: NZSMT.

Newman, J. M. (1998). Tensions of teaching: Beyond tips to critical reflection. Toronto: Canadian Scholars' Press.

Nordoff, P., \& Robbins, C. (1977). Creative music therapy. New York: John Day.

Nordoff, P., \& Robbins, C. (1983). Music therapy in special education. St Louis MO 63132: Magnamusic-Baton.

Nordoff, P., Robbins, C., \& Marcus, D. (2007). Creative music therapy : A guide to fostering clinical musicianship with CD (Audio). New York: MMB.

O'Brien, P., \& Ryba, K. (2005). Policies and systems in special education. In D. Fraser, R. Moltzen \& K. Ryba (Eds.), Learners with special education needs in Aotearoa New Zealand. Southbank, Victoria: Thomson Dunmore Press.

O'Regan, P. (2007). Making music connections. Kairaranga, 8(1), 36-39.

O'Toole, C., \& Kirkpatrick, V. (2007). Building collaboration between professionals in health and education through interdisciplinary training. Child Language Teaching and Therapy, 23, 325-352.

Ockelford, A., Welch, G., \& Zimmermann, S. (2002). Music education for pupils with severe or profound and multiple difficulties - current provision and future need. British Journal of Special Education, 29(4), 178-192.

Oldfield, A. (1993). A study of the way music therapists analyse their work. British Journal of Music Therapy, 7(1), 14-22.

Oldfield, A. (2000). Music therapy as a contribution to the diagnosis made by the staff team in child and family psychiatry - An initial description of a methodology. In T. 
Wigram (Ed.), Assessment and evaluation in the arts therapies: Art therapy, drama therapy, music therapy and dramatherapy. Radlett: Harper House Publications.

Orams, J. (2003). Strategies for implementing a music program in primary schools. MENZA, Retrieved from http://www.menza.org.nz/research/.

Park, P. (2001). Knowledge and participatory research. In P. Reason \& H. Bradbury (Eds.), Handbook of action research. Participative inquiry and practice (pp. 81-90). London: SAGE Publications.

Parsons, R. D., \& Kahn, W. J. (2005). The school counselor as consultant: An integrated model for school-based consultation. Belmont, CA: Thomson Brooks/Cole.

Pasiali, V. (2004). The use of prescriptive therapeutic songs in a home-based environment to promote social skills acquisition by children with autism: Three case studies. Music Therapy Perspectives, 22, 11-20.

Pavlicevic, M. (1999). Thoughts, words and deeds: Harmonies and counterpoints in music therapy theory. British Journal of Music Therapy, 13(2), 59-62.

Pavlicevic, M. (2004). Learning from Thembalethu: Towards responsive and responsible practice in community music therapy. In M. Pavlicevic \& G. Ansdell (Eds.), Community music therapy. London: Jessica Kinglsey Publishers.

Pavlicevic, M. (2005). Worksongs, playsongs: Communication, collaboration, culture and community. Paper presented at the 11th World Congress of Music Therapy. From Lullaby to Lament., Brisbane.

Pavlicevic, M., \& Ansdell, G. (2004a). Community music therapy. London: Jessica Kingley Publishers.

Pavlicevic, M., \& Ansdell, G. (2004b). Introduction: 'The ripple effect'. In M. Pavlicevic \& G. Ansdell (Eds.), Community music therapy. London: Jessica Kingsley.

Pellitteri, J. (2000). Music therapy in the special education setting. Journal of Educational and Psychological Consultation, 11(3\&4), 379-391.

Penman, M., \& Caswell, P. (2007). A journey into education. Kairaranga, 8(2), 3-5.

Pethybridge, E., \& Robertson, J. (2010). Education music therapy: Theoretical foundations explored in time-limited group work projects with children. In V. Karkou (Ed.), Arts therapies in schools. Research and practice (pp. 129-144). London: Jessica Kingsley Publishers.

Piggot-Irvine, E. (2003a). Key features of appraisal effectiveness. The International Journal of Educational Management, 17(4), 170-178.

Piggot-Irvine, E. (2003b). The nuts and bolts of action research. Paper presented at the NZARE, Auckland. 
Piggot-Irvine, E. (2004, 17/11/04). Action Research Paper presented at the Ministry of Education Workshop, Wellington.

Porter, J., Ouvry, C., Morgan, M., \& Downs, C. (2001). Interpreting the communication of people with profound and multiple learning difficulties. British Journal of Learning Disabilities, 29(1), 12.

Prizant, B. M., Wetherby, A. M., Rubin, E., Laurent, A. C., \& Rydell, P. J. (2006). The SCERTS model in practice: Program planning and intervention. Retrieved from http://www.scerts.com/frequently_asked_questions.htm. .

Proctor, S. (2004). Playing politics: Community music therapy and the therapeutic redistribution of music capital for mental health. In M. Pavlicevic \& G. Ansdell (Eds.), Community music therapy (pp. 214-232). London: Jessica Kingsley.

QSR International Pty Ltd. (2002). N6 reference guide (1 ed.). Doncaster, Victoria, Australia: QSR International Pty Ltd.

Rapoport, R. N. (1970). Three dilemmas in action research. Human Relations, 23, 488-513.

Reason, P., \& Bradbury, H. (2001). Handbook of action research. Participative inquiry and practice. London: Sage Publications.

Register, D. (2002). Collaboration and consultation: A survey of board certified music therapists. Journal of Music Therapy, 39(4), 305-321.

Register, D. (2004). Teaching child-care personnel to use music in the classroom: A comparison of workshop training versus on-site modeling. Music Therapy Perspectives, 22(2), 109-115.

Reuer, B. (1996). Posturing for the changing world: Consulting as a career option. Music Therapy Perspectives, 14, 16-20.

Rice, P. L., \& Ezzy, D. (1999). Qualitative Research Methods. A Health Focus. Victoria, Australia 3205.: Oxford University Press.

Rickson, D. J. (1997). The music therapist working in New Zealand schools with children who have disabilities. Annual Journal of the New Zealand Society for Music Therapy, 44-53.

Rickson, D. J. (1998). Sam: A new dawn on the horizon. Annual Journal of the New Zealand Society for Music Therapy, 37-41.

Rickson, D. J. (2000). Music: The way to developing a relationship with disturbed adolescents. Annual Journal of the New Zealand Society for Music Therapy, 2000, 19-33.

Rickson, D. J. (2001). A music therapy practice in special education settings in New 
Zealand. Journal of the New Zealand Society for Music Therapy, 2001, 26-47.

Rickson, D. J. (2002). Adam: A case study of an adolescent boy. Annual Journal of the New Zealand Society for Music Therapy, 51-59.

Rickson, D. J. (Artist). (2003). Oscar's Listening [CD Recording by The Oticon Foundation. Brian Ringrose, Producer].

Rickson, D. J. (2004). The boy with the glass flute - Journeying through the therapeutic process with a preadolescent who has been abused. Voices: A World Forum for Music Therapy, Retrieved from http://voices.no.

Rickson, D. J. (2006a). Instructional and improvisational music therapy approaches with adolescents who have ADHD: A comparison of the effects on motor impulsivity. Journal of Music Therapy, 43(1), 39-62.

Rickson, D. J. (2006b). Reflections on the nature of relationship in brief music therapy encounters with children who have developmental difficulties. New Zealand Journal of Music Therapy, 4, 23-45.

Rickson, D. J. (2008). The potential role of music in special education (The PROMISE) New Zealand music therapists consider collaborative consultation. New Zealand Journal of Music Therapy, 6, 76-97.

Rickson, D. J. (2009a). Community music therapy in new music therapy communities. Voices: A World Forum for Music Therapy, Retrieved 9/06/09 from http://www.voices.no/columnist/colrickson230309.php.

Rickson, D. J. (2009b). Researching ones own clinical practice: managing multiple roles in an action research project. Voices: A World Forum for Music Therapy, Retrieved March 5, 2009, from http://www.voices.no/mainissues/mi40009000307.php.

Rickson, D. J. (2009c). The use of music to facilitate learning and development in a school in Thailand: An exploratory case study. New Zealand Journal of Music Therapy, 7, 6187.

Rickson, D. J., \& McFerran, K. (2007). Music therapy in special education: Where are we now? Kairaranga, 8(1), 40-48.

Rickson, D. J., \& Watkins, W. G. (2003). Music therapy to promote prosocial behaviours in aggressive adolescent boys - A pilot study. Journal of Music Therapy, 40(4), 283301.

Ritchie, J. E. (1996). Using participartory research to enhance health in the work setting: An Australian experience. In K. de Koning \& M. Martin (Eds.), Participatory research in health: Issues and experiences. London: Zed Books.

Robbins, C., \& Robbins, C. (1991). Creative music therapy in bringing order, change, and communicativeness to the life of a brain-injured adolescent. In K. E. Bruscia (Ed.), 
Case studies in music therapy (pp. 231-251). Phoenixville, PA: Barcelona Publishers.

Robertson, J. (2000). An educational model for music therapy: The case for a continuum. British Journal of Music Therapy, 14(1), 41-46.

Rogers, P. J. (1995). Music therapy research in Europe: A context for the qualitative/quantitative debate. British Journal of Music Therapy, 9(2), 5-12.

Rolvsjord, R. (2006). Whose power of music? A discussion on music and power-relations in music therapy. British Journal of Music Therapy, 20(1), 5-12.

Rubin, H., \& Rubin, I. (1995). Qualitative interviewing: The art of hearing data. Thousand Oaks, CA: Sage Publications.

Ruud, E. (1998). Music therapy: Improvisation, communication and culture. Gilsum, NH: Barcelona Publishers.

Sabbatella, P. E. (1999). The process of evaluation in music therapy professional practice. Paper presented at the World Congress of Music Therapy., Washington, DC.

Sanders, M. G. (2003). Community involvement in schools - From concept to practice. Education and Urban Society, 35(2), 161-180.

Sapon-Shevin, M. (1996). Full inclusion as disclosing tablet: Revealing the flaws in our present system. Theory Into Practice, 35(1), 35-41.

Small, C. (1998). Musicking: The meanings of performing and listening. Hanover: University Press of New England.

Smeijsters, H. (1997). Multiple perspectives. A guide to qualitative research in music therapy. Gilsum, NH.: Barcelona Publishers.

Smith, D. S., \& Hairston, M. (1999). Music therapy in school settings: Current practice. Journal of Music Therapy, 36(4), 274-292.

Smith, M. (2002). Routines. Texas School for the Blind and Visually Impaired Retrieved from http://www.tsbvi.edu/index.htm

Smith, R. M., \& Nevin, A. (2005). Conceptualising liberatory roles for educational and psychological consultants: Implications for transition planning. Journal of Educational and Psychological Consultation, 16(4), 263-286.

Snell, A. M. (1996). Music therapy for learners with autism in a public school setting. In B. L. Wilson (Ed.), Models of music therapy interventions in school settings (pp. 211273). Silver Spring, MD 20910: American Music Therapy Assn, Inc.

Soanes, C., Spooner, A., \& Hawker, S. (Eds.). (2001). Oxford paperback dictionary thesaurus and wordpower guide. Oxford: Oxford University Press. 
Steele, A. L. (1977). Directive teaching and the music therapist as consultant. Journal of Music Therapy, 14(1), 17-26.

Stern, D. (1985). The interpersonal world of the infant: A view from psychoanalysis and developmental psychology. London: Academic Press.

Stevenson, K. (2003). Music therapy assisted communication with children with severe disabilities. New Zealand Journal of Music Therapy, 1, 82-92.

Stewart, D. (2004). Narratives in a new key: Transformational contexts in music therapy. In M. Pavlicevic \& G. Ansdell (Eds.), Community music therapy (pp. 281-297). London: Jessica Kingsley.

Stige, B. (1998). Perspectives on meaning in music therapy. British Journal of Music Therapy, 12(1), 20-27.

Stige, B. (2002). Culture-centered music therapy. Gilsum, NH: Barcelona Publishers.

Stige, B. (2004a). Community music therapy: Culture, care and welfare. In M. Pavlicevic \& G. Ansdell (Eds.), Community music therapy. London: Jessica Kingsley Publishers.

Stige, B. (2004b). On defining community music therapy [Contribution to Moderated Discussions]. Voices: A World Forum for Music Therapy, Retrieved from http://www.voices.no/discussions.

Stige, B. (2005a). Participatory action research. In B. L. Wheeler (Ed.), Music therapy research (2nd ed., pp. 404-415). Gilsum, NH: Barcelona Publishers.

Stige, B. (2005b). Researching community music therapy: Topics, objectives, and methods. Paper presented at the 11th World Congress of Music Therapy. From Lullaby to Lament, Brisbane.

Stige, B. (2006). On a notion of participation in music therapy. Nordic Journal of Music Therapy, 15(2), 121-139.

Strauss, A., \& Corbin, J. (1990). Basics of Qualitative Research: Grounded Theory Procedures and Techniques. Sage: Newbury Park.

Streeter, E. (1999). Finding a balance between psychological thinking and musical awareness in music therapy theory - A psychoanalytic perspective. British Journal of Music Therapy, 13(1), 5-20.

Susman, G., \& Evered, R. (1978). An assessment of the scientific merit of action research. Administrative Science Quarterly, 23, 582-603.

Sutton, J. (2002). Preparing a potential space for children with special needs. In A. Davies \& E. Richards (Eds.), Music therapy and group work: Sound company (pp. 188-201). London: Philadelphia: Jessica Kingsley. 
Swaggart, B. L., Gagnon, E., Bock, S. J., Earles, T. L., Quinn, C., \& Myles, B. S. (1995). Using social stories to teach social and behavioral skills to children with autism. Focus on Autistic Behaviour, 10(1), 1-16.

Tait, A. (2005). From lament to lullaby - Stories of indigenous community engagement with the arts for education and well being. Paper presented at the 11th World Congress of Music Therapy. From Lullaby to Lament, Brisbane.

Thomson, C. (2008). Collaborative consultation: A model for service delivery in the new paradigm for special education. Victoria University of Wellington, Wellington New Zealand.

Threlfall, C. (2005). Music therapy service delivery in education: Working effectively with classroom culture. Paper presented at the 11th World Congress of Music Therapy, Brisbane.

Tolich, M., \& Davidson, C. (1999). Starting fieldwork: An introduction to qualitative research in New Zealand. Auckland: Oxford University Press.

Trevarthen, C. (2000). Musicality and the intrinsic motive pulse: Evidence from human psychobiology and infant communication. Musicae Scientiae, Special Issue, 19992000, 1029-8649.

Trevarthen, C. (2002). Origins of musical identity. In R. A. R. MacDonald, D. J. Hargreaves \& D. Miell (Eds.), Musical identities. Oxford: Oxford University Press.

Trevarthen, C. (2005). First things first: Infants make good use of the sympathetic rhythm of imitation, without reason or language. Journal of Child Psychotherapy, 31(1), 91113.

Tutty, C., \& Hocking, C. (2004). A shackled heart: Teacher aides' experience of supporting students with high needs in regular classes. Kairaranga, 5(2), 3-9.

Twyford, K. (2007). Getting it together: Understanding the importance of collaboration. New Zealand Journal of Music Therapy, 5, 21-33.

Twyford, K. (2009). Finding a niche: Establishing a role for music therapy within the Ministry of Education, Special Education NZ. New Zealand Journal of Music Therapy, 78, 6-31.

Twyford, K., \& Watson, T. (2008a). Introduction. In K. Twyford \& T. Watson (Eds.), Integrated team working: Music therapy as part of transdisciplinary and collaborative approaches (pp. 11-28). London: Jessica Kingsley.

Twyford, K., \& Watson, T. (Eds.). (2008b). Integrated Team Working: Music Therapy as part of Transdisciplinary and Collaborative Approaches. London: Jessica Kingsley.

UNCRC. (1989). UN convention of the rights of the child, Article 12 (Publication., from http://www.everychildmatters.gov.uk): 
Underhill, K. K., \& Harris, L. M. (1974). The effect of contingent music on establishing imitation in behaviourally disturbed retarded children. Journal of Music Therapy, 11, 156-166.

UNESCO. (1989). United Nations convention on the rights of the child. Retrieved. from http://www.unicef.org/crc/.

UNESCO. (2009). Policy guidelines on inclusion in education. Retrieved. from http://www.unesco.org/en/education/.

Vygotsky, L. (1978). The development of higher psychological processes. In M. Cole, V. John-Steiner, S. Scribner \& E. Souberman (Eds.), Mind in society. Cambridge, MA: Harvard University Press.

Walsh Stewart, R. (2002). Combined efforts. Increasing social-emotional communication with children with Autistic Spectrum Disorder using psychodynamic music therapy and Division TEACCH communication programme. In A. Davies \& E. Richards (Eds.), Music therapy and group work: Sound company. London: Jessica Kingsley

Walworth, D. D. (2007). The use of music therapy within the SCERTS model for children with autism spectrum disorder. Journal of Music Therapy, 44(1), 2-22.

Warner, C. (2005). Music therapy with adults with learning difficulties and 'severe challenging behaviour': An action research inquiry into group music therapy within a community home Unpublished Unpublished Doctoral Thesis, University of West of England Bristol.

Warren, P. (1997). Music therapy with a child with cerebral palsy: A case study. Annual Journal of the New Zealand Society for Music Therapy, 29-43.

Warren, P. (2005). Personal Communication.

Webb, L. (2005). "Growing the expertise": The expectations of a new curriculum document. Music in pre-service primary teacher education. MENZA, Retrieved from http://www.menza.org.nz/research.

Wesley, P. W. (2002). Early intervention consultants in the classroom - Simple steps for building strong collaboration. Young Children, 57(4), 30-34.

Wesley, P. W., \& Buysse, V. (2004). Consultation as a framework for productive collaboration in early intervention. Journal of Educational and Psychological Consultation, 15(2), 127-150.

Wesley, P. W., \& Buysse, V. (2006). Ethics and evidence in consultation. Topics in Early Childhood Special Education, 26(3), 131-141.

Wesley, P. W., Buysse, V., \& Skinner, D. (2001). Early interventionists' perspectives on professional comfort as consultants. Journal of Early Intervention, 24(2), 112-128. 
Whitehurst, T. (2007). Changing perspectives on disability and inclusion: 'The Monkey King' arts project. In B. Carpenter \& J. Egerton (Eds.), New horizons in special education: Evidence-based practice in action: Sunfield Publications.

Wigram, T. (2000). A method of music therapy assessment for the diagnosis of autism and communication disorders in children. Music Therapy Perspectives, 18(1), 13-21.

Wigram, T., Pederson, I. N., \& Bond, O. L. (2002). A comprehensive guide to music therapy. London: Jessica Kingsley Publishers Ltd.

Williams, K. (2006). Action inquiry into the use of standardized evaluation tools for music therapy: A real life journey within a parent-child community program. Voices: A World Forum for Music Therapy, http://www.voices.no/mainissues/mi40006000208.html.

Wilson, B. L. (2002). Changing times: The evolution of special education. In B. L. Wilson (Ed.), Models of music therapy interventions in school settings (2nd ed., pp. 13-26). Silver Spring, MD: The American Music Therapy Association, Inc.

Wilson, B. L., \& Smith, D. S. (2000). Music therapy assessment in school settings. Journal of Music Therapy, 37(2), 95-117.

Wilson, S. (1991). Music therapy in education. British Journal of Music Therapy, 5(2), 1417.

Wolery, M., Sigalove Brashers, M., \& Neitzel, J. C. (2002). Ecological congruence assessment for classroom activities and routines: Identifying goals and intervention practices in childcare. Topics in Early Childhood Special Education, 22(3), 131-142.

Wood, S., Verney, R., \& Atkinson, J. (2004). From therapy to community: Making music in neurological rehabilitation. In M. Pavlicevic \& G. Ansdell (Eds.), Community music therapy (pp. 48-65). London: Jessica Kingsley Publishers.

Woodward, S. (2000). A response to James Robertson's 'An educational model for music therapy: The case for a continuum'. British Journal of Music Therapy, 14(2), 94-98.

World Federation of Music Therapy. (1996). FAQ music therapy. Retrieved from http://www.wfmt.info/WFMT/FAQ_Music_Therapy.html

World Health Organisation. (2001). International classification of functioning, disability and health. Geneva: WHO.

Wosch, T., \& Wigram, T. (Eds.). (2007). Microanalysis in music therapy : methods, techniques and applications for clinicians, researchers, educators and students London ; Philadelphia: Jessica Kingsley.

Wylie, C. (2000). Picking up the pieces. Review of special education 2000. Wellington: Ministry of Education. 
Yehuda, N. (2002). Multicultural encounters in music therapy - a qualitative research. Voices: A World Forum for Music Therapy, 2(3), Retrieved from http://www.voices.no/mainissues/Voices2(3)Yeuda.html.

Zins, J. E., \& Ponti, C. R. (1996). The influence of direct training in problem solving on consultee problem clarifiaciton, skills and attributions. Remedial and Special Education, 17, 370-377. 


\section{APPENDICES}

APPENDIX 1: Letter to New Zealand Society for Music Therapy

P.O. Box 756,

Massey University

Wellington.

College of Creative Arts

$10^{\text {th }}$ January 2006

Natali Allan,

Secretary,

New Zealand Society for Music Therapy,

12 The Yardarm,

Whitby,

Wellington

Dear Natali,

My research project towards the degree of PhD (Music) is entitled 'The Potential Role of Music in Special Education Settings in New Zealand - Developing a Music Therapy Assessment and Consultation Protocol'. Dr Allan Badley, College of Creative Arts, Massey University, is my primary supervisor.

The study will involve interviews with music therapists who have worked in special education settings and/or have undertaken assessments with children for consultation purposes. I would like to contact all music therapists to ask them if they are eligible for and interested in the study. I have enclosed a copy of an advertisement and ask if you would kindly publish this in MusT and Connections magazines. Please advise if there are any costs associated with this and I will forward a cheque accordingly. I would also like to forward the advertisement to you in electronic form and have it sent by email to all appropriately qualified music therapists on your NZSMT database. Please advise whether you are able to do this.

The project has been reviewed and approved by the Massey University Human Ethics Committee, WGTN Application - 05/59. If you have any concerns about the conduct of this research, please contact Professor Sylvia Rumball, Chair, Massey University Campus Human Ethics Committee: Wellington, 06350 5249, humanethicswn@massey.ac.nz.

If you have any further questions about the study feel free to contact me on 048012794 x6979, D.J.Rickson@massey.ac.nz or Allan Badley on 042794 x6974, A.D.Badley@ massey.ac.nz.

Yours faithfully, Daphne Rickson, Music Therapist / PhD Candidate. 


\section{Massey University \\ College of Creative Arts \\ Invitation to Participate in a Music Therapy Research Project}

My research project towards the degree of $\mathrm{PhD}$ (Music) is entitled 'The Potential Role of Music in Special Education Settings in New Zealand Developing a Music Therapy Assessment and Consultation Protocol'. If you are a qualified music therapist and have worked in special education settings, or have undertaken assessments for consultation in New Zealand, I would like to interview you. The interview would take place at a time and in a location convenient to you and would last about an hour. For further information or to express interest, please contact Daphne Rickson, College of Creative Arts, Massey University, P.O. Box 756, Wellington; Ph 048012794 x6979; D.J.Rickson@massey.ac.nz. 
APPENDIX 3: Information Sheet-Music Therapists

\section{Massey University}

P.O. Box 756,

College of Creative Arts

Wellington.

Dear

My research project towards the degree of $\mathrm{PhD}$ (Music) is entitled 'The Potential Role of Music in Special Education Settings in New Zealand - Developing a Music Therapy Assessment and Consultation Protocol'. Dr Allan Badley, College of Creative Arts, Massey University, is my primary supervisor. I am writing to you because you have expressed interest in the possibility of participating in the study.

I aim to develop and evaluate a Music Therapy Assessment and Consultation Protocol with members of 5-6 New Zealand special education teams (i.e. approximately 25-30 children, school staff, family/whanau and other specialists). The initial protocol is likely to involve data gathering, naturalistic participant observations, a clinical music therapy assessment undertaken by the music therapist (videoed), a review of the video by multidisciplinary team (MDT) members and the collaborative development of a programme of music experiences which will be especially planned to assist children to meet developmental goals from the Essential Skills Curriculum, and/or their Individual Education Plan. The music experiences will be introduced by one or more of the MDT. The primary focus of the project is to find out how the protocol is perceived, understood, valued, and used by each team of participants in turn, and with this knowledge to progressively develop and improve it. The study will be predominantly qualitative, and data will be gathered from multiple sources including participant observation, video of music therapy assessment sessions, focus groups, interviews, diaries, and logs.

You are invited to be interviewed along with approximately nine other music therapists because you have worked in special education settings, or have engaged in music therapy assessment for consultation work in New Zealand. All qualified NZ music therapists who fit these criteria and who are willing to be interviewed have been invited to participate in stage one of the study. The interviews will have a particular focus on assessment protocols - data gathering, planning, sessions, sharing and evaluation - as well as asking for more detailed information about the music therapy assessment sessions, so present and past practice can be described. The data from the interviews together with an analysis of video data of the researcher's assessment sessions will be used to plan an initial Developmental Music Therapy Assessment and Consultation Protocol. The protocol will then form the basis of an action research project with 5-6 special education teams.

All research data will be stored securely at Massey University, College of Creative Arts, for 
a period of five years after the completion of the study. It will then be destroyed.

I anticipate that interviews will take approximately one hour. A transcript of your interview will be returned to you for editing and you will need to set aside an hour to review the transcript. I will take all reasonable steps to maintain confidentiality. Participants' names will not be used in verbal discussion with anyone outside the research teams. All identifying features will be removed from written published material, and all material will be securely stored. Nevertheless, the special education and music therapy communities in New Zealand are small and there is a risk that you might be identifiable.

You are under no obligation to accept this invitation. If you decide to participate, you have the right to:

- decline to answer any question

- withdraw from the study up until the time you have reviewed your transcripts

- ask questions about the study at any time during participation

- provide information on the understanding that your name will not be used unless you give permission to the researcher

- be given access to a summary of the project findings when it is concluded

The project has been reviewed and approved by the Massey University Human Ethics Committee, WGTN Application - 05/59. If you have any concerns about the conduct of this research, please contact Professor Sylvia Rumball, Chair, Massey University Campus Human Ethics Committee: Wellington, 06350 5249, humanethicswn@ massey.ac.nz.

If you have any further questions about the study feel free to contact me on 048012794 x6979, D.J.Rickson@massey.ac.nz or Allan Badley on 042794 x6974, A.D.Badley@massey.ac.nz. If you are willing to participate, please sign the attached Consent Form and return it to me at the above address in the stamped and addressed envelope supplied.

Yours faithfully,

Daphne Rickson, Music Therapist / PhD Candidate. 
APPENDIX 4: Consent Form - Music Therapists

Massey University

College of Creative Arts

The Potential Role of Music in Special Education Settings in New Zealand -

Developing a Music Therapy Assessment and Consultation

Protocol

PARTICIPANT CONSENT FORM (Music Therapists)

This consent form will be held for a period of five (5) years

I have read the Information Sheet and have had the details of the study explained to me. My questions have been answered to my satisfaction, and I understand that I may ask further questions at any time.

I agree to being interviewed for this study under the conditions set out in the Information Sheet.

I agree to the interview being audio taped.

I wish/do not wish to have my tapes returned to me.

Signature:

Date:

Full Name - printed: 
APPENDIX 5: Interview Guidelines - Music Therapists

Guidelines for Semi-Structured Interview with Music Therapists

The interview questions would be used to "create a scaffolding for the interview, keep the questioning on topic, and link what is asked in individual interviews to the overall design" (Rubin \& Rubin, 1995, p.150). There will be flexibility to discuss ideas that emerge during the interview. Manuscripts will be returned to participants inviting their editing and additional comments, so the interpretation will not belong to me alone.

Interview Prompts:

1. Tell me about your work with children who have special education needs.

2. Describe what you do when you are informed about a new student who has been referred to you.

3. How do you find out all you need to know about the child?

4. Describe the music therapy assessment sessions.

5. Explain what you do with the information you gather about the student.

6. How is your work evaluated? 


\section{Massey University}

P.O. Box 756,

College of Creative Arts

Wellington.

\section{THE POTENTIAL ROLE OF MUSIC IN SPECIAL EDUCATION SETTINGS IN NEW ZEALAND - THE PROMISE: DEVELOPING A MUSIC THERAPY ASSESSMENT AND CONSULTATION PROTOCOL}

Massey University Human Ethics Committee Approved Protocol

(HEC: WGTN Application - 05/59).

$2^{\text {nd }}$ August 2006

Consent to use interview material - Music Therapists

Thank you for participating in interviews for my $\mathrm{PhD}$ research.

In the information sheet you received prior to consenting to be involved, you were advised that "Participants' names will not be used in verbal discussion with anyone outside the research teams. All identifying features will be removed from written published material, and all material will be securely stored. Nevertheless, the special education and music therapy communities in New Zealand are small there is a risk that you might be identifiable".

During the interviews most of you offered information about your work which will enable you, and/or your places of work, to be readily identified in the small New Zealand music therapy community. I have considered carefully with my supervisors what might be done to protect your anonymity. However you have given me information which is important and relevant to my research and which does need to be contextualised in order to preserve its meaning.

In view of this new understanding, I am giving you the opportunity to refuse or to re-consent to the use of your interview material, or parts thereof, for my research. After your interview you were given the opportunity to review the transcript and to delete or add information. Please take time to consider the content of the transcript (I can provide another copy of your edited transcript if you need it) and when you are ready, return the attached consent form to me in the stamped and addressed envelope provided.

Thank you again for your interest and support.

Daphne Rickson,

Researcher. 


\section{Massey University}

College of Creative Arts

The Potential Role of Music in Special Education Settings in New Zealand Developing a Music Therapy Assessment and Consultation Protocol HEC:WGTN Protocol - 05/59

PARTICIPANT CONSENT FORM (Music Therapists)

This consent form will be held for a period of five (5) years

I have read the Information Sheet and the additional letter from the researcher dated $2^{\text {nd }}$ August, and have had the details of the study explained to me. My questions have been answered to my satisfaction, and I understand that I may ask further questions at any time.

I have viewed a transcript of my interview, and have had an opportunity to edit it.

I understand that some of the things I have said may enable others to identify me or my place of work.

I agree that data from my transcript can be used for this research.

Signature:

Date:

Full Name - printed: 
APPENDIX 8: Advertisement - Special Education Team Members

\section{Massey University}

College of Creative Arts

\section{Invitation to Participate in a Music Therapy Research Project}

My research project towards the degree of $\mathrm{PhD}$ (Music) is entitled 'The Potential Role of Music in Special Education Settings in New Zealand Developing a Music Therapy Assessment and Consultation Protocol'. The project involves collaboration with special education teams to produce a comprehensive music therapy assessment for children who are eligible for Ongoing Reviewable Resource Scheme (ORRS) funding, and the introduction of resources and support for team members to help them to maximise the therapeutic use of music in a sustained way. The primary focus of the project is to find out how the protocol is perceived, understood, valued, and used by participants. I would like to hear from special education teams (of at least three members) who are working with a child who is ORRS funded, and who might be willing to participate in a music therapy assessment and consultation procedure. For further information please contact Daphne Rickson, New Zealand School of Music, Mt Cook Campus, P. O. Box 2332, Wellington; Ph 048012794 x6979; D.J.Rickson@massey.ac.nz. 
APPENDIX 9: Expression of Interest: Special Education Team Members

The Potential Role of Music in Special Education Settings in New Zealand - The PROMISE. Developing a Music Therapy Assessment and Consultation Protocol

\section{PhD Candidate}

Daphne Rickson, LTCL, MHealSc(MenH), MMusTher, RMT

College of Creative Arts, Massey University, P.O. Box 756, Wellington.

\section{REQUEST FOR EXPRESSIONS OF INTEREST}

My research project towards the degree of $\mathrm{PhD}$ (Music) is entitled 'The Potential Role of Music in Special Education Settings in New Zealand - Investigating a Developmental Music Therapy Assessment and Consultation Protocol'. I am seeking to recruit 5-6 special education multidisciplinary teams ${ }^{11}$ who are willing to be involved in a music therapy assessment procedure with one of their students. At least one member of the team will be asked to commit approximately 25 hours (over a period of 4-5 months) to the project. The others will be asked to attend multidisciplinary team meetings and a focus group.

I aim to introduce a music therapy assessment protocol that is useful and music strategies that are sustainable, by developing an understanding of the strengths and needs of all participants. In collaboration with special education teams I will develop a plan of music activities to help the child to meet his/her developmental or special education goals. Following the music therapy assessment session, again in collaboration with team members, I will analyse the material and provide information in order to assist teams with the development of IEP objectives. In addition I will produce an agreed plan of music activities, music therapy techniques and/or strategies to be implemented by one or more 'identified team member/s' with initial support from me if necessary. Strategies and resources will be offered to help identified team members to use music in an ongoing way to meet developmental or IEP objectives for the child. Support might include facilitation of spontaneous musical interaction between team member and child, provision of original compositions, recordings, recommendations of precomposed material, and/or demonstration of effective ways of using resources.

The primary focus of my study is to find out how the protocol is perceived, understood, valued, and used by participants. I aim to describe the experience from the perspective of team members, using

- clinical data gathered from multidisciplinary team members prior to the music therapy assessment session,

- data from naturalistic observations of children and team member/s in the context of their home and/or school setting,

- a music therapy assessment undertaken by the researcher (videoed),

\footnotetext{
${ }^{11}$ The multidisciplinary team includes parents, guardians, whanau, therapists, teachers, teacher aides and other specialists.
} 
- semi-structured interviews with at least one identified team member from each team prior to and immediately after the music therapy assessment session,

- focus group data from each multidisciplinary team meeting during video viewing of the music therapy assessment session,

- diary entries from identified team members over a four month period following the initial assessment session, as well as

- follow-up qualitative interviews with identified team members four months after their assessment and consultation procedure is initiated.

- Wherever possible, and with the consent of child, legal guardian and with general agreement from the multidisciplinary team, the child will also be interviewed to determine how he/she has experienced the procedure.

To be eligible to participate

The team must:

- be working with a child who is identified as having 'High' or 'Very High' needs according to the Ministry of Education's Ongoing Reviewable Resource Scheme

- have a written agreement with that child's parent or legal guardian, and the principal of the school the child attends, to complete the expression of interest form. The researcher will work through the principal to obtain informed consent from all potential participants if the team is selected to participate in the research project.

- consist of at least three professional people (parents and other professionals) who are willing to observe video of the music therapy assessment and to discuss the session with the music therapist in a focus group setting

- have at least one 'identified team member' who is willing to explore the implementation of therapeutic music strategies and to diary thoughts and feelings about the work. Identified team member/s will be asked to participate in semi structured interviews with the music therapist prior to and immediately after the music therapy assessment session plus a follow-up interview four months after the assessment and consultation procedure is initiated.

- It is not necessary for any of the team members to be trained musicians.

Selection Criteria

The following criteria will be taken into consideration when selecting teams for inclusion:

The order in which the expressions of interest are received

The number and variety of professionals in each multidisciplinary team

The geographical location of teams

The special education setting in which the learning takes place

Student diagnosis

Student gender

Supervisors

Dr Allan D. J. Badley, College of Creative Arts, Massey University, Wellington,

Dr Jill Bevan-Brown, College of Education, Massey University, Palmerston North

Professor Leslie Bunt, The Music Space Trust, St. Matthias Campus, University of West of England, Oldbury Court Road, Fishponds, Bristol BS16 2UP. 


\section{Expression of Interest - CONFIDENTIAL}

If you are interested in participating in this study, please complete the form below. Formal Information Sheets and Consent Forms will be sent to you if you are short listed for the study.

All Expression of Interest forms will be destroyed when participants have been recruited for the study.

Name and address of person completing this Expression of Interest

Are you working with a child who is ORRS funded?

Do you have written permission from the child's legal

YES / NO guardian, and the principal of the education facility the child attends, to complete this form?

If the answer is no please do not return this form.

Location of education facility - City/town only required at this stage

Type of education facility

How many team members are willing to participate?

What are their roles?

Do you have one or more 'identified team member/s' willing to explore the implementation of therapeutic music strategies and to diary thoughts and feelings about the work? 


\begin{tabular}{|l|l|}
\hline $\begin{array}{l}\text { Is/are your 'identified team member/s' able to participate in } \\
\text { semi-structured interviews with the music therapist prior to } \\
\text { and immediately after the music therapy assessment session, } \\
\text { plus a follow-up interview four months after the assessment } \\
\text { and consultation procedure is initiated? }\end{array}$ & \\
\hline $\begin{array}{l}\text { Does the student you are presenting for music therapy } \\
\text { assessment have 'High' or 'Very High' needs? }\end{array}$ & \\
\hline What is the student's diagnosis? & \\
\hline $\begin{array}{l}\text { Is the student male, or female? } \\
\text { For further information or to return your completed form, please contact: } \\
\text { Daphne Rickson, College of Creative Arts, Massey University, P.O. Box 756, Wellington. Ph } \mathbf{0 4 ~ 8 0 1 2 7 9 4} \\
\text { x69. D.J.Rickson@massey.ac.nz. }\end{array}$ & \\
\hline
\end{tabular}


P.O. Box 756,

\section{Massey University}

Wellington.

College of Creative Arts

The Chairperson,

Board of Trustees,

Dear

My research project towards the degree of $\mathrm{PhD}$ (Music) is entitled 'The Potential Role of Music in Special Education Settings in New Zealand - Developing a Music Therapy Assessment and Consultation Protocol'. Dr Allan Badley, College of Creative Arts, Massey University, is my primary supervisor. I am writing to you because the multidisciplinary special education team who supports one of your students who has high or very high special education needs according to the Ministry of Education Ongoing Reviewable Resources Scheme (ORRS) has expressed interest in the possibility of participating in the study.

The study aims to develop and evaluate a Developmental Music Therapy Assessment and Consultation Protocol to support members of New Zealand special education teams (school staff, family/whanau and other specialists) to use music experiences which have been especially planned to assist children to meet developmental goals from the Essential Skills Curriculum, and/or their Individual Education Plan (IEP). I will complete full music therapy assessment with participating children and assist one or more members of each team ('identified team member/s') to facilitate a programme of ongoing therapeutic music activity. The primary focus is to find out how the protocol is perceived, understood, valued, and used by participants. At least one member of each participating team would be involved in implementing planned music techniques, interviews, and keeping a diary of their experience. Others will be invited to participate in a focus group meeting. The school would receive a copy of the child's music therapy assessment report and would be entitled to retain a copy of all other research data related directly to the child's assessment, including video recordings.

The music therapist / researcher will

1. undertake observations of the child in school and/or home settings to gain an understanding of the child and team's needs

2. gather data relating to the child's developmental and educational progress

3. undertake a full music therapy assessment with the child in the presence of the 'identified team member/s'

4. interview 'identified team member/s before and after the music therapy assessment session

5. interview the child participant before and after the music therapy assessment session if appropriate

6. facilitate a focus group session with the multidisciplinary team who will view videotape of the assessment session and discuss 'How can (special education team members) use music and music therapy techniques to help (a child) to meet developmental or IEP goals?' 
7. collaborate with team members to plan and implement an ongoing programme of therapeutic music activities

8. interview the 'identified team member/s' at four month follow-up.

Because this is a special education study, it would be helpful if it could be undertaken at school, in school time and/or after school at a time convenient to team members. This letter seeks approval in principle for the study from you and your Board of Trustees. If you are able to give support in principle, details for implementation of the protocol will be negotiated with you as the Principal of the school.

All research data will be stored securely at Massey University, College of Creative Arts, for a period of five years after the completion of the study. It will then be destroyed.

I will take all reasonable steps to maintain confidentiality. Participants' names will not be used in verbal discussion with anyone outside the research teams. All identifying features will be removed from written published material, and all material will be securely stored. Nevertheless, the special education and music therapy communities in New Zealand are small and there is a risk that the school and participants might be identified.

You are under no obligation to accept this invitation. If you decide to allow staff to participate, you have the right to:

- withdraw your consent up until the time the music therapy assessment session has been undertaken

- provide information on the understanding that the school's name will not be used unless you give permission to the researcher

- be given access to a summary of the project findings when it is concluded

The project has been reviewed and approved by the Massey University Human Ethics Committee, WGTN Application - 05/59. If you have any concerns about the conduct of this research, please contact Professor Sylvia Rumball, Chair, Massey University Campus Human Ethics Committee: Wellington, 06350 5249, humanethicswn@ massey.ac.nz.

If you have any further questions about the study feel free to contact me on 048012794 x6979, D.J.Rickson@massey.ac.nz or Allan Badley on 042794 x6974, A.D.Badley@ massey.ac.nz. If you feel you have enough information, please complete the enclosed consent form and return it to me in the stamped and addressed envelope provided.

Yours faithfully,

Daphne Rickson, Music Therapist / PhD Candidate. 
APPENDIX 11: Consent Form - School Board of Trustees

\section{Massey University College of Creative Arts \\ The Potential Role of Music in Special Education Settings in New Zealand - \\ Developing a Music Therapy Assessment and Consultation \\ Protocol}

FACILITY CONSENT FORM (Board of Trustees)

This consent form will be held for a period of five (5) years

I have read the Information Sheet and have had the details of the study explained to me. My questions have been answered to my satisfaction, and I understand that I may ask further questions at any time. Subject to individual participants' informed consent, I am able to:

Support the study in principle

YES

Agree to the music therapy assessment process to be carried out at school subject to details being approved by the Principal

YES / NO

Signature:

Date:

Full Name - printed: 
P.O. Box 756,

\section{Massey University}

Wellington.

College of Creative Arts

Dear

My research project towards the degree of PhD (Music) is entitled 'The Potential Role of Music in Special Education Settings in New Zealand - Developing a Music Therapy Assessment and Consultation Protocol'. Dr Allan Badley, College of Creative Arts, Massey University, is my primary supervisor. I am writing to you because you have expressed interest in the possibility of participating in the study.

I aim to develop and evaluate a Music Therapy Assessment and Consultation Protocol with members of 5-6 New Zealand special education teams (i.e. approximately 25-30 children, school staff, family/whanau and other specialists). The initial protocol is likely to involve data gathering, naturalistic participant observations, a clinical music therapy assessment undertaken by the music therapist (videoed), a review of the video by multidisciplinary team (MDT) members and the collaborative development of a programme of music experiences which will be especially planned to assist children to meet developmental goals from the Essential Skills Curriculum, and/or their Individual Education Plan. The music experiences will be introduced by one or more of the MDT. The primary focus of the project is to find out how the protocol is perceived, understood, valued, and used by each team of participants in turn, and with this knowledge to progressively develop and improve it. The study will be predominantly qualitative, and data will be gathered from multiple sources including participant observation, video of music therapy assessment sessions, focus groups, interviews, diaries, and logs.

You are invited participate because you work with a child who has high or very high special education needs according to the Ongoing Reviewable Resource Scheme (ORRS) guidelines, and you are a member of a multidisciplinary team interested in the possibility of having a music therapy assessment completed for the child in your care. You have also been identified as a team member who might be willing to explore the implementation and monitoring of a therapeutic music programme. If you agree to participate, you will be invited to:

- be interviewed prior to the music therapy assessment (approximately one hour)

- attend a multidisciplinary team meeting to share information regarding the child prior to a music therapy assessment (approximately one hour)

- attend the music therapy assessment session (approximately one hour)

- be interviewed following the music therapy assessment session (approximately one hour)

- attend a focus group meeting with other members of your multidisciplinary team to view and discuss videotape of the music therapy assessment session (approximately two hours)

- collaborate on the design of a therapeutic music programme to help the child in your care to reach developmental or IEP goals (approx 2 hours)

- implement the therapeutic music programme as part of your workload for a period of at least four months following the music therapy assessment

- keep a diary of your interactions, experiences, thoughts and feelings about the process for at least four months following the music therapy assessment (at least one hour per week) 
- attend a follow-up interview four months after the implementation of the therapeutic music programme (approximately one hour)

Permission will requested from the Principal of your school for meetings to be held at school at a time convenient to you and other team members.

All research data will be stored securely at Massey University, College of Creative Arts, for a period of five years after the completion of the study. It will then be destroyed.

I will take all reasonable steps to maintain confidentiality. Participants' names will not be used in verbal discussion with anyone outside the research teams. All identifying features will be removed from written published material, and all material will be securely stored. Nevertheless, the special education and music therapy communities in New Zealand are small and there is a risk that you might be identified.

You are under no obligation to accept this invitation. If you decide to participate, you have the right to:

- decline to answer any questions

- withdraw from the study at any time before data collection has commenced

- discontinue participation at any time once data collection has commenced

- ask questions about the study at any time during participation

- provide information on the understanding that your name will not be used unless you give permission to the researcher

- be given access to a summary of the project findings when it is concluded

The project has been reviewed and approved by the Massey University Human Ethics Committee, WGTN Application - 05/59. If you have any concerns about the conduct of this research, please contact Professor Sylvia Rumball, Chair, Masey University Campus Human Ethics Committee: Wellington, 06350 5249, humanethicswn@ massey.ac.nz.

If you have any further questions about the study feel free to contact me on 048012794 x6979, D.J.Rickson@massey.ac.nz or Allan Badley on 042794 x6974, A.D.Badley@massey.ac.nz. If you are willing to participate, please sign the attached Consent Form and return it to me at the above address in the stamped and addressed envelope supplied.

Yours faithfully,

Daphne Rickson, Music Therapist / PhD Candidate. 
APPENDIX 13: Consent Form - Identified Team Member

\author{
Massey University \\ College of Creative Arts \\ The Potential Role of Music in Special Education Settings \\ in New Zealand - \\ Developing a Music Therapy Assessment and Consultation \\ Protocol
}

PARTICIPANT CONSENT FORM ('Identified' SE Team Member)

This consent form will be held for a period of five (5) years

I have read the Information Sheet and have had the details of the study explained to me. My questions have been answered to my satisfaction, and I understand that I may ask further questions at any time.

I agree to participate in the study under the conditions set out in the Information Sheet.

I agree to be present at a music therapy assessment session which is being videotaped.

I agree to be interviewed and I understand the interviews will be audiotaped.

I agree to participate in a focus group discussion and 1 understand the discussion will be audio taped.

I wish/do not wish to have my tapes returned to me.

Signature:

Date:

Full Name - printed: 
APPENDIX 14: Information Sheet - General Team Member

P.O. Box 756,

College of Creative Arts

Wellington.

Dear

My research project towards the degree of $\mathrm{PhD}$ (Music) is entitled 'The Potential Role of Music in Special Education Settings in New Zealand - Developing a Music Therapy Assessment and Consultation Protocol'. Dr Allan Badley, College of Creative Arts, Massey University, is my primary supervisor. I am writing to you because you have expressed interest in the possibility of participating in the study.

I aim to develop and evaluate a Music Therapy Assessment and Consultation Protocol with members of 5-6 New Zealand special education teams (i.e. approximately 25-30 children, school staff, family/whanau and other specialists). The initial protocol is likely to involve data gathering, naturalistic participant observations, a clinical music therapy assessment undertaken by the music therapist (videoed), a review of the video by multidisciplinary team (MDT) members and the collaborative development of a programme of music experiences which will be especially planned to assist children to meet developmental goals from the Essential Skills Curriculum, and/or their Individual Education Plan. The music experiences will be introduced by one or more of the MDT. The primary focus of the project is to find out how the protocol is perceived, understood, valued, and used by each team of participants in turn, and with this knowledge to progressively develop and improve it.

You are invited participate because you work with a child who has High or Very High special education needs according to the Ongoing Reviewable Resource Scheme (ORRS guidelines, and you are a member of a multidisciplinary team interested in exploring the therapeutic use of music with that child. If you agree to participate, you will be asked to attend a multidisciplinary team meeting to share information regarding the child prior to a music therapy assessment that I will undertake. The music therapy assessment will be videoed and you will be asked to attend a focus group meeting to view and discuss the video following the assessment session. The meeting will take about one hour, and the focus group is likely to take two hours of your time. The focus group discussion will be audiotaped and transcripts will be returned to you for editing. Editing might also involve approximately one hour of your time.

Permission will be requested from the Principal of your school for meetings to be held at school at a time convenient to team members. At least one other member of your team will be involved in implementing planned music techniques, keeping a diary of their experience, and interviews.

All research data will be stored securely at Massey University, College of Creative Arts, for 
a period of five years after the completion of the study. It will then be destroyed.

I will take all reasonable steps to maintain confidentiality. Participants' names will not be used in verbal discussion with anyone outside the research teams. All identifying features will be removed from written published material, and all material will be securely stored. Nevertheless, the special education and music therapy communities in New Zealand are small and there is a risk that you might be identified.

You are under no obligation to accept this invitation. If you decide to participate, you have the right to:

- decline to answer any question

- withdraw from the study up until the time you have reviewed your transcripts

- ask questions about the study at any time during participation

- provide information on the understanding that your name will not be used unless you give permission to the researcher

- be given access to a summary of the project findings when it is concluded

The project has been reviewed and approved by the Massey University Human Ethics Committee, WGTN Application - 05/59. If you have any concerns about the conduct of this research, please contact Professor Sylvia Rumball, Chair, Massey University Campus Human Ethics Committee: Wellington, 06350 5249, humanethicswn@massey.ac.nz.

If you have any further questions about the study feel free to contact me on 048012794 x6979, D.J.Rickson@massey.ac.nz or Allan Badley on 042794 x6974, A.D.Badley@massey.ac.nz. If you are willing to participate, please sign the attached Consent Form and return it to me at the above address in the stamped and addressed envelope supplied.

Yours faithfully,

Daphne Rickson, Music Therapist / PhD Candidate. 
APPENDIX 15: Consent Form - General Team Member

\section{Massey University}

College of Creative Arts

\section{The Potential Role of Music in Special Education Settings in New Zealand - \\ Developing a Music Therapy Assessment and Consultation Protocol}

PARTICIPANT CONSENT FORM (SE Team Member)

This consent form will be held for a period of five (5) years

I have read the Information Sheet and have had the details of the study explained to me. My questions have been answered to my satisfaction, and I understand that I may ask further questions at any time.

I agree to participate in the study under the conditions set out in the Information Sheet.

I agree to participate in a focus group discussion and $\mid$ understand that the discussion will be audiotaped.

I wish/do not wish to have my tapes returned to me.

Signature:

Date:

Full Name - printed: 
APPENDIX 16: Information Sheet - Parents/Caregivers

P.O. Box 756,

\section{Massey University}

College of Creative Arts

Wellington.

Dear

My research project towards the degree of $\mathrm{PhD}$ (Music) is entitled 'The Potential Role of Music in Special Education Settings in New Zealand - Developing a Music Therapy Assessment and Consultation Protocol'. Dr Allan Badley, College of Creative Arts, Massey University, is my primary supervisor. I am writing to you because you have expressed interest in the possibility of having your child participate in the study.

I aim to develop and evaluate a Music Therapy Assessment and Consultation Protocol with members of 5-6 New Zealand special education teams (i.e. approximately 25-30 children, school staff, family/whanau and other specialists). The initial protocol is likely to involve data gathering, naturalistic participant observations, a clinical music therapy assessment undertaken by the music therapist (videoed), a review of the video by multidisciplinary team (MDT) members and the collaborative development of a programme of music experiences which will be especially planned to assist children to meet developmental goals from the Essential Skills Curriculum, and/or their Individual Education Plan. The music experiences will be introduced by one or more of the MDT. The primary focus of the project is to find out how the protocol is perceived, understood, valued, and used by each team of participants in turn, and with this knowledge to progressively develop and improve it. The study will be predominantly qualitative, and data will be gathered from multiple sources including participant observation, video of music therapy assessment sessions, focus groups, interviews, diaries, and logs.

Your child is invited to participate because he/she has high or very high special education needs according to the Ministry of Education's Ongoing Reviewable Resource Scheme (ORRS) guidelines. If you agree to your child's participation, I will undertake some observations of him/her working at home and/or school, gather data from other professionals, and he/she will be invited to attend a music therapy assessment session, lasting approximately 30-45 minutes depending on the amount of time he/she can remain engaged in the process. The music therapy assessment will be videoed and you and other members of your child's multidisciplinary team will be invited to view and discuss it with me. The school will be able to obtain a copy of the video to keep with (your child's) school records.

I will provide a music therapy assessment report within two weeks of the assessment taking place, and will plan a programme of music activities with the multidisciplinary team to help your child meet his/her IEP goals. Permission will requested from the Principal of your school for the assessment to be held at school at a time convenient to your child and team members. At least one other member of your team will be involved in implementing planned music techniques with your child, keeping a diary of their experience, and I will 
interview that person to find out how the music programme is going.

I will provide a brief report to the school following the completion of the study to outline ways in which the Music Therapy Assessment and Consultation Protocol developed.

I would also like to do two interviews with your child, if you believe your child is able to be interviewed. In the first interview I would ask your child some questions about how he/she felt about the music therapy assessment. In the second I would ask him/her about how the music programme is going at home and/or school. You would be welcome to have the audiotapes returned once they have been transcribed.

All research data will be stored securely at Massey University, College of Creative Arts, for a period of five years after the completion of the study. It will then be destroyed.

I will take all reasonable steps to maintain confidentiality. Your child's name will not be used in verbal discussion with anyone outside the research teams. All identifying features will be removed from written published material, and all material will be securely stored. Nevertheless, the special education and music therapy communities in New Zealand are small and there is a risk that your child or the school he/she attends might be identified.

You are under no obligation to accept this invitation. If you agree to your child's participation you have the right to:

- withdraw him/her from the study up until the time the assessment session has taken place

- ask questions about the study at any time during your child's participation

- provide information on the understanding that your child's name will not be used unless you and your child give permission to the researcher

- be given access to a summary of the project findings when it is concluded

- ask for the audiotape to be turned off at any time during the interviews

The project has been reviewed and approved by the Massey University Human Ethics Committee, WGTN Application - 05/59. If you have any concerns about the conduct of this research, please contact Professor Sylvia Rumball, Chair, Massey University Campus Human Ethics Committee: Wellington, 06350 5249, humanethicswn@massey.ac.nz.

If you have any further questions about the study feel free to contact me on 048012794 x6979, D.J.Rickson@massey.ac.nz or Allan Badley on 042794 x6974, A.D.Badley@massey.ac.nz. If you are willing to participate, please sign the attached Consent Form and return it to me at the above address in the stamped and addressed envelope supplied.

Yours faithfully,

Daphne Rickson, Music Therapist / PhD Candidate. 
APPENDIX 17: Consent Form - Parents/Caregivers

\section{Massey University}

College of Creative Arts

The Potential Role of Music in Special Education Settings in New Zealand - Developing a

Music Therapy Assessment and Consultation Protocol

PARTICIPANT CONSENT FORM (Parent/Guardian)

This consent form will be held for a period of five (5) years

I have read the Information Sheet and have had the details of the study explained to me. My questions have been answered to my satisfaction, and I understand that I may ask further questions at any time.

I agree to my child (name ) participating in music under the conditions set out in the Information Sheet.

\begin{tabular}{|l|l|}
\hline YES & NO \\
\hline
\end{tabular}

I agree to my child being videotaped doing music so the people who work with him/her can view it and talk about it.

\begin{tabular}{|l|l|}
\hline YES & NO \\
\hline
\end{tabular}

I agree to the videotape being shown to other members of the school community, in workshops or presentations.

\begin{tabular}{|l|l|}
\hline YES & NO \\
\hline
\end{tabular}

I agree to the videotape being shown at music therapy or education conferences, and for the education of music therapy students.

\begin{tabular}{|l|l|}
\hline YES & NO \\
\hline
\end{tabular}

Signature:

Date:

Full Name - printed: 


\section{APPENDIX 18: Information Sheet - Child}

Ph: 048012794 x6979

\section{Massey University}

D.J.Rickson@massey.ac.nz

College of Creative Arts

Date:

Dear

My name is Daphne and I am a music lady. I like to do all kinds of music with children at school and sometimes at home to help them to do and learn things. When I am playing with children we sometimes listen to music, sing, play musical instruments, dance, and make up songs.

I am wondering whether your helpers at school or home would like to do new music things with you too. I would like to come to your school or home to meet you, and to play with you. Your mum or someone else you like can come with you to watch. I would have a video camera on to film us doing music together.

I would like to show the video of our music and talk to the people who work with you. We will talk about the kind of music games you like and what might help you to do and learn things. Then I would like to write a story about what happened and teachers, mums and dads and other music people will read it. The story will not have your real name in it.

You are not the only child who I am writing to. I am going to ask about another five (5) children if they want me to do music at their school too.

If you would like to play with me and be in my story you need to say "OK" to the principal and if you can, write your name on the paper behind this one. Someone will write your name for you if it is too hard.

If you say "OK" it will be all right to change your mind later. You can tell your mum and dad or school helpers you don't want to do any more. They will help you decide what the best thing to do is. It is also a good idea to ask lots of questions until you are sure you know what this is all about.

If you say "OK" I will be very pleased to meet you. If you don't say OK, I will still be happy. Thank you for reading or listening to my letter.

Best wishes,

Daphne Rickson,

Music Lady. 
APPENDIX 19: Consent Form - Child

\section{Massey University \\ College of Creative Arts}

The Potential Role of Music in Special Education Settings in New Zealand Developing a Music Therapy Assessment and Consultation Protocol

\section{PARTICIPANT CONSENT FORM (Child)}

This consent form will be held for a period of five (5) years

Someone has read me the letter about Daphne's music work and I understand what it is about. I have had time to think about the music work and I have asked all my questions. I know I am allowed to ask more questions when I think of them.

It is OK to do music with Daphne.

It is OK for Daphne to have a video camera on when we are doing music.

It is OK/not OK to have a long talk to Daphne.

It is OK/not OK for Daphne to have her tape recorder on when we are talking.

Signature:

Date:

Full Name - printed: 


\section{APPENDIX 20: Interview Guidelines - Identified Team Member}

\section{Guidelines for Semi-Structured Interview with Team members}

The interview questions would be used to "create a scaffolding for the interview, keep the questioning on topic, and link what is asked in individual interviews to the overall design" (Rubin \& Rubin, 1995, p.150). There will be flexibility to discuss ideas that emerge during the interview. Manuscripts will be returned to participants inviting their editing and additional comments, so the interpretation will not belong to me alone.

\section{Before the Assessment}

1. I want you to think about ALL music making (including spontaneous singing around the playground, house/yard; tapping rhythms etc. as well as planned music making) and tell me about how you use music in your work with (name)?

2 .

a. Tell me more about how you gained those skills OR

b. Tell me more about why you feel you don't use music with (name)?

3. What things do you think are important for (name)?

4. What are your concerns for (name) this week/today?

5. Tell me how you FEEL about the work you are doing to help (name) reach their IEP goals?

6. How do you think your child will respond in this music therapy assessment situation?

7. What do you expect from the music therapy assessment?

\section{$\underline{\text { Immediately After the Assessment }}$}

1. What are your immediate thoughts / impressions now that you have seen (name) interacting with the music therapist?

2. Tell me about the things that were difficult for you when watching assessment procedure?

3. Tell me about the things that were helpful for you when watching assessment procedure?

4. What did you find out about (name) while you were watching the assessment procedure?

5. Which of the activities or techniques you saw today do you think you would like to introduce yourself?

\section{At Four Month Follow-up}

1. Tell me how musically confident you feel now?

2. How do you think the music making is going with (name)?

3. Can you tell me how you FEEL about the work you are doing to help (name) reach their IEP goals? 


\section{Guidelines for Semi-Structured Interview - Child}

The interview questions would be used to "create a scaffolding for the interview, keep the questioning on topic, and link what is asked in individual interviews to the overall design" (Rubin \& Rubin, 1995, p.150). There will be flexibility to discuss ideas that emerge during the interview.

\section{After the Assessment}

6. Tell me what you think about the music we did together?

7. Which parts did you like best?

8. Which parts didn't you like?

9. Tell me if there are some music things you would like to keep doing?

10. Why do/don't you think it would be good to keep doing music?

\section{At Four Month Follow-up}

4. Tell me about the music you have been doing at school?

5. Tell me about the music you have been doing at home?

6. Which things do you like best?

7. Which things didn't you like?

8. Do you think you will keep doing music? 
APPENDIX 22: Authority for the Release of Tape Transcripts

\section{Massey University}

College of Creative Arts

\section{The Potential Role of Music in Special Education Settings in New Zealand - \\ Developing a Music Therapy Assessment and Consultation \\ Protocol}

\section{AUTHORITY FOR THE RESEASE OF TAPE TRANSCRIPTS}

I confirm that I have had the opportunity to read and amend the transcript of the interview/s conducted with me.

I agree that the edited transcript and the extracts from this may be used by the researcher, Daphne Rickson, in reports and publications arising from the research.

Signature:

Date:

Full Name - printed: 
APPENDIX 23: Interview Topic - Focus Group

\section{Guidelines for Focus Group Interview}

Focus groups enable in-depth discussion on a specific area of interest. It is a group discussion that relies heavily on the interaction between participants rather than individually answering the facilitator's questions. The facilitator introduces the topic and stimulates and assists participants to discuss it. The facilitator also needs to control the group to proceed in the direction that the focus group takes (Rice \& Ezzy, 1999).

\section{Topic}

How can (special education team members) use music and music therapy techniques to help (a child) to meet developmental or IEP goals? 


\section{APPENDIX 24: Interview Transcript, Petra}

Interview with Petra

Review of information and consents forms, highlighting research questions: "What do you do when assessing students for consultation purposes?", and "What can you learn about a child from just one assessment session and how can that information be used to help others use music in their work?"

DR: Can you tell me about your work with children who have special needs for a start?

PT: Yep. I've worked with children aged 5-21, so children and young

adults with a variety of learning disabilities in a school setting

which is - and I've been there for $4 \frac{1}{2}$ years...I set it up $4 \frac{1}{2}$ years ago

and its gone from part time to full time, back to part time, back to full

time... basically full time for the last three years. Um...

DR: And that's because you've chosen that isn't it?

PT: That's only because I... yeah through choice, yep. There's actually two

of us there now so that's pretty good. And we now access about five or

six other schools at present in the xxxxxxxxx area.

DR: Ok. Now um just before you move on - it's really interesting when you

say you "access those schools"... you go to those schools do you?

PT: They access us (Oh, they come into you.) Um, we do go to one other

unit which is a unit for physical disabilities and that's happened for

about $4 \frac{1}{2}$ years as well. So we actually go there, um but we have small

groups, large groups um come to us at the ah music therapy centre we call

it I suppose, or room, and that changes from term to term who we have

and some of it's short term contract. And often it just works that they

pay the school, the $\$ 60$ an hour and... um it's worked out that way. So

we've had quite a variety - I think we're probably looking at about six

DR: Cool. Any of that work that's um contracted, like short term 
PT: For a term or...mostly it's due to finances whether it stays or doesn't stay. In the past it has been to do with the money's run out or the 28 year's ended and it hasn't been restarted. (DR: Right). 29

$\mathrm{DR}$ : So, if that happens, or if the therapy stops for any other reason, do 30 you then put some kind of programme in place for other people to use - 31 PT: I haven't done that and I hesitate to do that - I don't believe in 32 that particularly. 33

DR: Can you tell me more about that because I am particularly interested 34 in that. 35

PT: I just don't think that music therapy works without a music 36 therapist. (DR: Right...) and whilst I'm happy to give advice as to how to 37 run it... I just don't believe that it's at all... I just think music therapy 38 is about the relationship between the therapist and the client. 39

DR: Yep. Can we go back to your thought though that you would be happy to 40 give advice? Do you do that? Because that's really what I am asking... 41 PT: But only very informally though - I only do it informally. I haven't 42 done it in a situation like; say a speech therapist would set up a 43 programme. I haven't done - and then the secretary of the school runs it, 44 things like that - I haven't done that. (DR: Right). And I haven't needed 45 to do that yet... if I was to go and work for Group special Ed or another 46 place, then sure I think that would happen. 
therapeutic way and people - you can't expect somebody just because they 56

can play two chords on a guitar to convey that. And a lot of people 57

wouldn't play two chords on a guitar. Most people you would be using, 58

like teacher aides, don't have those skills. And anyone can press the 59

'play' on a CD player but that's not music therapy. I'm just very 'anti' 60

it.

DR: Mm. I think what we probably should clarify before um we go any

further is that I am talking about helping people use music in more

therapeutic ways - I think we do differentiate music therapy, as a

profession, from the use of - the therapeutic use of music.

PT: Yeah absolutely.

DR: So what I am talking about in my study is whether we can empower

people to interact musically and to use music more therapeutically. And I

do have a basic premise that there are a lot of people out there doing

PT: Oh yeah there's a lot! Not in xxxxxxxxxxxx there's not but I am sure...

I know that there is out of...you know.

DR: Yes, and you know my thought is that we have skills as music

therapists to help others and at the moment... there's another whole side

Music Therapy, in contextualised music therapy, there's a big shift from

the medical model of music therapy to an empowerment model 
nothing, or is there something we can do? You did say that you thought -

PT: I'm sure that there is but then I would be very hesitant to call it

music therapy. Of course there's a huge amount that you can do. But would

I - by transferring all those ideas would I be using my therapeutic

skills or would I be using my education skills in doing that, in teaching

these teacher aides? Do you know what I mean?

DR: I think I do, but I don't think that we a talking about anything

different... you said "would you call it music therapy" and I have already

said I don't think you would. But you're still a music therapist - is

there another role for a music therapist to empower others to use music

PT: Yeah. I think could...there definitely could be. But then it becomes...

it becomes a little bit um frightening in that the fact that few music

therapists that we have would then be sitting in an um office writing

100

these programmes for... I think this is what we have been going round and

round in circles in xxxxxxxxxxxx about... is that ok, do we employ music

therapy through GSE - "yes" so that then I can be shared amongst the 300

103

people that have ORRS funding... I physically can't see 300 people. Um, do

104

I sit at a desk for five days a week and see them once a term like the

105

speech and physios and OTs do, and just check off "yes" you are doing

106

'Row Row Row Your Boat' and 'Mary Had a Little Lamb' - fantastic. Is

107

that... am I a music therapist then? Am I using my skills for the best?

DR: Well I don't know. Um, I wonder if we are still looking at it as an

'all or nothing' thing. I mean your thought that music therapists might

sit at a desk for five days a week and write programmes is definitely not

a realistic sort of thing. (PT: No it's not). But going back to your

thought that you know these children, you've worked with them and you've

PT: What's really well? Is that five weeks, is that three weeks...

$\mathrm{DR}$ : Or is it one assessment session?

$\mathrm{PT}$ : Is it one assessment session - that's not 'really well'!

DR: Tell me why.

PT: Well I mean anything can happen once. You're not going to get that 
PT: I think... I don't have... I don't use a formalised assessment - we work

DR: I do too. I don't think it is a 'should' at all! (PT: No) Definitely 
might lose some of our roles?

$\mathrm{PT}$ : Yeah.

DR: Right. Because that's certainly not - (PT: You throw a lot...) - not what I am anticipating.

PT: You throw a lot of expectation on...on to other people who are

transferring those programmes. I think it's easy for a person, and it's

'Joe Blow', to sit down and go through speech um sounds and programmes

DR: (Laughs). No. I totally agree with you.

PT: I just remember my first - one of my first sessions at xxxx and the 
really want to access these 300 people. (DR: Yeah) And I haven't even

touched the surface really because - on purpose - because I knew that I 
$\mathrm{DR}$ : Yes. Why have they not worked.

PT: (pause). I believe it's because music therapy can be so general, and

DR: Well to be so structured I guess.

PT: Yeah... to be so structured. And I mean we're not... do we aim to get all 
prior to -

PT: Getting in yeah, yeah, and even with parent discussion I want to make

DR: Mm. That's what I am calling in my piece of work the 'assessment

DR: - it's a relatively unstructured session... would you call

PT: Not always! If I knew that that student, if they were coming to me 
enough and I am wondering what "enough" is... I don't think we can ever

totally know somebody altogether - as you say assessment in music therapy

PT: And by the third week I was thinking reasonably differently to what I

DR: So tell me what it is about - what sort of things come out of your 
that a main goal that had come out of his psychiatrist's report up in

PT: Yeah! A formal assessment - it would be great. I think sometimes it

PT: Whether there's an outcome after the formal assessment I don't know.

PT: But in that situation, looking back, I'm thinking it probably 
know, when you read your transcript you might see that you have modified

PT: But I can see the pros and cons and I just think it changes with 
analysing what I do and just seeing "if you work flexibly, if you let the we learn if we go in without a tick chart" - (PT: And how do we transfer that to other people) - Exactly, exactly.

(Further discussion about analysis to date)

PT: I often...not often - no often - do feel I think that I'm sitting in 
DR: How do you write your social goals - can you give me one example?

PT: Um "Jacky will... learn to greet the child...um in the mainstream class...

PT: Yeah, I probably haven't...I don't do a tick chart though that says

PT: Well it's an overall... but I don't sit there and tick "Ok she's done

DR: I'm not saying (PT: But I haven't done it like that) whether it is

DR: I see what you're saying. I see what you're saying. Yep. 
report would simply report that -

PT: There would be that section - if there's a tick part. There would be

a general... the introduction section saying this is what she is doing, and

then the evaluation section at the end - recommendations.

DR: It's descriptive? How would you describe your report?

PT: Um I... we do... I do a few different types of reports obviously. I do

the ones that I have one on one, and they would be more specific and I do

the ones that I only see, you know, generally that I would see through a

large group and they would be group goals. I don't do individual goals

educational perspective - music education. And the become just tick

charts (sighs) and then the general comment at the end. So they not

particular specific. I can send you copies if you like?

DR: Oh no, no, no, no. That's fine. What you might do though is try to

describe for me - because I know you've had a lot of um political

discussions recently with your school, which is highly supportive, and

with Group Special Ed which I imagine you've had a bit of work to do. And

I know that you've had a lot of support from your Principal. But if you

500

had to sit right now and argue your case with um a GSE manager, what

501

would you say about your music therapy work? How would you produce the

PT: I wouldn't be able to. I wouldn't be able to. To actually sit there

DR: Let's not say statistics. Let's just say 'evidence'. How do you know

that you're doing a good job?

PT: (long pause). I wrote this all down the other day. We all had to do

it for something. Now it's all gone from my head.

DR: Well you could send those if you like to me - I'd be interested in

that.

PT: I guess um... I would use a variety of - I would probably show...I would

show reports from one... one or two students over a period of a few years.

I would present all the um things we've produced within the school. So

three CDs, all the song writing, all the productions... um... and I would 
haven't sat down and had this meeting with GSE yet and I've always

DR: I think one of the tricky things that you already identified earlier everyday and in the home and...being able to facilitate music all the time. 
PT: Oh no - I've always been on the same wavelength as you thinking that but I wasn't thinking that way before. I was more thinking more in the 
early childhood education, and music therapy - there's a real crossover

there in that situation. So yeah, definitely those skills could be

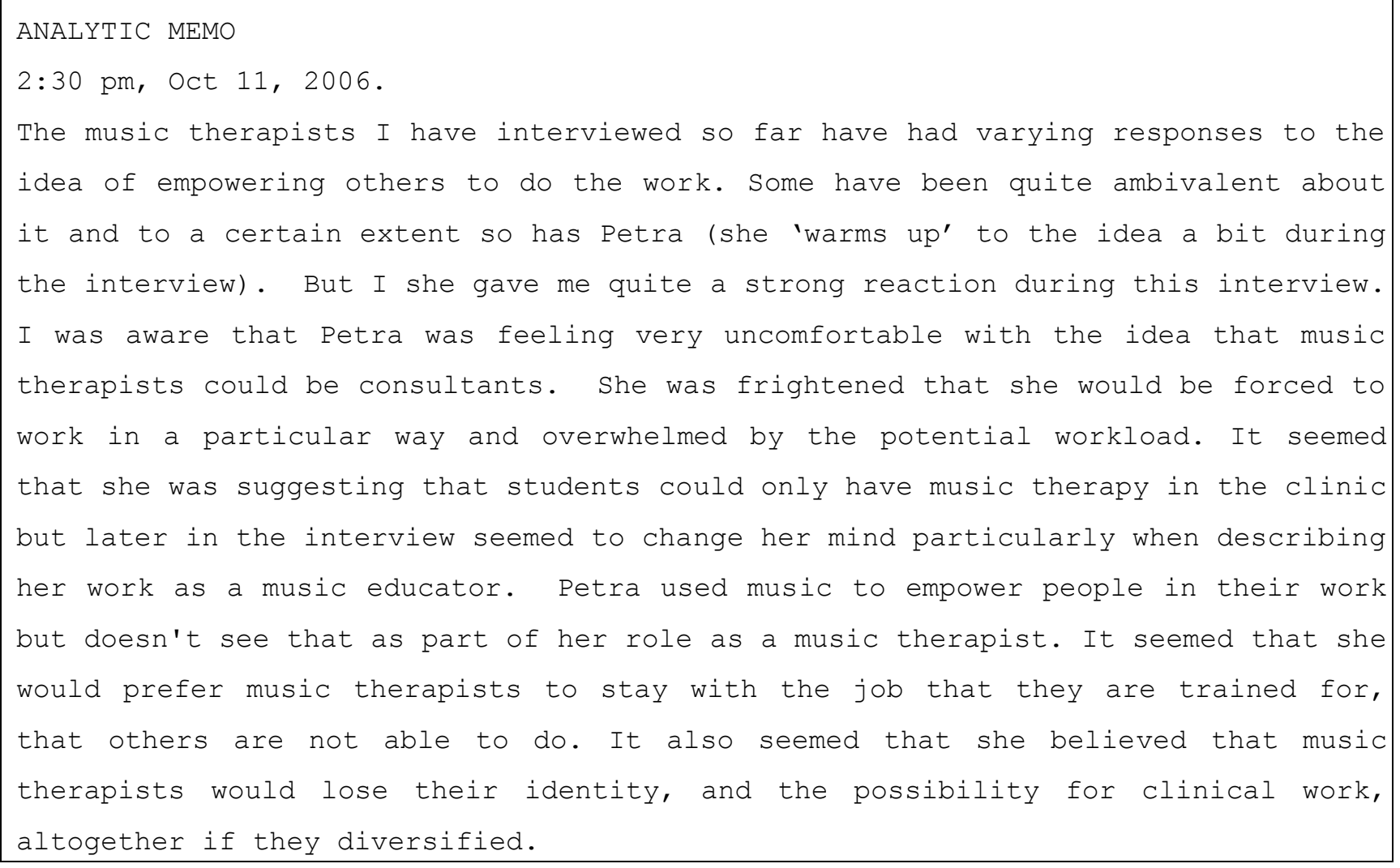


APPENDIX 25: Preliminary Definitions of Categories

\begin{tabular}{|l|l|}
\hline Work Context & $\begin{array}{l}\text { Include data on the context (e.g. special school } \\
\text { or inclusive setting) where the assessments } \\
\text { took place }\end{array}$ \\
\hline Music Therapists' Previous Experience & $\begin{array}{l}\text { Include data on any special skills the music } \\
\text { therapist bring to the assessment process, } \\
\text { including previous experience with SN } \\
\text { children, teaching qualifications }\end{array}$ \\
\hline Time & $\begin{array}{l}\text { Include comments regarding time allocated for } \\
\text { assessment purposes, time for consultations }\end{array}$ \\
\hline Working with MDT & $\begin{array}{l}\text { Include comments on music therapists' liaison } \\
\text { with the multidisciplinary team }\end{array}$ \\
\hline The Purpose of Assessments & $\begin{array}{l}\text { Include information on what music therapy } \\
\text { assessments were for and how they were used }\end{array}$ \\
\hline What is Consultation & $\begin{array}{l}\text { Include information on what music therapists } \\
\text { DO when they are 'consulting' }\end{array}$ \\
\hline Referral for Assessment & $\begin{array}{l}\text { Include information about how students get } \\
\text { referred for assessment, and the reasons for } \\
\text { referral }\end{array}$ \\
\hline Client Groups & $\begin{array}{l}\text { How does the music therapist conduct the } \\
\text { assessment session... does she use assessment } \\
\text { tools or improvisation }\end{array}$ \\
\hline $\begin{array}{l}\text { Include information about how the music } \\
\text { therapist accesses client or other information } \\
\text { when assessing or consulting }\end{array}$ \\
\hline \begin{tabular}{l} 
Describe the students and/or their diagnoses \\
\hline
\end{tabular}
\end{tabular}

\section{Definitions of Categories that Emerged}

\begin{tabular}{|l|l|}
\hline $\begin{array}{l}\text { Differences between Consultation and Longer } \\
\text { Term Work }\end{array}$ & $\begin{array}{l}\text { Include data that compares clinical work in } \\
\text { schools, to consultation work }\end{array}$ \\
\hline Funding & $\begin{array}{l}\text { Include data on who pays for music therapy } \\
\text { assessment/consultation, and any other issues } \\
\text { related to funding }\end{array}$ \\
\hline
\end{tabular}




\begin{tabular}{|l|l|}
\hline Evaluating Assessment/Consultation Practices & $\begin{array}{l}\text { Include information regarding how music } \\
\text { therapy consultation is assessed and/or how } \\
\text { MTs know their assessments are helpful }\end{array}$ \\
\hline What is Consultation & $\begin{array}{l}\text { Include information on what music therapists } \\
\text { understand of 'consulting' }\end{array}$ \\
\hline Attitudes to Consultation & $\begin{array}{l}\text { Include any comments that give an indication } \\
\text { of the MTs thoughts and feelings about } \\
\text { consultation, and the single session assessment } \\
\text { idea }\end{array}$ \\
\hline Negotiating Goals & $\begin{array}{l}\text { Include any comments about philosophical } \\
\text { differences that may exist between education } \\
\text { and therapy and how this might be managed. }\end{array}$ \\
\hline
\end{tabular}


APPENDIX 26: Category - What can the data tell me about helping others to use music...

What can the data tell me about helping others to use music in their work?

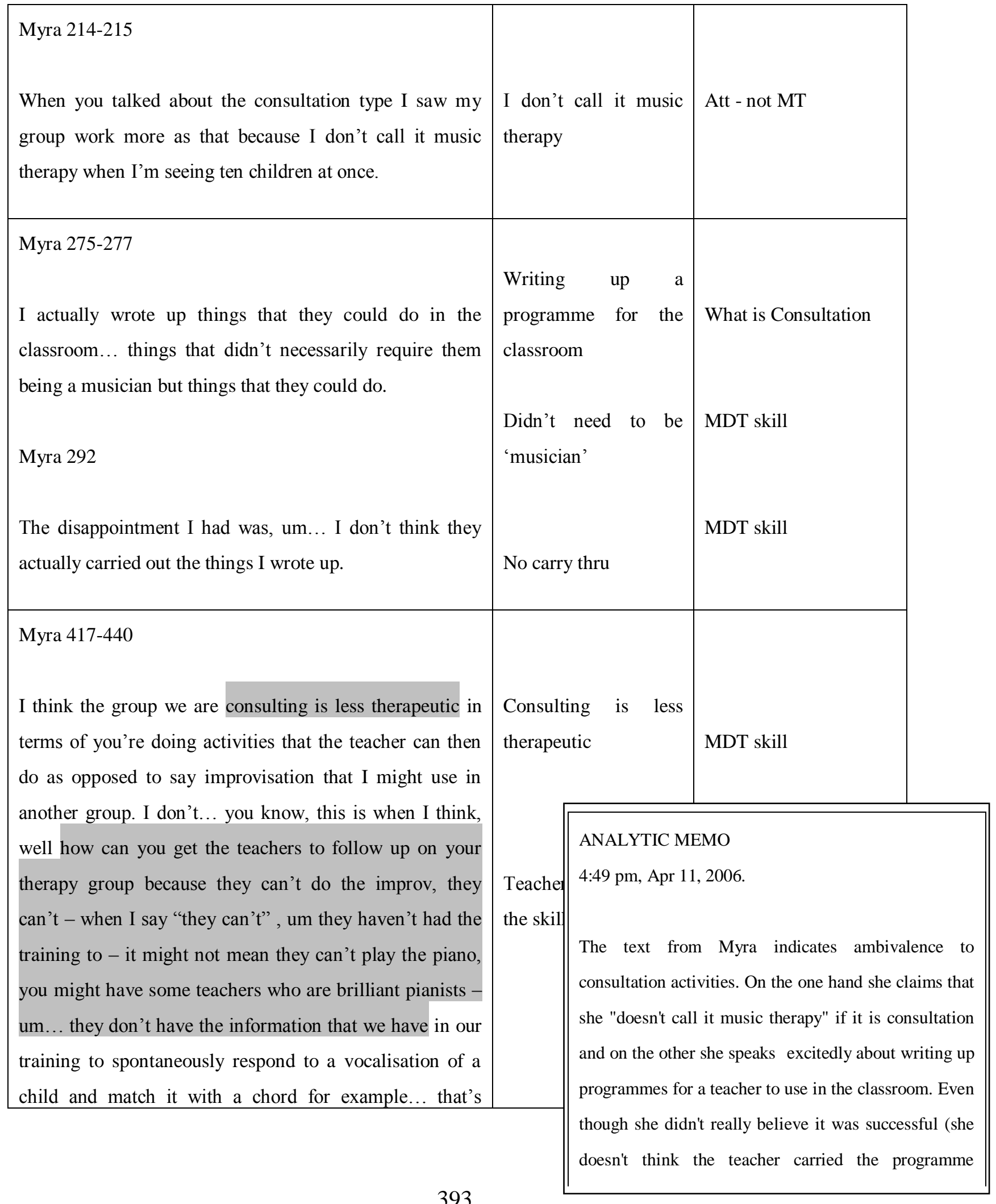




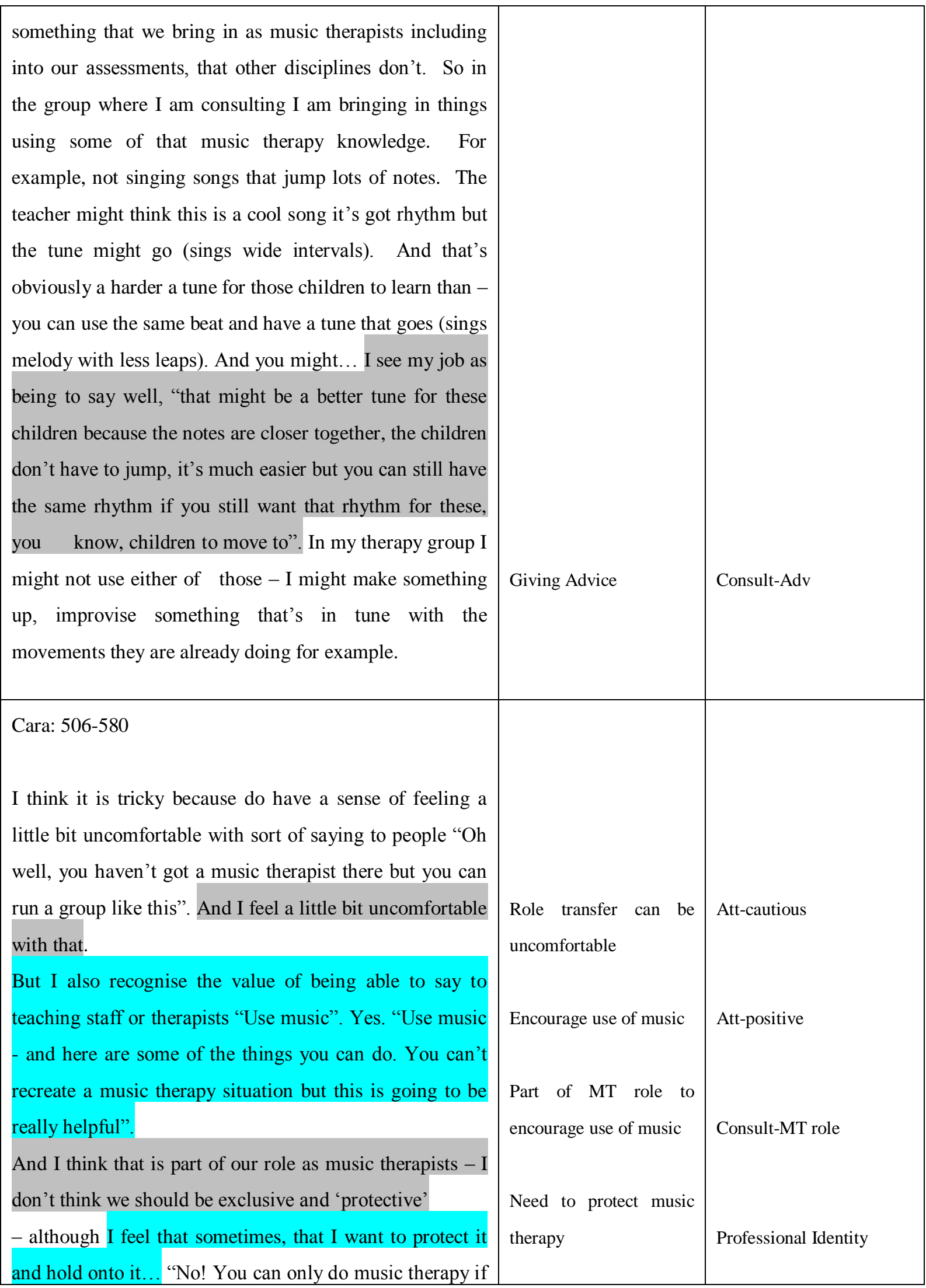




\begin{tabular}{|c|c|c|}
\hline you're a music therapist and trained in it!" & & \\
\hline $\begin{array}{l}\text { Kath 266-269 } \\
\text { We are far more collaborative now that we have ever been } \\
\text { over the last ten or twenty years, and this is very good. } \\
\text { There's a lot more sharing of skills and finance and } \\
\text { opportunities. }\end{array}$ & $\begin{array}{l}\text { Very good to } \\
\text { collaborate }\end{array}$ & $\begin{array}{l}\text { Att-increase } \\
\text { collaboration }\end{array}$ \\
\hline $\begin{array}{l}\text { Kath 277-301 } \\
\text { I've got a drumming group at a primary school. Anybody } \\
\text { can come. About four of those sixteen that turned up this } \\
\text { year are referred children on my caseload. So they are } \\
\text { referred for lack of attention, lack of motivation, unable to } \\
\text { express themselves in the classroom and so on... ...And } \\
\text { then I started pulling kids out who wanted to play drums } \\
\text { because they were just a disaster in terms of the school } \\
\text { orchestra if they don't have a little bit of training! } \\
\text { (Laughs). So I pulled them out, but these kids are part of } \\
\text { the orchestra which means they have access to assemblies, } \\
\text { going to old people's homes and stuff, and I take them } \\
\text { out. }\end{array}$ & $\begin{array}{l}\text { Drumming for all } \\
\text { Assemblies, outings }\end{array}$ & What is consult \\
\hline $\begin{array}{l}\text { Kath 333-338 } \\
\text { (If I wanted to collaborate) I had to make the advances. } \\
\text { But what I find is that those hats are really, really stifling. } \\
\text { And I found this as the 'music therapist'. If you're the } \\
\text { music therapist that's what you 'are'. If you're the RTLB, } \\
\text { that's what you 'are'. So, hopefully in this country we'll } \\
\text { start moving away from that. }\end{array}$ & $\begin{array}{l}\text { Role transfer can be } \\
\text { uncomfortable }\end{array}$ & $\begin{array}{l}\text { Consult-diff } \\
\text { relationships }\end{array}$ \\
\hline Lisa $336-353$ & & \\
\hline
\end{tabular}




\begin{tabular}{|c|c|c|}
\hline $\begin{array}{l}\text { Well the work that I am doing most of the time now with } \\
\text { adults who have intellectual disabilities, I'm doing more } \\
\text { and more consultation work and um I think that is } \\
\text { valuable because we have a waiting list and we can see } \\
\text { people for a limited amount of time. And it's a way to } \\
\text { extend things that have worked for them into other } \\
\text { environments, into their home and day programmes. Um } \\
\text { and it also can be a really good way to enhance the } \\
\text { relationships between the staff and the, and the people } \\
\text { because all the people we are working with are living in } \\
24 \text { hours a day staffed residential homes and ... ...(a staff } \\
\text { member asked for ideas) and I found some suggestions I } \\
\text { had written up for the last place he lived - these could be } \\
\text { used in his new home, so there was a way in to make } \\
\text { contact for someone who's got no speech, who's got } \\
\text { autism, you know. }\end{array}$ & $\begin{array}{l}\text { Consult to reduce } \\
\text { waiting list } \\
\text { A way to extend things } \\
\text { A really good way to } \\
\text { enhance relationships } \\
\text { Making suggestions }\end{array}$ & $\begin{array}{l}\text { What is consultation } \\
\text { What is consultation } \\
\text { What is consultation }\end{array}$ \\
\hline $\begin{array}{l}\text { Lisa 360-369 } \\
\text { For some people I've done consultation with, what it's } \\
\text { meant is that, where the staff are willing and have got the } \\
\text { time to do it, that that person is probably getting some } \\
\text { music input you know maybe four times a week, which is } \\
\text { always going to be more than I can offer! And some of } \\
\text { the people I work with, if the staff are able to ah um... } \\
\text { seize the opportunity, if the person is in the mood and } \\
\text { they can give them five minutes of quality interaction } \\
\text { using music, well that's great because I might go and see } \\
\text { that person and it might not be a good time for them, so } \\
\text { yeah... I think those things are really important. }\end{array}$ & $\begin{array}{l}\text { More musical input } \\
\text { Staff can seize the } \\
\text { opportunity for quality } \\
\text { interaction }\end{array}$ & $\begin{array}{l}\text { Why consult? } \\
\text { Att-positive } \\
\text { Why consult? } \\
\text { Att-really important }\end{array}$ \\
\hline $\begin{array}{l}\text { Lisa 377-389 } \\
\text { And for some people who um you know who are unable } \\
\text { to go out......it might be more effective for someone to }\end{array}$ & & \\
\hline
\end{tabular}




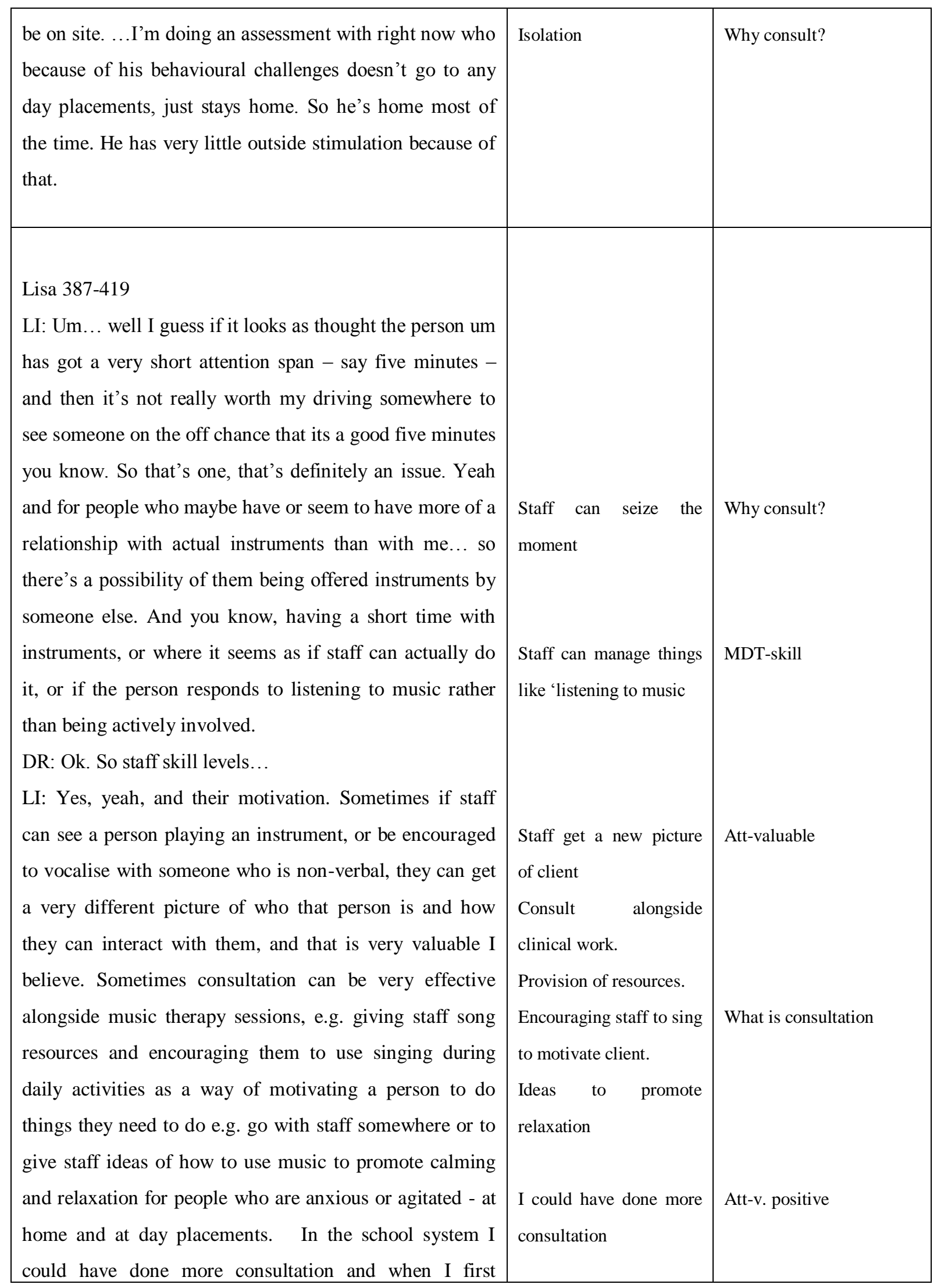




\begin{tabular}{|c|c|c|}
\hline $\begin{array}{l}\text { started um working I wasn't... that wasn't really in the } \\
\text { forefront of my mind to do consultation - I was very } \\
\text { focussed on doing the music therapy sessions - and I } \\
\text { think that it would of... it's something that needs to be } \\
\text { discussed as a possibility when you're starting you know } \\
\text { as a music therapist that this is something that you can } \\
\text { offer in these circumstances. }\end{array}$ & $\begin{array}{l}\text { MTs need to know about } \\
\text { consultation }\end{array}$ & Training \\
\hline 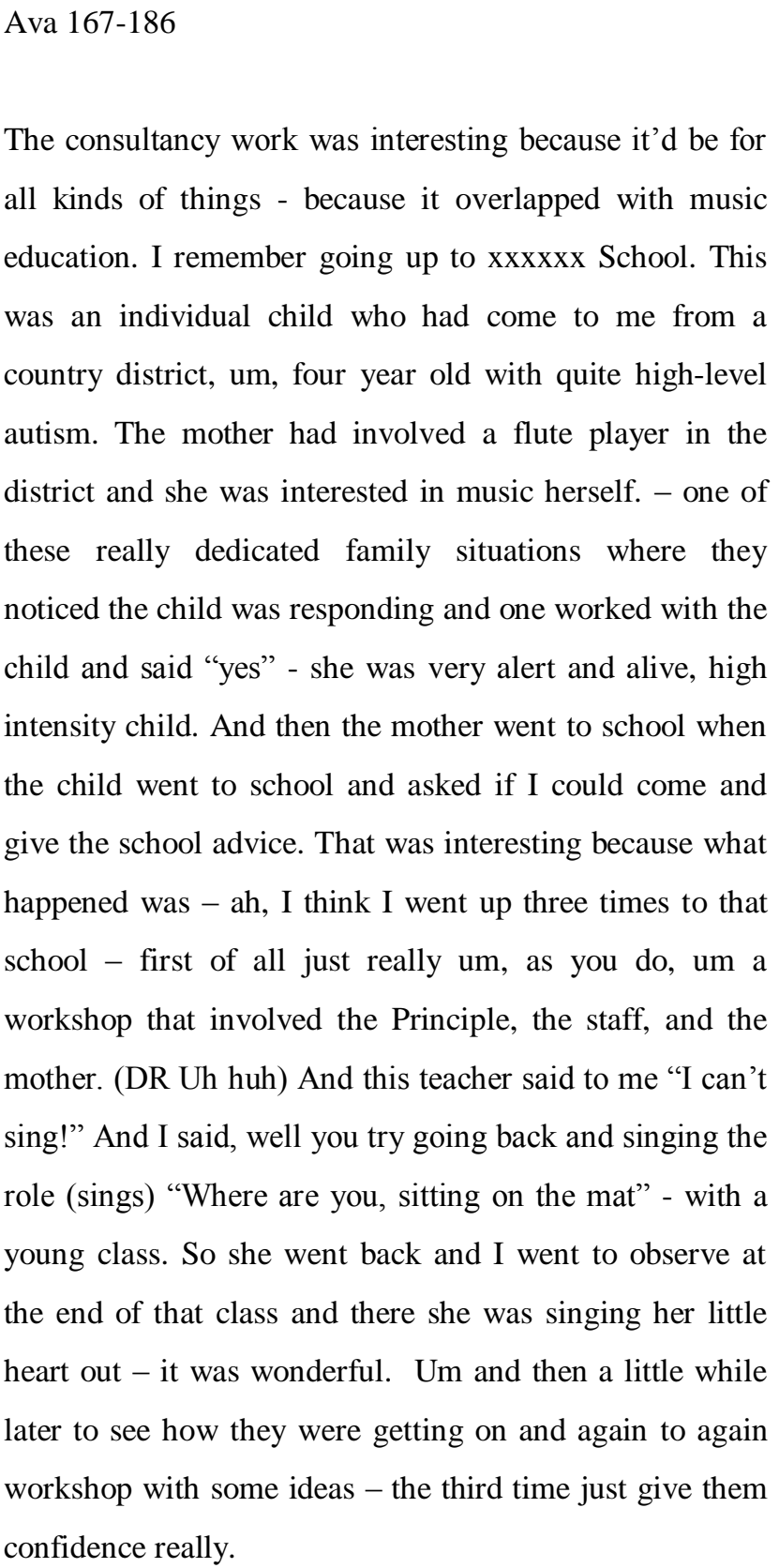 & $\begin{array}{l}\text { Workshop } \\
\text { Empowering staff to } \\
\text { sing }\end{array}$ & $\begin{array}{l}\text { What is consultation? } \\
\text { What is consultation? }\end{array}$ \\
\hline
\end{tabular}




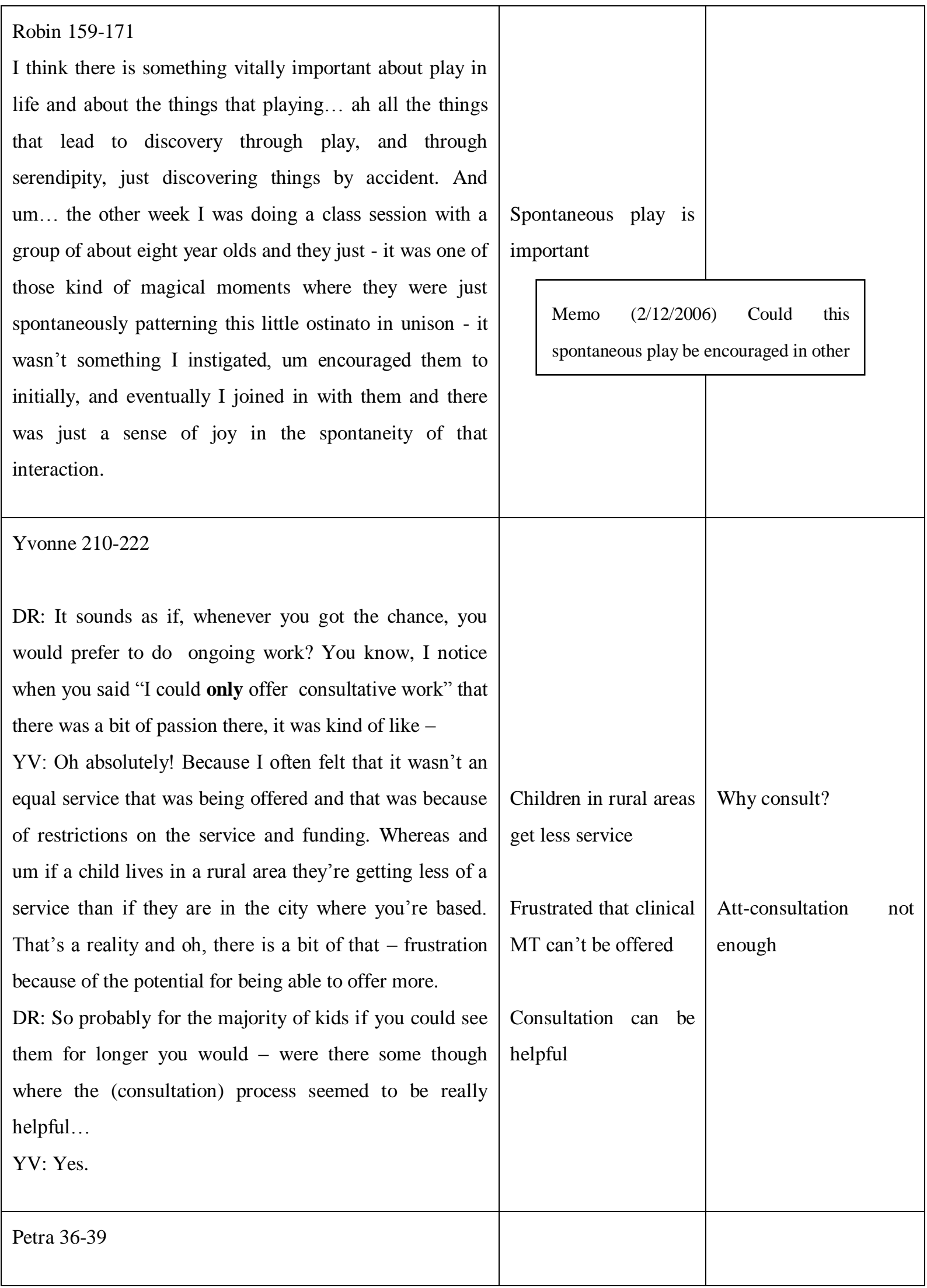




\begin{tabular}{|c|c|c|}
\hline $\begin{array}{l}\text { PT: I just don't think that music therapy works without a } \\
\text { music therapist... and whilst I'm happy to give advice as } \\
\text { to how to run it, I just don't believe in that at all. I just } \\
\text { think music therapy is about the relationship between the } \\
\text { therapist and the client. }\end{array}$ & $\begin{array}{l}\text { Only MTs can do music } \\
\text { therapy } \\
\text { MT relationship } \\
\text { Don't believe in it. }\end{array}$ & Att-negative \\
\hline $\begin{array}{l}\text { Petra 43-47 } \\
\text { (Giving advice) - I only do it informally. I haven't done it } \\
\text { in a situation like; say a speech therapist would set up a } \\
\text { programme... and then the secretary of the school runs it, } \\
\text { things like that - I haven't done that. And I haven't } \\
\text { needed to do that yet... if I was to go and work for } \\
\text { Group Special Ed or another place, then sure I think that } \\
\text { would happen. }\end{array}$ & $\begin{array}{l}\text { Setting } \\
\text { programmes }\end{array}$ & What is consultation? \\
\hline $\begin{array}{l}\text { Petra 55-61 } \\
\text { Our training is all about putting across the music and } \\
\text { using it in a therapeutic way and people - you can't } \\
\text { expect somebody just because they can play two chords } \\
\text { on a guitar to convey that. And a lot of people wouldn't } \\
\text { play two chords on a guitar. Most people you would be } \\
\text { using, like teacher aides, don't have those skills. And } \\
\text { anyone can press the 'play' on a CD player but that's not } \\
\text { music therapy. I'm just very 'anti'it. }\end{array}$ & $\begin{array}{l}\text { Most team members } \\
\text { don't have the skills to } \\
\text { use music. } \\
\text { That's not MT } \\
\text { I'm just very 'anti' it }\end{array}$ & $\begin{array}{l}\text { MDT Skills } \\
\text { Att-not MT } \\
\text { Att-very 'anti' }\end{array}$ \\
\hline $\begin{array}{l}\text { Petra } 87-92 \\
\text { PT: I'm sure that there is (something we can do) but then I } \\
\text { would be very hesitant to call it music therapy. } \\
\text {...Of course there's a huge amount that you can do. But } \\
\text { would I - by transferring all those ideas would I be using } \\
\text { my therapeutic skills or would I be using my education } \\
\text { skills in doing that, in teaching these teacher aides? }\end{array}$ & $\begin{array}{l}\text { Consultation is not } \\
\text { MT } \\
\text { Transferring skills } \\
\text { involves education } \\
\text { skills }\end{array}$ & What is consultation? \\
\hline
\end{tabular}




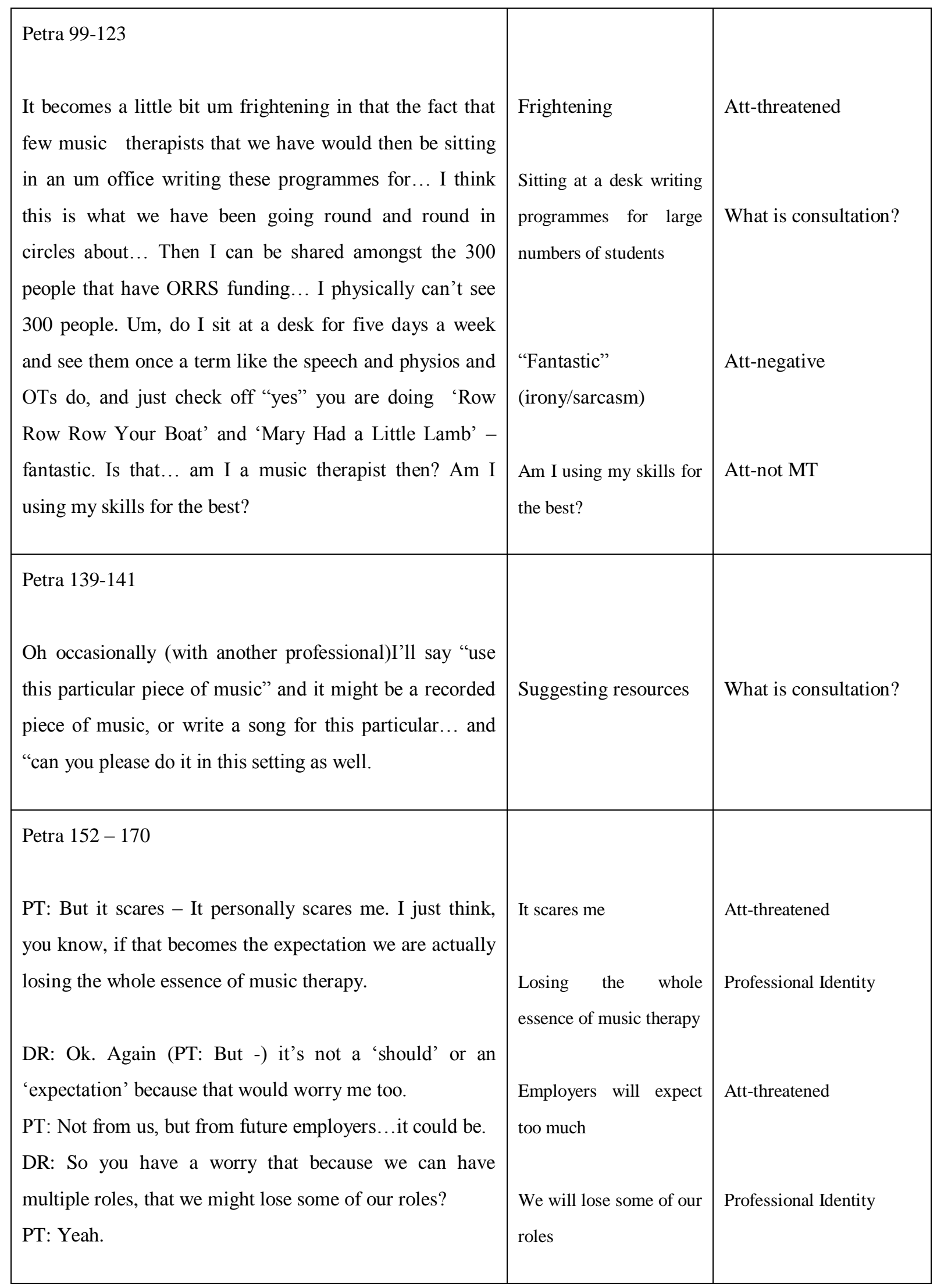




\begin{tabular}{|c|c|c|}
\hline $\begin{array}{l}\text { PT: You throw a lot of expectation on... on to other } \\
\text { people who are transferring those programmes. I think it's } \\
\text { easy for a person, and it's 'Joe Blow', to sit down and go } \\
\text { through speech um sounds and programmes and things, I } \\
\text { think that's very basic but to transfer anything musically } \\
\text { unless they're using all recorded music, which they would } \\
\text { be. And I think that um people get very overwhelmed by } \\
\text { music and very daunted by music...(pause) and I think it } \\
\text { could turn into bashing the drum away to the Beatles or... } \\
\text { and is that therapeutic? I don't think so. }\end{array}$ & $\begin{array}{l}\text { It's harder to transfer } \\
\text { music programmes to } \\
\text { MDT - they'll all use } \\
\text { recorded music } \\
\text { It won't be therapeutic }\end{array}$ & Att-negative \\
\hline $\begin{array}{l}\text { Petra 191-196 } \\
\text { But, yeah, I'm not saying definitely no, and I think there } \\
\text { is a definite need especially in a place of isolation like I'm } \\
\text { in. There's a definite need for me to access more people } \\
\text { than I have. And I'm definitely getting to that point now } \\
\text { where I really want to access these } 300 \text { people. And I } \\
\text { haven't even touched the surface really because - on } \\
\text { purpose - because I knew that I realistically couldn't do } \\
\text { it. }\end{array}$ & $\begin{array}{l}\text { There's a need to access } \\
\text { isolated students } \\
\text { I haven't even touched } \\
\text { the surface really }\end{array}$ & Att-overwhelmed \\
\hline $\begin{array}{l}\text { Petra 200-204 } \\
\text { And yeah become more of a referral person I suppose } \\
\text { person but there's a lot of hesitation. And I know that } \\
\text { that's where the GSE job is headed. And it's getting pretty } \\
\text { close now - we're getting pretty close to that. They } \\
\text { already made their decision... they have and it's gone } \\
\text { through. }\end{array}$ & $\begin{array}{l}\text { A referral person } \\
\text { They already made } \\
\text { their decision }\end{array}$ & $\begin{array}{l}\text { What is consultation } \\
\text { Feeling powerless }\end{array}$ \\
\hline $\begin{array}{l}\text { In a way I think of that as my music education hat...Next } \\
\text { week and the week after I'll be going to educate childcare }\end{array}$ & $\begin{array}{l}\text { I wear my education hat } \\
\text { when consulting }\end{array}$ & What is consultation? \\
\hline
\end{tabular}




\begin{tabular}{|c|c|c|}
\hline $\begin{array}{l}\text { educators about using music everyday and in the home } \\
\text { and...being able to facilitate music all the time. And even } \\
\text { if it is, as you said singing 'Twinkle Twinkle' or um just } \\
\text { using - realising how much music is part of everyday life } \\
\text { whether it's banging the saucepans with the wooden } \\
\text { spoons or whatever it is and yeah, becoming creative } \\
\text { beings again and... finding their inner music I suppose as } \\
\text { an adult, and transferring it to the child. }\end{array}$ & $\begin{array}{l}\text { Music is part of } \\
\text { everyday life } \\
\text { Helping people become } \\
\text { creative, finding their } \\
\text { inner music as an adult }\end{array}$ & 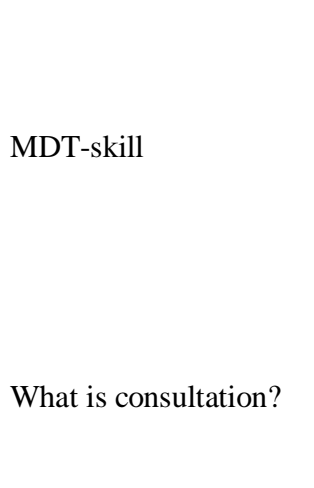 \\
\hline $\begin{array}{l}\text { Petra 556-562 } \\
\text { I was more thinking more in the fact that that shouldn't } \\
\text { become only our role. That it could be a very nice part of, } \\
\text { but certainly not to be expected as the only role. And } \\
\text { that's the one thing that scares me, is that we lose our } \\
\text { clinical... sessions and that clinical sessions are not... are } \\
\text { still valued as the most, as the ultimate music therapy } \\
\text { session. But if we need to be in a referral role, then that's } \\
\text { fine as well - but in addition. }\end{array}$ & $\begin{array}{l}\text { Consultation should not } \\
\text { replace clinical therapy } \\
\text { Clinic sessions are the } \\
\text { ultimate }\end{array}$ & $\begin{array}{l}\text { Professional Identity } \\
\text { Att-Consult } 2^{\text {nd }} \text { best }\end{array}$ \\
\hline $\begin{array}{l}\text { Petra 583-596 } \\
\text { Some of the best results I've had ever is in the last term } \\
\text { when I've had the mainstream kids in the mainstream } \\
\text { setting in their school hall. And we've had the most } \\
\text { amazing outcomes from that. }\end{array}$ & $\begin{array}{l}\text { Working in the } \\
\text { mainstream hall }\end{array}$ & CoMT \\
\hline $\begin{array}{l}\text { Petra 590-594 } \\
\text { I think there's a real crossover with music education and } \\
\text { early childhood education, and music therapy - there's a } \\
\text { real crossover there in that situation. So yeah, definitely } \\
\text { those skills could be definitely transferred to someone } \\
\text { with a little bit of musical skill... }\end{array}$ & $\begin{array}{l}\text { Consultation crosses } \\
\text { over with music and } \\
\text { early childhood } \\
\text { education }\end{array}$ & What is consultation? \\
\hline
\end{tabular}




\section{APPENDIX 27: Interview with Petra, from code to category}

Petra - Thinking about Consultation

ANALYTIC MEMO (from initial transcript)

$2: 30 \mathrm{pm}$, Oct 11, 2006.

The music therapists I have interviewed so far have had varying responses to the idea of empowering others to do the work. Some have been quite ambivalent about it and to a certain extent so has Petra (she 'warms up' to the idea a bit during the interview). But she gave me quite a strong reaction during this interview. I was aware that Petra was feeling very uncomfortable with the idea that music therapists could be consultants. She was frightened that she would be forced to work in a particular way and overwhelmed by the potential workload. It seemed that she was suggesting that students could only have music therapy in the clinic but later in the interview seemed to change her mind particularly when describing her work as a music educator. Petra used music to empower people in their work but doesn't see that as part of her role as a music therapist. It seemed that she would prefer music therapists to stay with the job that they are trained for, that others are not able to do. It also seemed that she believed that music therapists would lose their identity, and the possibility for clinical work, altogether if they diversified.

Petra $36-39$

PT: I just don't think that music therapy works without a music therapist... and whilst I'm happy to give advice as to how to run it, I just don't believe in that at all. I just think music therapy is about the relationship between the therapist and the client.

Petra $43-47$

(Giving advice) - I only do it informally. I haven't done it in a situation 
like; say a speech therapist would set up a programme... and then the secretary of the school runs it, things like that - I haven't done that. And I haven't needed to do that yet... if I was to go and work for Group special Ed or another place, then sure I think that would happen.

Petra $55-61$

Our training is all about putting across the music and using it in a therapeutic way and people - you can't expect somebody just because they can play two chords on a guitar to convey that. And a lot of people wouldn't play two chords on a guitar. Most people you would be using, like teacher aides, don't have those skills. And anyone can press the 'play' on a CD player but that's not music therapy. I'm just very 'anti'it.

Petra $87-92$

PT: I'm sure that there is (something we can do) but then I would be very hesitant to call it music therapy. .... of course there's a huge amount that you can do. But would I - by transferring all those ideas would I be using my therapeutic skills or would I be using my education skills in doing that, in teaching these teacher aides?

Petra 99-123

It becomes a little bit um frightening in that the fact that few music therapists that we have would then be sitting in an um office writing these programmes for... I think this is what we have been going round and round in circles about... Then I can be shared amongst the 300 people that have ORRS funding... I physically can't see 300 people. Um, do I sit at a desk for five days a week and see them once a term like the speech and physios and OTs do, and just check off "yes" you are doing 'Row Row Row Your Boat' and 'Mary Had a Little Lamb' - fantastic. Is that... am I a music therapist then? Am I using my skills for the best?

Petra $139-141$

Oh occasionally (with another professional)I'll say "use this particular piece of music" and it might be a recorded piece of music, or write a song for this particular... and "can you please do it in this setting as well. 
Petra $152-170$

PT: But it scares - It personally scares me. I just think, you know, if that becomes the expectation we are actually losing the whole essence of music therapy .

$\mathrm{DR}$ : Ok. Again (PT: But -) it's not a 'should' or an 'expectation' because that would worry me too.

PT: Not from us, but from future employers...it could be.

DR: So you have a worry that because we can have multiple roles, that we might lose some of our roles?

PT: Yeah.

PT: You throw a lot of expectation on... on to other people who are transferring those programmes. I think it's easy for a person, and it's 'Joe Blow', to sit down and go through speech um sounds and programmes and things, I think that's very basic but to transfer anything musically unless they're using all recorded music, which they would be. And I think that um people get very overwhelmed by music and very daunted by music... (pause) and I think it could turn into bashing the drum away to the Beatles or... and is that therapeutic? I don't think so.

Petra 191-196

But, yeah, I'm not saying definitely no, and I think there is a definite need especially in a place of isolation like I'm in. There's a definite need for me to access more people than I have. And I'm definitely getting to that point now where I really want to access these 300 people. And I haven't even touched the surface really because - on purpose - because I knew that I realistically couldn't do it.

Petra 200-204

And yeah become more of a referral person I suppose person but there's a lot of hesitation. And I know that that's where the GSE job is headed. And it's getting pretty close now - we're getting pretty close to that. They already made their decision... they have and it's gone through. 
Petra 539-546

In a way I think of that as my music education hat...Next week and the week after I'll be going to educate childcare educators about using music everyday and in the home and...being able to facilitate music all the time. And even if it is, as you said singing 'Twinkle Twinkle' or um just using - realising how much music is part of everyday life whether it's banging the saucepans with the wooden spoons or whatever it is and yeah, becoming creative beings again and... finding their inner music I suppose as an adult, and transferring it to the child.

Petra 556-562

I was more thinking more in the fact that that shouldn't become only our role. That it could be a very nice part of, but certainly not to be expected as the only role. And that's the one thing that scares me, is that we lose our clinical... sessions and that clinical sessions are not... are still valued as the most, as the ultimate music therapy session. But if we need to be in a referral role, then that's fine as well - but in addition.

Petra 583-596

Some of the best results I've had ever is in the last term when I've had the mainstream kids in the mainstream setting in their school hall. And we've had the most amazing outcomes from that.

Petra 590-594

I think there's a real crossover with music education and early childhood education, and music therapy - there's a real crossover there in that situation. So yeah, definitely those skills could be definitely transferred to someone with a little bit of musical skill... 


\section{APPENDIX 28: Category, Attitudes to consultation}

\section{Attitudes to consultation}

\begin{tabular}{|c|c|c|}
\hline $\begin{array}{l}\text { Myra 214-215 } \\
\text { I don't call it music therapy when I'm seeing ten children } \\
\text { at once. }\end{array}$ & $\begin{array}{l}\text { I don't call it music } \\
\text { therapy }\end{array}$ & $\begin{array}{l}\text { Myra worked in the } \\
\text { classroom with larger } \\
\text { groups of children, but } \\
\text { saw the work as } \\
\text { something she had to } \\
\text { do in addition to her } \\
\text { music therapy }\end{array}$ \\
\hline $\begin{array}{l}\text { Cara: } 506-580 \\
\text { I think it is tricky because do have a sense of feeling a } \\
\text { little bit uncomfortable with sort of saying to people "Oh } \\
\text { well, you haven't got a music therapist there but you can } \\
\text { run a group like this". And I feel a little bit uncomfortable } \\
\text { with that. } \\
\text { But I also recognise the value of being able to say to } \\
\text { teaching staff or therapists "Use music". Yes. "Use music } \\
\text { - and here are some of the things you can do. You can't } \\
\text { recreate a music therapy situation but this is going to be } \\
\text { really helpful". }\end{array}$ & $\begin{array}{l}\text { Role transfer can be } \\
\text { uncomfortable } \\
\text { Encourage use of music }\end{array}$ & $\begin{array}{l}\text { Cara was } \\
\text { uncomfortable about } \\
\text { some aspects of } \\
\text { consultation, yet valued } \\
\text { being able to support } \\
\text { others in their use of } \\
\text { music. }\end{array}$ \\
\hline Kath 266-269 & & \\
\hline $\begin{array}{l}\text { We are far more collaborative now that we have ever been } \\
\text { over the last ten or twenty years, and this is very good. } \\
\text { There's a lot more sharing of skills and finance and } \\
\text { opportunities. }\end{array}$ & $\begin{array}{l}\text { Very good } \\
\text { collaborate }\end{array}$ & $\begin{array}{l}\text { Kath appreciated the } \\
\text { opportunities she had to } \\
\text { collaborate with } \\
\text { colleagues }\end{array}$ \\
\hline Lisa $360-369$ & & \\
\hline For some people I've done consultation with, what & & Consultation is integral \\
\hline
\end{tabular}




\begin{tabular}{|c|c|c|}
\hline $\begin{array}{l}\text { meant is that, where the staff are willing and have got the } \\
\text { time to do it, that that person is probably getting some } \\
\text { music input you know maybe four times a week, which is } \\
\text { always going to be more than I can offer! And some of } \\
\text { the people I work with, if the staff are able to ah um... } \\
\text { seize the opportunity, if the person is in the mood and } \\
\text { they can give them five minutes of quality interaction } \\
\text { using music, well that's great because I might go and see } \\
\text { that person and it might not be a good time for them, so } \\
\text { yeah... I think those things are really important. }\end{array}$ & $\begin{array}{l}\text { Staff can seize the } \\
\text { opportunity for quality } \\
\text { interaction }\end{array}$ & $\begin{array}{l}\text { to Lisa's work and she } \\
\text { sees it as a way for her } \\
\text { clients to receive more } \\
\text { music input. Staff can } \\
\text { seize the opportunity } \\
\text { for quality interaction } \\
\text { and she thinks this is } \\
\text { really important. }\end{array}$ \\
\hline $\begin{array}{l}\text { Lisa } 387-419 \\
\text { LI: Yes, yeah, and their motivation. Sometimes if staff } \\
\text { can see a person playing an instrument, or be encouraged } \\
\text { to vocalise with someone who is non-verbal, they can get } \\
\text { a very different picture of who that person is and how } \\
\text { they can interact with them, and that is very valuable I } \\
\text { believe. }\end{array}$ & $\begin{array}{l}\text { I could have done more } \\
\text { consultation }\end{array}$ & $\begin{array}{l}\text { Lisa believes staff get a } \\
\text { new picture of their } \\
\text { client when they are } \\
\text { involved in music } \\
\text { activities with them, } \\
\text { and that this is really } \\
\text { valuable. } \\
\text { In retrospect she felt } \\
\text { she could have done } \\
\text { more consultation in } \\
\text { school settings. }\end{array}$ \\
\hline $\begin{array}{l}\text { Robin 159-171 } \\
\text { I think there is something vitally important about play in } \\
\text { life and about the things that playing... ah all the things } \\
\text { that lead to discovery through play, and through } \\
\text { serendipity, just discovering things by accident. And } \\
\text { um... the other week I was doing a class session with a } \\
\text { group of about eight year olds and they just - it was one of } \\
\text { those kind of magical moments where they were just } \\
\text { spontaneously patterning this little ostinato in unison - it } \\
\text { wasn't something I instigated, um encouraged them to } \\
\text { initially, and eventually I joined in with them and there } \\
\text { was just a sense of joy in the spontaneity of that }\end{array}$ & $\begin{array}{l}\text { Spontaneous play is } \\
\text { important }\end{array}$ & $\begin{array}{l}\text { Could this } \\
\text { ncouraged in other }\end{array}$ \\
\hline
\end{tabular}




\begin{tabular}{|c|c|c|}
\hline tion. & & \\
\hline $\begin{array}{l}\text { Yvonne 210-222 } \\
\text { DR: It sounds as if, whenever you got the chance, you } \\
\text { would prefer to do ongoing work? You know, I notice } \\
\text { when you said "I could only offer consultative work" that } \\
\text { there was a bit of passion there, it was kind of like - } \\
\text { YV: Oh absolutely! Because I often felt that it wasn't an } \\
\text { equal service that was being offered and that was because } \\
\text { of restrictions on the service and funding. Whereas and } \\
\text { um if a child lives in a rural area they're getting less of a } \\
\text { service than if they are in the city where you're based. } \\
\text { That's a reality and oh, there is a bit of that - frustration } \\
\text { because of the potential for being able to offer more. } \\
\text { DR: So probably for the majority of kids if you could see } \\
\text { them for longer you would - were there some though } \\
\text { where the (consultation) process seemed to be really } \\
\text { helpful... } \\
\text { YV: Yes. }\end{array}$ & $\begin{array}{l}\text { Children in rural areas } \\
\text { get less service } \\
\text { Frustrated that clinical } \\
\text { MT can't be offered } \\
\text { Consultation can be } \\
\text { helpful }\end{array}$ & $\begin{array}{l}\text { Yvonne valued ongoing } \\
\text { clinical work, and was } \\
\text { sometimes frustrated by } \\
\text { the increasing emphasis } \\
\text { on short-term work. } \\
\text { Nevertheless she agreed } \\
\text { that consultation can be } \\
\text { helpful. }\end{array}$ \\
\hline $\begin{array}{l}\text { Petra 36-39 } \\
\text { PT: I just don't think that music therapy works without a } \\
\text { music therapist... and whilst I'm happy to give advice as } \\
\text { to how to run it, I just don't believe in that at all. I just } \\
\text { think music therapy is about the relationship between the } \\
\text { therapist and the client. }\end{array}$ & $\begin{array}{l}\text { Only MTs can do music } \\
\text { therapy } \\
\text { MT relationship } \\
\text { Don't believe in it. }\end{array}$ & $\begin{array}{l}\text { Petra feels strongly that } \\
\text { music therapy must be } \\
\text { facilitated by a music } \\
\text { therapist, because of the } \\
\text { importance of the } \\
\text { relationship between } \\
\text { the therapist and client. }\end{array}$ \\
\hline $\begin{array}{l}\text { Our training is all about putting across the music and } \\
\text { using it in a therapeutic way and people - you can't } \\
\text { expect somebody just because they can play two chords } \\
\text { on a guitar to convey that. And a lot of people wouldn't }\end{array}$ & $\begin{array}{l}\text { Most team members } \\
\text { don't have the skills to } \\
\text { use music. }\end{array}$ & $\begin{array}{l}\text { She doesn't believe that } \\
\text { team members would } \\
\text { have the skills to } \\
\text { facilitate music } \\
\text { activities, and would be }\end{array}$ \\
\hline
\end{tabular}




\begin{tabular}{|c|c|c|}
\hline $\begin{array}{l}\text { play two chords on a guitar. Most people you would be } \\
\text { using, like teacher aides, don't have those skills. And } \\
\text { anyone can press the 'play' on a CD player but that's not } \\
\text { music therapy. I'm just very 'anti'it. }\end{array}$ & $\begin{array}{l}\text { That's not MT } \\
\text { I'm just very 'anti' it }\end{array}$ & $\begin{array}{l}\text { using recorded music. } \\
\text { She was "very 'anti"” to } \\
\text { this. }\end{array}$ \\
\hline $\begin{array}{l}\text { Petra } 87-92 \\
\text { PT: I'm sure that there is (something we can do) but then I } \\
\text { would be very hesitant to call it music therapy. }\end{array}$ & $\begin{array}{l}\text { Consultation is not } \\
\text { MT }\end{array}$ & $\begin{array}{l}\text { However, she thought } \\
\text { there might be some } \\
\text { things that a consultant } \\
\text { might be able to do but } \\
\text { would be "very hesitant } \\
\text { to call it music } \\
\text { therapy". }\end{array}$ \\
\hline $\begin{array}{l}\text { Petra 99-123 } \\
\text { It becomes a little bit um frightening in that the fact that } \\
\text { few music therapists that we have would then be sitting } \\
\text { in an um office writing these programmes for... I think } \\
\text { this is what we have been going round and round in } \\
\text { circles about... Then I can be shared amongst the } 300 \\
\text { people that have ORRS funding... I physically can't see } \\
300 \text { people. Um, do I sit at a desk for five days a week } \\
\text { and see them once a term like the speech and physios and } \\
\text { OTs do, and just check off "yes" you are doing 'Row } \\
\text { Row Row Your Boat' and 'Mary Had a Little Lamb' - } \\
\text { fantastic. Is that... am I a music therapist then? Am I } \\
\text { using my skills for the best? }\end{array}$ & $\begin{array}{l}\text { Frightening } \\
\text { Sitting at a desk writing } \\
\text { programmes for large } \\
\text { numbers of students } \\
\text { "Fantastic" } \\
\text { (irony/sarcasm) } \\
\text { Am I using my skills for } \\
\text { the best? }\end{array}$ & $\begin{array}{l}\text { Petra also described } \\
\text { consultations as "a little } \\
\text { bit frightening" because } \\
\text { she believed that she } \\
\text { would be sitting at a } \\
\text { desk writing } \\
\text { programmes for large } \\
\text { numbers of students. } \\
\text { The potential to be } \\
\text { writing programmes } \\
\text { and seeing students just } \\
\text { once a term was } \\
\text { sarcastically described } \\
\text { as "fantastic!" It } \\
\text { seemed that she felt her } \\
\text { current practice was } \\
\text { threatened, and if her } \\
\text { role changed she would } \\
\text { not be doing what she } \\
\text { had been trained for. }\end{array}$ \\
\hline & & \\
\hline
\end{tabular}


PT: But it scares - It personally scares me. I just think, you know, if that becomes the expectation we are actually losing the whole essence of music therapy.

DR: Ok. Again (PT: But -) it's not a 'should' or an 'expectation' because that would worry me too.

PT: Not from us, but from future employers... it could be.

DR: So you have a worry that because we can have multiple roles, that we might lose some of our roles?

PT: Yeah.

PT: You throw a lot of expectation on... on to other people who are transferring those programmes. I think it's easy for a person, and it's 'Joe Blow', to sit down and go through speech um sounds and programmes and things, I think that's very basic but to transfer anything musically unless they're using all recorded music, which they would be. And I think that um people get very overwhelmed by music and very daunted by music...(pause) and I think it could turn into bashing the drum away to the Beatles or... and is that therapeutic? I don't think so.

Petra 191-196

But, yeah, I'm not saying definitely no, and I think there is a definite need especially in a place of isolation like I'm in. There's a definite need for me to access more people than I have. And I'm definitely getting to that point now where I really want to access these 300 people. And I haven't even touched the surface really because - on purpose - because I knew that I realistically couldn't do it.

Petra 556-562
It scares me

Losing the whole essence of music therapy

Employers will expect

too much

We will lose some of our roles

It's harder to transfer music programmes to MDT - they'll all use recorded music

It won't be therapeutic

There's a need to access isolated students

I haven't even touched the surface really

On the other hand Petra accepted that students in isolated areas were entitled to music therapy service. But the thought of the large numbers of students that could potentially be on her case load felt overwhelming 


\begin{tabular}{|l|l|l|}
\hline $\begin{array}{l}\text { I was more thinking more in the fact that that shouldn't } \\
\text { become only our role. That it could be a very nice part of, } \\
\text { but certainly not to be expected as the only role. And } \\
\text { that's the one thing that scares me, is that we lose our } \\
\text { clinical... sessions and that clinical sessions are not... are } \\
\text { still valued as the most, as the ultimate music therapy } \\
\text { session. But if we need to be in a referral role, then that's } \\
\text { fine as well - but in addition. }\end{array}$ & $\begin{array}{l}\text { Consultion should not } \\
\text { replinical therapy }\end{array}$ & $\begin{array}{l}\text { If sesions are the } \\
\text { allowed, it should not } \\
\text { replace clinical music } \\
\text { therapy which is the } \\
\text { ultimate role for music } \\
\text { therapists }\end{array}$ \\
\hline
\end{tabular}


APPENDIX 29: Examples from Music Diary Entry Provided to Participants

Music Diary for PROMISE Research

Date: Monday 5/2/07 SAMPLE ONLY

\begin{tabular}{|l|l|l|}
\hline$\underline{\text { Time }}$ & What We Did & How I Feel / What I Will Do Next \\
\hline 8.45 & $\begin{array}{l}\text { I pushed him on the swing while singing } \\
\text { "up and down" and he gave me some good } \\
\text { eye contact }\end{array}$ & $\begin{array}{l}\text { It helped me relax and I was pleased with } \\
\text { his response... I think I will do this again } \\
\text { soon }\end{array}$ \\
\hline $\begin{array}{l}\text { The teacher was singing the roll... I noticed } \\
\text { that he really paid attention compared to } \\
\text { when she just called the names }\end{array}$ & $\begin{array}{l}\text { Talk to Mary about this and see if she is } \\
\text { happy to keep doing this while it is working } \\
\text { so well for Johnny }\end{array}$ \\
$\begin{array}{l}\text { We had class music and the kids all had } \\
\text { drums... it was too much for Johnny and he }\end{array}$ & $\begin{array}{l}\text { I took him into our quiet space with the } \\
\text { bongo drums and tried to engage him. It } \\
\text { was better with the 2 of us and he did calm } \\
\text { down and do a bit of turn taking on the } \\
\text { drum. (15 minutes) }\end{array}$ \\
\hline 11.15 & $\begin{array}{l}\text { Took him for a walk until music was } \\
\text { finished... sang a marching song and I } \\
\text { think it motivated him to walk steadily }\end{array}$ & $\begin{array}{l}\text { Talk to Daphne about how we can adapt the } \\
\text { music sessions so it is easier for Johnny to } \\
\text { manage staying with the class }\end{array}$ \\
\hline
\end{tabular}

Music Diary for PROMISE Research

Date: Tuesday 6/2/07 SAMPLE

ONLY

\begin{tabular}{|l|l|l|}
\hline$\underline{\text { Time }}$ & What We Did & How I Feel / What I Will Do Next \\
\hline 3.30 & I haven't written in my diary at all today! I & I'll try to be more aware but I feel pretty \\
& think I probably was doing a bit of chanting & stressed \\
& $\begin{array}{l}\text { but I was so busy with Johnny I forgot to } \\
\text { observe and record his responses. }\end{array}$ & $\begin{array}{l}\text { Maybe some background music will calm } \\
\text { us both down! }\end{array}$ \\
\hline
\end{tabular}


APPENDIX 30: Stage Two. Data sources and reasons for collection

\begin{tabular}{|c|c|c|}
\hline & Data Source & Primary Reason for Collecting Data \\
\hline 1 & Interview with music therapists & \multirow[t]{2}{*}{ Used to describe current practice and develop initial protocol. } \\
\hline 2 & Analysis of video of previous assessment material & \\
\hline 3 & First interviews with IDT & Used to determine their 'starting point' \\
\hline 4 & My observations from the field - diary & $\begin{array}{l}\text { Used to describe the environment/context, any issues } \\
\text { encountered }\end{array}$ \\
\hline 5 & Clinical assessment video & Evidence for objective observations (see below) \\
\hline 6 & Focus group & $\begin{array}{l}\text { An opportunity for the team to observe the student in a new } \\
\text { context - to identify strengths and potential? Used to share and } \\
\text { clarify observations before collaborative goal planning takes } \\
\text { place. }\end{array}$ \\
\hline 7 & Second Interviews with IDT & $\begin{array}{l}\text { To find out participants views about what happened during the } \\
\text { week i.e. whether the 'protocol' (the steps taken) seemed } \\
\text { helpful and what difficulties were encountered }\end{array}$ \\
\hline 8 & Journal from IDT & $\begin{array}{l}\text { What did the team do, and how did they feel about it? What } \\
\text { progress if any did the student make towards the agreed goals. }\end{array}$ \\
\hline & Follow-up Interviews & $\begin{array}{l}\text { What did the team do, and how did they feel about it? What } \\
\text { progress if any did the student make towards the agreed goals. } \\
\text { Any other issues - positive or negative that can help to } \\
\text { determine whether the process is helpful? }\end{array}$ \\
\hline
\end{tabular}




\begin{tabular}{|l|l|l|}
\hline Research Questions & Answered Primarily from Data Source: \\
\hline $\begin{array}{l}\text { Can music therapists empower MDT to use music } \\
\text { therapy strategies in the work with students who have } \\
\text { special education needs, in a sustained way? }\end{array}$ & All data sources \\
\hline $\begin{array}{l}\text { How? What is the best way of going about this, and what } \\
\text { are the important issues that need to be kept in mind? }\end{array}$ & Feveloping/evolving protocol; First Interview; Obs from the \\
\hline $\begin{array}{l}\text { What do team members observe? What might need to be } \\
\text { drawn out by the music therapist? }\end{array}$ & Focus group; Obs from the field \\
\hline $\begin{array}{l}\text { What do team members do, and is it sustainable? } \\
\text { relation to self? How does their perspective change over } \\
\text { time? }\end{array}$ & up Interviews \\
\hline $\begin{array}{l}\text { What do team members think/feel about the work in } \\
\text { relation to student? What progress if any do they } \\
\text { observe? }\end{array}$ & Second Interviews; Journal from IDT; Follow-up Interviews \\
\hline $\begin{array}{l}\text { Can this knowledge be presented in a form that is } \\
\text { helpful for music therapists (to draw from and to } \\
\text { share with teams) AND for potential employers? }\end{array}$ & The Protocol \\
\hline
\end{tabular}


APPENDIX 31: Field Log. Tim's Case

\section{FIELD LOG (TIM'S CASE)}

$10 / 02 / 07$

I have been anxious and worried about this week, but it seems that this concern is more about making sure I have prepared well and have what I need for the week ahead. Now that I am on my way I feel much better! The checklist I gave myself has been very helpful!

$12 / 02 / 07$

First day in the field! I am excited and enthusiastic to get on! What do I need to do first? 8.00am meeting with the Principal to sort out the timetable for the week, confirm that Angela is to be the ITM, ask whether Tim's mother is available for a chat, ask when staff meeting will be held and/or whether I can put a notice on the staff board so people know who I am, and orientation to school - where are the loos? Most important to build relationships today though! I didn't bring my IPOD and thought it was a disaster at first but wondered if it might be less threatening anyway if I were not so intrusive (or 'odd' if I am seemingly talking to myself as I make auditory notes!) on the first day... written notes will be fine!

Sandy was very warm and welcoming. She was very willing to give me her time to discuss the week and to give me an orientation, promising also to take me around the school at 10.30 to meet staff and children in their classrooms. This is an important thing to do... I had wondered about the notice for staff but it was great to be introduced directly to the children so they knew who I was and why I was 'wandering' in the grounds and making notes. There are six classrooms - Tim is in the same room he used last year but with a new class (year twos?).

\section{School Mission Statement:}

We aim to provide the highest quality teaching programmes within a caring environment which will enable each child to develop to the best of their ability in all aspects of education

\section{School Hours:}

Morning session 9-10.30, 11-12.30, 1.30-3pm 


\section{Tim's team:}

Mum: Lee (03 755 8590)

\begin{tabular}{|l|l|l|l|}
\hline & Morning & Afternoon & Teacher \\
\hline Monday & Valerie TA & Siobhan TA & Mary \\
\hline Tuesday & $\begin{array}{l}\text { Angela (ORRS T.) } \\
\text { Sally TA }\end{array}$ & Siobhan TA & Mary \\
\hline Wednesday & Sally TA & Siobhan TA & Mary \\
\hline Thursday & $\begin{array}{l}\text { Angela (ORRS T.) } \\
\text { Sally TA }\end{array}$ & $\begin{array}{l}\text { Angela (ORRS T.) } \\
\text { Siobhan TA }\end{array}$ & Mary \\
\hline Friday & & Siobhan TA & Mary \\
\hline
\end{tabular}

Timetable for Week:

\begin{tabular}{|c|c|c|c|}
\hline & Morning & Afternoon & After School \\
\hline Monday & $\begin{array}{l}\text { Meet Principal } \\
\text { Meet Tim and his team } \\
\text { Observations }\end{array}$ & Observations & Data gathering (records) \\
\hline Tuesday & $\begin{array}{l}\text { Meet Angela and Sally } \\
\text { Interview Angela } \\
\text { Complete Referral Form } \\
\text { Observations } \\
\text { Informal/Brief Meeting } \\
\text { with Jan (SEA) and } \\
\text { Nadia (OT) }\end{array}$ & $\begin{array}{l}\text { Meeting re Sensory Rm } \\
\text { Tim has gone swimming } \\
\text { 2pm Valerie!! }\end{array}$ & $\begin{array}{l}\text { Notes } \\
\text { Meet with GSE Adviser }\end{array}$ \\
\hline Wednesday & $\begin{array}{l}\text { Class Song } \\
\text { Interview Lee (and } \\
\text { Patrick) 9.30-9.45 } \\
\text { Observations } \\
\text { Informal meeting with }\end{array}$ & Observations & \\
\hline
\end{tabular}




\begin{tabular}{|c|c|c|c|}
\hline & Sue (SLT) & & \\
\hline Thursday & $\begin{array}{l}\text { Prepare for Assessment } \\
\text { Class Song } \\
\text { Assessment Session in } \\
\text { Room } 3 \\
\text { Observations }\end{array}$ & $\begin{array}{l}\text { Observations } \\
\text { Prepare for team meeting }\end{array}$ & $\begin{array}{l}\text { Team meeting (video) in } \\
\text { library } \\
\text { Angela } \\
\text { Sally } \\
\text { Lee } \\
\text { Mary - Was sick } \\
\text { Siobhan } \\
\text { Nadia (OT) - Didn't } \\
\text { arrive } \\
\text { Write report } \\
\text { Prepare programme based } \\
\text { on team decision - not } \\
\text { fully achieved }\end{array}$ \\
\hline Friday & $\begin{array}{l}\text { Class Song } \\
\text { Go over programme with } \\
\text { Angela / Sally / Lee / } \\
\text { Patrick? 9.30-9.45 }\end{array}$ & $\begin{array}{l}\text { Interview Sally } \\
\text { Interview Angela } \\
\text { Assembly } \\
\text { Class Song }\end{array}$ & $3 \mathrm{pm}$ Valerie \\
\hline
\end{tabular}

How will I have time to watch Angela trying out the programme, and to interview her? Can Angela come in on Friday?

I met Mary, classroom teacher, before school and my first impression was that she was 'worried' about how Tim might respond to me - she seemed to have expectations of what I might be looking for and/or doing. And her primary focus was on recorded music. She showed me the headphones Tim uses, and his 'own' CD player. When we talked about the possibility for me to help with classroom music, she told me that her programme involved the children bringing a CD to share with the class. She mentioned Tim's short attention span and his poor eye contact and in short seemed to have quite negative view of him. I 
reassured her that I would be as intrusive as possible but seemed to suggest that there would be no problem because she is able to "chat about things" with TA (seemingly during class time?). I wonder if this is a problem. She shows me Tim's desk and chair... the 'usual' kind (no adaptations) - does he need a foot stool?

The classroom is tidy. It is bright and stimulating. The desks are wooden but the chairs are a light shade of forest green and the room seems quite calm. The walls are dark blue clothboard from about a meter above the floor. Below that wooden walls are lined with shelves which contain books and puzzles, and other maths equipment. Some are also in tote trays. I notice a magnetic board which holds several key reading words. The classroom rules are written on a chart near the door: They read "we may look different but we feel the same", "We care about each other", "We play safely and fairly" and "We always so please and thankyou". I am particularly struck by a chart of 24 brightly coloured facial expressions. There is a red peanut shaped physio ball available for Tim. Outside the room, in a covered walkway are the children's pegs. I notice that Tim's peg has a picture of him rather than his printed name. On a seat in the covered walkway there is a plastic box labelled 'Tim's Container Box' full of plastic shapes (lots of wheels) and some toys. The children who are arriving are happy and several stop to chat to me - I tell them I am there to learn about Tim because I want to be his new friend.

I meet Sally and tell her how comfortable I am in the school already. She agrees and shares that the children really try to involve Tim in their play, and they really care about how he is feeling! But because it is a new class for him everyone is just learning about what will help him - it seems that what works one minute for Tim won't work the next. He is very tired sometimes - on the first day of school he slept for 20 minutes on the mat right after he arrived in the class!

Tim arrived just before 9.00am with his mum Lee. He is a good looking boy, with very short dark blonde hair and olive skin. His eye gaze is unusual - he does not give direct eye contact but seems to use peripheral vision? He is wearing his school uniform... a blue sweat shirt with the school logo over the left breast area, over a grey tee also with a logo on the chest. He has grey shorts and brown roman sandals on. He was being contained with physical support but walked independently and seemed keen to enter the classroom.

9.00 am. When class started, the children were at their desks and Tim was on the floor in the corner. He was smiling and approaching the TA who was working beside him making 
playdough letters. Tim vocalised "mmmm". He moved to the door and was taken back to his mat - at this time he was making protesting vocalisations. The other children were engaged in a chanting and clapping exercise... doing the 'Roll Rap'. Tim was calmly turning a plastic container in his hand. I wondered if he would be more involved if the rap was slower? He was attending when the rhythm was strong but lost it (and began to protest again) when it became 'messy' - Mary told me later that this activity is new to the class. She also shared that it is only for Mondays and that she has a different way of calling the roll on other days of the week, but seemed very willing to stick to it if it helped Tim. I have said that it would be good to see Tim's reaction/response to the other pieces first.

TA stands up and is holding Tim on her hips while the class say their prayers. Tim makes calm vocalisations, is sucking his finger, and is then quiet.

9.10am. Tim is taken from class room to the library. He led the way and entered willingly, sitting on a cushion spontaneously for his DVD. He watched/listened to 'Max', a musical video. He was smiling and attentive as he watched the screen. People were dancing and the scene was brightly coloured. They were demonstrating action songs but Tim made no attempt to imitate - does he ever? At one point Valerie helped Tim to clap and he accepted the co-active movement. (She said sometimes she is able to help him to move and he accepts several repetitions of an activity... but he needs to be in the mood). During this activity he smiled, and when I chanted "run, run, run" in time to the movement on the video, he turned and smiled at me. There was opportunity for the listener to choose the next activity by pointing to the screen - does Tim do this? Valerie said he didn't. I would suggest that we all wait for Tim to respond... even if he makes a protest sound because nothing is happening, it will be a communicative response to prompt 'more'.

For most of the time Tim was playing with a plastic container... Valerie says this is one thing he 'needs' to do. She also reported that music calms him - they use a range of CDs including Baby Mozart. Valerie talked about other children with SN that she works with and described how engaged they are with music... it seemed that she was comparing them a little and finding Tim wanting because "they respond so well to it". Tim likes bubbles, and disco lights. He is also interested in ME.

9.20am And he has paid attention to the DVD now for $7 \mathrm{mins}$. Now he is resting but not really watching - he could be listening to it OR other noises (there is a teacher in the corner of the library, working on the computer; and others are outside the room). Tim turned back 
to the video when they actors said "bye" though. They are eating 'imaginary' food on the video... would Tim understand that representation? When Tim appeared to loose attention the video was turned off - I might suggest they keep it on until a suitable transition time make sure of the 'end' of something before moving on.

9.27am Tim his invited to come to the hall. He stood but did not go to the door. Rather he was looking out the window. With verbal prompts (many), gestural prompt and talk of the motivating equipment there, he came spontaneously. Valerie did some good waiting for him and he came eventually! Tim is yawning already!

Tim walked steadily but with an unusual gait, his legs wide at the base. He needs help to negotiate steps and if coming down a steep grade will still go backwards. He accepts Valerie's hand and walked purposefully into the hall. He stamped his feet (LRL) straight away, seeming to enjoy the sound and the echo. He walks across the hall floor, looking at the light switch. Valerie goes behind the stage and Tim looks at the door she has gone through but does not follow her. Valerie plays peekaboo from behind the curtain. Tim laughs and runs to and fro, guessing where she might peep from. Tim takes his turn to hide behind the curtain, imitating Valerie, and his vocalisations increase considerably.... He is 'calling' to Valerie to find him! Valerie seems pleased with his response - she shares that her aim is to "make his day as enjoyable as possible". Tim has only been walking since he was five... She says he needs 'sensory' learning - and got out the bubbles. Tim watched them well and reached out to burst them. Arm movement was slow but accurate eyehand coordination... I could see him tracking each bubble he targeted. When I suggested to Valerie that we could use music with this game, she thought the CD could come over with him... what about live music? I will need to encourage her to sing about what he is doing! I think marching in the hall could be a good thing - he loved the sound of his feet! Valerie did a hoop rolling game with him... what about trying a chant (" $1,2,3 \ldots$ roll!")... Tim might vocalise for "roll"? Similarly for the scooter board... "Ready settee.... Go!"? What about a roll and catch song... especially if they can use it with a ball as well.

I noticed that Tim was flapping when he was on the scooter board. He was happy and laughing. It was very noisy on the floor but the noise did not seem to disturb him. He slid off the scooter and climbed back on by himself, vocalising for 'more'! The TA has the hoop around Tim's whole body and is pulling him by it. When she stops he can shuffle on the board to his favourite toy. I heard Tim do a nice imitation of Valerie's "Ah oh"! Tim makes a range of vocal sounds including "oom", "ah ah", “mm”, “oo oo", "yoi yoi yoi”, 
"wee da wee". He stopped vocalising when he saw Valerie pull out the mat. Tim suddenly walked out the door and shut it behind him... but was waiting behind the door.

9.53 Tim is walking on a felt mat, over the ladder on the floor, and along the beam. He manages the tasks really well but songs might help him some days? On ladder, movement is left, together, right, together etc. Tim climbed the steep steps onto the stage using his hands. Valerie is good at waiting for him! Valerie also acknowledged that Tim might be listening to sounds outside of the room... this is what I had suspected earlier. Valerie whistled - Tim pursed his lips and also made a whistling sound. Tim sang "Nn Nn" (up a perfect fourth!). Went to and climbed up a full flight of stairs at the back of the hall spontaneously... not afraid of heights! I asked Valerie if she is able to sing with Tim... she seems prepared and even confident to "have a go". I demonstrated "Walking Walking" and "Stamp and Stamp" and she picked up parts of it very quickly. Valerie tells me favourite songs have been Incy Wincy Spider and This Little Piggy.

10.20am. I was taken on my tour of the classrooms and introduced to staff and students. Again, this feels very comfortable and I know I am welcome in the school.

10.35am. Morning tea time. Valerie is spoon feeding Tim some yoghurt as he sits on a beanbag. He can drink independently from his cup. Tim watched DVD "On the Farm" and had a brief little flap. Tim appears to use his peripheral vision, perhaps especially when he is more tired... Valerie does not know of any recorded visual problems.

Valerie uses her 'gut' reaction and believes it is best to "go with what you are feeling" when working with Tim. It is hard to plan what might be the right thing to do because his responses change from moment to moment?

At morning tea time, Tim 'wanders'. Children are with him or follow him and 'mind' him, sometimes 'overhelping'. Perhaps that is because they know I am watching him? He still has his plastic jar with him. He lifts his arms readily for a carer to take his jersey off. He is happy in his own activity.

The classroom teacher seems more relaxed toward me. Valerie has shared that she is 'enjoying me' so I am perhaps trusted to be helpful to the team now?

After morning tea, Tim makes for room 6 where there are beds at the back of the room. 
Valerie brings him from the room as he protests. He didn't want to leave but got to the door of the room and sat on the step. Once he is out of the room she asks him for a kiss (appropriate? I think she wants me to see "what he can do").

11.10am We take the box if instruments into the library. Valerie held out a drum and Tim beat X xx X... Valerie didn't seem to notice. She was playing to him instead of with him. I asked her to listen to him and to respond to what she hears and she quickly realised that a nonverbal communication was possible. She began to copy Tim, to imitate him. It will be important for me to highlight all the ways in which he is communicating using beat, rhythm, various touch (twiddle vs solid tap), crescendo, and varying dynamics. I joined in and there was excellent communication via percussion instruments for at least10 minutes here! Then we allowed Tim some time on his own while Valerie set up another computer programme.

11.35 Tim was engaged in computer programmes by Astech - the first "Row Row Row the Boar" asked for him to move the mouse onto the boat at the end of each phrase. This challenges his eye-hand coordination at quite a fine level. Tim did not seem to look to the mouse at the end of each phrase but he DID look and attempt to manipulate at the end of the song. Thereafter he was consistent in looking at the mouse as the means for continuing. He appeared to be anticipating the sequence and was 'ready' for what would come next.

11.46 Tim is less attentive - looking away and sucking thumb. A new programme "Sights and Sounds" re-engaged him and with verbal prompts to "push the switch" he was activating the programme well. I asked Valerie to stop repeating "push the switch" because it was already articulated on the DVD and Tim was able to do it without further prompt. He is definitely aware of cause and effect! Waiting for him to process and act will be important. I would suggest letting the 'consequences' drive the behaviour rather than the 'instruction'. To sum up, Tim is learning to move the mouse, or to click - cannot do both tasks for one result yet.

11.57 Tim is finished on the computer after 22 mins. He walks outside to the toilet vocalising "Ba" "Bus" and a protest sounding "Na!" (?). Valerie is feeling comfortable enough with me to begin to share her personal story about family...talking as Tim walked and was toileted.

12.17 We are in the hall and Tim is being pushed on the 'wheely' chair. He is laughing and 
flapping. I liked the way Valerie stopped and waited until Tim indicated "more". He held is photographic story book ('The Day we Went to Shanty Town') while riding in the chair. His ability to request more increased - the time it took shortened.

12.30 Tim had his lunch in the library. Valerie fed him his sandwiches in bite sized pieces. I was surprised when she mentioned the PECS, as I had not seen her use them this morning. Also, I was noting how well Tim was able to choose from items in front of him and thought he might manage to choose from objects when they are available rather than from the pictures. However pictures provide the next level of symbolism that is important for reading... is this the reason for having Tim use them, or is it about basic more basic communication? The most frequently used PECS are 'toilet', 'lunch', 'sit at the desk', 'stand up', 'wash hands', 'go to lunch', 'go outside'... these are commands he does seem to understand verbally... but he needs to request.

Tim has strong contextual understanding... knew where to go, what to do when it was 'home time' especially when he saw Lee!

Reports to peruse as part of assessment (12.30pm, lunch time)

\author{
IEP \\ OT x 3 (not dated!) \\ PT/OT x 1 \\ Paediatrician's Report (at 16 months) \\ Champion Centre (EI report) \\ GSE Transition to school \\ GSE PACS communication report \\ ORRS Application
}

Give information and consent forms to Valerie, Siobhan and Lee?

1.30pm I met Tim's afternoon aide, Siobhan. He seemed to be really 'trying it on' for her it is only her third afternoon with him and he was refusing to walk. Perhaps he is tired too. Tim sat on the ground and would not weight bear when lifted. I sang "Stand up, Stand up..." and later "Walking Walking" which were not immediately successful but which did seem to help on second singing. As I sang the walking song phrase "Walking to the Classroom" (CDEFG) I heard Tim imitate the five notes of the scale vocally. 
$1.37 \mathrm{pm}$ Tim and Siobhan are reading large books in the classroom. Tim is settled on the floor and is smiling. Tim is demonstrating some joint attention skills. He is offering musical vocalisation, and Siobhan is chanting with him to ask him to turn the page. Tim holds his own book and does eventually turn the page independently. Siobhan continued to quietly sing/chant instructions and this seemed to be quite engaging for Tim. I did not observe Siobhan using PECs at this stage. The class is also reading books at their desk and the room is quiet. Tim is still holding a round yellow plastic object which he retrieved from his container box on the way past in the corridor. There is a balloon in the book. Siobhan takes a real balloon from her bag of resources and blows it up, calling Tim's attention to the task. Tim watches, takes it from her and gently lets it go down. He vocalises a very rhythmical "baba baba baba baba baba baba baa". Siobhan gives lovely simple reinforcement/instruction e.g. "good looking" "take the balloon". She then takes a party whistle (one that expands when blown) and demonstrates it for Tim. He is less interested the class are reading their news and it has become noisier in the room. They are chanting and Tim 'sings' but is "shushed". He seems to imitate the whistle noise? Tim makes a high pitched noise like the sound of a cock - I look in the book but it is not a cock or a hen but a truck... so the vocalisation is not relevant in this case! When I went to check the picture, Tim seem to get a fright - he pushed me away quickly and suddenly. He then flapped and tapped his chest fleetingly vocalising 'gu gu'. He 'dismissed' Siobhan with a wave of his hand - a very clear and effective gesture. He continues with "dada aaa" repetitively for a brief spell. Then he squeals "oooh delala". Siobhan waits (well done!) and Tim turns the page. Siobhan is now reinforcing ("good, you turned the page") what he is doing, using repetitive language, but is not intrusive. When she does lean forward Tim pushes her hand away. Sometimes he is not ready to turn the page! Tim claps 2 X5 times quickly. The children are now running, chanting, clapping, and counting out loud. The room is noisy. Tim joins in with rhythmic vocalisation.

Siobhan shares that she thinks Tim might be autistic. She believes it is important to give him time to process information. Siobhan has had some art therapy training in Germany not the full psychotherapeutic training but some initial work that enables her to work alongside other professionals.

$2 \mathrm{pm}$ The class is measuring. Siobhan tries to engage Tim in listening to a CD and he leaves his corner. Does he want to be involved, in his own way, with the other children and not isolated with different activities? He seems to be still 'in touch' with what is going on in 
the room sometimes. Siobhan uses the PECs to ask Tim if he would like Milo. Tim walks steadily from the classroom to the staffroom - Siobhan is trying a walking song! On the way Tim spots a new read sail in the playground. He looks at it carefully as he passes by keeps moving. He goes into the staffroom, clearly aware of where the Milo is made. He sits on a chair vocalising occasionally while Siobhan makes the Milo... "dubby dubby dubby da squeak squeak". The school secretary says "Hello Tim" and he vocalises in response she doesn't notice his communication and says "No?" as if he has not acknowledged her. Remember to remind staff to listen to him and read his responses positively!

Siobhan is still using the singing... I might have to suggest she doesn't overdo it to the point where it loses its impact.

Tim seems to like echoes, in the hall, in the toilet, in the playground and in the corridor. He began to vocalise as soon as he got to the hall but fell down screaming on the floor when asked to go to the toilet. I helped Siobhan to improvise/adapt songs using the 8 bar framework, keeping the lyrics clear and moderately loud - so they sounded purposeful. Tim responded immediately. The songs were used were:

1. "Go to the Toilet Tim; Sit on the Toilet Tim; Good Boy Tim...etc"

2. "Round \& Round" (with the Wheel he was holding while on toilet)

3. "You put your right foot in, you put your right foot in" ala Hoki Toki... (Get $\mathrm{L} \& \mathrm{R}$ right even if you think he doesn't understand!)

4. "This is the way we wash our hands; soap our hands; rinse our hands; dry our hands..."

Tim loves wheels! His reward for going to the toilet so well was another ride on the wheelie chair - I was sitting on it when he needed it and I got a very clear communication that it was his turn! It would be great to have a swing for Tim in the hall - he really responds to proprioceptive activities. When the chair stops he touches Siobhan's hand to indicate 'more'. We could have a song for turning on the chair... see if Tim can anticipate the "stop" and indicate "more" consistently? He is laughing, and flapping. He offers his legs for Siobhan to pull him! He helps to open the cupboard to get the scooter board. Enjoyed being pulled forward on the scooter but didn't seem to like going backwards? Tim did some more independent shuffling forward. He is continuing to vocalise.

We played "Oh Yoo Hoo" with the curtain again. Eye contact was good at times. It will take a while before he learns the sequence of activity... but if there are two people to model it will still interest him? Tim did not attempt to imitate our vocalisation... or to vocalise in 
the 'space' provided for him.

$2.45 \mathrm{pm}$ Tim left the hall compliantly and lead the way to the library. He sat immediately for his DVD. He is not looking directly at the TV but seems to be using peripheral vision in his right eye. He has his legs crossed and looks casually relaxed. He is twirling his yellow plastic object. Tim laughs at the DVD on which the actors are eating imaginary food - can Tim understand this sort of pretend play?

2.50pm. There are other students at a computer in the corner of the room - the music on their programme distracts Tim. He refocuses when it goes quiet again.

Siobhan and I both write notes in Tim's home notebook and we all walk to meet Lee. Tim is keen to go home!

\section{TUESDAY 13/02/07}

9.04 Tim is on the mat with the other students while they have roll call. There is no chanting today - one child is leading the group and calling others to say 'Hello' from another country. Tim has his plastic container. He is listening to the children; and watching(?). He also looks directly at me. He makes the occasional vocalisation but is mostly quiet.

9.07 Prayers. The children pass a candle round. Tim appears to ignore them as the candle passes directly in front of him.

9.10 Tim is making rhythmic vocalisations at a moderately loud volume. Sally helps Tim to hold the candle this time and says "Thankyou for friends in Room 2" on his behalf. Tim appears comfortable with this ritual.

9.15 The group disperses. Tim stands independently and follows! I meet and talk with Angela for the first time, and arrange an interview time.

9.25 The class is doing PE outside. Tim is playing "Here we go round the mulberry bush". He is holding hands with Sally and another child, in a group of six. He was laughing and managing to 'circle' with the group very well. However when the teacher calls the children to a larger class group, Tim does not manage the transition and resists joining them. He sucks his thumb, sits down and although the class waits for him for some time, they have to 
start without him. They are laughing and squealing loudly - it might be too noisy for Tim. He sits and watches.

9.30 The class go back to their room and Sally asks Tim to stand. Sally chats to Tim about what has been happening. She walks him to the toilet, talking about the rain and pointing out wet patches and equipment. Tim walks happily and is quiet in the loo too. I can hear Sally continuing to chat to him about what she is doing and what will happen during the day. Sally is singing and I hear Tim give a musical response "Aa aa aah aah aa aa aah" (rhythm X X X X xx X) - he's singing at the same tempo as Sallys Hoki Toki tune for "We put our right leg in..." When Sally finishes her song Tim continues with an " $\mathrm{mm} \mathrm{mm} \mathrm{mm}$ mm". Sue talks about 'cold' water and 'fast' water etc. She sings "This is the Way we Wash our Hands". Sally 'rewards' Tim with a spin in his wheelie chair. She starts singing "Slowly slowly slowly slowly, Slowly turning Tim around, Slowly, slowly, slowly, slowly" and finishes with a fast phrase "Turning Tim around!". They are engaged in 'slow' and 'fast' play for several minutes. Tim's vocalisation is communicative and with varied intonation, and Sally acknowledges his efforts by 'interpreting' what he might mean. Her very good commentary might need to reduce at times to give Tim a chance to receive clear instruction for example when going “Up, Up, Up the steps”. Watch Tim's movement and time your singing with strong rhythm to his movement. This is as much about sensory reinforcement (body awareness) as promoting cognitive understanding.

9.40 Tim is back in class in 'his' corner. He has a green plastic mould of a train. Sally uses PECs to indicate it's time for 'reading'. Tim takes a book from the shelf spontaneously. He starts at the back of the book and flips to the front before dropping it. He is looking away from Sally but engages briefly when she picks up the book and reads to him - then he resists, pushing her away. He begins a vocal protest when Sally insists he continue reading. He is clearly indicating he wants to leave. He gets up and struggles away. The classroom is noisy! The others are doing maths or reading in pairs or small groups. Tim hugs Sally. She tries another book and Tim pushes it away. WOULD HE PREFERR MUSIC BOOKS (TAPES WITH BOOK?). Sally gave up declaring there is "no point persevering with reading".

9.43 Tim walks to the library and sits on his 'working chair' - the bean bag is for when the work is done! He vocalises C cd E E, D D C C using "Aa". I was able to notate it and Sally simultaneously realised that he was singing "Down at the Station" a favourite DVD (which was not at school today!). 'Max' is inserted and Tim looks immediately when it comes on. 
He smiles, has a brief flap, vocalises and flaps again. He has his feet up on the chair in a relaxed manner. He is listening to the clapping song and during the second playing Sally patterns Tim through 'Clap Your Hands' C D E - / f F f F D/ G gg G A / G - E - / C D E - / f F f F D / G F ee D C.

Clap your hands, clap to the music, . .......... Clap your hands, clap to the music, Now get ready to stop!

9.55 Tim goes to the TV to touch Max. He is tapping his knees with Sally's help. The command is "Stand up Straight" but Tim is still sitting - can he be encouraged to stand at this point? He continues to allow co-active movement. He allows Sally to facilitate his hands to his hips and leaves them there X2. He seems aware that he is 'imitating'. He can't choose an activity from the TV but could he choose from two objects (instruments?) or picture cards for activities? Tim laughs when Sally is relaying stories about him - he seems to know we are talking about his antics. She says he is choosing, listening, and seeming to understand more this term.

\subsection{Tim goes to morning tea.}

\subsection{0am Interview with Angela}

11.10am Tim is on the mat with the class group for reading. He is holding a red plastic mould. He is not looking at the child who is reading but is vocalising happily, rocking, twisting from side to side occasionally and tapping his object. He vocalises "Ah ah ah Baa!" (X X X X----) using rising intonation. Angela removed him from the group - was he distracting the other children? She hides a toy behind a cushion. Tim looks out the side of his right eye and pushes the cushion aside. Tim picks up a picture book of transport. Angela blows a balloon and Tim takes it from her, letting it go down slowly. This is repeated. Angela allows the air on Tim's face. He is calm, looking with his peripheral vision. The other children clap and Tim listens. Angela tries to engage Tim again with some soundmaking toys behind her back and also hiding behind the cushion. Tim crawls away and takes a large picture book (Happy Birthday) from the shelf. He also chose this on yesterday? He allows Angela to read it to him, but just the first few pages. He tries to choose another book - his attention is gone from the first. He gives Angela a new book and engages as she reads from it. He is not looking at her or the book but seems to be listening carefully to her rhythmic reading. Next he chooses Hairy McLarey... Angela brings a photo book and Tim allows the exchange. In the book there is a photo of a bus - Angela sings 
"The Wheels on the Bus". Tim looks at the photo for three verses. Angela tries to tickle him to stimulate laughter and/or vocal response - he is seeming to be rather 'flat' (Lee reports he was very hard to wake today). He pulled away from Angela without laughing and has chosen another Hairy McLarey book, this time a small version. He is still holding his bus photo and is making a noise with it but is still listening to Angela. He also did some good looking, with central as well as peripheral vision. Tim indicated 'more' when the book was finished. The book was partially repeated and during this reading Tim allowed Angela to put the photo away.

Tim takes the Happy Birthday book again. Angela taps two plastic toys together. Tim looks and reaches, taking them two times. He allows Angela to take them from him, listens and reaches. Tim is rewarded by being allowed time to 'twirl' them. Then he goes back to his Happy Birthday book. Angela blows a whistle which extends and Tim smiles and looks. He laughs as it extends, twice. Angela continues but does not get the same response so stops. But Tim is still interest - it seems he needed longer to process that she thought he might. Tim sings "Ba ba ba ba ba". Angela blows the balloon up again and allows the air to come out on his face. Tim smiles.

11.33 'Hall time'. Tim stands and walks with Angela. As we walk, Sally reports that she noticed Tim would not take his lunch box in his right hand, but would carry it in his left. (IS DOMINANCE ESTABLISHED?). Tims vocalisations increase - I think it is the echoe in the hall. Angela rolls the physio ball and the peanut ball. He does not put his hand out to the ball as it comes towards him. ROLL AND STOP SONG? He chooses to lie on the ball, and Angela rocks him. She warns him “one more' before asking him to go to the toilet.

Tim sits down in protest. Both Angela and Sally are trying to coax and lift Tim but he will not weight bear. I sing "Stand up, Stand up, Stand up" using rising intervals. The first time I stamp the beat. The second time I tap Tim's back, and the third time I give him under arm support. He still does not weight bear but laughs and is vocalising a rhythmic "u" "u" "u". I laugh with him and repeat the sequence and this time he stands. He walked to the toilet to the walking song, which offered him a strong clear beat to follow.

Angela is impressed and wants the whole team to try the "Stand up" Chant, and the "Walking Song". While she is toileting Tim I also show Sally "Oh Yoo Hoo".

When Tim returns from the toilet he engages in his textured walk. He is tracking the 
'path' to his reinforcer (a plastic object) very well and appears to be enjoying the activity and his praise (clapping and verbal praise) very much. At one point (can't remember why) he verbalised a clear "No!".

$1.00 \mathrm{pm}$ I attended a preliminary meeting to plan a sensory room for Tim and others who have special needs. Send catalogue of equipment that might be useful, to Lee before 21/3/07.

\section{WEDNESDAY 14/02/07}

9.00am I joined the class on the floor for roll call. One child was calling each child's name and they were to respond with "something beginning with ' $r$ ". After prayers I introduced the class to 'A song for Tim and his Friends'. Tim laughed and looked around, stayed with the group for about another 5 minutes unsupported but got up to look at a book as other children took their turn - it might be a bit long... or perhaps we could try the children standing up in pairs to give more visual cues about whose turn it is? Tim sat down again easily when guided though and Mum spoke/signed "Hello" for Tim when it was his turn.

9.30am PE time - Tim walked willingly but slowly to the playground. He was twiddling a green container. He walked steadily and did not protest, but was distracted several times and had to be guided to keep working in the direction of the playground. Tim needs help to throw a ball - he could be assisted by the inclusion of rhythm? He is not able to stay in line - some of this will be inattention but it seems also body awareness... he might need to keep moving to know where he is in space. He is less interested in the other children than his TA, Sally. Tim protests when she tries to keep him in line - perhaps quiet chanting "waiting, waiting, waiting" might help? When it was time to run, Tim began well but only went a few metres - the grass was wet and he had sandals on (!?).

\subsection{5am Interview with Tim's parents}

10.30am Tim is in the playground. He is holding a green container and is twirling it. $\mathrm{He}$ sees an aeroplane and looks up and smiles. He is shifting his weight from side to side, rocking. Tim's dad is in the playground and calls to Tim but he does not observe and/or respond to him. He lets out a high pitched squealing noise - there is quite a lot of that going on in the playground! Dad approaches him and calls "Oi!". Tim stills, stops twirling the container, and steps forward putting his thumb in his mouth. Dad was with him briefly but 
had to go. Tim noticed the car key in Dad's hand and wanted to go with him. He began to cry and to flap. He cuddled into Sally who is trying to calm him by talking about other good things that are going to happen this morning - can Tim understand that far into the future? He seems tired and is really snuggling in to Sally with his thumb in his mouth. She encourages him to the playground. Tim has his hands over his ears, is still flapping and making crying noises (“" $\mathrm{n} n \mathrm{n} n$ "). When Sally turns away, Tim screams.

\section{Reported later}

11.00am Tim rides his three wheel bike to swimming. He swims independently (with armbands) and is able to kick to help himself move in the water. Sally reported that he seemed to be saying "look at me, I'm swimming!"

12.05pm Tim is back from swimming. He is in the group space (on his own) pulling out reading books from a plastic box... he pulls out 7 and glances at each. He engages for longer with the $8^{\text {th }}$ book, 'Postman Pat' but it is still brief.

\subsection{Computer}

\subsection{Toilet}

12.30 Lunch. Tim gets up quickly to get his lunch box but doesn't go directly to his bag. Would a song be helpful here?

1pm Afternoon with Tim in hall, trialling some ideas that Siobhan wanted help with. She has her own strong ideas about what she might do based on her training. She is open to hearing/observing new things but perhaps not finding it so easy to adapt what she is already trying. This is understandable since she is just starting work with him (it is her $5^{\text {th }}$ day) because she will want to be consistent about some things (e.g. she has decided that she won't tell him that he is going to the toilet; rather that he is going to the wheelie chair which he gets as a reward afterward). At home he is beginning to understand "shoes THEN car" so he might manage "Toilet THEN wheelie"?

I am beginning to get a feeling of being rushed - is this how it is for Tim?

Let's WAIT for Tim to communicate. Give him some TIME to decide and act. Watch for ANY communication and interpret it overtly for him. When 'left to his own devices' Tim found the bubbles and threw them through the hatch to us; opened the cupboard and indicated that he wanted the scooter. When he is SAFE he can be given more SPACE. 
Tim held the ring with two hands so he could be pulled on the scooter... other carers were putting the ring right round his back?

Siobhan is having some personal and professional difficulties. She was upset and unable to give Tim the energy he demands of a carer.

THURSDAY 15/2/07

9.00am

I joined the class for mat time and we did the song again - this time asking the children to stand up with their peer when it was their turn to sing. This helped the group to focus on others and to remember to respond. Sally responded quickly for Tim, not waiting and allowing the space to prompt him. I spoke to her later about the importance of the rhythm of the song and the possibility that Tim would become increasingly aware of the pattern that is set up and the opportunity he has to give SOME kind of response.

\subsection{0am}

Music therapy assessment (see video data)

\subsection{0am}

At morning tea time I observed Sally giving Tim his morning tea, initially in the playground where he was sitting at the picnic table with other children. He seemed to be enjoying their company and the children were happy to be with us... it could be the presence of adults that is attractive but they did seem to be interested in being with Tim too. However Tim was not able to eat his morning tea in such a stimulating environment today... he was very clearly saying "no!" (verbally!). The boundaries around this behaviour are difficult for staff - when he refuses to do what he is asked he but communicating so well it seems important to acknowledge the communication but to insist that he complete the task.

Sally took Tim to the hall where it was less stimulating. He initially continued to protest when he was seated on a chair, but when allowed to sit on the floor he engaged well and complied with eating his morning tea. Sally sang Winnie the Pooh (as Tim ate his honey sandwiches) in a warm and calming voice. She moved her body to the gentle rhythm, employed an appropriate level of accent, paused occasionally and kept him calm and 
interested. When he moved on to eating his yoghurt Sally changed her song to the yoghurt jingle. This music was very relevant to the situation and was well executed!

When it was time to go outside again Sally 'teased' Tim with 'reverse psychology' telling him in an animated voice "Don't go outside Tim... don't go out the door...". This seemed to be a very inappropriate interaction with a student who had such significant communication impairments... it made me quite anxious because I didn't quite know what to do about it even though it had provoked a strong reaction in me. I didn't want to interrupt her when she was working with Tim but it was something that would be hard to go back to later...

Sally noted that Tim was quiet today and advised that some days he is very vocal and other days he is not. Today he is fairly unsettled overall and she wondered if he had a sore tummy - he had been to the loo a lot. From being almost euphoric when he came in by the end of the morning he was very tired and out of sorts. However even though he was not playing with his voice as observed on other days, Tim verbally communicated "no!" several times this morning. He also had a 'cheeky' demeanour and was challenging boundaries in a way that suggested he was aware of the power he could exert over his carers. Sally was aware that it could be his response to being back at school after a long break (it is now only week two of term one).

After morning tea Tim was invited to the library to watch his 'Love to Sing' video. This is a great teaching resource (designed by SLTs) which is bright, simple, repetitive, and easy to follow. It contained action songs presented at a tempo which was slow enough to allow Sally to do some coactive movement with Tim. He was able to tolerate this activity for a few bars of three separate songs.

After lunch, while Tim was at swimming, I prepared for the focus group meeting. I was aware as people began to arrive for the meeting that it was particularly difficult for families to attend an after school meeting. Patrick and Lee both wanted to be there but they were unable to find care for Tim. He was very upset about the change in his routine and insisted on getting in the car as usual at $3 \mathrm{pm}$ and refused to play at school with his sister. The car was eventually positioned within the school grounds outside the library where we could see him, and Tim was allowed to sleep in the car (with all the windows down). Although it can be problematic for school staff to have meetings in school time, the difficulties that parents encounter are often much worse - RECOMMEND THAT TEAMS SHOULD AIM TO 
MEET IN SCHOOL TIME.

\section{$3.30 \mathrm{pm}$}

Focus Group Meeting (see transcript)

My thought for the day... 'It seems as if we have to put more play into Tim's work, and more work into his play" (!?).

In the evening I prepared an initial report for Tim.

\section{FRIDAY 16/2/07}

Tim went to sleep during mat time. He snuggled up into Sally with his finger in his mouth as the team (Lee, Angela, Siobhan and I) waited for them to be ready to go to the gym to trial some music activities.

As I thought about the week, I felt that the observations I have made of Tim had been at least as important as the direct music therapy assessment in helping me to understand him. I also became aware that my assessment session probably was different because of this understanding and because I was planning consultation work rather than ongoing therapy. On the other hand, perhaps it was still Tim who guided what happened in the assessment (i.e. the unaccompanied 1:1 work) - I certainly felt that he needed the prompt and containment that my close presence provided - although I can't help thinking that I might have subconsciously been thinking of the sorts of things that other SE team members could do that demanded less formal music skills.

\subsection{0am}

In the gym...We invited Tim to tell us what he would like to do. He is familiar with a range of Perceptual Motor Activities that he does in regularly in this setting. Although he enjoys and seeks vestibular stimulation (particularly his wheelie chair) he usually requires prompting to begin and to remain engaged. Today Tim stands outside the door watching as Angela uses the wobble board and improvises a song. Although she is inviting him to come in he seems to be 'entertained' and is enjoying watching/listening. I advised the team to wait quietly for Tim and he eventually took a few steps forward. Angela and I played 'roll and catch' with the physio ball, singing the song composed for this purpose. Tim remains in the doorway - he has no adults with him and he is attending to the play. 
When an adult approaches him he seems to prepare himself to run and when they reach out he pulls quickly away. He shuts the door of the gym... now he is outside and we are in this is different! I move quickly to the door and open it carefully... Tim is still there, waiting cheekily. I begin the 'Oh Yoo Hoo' song. Tim is smiling as I peep around at him, and vocalises $2 \mathrm{X}$ when I wait for his response. When the song suggests it is time to "come out!" I move quickly to Tim, touch his shoulders and prompt him towards the hall. He moves in and the door is shut behind him. Angela, Siobhan, and Sally all have a go at singing the precomposed songs, or improvising song for movement. I have to reinforce the importance of the rhythm, of observing Tim and timing the music to match his movement. I also stress how helpful accent can be, and also the way a simple rising melody can support movement. Tim's response seems variable - he engages briefly in the activities but still needs prompting to return for repetitions or to begin a new activity. I encourage the 'hands off approach though... Tim IS motivated to play and given time is able to respond independently. Later the team advised that they believed that he was demonstrating very good levels of engagement and that the length of time he was able to stay in the gym i.e. 45 minutes, was excellent. They are keen to continue with the PMP music programme. Songs for Tim have been notated and recorded on $\mathrm{CD}$ - four copies were presented to various team members (Angela and Lee have copies).

\subsection{0am}

Angela's interview (see transcript)

\section{$11.45 \mathrm{am}$}

Sally's interview (see transcript)

1. Angela has advised that she would like to use the home/school notebook to record music work and Tim's responses to that. She will draw up a section on each page for that purpose. Lee has given permission for information in the H/S notebook to be photocopies and forwarded regularly to me.

2. Tim's IEP review is not until April

3 . In the week beginning $19^{\text {th }}$ February, the team will be having a programme meeting and at that time they will consider the music and set goals. Copies of the meeting notes will be sent to me.

4. Siobhan is interested in recording things in more detail and would like to use the research diary format.

5. I will make copies of the music therapy assessment video for Lee/Patrick and for the school (via Angela).

6. I will need to make up a book of the songs for the team and draw attention to the important elements in each (e.g. point out strong/accented notes etc.) 
7. The Rickson/Krout resource might be useful - include it in the package

8. But how can we agree a specific plan? How can we tie what we know about Tim and his relationship with music into the IEP goals? How will the goals be evaluated? It mightn't happen while I am on site ... it feels as if I might be overloading the team with information and/or requests now? Make sure Angela sees the sheet from the protocol (email it)

9. My contribution to ideas about specific plan can include thoughts about class music and the specific song to help with greetings; the PMP programme; and ideas for improv with the bongo drums as indicated in the MT assessment session.

10. Angela is going to video ongoing work and send it to me for comment

\section{AFTER I LEFT THE FIELD}

\section{$17 / 2 / 07$}

I feel as if I haven't given enough time to observing individuals trying out our ideas. The visit seemed short and I was feeling pressured to watch and learn and to give input; but the third part, the process of assimilation, was missing. It's great that Angela will be able to video ongoing work though - maybe we can do it that way?

Would a staff workshop about $1 / 2$ way through have been helpful?

I wondered if I had timed things well overall - should the assessment be earlier? But the way I did it did suit the 'natural' timetable (e.g. when particular staff were available) well. If we did a workshop straight after the assessment (for all available staff) before I modelled ideas with Tim; then staff 'had a go', would we learn more?

The balance between planning a programme for the week that 'covers what I want to do' versus what works well for the team within their usual routines is tricky.

Maybe some of the 'pressure' I am left with is transference from the team? They have "so much to do and so little time"... I have to remember that a little can be a lot. This seemed to come through in the interview (the team believe they are benefiting from my presence) so I have to leave it there to a certain extent. "Trust in the process". All in all I felt really valued and appreciated... time will tell what impact my presence has had in the medium term.

I remember that Lee told me she had been to a workshop I facilitated in 2004. She said she 
had been excited about some of the strategies that I had suggested. She had asked me later about specific ideas for Tim and had gone home and tried them and found some very helpful. However she also recalled that I had responded that it was difficult to advise Tim's specific needs without seeing and that I had reinforce the importance of the 'timing' of music interventions. On Friday she told me that she could see how true that thought seemed to be now that she had seen me with Tim - the assessment and consultation process had brought things together for her.

\section{$19 / 2 / 07$}

I am anxious that the team won't develop a 'project'? (!) See Angela's emails. Does it HAVE to be a 'project' - action research highlights 'how it is' not 'how it should be'. (Supervision with Jill?)

$25 / 2 / 07$

I am increasingly aware of how blurred the boundaries were in my various 'roles' in the field. My interview with parents is a real mix of me as therapist/consultant AND researcher, but predominantly the former.

And yet I did the interview because the parents had expressed interest in being involved in the research. However it's also hard for them to take a leadership role - it was my responsibility to 'lose' the expert role and I don't think I have done that in this interview. I think I want to engage participants in discussion but am I offering advice in a way that shuts them down?

The research process might get in the way of the 'consultation' (!). I am returning transcripts to participants and fear they will be overwhelmed by data.

I am increasingly aware of the confusion/intermingling of roles - when was I a clinician and when a researcher? The interviews are an example - I was clear about why I was doing them before I went into the field but later they became not a research method but a clinical data gathering method. I reviewed them to get information on what the team wanted for Tim for programme planning. But perhaps this was an unexpected necessity because there was not enough time in the week to feel as if I had 'empowered' the team to write/discuss their won goals for him... I needed to feed back what I had understood their priorities to be to ensure goals were team led?? Or is it because I wanted goals for MT to be 'appropriate' WHOSE goals? Have I decided what the options might be? How can I get away from the 
'expert' role? And what of the focus group !?! (See typed comments with the interview data). Similarly in the focus group I was a participant... too expert - but what if I hadn't contributed...?

$26 / 2 / 07$

As I am typing focus group notes I remember that mum brought a foot stool into school on Fri am to try for size at the desk... this is important given the conversation re seating during the assessment

(Transcribing focus group data)

The analysis looks closely at what's happening and one loses sight of the whole experience. What talk was there of the emotional experience, the playfulness, the joy? THE WHOLE IS GREATER THAN THE SUM OF ITS PARTS.

The focus group transcript is 6190 words and 2359 (38\%!!) are therapist input. I am definitely a clinician in this context but even so this is a lot of input!! Analyse according to type of interaction... am I answering direct questions?

$2 / 3 / 07$

The process of applying for ORRS review from High to Very High needs seemed to have caused a riff between the school team (or at least parents... who were good personal friends of staff at school especially Angela and Sally) and GSE. Are they not using the GSE staff? Parents have high expectations and "see things others don't"

\section{SEE JO’S EMAIL!}

Thoughts:

1. Does the 'visiting music therapist' become the 'angel' and the local music therapist 'the devil'

2. What of the assessment process being exciting, new, comparatively successful and later when things settle down music therapy can become more 'mundane'

3. Interagency communication

$7 / 3 / 07$

I mustn't forget my role as 'parent advocate' in the past... I developed quite a passion for ensuring the needs of my child were being met and recognised a lot of myself in Lee. I was also a parent support for parents of children newly diagnosed with hearing impairment - the 
empathic stance is familiar.

$12 / 3 / 07$

I am thinking a lot about the importance the team placed on my "spending time". Watson, Abbot and Townsley too identify this as a crucial aspect of care "through the "voices" of children who have complex health needs'. It made a "real difference if the professionals made an effort to get to know and talk to them directly" (p93). While I believe the relationship with tom was established during my music session I didn't involve him in my daily observations - I kept myself 'distant' as a researcher. I need to talk to people (including the child) more in future visits to schools. Get more field notes that are not passive but drawn on my 'interaction with people and their responses...1 including the child's responses to me. TALK TO THE STUDENT.

\section{SEND PICTURES, PHOTOS, NOTES ETC. TO THE STUDENT BEFORE GOING INTO THE FIELD.}

PhD notes 15/3/07:

Thoughts from discussion with Sarah after she had viewed Tim's assessment video:

1. A music therapist should look at the data

2. What was I thinking from a Methodological perspective when I was working?

3. What is the nature of the frame for the work?

4. Think about autism as 'motivational conflict' - a music therapy assessment would usually test different 'styles' of intervention (non-intrusive vs intrusive)

5. I felt as if I was working 'intuitively' and that my interactive style was appropriate for Tim (WAS it intrusive?)... was my 'decision' to work with the student in this way due to the knowledge/understanding of him that came from observations and data gathering?

6. Would it be different if the relationship was ongoing? I DID establish a therapeutic relationship but the strength of the bond would increase over time?

7. I wanted to HIGHLIGHT the student's POTENTIAL to demonstrate where had STRENGTHS... my intuition was telling me that if I 'backed off' he would not be able to show me what he could do.

8. I believe, even more strongly that the consulting music therapist DOES need training / experience to be able to connect quickly with the student (based on his/her considerable experience of interacting with other children who have SN)? While it might look easy "don't try this at home".

9. Is my approach tending towards a 'behavioural' framework - wanting to find opportunities to 'reinforce' his strengths, trying to achieve a lot in a short time frame rather than 'waiting' for him to develop motivation over time (as one might with longer term work). 
10. Does the depth/strength of 'change' correlate with the length of time allowed for the change to occur?

11. Look to Richer's work for information about 'attention' 'listening', 'the indirect route', 'animated baby play'

12. Break down and reconstruct 
APPENDIX 32 : Thoughts from reflective journal after case study one

\section{THOUGHTS FROM REFLECTIVE JOURNAL AFTER CASE STUDY ONE}

- The environmental assessment is central to this approach

- Team members gained skill and knowledge regarding the therapeutic use of music, through interactions in natural settings

- The clinical assessment was crucial in helping team members identify student's strengths and gain new perspectives of them

- Developing positive relationships with adults was a fundamental task. Although I initially felt disempowered because the work seemed to rely more on verbal than musical communication, I believe as a music therapist I have a particular way of being which will be highly valued by team members.

- Even within a collaborative framework I can be perceived as an expert in my field

- Safety in consultation processes can be maintained by employing an ethical framework associated with action research

- In this model the emphasis is on helping the adults to help the student

- A careful balance of direct work with students, and indirect work for students, is required

- The music therapy consultation protocol thus far developed appears to have 'social validity' and be of 'functional value' for students and team members

- How much should I be concerned with systemic issues?

- Should I respond to requests for help with matters that are outside my training but within my realm of experience?

- Pre-consultation workshops might improve consultation outcomes

- Pragmatic issues need to be recorded and attended to. 
APPENDIX 33: Themes from second interview with Angela

Themes from Second Interview, Angela

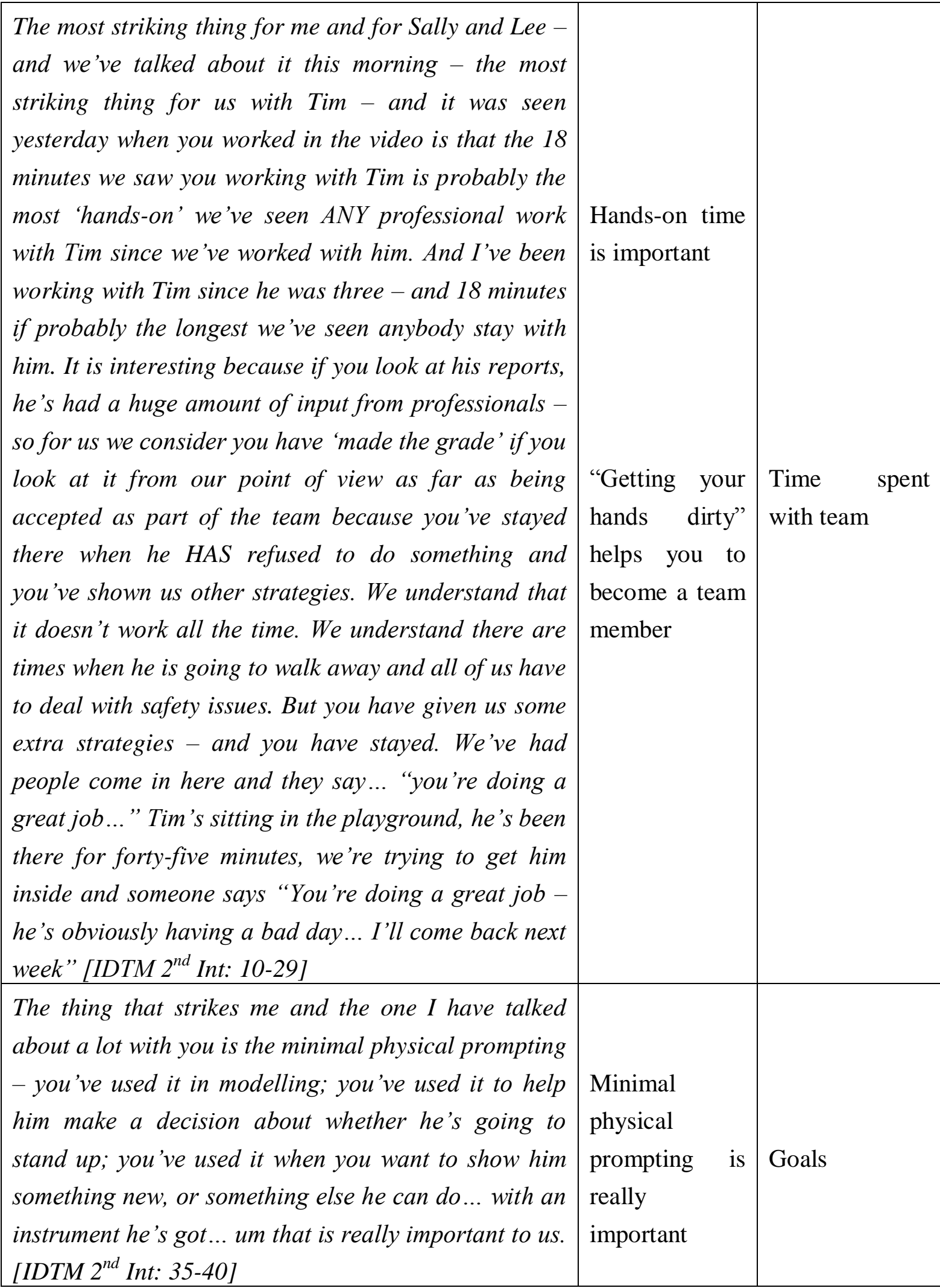




\begin{tabular}{|c|c|c|}
\hline $\begin{array}{l}\text { We've tried a number of different strategies over the } \\
\text { three years and the LEAST physical prompting is a } \\
\text { priority for us. So I think that's a really positive thing. } \\
\text { Even if that was the one thing I learnt this week that } \\
\left.\text { would be enough for me. [IDTM } 2^{\text {nd }} \text { Int: } 44-47\right]\end{array}$ & $\begin{array}{l}\text { One helpful } \\
\text { strategy can be } \\
\text { enough }\end{array}$ & Goals \\
\hline $\begin{array}{l}\text { I like the that way you were able to give him the same } \\
\text { messages but change your tempo - that was quite a } \\
\text { difficult thing I saw this morning for all of us to pick } \\
\text { up... but we'll get there with it. It's just that we've got } \\
\text { to read him as well and read what type of message } \\
\left.\text { we're sending to him. [IDTM } 2^{\text {nd }} \text { Int: } 46-49\right]\end{array}$ & $\begin{array}{l}\text { Notice non- } \\
\text { verbal } \\
\text { communication } \\
\text { strategies }\end{array}$ & $\begin{array}{l}\text { Communicating } \\
\text { with student }\end{array}$ \\
\hline $\begin{array}{l}\text { The imitating of Tim's efforts is something that we've } \\
\text { done occasionally but we've never used it in the way } \\
\text { we've seen it this week. So that is REALLY important } \\
\text { for us because that is actually giving him some } \\
\text { recognition of his communication attempts.[IDTM } 2^{\text {nd }} \\
\text { Int: 51-55] }\end{array}$ & $\begin{array}{l}\text { Acknowledge } \\
\text { communication } \\
\text { attempts }\end{array}$ & $\begin{array}{l}\text { Communicating } \\
\text { with student }\end{array}$ \\
\hline $\begin{array}{l}\text { What struck me yesterday was the change in Tim's } \\
\text { positioning particularly with his feet - but his } \\
\text { positioning as he grew in confidence... and I DID } \\
\text { realise that you sat in a chair and then you } \\
\text { deliberately moved closer which is something that } \\
\text { sometimes works and sometimes doesn't for us. } \\
\left.\text { [IDTM } 2^{\text {nd }} \text { Int: } 65-69\right]\end{array}$ & $\begin{array}{l}\text { It doesn't } \\
\text { always work }\end{array}$ & Goals \\
\hline $\begin{array}{l}\text { Seeing somebody else do it and seeing somebody else } \\
\text { WAIT for him and allow him the time to process what } \\
\text { is going on has given US enough skill to show us how } \\
\text { we might go forward. [IDTM } 2^{\text {nd }} \text { Int: 69-71] }\end{array}$ & $\begin{array}{l}\text { Modelling is } \\
\text { important } \\
\text { Waiting is } \\
\text { important } \\
\end{array}$ & $\begin{array}{l}\text { Time spent } \\
\text { with team } \\
\text { Allowing time } \\
\text { and space }\end{array}$ \\
\hline $\begin{array}{l}\text { Leaving a gap for predicting what will come next - I } \\
\text { REALLY like that idea. I think we've done it with } \\
\text { rhyme before but we've never taken it further than } \\
\text { that. So we've done it in a rhyme with him where } \\
\text { we're playing in Floortime - where we might be doing } \\
\text { finger puppets and we've left a gap for prediction BUT } \\
\text { where Tim may have answered in some way we } \\
\left.\text { haven't acknowledged it. [IDTM } 2^{\text {nd }} \text { Int: } 78-83\right]\end{array}$ & $\begin{array}{l}\text { Leave a space } \\
\text { for student } \\
\text { communication }\end{array}$ & $\begin{array}{l}\text { Allowing time } \\
\text { and space }\end{array}$ \\
\hline We've left a gap for prediction BUT where Tin & & \\
\hline
\end{tabular}




\begin{tabular}{|c|c|c|}
\hline $\begin{array}{l}\text { have answered in some way we haven't acknowledged } \\
\text { it. We might have said "yes", or "good work" or } \\
\text { something but we haven't acknowledged it in the way } \\
\text { that you've specifically acknowledged his efforts - and } \\
\text { I like that! So the prediction will be an area that we'll } \\
\text { work on as well - like it's a simple thing... it doesn't } \\
\left.\text { take much to do! [IDTM } 2^{\text {nd }} \text { Int: } 83-87\right]\end{array}$ & $\begin{array}{l}\text { Acknowledge } \\
\text { student } \\
\text { communication } \\
\text { attempts }\end{array}$ & $\begin{array}{l}\text { Communicating } \\
\text { with student }\end{array}$ \\
\hline $\begin{array}{l}\text { In fact what I really think I've learnt most is to stop. } \\
\text { And to be patient rather than to do ANYthing else. } \\
\text { [IDTM } 2^{\text {nd }} \text { Int: } 87-89 \text { ] }\end{array}$ & $\begin{array}{l}\text { Learnt to be } \\
\text { patient }\end{array}$ & $\begin{array}{l}\text { Allowing time } \\
\text { and space }\end{array}$ \\
\hline $\begin{array}{l}\text { And the bongo drums - I know we talked about those! } \\
\text { I think they could be a REAL asset to us. I particularly } \\
\text { like the way that it can differentiate territory and then } \\
\text { you can 'intrude' on someone's territory and they can } \\
\text { 'intrude' on yours - and then it can become a shared } \\
\text { territory. I really like that idea for Tim... and some of } \\
\text { the other special needs kids I work with. } \\
\text { [IDTM } 2^{\text {nd }} \text { [9-94] }\end{array}$ & $\begin{array}{l}\text { Thinking about } \\
\text { helpful } \\
\text { instruments }\end{array}$ & Resources \\
\hline $\begin{array}{l}\text { You made the echoes or you spoke and so he could } \\
\text { actually feel on the other side the vibrations of the } \\
\text { speech... um or you tapping, or anything else. I've } \\
\text { never done anything like that with him before and he } \\
\text { responded really nicely in that situation - it's that turn } \\
\text { taking once again indicating that he's communicating } \\
\text { something. [IDTM } 2^{\text {nd }} \text { Int: 98-103] }\end{array}$ & $\begin{array}{l}\text { Notice and } \\
\text { encourage non- } \\
\text { verbal } \\
\text { communication }\end{array}$ & $\begin{array}{l}\text { Communication } \\
\text { with student }\end{array}$ \\
\hline $\begin{array}{l}\text { The other thing that I think is important for ALL of us } \\
\text { is looking at the fact that, that - and I noticed today } \\
\text { that we were already doing it when we brought him } \\
\text { across here so we've obviously taken it on board } \\
\text { already... Sally and I were doing it - is taking his } \\
\text { hand and guiding him but with the minimal input... It } \\
\text { may be a finger, it may be the tip of a finger - } \\
\text { whatever. But it's still allowing him the freedom to } \\
\text { know that this is where we're going... and sometimes } \\
\text { Tim seeks your hand and that's fine if he seeks it-but } \\
\text { at other times that idea of your hands behind his back } \\
\text { - again or the minimal... once again the 'minimal }\end{array}$ & $\begin{array}{l}\text { Developing } \\
\text { trust }\end{array}$ & Goals \\
\hline
\end{tabular}




\begin{tabular}{|c|c|c|}
\hline $\begin{array}{l}\text { physical prompt' required... I think is essential. } \\
\left.\text { [IDTM } 2^{\text {nd }} \text { Int: } 103-113\right]\end{array}$ & prompts & \\
\hline 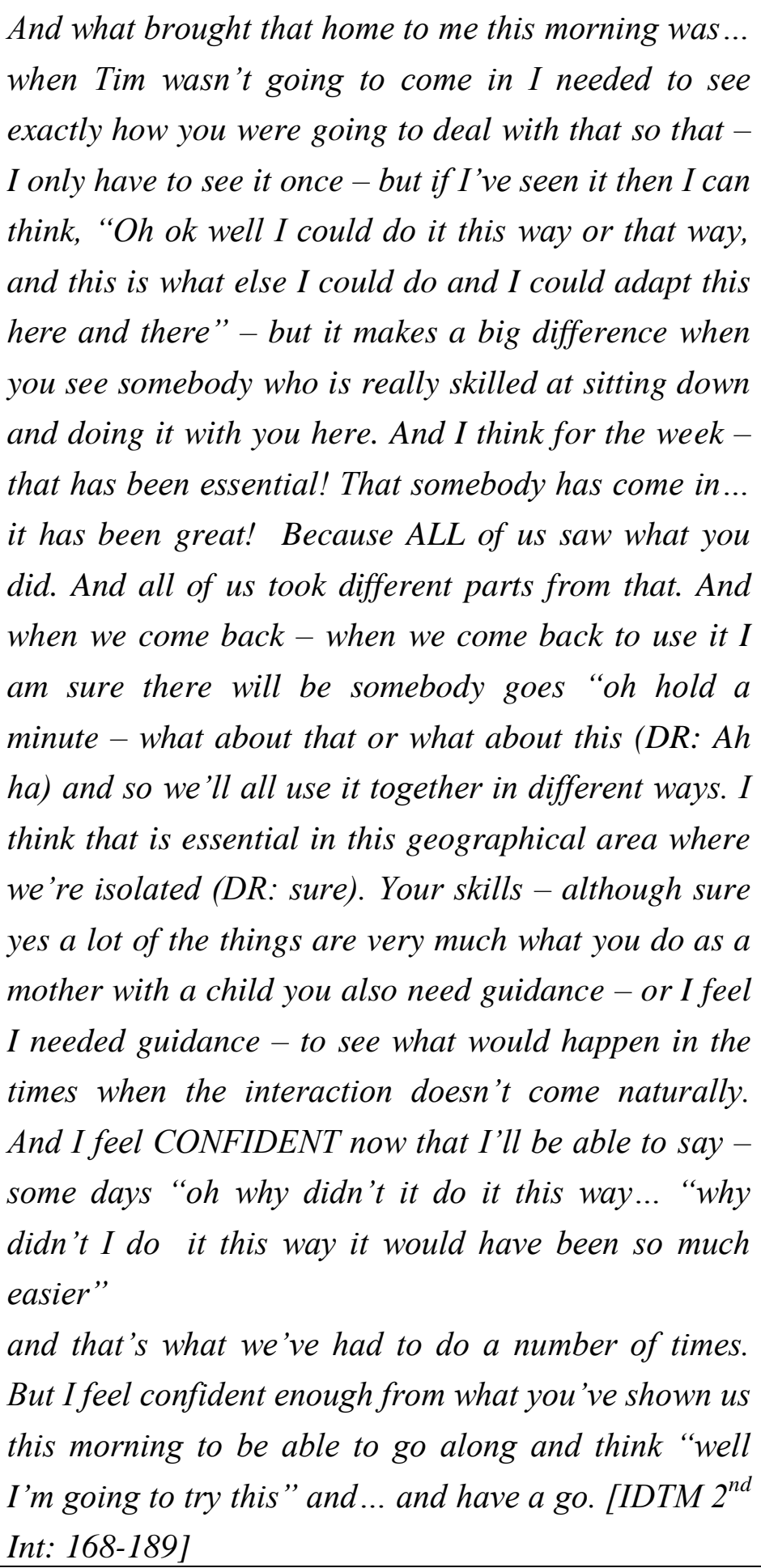 & $\begin{array}{l}\text { It might be } \\
\text { 'natural' but } \\
\text { we need } \\
\text { guidance } \\
\text { Reflective } \\
\text { practice } \\
\text { Confidence to } \\
\text { have a go }\end{array}$ & $\begin{array}{l}\text { Time spent } \\
\text { with team }\end{array}$ \\
\hline $\begin{array}{l}\text { Well for us it's great. We've got - we've got things } \\
\text { now we can do. We've got strategies we can use and... } \\
\text { it doesn't matter if } 50 \% \text { of them don't work. One new } \\
\text { strategy with Tim is really important because we're }\end{array}$ & $\begin{array}{l}\text { We will learn } \\
\text { more about }\end{array}$ & Sustainability \\
\hline
\end{tabular}


here on our own going “what DO we do next?" And I Tim by trying believe that we will learn more about Tim from using new strategies new strategies. [IDTM $2^{\text {nd }}$ Int: 218-222] 
APPENDIX 34: Themes from follow-up interview, Angela

Themes from Follow-up Interview, Angela

\begin{tabular}{|c|c|c|}
\hline $\begin{array}{l}\text { "Little tiny strategies like a little song make a } \\
\text { big difference to Tim - we can get him to do } \\
\text { things now because we can just jolly him along } \\
\text { with a song. It's a little thing" [IDTM Follow- } \\
\text { up: } 337 \text { - 340] }\end{array}$ & $\begin{array}{l}\text { Little strategies } \\
\text { make a big } \\
\text { difference }\end{array}$ & Strategies-value \\
\hline $\begin{array}{l}\text { "The process has made us stop and look at his } \\
\text { education from another viewpoint - from } \\
\text { another lens if I want to think about it. It's } \\
\text { probably from another lens. Because we know } \\
\text { that there were gaps that we were not getting to. } \\
\text { It's helped US identify some. YOU were able to } \\
\text { identify some when you were here but we are } \\
\text { also able to identify some now that you are not } \\
\text { here because we have a platform to work from" } \\
\text { [IDTM Follow-up: 30-36]. }\end{array}$ & $\begin{array}{l}\text { Looking at his } \\
\text { education } \\
\text { through another } \\
\text { lens }\end{array}$ & Strategies-value \\
\hline $\begin{array}{l}\text { A: "I think the process of looking at the goals for } \\
\text { the IEP (over past years) has been quite difficult } \\
\text { because we have had the same goals...and when } \\
\text { you suddenly go a step further and we (are now) } \\
\text { considering the types of strategies we can use } \\
\text { particularly in sensory and music therapy } \\
\text { strategies, it changes the WAY that we teach" } \\
\text { [IDTM Follow-up: } 44-49] \text {. }\end{array}$ & $\begin{array}{l}\text { Sensory and } \\
\text { music therapy } \\
\text { strategies change } \\
\text { the way we teach }\end{array}$ & $\begin{array}{l}\text { Strategies- } \\
\text { change }\end{array}$ \\
\hline $\begin{array}{l}\text { A: "So suddenly for us a big light's gone on for } \\
\text { us and oh my gosh it's fabulous. I know that there } \\
\text { is years and years of support that's gone on there } \\
\text { but I do believe that the strategies we've used, } \\
\text { particularly of echoing back or of expecting or } \\
\text { waiting for him to imitate, um the fact that we } \\
\text { actually talk back to him if he screams saying } \\
\text { "yes I know you don't like that - I don't like it }\end{array}$ & $\begin{array}{l}\text { New strategies } \\
\text { pushed the door } \\
\text { open for us }\end{array}$ & Strategies-value \\
\hline
\end{tabular}




\begin{tabular}{|c|c|c|}
\hline $\begin{array}{l}\text { either but we have to do it", um those types of } \\
\text { things have pushed that door open a little bit } \\
\text { wider for us" [IDTM follow-up: 214-221]. }\end{array}$ & & \\
\hline $\begin{array}{l}\text { A: "when we're doing music if we're actually } \\
\text { copying his physical gestures he becomes } \\
\text { incredibly excited. He's incredibly excited by it. } \\
\text { And he will actually stop what he's doing and } \\
\text { laugh and laugh. So instead of Sue doing the } \\
\text { waving right up high, she's doing it low like he } \\
\text { would do. And he will actually stop what he is } \\
\text { doing and laugh. And the same with "Row Row } \\
\text { the Boat" and those sorts of rhymes, if we } \\
\text { actually copy HIS movements we are finding he is } \\
\text { stopping and engaging with us - he is taking } \\
\text { notice of it. Now that's huge for us because... we } \\
\text { haven't DONE it in the past...it is a strategy we } \\
\text { haven't used. We haven't copied him; we've } \\
\text { expected him to copy us... It doesn't mean that he } \\
\text { always copes but it means that he does cope a lot } \\
\text { better. So sometimes I'll echo him - and we chop } \\
\text { and change depending on what's happening - } \\
\text { we're not copying him all the time! But when we } \\
\text { DO echo what he is saying, he'll stop and listen"” } \\
\text { [IDTM Follow-up: 167-182]. }\end{array}$ & $\begin{array}{l}\text { We haven't done } \\
\text { it in the past }\end{array}$ & $\begin{array}{l}\text { Strategies- } \\
\text { change }\end{array}$ \\
\hline $\begin{array}{l}\text { A: “....that's one thing with the music therapy } \\
\text { strategies - they have opened up the potential for } \\
\text { communication and that's HUGE for us. Because } \\
\text { in some ways we shut it down. And that's part of } \\
\text { us learning about what we're doing as well. And } \\
\text { part of it is trying to cope with Tim's behaviour } \\
\text { as well but I think that has been huge for us to try } \\
\text { to open up that communication area" [IDTM } \\
\text { Follow-up: } 61-66] \text {. }\end{array}$ & $\begin{array}{l}\text { Opened the } \\
\text { potential for } \\
\text { communication }\end{array}$ & $\begin{array}{l}\text { Strategies- } \\
\text { change }\end{array}$ \\
\hline
\end{tabular}




\begin{tabular}{|c|c|c|}
\hline $\begin{array}{l}\text { A: “...sometimes we have questioned ourselves } \\
\text { just because we hear that 'he's at this end of the } \\
\text { scale'... so suddenly the music therapy strategies } \\
\text { have justified to us our thoughts. We actually } \\
\text { believe in the potential that he is showing..." } \\
\text { [IDTM Follow-up: } 69-72] .\end{array}$ & $\begin{array}{l}\text { Justified our } \\
\text { thoughts }\end{array}$ & $\begin{array}{l}\text { Strategies- } \\
\text { affirmed }\end{array}$ \\
\hline $\begin{array}{l}\text { A:" I (emphasis on 'I') think it's giving him a } \\
\text { new awareness of himself and it's giving US a } \\
\text { new awareness of OURselves and OUR role. You } \\
\text { know it's made us realise how limiting we can be } \\
\text { without even meaning to be" [IDTM Follow-up: } \\
185 \text { - 188]"We are seeing this huge spike in } \\
\text { interactive intent. So I do think the (music } \\
\text { therapy) visit has made a difference - the whole } \\
\text { process of us looking at ourselves as well" } \\
\text { [IDTM Follow-up: } 244-246] \text {. }\end{array}$ & $\begin{array}{l}\text { A new awareness } \\
\text { of ourselves }\end{array}$ & $\begin{array}{l}\text { Strategies- } \\
\text { reflection }\end{array}$ \\
\hline "we're listening to him more" [357]. & Listening more & $\begin{array}{l}\text { Student } \\
\text { progress }\end{array}$ \\
\hline $\begin{array}{l}\text { "he's even talking to the speech language } \\
\text { therapist" [IDTM Follow-up: } 85-86]\end{array}$ & Talking to SLT & $\begin{array}{l}\text { Student } \\
\text { progress }\end{array}$ \\
\hline $\begin{array}{l}\text { A: "He's getting his hands ready for the song } \\
\text { that's coming on. So you know that is awesome } \\
\text { for us because we now know that he understands } \\
\text { the routine of how the lesson is going to go. And } \\
\text { he also understands - he predicts that the song is } \\
\text { coming on and he gets his hands ready for that } \\
\text { music so for us that's fabulous because it shows } \\
\text { he's thinking about it" [IDTM Follow-up: 136- } \\
\text { 142]. }\end{array}$ & $\begin{array}{l}\text { Anticipating } \\
\text { Predicting }\end{array}$ & $\begin{array}{l}\text { Student } \\
\text { progress }\end{array}$ \\
\hline $\begin{array}{l}\text { A: “There were some boys being 'naughty' in the } \\
\text { class. And so Tim was looking from the side as he } \\
\text { does. And he stopped what he was doing and } \\
\text { watched the boys and he laughed. And he looked } \\
\text { back again. He laughed again. He knew they }\end{array}$ & $\begin{array}{l}\text { Attending } \\
\text { Socialising }\end{array}$ & \\
\hline
\end{tabular}


were going to get in trouble. He knew that what they were doing was wrong. He understood that those three boys were being 'naughty' and were really pushing it as to whether they were going to get caught. And three times he looked, and three times he laughed"

DR: And this is different from the way things have been before?

A: Yes it is because what's happening now, and it came through in the IEP really clearly from everybody who works in the school, is that Tim is actually seeking other children. And that's a massive step forward for us... to see any sign that he is actually seeking play with somebody else. So suddenly for us a big light's gone on for us and oh my gosh it's fabulous. I know that there is years and years of support that's gone on there but I do believe that the strategies we've used, particularly of echoing back or of expecting or waiting for him to imitate, the fact that we actually talk back to him if he screams saying 'yes I know you don't like that - I don't like it either but we have to do it', those types of things have pushed that door open a little bit wider for us. And the fact now that he is seeking people to play with means that we just WANT to open that door for him" [IDTM Follow-up: 198-222].

A: "And we're now looking at - and I think this is a VERY important thing - since this process started. When we're looking at the goals for the IEP instead of using the MOST highly reinforcing thing on his card we are now saying "ok, let's look at the choices at a lower level". For example when he's sitting at his desk will he choose to work on his writing or read a book? Now that would simply not have been possible -

Understanding

Socialising

Student

progress Increase in

intrinsic

motivation 
you've seen him on a bad day - that would not have been possible. So we are now looking at that level and that is a HUGE HUGE thing for us".

DR: So you think his motivation may have increased then for doing things -

A: I KNOW it has. I KNOW it has. Because I have also videoed him sitting at his desk when he first came to school, I have videoed him at his desk in the last two years, and we now - in the last four weeks - he has doubled the time he can stay at his desk. So I actually have clear data that has shown me that he has made that potential - it has become a realistic goal and we're aiming for one minute more each week and we're getting it! Now Sue's using a song to settle him at his desk and then she's moving away. So when I'm talking $41 \frac{1}{2}$ minutes of sitting - I'm talking about no teacher aide support. It is fantastic - it's absolutely amazing. But I know she's using song to keep him there, to get him settled there and then she's moving away. Now I also know if he became restless she would step back in and do the same thing and that appears to be working really well. If he's working on a train picture she'll just make up a train song, you know. And that's a VERY CLEAR indicator for us. We can't argue with that because we have the data that shows where he started and we have the data to show where he is now" [IDTM Follow-up:89-120].

A: "He's just been fabulous! He's just been gorgeous doing all these little incidental things and just letting us know that he is part of our world" [IDTM Follow-up: 524 - 526].

Increase in time on task

Student progress

“real hope” [386]

Increased

Student

awareness of self progress and others 
A: "So they're organisational matters but it has brought up a bigger issue of when a child is mainstreamed, inclusion means they are part of the class - that they will be included. Now that has become a big issue because on the one hand we have a curriculum where the big issues for the parents are toileting and communication... so that requires him being out of the classroom. But on the other hand he is part of the class - so how is he included in the class? Simple things like "ok lets sing the 'Hello' song to the whole class" - that's not a difficult strategy to put in place - BUT there is resistance from some people at times who see that as interfering with the daily timetable. It's important to understand that small strategies can make a big difference... We've had to work through these issues and I think that the school, the principal in particular, has picked them up and gone with them and made it very clear that Tim is to be included in the school and that Tim's needs will be considered in this way. And so that has been really clearly stated in the IEP - I think that's part of mainstreaming! That's part of inclusion and we have to deal with (these issues). Because we've been saying 'ok let's have a look at where he is up to' we've had to tighten up a few things. Little tiny strategies like a little song make a big difference to Timwe can get him to do things now because we can just jolly him along with a song. It's a little thing but it's a huge thing. But does it make a big impact on anyone else's day? No! So it doesn't matter. It's not going to change the class timetable; it's not going to change the world for all the other children in the class; but it is going to change the social interaction; it's going to change Tim's world; it's going to change the

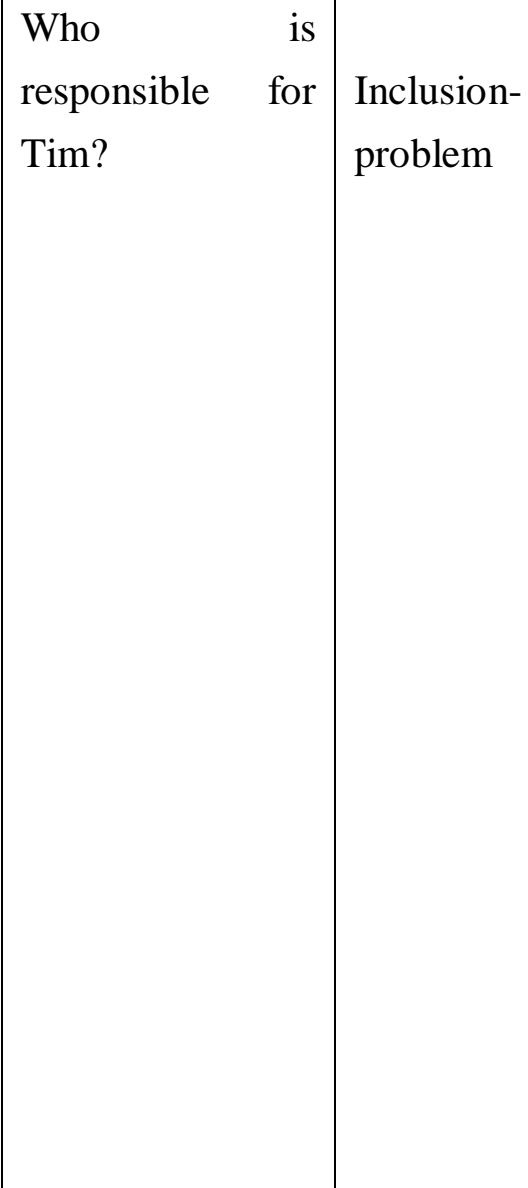

Analytic Memo 28/4/2007

O'Brien and Ryba (2005) suggest that "a lack of capacity and capability within the regular school system is at the root of perpetuating a separate system". Timothy's team worked within a miniature social system elements of which resonated with this. 
way we deal with him; and change the way he interactions with his peers. DR: So the little 'Hello' song - has it been used in class?

A: It has been used in class, yeah. But I think we have to keep working. Inclusion is one of those things you have to keep... you can never sit back and say "yeah we've done it" - it moulds itself around the people and what's happening" [IDTM Follow-up: 254-354].

A: I think the most valuable thing was having someone here to model and also for us to be able to see that there were other strategies in the same situation. Because we've been in the same situation a million times but seeing that there could be another strategy as simple as the gesture on the back and the 'stand up' (song). Now that's a simple strategy but that's a powerful strategy. And that was the start for me. As soon as I saw that I knew we were on our way" [IDTM Follow-up: 422 - 431].

A: "..I believe that the family and school have not received as much support (as they deserve)... I am really clear about that. I do believe we have really done it on our own...And I have found it really difficult at times to watch Lee and Patrick search and search and search for support and it is not always forthcoming ... We contribute to this in that people will ask "what do you need" and we'll say "nothing" because we've had nothing so we don't see any point in somebody extra coming around if it's not going to make any difference. So in a way it becomes a cycle where we actually do it on our own anyway...we actually contribute to the problem too if you sit back and look at it. But at the last IEP meeting

Analytic Memo: 28/4/2007

Angela mentioned that having someone on site, and the need for ongoing support, in her earlier interview too. So this seems really important to look at again.

\section{Having someone}

here to model

was valuable

\section{Simple}

powerful
Desperate for Reiteration ongoing support 
we were asked exactly what we wanted in the way of support for Tim in the next six months. Now I think that is a big change. ...there is apparently a new contract coming out where you have to ask exactly what you want and it is around delivery of service. Obviously there must be some sort of achievement goals around that as well. I believe that that will lead to better outcomes" [IDTM Follow-up: 433 - 470].

A: "The music therapy report was fabulous. I thought for me the amount of information you gave was almost a difficulty for me because there was SO much that we needed to do and yet I couldn't see all of the ways I could fit it in. So I've had to step back and say 'Ok, no. Let's go back to one goal and work from one goal. Let's use it for one goal' but the reality is that it's just become immersed in his day anyway - it's used in behaviour management; it's used in communication; it's used in sensory. So it's just become immersed in his day and that's the way I think a timetable should work for Timothy" [IDTM Follow-up: 488-497]

A: "(Sally and Siobhan) are now searching out the songs that appeal more to him so they can use those in the Floortime activities... So that shows for me too that the teacher aides are actually taking it a step further by saying 'hold on... I know that this worked really well so why don't we bring in this song?' and 'why are we using this?' So they're starting to reflect. They've always worked in that manner but I think they feel much more confident because they've seen you do it" [IDTM Follow-up: 148 - 155] "and I think that we've actually got enough

Music immersed Future-positive in his day
Teachers' aides Futureare reflecting sustainable? 


\begin{tabular}{|c|c|c|}
\hline $\begin{array}{l}\text { initiative now to read the situation and go 'Oh. } \\
\text { Now let's trial NOT singing in the toilet to see } \\
\text { what happens' and then we would talk amongst } \\
\text { ourselves which we're very good at } \\
\text { doing...talking about what was working and not } \\
\text { working" [IDTM Follow-up: 361-364] }\end{array}$ & $\begin{array}{l}\text { Team members } \\
\text { are evaluating } \\
\text { and planning }\end{array}$ & $\begin{array}{l}\text { Future- } \\
\text { sustainable? }\end{array}$ \\
\hline $\begin{array}{l}\text { "We're doing it enough new to say 'oh well, } \\
\text { sometimes it works and sometimes it doesn't' and } \\
\text { it doesn't work you go 'hold on - was it me... } \\
\text { what could I do differently? Or is it one of those } \\
\text { days where we are just going to have to keep } \\
\text { trying and trying and trying?", [IDTM Follow- } \\
\text { up: 373-377]. }\end{array}$ & $\begin{array}{l}\text { Team members } \\
\text { are evaluating } \\
\text { and planning }\end{array}$ & $\begin{array}{l}\text { Future- } \\
\text { sustainable? }\end{array}$ \\
\hline $\begin{array}{l}\text { "(we will consult a music therapist again) } \\
\text { because I think that as he goes through these } \\
\text { different steps I can already see that his levels of } \\
\text { compliance are much higher but I think that we } \\
\text { are listening to him more - so therefore that } \\
\text { might be why they are. But I think that as he goes } \\
\text { forward we probably will want some more } \\
\text { support" [IDTM Follow-up: 395-400]. }\end{array}$ & $\begin{array}{l}\text { Will need some } \\
\text { more support in } \\
\text { future }\end{array}$ & $\begin{array}{l}\text { Future- } \\
\text { sustainable? }\end{array}$ \\
\hline $\begin{array}{l}\text { A: "Well the thing is for us is that, we're isolated, } \\
\text { this is working, and it's the first time we've seen } \\
\text { his communication really open up so this is } \\
\text { working. So we already think it has enough } \\
\text { credibility and I know that (all of the team } \\
\text { members agree) because we've talked amongst } \\
\text { ourselves about different incidents that are } \\
\text { happening - so we think it has the credibility, and } \\
\text { I've told the principal that it has credibility. So } \\
\text { we know that we would search that information } \\
\text { (out again) at another time" [IDTM Follow-up: } \\
\text { 507-514]. }\end{array}$ & $\begin{array}{l}\text { We think this has } \\
\text { credibility }\end{array}$ & $\begin{array}{l}\text { Future- } \\
\text { sustainable? }\end{array}$ \\
\hline $\begin{array}{l}\text { 'But we are now looking at this (work with the } \\
\text { music therapist) as the 'START' of a journey with } \\
\text { Tim - It's not going to be just a 'project' it's }\end{array}$ & It's just the start & Future- \\
\hline
\end{tabular}




\begin{tabular}{|l|l|l|}
\hline $\begin{array}{l}\text { going to be part of how we look at things in the } \\
\text { future..." }\end{array}$ & of a journey & sustainable? \\
\hline "He has set music therapy strategy times but he & & \\
$\begin{array}{l}\text { actually has it immersed in the rest of his day in } \\
\text { lots of ways - and it's perfectly natural" [IDTM }\end{array}$ & natural perfectly & Future- \\
Follow-up: 541-543]. & sustainable? \\
\hline
\end{tabular}


APPENDIX 35: 'Angela' [Tim's Case] example of category development

TEAM MEMBER 'ANGELA' (CASE STUDY TIM) EXAMPLE OF CATEGORY DEVELOPMENT

\section{DATA FROM WEEK IN THE FIELD}

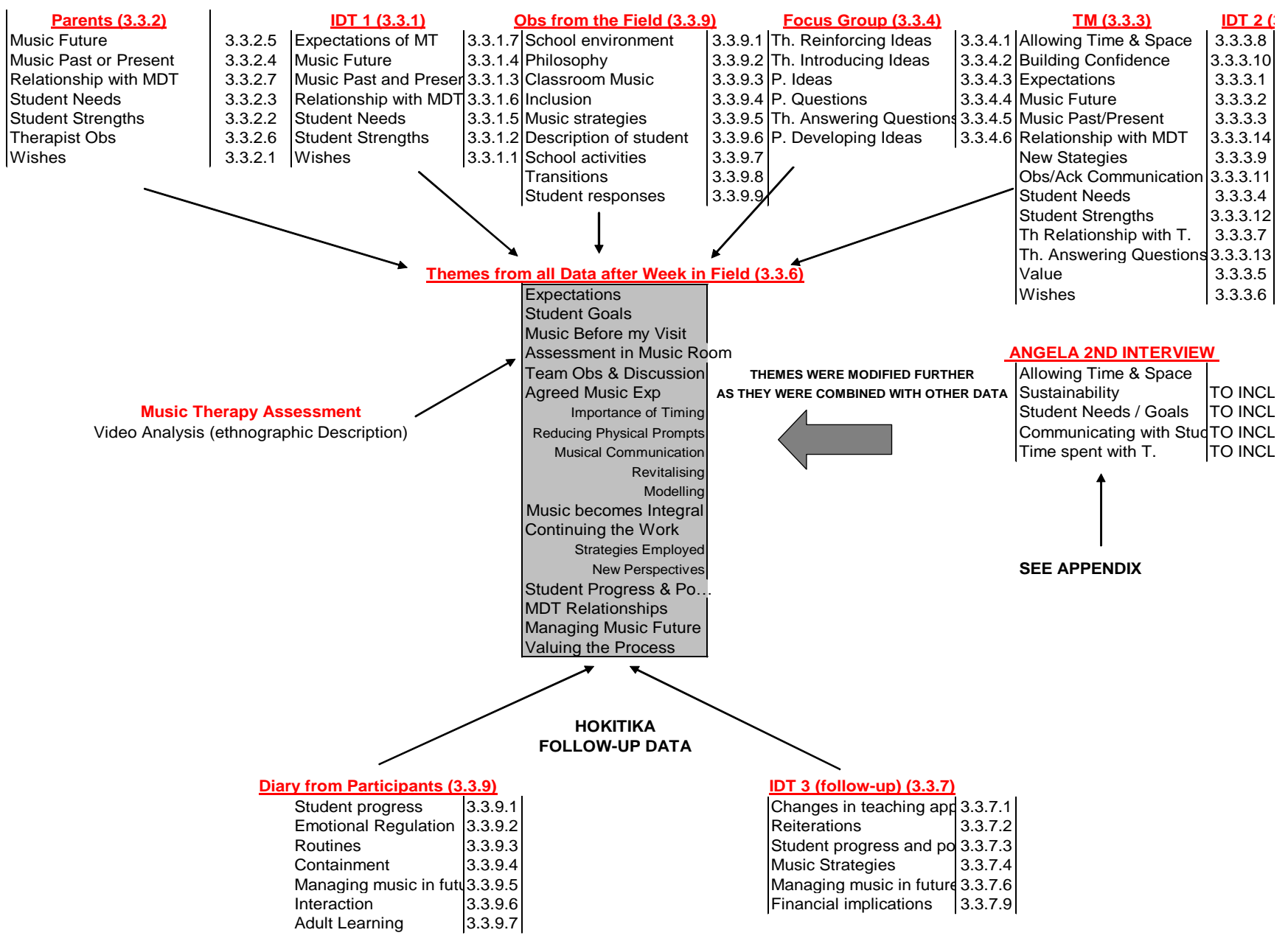




\begin{tabular}{|c|c|c|}
\hline & Team members noticed, or wondered about: & Therapist response \\
\hline $\begin{array}{l}\text { Category: Therapist } \\
\text { Agreeing with or } \\
\text { Reinforcing Participant } \\
\text { Ideas }\end{array}$ & $\begin{array}{l}\text { Whether Tim knew what to do with some of the } \\
\text { instruments. }\end{array}$ & $\begin{array}{l}\text { It could be helpful to accept his contribution, as long as } \\
\text { it was safe. In music therapy there is usually no 'right' } \\
\text { or 'wrong' way to play instruments. }\end{array}$ \\
\hline $\begin{array}{l}\text { Category: Therapist } \\
\text { Agreeing with or } \\
\text { Reinforcing Participant } \\
\text { Ideas }\end{array}$ & $\begin{array}{l}\text { They needed to keep their language simple, clear and } \\
\text { really specific. }\end{array}$ & \\
\hline $\begin{array}{l}\text { Category: Therapist } \\
\text { Agreeing with or } \\
\text { Reinforcing Participant } \\
\text { Ideas }\end{array}$ & $\begin{array}{l}\text { Tim seemed initially to move away from me when I } \\
\text { leaned toward him in an attempt to invite him to play } \\
\text { the bongo drums. }\end{array}$ & $\begin{array}{l}\text { His reaction might be considered to be very appropriate } \\
\text { because I had encroached relatively intrusively on his } \\
\text { personal space. The bongo drums are useful as an } \\
\text { instrument that provides both a 'barrier' and a 'bridge' } \\
\text { between players. The idea that the barrier between us } \\
\text { enabled Tim to interact 'safely' with me was supported } \\
\text { by his ability to engage quickly and comfortably in the } \\
\text { activity, to remain with me, and to engage in playing } \\
\text { the drums }\end{array}$ \\
\hline $\begin{array}{l}\text { Category: Therapist } \\
\text { Agreeing with or } \\
\text { Reinforcing Participant } \\
\text { Ideas }\end{array}$ & $\begin{array}{l}\text { The importance of finding strategies for working with } \\
\text { Tim that involved the least physical prompts, while } \\
\text { also keeping him safe, and the possibility that music } \\
\text { would be helpful in this regard. 'You used the } \\
\text { minimum physical prompting to help him to play the } \\
\text { bells, you used minimal prompting to say 'Let's go to } \\
\text { another activity' and when he didn't choose to do it } \\
\text { you left him... there are some strategies (here) that we } \\
\text { can use... because we don't WANT to be particularly } \\
\text { tough on Tim - we want him as independent as } \\
\text { possible... And I think that THAT'S part of the } \\
\text { potential for this" [Focus Group: 226-236]. }\end{array}$ & $\begin{array}{l}\text { I knew he was motivated by music and I could } \\
\text { communicate musically that I trusted him to respond. } \\
\text { (I was hopeful that having seen Tim's responses to } \\
\text { music, team members would also develop confidence } \\
\text { in his ability to respond positively to their musical } \\
\text { prompts, that they would observe his attempts to } \\
\text { communicate and that this in turn would increase their } \\
\text { ability to give him more time and space to respond. I } \\
\text { was able to make connections with the way in which } \\
\text { music therapists match, mirror, imitate and extend } \\
\text { musical contributions from clients thus increasing their } \\
\text { awareness of what they have offered, and letting them }\end{array}$ \\
\hline
\end{tabular}




\begin{tabular}{|c|c|c|}
\hline & & $\begin{array}{l}\text { know that their musical communication has been heard } \\
\text { and is valued. By focusing on the musical aspects of } \\
\text { the communication it was possible for me to give a } \\
\text { relatively direct appraisal of what I had observed of } \\
\text { team members' interactions with Tim in various } \\
\text { contexts). "... I would like you to see or hear more of } \\
\text { what Tim is communicating, making sure that he knows } \\
\text { that you've 'heard what he has said' or seen what he is } \\
\text { communicating" [271-275]. }\end{array}$ \\
\hline $\begin{array}{l}\text { Category: Therapist } \\
\text { Agreeing with or } \\
\text { Reinforcing Participant } \\
\text { Ideas }\end{array}$ & $\begin{array}{l}\text { Tim was anticipating the end of the song i.e. that he } \\
\text { tended to punctuate cadence points }\end{array}$ & $\begin{array}{l}\text { I was able to make links with songs that I had } \\
\text { suggested they use with him in other contexts, because } \\
\text { the eight-bar structure that Tim appeared to be aware of } \\
\text { could be used to frame simple activities and help him } \\
\text { to increase time on task. }\end{array}$ \\
\hline $\begin{array}{l}\text { Category: Therapist } \\
\text { Agreeing with or } \\
\text { Reinforcing Participant } \\
\text { Ideas }\end{array}$ & $\begin{array}{l}\text { Tim's mum and dad were working hard to help him to } \\
\text { understand that he needed to wait sometimes in order } \\
\text { to accommodate his sibling's needs. }\end{array}$ & $\begin{array}{l}\text { Turn taking in improvised music might be important } \\
\text { too, to give him the chance to be the 'leader', to be in } \\
\text { control and to 'say' what it is he wants to do and how } \\
\text { things should run. }\end{array}$ \\
\hline $\begin{array}{l}\text { Category: Therapist } \\
\text { Agreeing with or } \\
\text { Reinforcing Participant } \\
\text { Ideas }\end{array}$ & $\begin{array}{l}\text { Turn taking, especially saying Hello in response to a } \\
\text { greeting, felt like an important and realistic } \\
\text { medium/long-term goal for Tim. }\end{array}$ & $\begin{array}{l}\text { True, but keep the interaction playful to ensure that } \\
\text { Tim had the underpinning skills necessary for } \\
\text { spontaneous communication. } \\
\text { " That reciprocal turn taking and that ANTICIPATING } \\
\text { when it's "my turn" to say Hello - that whole give and } \\
\text { take and backwards and forwards sort of thing } \\
\text { underpins (General agreement)... being able to say } \\
\text { 'Hello' and saying 'Hello' back. (L: yes) I would hate } \\
\text { for us to get to any kind of place where we expect Tim } \\
\text { to say 'Hello' and nothing happens until he says } \\
\text { does... that sort of thing. That's why there is such an } \\
\text { emphasis on there (indicating the video) what HE } \\
\text { communicates... and the way HE communicates being }\end{array}$ \\
\hline
\end{tabular}




\begin{tabular}{|c|c|c|}
\hline & & $\begin{array}{l}\text { acceptable. He vocalised to me (L: Yes) and that would } \\
\text { be enough for now?" }\end{array}$ \\
\hline $\begin{array}{l}\text { Category: Introducing } \\
\text { New Ideas or Giving } \\
\text { Direct Advice }\end{array}$ & $\begin{array}{l}\text { Tim's mum was already a strong advocate for singing } \\
\text { songs to help Tim with daily routines. She reminded } \\
\text { the team that he is calmed by singing, and that certain } \\
\text { songs have been associated with particular activities at } \\
\text { home. Her contribution was invaluable for encouraging } \\
\text { the team to continue this work }\end{array}$ & $\begin{array}{l}\text { Try not to become pedantic about having to sing a } \\
\text { particular song at a given time. Consistency is } \\
\text { important but needs to be balanced with a thought } \\
\text { about singing spontaneously - perhaps an improvised } \\
\text { song - to help with a task, keeping relaxed, and singing } \\
\text { and listening to music for FUN. }\end{array}$ \\
\hline $\begin{array}{l}\text { Category: Therapist } \\
\text { Agreeing with or } \\
\text { Reinforcing Participant } \\
\text { Ideas }\end{array}$ & & $\begin{array}{l}\text { It is important for children to have the opportunity to } \\
\text { explore any instruments that are introduced in a music } \\
\text { therapy session and we need to be ready to share } \\
\text { anything with them. Some children need to mouth } \\
\text { instruments and although we might encourage them to } \\
\text { explore it in other ways by demonstrating how it might } \\
\text { be played, their need to mouth would be accepted if } \\
\text { that was the developmental level they were at. }\end{array}$ \\
\hline $\begin{array}{l}\text { Category: Therapist } \\
\text { Agreeing with or } \\
\text { Reinforcing Participant } \\
\text { Ideas }\end{array}$ & $\begin{array}{l}\text { Lee sang to Tim a lot but Patrick felt less able to and } \\
\text { during the meeting he joked about going off to karaoke } \\
\text { bars to prepare for the challenge. }\end{array}$ & $\begin{array}{l}\text { I laughingly reminded him that Tim would enjoy his } \\
\text { voice and that it would be really good if he would } \\
\text { forgo the karaoke and "sing to Tim" instead. }\end{array}$ \\
\hline $\begin{array}{l}\text { Category: Direct } \\
\text { Questions }\end{array}$ & $\begin{array}{l}\text { Is it important to make sure the therapist and student } \\
\text { each have access to instruments that look/sound the } \\
\text { same? [Focus Group: 95-101] }\end{array}$ & $\begin{array}{l}\text { "We have to share our instruments with the children as } \\
\text { well. So having them the same or being able to say 'if } \\
\text { you want to try mine here it is', (is important). You } \\
\text { have to be able to give it up" [Focus Group: } 110 \text { - } \\
117] \text {. }\end{array}$ \\
\hline $\begin{array}{l}\text { Category: Direct } \\
\text { Questions }\end{array}$ & $\begin{array}{l}\text { Is it important for the student to be on a chair, or } \\
\text { can you work with them on the floor? [Focus } \\
\text { Group: 296-298] }\end{array}$ & $\begin{array}{l}\text { "I would have worked with Tim on the floor (if he'd } \\
\text { gone there). However the reason I have chairs is that I } \\
\text { think that a lot of children respond well to those } \\
\text { boundaries" [Focus Group: } 299 \text { - 302] }\end{array}$ \\
\hline
\end{tabular}




\begin{tabular}{|c|c|c|}
\hline & & $\begin{array}{l}\text { "I certainly wouldn't physically restrain him .... (but) } \\
\text { boundaries do help children to feel safe if they know ... } \\
\text { where the 'spot' is" [Focus Group: } 312 \text { - 318] }\end{array}$ \\
\hline \multirow[t]{2}{*}{$\begin{array}{l}\text { Category: Direct } \\
\text { Questions }\end{array}$} & $\begin{array}{l}\text { You are using minimal physical prompts but Tim } \\
\text { needs deep pressure. How do we manage the } \\
\text { balance of that? [Focus Group: } 319-325 \text { ] }\end{array}$ & $\begin{array}{l}\text { "(I think) he will like pressure through his shoulders } \\
\text { (and perhaps) his head... ... his body awareness is } \\
\text { such that if you touch his shoulders or touch his arm } \\
\text { symmetrically from behind it helps him know where he } \\
\text { is in space... So I would just give him a bit of a } \\
\text { reminder about where he is - here's your legs, and } \\
\text { here's your feet - one of the little songs on the tape is } \\
\text { "Feet, feet, feet, feet," (demonstrating pushing on feet } \\
\text { while singing) and then you could go back to saying or } \\
\text { doing the stand up song..."[ Focus Group : } 326 \text { - 347] }\end{array}$ \\
\hline & $\begin{array}{l}\text { Tim needs 'free' time and 'structured' time. How } \\
\text { can I help him to realise when he needs to be here or } \\
\text { there, or to do this or that, and when he can choose } \\
\text { what he would like to do? [Focus Group: } 437-442 \text { ] }\end{array}$ & $\begin{array}{l}\text { "If I was doing ongoing music therapy with Tim I think } \\
\text { the thing I would ask him to do next would be to sit } \\
\text { down for the Goodbye song. He sat for the Hello song } \\
\text { and then we did all this lovely stuff which had no } \\
\text { demands really - we were communicating together... } \\
\text { (And he was choosing) but then he walked out. So the } \\
\text { next thing would be "Ok... you've said you're finished } \\
\text { - if you've finished we sing Goodbye and then we go". } \\
\text { So there is definitely a place for those boundaries and } \\
\text { for asking for specific responses as well" [Focus } \\
\text { Group: } 443 \text { - 450]. }\end{array}$ \\
\hline $\begin{array}{l}\text { Category: Participants } \\
\text { Developing Ideas }\end{array}$ & $\begin{array}{l}\text { The team talked about the ways in which Tim's } \\
\text { responses might be different in another situation, or at } \\
\text { another time. For example, Patrick suggested that if I } \\
\text { was working on the floor with Tim, he might have } \\
\text { gotten up and walked away from me. And although } \\
\text { Tim swapped instruments with me, and another time }\end{array}$ & \\
\hline
\end{tabular}




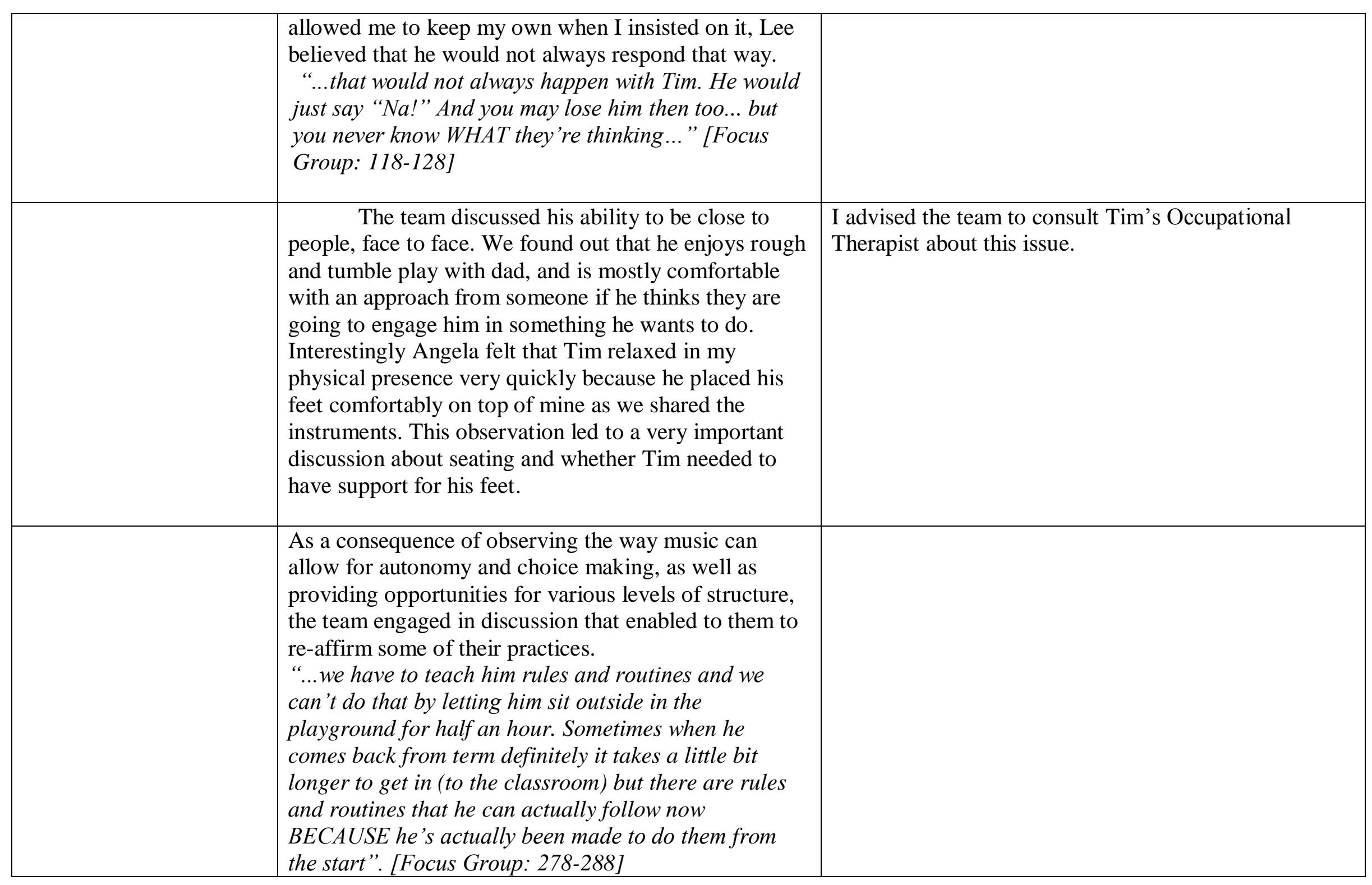




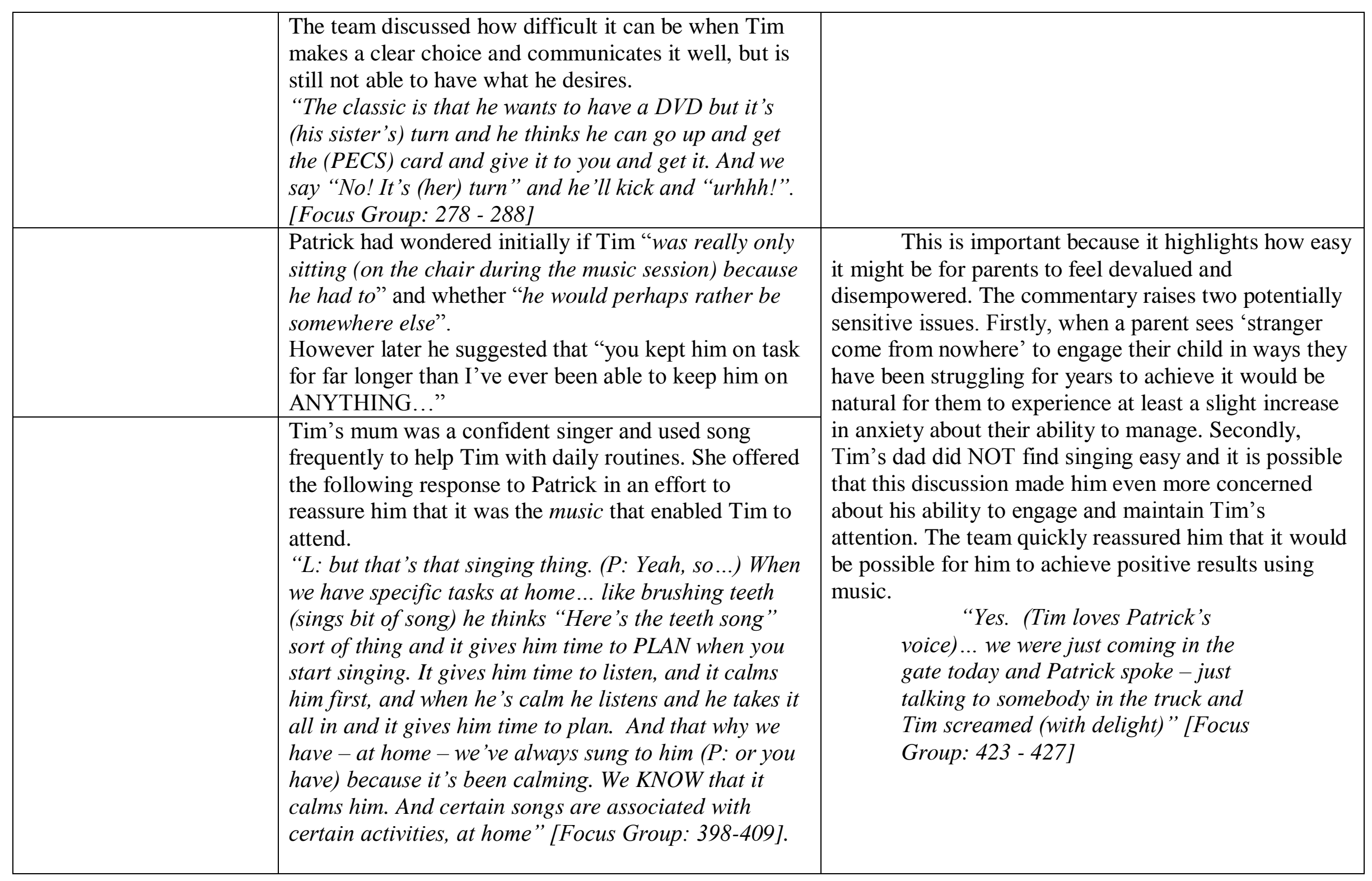




\begin{tabular}{|l|l|}
\hline \multicolumn{1}{|c|}{ The enthusiasm the rest of the team had for } \\
music making is demonstrated in the way they \\
developed their ideas for ongoing programming. It was \\
particularly encouraging that they saw the importance \\
of timetabling to enable all teacher's aides to have time \\
to play with Tim in music. \\
"A: we will look at making a time for (three of us) to \\
do the 10-15 minute things and if it is working for the \\
day that's fine and if it's not working for the day we \\
just have to accept that that's the way it is sometimes. \\
... We will obviously be using music therapy (strategies) \\
in both structured and unstructured ways..." [Focus \\
Group: 454 - 467]
\end{tabular} \mid


APPENDIX 37: Analysis of Tim's Music Therapy Assessment Session - Timeline

\section{VIDEO ANALYSIS OF TIM'S MUSIC THERAPY SESSION}

$1 \mathrm{~min} 50$ secs

Daphne Singing Tim's response/initiation

\begin{tabular}{|c|c|c|}
\hline \\
\hline \multicolumn{3}{|l|}{$\begin{array}{l}1.50 \\
1.51\end{array}$} \\
\hline \multicolumn{3}{|l|}{1.52} \\
\hline 1.53 & Tim & \\
\hline 1.54 & has the & \\
\hline 1.55 & bells & \\
\hline 1.56 & & Tim is turning his bells but looking to the left \\
\hline 1.57 & Tim & \\
\hline 1.58 & has the & \\
\hline 1.59 & bells & \\
\hline 2.00 & & Tim smiles (still looking to the left) \\
\hline 2.01 & Tim & \\
\hline 2.02 & has the & Tim is turning towards Daphne \\
\hline 2.03 & bells & Giggles \\
\hline 2.04 & at music time & The way he is turning his bells has changed \\
\hline 2.05 & today & \\
\hline 2.06 & & Tim is mouthing lips open and closed \\
\hline
\end{tabular}

Notation

2.07 


\begin{tabular}{|c|c|c|}
\hline 2.08 & Tim & Tim vocalises \\
\hline 2.09 & turns the & \\
\hline 2.10 & bells & Continues to vocalise 'mm' \\
\hline 2.11 & Tim & \\
\hline 2.12 & turn the & \\
\hline 2.13 & bells & \\
\hline 2.14 & & Tim stills... he stops turning his bells \\
\hline 2.15 & & Seems to be concentrating... waiting \\
\hline 2.16 & & Begins to turn bells then puts them to mouth \\
\hline 2.17 & Tim & \\
\hline 2.18 & play the bells & Tim takes bells away from mouth and turns them \\
\hline 2.19 & at mu- & \\
\hline 2.20 & sic time & \\
\hline 2.21 & today & \\
\hline 2.22 & $\operatorname{Tim} \ldots$ & \\
\hline 2.23 & & Tim is looking to the right - he stills and waits \\
\hline & Shake & \\
\hline 2.24 & (whispered) & \\
\hline 2.25 & bells & \\
\hline 2.26 & & Turns towards therapist, and begins to turn bells \\
\hline 2.27 & Shake the bells & Tim squeals, seemingly with excitement \\
\hline 2.28 & & Vocalising "ooh" \\
\hline 2.29 & Tim & Puts bells to mouth \\
\hline 2.30 & shake the bells & Pulls away slightly as Daphne reaches towards the bells \\
\hline
\end{tabular}


2.32 Shake shake

2.33

2.34

2.35 Shake the

2.36 bells at music

2.37 time

2.38 Tim shake

2.39 the bells

2.40 Shake shake

2.41

2.42 Tim

2.43 shake the

2.44 bells

2.45 Shake shake

2.46

2.47 Tim shake the

2.48 bells

2.49 Shake shake

2.50

$2.51 \mathrm{Tim}$

2.52 shake the

2.53 bells,

2.54 At
Laughs

Is turning the bells at moderate pace

Daphne models a shaking motion

Tim allows Daphne to hold the bells with him and shake them

Laughs

Continues to smile

Daphne models a shaking motion

Giggles

Continues to giggle - appears to be anticipating the next 'shake shake'

Giggles increase and he pulls away in fun as Daphne demonstrates 'shake shake'

Gigglesincrease and he pulls away in fun as Daphnedemonstrates'shake shake'

Is calm and appears to be listening during last phrase, turning bells only occassionally. Head is facing Daphne but eyes are down looking at bells and his container which he has between his knees. 


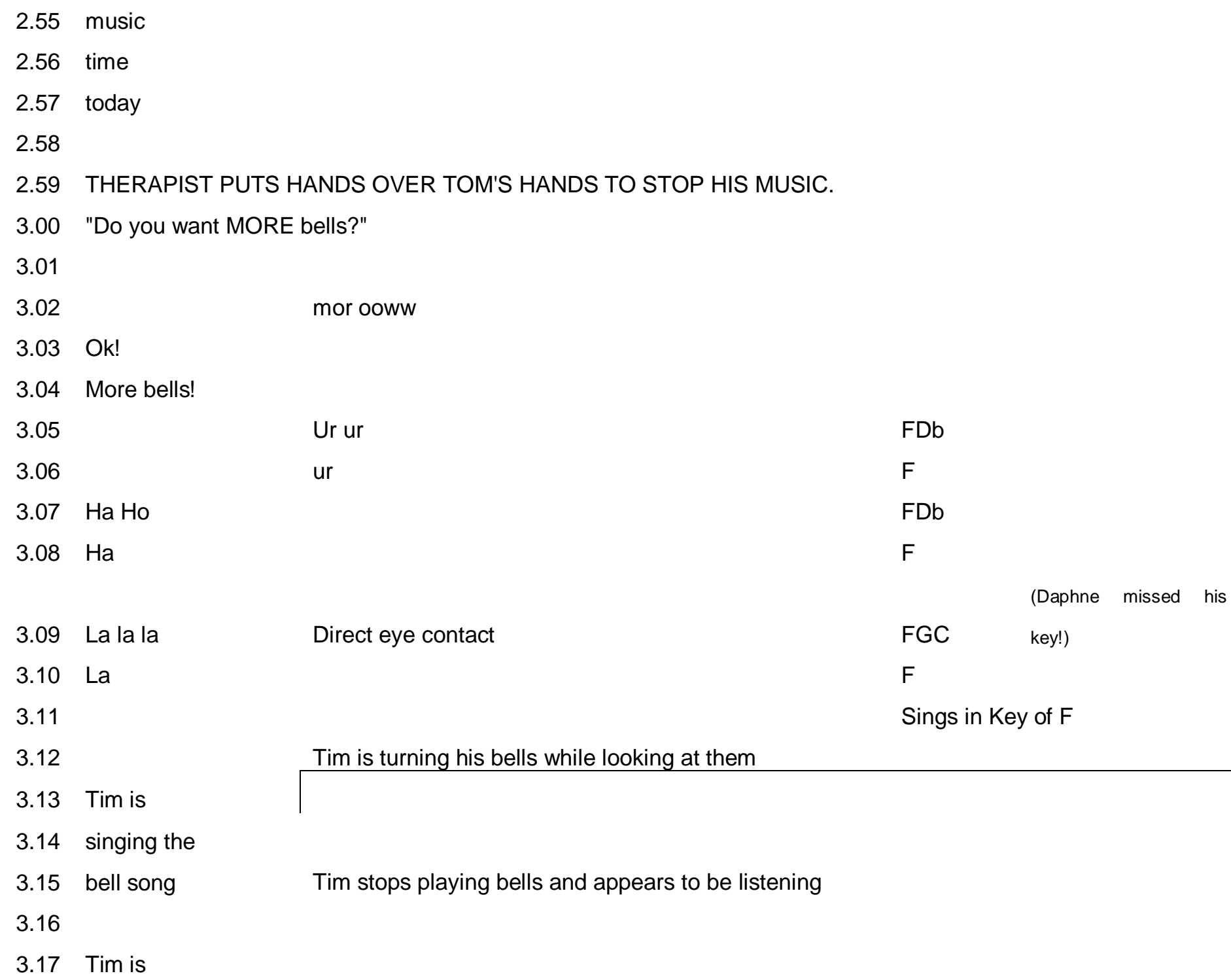


3.18 singing the

3.19 bell song

3.20

Turns the bells

3.21 Tim is

3.22 singing the

3.23 bell

3.24 song

3.25 at

3.26 music

3.27 today

3.28

3.29 Tim

3.30 has the

3.31 bells

3.33 Tim

3.34 has the

3.35 bells

3.36

3.37 Tim

3.38 has the bells

Tim is turning his bells 'absent mindedly'

3.39 at mu-

3.40 sic time

3.41 today

Daphne reaches out with index finger and touches bells to give visual prompt at 3.35. Throughout this period Tim does not seem to be playing his bells with musical intent but is sounding them occassionally. He is however focusing on them as if acknowledging the song. 
3.44 has the bells

3.45

3.46

3.47

3.48 Tim

3.49 has the bells

Tim laughs and picks up his container as if to say "no I don't!"

3.52

3.53 Bell time!

3.54

3.55

3.56 It's bell

3.57 time!

$3.58 \mathrm{Tim}$

3.59 has the bells

4.00 at mu-

4.01 sic time

4.02 today.

$4.03 \mathrm{Tim}$ -

4.04 shake the

4.05 bells

Daphne challenges him light heartedly and Tim clutches his container protectively, laughing

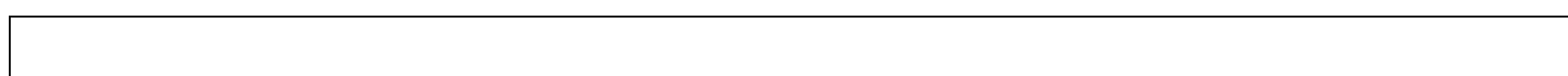

Daphne takes the container quickly and places it under her chair. Tim begins flapping excitedly.

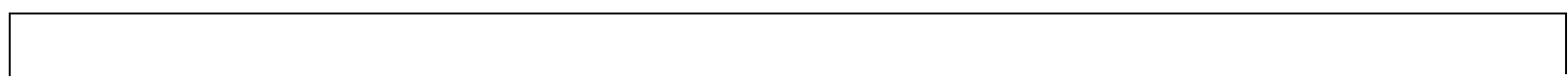

Tim laughs refocuses and begins to turn bells.

Tim vocalises around the tonic note 
4.06 shake the

4.07 bells

4.08

4.09 Tim

4.10 shake the bells

4.11

4.12 Shake the

4.13 bells at music

4.14 time

$4.15 \mathrm{Tim}$

4.16 shake -

4.17

4.18 shake -

4.19 the bells

4.20

4.21 Hey! (softly)

4.22

4.23 shake the

4.24 bells

4.25

4.26 shake the

4.27 bells

4.28

$4.29 \mathrm{Tim}$
Daphne reaches out with pincer grip and helps to shake the bells

Tim laughs and holds them protectively

Oooh! (Leans back stretching and almost yawns)

\section{Ee oh}

Tim laughs and holds bells very still

Continues to laugh

Tim is laughing and looking away appearing to tease

Tim shakes his head

Daphne reaches out with pincer grip and helps to shake the bells and Tim is observing her carefully

Daphne reaches out with pincer grip and helps to shake the bells and Tim is observing her carefully

Tim begins turning his bells again and vocalising for several seconds in a 'shivering' fashion 
4.30 shake the

4.31 bells

4.32

4.33000000

4.34

4.35

Shake

4.36 (staccato)

4.37 bells at (staccato)

4.38 mu-sic (staccato)

4.39 time -

4.40 Thom-as (staccato)

4.41 shake (staccato)

4.42 the (staccato)

Tim looks towards Daphne

4.43

4.44

4.45

4.46

4.47 bells ( $p$ \& legato)

4.48

4.49

4.50 Woooooo...

$4.51 \quad \ldots 000000$

4.52

Daphne reaches out and taps the bells several times. Tim gives a relatively prolonged squeal and Daphne joins him. 
4.53 We're going to

4.54 swap the bells

Tim allows Daphne to take the bells and puts his hands behind his back. He is giving good quality eye

4.55 over. We're

4.56 going to find

4.57 something else.

4.58 Come Tim contact.

Daphne reaches out inviting Tim to rise - he gives

laugh

Daphne gets up, Tim remains seated but is visually tracking her

4.59

5.00 (Continuous rhythm with

5.01 pocket shaker below)

$5.02 \mathrm{xx}$

$5.03 x x$

$5.04 x x x$

5.05

$5.06 \mathrm{xx}$

$5.07 x x$

$5.08 \quad x x x$

5.09

Tim is slouching back in his chair in a relaxed way and appears for the most part to be watching and

5.10 listening to Daphne. However there is a lot of noise in the corridor and Daphne suddenly wonders if indeed he is concentrating on sounds from outside.

5.11

5.12

5.13

5.14

5.15 Isn't it noi-

Daphne chooses a pocket shaker, turns to face Tim and 'invites' him to come and take it (or another instrument) by remaining at the instrument table. 
5.16 sy outside

5.17 today

5.18

Tim looks away from Daphne towards where she had previously been sitting.

5.19

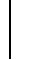

$5.20 \mathrm{xx}$

5.21

$5.22 x x$

5.23

5.24 Come Tim

5.25

5.26

$5.27 \quad x x$

5.28

Tim continues to 'look' straight ahead and does not respond to any of my soundmaking. He is

5.30 wriggling in his chair, sometimes putting his feet up on the seat, possibly still listening to sounds outside but seems generally inattentive.

5.31

5.32

5.33

5.34

5.35

5.36

5.37

5.38

5.39 


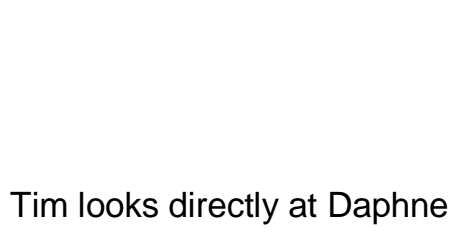

5.47

5.48 Thom-as

5.49 wants to

5.50 sing

\subsection{1}

5.52 uho

5.53 uh oh

5.54 uho

5.55 uh oh

5.56

5.57 Thom-as

5.58 wants

5.59 to

6.00 sing

6.01 and

6.02 shake

\section{Tim looks directly at Daphne}

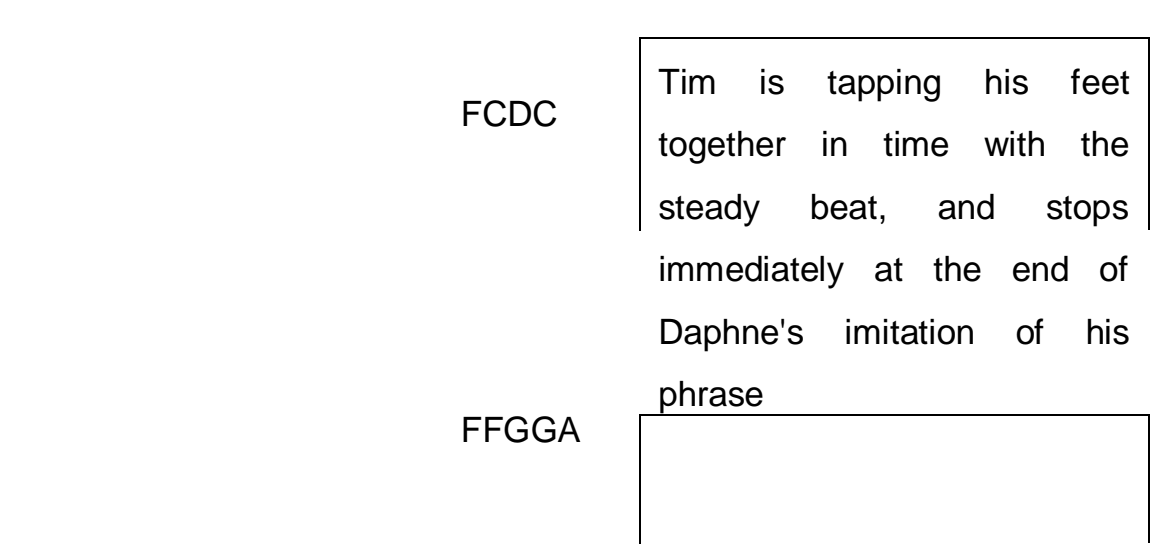

FCDC

FCDC

(low)

ACGCF

Tim is still and listening, as Daphne moves from the table back to the chair in front of him. He gives her eye contact and waits until the end of her improvised song before standing and turning as if to leave. 
(Sharp intake of breath)

6.03

On

Tim is smiling

6.04 Shake shake

6.05 Shake shake

6.06 shake shake shake

6.07 Shaka shake

Tim is 'flapping' (dancing?) in time with Daphne's singing, still smiling

6.08 Shake shake

6.09 shake shake shake

6.10 shake

6.11 shake

6.12 shake

6.13 shake shake shake

6.14 and stop!

6.15

6.16

6.17

6.18

6.19

6.20

6.21

6.22

6.23 Aaa!

6.24

6.25 Sing some more...!

F, G

G Tim is sitting still and looking slightly to the right of Daphne.

GGG... 
6.26 We can

6.27 Shake shake

6.28 Shake shake

6.29 shake shake shake

6.30 Shaka shake

6.31 Shake shake

6.32 shake shake shake

6.33 shake

6.34 shake

6.35 shake

6.36 shake shake shake

6.37 and stop!

6.38

6.39

$6.40 \times$

6.41

6.42 oh

6.43 we

6.44 can..

6.45

6.46

6.47

6.48

6.49

G G

As the original tempo of the Shaking Song returns Tim turns to Daphne and resumes his dance. Although his hands are 'flapping' the movements are very similar to those Daphne is using to sound the pocket shaker

Tim leans forward take Daphne's shaker, laughing.

Daphne also leans forward to pick up the other shaker from the side of Tim's chair

Tim holds his shaker with two hands, close to his chest, not playing but tapped it just after 'stop'.

$\mathrm{X}$

$\mathrm{xX}$

Daphne is waiting to see what Tim will do. Eventually he puts one corner of the pocket shaker in his mouth and, still holding it with two hands, begins to bend the instrument so it makes a clicking sound 
As soon as Daphne begins to imitate him, Tim stops his movement. However at the end of the phrase he begins to turn the instrument using two hands in the motion of turning a steering wheel

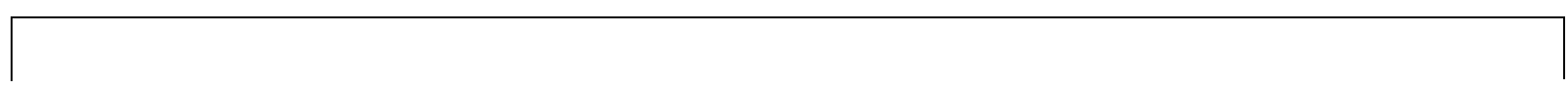

Tim continues his turning movement, in time with the music, for a complete phrase, stopping precisely at the semi-cadence point

Tim mouths and bends the instrument

Tim is looking at me and seems to be concentrating

Tim seems to make quite a deliberate attempt to bend the instrument but misses his 'cue'

I put one hand on Tim's instrument and shake both pocket shakers at the same time as the tempo of the music increase. Tim smiled gave good quality eye contact but released his shaker

Tim looks as if he is going to leave his chair again. I lean forward putting my face in front of his in an attempt to gain his attention and hand him the shaker which he accepts and mouths 
7.16 Shaka shaka

Tim is sitting sideways on his chair, and does not appear to be attending to me. But quite precisely at

7.17 shake shake shake

the end of this phrase he begins to turn his instrument steadily

7.18

7.19 Shake and

7.20 shake and

7.21 turn and

7.22 turn

7.23 turn turn

7.24 turn and

7.25 turn

7.26 mine...

7.27 that's mine..

Tim is turning his pocket shaker steadily in time to the music

Tim is still and listening to me turn my pocket shaker at moderately loud volume. At the end of the phrase he reaches out to take it, almost as if he's thinking "yours works better than mine"

turns it over once, half heartedly

\begin{tabular}{|l|l}
$\begin{array}{l}\text { All of this } \\
\text { while Tim }\end{array}$ & Tim 'rocks' his pocket shaker \\
is looking & Daphne replies \\
pretty &
\end{tabular}




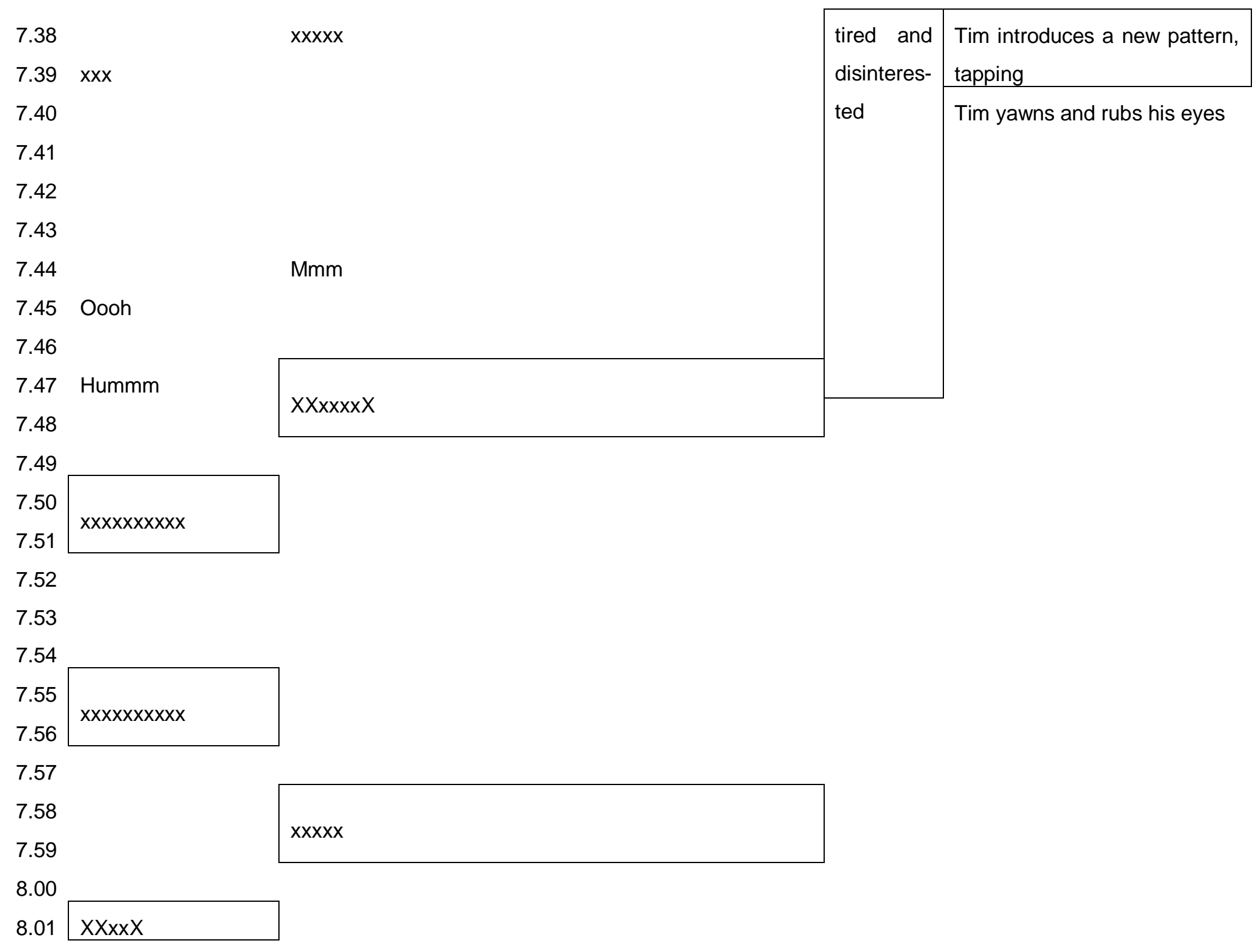




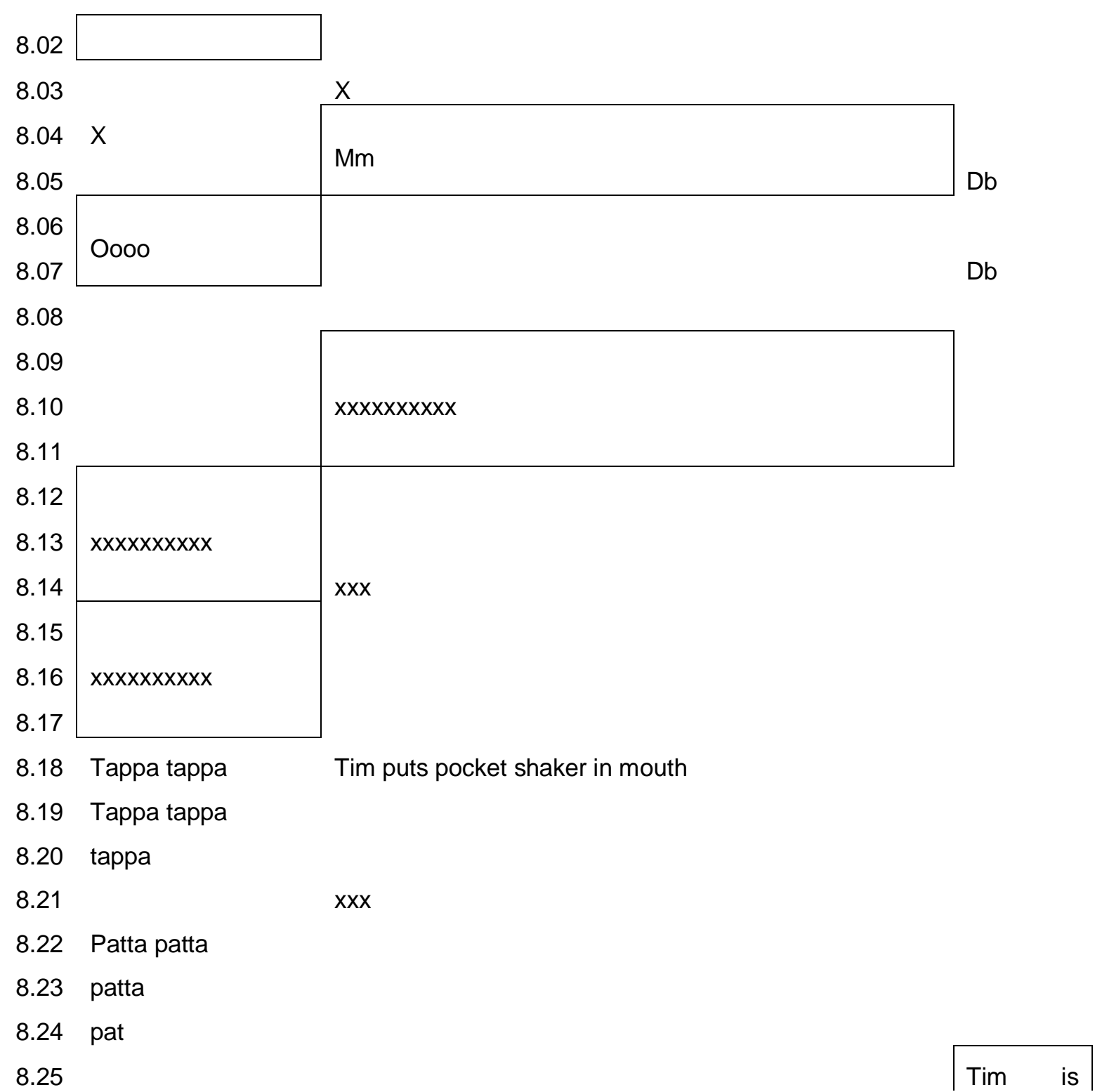




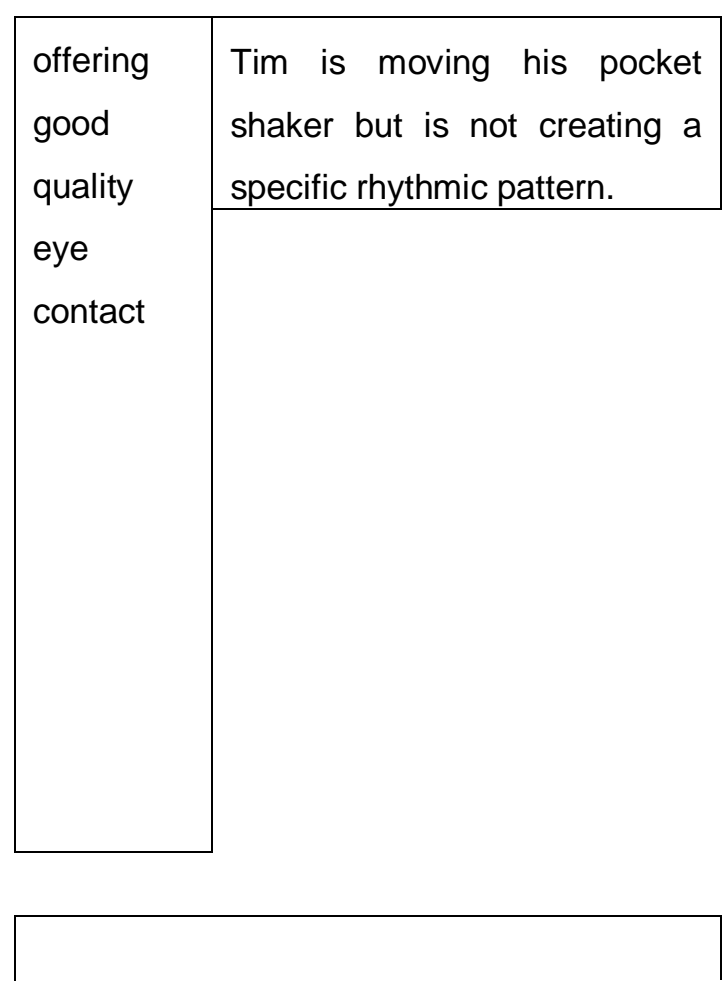

Tim is looking away to his right. He is still and silent. He does not appear to be listening to me. At 8.47 he swings quickly around to his left and looks behind him to the door as if looking for children in the corridor. 
8.51 Who's that?

What can you

8.52 hear?

Tim turns back disinterestly to his shaker

8.53

$x x x x$

8.54 Tappa tappa

8.55 tap

8.56 Tappa tappa

8.57 tap...and shake!

8.58 Good boy!

8.59 Shake! (laughs)

9.00

9.01 Tappa tappa

9.02 tap

9.03 Tappa tappa

9.04 tap...and

9.05 tuuur

9.06 uuuur

9.07 uuuurn

$9.08 \times X$

9.09

9.10

9.11

9.12

Tim is rocking his shaker gently from side to side as if steering a car. His is sitting in a very relaxed position with legs extended and crossed, resting on my feet. At 9.03 he smiles begins to turn his shaker in full circles almost in time to my chant.

Tim stops and is watching me 
9.40

9.41

9.42

9.43

9.44

9.45

9.46

9.47

9.48

9.49

9.50

9.51 mine..

9.52 that's mine...

9.53 Shall we swap

9.54 You can have

9.55 that one

9.56 and I'll have

9.57 this one. Ok.

9.58

9.59

10.00 


\section{APPENDIX 38: Indicators of Efficiency, John's Case}

\section{Resources to draw on}

Music had been available to John throughout his life and he expressed sensitivity and "real joy" in music making. At least two of his teacher's aides "loved music" and were willing to take what may have felt like personal risks to use music strategies with him. The team was reminded about the possibility for using the resources already available to them e.g. the fully equipped music room and the specialist music teacher. Music strategies and the IEP

The music therapy strategies were closely linked to programmes already being used, e.g. Social Stories, and the reasons behind the introduction of the strategies were clear. The strategies focused on John's interest and strength and were viewed as being practical. John's father requested that music therapy strategies be included in his IEP.

The value of the team meeting

The team was able to observe the student's positive interaction with the music therapist, and began to view his musicality as his strength. The video material and subsequent discussion provided opportunities for the music therapist to give direct instruction regarding the use of music therapy techniques.

\section{Increasing confidence and feeling empowered}

Team members felt that the work they were already doing was affirmed through positive feedback, and they gained confidence during the week of the consultation as they observed and participated in the use of music therapy strategies. The teacher's aides were increasingly aware that they had already been singing spontaneously to John, and began overtly building on their natural musicality. They were able to continue to use Musically Adapted Social Story Songs, and began to spontaneously make up their own songs to support John's work.

\section{Student Progress}

John responded immediately to the music therapy strategies. He used the Musically Adapted Social Stories well (e.g. learnt to "stop" on command; manage his tee shirt routine etc.) and began to make up his own songs and to sing himself through tasks. He was more able to regulate his emotions with musical support. Furthermore, the team noted that he developed peer relationships through music which were generalised to the playground environment. John was learning to play a xylophone by ear and from simple score, and 
loving it.

New Perspectives

After watching the video of the music therapy session, the team recognised John's delight in music making, noted his passion and enthusiasm and rarely heard laughter and, later, recognised that music could be used to "turn John's mood" and "change his attitude". The teacher's aides "experienced a new way of communicating with John" (SLT) and became committed to continuing the work.

\section{Belief in Music Strategies}

John's teacher's aides confessed that they learnt more that they thought they might. They recognised that John engaged more readily and understood more when offered musical communication and were convinced that the music therapy strategies would be helpful after observing John's positive response. Further, they believed that the music therapy strategies had benefits for other students. The speech and language therapist on the team was convinced that music strategies would support the communication programme.

\section{Commitment to Continuing the Work}

The team worked collaboratively to determine which music therapy strategies would be used. The teacher's aides were willing to take risks to use music spontaneously, in everyday situations in the school context. They enjoyed sharing musical instruments with John and this helped them to develop positive relationships with him. They were excited about the ongoing work. Furthermore, the team invested in further resources (musical instruments for home and school) for the ongoing music programme. John's dad thought the consultation was like a "breath of fresh air". He requested that music therapy strategies be included in his IEP, and the team agreed they would consider requesting a further music therapy consultation in future. 


\section{APPENDIX 39: Reflections and Learning Outcomes from John's Case}

John was trying to manage in an environment that was not always conducive to his learning. It seemed that his behaviour was having a significant impact on staff members' ability to cope, and they in turn were responding in ways that at times exacerbated his difficult behaviour. I needed to consider carefully whether my focus of support should be on the staff or the student. It seemed important that I had enough time to build positive relationships with team members so they could look more objectively at the work they do and need to do with him. They did seem to develop a more cohesive approach to their work with him.

I felt really challenged to do and say the right thing. I felt as if I was in a moderator role at times, especially when team members talked to me about their co-workers. I reminded myself it was important to respond to their need to be heard, to acknowledge the difficult situation that were all in, to highlight positive observations and to keep the focus on the work with John. Team members, particularly John's dad shared a significant amount of personal information and it was important to respond in a way that was supportive but to keep boundaries around the role i.e. to remember I was there to support John and his team (dad of course is part of the team), not to provide therapy for dad or other team members. Once again I wondered how I would have managed this with less experience. I also knew that my experience as a parent of a special needs child allowed me to have genuine empathy and helped me to respond appropriately.

John's team expected that my role would be to help revise an existing programme of music for a group of children who have special needs. I tried to include that in the programme, but the assessment indicated that there were other areas where music could be used that were likely to be more directly helpful for John. The team's initial thoughts and ideas for music therapy strategies changed after the individual music session identified possibilities for using the xylophone to address several developmental and academic goals. It seemed important to use this strength to help team members to develop a positive view of John and to facilitate inclusion.

John liked to take control in the music session, just as he did in other settings. Nevertheless he was able to demonstrate more of his strengths and his team were impressed with his responses, seemed to become more respectful of him, and increased their expectations of his potential. On the other hand the environmental assessment suggested that 
providing strategies to help staff to work a) in a more relaxed way with him through spontaneous musical games and songs and b) in a more consistent way with him through the use of adapted social story songs, would be beneficial for everyone. I had to limit suggestions to ensure realistic and effective intervention.

Although they enjoyed music, John's teacher's aides began the process believing that their singing might not be attractive and one was quite judgmental about what constituted 'good' or 'real' music. I left the field wondering if they would be comfortable continuing the work. However although they initially felt embarrassed singing in front of other children/teachers they have become enthusiastic about it, especially after observing John's positive responses to their music.

The sudden illness of a key liaison person meant communication difficulties occurred within John's team. Most significantly the teacher wasn't expecting me. I need to be particularly sensitive regarding the potential for my visit to be intrusive. Further, there was a specialist music teacher in the school and I didn't know about this until I arrived. I was reminded that before future consultations I would need to ensure that information regarding my visit has been received by all interested parties, and also to find a way to ensure that I have all the important information I need.

John was not being included in classroom or specialist music programmes. How can classroom or specialist music teachers be empowered to include students who have special needs in their programmes? I could have had a discussion with the team regarding the principles of inclusion, yet it did not seem appropriate to confront them directly on that issue. Instead, the team decided to be consistent about when and where he wore his Red Tee Shirt, and the music therapy strategies supported that. While this might have signaled him as being 'different' at least John was aware of what was expected of him and managed the task more easily. In doing so he reduced his emotional outbursts which in turn would make him more acceptable to his peers and aid his social inclusion. How could I facilitate John's inclusion when the team want me to work with musically adapted social stories? I was still working within my code of ethics. But I was challenged to consider how I accommodate the school's culture and still feel as if I was maintaining my personal and professional integrity with regard to how I would manage John's 'inclusion'.

Two classroom teachers were unable to manage having John in class and appeared to designate programme development and management to the teacher's aides. I know I haven't been able to see the 'whole picture' in just one week (especially since the designated Special 
Needs Teacher was absent at the time of the consultation) but it seems that the teacher's aides are shouldering too much responsibility... and it was really hard to increased the teachers' involvement in the work. The teacher's aides appeared to be working independently this week but I don't know if this is indicative of usual practice.

It was helpful having John's speech and language therapist support the introduction of adapted social story songs. 


\section{APPENDIX 40: Interview with John}

We were in the staffroom. I put the xylophone and bongo drums on the floor and I was sitting beside Jay with the instruments in front of us. I told Jay several times that I would like to talk about music and have shown him the IPOD. We have watched the counter go round on the IPOD and Jay has had a turn at pushing the buttons. Although I have informed him several times that the IPOD will be listening to and recording us, I don't believe he understood that concept. His parents have given consent on his behalf for the recording to be undertaken and used for research purposes. We were together for almost one hour and I have approximately twenty five minutes of audio recording. During both of these time-frames we were playing the instruments and the teacher's aides were observing how they might interact musically with Jay in future. I occasionally talked them through something and/or encouraged them to work with him independently. I reminded Jay regularly that the IPOD was listening to him and that I would like him to talk with me about music. From the twenty-five minutes of audio recording I have selected two minutes and twenty-one seconds which could be transcribed as 'interview' data.

DR: (Looking at the IPOD counter with Jay) There we go - now it's counting... 22, 23... We played the bongo drums didn't we?

$\mathrm{J}$ : And I played - and we did some marching!

DR: We did do marching. What did you like best - marching or bongo drums?

J: Look it's going to 38 !

DR: It is! Thirty-nine and 40! I like bongo drums.

J: Sometimes.

DR: Sometimes you like bongo drums?

$\mathrm{J}$ : I'll push again.

DR: No. Not yet. No.

J: Have a look first.

DR: Yes. We'll watch it for a wee while and we'll talk about music. We did music with the xylophone didn't we?

J: No I like it!

DR: Yes you did like it!

$\mathrm{J}$ : Please can we have it again?

DR: I think we can have it again after. Yes.

J: Please! Please!

DR: Yeah. Nice asking. What else did you like? Did you like the piano?

J: No. You had the piano.

DR: I did have the piano. What about the drum? Did you play the drum?

Jay sneezes. 
DR: Oh! Bless you.

Jay sneezes twice more.

DR: Ooh!

$\mathrm{J}$ : Listen to me (names both teacher's aides)

DR: Bless you, yeah! Did you play the drums? (Pause) Did you play the drums?

J: Oh wait. I'll push the button.

DR: Oh go on then - stop it for a minute.

Recording is stopped and restarted.

DR: Ok! Did we play the drums?

J: Yip!

DR: Did you like the drums?

J: (strikes the drum once) I'll...I'll play it on here. I'll play you ok?

DR: Ok.

Jay turns the recording off again and it is restarted soon after.

$\mathrm{J}$ : and it's going to seven and eight, eh?

DR: When the numbers are going round I'm going to talk about music.

$\mathrm{J}$ : You have it now.

DR: I like playing the piano.

$\mathrm{J}$ : And I play the xylophone.

DR: You did! And you -

$\mathrm{J}$ : And see if we can do it again one more time.

DR: Yes we'd like to do it again. What about music with (names teacher's aide)?

$\mathrm{J}$ : She was great.

DR: She WAS great wasn't she?

$\mathrm{J}$ : She was doing some marching.

DR: Yeah, yeah.

J: (To teacher's aide). You tried to do some marching didn't you?

W: Yes I did, yeah.

DR: And this morning -

J: And I hope you did though. And you did some marching didn't you? And I'll play the drums. We did eh?

DR: (Laughs) and we sang “I'm a Little Hunk of Tin..." didn't we? We did music with the group.

I try to engage Jay in discussion about group music but he does not respond. I tell him that we are finished talking about music and encourage him to turn the recorder off and he does so. 
APPENDIX 41: First five pages of participant diary (Tim's case)

JOURNAL OF ACTIVITIY - DATA FROM TOM'S TEAM TAKEN FROM HOME/SCHOOL BOOK PLUS MUSIC JOURNAL

12/02/2007 Morning

12/02/2007 Afternoon

13/02/2007 From home

13/02/2007 Morning
Brilliant morning. Tim was really well behaved. He really worked hard, 20mins max. 20 mins computer - he concentrated really well. We did a lot of work with Daphne. We also worked in the hall with the ladder, beam etc. Tim tracked and stepped really well. Lots of great work. he worked really well to my instructions. Wonderful work. Tim ate a good morning tea. he also had a lot to drink out of his cup. I fed him his morning tea and lunch in the library when he settled well without being distracted from the other kids. Changed him at $11.00 \mathrm{pm}$. The manual kids left the gate open which Tim spotted really quickly. Please mention to other teachers, duty teacher etc.

It was a lovely day with Tim. Daphne was here and together we had a lot of fun. I learnt a lot of interesting new methods from her and Tim responded to her very well. We did a lot of singing. Especially on the toilet the songs are very useful and I think it will help a lot to develop his daily routine further. Apart from that we looked at several books and played with the rolling board. He concentrated very well and focused on things. Reached to me more than I have seen him doing before. Enjoyed it lots.

Everyone is really enjoying having Daphne here making suggestions. Tim was very hard to wake this morning and seems quite tired. Hope he has a good day. Thanks for the great work ladies!

Tim has been happy but seemed tired and not settled for long for things today. He sat well on the mat and he LOVED PE today. That chair is great inspiration for toileting. Very clever Tim is helping me make his milo today. Using his left hand seems to be the trick to get him to carry his lunch box. Starting a wee bit earlier too. Loved 'Max' but not keen to read. Awesome work in the 
hall with Angela and Daphne.

13/2/07 Afternoon

14/02/2007 From home

Sally and Angela showed me the swimming lessens today. Enjoyed it lots with Tim. He seemed to have a lot of fun. At the end a little wave hit him and Tim got a wee fright. I held him for a moment but he didn't need long to get used to floating in the water by himself again. When he rode his bike back today he was really quick and he seemed to be quite proud to be peddling his bike by himself. When I tried to help him put his bike away at the end he pushed my hand away and had a wee ride at the school ground.

Many thanks for making Tim's transition back to school so successful. Thank you too Sally for helping Siobhan with Tim's routine for swimming. Can be a bit of a mission when you are unsure of the routine. I have bought you all a folder each to put info about Tim or anything you may want to add about Special Needs. Have put an article about Autism from Time magazine which is excellent! So any timetables/programmes/ IEPs/sensory notes, etc... Thought it might be easier for all of you. Have been having a short discussion with Sally about objectives for Tim this Term/Year. We talked about independence being a huge focus. Sally mentioned taking his school bag out to lunch, making Milo, pulling up pants. Will talk about other independence activities and what motivation may encourage Tim. If either of you can think of small, manageable independent activities we could look at, at perhaps a meeting with Angela next week, that would be great. Just jot down any ideas in the back of this book, etc. Have a great day. 
14/02/2007 Morning

15/02/2007 From home

15/2/07 Morning
Tim was great when he saw you and Patrick in the staffroom, but we had a big cup of juice in the library so we didn't disturb you. Tim enjoyed 'Max' and also some reading this morning. He LOVED PE on the field and threw the ball for shot-put really well. As usual his running was variable. Big mistake with Patrick at morning tea - Tim was very sad when he couldn't go with Patrick but cheered up when we swam, did some great work and Fiona introduced him to the float board and he had a big play with this. Big real also on return to class and we set up computer position for Tim to see screen properly - will talk to Mary about this. He seemed quite tired but awesome listening today and made some great choices.

Yes, wasn't Tim great. He just waited for you Sally, at the staffroom door - though we said he could come in... just wonderful! Thank you for setting up the computer. He will love that! Neat that Tim was keen to throw - have to get practicing his running song for the athletics and maybe a new toy to motivate. Great that he used the kickboard. Will sneak down next week to have a look. Did bring Tim's togs but I know he is getting videoed today - not sure how long that will take. Great work by Tim on his 'read all about me' sheet. No hesitation at the start to colour on the paper. Small pencils too which was hard. That's all your hard work Sally - was keen to draw and knew where to draw as well.

Tim became unsettled while on the mat. Very keen to be out - can't blame him - toilet then music session with Daphne which went really well. Became unsettled after 20mins. We went for a milo which he wasn't interest in making and tried more music but he wasn't keen at all. Not keen on returning to class, but when we did I told him we could read but he refused ANY involvement from me. Sat and happily read on own. Not keen for any MT so we went to supper room where he happily ate with some songs. tom because very unsettled and had a few 
'nos' for hitting computer and trying to bite etc. We prevailed and on return sat happily for 10 mins. Had another read and showed Daphne how much he loves 'Love to sing' video. Much happier at lunchtime. Lots of toilets. Seems tired.

Didn't go swimming today. Tim tested a lot. Drove to the pool and back to change his trousers. Needed a while till he stood up from the toilet floor. I tempted him with the turning chair. After a while he got up and listed very well. Changed his trousers. He sat on the toilet for a while and laughed. Another sign he was 'testing' before. He put his trousers on really well - I sang to him and he lifted them up himself almost all the way. At the end I told him we are finished and he was doing well going to the toilet - that is when he walked to the chair. I turned him a bit - when we stopped he showed me that he wanted bubbles. He ran into the kitchen and grabbed them so we blew a a few bubbles.

Many thanks everyone for taking the time to view the video etc. Isn't it great for once to be able to stand back and just watch Tim (without worrying about safety) just really focusing on how he is responding/communicating! When Erin comes next Thursday can we all remind each other if she can check Tim's posture when sitting at his 16/2/07 From home desk. Will check today how high the stool needs to be for his feet and what he needs on the chair! A huge thank you Sally, Siobhan, Vicki, for working so hard with Daphne being here. You were all so welcoming to her and it is an added pressure at times to have someone there, watching the whole time. If it wasn't for your cooperation her visit would not have been the success that it has been - so well done and many, many thanks. 
Can you remind me about asking Mary for reading homework for Tim - I'm not sure if the other children have received any or not. Have a great day!

\section{$16 / 02 / 2006$}

Had lunch. Tim was sitting nicely and eating. After food he spent the rest of his

12.30pm lunchtime testing out whether he could get back into the school (classroom) or not.

1.30pm We went to the toilet

$1.45 \mathrm{pm}$

2.00pm

Went to the music room. Tim had a spin.

Tim was sitting on the yellow line. Daphne started to walk up to the yellow line and stop. She sang a song

Spinning the chair with a song. Tim showed very early what he wanted
Sometimes I felt a bit helpless because it was very physical to try to keep him out of the classroom. I will try to figure out if he wants something special or if he is testing me.

Worked well. We are getting more structure going in the toilet routine. Songs and short requests seem to work well. I want to start to make a proper finish to the toilet routine before he can get to the spinning chair.

I might step back a bit next time so he needs to show me even more what he wants to do next

It felt nice to get Tim's attention. Unfortunately I couldn't make him sit on the chair. He did get up from the turning chair very well. I needed to give him my hand because the chair is a bit high. Daphne came. Tim was trying to get passed me all the time and we let him run into the playground where he indicated that he wanted to go home by crossing the yellow line (to the gate).

I can see that this is one of the best learning opportunities for Tim. The learning situation is chosen in the context. I will try to do more of those learning games then try to bring him back to my idea of the day (i.e. the 
while she did that ("I can task at hand).

walk and walk and stop!")

To help Tim to assembly, I feel like the 'Standing Up' song and the help is working. Daphne etched her guitar. I just need to start to figure out what is most appropriate

2.20pm Tim needed a bit of support (at any given time), when I need to be patient and when to stand up but walked I need to be more firm, and when he is communicating without a problem then. little things.

Assembly: Tim watched

from outside (the door). It I feel like he sometimes enjoys going where the other took him a while to settle children and he liked watching them. I think I will try to

2.30pm down. When the children let him be a part of the assembly more often. When started singing he turned Daphne sang her goodbye song it took him a while but around to tome, moved with then he seemed to understand it and we shook hands.

it and laughed.

19/2/07 From home

19/2/07 Morning
This week Angela is going to put together a daily programme including a music session in for us all to look at and follow, when possible. Though it will be flexible depending on Tim of course! Had a great week with Daphne and she is sending a copy of the video she took of the therapy session with Tim for all of us to look at, so Vicki, you are most welcome to view this when it comes. Also Vicki, you'll notice I've put a copy for you of Daphne's report for you to keep. Have got Sandra (OT) to organise a box to place under Tim's feel and will get a pillow as well, so Tim has better posture and stability when working at his desk. Have brought along Tim's weighted vest as well.

Tim had a 'no' day. We got there eventually but we had to persevere. Tim threw a book on the ground and I made him pick it up and put it back. It took ten minutes but was worth it. Lots of smiles, loved having me on. Tim had a great swim. He floated on his back and tummy really well and independently. 
19/2/07 Afternoon

\section{$1.00 \mathrm{pm}$}

$1.15 \mathrm{pm}$

19/02/2007
Tim was quite sure about what he didn't want to do today but he was in a good mood and very switched on. He grabbed the little tractor after we had looked at tractors in his book and when I asked him to show me a car he took his toy car. Even when I asked him for wheels he seemed to search in his box. I just stared to use 'yes' and 'no' cards. He chooses the card if he wants something, but he hasn't figured out yet that 'yes' is green and 'red' no. Made him a bit upset. We did the cards when we watch 'Max' and at the end he seemed to grab the green card directly (I held them very close) but I said to him "take the green card!" - so that will need a bit of work.

Valerie told me Tim has been a bit naughty today and he doesn't want to do whatever you ask him to. When I started Tim was about to climb the steps at the back of the hall. He didn't want to come off, but it wasn't a big fight. He didn't resist me guiding him down but he wouldn't step down himself. He laughed and took my hand and brought me to the the kickboard. He opened the door, dragged everything out and sat on it. Ran around outside and didn't try for the door most of the time. Tried it once. I sang the walking song as we walked 0 the door and ended with 'stop because it's lunchtime'.
I feel that Tim is a bit naughtier now. When I mirrored his anger back to him today he seemed as if he enjoyed getting even louder and angrier but then he seem to quickly regulate himself too.

The song worked well. I did hold his shoulder when I said stop, but he didn't resist. He wasn't upset as he usually would be when I don't let him go somewhere. I guess he wants to get the security about where he can go and when. I want to try to have a lot less physical contact though. 
Sat in classroom. Tim took many of his toys and books out. Looked at the tractors.

Got a yellow play tractor from his toys and said "well I think picking up the tractor was not random. I was quite done this is a tractor like in impressed with that and with finding a car as well. I feel

1.30pm the book!" He laughed and I a bit lost in the classroom though because I didn't know asked can you find me a what to help him with. I think the company with the other car. He LOOKED AROUND children is most important. Specially mat time. and picked up a red car. I asked him for wheels and he seemed to search. He looked in his empty play box 
APPENDIX 42: Findings, Themes from All Four Case Studies

\section{How was the consultation process perceived and understood?}

- to be different to what they anticipated. Some team members had little initial understanding of the possibilities for music to support a student's developmental or academic programme, and their initial perception of what the consultation could involve did not match the eventual process;

- as empowering. They were empowered to use music regardless of their previous musical experience or training;

- provided new perspectives. They discovered different ways of thinking about the student, gained new insights to their own strengths, and found new ways to approach their work e.g. "it changed the way we teach";

- instilled hope and optimism. Students' strengths were identified and progress was anticipated. Expectations were therefore raised;

- energised and enthused. They were excited and motivated to get on with the work. A parent described it as "a breath of fresh air";

- as affirming. The process provided much needed confirmation that their hunches were right, especially with regard to student response to music and developmental potential, and affirmation that the work they were already doing was valuable and could be built on;

- to be somewhat generalisable. In several cases planned music experiences were seen to have benefits for other students;

- to be unobtrusive and non-threatening. The music therapist's presence was perceived to have a positive impact on the environmental milieu;

- increased their self awareness. Team members gained a heightened awareness of what they were already doing, and how their interventions, or lack of intervention, impacted on their students;

- instilled confidence. Team members came to believe they could successfully introduce planned music experiences, and were committed to continuing the work;

- to be successful. Team members believed planned music experiences contributed appreciably to students' progress towards, or achievement of, developmental or 
academic goals, particularly in the areas of communication, and/or emotional regulation. Further, specialists from outside the core school teams, e.g. Speech Language Therapists and the Neurodevelopmental Therapist, were also convinced that planned music experiences would support communication programmes.

\section{How was the consultation process used?}

- Practical support was offered as needed prior to visits. For example it was important to send a photograph of the music therapist to students who had autism, so they could anticipate the visit and know a little more about what to expect.

- Teams drew on a range of existing physical and personal resources to do the work.

- Team members drew on their own confidence and enthusiasm, as well as the encouragement of the music therapy consultant, in order to take risks and try new ways of working. The music therapist instilled musical confidence in team members.

- The music therapist's advice was considered alongside local knowledge and skill. Teams found their own unique way of working as a team, and as individuals within the team. Team members took different, but equally successful, approaches to the work. Their initial levels of confidence and previous experience of using music had an impact on the way in which they initially approached the work.

- The environmental assessment was central to the work, and the individual music therapy assessment session was less important than was originally anticipated. The consultant observed first-hand students' strengths and needs in context, and began to interact with them and their teams as they went about their daily routines.

- The music therapist was able to facilitate a music therapy assessment session with each of the students, and to communicate musically with them using improvisatory techniques which are precisely delivered, and learnt from years of training and experience. The encounter was different to the way they were able to experience music in other settings.

- The music therapist consultant allowed time, and maintained a level of flexibility, to enable positive relationships to develop with team members. 
- Teachers mostly had plenty of advance warning in order to plan for the consultation visit, apart from one case where the absence of a key team member resulted in a communication breakdown.

- Each visit was carefully planned and structured to ensure best use was made of the time available. The music therapy session and team meetings were scheduled mid-week, to enable team members to make decisions regarding the activities and strategies they intended to implement, to prepare resources, and to trial the strategies. In one case, a plan for the music therapist to collaborate with SLT during the follow-up period did not eventuate.

- Team members felt the time allowed for the consultation enabled them to absorb information, read, observe their students, and try out ideas.

- The process was highly collaborative, with the music therapist consultant working alongside colleagues as their equal, rather than as a 'specialist' or 'expert'. The music therapist consultant encouraged all team members to contribute at levels they were comfortable with, at all times.

- Team members gave each other space. The music therapist took time away from the classroom to make resources, write reports etc. One teacher also took the opportunity to do other things while her students were involved in music sessions with the therapist.

- Team members worked together in an attempt to understand and interpret their student's responses.

- Members warmed quickly to the team meetings during which they viewed video of the individual session, and developed in-depth conversation about a variety of issues that affected their students, from general developmental progress to specific strategies that might be considered, and from political issues to their own team dynamics. Generally observations were diverse, but each team noted their student's positive interaction with the music therapist, and viewed their engagement with music as one of their strengths. In all cases, viewing video material as a team, and the subsequent discussion, resulted in teams asking the music therapist consultant direct questions and requesting direct advice about the use of music therapy techniques, as well as other developmental issues. 
- Team members asked the music therapist's advice about a wide range of education and developmental issues, and being able to respond increased their trust in her.

- The music therapist drew on the knowledge and experience of colleagues in order integrate musically adapted social stories; use of alternative communication systems and so on, in music programmes.

- Teams chose the planned music experiences they would introduce. This ensured they maintained a sense of ownership of the work, and their interventions were realistic and effective.

- The environmental and clinical assessment processes uncovered unexpected opportunities for music to support student growth and learning.

- Music strategies were used to support existing programmes i.e. SCERTS, Floortime, Social Stories, and 'Routines' associated with Orientation and Mobility, and addressed current teaching targets.

- Team members variously learnt to use songs to motivate and support action, to teach routines, and as a communicative medium in spontaneous play. During planned sessions with instruments they learnt to improvise and to maintain playful interaction, to develop musical turn-taking with the student, and to use predictable and familiar music (sometimes recorded) to enable students to anticipate and prepare a response. Across teams, members noted that the consultation helped them to recognise the importance of observing their students more closely, identifying and acknowledging their communication attempts, of taking their lead from him or her, and allowing time for a response. Seeing what their students were capable of, or noticing their joy in music making, reinforced what they were already doing, and motivated team members to continue with the work.

- Work was undertaken in inclusive settings with peers as much as possible.

- While music was used to reinforce the IEP goals that were already in place, articulation of how students might progress towards these goals was generally loose.

- In most cases, team members began to gently but firmly increase their demands and expectations of what the student could manage on a daily basis.

- Several team members, and one of the students, began to develop and use their own planned music experiences following the consultation. Two classroom 
teachers used their experience of being involved in the music therapy consultation to reconsider their use of music in the classroom to accommodate the participant student. One classroom teacher had begun to empower teacher's aides to use planned music experiences in their work.

- During the follow-up period of the consultation, three of the students developed various cognitive skills essential to communication including attending, making requests, imitating, and sequencing. The fourth student, who was verbal, began to make up his own songs and to sing himself through tasks. One began seeking the company of other children, and another developed peer relationships through music which were generalised to the playground environment. All four students were reportedly less anxious or more relaxed, and three were more able to regulate emotions, comply with requests, and manage routines. In two cases, toileting skills improved. It is possible that this improvement was related to students' decreasing anxiety. One student was increasingly alert when music was used as a stimulus, and had begun to demonstrate a new quality of listening. She was vocalising more, and her purposeful movements increased. Another's tolerance for auditory stimulation appeared to improve, and his team believed that the gentle increased exposure to a wide variety of sounds and music during and following the music therapy consultation, contributed to this. This student also learnt to read music from simple score while playing the xylophone.

- Tasks for some team members (as well as the student's tasks) were broken down into small steps which were recorded in the reporting package, extensive resources were made to support the work, and some were adapted or added to in the follow-up period. Other team members were comfortable working in more flexible ways.

- In the beginning the amount of information provided for teams had the potential to be overwhelming, and was therefore reduced accordingly.

- Team members were aware that despite their positive response to music, students with very high levels of need are likely to take small steps forward, and progress is expected to be slow.

- Team members learnt to be more discerning about the level of support students required in order to participate in music activities. 
- The music therapist consultant worked within the culture of the school, yet challenged team members to introduce new ideas when they seemed important.

- A relationship based on trust and respect enabled team members to accept constructive criticism gracefully.

- Teachers' aides had a dominant role in working alongside the consultant music therapist.

- The process enabled teams to examine their own dynamic functioning.

- Team members used the music therapist consultant as a 'sounding board', sharing feelings of isolation and frustration due to the lack of support they perceived they received.

- The primary focus of consultation is on the adults on the team, and the level of direct and indirect work with student needed to be carefully balanced.

- The music therapist consultant occasionally took a mediator role to avoid team members 'splitting', and to gain their support and commitment to the work.

- Some family and other team members disclosed personal information and treated the music therapist consultant as a confidant. The consultant was careful to listen sensitively, but to ensure the emphasis was on sharing information that related to consultee and/or student wellbeing in the school context.

- The collaborative nature of the music therapy consultation enabled team members to draw on each other for ongoing support. Team members who sought advice during the follow-up period acted upon the advice given.

- All teams indicated that they would continue to use music with students in an ongoing way, and would include music strategies on their student's IEPs as a tool for learning.

- Team members demonstrated commitment by purchasing new equipment or resources for their ongoing work.

\section{How was the consultation process valued?}

Team members valued: 
- the time allowed for the consultation, especially since it enabled the music therapist consultant to observe and work with the student and his/her team when they were having a more difficult time;

- having the music therapist on site so they could directly observe strategies being modelled, be supported in their attempts to facilitate, and could ask questions or make suggestions about the work;

- the holistic nature of music therapy which encourages physical, emotional, social and cognitive development in students;

- programmes that were realistic, meaningful, and purposeful, and fit with each individual team member's way of working;

- being able to choose from a range of music experiences to promote stimulation or relaxation, active participation or shared listening, and so on;

- strategies that helped to address current teaching targets;

- knowing the reasons behind the introduction of the strategies;

- clarity regarding the way in which the strategy was to be introduced;

- strategies that built on what team members were already doing;

- strategies that were uncomplicated and therefore easy to manage;

- fresh ideas that added interest, motivation, and vitality for themselves and their students, and activities or programmes that contained intrinsic goals;

- students being able to manage at least some of their tasks more easily when supported by music;

- being given 'permission' to take time to build a therapeutic relationship with their student;

- gaining new and positive perspectives of their student/s;

- the opportunity to see what their students are capable of, and their joy in music making;

- knowing that raising expectations and challenging students to achieve their potential can enhance their relationship with that student;

- being challenged to look at themselves, as well as their student;

- having new ways to communicate with students;

- experiencing "real hope for the future"; 
- having a school term of follow-up, and receiving feedback on their work during this period;

- the music therapist's 'musical way of being' (including musical skills, empathy, caring, careful listening and so on). 


\section{Indicators of Efficiency}

- A collaborative approach was able to be maintained. The music therapist consultant was able to work alongside colleagues as an equal, rather than as a 'specialist' or 'expert', and participants were therefore empowered and eager to attempt new tasks.

- The music therapist was on site and for a length of time conducive to effective learning. Team members could directly observe strategies being modelled, were supported in their attempts to facilitate, and could ask questions or make suggestions about the work.

- Activities were deemed to be realistic, meaningful, and purposeful, and built on what team members were already doing. The activities and strategies were uncomplicated and therefore easy to manage, and were valued for their 'practicality' and 'accessibility'. The reasons for their introduction and the way they would be introduced were clear.

- Teams believed planned music experiences benefited their students with SN, and peers. Team members documented progress towards academic or developmental goals, and the music strategies were believed to have contributed to students' progress towards, or achievement of those goals particularly in the areas of communication, or emotional regulation.

- Team members developed new perspectives of their students and of their work with the student. Teams consistently reported that they had gained new and positive perspectives of their students from the music therapy consultation, and this positively affected their work in fundamental ways. In recognising their strengths, team members seemed to become more respectful of their students, to have a more positive view of them, and higher expectations of what they might be able to manage.

- Student and staff responses to music significantly motivated the sustained use of planned music experiences. Students were observed to manage at least some of their tasks more easily when music strategies were applied and a range of positive student outcomes were described. But staff found that music energised their work too. Moreover, being given 'permission' to take time to build a therapeutic 
relationship, and gaining new and positive perspectives of their student/s were highly valued outcomes. Team members appreciated being challenged to look at themselves, as well as their student, and in some cases learnt that raising expectations and challenging students to achieve their potential could enhance their relationship with that student.

- Teams were committed to continuing the work. All teams indicated that they would continue to use music with students in an ongoing way. The music therapy consultation process gave several team members 'real hope for the future'.

\section{Learning Outcomes}

The research also highlighted:

- the importance of drawing on teams' existing physical and personal resources. Teams had space to work in, musical instruments they could use, and considerable personal resources to draw on, and these all had an impact on the success of the consultation;

- the value of time, and its careful management. Having the time to build relationships with team members and students and coming to know them in the context of their special education communities, in good and bad times, was crucial to the success of the work. The time was carefully managed to ensure that observations, data gathering, and planning meetings were balanced with opportunities for modelling and trialling, and empowering team members to facilitate strategies. Follow-up was also an important aspect of the music therapy consultation protocol;

- the need to provide adequate support for teams. Teams expressed frustration with existing support systems and it was important to acknowledge their distress and to consider the implications of their concerns for the music therapy consultation. Specifically teams needed to be given realistic expectations of what could be achieved through the consultation and to be left feeling that they had learnt something useful and sustainable, so that they did not experience feelings of abandonment once it was over;

- the importance of making time for team meetings. Team members used the multidisciplinary team meetings to focus on videotape of music therapy work and developed in-depth conversations about a variety of issues that affected their students, 
from general developmental progress to specific strategies that might be considered, and from political issues to their own team dynamics;

- issues in building therapeutic relationships. Building positive relationships with team members was essential but not unproblematic due to friction within teams and between teams and outside agencies; families choosing to disclose personal information which highlighted professional boundary issues; and the variety of personalities and workings styles that were encountered among the team members;

- the desirability of ongoing music therapy for many students. The clinical music therapy sessions gave each of the students a new communicative experience which had the potential to be developed further in ongoing therapy;

- the need to recognise and work with differing approaches in therapy and education, including the dilemma of inclusion. In this work the music therapist encountered team members who had differing philosophical beliefs, or who practiced in ways that differed from the approach the music therapist consultant might take in the same situation;

- issues with regard to ethnicity. Cultural differences may have affected Māori participation, and it is possible that more emphasis on oral rather than written communication would have facilitated this;

- the need to be clear about the responsibilities of each team member. Care needs to be taken when delegating responsibilities to teachers' aides;

- that collaborative work generates innovative and unique strategies that are responsive to individual needs. A wide range of activities and strategies were employed across cases, and were chosen to fit within existing approaches or programmes of work and to address current teaching targets. Building music strategies into, or making links with existing programmes i.e. the SCERTS, Floortime, Social Stories, and 'Routines' associated with Orientation and Mobility, and fitting them with each team member's individual way of working contributed to the success of the consultations;

- that music therapists may need to consider ways in which they can practically support students prior to consultation visits.

- that care needs to be taken to ensure information regarding the impending music therapy consultation is received by all interested parties. The sudden illness of a key liaison person led to a communication breakdown which had the potential to be 
catastrophic. The music therapist needed to be particularly sensitive regarding her potential to be intrusive in classroom or other settings, especially when she had not been expected. Moreover, on one occasion there was a specialist music teacher in the school and it would have been helpful to know about this so he/she could have been more involved in the work. Further, it is likely that contact will need to be made with professional colleagues who work outside the school (e.g. MoE SE staff), prior to the visit;

- that technical equipment cannot always be relied upon. Having the opportunity to review the videotape of students' individual music therapy session was highly valued by teams, but the music therapist needed to be prepared to relay, explain and lead a discussion based on the events of the individual music session without the aid of audiovisuals;

- that consultation work is difficult and should be undertaken by experienced music therapists. 